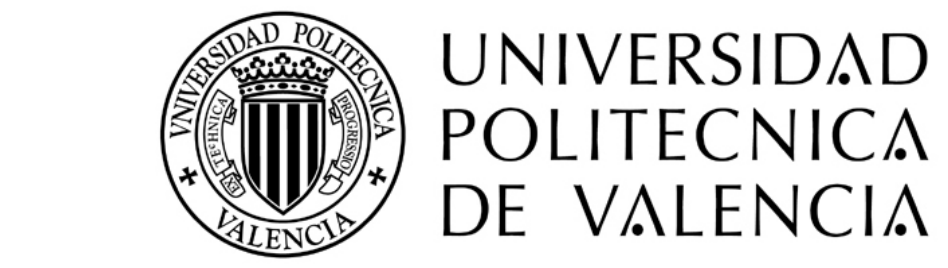

UNIVERSIDAD POLITÉCNICA DE VALENCIA

Departamento de Comunicaciones

\title{
ANÁLISIS Y DISEÑO DE DISPOSITIVOS PASIVOS DE MICROONDAS Y MILIMÉTRICAS EN GUÍA DE ONDAS CON EXCITACIÓN COAXIAL
}

\author{
Ángel Antonio San Blas Oltra \\ Directores: Dr. Vicente E. Boria Esbert \\ Dr. Benito Gimeno Martínez
}

Tesis Doctoral presentada en la Universidad Politécnica de Valencia para la obtención del título de Doctor Ingeniero de Telecomunicación

Valencia, Mayo 2008 

A Celia y a mis padres. 



\section{Agradecimientos}

Todavía recuerdo la tarde en que conocí a Vicente y a Benito. Era un día de otoño que me pasé por el despacho de Vicente en busca de algún proyecto fin de carrera que me despertara cierto interés. De la charla con mis actuales directores de tesis me llamó mucho la atención el hecho de que Benito me preguntara, nada más sentarme, si me gustaban las matemáticas. En ese momento no podía ni imaginar la enorme trascendencia de su pregunta. Desde ese día ya han transcurrido algunos años y, fruto de todo el trabajo realizado, ha surgido esta tesis doctoral. Y es precisamente hoy, escribiendo estas líneas, cuando empiezo a entender la pregunta que me hizo Beni.

Vicente y Beni me han ayudado en todo, tanto desde un punto de vista profesional como desde un punto de vista personal, para que este momento sea una realidad. Sin ninguna duda, sin su amistad y constante apoyo no estaría donde estoy. De ellos me gustaría destacar su enorme entrega en todo lo que hacen, su infinita habilidad para resolver problemas, y su gran capacidad de trabajo. A día de hoy, ya no son solamente mis directores de tesis; son también unos grandes amigos. Gracias a ellos he aprendido todo lo que sé. Muchas gracias a los dos por todo: sois los mejores directores de tesis que hubiera podido tener.

Mis padres siempre me lo han dado todo. En todos estos años, hemos tenido momentos de dificultades pero también momentos inolvidables. Vosotros me habéis enseñado que, con esfuerzo y con tesón, se puede conseguir todo en esta vida. Los dos sois unos auténticos luchadores y, para mí, siempre seréis un ejemplo a seguir. Sin vosotros, este trabajo nunca hubiera salido adelante. Muchas gracias por todo. Os quiero muchísimo.

Celia, mi mujer, mi amor, eres el pilar más importante de mi vida. A pesar de ser más joven que yo, siempre has sido mucho más madura que yo en todos los aspectos, y me has enseñado muchas cosas durante los más de 10 años que estamos juntos. Quería darte las gracias por haber creído en mis posibilidades, y por haberme apoyado en todas las decisiones que he tomado. Sin tu cariño diario, sin tu enorme sensibilidad e inocencia, sin tu infinita paciencia, nada de esto tendría sentido. Muchas gracias por estar siempre ahí y por aportarme esa estabilidad que tanto necesito. Te vull cada dia més, solet meu.

La realización de esta tesis tampoco hubiera sido posible sin la ayuda de otras muchas personas. En primer lugar, quisiera destacar a los profesores Marco Bressan y Giuseppe Conciauro de la Università degli Studi di Pavia, por su inestimable ayuda durante mi estancia en Italia. Igual de importante ha sido la ayuda y paciencia de mi compañero Fermín Mira, quien ha tenido que leer y contestar cientos de correos electrónicos. Me acuerdo también mucho de los compañeros de trabajo en Italia, en especial de Luca, Francesco, Marco y Davide. Asimismo, me gustaría mencionar a todos los compañeros y amigos que he ido dejando atrás en mi recorrido como becario por la Universidad de Valencia y la Universidad Politécnica de Valencia: Daniele (il Tutor), Pepe, Pere, Morro, Jordi, Máriam, Luis Javier, Santi, Héctor, Pablo, Ana y Stephan. Muchas gracias a todos. 
Por último, me gustaría también mencionar a mis actuales compañeros del Área de Teoría de la Señal de la Universidad Miguel Hernández de Elche: Adrián, Alberto, Andrés, Ángela, Carlos, Enrique, Germán, Javier, Javier, José Antonio, Juan, Pablo, Ricardo y Rubén. Gracias a todos.

Kiri y Pipo, gracias por todos los buenos momentos. Gracias también a Servi y a Panxi, y a toda la colla. Siempre os llevaré en el corazón.

Ángel A. San Blas Oltra 


\section{Resumen}

Las guías coaxiales se emplean frecuentemente en la alimentación e interconexión de un elevado número de dispositivos de microondas y ondas milimétricas utilizados en numerosas aplicaciones prácticas (filtros, diplexores, multiplexores, etc.). Por ello, la caracterización rigurosa y eficiente de la excitación coaxial de dispositivos implementados en tecnología guiada es un tema de investigación muy importante que ha sido tratado ampliamente en la literatura técnica. Sin embargo, el número de contribuciones que se dedican a investigar este tema desde un punto de vista multimodal es realmente reducido.

El objetivo fundamental de este trabajo consiste en la caracterización multimodal eficiente de la excitación coaxial de dispositivos implementados en guía rectangular utilizando el método BI-RME (Boundary Integral - Resonant Mode Expansion) 3D. En concreto, en este trabajo se caracterizan dos tipos de configuraciones ampliamente utilizadas en la práctica: la excitación coaxial clásica, y la excitación coaxial en configuración colineal. Este último tipo de excitación se emplea comúnmente en los denominados filtros en configuración comb-line, los cuales también son objeto de investigación en el presente trabajo. En particular, se ha desarrollado una herramienta de análisis y diseño muy eficiente desde un punto de vista computacional que permite caracterizar de forma precisa este tipo de filtros.

Otro de los objetivos del presente trabajo es el análisis y el diseño de uniones en guía rectangular compensadas con postes conductores de geometría cilíndrica utilizando la técnica BI-RME 3D. Las uniones diseñadas (codos rectos, uniones en $\mathrm{T}$, uniones $\mathrm{T}$ mágica y uniones turnstile) presentan una respuesta eléctrica óptima y, además, el trabajo desarrollado demuestra que la posición relativa del poste en la estructura es un parámetro de diseño muy importante que no se había considerado previamente en la literatura técnica dedicada a este tema. 



\section{Resum}

Les guies coaxials es fan servir freqüentment en l'alimentació i la interconnexió d'un gran nombre de dispositius de microones i ones mil.limètriques utilizats en diverses aplicacions pràctiques (filtres, diplexors, multiplexors, etc.). Per aquesta raó, la caracterització rigurosa i eficient de l'excitació coaxial de dispositius implementats en tecnologia guiada és un tema d'investigació molt important que ha sigut àmpliament tractat en la literatura tècnica. A pesar d'això, el nombre de contribucions dedicades a investigar aquest problema des d'un punt de vista multimodal es realment reduit.

L'objectiu fonamental d'aquest treball consisteix en la caracterització multimodal eficient de l'excitació coaxial de dispositius implementats en guia rectangular utilitzant el mètode BI-RME (Boundary Integral - Resonant Mode Expansion) 3D. En concret, en aquest treball es caracteritzen dos tipus de configuracions àmpliament utilitzades en la pràctica: l'excitació coaxial clàssica, i l'excitació coaxial en configuració colinial. Aquest últim tipus d'excitació s'empra comunament en els denominats filtres en configuració comb-line, els quals també són investigats en aquest treball. En particular, s'ha desenvolupat una ferramenta d'anàlisi i disseny molt eficient des d'un punt de vista computacional que permet caracteritzar de forma precisa aquest tipus de filtres.

Altre dels objectius d'aquest treball és l'anàlisi i el disseny d'unions en guia rectangular compensades amb pals conductors de geometria cilíndrica utilitzant la tècnica BI-RME 3D. Les unions dissenyades (colzes rectes, unions en $\mathrm{T}$, unions $\mathrm{T}$ màgica i unions turnstile) presenten una resposta elèctrica òptima i, a més, el treball desenvolupat demostra que la posició relativa del pal en l'estructura és un paràmetre de disseny molt important que no s'havia considerat prèviament en la literatura tècnica dedicada a aquest tema. 



\section{Abstract}

Coaxial waveguides are frequently used in order to feed and interconnect a wide variety of microwave and millimeter wave devices (filters, diplexers, multiplexers, etc.). As a consequence, the rigorous and efficient characterization of the coaxial excitacion of waveguide structures is a very important topic that has been widely studied in the technical literature. However, a few number of these contributions investigate this problem from a multimodal point of view.

The main objective of this work is the efficient multimodal characterization of the coaxial excitation of waveguide components using the 3D BI-RME (Boundary Integral - Resonant Mode Expansion) method. In particular, two types of configurations widely used in practical applications are discussed: the classical coaxial excitation, and the coaxial excitation based on an end-launcher configuration. The end-launcher configuration is commonly used to feed comb-line filters, which are also investigated in this work. Concretely, a very efficient CAD tool for the analysis and design of comb-line filters has been developed.

The analysis and design of compensated waveguide junctions using partial-height metallic posts is also a main objective of this investigation. Following the 3D BI-RME method, we present the design of a great variety of compensated waveguide junctions (right-angled bends, $\mathrm{T}$ junctions, magic $\mathrm{T}$ junctions, and turnstile junctions) with an optimum electrical performance. Moreover, we demonstrate that the relative position of the metallic post in the structure is a very important design parameter that has not been taken into account in previous works concerning compensated junctions using partial-height metallic posts. 



\section{Índice}

$\begin{array}{lll}\text { Resumen } & \text { VII }\end{array}$

Resum IX IX IX I

$\begin{array}{lll}\text { Abstract } & \text { XI }\end{array}$

1. Introducción 1

1.1. Motivación y Objetivos de la Tesis . . . . . . . . . . . . . . . . . 1

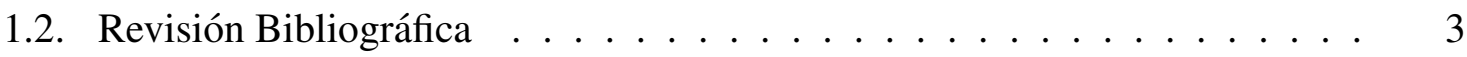

1.3. Estructura de la Tesis . . . . . . . . . . . . . . . . . 3

2. Contribuciones al Análisis Eficiente de Uniones Planares Complejas 7

2.1. Introducción . . . . . . . . . . . . . . . . 7

2.2. Análisis de Uniones Planares mediante la Técnica de la Ecuación Integral . 9

2.2.1. Aceleración del cálculo del núcleo de la ecuación integral . . . . . 14

2.2.2. Resolución de la ecuación integral planteada . . . . . . . . . . . . 17

2.3. Discontinuidades entre Guías Rectangulares y Guías Coaxiales . . . . . . . 20

2.3.1. Obtención de los modos de una guía coaxial utilizando el método de los momentos . . . . . . . . . . . . . . . . 21

2.3.2. Análisis de la unión planar entre una guía rectangular y una guía coaxial . . . . . . . . . . . . . . . . 34

2.3.3. Ejemplos de validación . . . . . . . . . . . . . . . . 39

2.4. Discontinuidades entre Guías Rectangulares y Guías Circulares . . . . . . . 42

2.4.1. Obtención de los modos de una guía circular utilizando el método de los momentos . . . . . . . . . . . . . . . . . 43

2.4.2. Análisis de la unión planar entre una guía rectangular y una guía circular ...................... 55

2.4.3. Ejemplos de validación . . . . . . . . . . . . . . . 58

2.5. Discontinuidades entre Guías Coaxiales . . . . . . . . . . . . . 60

2.5.1. Análisis de la unión planar entre dos guías coaxiales concéntricas . 61

2.5.2. Ejemplos de validación . . . . . . . . . . . . . . . . 65

2.6. Discontinuidades Múltiples entre Guías Rectangulares . . . . . . . . . . . 67

2.6.1. Análisis de una discontinuidad múltiple entre guías rectangulares . 69 
2.6.2. Discontinuidades múltiples en estructuras inductivas implementadas en guía rectangular . . . . . . . . . . . . . . . 71

2.6.3. Ejemplos de validación . . . . . . . . . . . . . . . . 72

3. Análisis y Diseño de Uniones Multi-Puerto en Guía Rectangular Compensadas con Postes Cilíndricos de Geometría Arbitraria

3.1. Introducción . . . . . . . . . . . . . . . . . . . . . . 75

3.2. Generalización del Método BI-RME 3D para el Análisis de Uniones Compensadas en Guía Rectangular . . . . . . . . . . . . . . . . . . 76

3.2.1. Revisión del método BI-RME 3D . . . . . . . . . . . . . . . 77

3.2.2. Análisis multimodal eficiente de uniones multi-puerto compensadas en guía rectangular . . . . . . . . . . . . . . . . 81

3.3. Ejemplos de Aplicación . . . . . . . . . . . . . . . . . . . . . . . . . . . 94

3.3.1. Codos rectos en plano $H$ y en plano $E \ldots \ldots$. . . . . . . . . 95

3.3.2. Uniones en $\mathrm{T}$ en plano $H$ y en plano $E \ldots \ldots$. . . . . . . . . 100

3.3.3. Uniones en T mágica . . . . . . . . . . . . . . . . . . 106

3.3.4. Uniones turnstile . . . . . . . . . . . . . . . . . . 108

4. Análisis Riguroso de la Excitación Coaxial de Dispositivos en Guía de Ondas 113

4.1. Introducción . . . . . . . . . . . . . . . . . . . . 113

4.2. Análisis Multimodal de la Excitación Coaxial de Dispositivos en Configuración Clásica mediante el Método BI-RME 3D . . . . . . . . . . . . . . . . 119

4.2.1. Cálculo de los elementos de la matriz G . . . . . . . . . . . . . . . 122

4.2.2. Cálculo de los elementos de la matriz T . . . . . . . . . . . . . . . . 129

4.2.3. Cálculo de los elementos de la matriz L . . . . . . . . . . . . . . . 142

4.2.4. Cálculo de los elementos de la matriz F . . . . . . . . . . . . . 145

4.2.5. Ejemplos de validación . . . . . . . . . . . . . . . . . 150

4.3. Análisis Multimodal de la Excitación Coaxial de Dispositivos en Configuración Colineal mediante el Método BI-RME 3D . . . . . . . . . . . . . . 159

4.3.1. Cálculo de los elementos de la matriz G . . . . . . . . . . . . . . . 162

4.3.2. Cálculo de los elementos de la matriz T . . . . . . . . . . . . 165

4.3.3. Cálculo de los elementos de la matriz L . . . . . . . . . . . . . . . . . 175

4.3.4. Cálculo de los elementos de la matriz F . . . . . . . . . . . . . . 175

4.3.5. Ejemplos de validación . . . . . . . . . . . . . . . 176

5. Herramienta de Análisis de Filtros y Dispositivos en Configuración Comb-Line 185

5.1. Introducción . . . . . . . . . . . . . . . . . . . 185

5.2. Descripción de la Herramienta Implementada . . . . . . . . . . . . . 187

5.3. Fundamentos Teóricos . . . . . . . . . . . . . . . . . . . . . . . 194

5.4. Ejemplos de Aplicación . . . . . . . . . . . . . . . . . . . 201

5.4.1. Validación de la herramienta de la etapa $3 \ldots . . . . . . .202$

5.4.2. Validación de la herramienta de la etapa $4 \ldots . . . . . . .206$

5.4.3. Validación de la herramienta de la etapa 5 . . . . . . . . . . . 212

5.4.4. Otros ejemplos de validación . . . . . . . . . . . . . . 216 
6. Conclusiones $\quad \mathbf{2 2 3}$

6.1. Conclusiones y Principales Aportaciones . . . . . . . . . . . . . . . . . . . 223

6.2. Líneas Futuras de Investigación . . . . . . . . . . . . . . . . . . 225

$\begin{array}{ll}\text { APÉNDICES } & 227\end{array}$

A. Matrices P y Q del Cálculo de los Modos de una Guía Coaxial usando el Método de los Momentos $\quad 227$

A.1. Modos $\mathrm{TM}^{z} \ldots \ldots \ldots \ldots \ldots \ldots$. . . . . . . . . . 227

A.2. Modos $\mathrm{TE}^{z} \ldots \ldots \ldots \ldots \ldots \ldots \ldots$

B. Matrices P y Q del Cálculo de los Modos de una Guía Circular usando el Método de los Momentos

B.1. Modos $\mathrm{TM}^{z} \ldots \ldots \ldots \ldots \ldots \ldots \ldots$

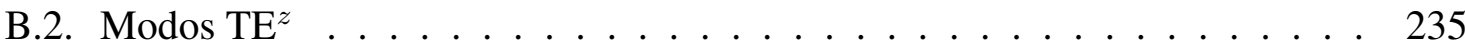

C. Análisis Modal de Guías y Cavidades Rectangulares 239

C.1. Funciones Vectoriales Modales de Campo Magnético de una Guía Rectangular239

C.2. Funciones de Green de tipo Magnético de una Cavidad Rectangular . . . . 241

D. Series y Funciones Auxiliares utilizadas en el Análisis de Uniones Compensadas243

D.1. Series utilizadas en el Análisis de Uniones Compensadas . . . . . . . . . . 243

D.2. Funciones Auxiliares utilizadas en el Análisis de Uniones Compensadas . . 244

E. Conexión de Matrices de Impedancias y Admitancias Generalizadas 247

F. Series Auxiliares utilizadas en el Análisis de la Excitación Coaxial de Dispositivos

G. Integrales Auxiliares utilizadas en el Análisis de la Excitación Coaxial de Dispositivos

G.1. Excitación Coaxial de Dispositivos en Configuración Clásica . . . . . . . . 255

G.2. Excitación Coaxial de Dispositivos en Configuración Colineal . . . . . . . 257

H. Publicaciones realizadas relacionadas con la Tesis Doctoral 263 


\section{Índice de Figuras}

2.1. Unión planar entre dos guías de onda de sección transversal arbitraria. . . . . . .

2.2. Red multimodal equivalente que caracteriza a una unión planar entre dos guías de onda de sección transversal arbitraria mediante la técnica de la ecuación integral. .

2.3. Sección transversal de una guía coaxial de radios externo $r_{1}$ e interno $r_{2}$. Se asume que la permitividad dieléctrica relativa es $\varepsilon_{r}$ y que la permeabilidad magnética

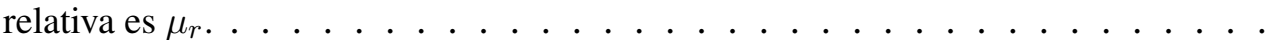

2.4. Unión planar entre una guía rectangular de dimensiones $a$ y $b$, y una guía coaxial de radio externo $r_{1}$ y radio interno $r_{2}$. Los parámetros $\left(x_{s}, y_{s}\right)$ sitúan el centro de la guía coaxial con respecto a la esquina inferior derecha de la guía rectangular. . . .

2.5. Módulo en dB del parámetro de transmisión $S_{21}$ relativo a una unión planar entre una guía rectangular WR-75 ( $a=19,05 \mathrm{~mm}, b=9,525 \mathrm{~mm})$ y una guía coaxial $\left(r_{1}=1,9 \mathrm{~mm}, r_{2}=0,6 \mathrm{~mm}, \varepsilon_{r}=1,7\right)$. La guía coaxial se encuentra en una posición centrada con respecto a la guía rectangular. El modo de excitación es el modo $\mathrm{TM}_{11}^{z}$ de la guía rectangular WR-75 y el modo de respuesta es el modo $\mathrm{TEM}^{z}$ de la guía coaxial. . . . . . . . . . . . . . . . . . . .

2.6. Módulo en dB del parámetro de transmisión $S_{21}$ relativo a una unión planar entre una guía rectangular WR-75 ( $a=19,05 \mathrm{~mm}, b=9,525 \mathrm{~mm}$ ) y una guía coaxial $\left(r_{1}=1,9 \mathrm{~mm}, r_{2}=0,6 \mathrm{~mm}, \varepsilon_{r}=1,7\right)$. La guía coaxial se encuentra descentrada con respecto a la guía rectangular $\left(x_{s}=4,25 \mathrm{~mm}, y_{s}=6,0 \mathrm{~mm}\right)$. El modo de excitación es el modo $\mathrm{TM}_{11}^{z}$ de la guía rectangular WR-75 y el modo de respuesta es el modo TEM ${ }^{z}$ de la guía coaxial. . . . . . . . . . . . . . . . . .

2.7. Módulo en dB del parámetro de transmisión $S_{21}$ relativo a una unión planar entre una guía rectangular WR-90 ( $a=22,90 \mathrm{~mm}, b=10,20 \mathrm{~mm})$ y una guía coaxial $\left(r_{1}=3,2 \mathrm{~mm}, r_{2}=0,9 \mathrm{~mm}, \varepsilon_{r}=1,0\right)$. La guía coaxial se encuentra centrada con respecto a la guía rectangular. El modo de excitación es el modo $\mathrm{TM}_{11}^{z}$ de la guía rectangular WR-90 y el modo de respuesta es el modo $\mathrm{TEM}^{z}$ de la guía coaxial. . .

2.8. Sección transversal de una guía circular de radio $r_{1} \ldots \ldots \ldots \ldots$. . . . . .

2.9. Unión planar entre una guía rectangular de dimensiones $a$ y $b$, y una guía circular de radio $r_{1}$. Los parámetros $\left(x_{s}, y_{s}\right)$ sitúan el centro de la guía circular con respecto a la esquina inferior derecha de la guía rectangular. . . . . . . . . . . . . . . 
2.10. Módulo en dB del parámetro de transmisión $S_{21}$ relativo a una unión planar entre una guía rectangular WR-75 $(a=19,05 \mathrm{~mm}, b=9,525 \mathrm{~mm})$ y una guía circular de radio $r_{1}=3,0 \mathrm{~mm}\left(\varepsilon_{r}=8,0\right)$. La guía circular se encuentra centrada con respecto a la guía rectangular. . . . . . . . . . . . . . . . .

2.11. Módulo en dB del parámetro de transmisión $S_{21}$ relativo a una unión planar entre una guía rectangular WR-90 ( $a=22,90 \mathrm{~mm}, b=10,20 \mathrm{~mm})$ y una guía circular de radio $r_{1}=2,7 \mathrm{~mm}\left(\varepsilon_{r}=14,0\right)$. La guía circular se encuentra centrada con respecto a la guía rectangular. . . . . . . . . . . . . . .

2.12. Módulo en dB del parámetro de transmisión $S_{21}$ relativo a una unión planar entre una guía rectangular WR-90 ( $a=22,90 \mathrm{~mm}, b=10,20 \mathrm{~mm})$ y una guía circular de radio $r_{1}=2,7 \mathrm{~mm}\left(\varepsilon_{r}=14,0\right)$. La guía circular se encuentra descentrada con respecto a la guía rectangular $\left(x_{s}=6,45 \mathrm{~mm}, y_{s}=3,1 \mathrm{~mm}\right) \ldots \ldots \ldots$. . . .

2.13. Unión planar entre dos guías coaxiales concéntricas $(a)$ y $(b)$ de radios externos $r_{1}^{(a)}$ y $r_{1}^{(b)}$, y de radios internos $r_{2}^{(a)}$ y $r_{2}^{(b)}$, respectivamente. . . . . . . . .

2.14. Módulo en dB del parámetro de reflexión $S_{11}$ relativo a una unión planar entre dos guías coaxiales $(a)$ y $(b)$. Las dimensiones de las guías son las siguientes: $r_{1}^{(a)}=6,0$ $\mathrm{mm}, r_{2}^{(a)}=0,65 \mathrm{~mm}, r_{1}^{(b)}=5,0 \mathrm{~mm} \mathrm{y} r_{2}^{(b)}=2,65 \mathrm{~mm}\left(\varepsilon_{r}^{(a)}=\varepsilon_{r}^{(b)}=1,0\right)$. Los resultados de la simulación se comparan con los proporcionados por el método directo descrito en [36] . . . . . . . . . . . . . . . . . . .

2.15. Módulo en dB del parámetro de reflexión $S_{11}$ relativo a una unión planar entre dos guías coaxiales $(a)$ y $(b)$. Las dimensiones de las guías son las siguientes: $r_{1}^{(a)}=8,6$ $\mathrm{mm}, r_{2}^{(a)}=0,65 \mathrm{~mm}, r_{1}^{(b)}=5,0 \mathrm{~mm} \mathrm{y} r_{2}^{(b)}=1,42 \mathrm{~mm}\left(\varepsilon_{r}^{(a)}=4,25 \mathrm{y} \varepsilon_{r}^{(b)}=7,2\right)$. Los resultados de la simulación se comparan con los proporcionados por el método directo descrito en [36] . . . . . . . . . . . . . . . . . . . .

2.16. Módulo en dB del parámetro de transmisión $S_{21}$ relativo a una unión planar entre dos guías coaxiales $(a)$ y $(b)$. Las dimensiones de las guías son las siguientes: $r_{1}^{(a)}=$ $15,2 \mathrm{~mm}, r_{2}^{(a)}=7,41 \mathrm{~mm}, r_{1}^{(b)}=11,5 \mathrm{~mm} \mathrm{y} r_{2}^{(b)}=9,24 \mathrm{~mm}\left(\varepsilon_{r}^{(a)}=2,33 \mathrm{y}\right.$ $\left.\varepsilon_{r}^{(b)}=15,1\right)$. Los resultados de la simulación se comparan con los proporcionados por el método directo descrito en $[36] \ldots \ldots \ldots \ldots$

2.17. Discontinuidad múltiple entre guías rectangulares. . . . . . . . . . . . .

2.18. Vista en planta de una discontinuidad múltiple en una estructura inductiva implementada en guía rectangular. . . . . . . . . . . . . . . . .

2.19. Vista en planta de una discontinuidad múltiple en una estructura inductiva implementada en guía rectangular. . . . . . . . . . . . . . . . . .

2.20. Módulo en dB de los parámetros de dispersión de la estructura inductiva implementada en guía rectangular cuya vista en planta se detalla en la figura 2.19. Comparación con los resultados proporcionados por HFSS. . . . . . . . . . . . .

2.21. Vista en planta de un filtro inductivo de tres resonadores implementados en guía estándar WR-12 con inserciones metálicas en plano $E$. Las dimensiones del filtro se han extraído de [45]. . . . . . . . . . . . . . . . . .

2.22. Módulo en dB del parámetro $S_{21}$ del filtro inductivo con inserciones metálicas en plano $E$ de la figura 2.21. Comparación de los resultados obtenidos utilizando la técnica de la ecuación integral con los datos experimentales extraídos de [45]. . . . 
3.1. Uniones multi-puerto en guía rectangular compensadas con un poste conductor cilíndrico de altura variable situado en una posición arbitraria. (a) Codo recto en plano $H$; (b) codo recto en plano $E$ con un iris de acoplo en uno de los puertos; (c) unión en T en plano $H$; (d) unión en T en plano $E$ con un iris de acoplo en uno de los puertos; (e) unión T-mágica con un iris de acoplo en uno de los puertos; (f) unión turnstile. . . . . . . . . . . . . . . . . . . . . . .

3.2. Geometría básica bajo análisis: unión en guía rectangular de 5 puertos cargada con un poste conductor cilíndrico de altura arbitraria situado en la base de la cavidad en la posición $\left(x_{0}, 0, z_{0}\right) \ldots \ldots \ldots \ldots \ldots \ldots \ldots$

3.3. Representación de los sistemas de coordenadas utilizados para los puertos de acceso $\xi=3,4,5 \ldots \ldots \ldots \ldots \ldots \ldots \ldots$

3.4. Pérdidas de retorno de un codo recto en plano $H$ implementado en guía rectangular WR-90 compensado con un poste conductor cilíndrico $(r=0,5 \mathrm{~mm}, h=9,0 \mathrm{~mm})$. Comparación del caso en el que el poste está en una posición descentrada $\left(x_{0}=9,45\right.$ $\mathrm{mm}, z_{0}=17,45 \mathrm{~mm}$ ) con el caso centrado. . . . . . . . . . . . .

3.5. Pérdidas de retorno de un codo recto en plano $H$ implementado en guía rectangular WR-90 compensado con un poste conductor cilíndrico $(r=0,5 \mathrm{~mm}, h=9,0 \mathrm{~mm})$. Influencia de la posición relativa del poste en la respuesta en frecuencia de la estructura. 96

3.6. Pérdidas de retorno de un codo recto en plano $H$ implementado en guía rectangular WR-90 compensado con un poste conductor cilíndrico $(h=9,0 \mathrm{~mm})$ situado en una posición descentrada $\left(x_{0}=9,45 \mathrm{~mm}, z_{0}=17,45 \mathrm{~mm}\right)$. Influencia del radio del poste en la respuesta en frecuencia de la estructura. . . . . . . . . . . . . .

3.7. Pérdidas de retorno de un codo recto en plano $H$ implementado en guía rectangular WR-90 compensado con un poste conductor cilíndrico $(r=0,5 \mathrm{~mm})$ situado en una posición descentrada $\left(x_{0}=9,45 \mathrm{~mm}, z_{0}=17,45 \mathrm{~mm}\right)$. Influencia de la altura del poste en la respuesta en frecuencia de la estructura. . . . . . . . . . . . .

3.8. Pérdidas de retorno de un codo recto en plano $E$ implementado en guía rectangular WR-90 compensado con un poste conductor cilíndrico ( $r=3,75 \mathrm{~mm}, h=1,5 \mathrm{~mm}$ ) en posición descentrada $\left(x_{0}=11,45 \mathrm{~mm}, z_{0}=4,10 \mathrm{~mm}\right)$. En el puerto (5) del codo se ha introducido un iris de adaptación $\left(h_{\text {iris }}=7,0 \mathrm{~mm}, l_{\text {iris }}=20,5 \mathrm{~mm}, t_{\text {iris }}=1,5\right.$ $\mathrm{mm}) \ldots \ldots \ldots \ldots \ldots \ldots \ldots$

3.9. Pérdidas de retorno de un codo recto en plano $E$ implementado en guía rectangular WR-90 compensado con un poste conductor cilíndrico $(h=1,5 \mathrm{~mm})$ situado en una posición descentrada. En el puerto (5) del codo se ha introducido un iris de adaptación. Influencia del radio del poste en la respuesta en frecuencia de la estructura.100

3.10. Pérdidas de retorno de un codo recto en plano $E$ implementado en guía rectangular WR-90 compensado con un poste conductor cilíndrico $(r=3,75 \mathrm{~mm})$ situado en una posición descentrada $\left(x_{0}=11,45 \mathrm{~mm}, z_{0}=4,10 \mathrm{~mm}\right)$. En el puerto (5) del codo se ha introducido un iris de adaptación. Influencia de la altura del poste en la respuesta en frecuencia de la estructura. . . . . . . . . . . . . . .

3.11. Pérdidas de retorno de un codo recto en plano $E$ implementado en guía rectangular WR-90 compensado con un poste situado en una posición descentrada. Influencia de la altura a la que se sitúa el iris $\left(h_{\text {iris }}\right)$ en la respuesta en frecuencia de la estructura.101 
3.12. Pérdidas de retorno de un codo recto en plano $E$ implementado en guía rectangular WR-90 compensado con un poste situado en una posición descentrada. Influencia de la longitud del iris $\left(l_{\text {iris }}\right)$ en la respuesta en frecuencia de la estructura. . . . . .

3.13. Parámetros de dispersión de una unión en T en plano $H$ en guía WR-75 compensada con un poste cilíndrico $(r=0,254 \mathrm{~mm}, h=7,62 \mathrm{~mm})$. Comparación entre el caso en que el poste está centrado con el caso en que está descentrado $\left(x_{0}=9,525 \mathrm{~mm}\right.$, $z_{0}=12,525 \mathrm{~mm}$ ). Los resultados obtenidos para el caso en que el poste está centrado se comparan con los datos presentados en [59]. . . . . . . . . . . . . .

3.14. Pérdidas de retorno de una unión en T en plano $H$ en guía WR-75 compensada con un poste cilíndrico $(r=0,254 \mathrm{~mm}, h=7,62 \mathrm{~mm})$ situado en la posición $\left(x_{0}=\right.$ $\left.a / 2, z_{0}\right)$. Influencia de la posición relativa del poste en la respuesta en frecuencia del divisor. . . . . . . . . . . . . . . . . . . .

3.15. Parámetros de dispersión de una unión en T en plano $H$ en guía WR-28 compensada con un poste cilíndrico $(r=0,635 \mathrm{~mm}, h=3,048 \mathrm{~mm})$. Comparación entre el caso en que el poste está centrado con el caso en que está descentrado $\left(x_{0}=9,525 \mathrm{~mm}\right.$, $z_{0}=5,362 \mathrm{~mm}$ ). Los resultados obtenidos para el caso en que el poste está centrado se comparan con los datos presentados en [59]. . . . . . . . . . . . . .

3.16. Parámetros de dispersión de una unión en T en plano $E$ en guía WR-75 compensada con un poste cilíndrico $(r=2,9 \mathrm{~mm}, h=4,65 \mathrm{~mm})$ situado en una posición centrada. En el puerto (5) se ha introducido un iris de adaptación $\left(h_{\text {iris }}=1,5 \mathrm{~mm}\right.$, $\left.l_{\text {iris }}=15,3 \mathrm{~mm}, t_{\text {iris }}=1,25 \mathrm{~mm}\right) \ldots \ldots \ldots \ldots \ldots$

3.17. Parámetros de dispersión de una unión en T mágica sin compensar implementada en guía rectangular WR-90. Comparación con las medidas experimentales extraídas de [9]. (a) Parámetros de reflexión. (b) Parámetros de transmisión. . . . . . . . . . .

3.18. Parámetros de dispersión de una unión en T mágica implementada en la guía rectangular WR-90 compensada con un poste situado en posición centrada $(r=0,65$ $\mathrm{mm}, h=9,5 \mathrm{~mm}$ ). En el puerto (5) de la estructura se ha introducido un iris de adaptación $\left(h_{\text {iris }}=4,0 \mathrm{~mm}, l_{\text {iris }}=16,11 \mathrm{~mm}, t_{\text {iris }}=1,5 \mathrm{~mm}\right.$ ). (a) Parámetros de reflexión. (b) Parámetros de transmisión. . . . . . . . . . . . . . . . . .

3.19. Parámetros de dispersión de una unión en T mágica implementada en la guía rectangular WR-90 compensada con un poste $(r=0,65 \mathrm{~mm}, h=9,5 \mathrm{~mm})$ situado en posición descentrada $\left(x_{0}=a / 2, z_{0}=14,45 \mathrm{~mm}\right)$. En el puerto (5) de la estructura se ha introducido un iris de adaptación $\left(h_{\text {iris }}=4,0 \mathrm{~mm}, l_{\text {iris }}=16,11 \mathrm{~mm}\right.$, $t_{\text {iris }}=1,5 \mathrm{~mm}$ ). (a) Parámetros de reflexión. (b) Parámetros de transmisión. . . . .

3.20. Parámetros de dispersión de una unión turnstile sin compensar implementada en la guía rectangular WR-62. La guía circular es de radio $\rho=6,99 \mathrm{~mm}$. Comparación con los resultados presentados en [10] . . . . . . . . . . . . . . . 110

3.21. Parámetros de dispersión de una unión turnstile compensada (poste centrado; $r=2,8 \mathrm{~mm}$, $h=3,7 \mathrm{~mm}$ ) implementada en la guía rectangular WR-62. La guía circular es de radio $\rho=6,99 \mathrm{~mm}$. El caso (a) se corresponde con la unión sin compensar, mientras que el caso (b) se corresponde con la unión compensada. Los resultados de la simulación se comparan con HFSS. 
4.1. Excitación clásica de dispositivos implementados en guía rectangular mediante sonda coaxial terminada en disco. . . . . . . . . . . . . . . . . . . . . . .

4.2. Excitación de dispositivos implementados en guía rectangular mediante sonda coaxial en configuración colineal. . . . . . . . . . . . . . . . . . . .

4.3. Vistas frontal y lateral de la excitación coaxial clásica de una guía rectangular semiinfinita mediante sonda terminada en disco. $\ldots \ldots \ldots \ldots$

4.4. Vista superior de la excitación clásica de dispositivos implementados en guía rectangular. Representación del sistema de referencia en coordenadas cilíndricas utilizado.

4.5. Vista frontal de la cavidad perturbada obtenida tras cortocircuitar los puertos de acceso de la estructura bajo análisis. La cavidad rectangular es de longitud $c$ (en el eje $\hat{\mathbf{z}}) . \ldots \ldots \ldots \ldots \ldots$

4.6. Pérdidas de retorno para una guía rectangular semi-infinita implementada en la guía estándar WR-62 excitada mediante sonda coaxial convencional $\left(r_{1}=1,6764 \mathrm{~mm}\right.$, $r_{2}=0,635 \mathrm{~mm}, Z_{0}=50 \Omega, d_{z}=5,0 \mathrm{~mm}, d_{x}=a / 2$ ). En la figura se estudian dos casos en función del valor de la profundidad de penetración de la sonda $h_{p}$. Los resultados de la simulación se comparan con los datos experimentales presentados en $[17] \ldots \ldots \ldots \ldots \ldots \ldots \ldots \ldots$

4.7. Estudio de convergencia para el módulo del parámetro $S_{11}(f=15 \mathrm{GHz})$ relativo a la estructura simulada en la figura 4.6 con $h_{p}=1,2954 \mathrm{~mm}$. El estudio se realiza en función del número de modos $Q^{\mathrm{TE}}=Q^{\mathrm{TM}}$ utilizados en la guía rectangular de

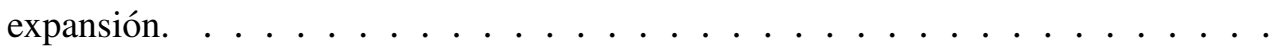

4.8. Pérdidas de retorno para una guía rectangular semi-infinita implementada en la guía estándar WR-75 excitada mediante sonda coaxial convencional $\left(r_{1}=3,18 \mathrm{~mm}\right.$, $\left.r_{2}=0,3 \mathrm{~mm}, \varepsilon_{r}=2,09, h_{p}=4,7625 \mathrm{~mm}, d_{z}=6,8 \mathrm{~mm}, d_{x}=a / 2\right)$. Los resultados de la simulación se comparan con los datos proporcionados por HFSS. .

4.9. Pérdidas de retorno para una guía rectangular semi-infinita ( $a=57,04 \mathrm{~mm}, b=28,8 \mathrm{~mm})$ excitada mediante sonda coaxial convencional $\left(r_{1}=2,12 \mathrm{~mm}, r_{2}=0,635 \mathrm{~mm}\right.$, $\left.\varepsilon_{r}=2,09, h_{p}=14,4 \mathrm{~mm}, d_{z}=19,2 \mathrm{~mm}, d_{x}=a / 2\right)$. Los resultados de la simulación se comparan con los datos proporcionados por HFSS. . . . . . . . . . . . .

4.10. Pérdidas de retorno para una guía rectangular semi-infinita implementada en la guía estándar WR-90 excitada mediante sonda coaxial convencional $\left(r_{1}=3,45 \mathrm{~mm}\right.$, $\left.r_{2}=0,55 \mathrm{~mm}, \varepsilon_{r}=2,09, h_{p}=5,5 \mathrm{~mm}, d_{z}=8,6 \mathrm{~mm}, d_{x}=a / 2\right)$. Los resultados de la simulación se comparan con los datos proporcionados por HFSS. . . . . . .

4.11. Pérdidas de retorno para una guía rectangular semi-infinita implementada en la guía estándar WR-75 excitada mediante una sonda coaxial terminada en disco $\left(r_{1}=3,18\right.$ $\mathrm{mm}, r_{2}=0,3 \mathrm{~mm}, \varepsilon_{r}=2,09, h_{p}=4,7625 \mathrm{~mm}, h_{d}=1,5 \mathrm{~mm}, d_{z}=6,8 \mathrm{~mm}, d_{x}=$ $a / 2$ ). Los resultados de la simulación se comparan con los datos proporcionados por HFSS. 
4.12. Pérdidas de retorno para una guía rectangular semi-infinita implementada en la guía estándar WR-137 excitada mediante una sonda coaxial terminada en disco $\left(r_{1}=\right.$ $4,8768 \mathrm{~mm}, r_{2}=1,524 \mathrm{~mm}, r_{d}=4,0 \mathrm{~mm}, h_{p}=3,4 \mathrm{~mm}, h_{d}=4,5 \mathrm{~mm}, Z_{0}=$ $\left.50 \Omega, d_{z}=11,6 \mathrm{~mm}, d_{x}=a / 2\right)$. Los resultados de la simulación se comparan con los datos proporcionados por HFSS y con los datos extraídos de [19]. En la misma figura se presenta en trazo punteado la respuesta de una sonda coaxial convencional con $h_{p}^{\prime}=7,9 \mathrm{~mm} . \ldots \ldots \ldots \ldots \ldots$

4.13. Estudio de convergencia para el módulo del parámetro $S_{11}(f=7,5 \mathrm{GHz})$ relativo a la estructura simulada en la figura 4.12. El estudio se realiza en función del número de modos $Q^{\mathrm{TE}}=Q^{\mathrm{TM}}$ utilizados en la guía rectangular de expansión. . . . . . .

4.14. Pérdidas de retorno de la estructura analizada en la figura 4.12 en función de la distancia normalizada $d_{z} /\left(\lambda_{g} / 4\right)$ a la pared cortocircuitada de la guía rectangular. Los resultados se comparan con los datos proporcionados por HFSS. . . . . . . .

4.15. Filtro inductivo de 7 cavidades excitado mediante sonda coaxial convencional. . .

4.16. Pérdidas de retorno asociadas al filtro inductivo excitado mediante sonda coaxial representado en la figura $4.15 \ldots \ldots \ldots$. . . . . . . . . . .

4.17. Excitación coaxial en configuración colineal de dispositivos implementados en guía rectangular. . . . . . . . . . . . . . .

4.18. Vistas lateral y frontal del bloque básico relativo a la excitación coaxial en configuración colineal de dispositivos implementados en guía rectangular. . . . . . . . .

4.19. Sistema de referencia en coordenadas cilíndricas utilizado en el análisis de la excitación coaxial en configuración colineal de dispositivos implementados en guía rectangular. . . . . . . . . . . . . . . . . .

4.20. Estructura utilizada para validar la herramienta de análisis de la excitación coaxial de dispositivos en configuración colineal. En este primer ejemplo, se abren los accesos (1) y (2) del bloque básico. . . . . . . . . . . . . . . . . . .

4.21. Parámetros de dispersión asociados a la estructura representada en la figura 4.20. Los datos obtenidos se comparan con los que proporciona HFSS. . . . . . . . . .

4.22. Estructura utilizada para validar la herramienta de análisis de la excitación coaxial de dispositivos en configuración colineal. En este segundo ejemplo, se abren los accesos (1) y (3) del bloque básico. . . . . . . . . . . . . . . . . .

4.23. Parámetros de dispersión asociados a la estructura representada en la figura 4.22. Los datos obtenidos se comparan con los que proporciona HFSS. . . . . . . . . .

4.24. Estructura utilizada para validar la herramienta de análisis de la excitación coaxial de dispositivos en configuración colineal. En este tercer ejemplo, se abren los accesos (1), (2) y (3) del bloque básico. . . . . . . . . . . . . . . . .

4.25. Parámetros de dispersión asociados a la estructura representada en la figura 4.24. Los datos obtenidos se comparan con los que proporciona HFSS. . . . . . . . . .

4.26. Estructura utilizada para validar la herramienta de análisis de la excitación coaxial de dispositivos en configuración colineal. En este cuarto ejemplo, se abren los accesos (1), (3) y (4) del bloque básico. . . . . . . . . . . . . . . . .

4.27. Parámetros de dispersión asociados a la estructura representada en la figura 4.26. Los datos obtenidos se comparan con los que proporciona HFSS. . . . . . . . . . 
4.28. Estructura utilizada para validar la herramienta de análisis de la excitación coaxial de dispositivos en configuración colineal. En este quinto ejemplo, se abren los 4 accesos del bloque básico. . . . . . . . . . . . . . . . .

4.29. Parámetros de dispersión asociados a la estructura representada en la figura 4.28. Los datos obtenidos se comparan con los que proporciona HFSS. . . . . . . . . .

4.30. Vistas lateral y superior de la conexión del bloque básico de la figura 4.17 con una guía rectangular de dimensiones $a^{(2)} \times b^{(2)}$ a través de un iris rectangular de sección transversal $a^{(1)} \times b^{(1)}$ y longitud $l_{\text {iris }} \ldots \ldots \ldots \ldots \ldots$

4.31. Parámetro de transmisión asociado a la estructura representada en la figura 4.30. Los datos obtenidos se comparan con los que proporciona HFSS.

5.1. Diplexor en configuración comb-line implementado en tecnología guiada. El dispositivo consta de 12 resonadores rectangulares cargados con postes conductores de geometría cilíndrica (realmente, se trata de 2 filtros, cada uno con 6 resonadores

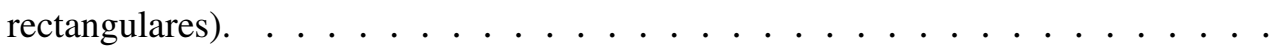

5.2. Filtro genérico en configuración comb-line constituido por 10 resonadores y 2 puertos de acceso en guía coaxial. En tres de los resonadores se ha incluido un tornillo de sintonía. . . . . . . . . . . . . . . . . . .

5.3. Vista en planta y vista lateral de un resonador comb-line de dimensiones $a \times b \times c$ cargado con un poste conductor cilíndrico situado en la posición relativa $\left(-d_{x, c}, 0, d_{z, c}\right) .190$

5.4. Vistas laterales de los diferentes tipos de postes conductores soportados por la herramienta de simulación: poste cilíndrico (arriba izquierda); poste en forma de champiñón (arriba derecha); y poste en forma de vaso (abajo).

5.5. Sistema de referencia utilizado para definir la posición relativa de los irises de acoplamiento entre resonadores comb-line. . . . . . . . . . . . . . . .

5.6. Vista en planta y vista lateral de un resonador comb-line con un tornillo de sintonía de radio $r_{t}$ y altura $h_{t}$ situado en la posición relativa $\left(d_{x, t}, b, d_{z, t}\right) . \ldots \ldots$.

5.7. Puerto de acceso de sección transversal rectangular $\left(a_{p} \times b_{p}\right)$ cuyas dimensiones no coinciden con las de la cara lateral del resonador $(a \times b) \ldots \ldots \ldots \ldots$

5.8. Puerto de acceso de sección transversal rectangular de dimensiones $a_{p}^{\prime} \times b_{p}^{\prime}$ a través de un iris de tipo rectangular de sección transversal $a_{i r, p} \times b_{i r, p}$ y longitud $l_{i r, p} . \ldots 194$

5.9. Puerto de acceso mediante sonda coaxial terminada en disco. En caso de que $r_{d}=h_{d}=0$ se obtiene la sonda coaxial convencional. . . . . . . . . . . . . . . . . 195

5.10. Conexión de todas las matrices multimodales implicadas en la caracterización del resonador $(2,2)$ del filtro comb-line genérico de la figura 5.2 . . . . . . . . . .

5.11. Matrices multimodales implicadas en la caracterización del resonador $(2,3)$ del filtro comb-line genérico de la figura $5.2 \ldots \ldots \ldots$. . . . . . . . .

5.12. Obtención de una MIG equivalente para la representación multimodal del resonador $(2,2)$ del filtro de la figura 5.2.

5.13. Interconexión de las MIG equivalentes para el filtro comb-line genérico de la figura 5.2.198

5.14. Conexión de la MIG correspondiente a un resonador comb-line de 4 accesos con la MIG asociada a la unión planar que se produce al introducir el iris de acoplamiento. 
5.15. Filtro comb-line de 4 cavidades con dos puertos de acceso, siendo uno de ellos de tipo coaxial y el otro de tipo rectangular. Esta estructura se utiliza para validar la herramienta de simulación relativa a la etapa $3 \ldots \ldots$. . . . . . . .

5.16. Módulo del parámetro $S_{21}$ para el filtro comb-line de la figura 5.15. Los resultados obtenidos con la herramienta de simulación se comparan con los datos proporcionados por HFSS.

5.17. Dispositivo comb-line de 7 cavidades con tres puertos de acceso, siendo dos de ellos de tipo coaxial y el otro de tipo rectangular. Esta estructura se utiliza para validar la herramienta de simulación relativa a la etapa $3 \ldots \ldots \ldots$. . . . . . .

5.18. Módulo de los parámetros $S_{21}$ y $S_{31}$ (líneas continuas) para el dispositivo combline de la figura 5.17. Los resultados obtenidos con la herramienta de simulación se comparan con los datos proporcionados por HFSS. . . . . . . . . . . .

5.19. Dispositivo comb-line de 11 cavidades y 5 puertos de acceso. Esta estructura se utiliza para validar la herramienta de simulación relativa a la etapa 3. . . . . . .

5.20. Módulo de los parámetros de dispersión relativos al dispositivo comb-line de 11 cavidades y 5 puertos de acceso representado en la figura 5.19. (a) $\left|S_{21}\right|$ (dB); (b) $\left|S_{31}\right|(\mathrm{dB})$; (c) $\left|S_{41}\right|(\mathrm{dB}) ;(\mathrm{d})\left|S_{51}\right|(\mathrm{dB}) \ldots \ldots \ldots \ldots \ldots$

5.21. Fase de los parámetros de dispersión relativos al dispositivo comb-line de 11 cavidades y 5 puertos de acceso representado en la figura 5.19. (a) $\arg \left(S_{11}\right)$ (grados); (b) $\arg \left(S_{21}\right)$ (grados); (c) $\arg \left(S_{31}\right)$ (grados); (d) $\arg \left(S_{41}\right)$ (grados). . . . . . . .

5.22. Filtro comb-line de 2 cavidades y 2 puertos de acceso, siendo uno de ellos de tipo coaxial y el otro de tipo rectangular. Esta estructura se utiliza para validar la herramienta de simulación relativa a la etapa $4 \ldots$. . . . . . . . . . . .

5.23. Módulo del parámetro $S_{21}$ para el filtro comb-line de la figura 5.22. Los resultados obtenidos con la herramienta de simulación se comparan con los datos proporcionados por HFSS.

5.24. Dispositivo comb-line de 8 cavidades y 3 puertos de acceso, siendo uno de ellos de tipo coaxial y dos de tipo rectangular. Esta estructura se utiliza para validar la herramienta de simulación relativa a la etapa $4 . \ldots$. . . . . . . . . . .

5.25. Módulo de los parámetros $S_{21}$ y $S_{31}$ para el dispositivo comb-line de la figura 5.24. Los resultados obtenidos con la herramienta de simulación se comparan con los datos proporcionados por HFSS. . . . . . . . . . . . . . . . .

5.26. Fase de los parámetros de dispersión relativos al dispositivo comb-line de 8 cavidades y 3 puertos de acceso representado en la figura 5.24. (a) $\arg \left(S_{11}\right)$ (grados); (b) $\arg \left(S_{21}\right)($ grados $) ;\left(\right.$ c) $\arg \left(S_{31}\right)$ (grados) . . . . . . . . . . . .

5.27. Filtro comb-line de 2 cavidades y 2 puertos de acceso, siendo uno de tipo coaxial y el otro de tipo rectangular. Esta estructura se utiliza para validar la herramienta de simulación relativa a la etapa $5 . \ldots \ldots \ldots \ldots$

5.28. Módulo del parámetro $S_{21}$ para el filtro comb-line de la figura 5.27. Los resultados obtenidos con la herramienta de simulación se comparan con los datos proporcionados por HFSS. . . . . . . . . . . . . . . . . . . . 
5.29. Dispositivo comb-line de 8 cavidades y 3 puertos de acceso, siendo uno de tipo coaxial y el resto de tipo rectangular. Esta estructura se utiliza para validar la herramienta de simulación relativa a la etapa $5 . \ldots$. . . . . . . . . . . .

5.30. Módulo de los parámetros de dispersión del dispositivo comb-line de la figura 5.29. Los resultados obtenidos con la herramienta de simulación se comparan con los datos proporcionados por HFSS. . . . . . . . . . . . . . . .

5.31. Fase de los parámetros de dispersión relativos al dispositivo comb-line de 8 cavidades y 3 puertos de acceso representado en la figura 5.29. (a) $\arg \left(S_{11}\right)$ (grados); (b) $\arg \left(S_{21}\right)$ (grados); (c) $\arg \left(S_{31}\right)$ (grados). . . . . . . . . . . . .

5.32. Filtro comb-line de 4 cavidades y 2 puertos de acceso de tipo coaxial. Los resonadores están cargados con postes conductores en forma de vaso y poseen todos ellos tornillos de sintonía. . . . . . . . . . . . . . . . . .

5.33. Módulo del parámetro de transmisión $S_{21}$ del filtro comb-line de la figura 5.32. Los resultados obtenidos con la herramienta de simulación se comparan con los datos proporcionados por HFSS. . . . . . . . . . . . . . .

5.34. Filtro comb-line de 6 cavidades en línea y 2 puertos de acceso de tipo coaxial. Las dimensiones del filtro se han extraído de [78]. Los resonadores están cargados con postes conductores de geometría cilíndrica. . . . . . . . . . . . . . .

5.35. Módulo de los parámetros de dispersión del filtro comb-line de la figura 5.34. Los resultados obtenidos con la herramienta de simulación se comparan con los datos proporcionados por HFSS. . . . . . . . . . . . . . . . .

E.1. Conexión de una matriz de admitancias generalizada y una matriz de impedancias generalizada. . . . . . . . . . . . . . . . 


\section{Índice de Tablas}

2.1. Valores de los coeficientes $\beta_{r, m}(r=1,2,3)$ para los modos $\mathrm{TE}^{z}$ y $\mathrm{TM}^{z} \ldots \ldots$. . .

2.2. Comparación del número de onda de corte obtenido con la herramienta implementada con los resultados presentados en [28]. La guía coaxial analizada es de radio externo $r_{1}=1,6 \mathrm{~m}$ y de radio interno $r_{2}=1,0 \mathrm{~m}\left(\varepsilon_{r}=1,0\right)$. Los resultados presentados se refieren a los modos $\mathrm{TM}_{s, q}^{z} \operatorname{con}(s, q)=(0,1),(2,1),(0,2),(3,2),(0,3)$, $(2,3),(0,4),(3,4) \ldots \ldots \ldots \ldots \ldots \ldots$

2.3. Comparación del número de onda de corte obtenido con la herramienta implementada con los resultados presentados en [40]. La guía coaxial analizada es de radio externo $r_{1}=1,2222 \mathrm{~m}$ y de radio interno $r_{2}=1,0 \mathrm{~m}\left(\varepsilon_{r}=1,0\right)$. Los resultados presentados se refieren a los modos $\mathrm{TM}_{s, 1}^{z}$ con $s=1,2,5,10,100,200 . \quad \ldots$.

2.4. Convergencia del número de onda de corte expresado en $\mathrm{rad} / \mathrm{m}$ de los modos $\mathrm{TM}_{s, q}^{z}$ para una guía coaxial de radio externo $r_{1}=1,6 \mathrm{~m}$ y de radio interno $r_{2}=1,0 \mathrm{~m}$ $\left(\varepsilon_{r}=1,0\right)$ en función del número de términos $N_{\rho}$ considerados en la expansión radial de la solución modal. Los índices modales analizados son $(s, q)=(0,1)$, $(2,1),(0,2),(3,2),(0,3),(2,3),(0,4),(3,4) \ldots \ldots \ldots \ldots \ldots$

2.5. Comparación del número de onda de corte obtenido con la herramienta implementada con los resultados presentados en [28]. La guía coaxial analizada es de radio externo $r_{1}=1,6 \mathrm{~m}$ y de radio interno $r_{2}=1,0 \mathrm{~m}\left(\varepsilon_{r}=1,0\right)$. Los resultados presentados se refieren a los modos $\mathrm{TE}_{s, q}^{z}$ con $(s, q)=(0,2),(2,2),(0,3),(3,3),(0,4)$, $(2,4),(0,5),(3,5) \ldots \ldots \ldots \ldots \ldots \ldots$

2.6. Comparación del número de onda de corte obtenido con la herramienta implementada con los resultados presentados en [40]. La guía coaxial analizada es de radio externo $r_{1}=1,0202 \mathrm{~m}$ y de radio interno $r_{2}=1,0 \mathrm{~m}\left(\varepsilon_{r}=1,0\right)$. Los resultados presentados se refieren a los modos $\mathrm{TE}_{s, 2}^{z}$ con $s=1,2,5,10,100,200 \ldots \ldots$. .

2.7. Convergencia del número de onda de corte de los modos $\mathrm{TE}_{s, q}^{z}$ expresado en rad $/ \mathrm{m}$ para una guía coaxial de radio externo $r_{1}=1,6 \mathrm{~m}$ y de radio interno $r_{2}=1,0$ m $\left(\varepsilon_{r}=1,0\right)$ en función del número de términos $N_{\rho}$ considerados en la expansión radial de la solución modal. Los índices modales de los modos analizados son: $(s, q)=(0,2),(2,2),(0,3),(3,3),(0,4),(2,4),(0,5),(3,5) \ldots \ldots \ldots$

2.8. Número de onda de corte relativo a los modos $\operatorname{TM}_{s, 1}^{z}(s=0,1,2,5,6,7,8)$ de una guía circular de radio $r_{1}=1,0 \mathrm{~m}$. Los resultados obtenidos con la herramienta implementada se comparan con los datos que se recogen en [44] . . . . . . . . . . 
2.9. Estudio de convergencia para el número de onda de corte expresado en $\mathrm{rad} / \mathrm{m}$ de los modos $\mathrm{TM}_{s, 1}^{z}(s=0,1,2,5,6,7,8)$ de una guía circular de radio $r_{1}=1,0 \mathrm{~m}$ en función del número de términos $N_{\rho}$ considerados en la expansión radial de la solución modal. . . . . . . . . . . . . . . . . . . . . . .

2.10. Número de onda de corte relativo a los modos $\mathrm{TE}_{s, 3}^{z}(s=0,1,2,5,6,7,8)$ de una guía circular de radio $r_{1}=1,0 \mathrm{~m}$. Los resultados obtenidos con la herramienta implementada se comparan con los datos extraídos de [44] . . . . . . . . . . .

2.11. Estudio de convergencia para el número de onda de corte expresado en $\mathrm{rad} / \mathrm{m}$ de los modos $\mathrm{TE}_{s, 3}^{z}(s=0,1,2,5,6,7,8)$ de una guía circular de radio $r_{1}=1,0 \mathrm{~m}$ en función del número de términos $N_{\rho}$ considerados en la expansión radial de la solución modal. . . . . . . . . . . . . . . . . . . . .

3.1. Definición de las matrices del método BI-RME 3D. . . . . . . . . . . . .

4.1. Valores de las anchuras y de las longitudes de las 17 guías rectangulares que integran el filtro inductivo excitado mediante sonda coaxial convencional representado en la figura 4.15 (se asume que las guías se numeran de izquierda a derecha). La altura de todas las guías que integran la estructura se mantiene constante e igual a $b=12,624$ mm. . . . . . . . . . . . . . . . . .

5.1. Dimensiones y posición relativa $(\mathrm{mm})$ de los postes conductores de geometría cilíndrica del filtro comb-line de 4 resonadores de la figura $5.15 \ldots \ldots \ldots$. . . . . . .

5.2. Dimensiones y posición relativa $(\mathrm{mm})$ de los irises de acoplamiento del filtro combline de 4 resonadores de la figura $5.15 \ldots . . \ldots 203$

5.3. Dimensiones y posición relativa $(\mathrm{mm})$ de los postes conductores de geometría cilíndrica del dispositivo comb-line de 7 resonadores de la figura $5.17 \ldots \ldots . . . .$.

5.4. Dimensiones y posición relativa $(\mathrm{mm})$ de los irises de acoplamiento del dispositivo comb-line de 7 resonadores de la figura 5.17 . . . . . . . . . . . 205

5.5. Dimensiones y posición relativa $(\mathrm{mm})$ de los postes conductores de geometría cilíndrica del dispositivo comb-line de 11 resonadores de la figura 5.19 . . . . . . . . . .

5.6. Dimensiones y posición relativa $(\mathrm{mm})$ de los irises de acoplamiento del dispositivo comb-line de 11 resonadores de la figura 5.19. . . . . . . . . . . . . . 208

5.7. Dimensiones y posición relativa $(\mathrm{mm})$ de los postes conductores de geometría cilíndrica del dispositivo comb-line de 8 resonadores de la figura 5.24 . . . . . . . . .

5.8. Dimensiones y posición relativa $(\mathrm{mm})$ de los postes conductores en forma de vaso del dispositivo comb-line de 8 resonadores de la figura 5.24. . . . . . . . . . .

5.9. Dimensiones y posición relativa $(\mathrm{mm})$ de los postes conductores en forma de champiñón del dispositivo comb-line de 8 resonadores de la figura 5.24 . . . . . . . . .

5.10. Dimensiones y posición relativa $(\mathrm{mm})$ de los tornillos de sintonía del dispositivo comb-line de 8 resonadores de la figura $5.24 \ldots \ldots \ldots \ldots$. . . . . . .

5.11. Dimensiones $(\mathrm{mm})$ de los irises de sección transversal circular del dispositivo combline de 8 resonadores de la figura $5.29 \ldots \ldots \ldots \ldots \ldots$ 
5.12. Dimensiones $(\mathrm{mm})$ del filtro comb-line de 6 resonadores en línea de la figura 5.34. Se asume que el iris de cada resonador es el que se conecta al puerto local (2) de dicho resonador. 


\section{Capítulo 1}

\section{Introducción}

\subsection{Motivación y Objetivos de la Tesis}

La caracterización multimodal rigurosa de la excitación coaxial de dispositivos de microondas implementados en tecnología guiada es un tema de investigación que, desde hace décadas, ha suscitado un enorme interés en la comunidad científica. Un gran número de dispositivos utilizados actualmente en las bandas de microondas y ondas milimétricas (filtros, diplexores, multiplexores, transductores ortomodales, etc.) se alimentan frecuentemente utilizando una excitación mediante sonda coaxial. Asimismo, las guías coaxiales se emplean comúnmente como elemento de interconexión de los diferentes subsistemas que integran un gran número de dispositivos. Sin embargo, a pesar de la gran relevancia que han adquirido las guías coaxiales en el proceso de diseño de numerosos dispositivos de enorme interés práctico, en la literatura técnica existen muy pocos trabajos de investigación dedicados a realizar un análisis multimodal riguroso de la excitación coaxial de estructuras implementadas en tecnología guiada. De hecho, la gran mayoría de las contribuciones realizadas hasta la fecha, se centran, fundamentalmente, en el cálculo de la impedancia de entrada de la estructura bajo análisis vista desde la guía coaxial de excitación con el objetivo de maximizar el grado de adaptación entre los diferentes medios guiados.

Por otra parte, es importante comentar que, en la actualidad, la excitación mediante sonda coaxial no suele considerarse en la fase inicial del proceso de análisis y diseño de muchos dispositivos utilizados en las bandas de microondas y ondas milimétricas; es decir, suele ser habitual integrar la excitación coaxial en el dispositivo tras finalizar el proceso de diseño del mismo. Este procedimiento puede originar que, una vez integrada dicha excitación, la respuesta eléctrica del dispositivo no coincida con la que inicialmente se había diseñado. En consecuencia, sería muy ventajoso disponer de una herramienta de simulación que, desde un primer momento, integrase la excitación coaxial en la caracterización multimodal del dispositivo bajo análisis. De esta forma, no sólo se lograría un ahorro importante en el tiempo invertido en el proceso de diseño, sino también una importante reducción en el volumen del subsistema finalmente diseñado.

En esta misma línea, la industria dedicada al sector de las telecomunicaciones espaciales 
ha mostrado un especial interés en el desarrollo de una herramienta de simulación, flexible y muy eficiente desde un punto de vista computacional, que les permita disminuir de manera sustancial el tiempo invertido en el proceso de diseño de diferentes dispositivos de microondas que suelen excitarse mediante sonda coaxial, como los denominados filtros en configuración comb-line (actualmente, muchos de estos dispositivos suelen diseñarse utilizando programas de simulación de propósito general ineficientes computacionalmente para el diseño de este tipo de estructuras).

Así pues, todos los hechos comentados anteriormente han motivado la realización de este trabajo de investigación. En este sentido, el objetivo fundamental de la presente tesis doctoral es la caracterización multimodal rigurosa de la excitación coaxial de dispositivos implementados en guía rectangular. Además, con el objetivo de que la herramienta de simulación implementada pueda ser posteriormente integrada en otras herramientas destinadas al diseño automatizado de dispositivos, es de vital importancia que el método de análisis utilizado sea muy eficiente desde un punto de vista computacional. Por este motivo, se ha optado por escoger una técnica de análisis multimodal basada en el método BI-RME 3D (Boundary Integral - Resonant Mode Expansion), el cual permite el análisis electromagnético eficiente de estructuras de geometría 3D arbitraria obteniendo una representación multimodal equivalente de las mismas en términos de una matriz de admitancias generalizada. De este modo, la técnica de análisis mencionada se empleará con el fin de obtener una caracterización multimodal rigurosa de dos tipos diferentes de excitación mediante sonda coaxial, ambas muy utilizadas en la práctica: la excitación coaxial estándar y la excitación coaxial en configuración colineal. Este último tipo de excitación, caracterizada por el hecho de que la guía coaxial se mantiene colineal con la guía rectangular a la que alimenta, se utiliza frecuentemente en la alimentación de los denominados filtros en configuración comb-line, los cuales son también objeto de análisis en esta tesis doctoral.

Por otra parte, con el fin de caracterizar de forma rigurosa la excitación coaxial de dispositivos implementados en guía rectangular, ha sido necesario extender la teoría del método BI-RME 3D para que se permita el análisis de dispositivos con puertos de acceso situados en planos ortogonales, siendo uno de dichos puertos de tipo coaxial. La razón de ello es que, hasta la fecha, el método BI-RME 3D se ha utilizado para caracterizar dispositivos con puertos de acceso de sección transversal rectangular situados en planos paralelos. Esta extensión del método BI-RME 3D, que constituye una de las principales aportaciones originales de este trabajo, ha permitido el análisis y diseño de diferentes tipos de uniones en guía rectangular (codos rectos y uniones en $\mathrm{T}$ en plano $H$ y en plano $E$, uniones en T mágica y uniones turnstile) compensadas con postes conductores de geometría cilíndrica.

Finalmente, es importante destacar que el análisis multimodal de la excitación coaxial de estructuras implementadas en guía rectangular ha requerido el cálculo de los coeficientes de acoplamiento modal entre las soluciones modales asociadas a una guía coaxial y las relativas a la guía rectangular a la que el coaxial alimenta. Este cálculo ha originado una sección de esta tesis doctoral dedicada al análisis eficiente de uniones planares complejas entre guías de onda de sección transversal arbitraria utilizando la técnica de la ecuación integral. Así por ejemplo, en dicha sección se estudian uniones planares entre dos guías coaxiales, entre una 
guía rectangular y una guía coaxial, y entre una guía rectangular y una guía circular (el análisis de este último tipo de unión resulta de interés para analizar filtros en configuración comb-line que incluyan irises de acoplamiento de sección transversal circular).

\subsection{Revisión Bibliográfica}

En este apartado se realiza una revisión bibliográfica breve de las principales aportaciones realizadas en los diferentes temas de investigación que se tratan en esta tesis doctoral. De esta manera, se ha optado por presentar una revisión bibliográfica más exhaustiva en los apartados de introducción de cada uno de los capítulos que integran este trabajo.

En relación con el análisis multimodal de uniones planares entre guías de onda de diferente sección transversal, merecen especial mención la técnica de adaptación modal [1], el denominado método directo [2], [3], el método de los elementos finitos [4] y la técnica de la ecuación integral [5], [6], [7]. Esta última, que es la que se ha escogido para caracterizar de manera rigurosa las diferentes uniones planares analizadas, es la más eficiente desde un punto de vista computacional, aunque es la que requiere un desarrollo teórico más complejo.

Por otro lado, en la literatura técnica pueden encontrarse numerosas contribuciones dedicadas al análisis y diseño de uniones compensadas en guía rectangular como las que son objeto de análisis en este trabajo. De entre ellas, destacamos las que se basan en obtener una matriz de admitancias generalizada o una matriz de dispersión generalizada equivalente que caracterice la unión bajo análisis [8], [9], [10]. En nuestro caso, se ha optado por implementar el método BI-RME 3D, dada su enorme flexibilidad y su elevada eficiencia computacional [11], [12].

Finalmente, en relación con la caracterización de la excitación coaxial de dispositivos implementados en tecnología guiada, es posible encontrar un elevado número de contribuciones dedicadas a mejorar el grado de adaptación entre la guía coaxial y el subsistema al que ésta alimenta [13], [14], [15], [16]. Sin embargo, existen muy pocas aportaciones que estudien el problema desde un punto de vista multimodal. Así por ejemplo, aunque en [17] y [18] se realiza un análisis riguroso de este tema, no se puede considerar que la caracterización realizada sea estrictamente multimodal ya que, en la guía coaxial, sólo se considera el modo fundamental TEM. Por otro lado, aunque en [19] sí se realiza un análisis multimodal de la excitación coaxial de dispositivos, la técnica empleada tiene la gran desventaja de ser poco eficiente desde un punto de vista computacional. En este trabajo de tesis, con el fin de utilizar una técnica de análisis rigurosa y eficiente, se ha optado por caracterizar la excitación coaxial utilizando el método BI-RME 3D.

\subsection{Estructura de la Tesis}

La presente tesis doctoral se encuentra dividida en seis capítulos. El primero de ellos es el presente capítulo de introducción, en el que se han destacado las principales motivaciones 
y objetivos de la tesis, y se ha realizado una breve revisión bibliográfica de los temas de investigación tratados en este trabajo.

El capítulo 2 se dedica al análisis multimodal eficiente de uniones planares complejas. En este capítulo se presenta, en primer lugar, la técnica de la ecuación integral utilizada para caracterizar uniones planares entre guías de onda de diferente sección transversal. Posteriormente, la técnica se aplica para analizar diferentes tipos de discontinuidades. En particular, se estudian discontinuidades entre guías rectangulares y guías coaxiales, entre guías rectangulares y guías circulares, discontinuidades entre guías coaxiales de diferente sección transversal, y uniones planares entre múltiples guías rectangulares. Por otro lado, con el fin de maximizar la eficiencia computacional del método implementado, se propone una técnica alternativa para calcular las soluciones modales de una guía coaxial y de una guía circular. En concreto, se propone expresar la variación radial de las soluciones modales relativas a los medios guiados mencionados, en términos de funciones sinusoidales que satisfacen las correspondientes condiciones de contorno. De esta manera, se evita el uso de las clásicas funciones de Bessel, con lo que se acelera el cálculo de las diferentes integrales numéricas que surgen al caracterizar este tipo de discontinuidades (en particular, aquellas discontinuidades en las que interviene la guía coaxial).

En el capítulo 3 se utiliza el método BI-RME 3D para el análisis riguroso y eficiente de uniones compensadas en guía rectangular. Así, en primer lugar, se realiza una revisión del método BI-RME 3D y, posteriormente, se presenta una generalización de dicho método que permite el análisis de uniones en guía rectangular que posean puertos de acceso situados en planos ortogonales. De esta manera, se posibilita el diseño de diferentes tipos de uniones en guía rectangular compensadas con postes conductores de geometría cilíndrica. En concreto, se diseñan codos rectos en plano $H$ y en plano $E$, uniones en $\mathrm{T}$ en plano $H$ y en plano $E$, uniones T mágica y uniones turnstile. Es importante destacar que en este capítulo, aparte de investigar cómo influyen el radio y la altura del poste conductor en la respuesta eléctrica de las diferentes uniones analizadas, se demuestra que la posición relativa del poste conductor en la estructura es un parámetro de diseño muy importante que, hasta ahora, no se había tenido en cuenta en la literatura técnica dedicada a este tema. Este nuevo parámetro de diseño permitirá la optimización de la respuesta eléctrica de muchas de las uniones estudiadas.

En el capítulo 4 se trata el análisis multimodal riguroso de la excitación coaxial de dispositivos en guía de ondas. Para abordar este objetivo, se utiliza el método BI-RME 3D. En este capítulo se analizan dos tipos de excitación mediante sonda coaxial muy utilizados en la práctica: la excitación coaxial clásica, y la excitación coaxial en configuración colineal, en la que la sonda coaxial permanece colineal con la guía rectangular a la que alimenta. Para ambos tipos de configuración, se detalla el análisis multimodal correspondiente y se presentan diferentes ejemplos de verificación que validan completamente la precisión y la eficiencia computacional de la técnica multimodal empleada. Por otro lado, la gran versatilidad del método BI-RME 3D utilizado permite considerar excitaciones mediante sondas coaxiales terminadas en disco, las cuales consiguen mejorar las pérdidas de retorno del dispositivo y aumentar el ancho de banda de adaptación. Finalmente, como ejemplo de aplicación, se presenta el diseño de un filtro inductivo implementado en guía rectangular excitado mediante 
sonda coaxial.

En el capítulo 5 se presenta una herramienta de simulación destinada al análisis eficiente de los denominados filtros en configuración comb-line. Este tipo de filtros, muy utilizados en diversas aplicaciones prácticas en el ámbito de las comunicaciones espaciales, están constituidos por la asociación de diferentes resonadores rectangulares cargados con postes conductores de geometría cilíndrica. Los diversos resonadores se acoplan entre sí mediante los correspondientes irises de acoplamiento, los cuales pueden ser de sección transversal rectangular o circular, y estar situados en una posición arbitraria. Por otro lado, este tipo de filtros suele estar alimentado por sondas coaxiales en configuración colineal que pueden contactar o no con el poste conductor presente en los resonadores comb-line. En este capítulo se analiza el caso en el que la sonda coaxial no llega a contactar con el poste conductor. Así pues, el objetivo de este capítulo consiste en la implementación de una herramienta de simulación eficiente que consiga analizar con precisión este tipo de filtros. La herramienta desarrollada permite considerar diferentes tipos de puertos de acceso (entre ellos, el de tipo coaxial), permite introducir tornillos metálicos de sintonía en los resonadores, así como considerar diferentes tipos de postes conductores (en forma de vaso o en forma de champiñón) además del poste clásico de geometría cilíndrica. La herramienta desarrollada se ha validado con diferentes ejemplos de aplicación, entre ellos, un filtro comb-line paso-banda de 4 resonadores que trabaja en banda $\mathrm{C}$.

Finalmente, en el capítulo 6 se recogen las conclusiones más relevantes del trabajo de investigación realizado, y se apuntan las líneas futuras de investigación que origina la presente tesis doctoral. Las publicaciones más relevantes relacionadas con la labor investigadora desarrollada en este trabajo se presentan en el Apéndice $\mathrm{H}$. 


\section{Capítulo 2}

\section{Contribuciones al Análisis Eficiente de Uniones Planares Complejas}

\subsection{Introducción}

En la actualidad, los sistemas de comunicaciones utilizados en diversas aplicaciones en las bandas de microondas y de ondas milimétricas (por ejemplo, sistemas radar, sistemas inalámbricos y sistemas embarcados para aplicaciones espaciales) están basados en una gran variedad de componentes implementados en tecnología guiada [20]. La gran mayoría de dichos componentes, a causa de unas especificaciones de diseño de tipo eléctrico y mecánico cada vez más restrictivas, se implementan mediante la conexión en cascada de guías de onda de sección transversal arbitraria [21]. Una forma eficiente de caracterizar electromagnéticamente este tipo componentes es mediante la denominada técnica de segmentación [22], [23], [24]. Este método permite el análisis eficiente de una estructura completa mediante la caracterización multimodal de cada uno de los bloques fundamentales que componen dicha estructura. En concreto, el análisis de un dispositivo basado en la conexión en cascada de diferentes guías de onda de cualquier sección transversal puede descomponerse en la caracterización parcial de cada una de las uniones planares y de los distintos tramos de guía uniforme que integran la estructura.

En consecuencia, el análisis electromagnético de cualquier dispositivo implementado en tecnología guiada debe partir, necesariamente, de la caracterización rigurosa y eficiente de las diferentes uniones planares que estén presentes en la estructura. Así por ejemplo, en un filtro en línea inductivo implementado en guía rectangular, encontramos uniones entre guías de onda de sección transversal rectangular [25]; en las denominadas uniones turnstile tenemos una discontinuidad planar entre una guía rectangular y una guía circular [10]; en los filtros implementados en tecnología guiada es bastante común encontrar irises de acoplamiento circulares, con lo que en este tipo de aplicaciones también encontraríamos discontinuidades entre guías rectangulares y guías circulares [26]; y en los filtros en modo dual implementados en guía circular podemos encontrar discontinuidades entre guías circulares de diferente 
sección transversal [27].

Así pues, el aspecto clave en la caracterización electromagnética de un gran número de dispositivos utilizados frecuentemente en diversas aplicaciones prácticas radica en una modelización rigurosa y eficiente de las diferentes discontinuidades que existan en la estructura. En este sentido, el estudio de las uniones planares entre guías de onda de cualquier sección transversal constituye un problema clásico en teoría de microondas. A pesar de que se ha realizado un enorme esfuerzo para desarrollar algoritmos de simulación muy eficientes durante los últimos años [7], existe todavía un gran interés en este área a medida que va aumentando la complejidad de los dispositivos y, paralelamente, se va requiriendo una mayor eficiencia computacional a la herramienta de simulación.

Una de las primeras aportaciones relevantes al análisis de las uniones planares fue realizada por Marcuvitz [28], quien caracterizó diferentes tipos de uniones en términos de redes circuitales equivalentes. La gran ventaja de este tipo de representación radica en la sencillez de la red circuital equivalente proporcionada. Sin embargo, es importante destacar que estas redes circuitales equivalentes únicamente se han derivado para el modo fundamental, con lo que resultan poco útiles para todas aquellas aplicaciones que requieran un tratamiento de tipo multimodal. Por otro lado, la mayor parte de los esfuerzos recientes dedicados al análisis de las uniones planares entre guías de onda arbitrarias se ha centrado en la representación de estas uniones mediante redes multimodales equivalentes. De esta manera, la discontinuidad que se produce en el plano de conexión de dos guías de onda de diferente sección transversal se modela mediante una red multimodal, en la que el acoplamiento electromagnético que se produce entre los diferentes modos asociados a las dos guías de la unión queda caracterizado mediante un conjunto de parámetros dados. Así por ejemplo, el método de la adaptación modal (en inglés, mode-matching) logra caracterizar la unión planar bajo análisis en términos de una red multimodal equivalente utilizando parámetros de dispersión [1]. Aunque la formulación teórica de este método no es excesivamente compleja, uno de sus grandes inconvenientes es el conocido problema de la convergencia relativa [29], [30], [31]. Otro método multimodal que merece ser destacado es el denominado método directo [2], [3], [32]. Esta técnica permite representar una unión planar entre dos guías de onda arbitrarias mediante una red equivalente multimodal formulada en términos de una matriz de admitancias generalizada. Para ello, el método se basa en la teoría general de líneas de transmisión. Si bien este método es sumamente riguroso y su formulación teórica es sencilla, su eficiencia computacional no es muy elevada y exige separar un acceso de referencia del plano de la unión.

Por otro lado, el método de los elementos finitos (en inglés, finite-element method) [4] puede también utilizarse para al análisis multimodal de uniones planares. Aunque la gran ventaja de esta técnica es su enorme versatilidad, su eficiencia computacional es bastante inferior a la que proporcionan los métodos multimodales comentados anteriormente en este apartado de introducción. Por último, otro método ampliamente utilizado en la caracterización electromagnética de uniones planares es la técnica de diferencias finitas en el dominio del tiempo (en inglés, FD-TD, Finite-Difference Time-Domain). Este método, cuya formulación original puede encontrarse en [33], se basa en la discretización de las ecuaciones 
diferenciales de Maxwell en los dominios espacial y temporal, estableciendo un conjunto de ecuaciones en diferencias para las seis componentes del campo electromagnético. A pesar de que esta técnica es muy versátil y ofrece una gran flexibilidad de análisis, su principal limitación vuelve a ser la escasa eficiencia computacional del método.

El objetivo de este capítulo es el análisis multimodal riguroso y eficiente de uniones planares complejas utilizando la técnica de la ecuación integral, cuya formulación se encuentra detallada en [5], [6], [7], [34]. Este método permite la representación de una unión planar en términos de una red multimodal equivalente basada en una matriz de impedancias generalizada. La gran aportación de esta nueva técnica radica en la distinción que introduce entre los denominados modos accesibles, que se utilizan para conectar transiciones, y los denominados modos localizados, usados para describir el campo electromagnético en la unión. Gracias a esta aportación, la red multimodal equivalente queda formulada, únicamente, en términos de los modos accesibles, reduciendo así enormemente el esfuerzo computacional asociado a la herramienta de simulación, pues la técnica logra resultados convergentes con un número reducido de modos accesibles. Por otra parte, el inconveniente más importante de esta técnica de análisis multimodal es la complejidad asociada a la formulación teórica del método.

En la siguiente sección, se presentan las diferentes contribuciones que este capítulo aporta a la técnica de la ecuación integral. Así, hasta la fecha, dicha técnica se había aplicado, típicamente, a la caracterización de uniones entre guías rectangulares. Sin embargo, en este capítulo se analizan de manera rigurosa diferentes tipos de uniones planares en las que intervienen también guías circulares y guías coaxiales. En concreto, las uniones planares que se estudian en este capítulo utilizando dicha técnica son las siguientes: discontinuidades entre guías rectangulares y guías coaxiales (sección 2.3), uniones entre guías rectangulares y guías circulares (sección 2.4), discontinuidades entre guías coaxiales (sección 2.5) y discontinuidades múltiples entre guías rectangulares (sección 2.6). Para cada una de estas uniones, se proporciona una herramienta multimodal eficiente y rigurosa que puede ser utilizada en herramientas más complejas destinadas al análisis y diseño de dispositivos de microondas y ondas milimétricas. Por otra parte, el análisis de las uniones planares en las que intervengan la guía circular o la guía coaxial, requerirá la obtención de la carta modal de este tipo de guías. Para ello, se presentará un técnica alternativa a la solución modal clásica en la que la variación radial de las soluciones modales se expresará en términos de funciones sinusoidales.

\subsection{Análisis de Uniones Planares mediante la Técnica de la Ecuación Integral}

Consideremos una unión planar entre dos guías de onda uniformes de sección transversal arbitraria, como la representada en la figura 2.1. En dicha figura se distinguen dos guías que consideraremos semi-infinitas y sin pérdidas: la guía (1), definida para $z<0$; y la guía (2), definida para $z>0$. La unión se encuentra en el plano $z=0$ y la sección transversal de la 
guía (2) está completamente contenida en la sección transversal de la guía (1). Además, se asume que en la región de la unión no existen pérdidas.

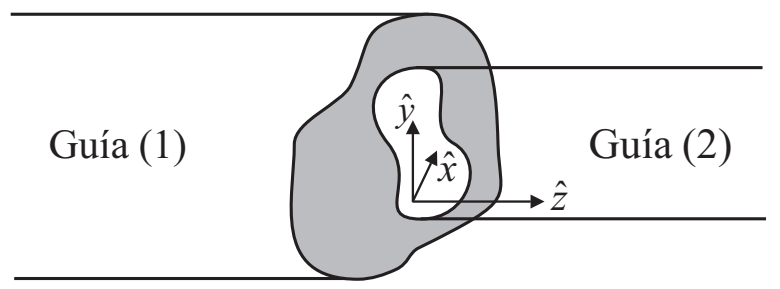

Figura 2.1: Unión planar entre dos guías de onda de sección transversal arbitraria.

El campo electromagnético transversal en el plano de la unión puede expresarse de la siguiente manera [28]:

$$
\begin{aligned}
& \mathbf{E}_{t}(x, y)=\sum_{m=1}^{\infty} V_{m} \mathbf{e}_{m}(x, y) \\
& \mathbf{H}_{t}(x, y)=\sum_{m=1}^{\infty} I_{m} \mathbf{h}_{m}(x, y)
\end{aligned}
$$

siendo $\mathbf{e}_{m}(x, y) \mathbf{y} \mathbf{h}_{m}(x, y)$, respectivamente, las funciones vectoriales modales normalizadas de campo eléctrico y de campo magnético relativas al modo $m$-ésimo; y $V_{m}$ e $I_{m}$ el voltaje y la corriente modal equivalentes asociados al $m$-ésimo modo de las guías en el plano de la unión, respectivamente, y que se definen como sigue:

$$
\begin{aligned}
& V_{m}=V_{m}^{+}+V_{m}^{-} \\
& I_{m}=I_{m}^{+}-I_{m}^{-}
\end{aligned}
$$

Obsérvese que la dependencia con la coordenada $z$ ha desaparecido en las expresiones (2.1)(2.4) por considerar que el plano de la unión es el plano $z=0$.

Asumamos ahora que el modo $p$-ésimo de la guía (1) incide sobre la unión planar, originándose una señal transmitida hacia la guía (2) y una señal reflejada en la guía (1). Teniendo en cuenta que el campo magnético transversal en el plano de la unión debe ser continuo, la consideración anterior implica que ${ }^{1}$ :

$$
I_{p}^{+(1)} \mathbf{h}_{p}^{(1)}-\sum_{m=1}^{\infty} I_{m}^{-(1)} \mathbf{h}_{m}^{(1)}=\sum_{m=1}^{\infty} I_{m}^{+(2)} \mathbf{h}_{m}^{(2)}
$$

Seguidamente, debemos introducir el concepto de modos accesibles y modos localizados. Los modos accesibles son aquellos que se utilizan para conectar transiciones y son los primeros modos de cada guía, incluyendo todos los modos propagativos y los primeros modos

\footnotetext{
${ }^{1}$ Por simplicidad en la notación, de aquí en adelante omitiremos la dependencia con $(x, y)$ de las funciones vectoriales modales normalizadas.
} 
evanescentes. Por tanto, los modos accesibles definen el rango de la matriz de impedancias generalizada que caracteriza la unión planar. Por su parte, los modos localizados son el resto de modos excitados en la unión. Todos ellos son de tipo evanescente y su contribución es relevante en el análisis electromagnético de la unión, pero no lo es en la conexión con las guías de onda adyacentes a la unión analizada.

Así pues, sea $N^{(1)}$ el número de modos accesibles considerados en la guía (1), y sea $N^{(2)}$ el número de modos accesibles tomados en la guía (2). Si ahora recuperamos la expresión (2.5) y separamos los modos accesibles de los localizados obtendremos que ${ }^{2}$ :

$I_{p}^{+(1)} \mathbf{h}_{p}^{(1)}-\sum_{m=1}^{N^{(1)}} I_{m}^{-(1)} \mathbf{h}_{m}^{(1)}-\sum_{m=N^{(1)}+1}^{\infty} Y_{m}^{(1)} V_{m}^{-(1)} \mathbf{h}_{m}^{(1)}=\sum_{m=1}^{N^{(2)}} I_{m}^{+(2)} \mathbf{h}_{m}^{(2)}+\sum_{m=N^{(2)}+1}^{\infty} Y_{m}^{(2)} V_{m}^{+(2)} \mathbf{h}_{m}^{(2)}$

donde $Y_{m}^{(\xi)}(\operatorname{con} \xi=1,2)$ representa la admitancia modal característica del $m$-ésimo modo de la guía $(\xi)$ definida como sigue:

$$
Y_{m}^{(\xi)}=\frac{I_{m}^{+(\xi)}}{V_{m}^{+(\xi)}}=\frac{I_{m}^{-(\xi)}}{V_{m}^{-(\xi)}} \quad(\xi)=(1),(2)
$$

Por otro lado, es importante destacar que en la guía (2) únicamente existen modos transmitidos, por lo que podemos afirmar que:

$$
I_{m}^{+(2)}=I_{m}^{(2)} \quad V_{m}^{+(2)}=V_{m}^{(2)}
$$

Igualmente, todos los modos que se excitan en la guía (1), a excepción del modo $p$-ésimo, son modos reflejados. Por ello, podemos escribir que:

$$
\begin{aligned}
I_{p}^{(1)}= & I_{p}^{+(1)}-I_{p}^{-(1)}, \quad & V_{p}^{(1)}=V_{p}^{+(1)}+V_{p}^{-(1)} ; \quad m=p \\
& -I_{m}^{-(1)}=I_{m}^{(1)}, \quad & V_{m}^{-(1)}=V_{m}^{(1)} ; \quad \forall m \neq p
\end{aligned}
$$

Con todo ello, la ecuación (2.6) puede reescribirse como:

$$
\sum_{m=1}^{N^{(1)}} I_{m}^{(1)} \mathbf{h}_{m}^{(1)}-\sum_{m=N^{(1)}+1}^{\infty} Y_{m}^{(1)} V_{m}^{(1)} \mathbf{h}_{m}^{(1)}=\sum_{m=1}^{N^{(2)}} I_{m}^{(2)} \mathbf{h}_{m}^{(2)}+\sum_{m=N^{(2)}+1}^{\infty} Y_{m}^{(2)} V_{m}^{(2)} \mathbf{h}_{m}^{(2)}
$$

Seguidamente se introduce el concepto de admitancia modal asintótica $\hat{Y}_{m}^{(\xi)}$, la cual representa la admitancia modal de los modos de orden superior, es decir, de la gran parte de los modos localizados:

$$
\hat{Y}_{m}^{(\xi)}=\lim _{\frac{k}{k_{t, m}^{(\xi)} \rightarrow 0}} Y_{m}^{(\xi)} \approx \begin{cases}-j \frac{k_{t, m}^{(\xi)}}{\omega \mu} & \text { si } m \text { es un modo } \mathrm{TE}^{z} \\ j \frac{\omega \varepsilon}{k_{t, m}^{(\xi)}} & \text { si } m \text { es un modo } \mathrm{TM}^{z}\end{cases}
$$

\footnotetext{
${ }^{2}$ En lo que sigue se asume que el modo $p$-ésimo incidente es un modo accesible de la guía (1).
} 
En la expresión anterior, $\omega=2 \pi f$ es la pulsación angular; $k_{t, m}^{(\xi)}$ representa el número de onda de corte asociado al $m$-ésimo modo de la guía $(\xi)$; y $k$ es el número de onda del medio que rellena uniformemente la guía, $k=\omega \sqrt{\mu \varepsilon}$. Ahora, definimos las siguientes corrientes modales $^{3}$ :

$$
\begin{aligned}
& \bar{I}_{m}^{(1)}=I_{m}^{(1)}+\hat{Y}_{m}^{(1)} V_{m}^{(1)} \\
& \bar{I}_{m}^{(2)}=I_{m}^{(2)}-\hat{Y}_{m}^{(2)} V_{m}^{(2)}
\end{aligned}
$$

Si ahora sumamos a cada miembro de (2.11) la expresión:

$$
\sum_{m=1}^{N^{(1)}} V_{m}^{(1)} \hat{Y}_{m}^{(1)} \mathbf{h}_{m}^{(1)}-\sum_{m=1}^{N^{(2)}} V_{m}^{(2)} \hat{Y}_{m}^{(2)} \mathbf{h}_{m}^{(2)}
$$

y tenemos en cuenta las nuevas corrientes definidas en (2.13)-(2.14), la ecuación (2.11) se transforma en:

$$
\begin{aligned}
\sum_{m=1}^{N^{(1)}} \bar{I}_{m}^{(1)} \mathbf{h}_{m}^{(1)}-\sum_{m=1}^{N^{(2)}} \bar{I}_{m}^{(2)} \mathbf{h}_{m}^{(2)}= & \sum_{m=N^{(1)}+1}^{\infty} Y_{m}^{(1)} V_{m}^{(1)} \mathbf{h}_{m}^{(1)}+\sum_{m=N^{(2)}+1}^{\infty} Y_{m}^{(2)} V_{m}^{(2)} \mathbf{h}_{m}^{(2)} \\
& +\sum_{m=1}^{N^{(1)}} \hat{Y}_{m}^{(1)} V_{m}^{(1)} \mathbf{h}_{m}^{(1)}+\sum_{m=1}^{N^{(2)}} \hat{Y}_{m}^{(2)} V_{m}^{(2)} \mathbf{h}_{m}^{(2)}
\end{aligned}
$$

El campo electromagnético transversal en la unión $\mathbf{E}_{t}\left(x^{\prime}, y^{\prime}\right)$ es desconocido, pudiéndose asegurar por continuidad que:

$$
\mathbf{E}_{t}\left(x^{\prime}, y^{\prime}\right)=\sum_{m=1}^{\infty} V_{m}^{(1)} \mathbf{e}_{m}^{(1)}\left(x^{\prime}, y^{\prime}\right)=\sum_{m=1}^{\infty} V_{m}^{(2)} \mathbf{e}_{m}^{(2)}\left(x^{\prime}, y^{\prime}\right)
$$

donde los voltajes modales equivalentes $V_{m}^{(\xi)}$ pueden expresarse como [28]:

$$
\begin{aligned}
V_{m}^{(\xi)} & =\int_{S^{(2)}} \mathbf{E}_{t}\left(x^{\prime}, y^{\prime}\right) \cdot \mathbf{e}_{m}^{(\xi) *}\left(x^{\prime}, y^{\prime}\right) d S^{\prime}=\int_{S^{(2)}} \mathbf{E}_{t}\left(x^{\prime}, y^{\prime}\right) \cdot\left(\mathbf{h}_{m}^{(\xi)}\left(x^{\prime}, y^{\prime}\right) \times \hat{\mathbf{z}}\right)^{*} d S^{\prime} \\
& =\int_{S^{(2)}}\left(\hat{\mathbf{z}} \times \mathbf{E}_{t}\left(x^{\prime}, y^{\prime}\right)\right) \cdot \mathbf{h}_{m}^{(\xi) *}\left(x^{\prime}, y^{\prime}\right) d S^{\prime}
\end{aligned}
$$

siendo $(\xi)=(1),(2)$. Además, es importante destacar que las integrales de superficie anteriores se restringen a la sección transversal $S^{(2)}$ de la guía (2) ya que, tal y como se ha apuntado anteriormente, dicha sección transversal está contenida completamente en la de la

\footnotetext{
${ }^{3}$ Resulta sencillo comprobar que las corrientes $\bar{I}_{m}^{(1)}$ y $\bar{I}_{m}^{(2)}$ tienden a cero cuando $m$ es un modo de orden superior (modo localizado). Esto implica que estas nuevas corrientes sólo tienen sentido para los modos accesibles.
} 
guía $(1)^{4}$. Por otra parte, resulta de especial relevancia hacer notar que se ha forzado a que las funciones vectoriales presentes en la expresión anterior (2.18) dependan de variables primadas, para evitar así futuras confusiones en la notación cuando las relaciones que se deduzcan posteriormente sean utilizadas en la ecuación (2.11) deducida con anterioridad.

A continuación, se expande el campo $\hat{\mathbf{z}} \times \mathbf{E}_{t}\left(x^{\prime}, y^{\prime}\right)$ en términos de una combinación lineal de modos accesibles de las guías (1) y (2):

$$
\hat{\mathbf{z}} \times \mathbf{E}_{t}\left(x^{\prime}, y^{\prime}\right) \approx \sum_{n=1}^{N^{(1)}} \bar{I}_{n}^{(1)} \mathbf{M}_{n}^{(1)}\left(x^{\prime}, y^{\prime}\right)-\sum_{n=1}^{N^{(2)}} \bar{I}_{n}^{(2)} \mathbf{M}_{n}^{(2)}\left(x^{\prime}, y^{\prime}\right)
$$

donde $\mathbf{M}_{n}^{(1)}\left(x^{\prime}, y^{\prime}\right)$ y $\mathbf{M}_{n}^{(2)}\left(x^{\prime}, y^{\prime}\right)$ son funciones vectoriales desconocidas. Si ahora actualizamos la expresión (2.18) con la nueva expansión indicada en (2.19), e introducimos el resultado en la ecuación (2.11) cambiando la expresión de $V_{m}^{(\xi)}$ que allí aparece por la nueva relación deducida, es posible escribir la siguiente ecuación integral de Fredholm de primera especie:

$$
\begin{aligned}
\mathbf{h}_{n}^{(\gamma)}(x, y)= & \int_{S^{(2)}} \mathbf{M}_{n}^{(\gamma)}\left(x^{\prime}, y^{\prime}\right)\left[\sum_{m=1}^{N^{(1)}} \hat{Y}_{m}^{(1)} \mathbf{h}_{m}^{(1)}(x, y) \cdot \mathbf{h}_{m}^{(1) *}\left(x^{\prime}, y^{\prime}\right)\right. \\
& +\sum_{m=N^{(1)}+1}^{\infty} Y_{m}^{(1)} \mathbf{h}_{m}^{(1)}(x, y) \cdot \mathbf{h}_{m}^{(1) *}\left(x^{\prime}, y^{\prime}\right)+\sum_{m=1}^{N^{(2)}} \hat{Y}_{m}^{(2)} \mathbf{h}_{m}^{(2)}(x, y) \cdot \mathbf{h}_{m}^{(2) *}\left(x^{\prime}, y^{\prime}\right) \\
& \left.+\sum_{m=N^{(2)}+1}^{\infty} Y_{m}^{(2)} \mathbf{h}_{m}^{(2)}(x, y) \cdot \mathbf{h}_{m}^{(2) *}\left(x^{\prime}, y^{\prime}\right)\right] d S^{\prime} \quad(\gamma)=(1),(2)
\end{aligned}
$$

La expresión anterior es una ecuación integral de dos variables cuya incógnita es la función vectorial $\mathbf{M}_{n}^{(\gamma)}\left(x^{\prime}, y^{\prime}\right)$. Además, de forma compacta, dicha ecuación puede reescribirse como:

$$
\mathbf{h}_{n}^{(\gamma)}(x, y)=\int_{S^{(2)}} K\left(x, y, x^{\prime}, y^{\prime}\right) \mathbf{M}_{n}^{(\gamma)}\left(x^{\prime}, y^{\prime}\right) d S^{\prime}
$$

donde $K\left(x, y, x^{\prime}, y^{\prime}\right)$ es el núcleo de la ecuación integral, que se corresponde con el término entre corchetes de (2.20). Una vez que hayamos resuelto la ecuación integral anterior, es importante observar que, partiendo de (2.18), es posible encontrar una relación entre los voltajes modales $V_{m}^{(\xi)}$ y las corrientes modales $\bar{I}_{m}^{(\xi)}$, permitiendo así caracterizar la unión planar a través de una matriz de impedancias generalizada (MIG) definida para los modos accesibles considerados. En efecto:

$V_{m}^{(\xi)}=\sum_{n=1}^{N^{(1)}} \bar{I}_{n}^{(1)} \int_{S^{(2)}} \mathbf{M}_{n}^{(1)}\left(x^{\prime}, y^{\prime}\right) \cdot \mathbf{h}_{m}^{(\xi) *}\left(x^{\prime}, y^{\prime}\right) d S^{\prime}-\sum_{n=1}^{N^{(2)}} \bar{I}_{n}^{(2)} \int_{S^{(2)}} \mathbf{M}_{n}^{(2)}\left(x^{\prime}, y^{\prime}\right) \cdot \mathbf{h}_{m}^{(\xi) *}\left(x^{\prime}, y^{\prime}\right) d S^{\prime}$

\footnotetext{
${ }^{4}$ Puesto que las guías no tienen pérdidas, el campo $\mathbf{E}_{t}\left(x^{\prime}, y^{\prime}\right)$ es nulo fuera de la zona de unión entre las dos guías.
} 
De esta manera, la MIG de la unión planar queda definida de la siguiente manera:

$$
Z_{m, n}^{(\xi, \gamma)}=\int_{S^{(2)}} \mathbf{M}_{n}^{(\gamma)}\left(x^{\prime}, y^{\prime}\right) \cdot \mathbf{h}_{m}^{(\xi) *}\left(x^{\prime}, y^{\prime}\right) d S^{\prime}
$$

con $(\gamma),(\xi)=1,2$. Así pues, finalmente, podemos expresar (2.22) como:

$$
V_{m}^{(\xi)}=\sum_{n=1}^{N^{(1)}} \bar{I}_{n}^{(1)} Z_{m, n}^{(\xi, 1)}+\sum_{n=1}^{N^{(2)}}\left(-\bar{I}_{n}^{(2)}\right) Z_{m, n}^{(\xi, 2)} \quad m=1, \ldots, N^{(\xi)}
$$

La red multimodal equivalente caracterizada por la ecuación (2.22) se ha representado en la figura 2.2.

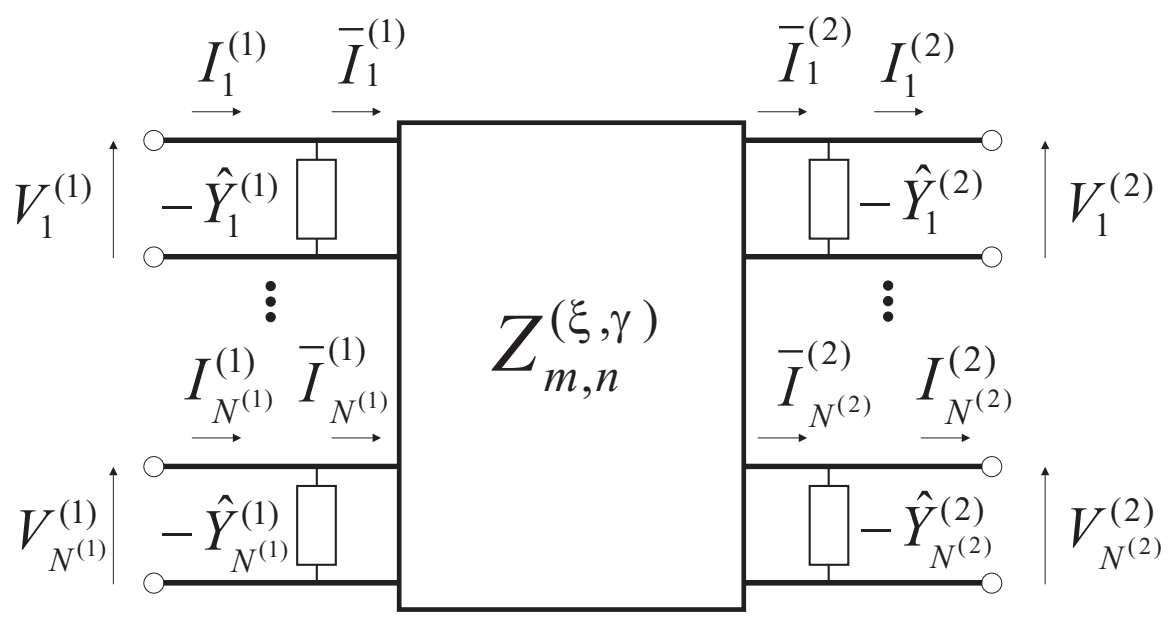

Figura 2.2: Red multimodal equivalente que caracteriza a una unión planar entre dos guías de onda de sección transversal arbitraria mediante la técnica de la ecuación integral.

\subsubsection{Aceleración del cálculo del núcleo de la ecuación integral}

Con el fin de incrementar la eficiencia computacional del algoritmo, el cálculo del núcleo $K\left(x, y, x^{\prime}, y^{\prime}\right)$ de la ecuación integral (2.21) se puede acelerar extrayendo la dependencia con la frecuencia que dicho núcleo presenta. Con ello se pretende que una parte importante de los cálculos se realice fuera del bucle en frecuencia del código implementado. Para lograr este objetivo, se parte del término entre corchetes de (2.20) y se reescribe de la siguiente 
manera ${ }^{5}$ :

$$
\begin{aligned}
K\left(x, y, x^{\prime}, y^{\prime}\right)= & \sum_{m=1}^{\infty} \hat{Y}_{m}^{(1)} \mathbf{h}_{m}^{(1)}(x, y) \cdot \mathbf{h}_{m}^{(1) *}\left(x^{\prime}, y^{\prime}\right) \\
& -\sum_{m=N^{(1)}+1}^{\infty} \hat{Y}_{m}^{(1)}\left(1-\frac{Y_{m}^{(1)}}{\hat{Y}_{m}^{(1)}}\right) \mathbf{h}_{m}^{(1)}(x, y) \cdot \mathbf{h}_{m}^{(1) *}\left(x^{\prime}, y^{\prime}\right) \\
& +\sum_{m=1}^{\infty} \hat{Y}_{m}^{(2)} \mathbf{h}_{m}^{(2)}(x, y) \cdot \mathbf{h}_{m}^{(2) *}\left(x^{\prime}, y^{\prime}\right) \\
& -\sum_{m=N^{(2)}+1}^{\infty} \hat{Y}_{m}^{(2)}\left(1-\frac{Y_{m}^{(2)}}{\hat{Y}_{m}^{(2)}}\right) \mathbf{h}_{m}^{(2)}(x, y) \cdot \mathbf{h}_{m}^{(2) *}\left(x^{\prime}, y^{\prime}\right)
\end{aligned}
$$

En esta nueva expresión del núcleo se observa que la primera y la tercera serie presentan una dependencia explícita con la frecuencia (recuerde la expresión (2.12) para las admitancias modales asintóticas). Este aspecto resulta muy ventajoso desde un punto de vista computacional, ya que, inicialmente, puede calcularse una versión estática (independiente de la frecuencia) de dichas series fuera del bucle en frecuencia y, posteriormente, ya en el bucle, recuperar la dependencia frecuencial de las series atendiendo a la expresión $(2.12)^{6}$.

Por otro lado, en la segunda y en la cuarta serie de (2.25), encontramos un término que cumple la siguiente propiedad:

$$
\lim _{m \rightarrow \infty}\left(1-\frac{Y_{m}^{(\xi)}}{\hat{Y}_{m}^{(\xi)}}\right)=0
$$

ya que, por definición, la admitancia modal característica es igual a la asintótica a medida que el orden del modo considerado tiende a infinito. Este hecho posibilita la aproximación del término anterior mediante un desarrollo en serie de Taylor rápidamente convergente. En particular se tiene que:

$$
1-\frac{Y_{m}^{(\xi)}}{\hat{Y}_{m}^{(\xi)}}=\left\{\begin{array}{c}
1-\sqrt{1-\left(\frac{k}{k_{t, m}^{(\xi)}}\right)^{2}} \text { si } m \text { es un modo } \mathrm{TE}^{z} \\
1-\frac{1}{\sqrt{1-\left(\frac{k}{k_{t, m}^{(\xi)}}\right)^{2}}} \text { si } m \text { es un modo } \mathrm{TM}^{z}
\end{array}\right.
$$

\footnotetext{
${ }^{5}$ Observe que, en la expresión (2.25), la primera y la tercera serie del núcleo de la ecuación integral (2.20) se han completado hasta infinito, restando después los términos añadidos.

${ }^{6}$ Es interesante observar que cada una de las series estáticas presentes en (2.25) se debe descomponer, a su vez, en otras dos series estáticas. Una de ellas será la relativa a los modos $\mathrm{TE}^{z}$ y la otra será la asociada a los modos $\mathrm{TM}^{z}$.
} 
Así pues, el desarrollo en serie de Taylor de la función anterior permite expresar que ${ }^{7}$ :

$$
1-\frac{Y_{m}^{(\xi)}}{\hat{Y}_{m}^{(\xi)}} \approx \sum_{r=1}^{R} \beta_{r, m}\left(\frac{k}{k_{t, m}^{(\xi)}}\right)^{2 r}
$$

En la tabla 2.1 se presentan los valores que adopta el coeficiente de expansión de Taylor $\beta_{r, m}$ para $r=1,2,3$ en función de que $m$ sea un modo de tipo $\mathrm{TE}^{z}$ o $\mathrm{TM}^{z}$.

\begin{tabular}{|c|c|c|}
\hline \hline$r$ & $\mathrm{TE}^{z}$ & $\mathrm{TM}^{z}$ \\
\hline 1 & $1 / 2$ & $-1 / 2$ \\
\hline 2 & $1 / 8$ & $-3 / 8$ \\
\hline 3 & $1 / 16$ & $-5 / 16$ \\
\hline
\end{tabular}

Tabla 2.1: Valores de los coeficientes $\beta_{r, m}(r=1,2,3)$ para los modos $\mathrm{TE}^{z}$ y $\mathrm{TM}^{z}$.

Con todo ello, el núcleo de la ecuación integral podemos reescribirlo de la siguiente manera:

$$
K\left(x, y, x^{\prime}, y^{\prime}\right)=\hat{K}\left(x, y, x^{\prime}, y^{\prime}\right)-\sum_{r=1}^{R} k^{2 r} \hat{\tilde{K}}\left(x, y, x^{\prime}, y^{\prime}\right)
$$

donde:

$$
\begin{aligned}
\hat{K}\left(x, y, x^{\prime}, y^{\prime}\right)= & \sum_{m=1}^{\infty} \hat{Y}_{m}^{(1)} \mathbf{h}_{m}^{(1)}(x, y) \cdot \mathbf{h}_{m}^{(1) *}\left(x^{\prime}, y^{\prime}\right)+\sum_{m=1}^{\infty} \hat{Y}_{m}^{(2)} \mathbf{h}_{m}^{(2)}(x, y) \cdot \mathbf{h}_{m}^{(2) *}\left(x^{\prime}, y^{\prime}\right) \\
\hat{\tilde{K}}\left(x, y, x^{\prime}, y^{\prime}\right)= & \sum_{m=N^{(1)}+1}^{\infty} \hat{Y}_{m}^{(1)} \beta_{r, m} \frac{1}{\left(k_{t, m}^{(1)}\right)^{2 r}} \mathbf{h}_{m}^{(1)}(x, y) \cdot \mathbf{h}_{m}^{(1) *}\left(x^{\prime}, y^{\prime}\right) \\
& +\sum_{m=N^{(2)}+1}^{\infty} \hat{Y}_{m}^{(2)} \beta_{r, m} \frac{1}{\left(k_{t, m}^{(2)}\right)^{2 r}} \mathbf{h}_{m}^{(2)}(x, y) \cdot \mathbf{h}_{m}^{(2) *}\left(x^{\prime}, y^{\prime}\right)
\end{aligned}
$$

Es importante destacar que estas dos nuevas componentes del núcleo son estáticas en el sentido de que presentan una dependencia explícita con la frecuencia. En consecuencia, su cálculo efectivo se puede trasladar fuera del bucle en frecuencia del código implementado, optimizando así la eficiencia computacional global del método.

\footnotetext{
${ }^{7}$ Observe que los coeficientes del desarrollo en serie de Taylor $\beta_{r, m}$ no dependen de la guía ( $\xi$ ) considerada. Además, el cálculo de estos coeficientes - que, por ejemplo, puede llevarse a cabo utilizando el programa comercial Mathematica 3.0 - revela que los coeficientes ligados a las potencias impares son nulos. Por otro lado, en un caso práctico, el número de términos $R=3$ suele proporcionar resultados convergentes.
} 


\subsubsection{Resolución de la ecuación integral planteada}

En virtud de los resultados obtenidos en la sección anterior, la ecuación integral a resolver presenta el siguiente aspecto:

$$
\mathbf{h}_{n}^{(\gamma)}(x, y)=\int_{S^{(2)}}\left[\hat{K}\left(x, y, x^{\prime}, y^{\prime}\right)-\sum_{r=1}^{R} k^{2 r} \hat{\tilde{K}}\left(x, y, x^{\prime}, y^{\prime}\right)\right] \mathbf{M}_{n}^{(\gamma)}\left(x^{\prime}, y^{\prime}\right) d S^{\prime}
$$

De manera equivalente, la ecuación integral anterior puede expresarse en términos del siguiente operador integral $\mathcal{L}$ :

$$
\mathcal{L} \mathbf{v}\left(x^{\prime}, y^{\prime}\right)=\int_{S^{(2)}}\left[\hat{K}\left(x, y, x^{\prime}, y^{\prime}\right)-\sum_{r=1}^{R} k^{2 r} \hat{\tilde{K}}\left(x, y, x^{\prime}, y^{\prime}\right)\right] \mathbf{v}\left(x^{\prime}, y^{\prime}\right) d S^{\prime}
$$

donde $\mathbf{v}\left(x^{\prime}, y^{\prime}\right)$ es una función vectorial arbitraria. Entonces, la ecuación integral puede ahora escribirse como:

$$
\mathcal{L} \mathbf{M}_{n}^{(\gamma)}\left(x^{\prime}, y^{\prime}\right)=\mathbf{h}_{n}^{(\gamma)}(x, y)
$$

Para resolver la ecuación integral anterior utilizaremos el método de los momentos, el cual logra transformar la ecuación integral bajo análisis en un problema matricial lineal equivalente. En particular trabajaremos con el denominado procedimiento de Galerkin. Según este método, en primer lugar, la función incógnita debe expandirse en términos de un conjunto completo de funciones base. En este caso, las funciones base escogidas se corresponden con las funciones vectoriales modales de campo magnético de la guía $(2)^{8}$ :

$$
\mathbf{M}_{n}^{(\gamma)}\left(x^{\prime}, y^{\prime}\right) \approx \sum_{q=1}^{Q^{(\gamma)}} \alpha_{q}^{(n, \gamma)} \mathbf{h}_{q}^{(2)}\left(x^{\prime}, y^{\prime}\right)
$$

donde $Q^{(\gamma)}$ es el número de funciones base escogidas en cada guía y $\alpha_{q}^{(n, \gamma)}$ son unos coeficientes de expansión desconocidos. Si aplicamos a continuación el método de Galerkin a la expresión (2.33), debemos ponderar cada uno de los miembros de dicha expresión por las funciones base elegidas, por lo que tendremos que:

$$
\begin{aligned}
\sum_{q=1}^{Q^{(\gamma)}} \alpha_{q}^{(n, \gamma)} \mathcal{L} \mathbf{h}_{q}^{(2)}\left(x^{\prime}, y^{\prime}\right) & \approx \mathbf{h}_{n}^{(\gamma)}(x, y) \\
\sum_{q=1}^{Q^{(\gamma)}} \alpha_{q}^{(n, \gamma)}\left\langle\mathcal{L} \mathbf{h}_{q}^{(2)}\left(x^{\prime}, y^{\prime}\right) \mid \mathbf{h}_{p}^{(2)}\left(x^{\prime}, y^{\prime}\right)\right\rangle & \approx\left\langle\mathbf{h}_{n}^{(\gamma)}(x, y) \mid \mathbf{h}_{p}^{(2)}(x, y)\right\rangle
\end{aligned}
$$

\footnotetext{
${ }^{8}$ Del análisis de la ecuación (2.19) se desprende que $\hat{\mathbf{z}} \times \mathbf{E}_{t}\left(x^{\prime}, y^{\prime}\right)$ tiene forma de campo magnético. Además, $\mathbf{E}_{t}\left(x^{\prime}, y^{\prime}\right)$ debe ser nulo fuera de la zona de unión de las dos guías. De esta forma, la elección de las funciones base queda completamente justificada.
} 
En esta última expresión, $p=1, \ldots, Q^{(\gamma)} ; \mathrm{y}\langle\mathbf{a} \mid \mathbf{b}\rangle$ representa el siguiente producto interno:

$$
\langle\mathbf{a} \mid \mathbf{b}\rangle=\int_{S^{(2)}} \mathbf{a} \cdot \mathbf{b}^{*} d S
$$

El miembro de la derecha de (2.36) se puede calcular fácilmente de la siguiente manera:

$$
\left\langle\mathbf{h}_{n}^{(\gamma)}(x, y) \mid \mathbf{h}_{p}^{(2)}(x, y)\right\rangle=\left\{\begin{array}{cc}
\int_{S^{(2)}} \mathbf{h}_{n}^{(1)}(x, y) \cdot \mathbf{h}_{p}^{(2) *}(x, y) d S & \text { si }(\gamma)=(1) \\
\delta_{n, p} & \text { si }(\gamma)=(2)
\end{array}\right.
$$

donde $\delta_{n, p}$ representa la conocida delta de Kronecker:

$$
\delta_{n, p}= \begin{cases}1 & \text { si } n=p \\ 0 & \text { si } n \neq p\end{cases}
$$

Por otro lado, observamos que en el miembro de la izquierda de (2.36) debe calcularse la expresión $\mathcal{L} \mathbf{h}_{q}^{(2)}\left(x^{\prime}, y^{\prime}\right)$. Así, teniendo en cuenta (2.30) y (2.31) se deduce que:

$$
\begin{aligned}
\mathcal{L} \mathbf{h}_{q}^{(2)}\left(x^{\prime}, y^{\prime}\right)= & \int_{S^{(2)}}\left(\sum_{m=1}^{\infty} \hat{Y}_{m}^{(1)} \mathbf{h}_{m}^{(1)}(x, y) \cdot \mathbf{h}_{m}^{(1) *}\left(x^{\prime}, y^{\prime}\right)\right. \\
& \left.+\sum_{m=1}^{\infty} \hat{Y}_{m}^{(2)} \mathbf{h}_{m}^{(2)}(x, y) \cdot \mathbf{h}_{m}^{(2) *}\left(x^{\prime}, y^{\prime}\right)\right) \mathbf{h}_{q}^{(2)}\left(x^{\prime}, y^{\prime}\right) d S^{\prime} \\
& -\sum_{r=1}^{R} k^{2 r} \int_{S^{(2)}}\left(\sum_{m=N^{(1)}+1}^{\infty} \hat{Y}_{m}^{(1)} \beta_{r, m} \frac{1}{\left(k_{t, m}^{(1)}\right)^{2 r}} \mathbf{h}_{m}^{(1)}(x, y) \cdot \mathbf{h}_{m}^{(1) *}\left(x^{\prime}, y^{\prime}\right)\right. \\
& \left.+\sum_{m=N^{(2)}+1}^{\infty} \hat{Y}_{m}^{(2)} \beta_{r, m} \frac{1}{\left(k_{t, m}^{(2)}\right)^{2 r}} \mathbf{h}_{m}^{(2)}(x, y) \cdot \mathbf{h}_{m}^{(2) *}\left(x^{\prime}, y^{\prime}\right)\right) \mathbf{h}_{q}^{(2)}\left(x^{\prime}, y^{\prime}\right) d S^{\prime}
\end{aligned}
$$

A continuación, definimos la integral de acoplamiento entre los modos $m$-ésimo y $q$-ésimo de las guías involucradas en la unión planar como:

$$
A_{m, q}=\int_{S^{(2)}} \mathbf{h}_{m}^{(1)}\left(x^{\prime}, y^{\prime}\right) \cdot \mathbf{h}_{q}^{(2) *}\left(x^{\prime}, y^{\prime}\right) d S^{\prime}
$$


Así pues, la expresión (2.40) se puede reescribir de la siguiente manera:

$$
\begin{aligned}
\mathcal{L} \mathbf{h}_{q}^{(2)}\left(x^{\prime}, y^{\prime}\right)= & \sum_{m=1}^{\infty} \hat{Y}_{m}^{(1)} A_{m, q}^{*} \mathbf{h}_{m}^{(1)}(x, y)+\hat{Y}_{q}^{(2)} \mathbf{h}_{q}^{(2)}(x, y) \\
& -\sum_{r=1}^{R} k^{2 r} \sum_{m=N^{(1)}+1}^{\infty} \hat{Y}_{m}^{(1)} \beta_{r, m} \frac{1}{\left(k_{t, m}^{(1)}\right)^{2 r}} A_{m, q}^{*} \mathbf{h}_{m}^{(1)}(x, y) \\
& -\sum_{r=1}^{R} k^{2 r} \hat{Y}_{q}^{(2)} \beta_{r, q} \frac{1}{\left(k_{t, q}^{(2)}\right)^{2 r}} \mathbf{h}_{q}^{(2)}(x, y) F_{q}
\end{aligned}
$$

donde hemos definido:

$$
F_{q}= \begin{cases}0 & \text { si } q<N^{(2)}+1 \\ 1 & \text { si } q \geq N^{(2)}+1\end{cases}
$$

Finalmente, el producto interno presente en el miembro de la izquierda de (2.36) se calcula como:

$$
\begin{aligned}
\left\langle\mathcal{L} \mathbf{h}_{q}^{(2)}\left(x^{\prime}, y^{\prime}\right) \mid \mathbf{h}_{p}^{(2)}\left(x^{\prime}, y^{\prime}\right)\right\rangle= & \sum_{m=1}^{\infty} \hat{Y}_{m}^{(1)} A_{m, q}^{*} A_{m, p}+\hat{Y}_{q}^{(2)} \delta_{p, q} \\
& -\sum_{r=1}^{R} k^{2 r} \sum_{N^{(1)}+1}^{\infty} \hat{Y}_{m}^{(1)} \beta_{r, m} \frac{1}{\left(k_{t, m}^{(1)}\right)^{2 r}} A_{m, q}^{*} A_{m, p} \\
& -\sum_{r=1}^{R} k^{2 r} \hat{Y}_{q}^{(2)} \beta_{r, q} \frac{1}{\left(k_{t, q}^{(2)}\right)^{2 r}} F_{q} \delta_{p, q}
\end{aligned}
$$

siendo $\delta_{p, q}$ la delta de Kronecker.

Llegados a este punto, podemos transformar (2.36) en un problema matricial lineal equivalente. Para ello, definimos las siguientes matrices:

$$
\begin{aligned}
B_{p, q} & =\left\langle\mathcal{L} \mathbf{h}_{q}^{(2)}\left(x^{\prime}, y^{\prime}\right) \mid \mathbf{h}_{p}^{(2)}\left(x^{\prime}, y^{\prime}\right)\right\rangle \\
C_{p}^{(n, \gamma)} & =\left\langle\mathbf{h}_{n}^{(\gamma)}(x, y) \mid \mathbf{h}_{p}^{(2)}(x, y)\right\rangle= \begin{cases}A_{n, p} & \text { si }(\gamma)=(1) \\
\delta_{n, p} & \text { si }(\gamma)=(2)\end{cases} \\
D_{q}^{(n, \gamma)} & =\alpha_{q}^{(n, \gamma)}
\end{aligned}
$$

Así pues, el problema matricial que se obtiene finalmente es:

$$
\mathbf{B} \cdot \mathbf{D}^{(n, \gamma)}=\mathbf{C}^{(n, \gamma)}
$$


donde B es una matriz compleja de dimensiones $Q^{(\gamma)} \times Q^{(\gamma)}$; y $\mathbf{C}^{(n, \gamma)}$ y $\mathbf{D}^{(n, \gamma)}$ son vectores columna, siendo este último el que representa las incógnitas del sistema.

Una vez resuelto el problema matricial (2.48), se puede retomar la expresión (2.23) para calcular los elementos de la matriz de impedancias generalizada que caracteriza a la unión planar bajo estudio:

$$
\begin{aligned}
Z_{m, n}^{(\xi, \gamma)} & =\int_{S^{(2)}} \mathbf{M}_{n}^{(\gamma)}\left(x^{\prime}, y^{\prime}\right) \cdot \mathbf{h}_{m}^{(\xi) *}\left(x^{\prime}, y^{\prime}\right) d S^{\prime} \approx \int_{S^{(2)}} \sum_{q=1}^{Q^{(\gamma)}} \alpha_{q}^{(n, \gamma)} \mathbf{h}_{q}^{(2)}\left(x^{\prime}, y^{\prime}\right) \cdot \mathbf{h}_{m}^{(\xi) *}\left(x^{\prime}, y^{\prime}\right) d S^{\prime} \\
& =\sum_{q=1}^{Q^{(\gamma)}} \alpha_{q}^{(n, \gamma)} \int_{S^{(2)}} \mathbf{h}_{q}^{(2)}\left(x^{\prime}, y^{\prime}\right) \cdot \mathbf{h}_{m}^{(\xi) *}\left(x^{\prime}, y^{\prime}\right) d S^{\prime} \\
& =\left\{\begin{array}{cc}
\sum_{q=1}^{Q^{(\xi)}} \alpha_{q}^{(n, \gamma)} A_{m, q}^{*} & \text { si }(\xi)=(1) \\
\alpha_{m}^{(n, \gamma)} & \operatorname{si}(\xi)=(2)
\end{array}\right.
\end{aligned}
$$

\subsection{Discontinuidades entre Guías Rectangulares y Guías Coaxiales}

En esta sección nos ocuparemos de caracterizar una unión planar entre una guía de sección transversal rectangular y una guía coaxial utilizando la técnica de la ecuación integral descrita anteriormente. Si se analizan las expresiones de la técnica de la ecuación integral descritas en la sección anterior correspondientes a la expresión general para la matriz de impedancias generalizada que caracteriza a una unión planar, se desprende que en su cálculo intervienen, de una parte, los números de onda de corte de los modos asociados a las guías involucradas en la unión y, de otra, unas integrales de acoplamiento entre las funciones vectoriales modales de campo magnético de dichas guías. Así pues, en primer lugar, será necesario obtener la carta modal de una guía rectangular y de una guía coaxial para, posteriormente, utilizar estos resultados en el cálculo de las integrales de acoplamiento.

A este respecto, sabemos que la variación radial de la solución modal clásica para una guía coaxial se expresa en términos de una combinación de funciones de Bessel de primera y de segunda especie, mientras que la variación angular es de tipo sinusoidal [35]. Con el propósito de maximizar la eficiencia computacional del código implementado, sería interesante evitar el uso de funciones de Bessel para la descripción modal de la guía coaxial ya que, de esta forma, se aceleraría el cálculo de las integrales de acoplamiento, las cuales deben evaluarse de forma numérica. Por ello, en esta sección se propone un método alternativo para la obtención de los modos de una guía coaxial basado en expresar la variación radial de las soluciones modales en términos de funciones sinusoidales. Por su parte, las expresiones 
de las funciones vectoriales modales de una guía rectangular son sobradamente conocidas y pueden encontrarse, por ejemplo, en [28].

Finalmente, es importante comentar que las conclusiones de este apartado resultarán cruciales para los objetivos del capítulo 4, en el que se pretende caracterizar la excitación coaxial de dispositivos pasivos de microondas implementados en guía rectangular. En particular, en dicho capítulo se utilizarán las integrales de acoplamiento obtenidas en esta sección con el objetivo de expresar los modos de la guía coaxial de excitación utilizando una combinación lineal de los modos de la guía rectangular a la que el coaxial alimenta. De esta manera, tal y como se comprobará en el capítulo correspondiente, se logrará simplificar al máximo la aplicación del método BI-RME 3D a la caracterización de la excitación coaxial de dispositivos.

\subsubsection{Obtención de los modos de una guía coaxial utilizando el método de los momentos}

El objetivo de esta sección es la obtención de la carta modal de una guía coaxial utilizando para ello una formulación alternativa a la desarrollada en la solución modal clásica [28]. La nueva técnica propuesta expresa la variación radial de las soluciones modales en términos de funciones sinusoidales, y transforma la ecuación de Helmholtz en un problema de autovalores equivalente utilizando el método de los momentos [21], [36]. Así pues, consideremos una guía coaxial de radio externo $r_{1}$ y de radio interno $r_{2}$, como la representada en la figura 2.3. Por otro lado, asumiremos que la guía está rellena uniformemente de un material dieléctrico de permitividad relativa $\varepsilon_{r}$, y permeabilidad magnética relativa $\mu_{r}$. En la figura 2.3 se han representado, además, los sistemas de ejes coordenados en cartesianas y en cilíndricas que se van a utilizar en el análisis (la coordenada axial $z$ no se ha representado en la figura y se satisface $\hat{\mathbf{x}} \times \hat{\mathbf{y}}=\hat{\mathbf{z}}$ y $\hat{\boldsymbol{\rho}} \times \hat{\boldsymbol{\phi}}=\hat{\mathbf{z}})$.

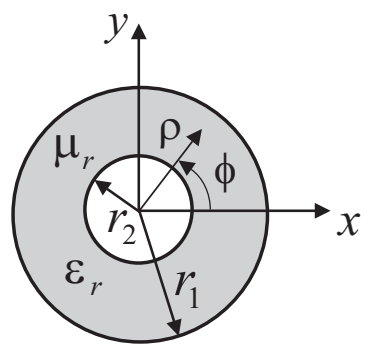

Figura 2.3: Sección transversal de una guía coaxial de radios externo $r_{1}$ e interno $r_{2}$. Se asume que la permitividad dieléctrica relativa es $\varepsilon_{r}$ y que la permeabilidad magnética relativa es $\mu_{r}$.

A continuación, el estudio se particulariza para las diferentes soluciones modales admitidas en una guía coaxial: modo $\mathrm{TEM}^{z}$, modos $\mathrm{TM}^{z}$ y modos $\mathrm{TE}^{z}$.

\section{Modo TEM ${ }^{z}$}

Para el modo $\mathrm{TEM}^{z}$ se cumple que las componentes axiales de los campos eléctrico y magnético son nulas: $E_{z}=H_{z}=0$. Por su parte, las funciones vectoriales modales asociadas 
a los campos transversales satisfacen las siguientes ecuaciones [37]:

$$
\begin{aligned}
\nabla_{t} \cdot \mathbf{e}_{t}^{\mathrm{TEM}^{z}}(\rho, \phi) & =0 \\
\nabla_{t} \times \mathbf{e}_{t}^{\mathrm{TEM}^{z}}(\rho, \phi) & =0 \\
\nabla_{t} \cdot \mathbf{h}_{t}^{\mathrm{TEM}^{z}}(\rho, \phi) & =0 \\
\nabla_{t} \times \mathbf{h}_{t}^{\mathrm{TEM}^{z}}(\rho, \phi) & =0
\end{aligned}
$$

De (2.51) se deduce que la función vectorial modal de campo eléctrico puede expresarse de la siguiente manera:

$$
\mathbf{e}_{t}^{\mathrm{TEM}^{z}}(\rho, \phi)=-\nabla_{t} \psi(\rho, \phi)
$$

siendo $\psi(\rho, \phi)$ una función potencial desconocida. Además, teniendo en cuenta (2.50), se concluye que $\nabla_{t}^{2} \psi(\rho, \phi)=0$. Esta última ecuación es la conocida ecuación de Laplace, la cual proporcionará una solución modal de tipo estático (la frecuencia de corte de los modos $\mathrm{TEM}^{z}$ es nula). Si ahora desarrollamos el operador laplaciano transversal y expresamos la ecuación de Laplace en coordenadas cilíndricas obtendremos que:

$$
\left(\frac{1}{\rho} \frac{\partial}{\partial \rho}\left(\rho \frac{\partial}{\partial \rho}\right)+\frac{1}{\rho^{2}} \frac{\partial^{2}}{\partial \phi^{2}}\right) \psi(\rho, \phi)=0
$$

A continuación, empleamos el método de separación de variables para resolver la ecuación diferencial anterior, por lo que podemos escribir inicialmente que:

$$
\psi(\rho, \phi)=R^{\mathrm{TEM}^{z}}(\rho) \Phi(\phi)
$$

donde $\Phi(\phi)$ será constante debido a la simetría de la estructura. Con ello, retomando la ecuación (2.55), es fácil concluir que la función que aporta la variación radial $R^{\mathrm{TEM}^{z}}(\rho)$ será de la forma:

$$
R^{\mathrm{TEM}^{z}}(\rho)=A \ln \rho+B
$$

siendo $A$ y $B$ constantes. Por lo tanto, recuperando la expresión (2.54) y tomando $A=1$, se concluye que:

$$
\widetilde{\mathbf{e}}_{t}^{\mathrm{TEM}}(\rho)=\frac{1}{\rho} \hat{\boldsymbol{\rho}}
$$

donde $\widetilde{\mathbf{e}}_{t}^{\mathrm{TEM}^{z}}(\rho)$ se corresponde con la función vectorial modal de campo eléctrico no normalizada. Por otro lado, la función vectorial de campo magnético no normalizada puede calcularse como [37]:

$$
\widetilde{\mathbf{h}}_{t}^{\mathrm{TEM}^{z}}(\rho)=\hat{\mathbf{z}} \times \widetilde{\mathbf{e}}_{t}^{\mathrm{TEM}^{z}}(\rho)=\frac{1}{\rho} \hat{\boldsymbol{\phi}}
$$

Con el objetivo de conseguir unas expresiones normalizadas para las funciones vectoriales modales, utilizamos la siguiente condición de ortonormalidad:

$$
\int_{S}\left(\mathbf{e}_{t}^{\mathrm{TEM}^{z}}(\rho) \times \mathbf{h}_{t}^{\mathrm{TEM}^{z}}(\rho)\right) \cdot \hat{\mathbf{z}} d S=1
$$


donde $\mathbf{e}_{t}^{\mathrm{TEM}^{z}}(\rho)$ y $\mathbf{h}_{t}^{\mathrm{TEM}}(\rho)$ son las funciones vectoriales modales normalizadas, y la región de integración $S$ se extiende sobre la sección transversal de la guía coaxial. Por tanto, el factor de normalización para los modos $\mathrm{TEM}^{z}$ puede calcularse como:

$$
\mathcal{N}_{\mathrm{TEM}^{z}}=\left(\int_{S}\left(\widetilde{\mathbf{h}}_{t}^{\mathrm{TEM}^{z}}(\rho) \times \hat{\mathbf{z}}\right) \cdot \widetilde{\mathbf{e}}_{t}^{\mathrm{TEM}^{z}}(\rho) d S\right)^{-\frac{1}{2}}=\frac{1}{\sqrt{2 \pi \ln \left(r_{1} / r_{2}\right)}}
$$

Así pues, las expresiones definitivas para las funciones vectoriales modales normalizadas son las siguientes:

$$
\begin{aligned}
\mathbf{e}_{t}^{\mathrm{TEM}^{z}}(\rho) & =\frac{1}{\sqrt{2 \pi \ln \left(r_{1} / r_{2}\right)}} \frac{1}{\rho} \hat{\boldsymbol{\rho}} \\
\mathbf{h}_{t}^{\mathrm{TEM}^{z}}(\rho) & =\frac{1}{\sqrt{2 \pi \ln \left(r_{1} / r_{2}\right)}} \frac{1}{\rho} \hat{\boldsymbol{\phi}}
\end{aligned}
$$

Estas funciones vectoriales satisfacen, lógicamente, la condición de ortonormalidad (2.60). Finalmente, la impedancia modal característica para los modos $\mathrm{TEM}^{z}$ es:

$$
Z_{\mathrm{TEM}^{z}}=\sqrt{\frac{\mu_{0} \mu_{r}}{\varepsilon_{0} \varepsilon_{r}}}
$$

\section{$\underline{\operatorname{Modos}} \mathbf{T M}^{z}$}

Los modos $\mathrm{TM}^{z}$ satisfacen que la componente axial del campo magnético $h_{z}$ es nula. La ecuación de Helmholtz que debe satisfacer el campo eléctrico axial $\psi=e_{z}$ para estos modos es la siguiente:

$$
\left(\nabla^{2}+\omega^{2} \mu \varepsilon\right) \psi(\rho, \phi, z)=0
$$

donde, por el método de separación de variables, podemos afirmar que:

$$
\psi(\rho, \phi, z)=R^{\mathrm{TM}^{z}}(\rho) \Phi(\phi) e^{-j \beta z}
$$

De esta manera, teniendo en cuenta que $\nabla^{2} \psi=\nabla_{t}^{2} \psi+\nabla_{z}^{2} \psi$, se puede reescribir la expresión (2.65) como:

$$
\left(\nabla_{t}^{2}+k_{t}^{2}\right)\left(R^{\mathrm{TM}^{z}}(\rho) \Phi(\phi)\right)=0
$$

donde, por comodidad en la notación, se ha omitido la dependencia con la coordenada axial. Además, en la ecuación anterior, $k_{t}^{2}=\omega^{2} \mu \varepsilon-\beta^{2}$ representa el número de onda de corte asociado a los modos $\mathrm{TM}^{z}$ de la guía coaxial. Si ahora se desarrolla el operador laplaciano con respecto a las coordenadas transversales y se manipula adecuadamente la expresión resultante, es posible llegar al siguiente resultado partiendo de (2.67):

$$
\frac{\rho}{R^{\mathrm{TM}^{z}}(\rho)} \frac{d}{d \rho}\left(\rho \frac{d R^{\mathrm{TM}^{z}}(\rho)}{d \rho}\right)+k_{t}^{2} \rho^{2}=-\frac{1}{\Phi(\phi)} \frac{d^{2} \Phi(\phi)}{d \phi^{2}}
$$


El miembro izquierdo de (2.68) sólo depende de $\rho$, mientras que el miembro de la derecha únicamente depende de $\phi$. En consecuencia, cada uno de estos miembros debe ser igual a una constante que denominaremos $s^{2}$. Así pues, se concluye que:

$$
\begin{aligned}
\frac{d^{2} R^{\mathrm{TM}^{z}}(\rho)}{d \rho^{2}}+\frac{1}{\rho} \frac{d R^{\mathrm{TM}^{z}}(\rho)}{d \rho}+\left(k_{t}^{2}-\frac{s^{2}}{\rho^{2}}\right) R^{\mathrm{TM}^{z}}(\rho) & =0 \\
\frac{d^{2} \Phi(\phi)}{d \phi^{2}}+s^{2} \Phi(\phi) & =0
\end{aligned}
$$

La solución de (2.70) es bien conocida [38] y puede expresarse como:

$$
\Phi_{(\xi), s}(\phi)=\left\{\begin{array}{cc}
\cos (s \phi), s=0,1,2, \ldots & (\xi)=(c) \\
\sin (s \phi), s=1,2,3, \ldots & (\xi)=(s)
\end{array}\right.
$$

Es importante destacar que $s$ representa el índice modal asociado a la variable angular de las soluciones modales. En caso de que $s \neq 0$, se tendrá un modo degenerado. El modo degenerado de tipo coseno (o también denominado modo par) lo denotaremos mediante $(\xi)=(c)$, mientras que el de tipo seno (o también denominado modo impar) lo representaremos mediante $(\xi)=(s)$.

Por su parte, la ecuación (2.69) puede reescribirse de la siguiente forma:

$$
\left(\frac{d^{2}}{d \rho^{2}}+\frac{1}{\rho} \frac{d}{d \rho}-\frac{s^{2}}{\rho^{2}}\right) R^{\mathrm{TM}^{z}}(\rho)=-k_{t}^{2} R^{\mathrm{TM}^{z}}(\rho)
$$

Seguidamente, definimos el operador diferencial $\mathcal{L}_{s}$ como:

$$
\mathcal{L}_{s}=\frac{d^{2}}{d \rho^{2}}+\frac{1}{\rho} \frac{d}{d \rho}-\frac{s^{2}}{\rho^{2}}
$$

De esta manera, la ecuación diferencial (2.72) se transforma en el siguiente problema de autovalores equivalente:

$$
\mathcal{L}_{s} R^{\mathrm{TM}^{z}}(\rho)=-k_{t}^{2} R^{\mathrm{TM}^{z}}(\rho)
$$

Para la obtención de los modos $\mathrm{TM}^{z}$ escogemos la siguiente función potencial:

$$
e_{z_{(\xi), m}}^{\mathrm{TM}^{z}}(\rho, \phi)=\left\{\sum_{r=1}^{\infty} d_{s, q}^{(r)} \sin \left(r \pi \frac{\rho-r_{2}}{r_{1}-r_{2}}\right)\right\} \Phi_{(\xi), s}(\phi)=R_{s, q}^{\mathrm{TM}^{z}}(\rho) \Phi_{(\xi), s}(\phi)
$$

donde $m$ denota el orden del modo; $(s, q)$ es la pareja de índices modales asociados a las variables angular y radial, respectivamente; y $d_{s, q}^{(r)}$ son unos coeficientes de expansión desconocidos que representan los autovectores del problema (2.74). Es importante notar que la variación radial de la solución modal se ha expresado en términos de funciones sinusoidales que satisfacen las condiciones de contorno de Dirichlet, ya que se cumple que:

$$
\left.e_{z_{(\xi), m}}^{\mathrm{TM}^{z}}(\rho, \phi)\right|_{\rho=r_{1}}=\left.e_{z_{(\xi), m}}^{\mathrm{TM}^{z}}(\rho, \phi)\right|_{\rho=r_{2}}=0
$$


A continuación, se debe resolver el problema de autovalores (2.74) teniendo en cuenta la expresión que ahora adopta la función de variación radial $R_{s, q}^{\mathrm{TM}^{z}}(\rho)$ :

$$
R_{s, q}^{\mathrm{TM}^{z}}(\rho)=\sum_{r=1}^{\infty} d_{s, q}^{(r)} \sin \left(r \pi \frac{\rho-r_{2}}{r_{1}-r_{2}}\right)=\sum_{r=1}^{\infty} d_{s, q}^{(r)} \alpha_{r}^{\mathrm{TM}^{z}}(\rho)
$$

Para la resolución del problema de autovalores se utilizará el método de los momentos (procedimiento de Galerkin). El producto interno que utilizaremos en la aplicación del procedimiento de Galerkin será el siguiente:

$$
\langle f(\rho) \mid g(\rho)\rangle=\int_{r_{2}}^{r_{1}} f(\rho) g^{*}(\rho) \rho d \rho
$$

Así pues, si se aplica el procedimiento de Galerkin sobre el problema (2.74) se obtiene:

$$
\left\langle\mathcal{L}_{s} R_{s, q}^{\mathrm{TM}^{z}}(\rho) \mid \alpha_{t}^{\mathrm{TM}^{z}}(\rho)\right\rangle=-k_{t, m}^{2}\left\langle R_{s, q}^{\mathrm{TM}^{z}}(\rho) \mid \alpha_{t}^{\mathrm{TM}^{z}}(\rho)\right\rangle
$$

donde $\alpha_{t}^{\mathrm{TM}^{z}}(\rho)$ representan las funciones de test del procedimiento de Galerkin definidas en (2.77). Si empezamos desarrollando el miembro de la derecha de (2.79) obtendremos:

$$
\left\langle R_{s, q}^{\mathrm{TM}^{z}}(\rho) \mid \alpha_{t}^{\mathrm{TM}^{z}}(\rho)\right\rangle=\sum_{r=1}^{\infty} d_{s, q}^{(r)} \int_{r_{2}}^{r_{1}} \rho \sin \left(r \pi \frac{\rho-r_{2}}{r_{1}-r_{2}}\right) \sin \left(t \pi \frac{\rho-r_{2}}{r_{1}-r_{2}}\right) d \rho
$$

Con respecto al miembro de la izquierda de (2.79), empezamos aplicando el operador diferencial $\mathcal{L}_{s}$ sobre la función radial $R_{s, q}^{\mathrm{TM}}{ }^{z}(\rho)$ :

$$
\begin{aligned}
\mathcal{L}_{s} R_{s, q}^{\mathrm{TM}^{z}}(\rho)= & \left(\frac{d^{2}}{d \rho^{2}}+\frac{1}{\rho} \frac{d}{d \rho}-\frac{s^{2}}{\rho^{2}}\right) R_{s, q}^{\mathrm{TM}^{z}}(\rho) \\
= & \sum_{r=1}^{\infty} d_{s, q}^{(r)}\left[\left(\frac{d^{2}}{d \rho^{2}}+\frac{1}{\rho} \frac{d}{d \rho}-\frac{s^{2}}{\rho^{2}}\right) \sin \left(r \pi \frac{\rho-r_{2}}{r_{1}-r_{2}}\right)\right] \\
= & \sum_{r=1}^{\infty} d_{s, q}^{(r)}\left[-\left(\left(\frac{r \pi}{r_{1}-r_{2}}\right)^{2}+\frac{s^{2}}{\rho^{2}}\right) \sin \left(r \pi \frac{\rho-r_{2}}{r_{1}-r_{2}}\right)\right. \\
& \left.+\frac{r \pi}{r_{1}-r_{2}} \frac{1}{\rho} \cos \left(r \pi \frac{\rho-r_{2}}{r_{1}-r_{2}}\right)\right]
\end{aligned}
$$

Por tanto, se tiene que:

$$
\begin{aligned}
\left\langle\mathcal{L}_{s} R_{s, q}^{\mathrm{TM}^{z}}(\rho) \mid \alpha_{t}^{\mathrm{TM}^{z}}(\rho)\right\rangle= & -\sum_{r=1}^{\infty} d_{s, q}^{(r)} \int_{r_{2}}^{r_{1}}\left[\left(\rho\left(\frac{r \pi}{r_{1}-r_{2}}\right)^{2}+\frac{s^{2}}{\rho}\right) \sin \left(r \pi \frac{\rho-r_{2}}{r_{1}-r_{2}}\right)\right. \\
& \left.-\frac{r \pi}{r_{1}-r_{2}} \cos \left(r \pi \frac{\rho-r_{2}}{r_{1}-r_{2}}\right)\right] \sin \left(t \pi \frac{\rho-r_{2}}{r_{1}-r_{2}}\right) d \rho
\end{aligned}
$$


Seguidamente, definimos las siguientes matrices:

$$
\begin{aligned}
P_{r, t}^{\mathrm{TM}^{z}}= & \int_{r_{2}}^{r_{1}}\left[\left(\rho\left(\frac{r \pi}{r_{1}-r_{2}}\right)^{2}+\frac{s^{2}}{\rho}\right) \sin \left(r \pi \frac{\rho-r_{2}}{r_{1}-r_{2}}\right)\right. \\
& \left.-\frac{r \pi}{r_{1}-r_{2}} \cos \left(r \pi \frac{\rho-r_{2}}{r_{1}-r_{2}}\right)\right] \sin \left(t \pi \frac{\rho-r_{2}}{r_{1}-r_{2}}\right) d \rho \\
Q_{r, t}^{\mathrm{TM}^{z}}= & \int_{r_{2}}^{r_{1}} \rho \sin \left(r \pi \frac{\rho-r_{2}}{r_{1}-r_{2}}\right) \sin \left(t \pi \frac{\rho-r_{2}}{r_{1}-r_{2}}\right) d \rho
\end{aligned}
$$

donde $r, t=1,2, \ldots, N_{\rho}$, siendo $N_{\rho}$ el número de términos que se consideran en el sumatorio de la expansión (2.77). De esta forma, nos quedará que:

$$
\begin{aligned}
\left\langle R_{s, q}^{\mathrm{TM}^{z}}(\rho) \mid \alpha_{t}^{\mathrm{TM}^{z}}(\rho)\right\rangle & =\sum_{r=1}^{N_{\rho}} Q_{r, t}^{\mathrm{TM}^{z}} d_{s, q}^{(r)} \\
\left\langle\mathcal{L}_{s} R_{s, q}^{\mathrm{TM}^{z}}(\rho) \mid \alpha_{t}^{\mathrm{TM}^{z}}(\rho)\right\rangle & =-\sum_{r=1}^{N_{\rho}} P_{r, t}^{\mathrm{TM}^{z}} d_{s, q}^{(r)}
\end{aligned}
$$

Así pues, el problema de autovalores inicial queda formulado de la siguiente manera:

$$
\sum_{r=1}^{N_{\rho}} P_{r, t}^{\mathrm{TM}^{z}} d_{s, q}^{(r)}=k_{t, m}^{2} \sum_{r=1}^{N_{\rho}} Q_{r, t}^{\mathrm{TM}^{z}} d_{s, q}^{(r)}
$$

Finalmente, la ecuación anterior puede escribirse de manera equivalente usando una notación matricial:

$$
\mathbf{P}^{\mathrm{TM}^{z}} \cdot \mathbf{D}_{m}=k_{t, m}^{2} \mathbf{Q}^{\mathrm{TM}^{z}} \cdot \mathbf{D}_{m}
$$

En esta última expresión podemos identificar que $\mathbf{P}^{\mathrm{TM}^{z}}=\left(P_{r, t}^{\mathrm{TM}^{z}}\right), \mathbf{Q}^{\mathrm{TM}^{z}}=\left(Q_{r, t}^{\mathrm{TM}^{z}}\right)$, y que $\mathbf{D}_{m}=\left(d_{s, q}^{(r)}\right)$, con $r, t=1,2, \ldots, N_{\rho}$. Además, $(s, q)$ es la pareja de índices modales asociados a la $m$-ésima solución modal. Obsérvese que las matrices $\mathbf{P}^{\mathrm{TM}^{z}}$ y $\mathrm{Q}^{\mathrm{TM}^{z}}$ son reales y simétricas, por lo que los autovalores obtenidos serán reales. Las soluciones de los elementos de estas matrices pueden consultarse en el Apéndice A. Una vez resuelto el problema de autovalores (2.88), se obtienen tanto los números de onda de corte $k_{t, m}$ asociados a los modos $\mathrm{TM}^{z}$ (autovalores del problema), así como los coeficientes de expansión $d_{s, q}^{(r)}$ utilizados en la función potencial definida en (2.77) (autovectores del problema).

El siguiente paso consiste en obtener las funciones vectoriales modales de campo eléctrico y de campo magnético asociadas a los modos $\mathrm{TM}^{z}$. Para ello, se recuerda que [39]:

$$
\begin{aligned}
& \widetilde{\mathbf{e}}_{t_{(\xi), m}}^{\mathrm{TM}^{z}}(\rho, \phi)=-\nabla_{t} e_{z_{(\xi), m}}^{\mathrm{TM}^{z}}(\rho, \phi) \\
& \widetilde{\mathbf{h}}_{t_{(\xi), m}}^{\mathrm{TM}^{z}}(\rho, \phi)=\hat{\mathbf{z}} \times \widetilde{\mathbf{e}}_{t_{(\xi), m}}^{\mathrm{TM}^{z}}(\rho, \phi)
\end{aligned}
$$


siendo $\widetilde{\mathbf{e}}_{t_{(\xi), m}}^{\mathrm{TM}^{z}}(\rho, \phi)$ y $\widetilde{\mathbf{h}}_{t(\xi), m}^{\mathrm{TM}}(\rho, \phi)$ las correspondientes funciones vectoriales modales no normalizadas asociadas al modo $m$-ésimo. Por tanto, se puede concluir fácilmente que:

$$
\begin{aligned}
\widetilde{\mathbf{e}}_{t_{(\xi), m}}^{\mathrm{TM}^{z}}(\rho, \phi)= & \frac{\pi}{r_{1}-r_{2}}\left(\sum_{r=1}^{N_{\rho}} r d_{s, q}^{(r)} \cos \left(r \pi \frac{\rho-r_{2}}{r_{1}-r_{2}}\right)\right) \Phi_{(\xi), s}(\phi) \hat{\boldsymbol{\rho}} \\
& +\frac{1}{\rho}\left(\sum_{r=1}^{N_{\rho}} d_{s, q}^{(r)} \sin \left(r \pi \frac{\rho-r_{2}}{r_{1}-r_{2}}\right)\right) \Phi_{(\xi), s}^{\prime}(\phi) \hat{\boldsymbol{\phi}} \\
\widetilde{\mathbf{h}}_{t_{(\xi), m}}^{\mathrm{TM}^{z}}(\rho, \phi)= & -\frac{1}{\rho}\left(\sum_{r=1}^{N_{\rho}} d_{s, q}^{(r)} \sin \left(r \pi \frac{\rho-r_{2}}{r_{1}-r_{2}}\right)\right) \Phi_{(\xi), s}^{\prime}(\phi) \hat{\boldsymbol{\rho}} \\
& +\frac{\pi}{r_{1}-r_{2}}\left(\sum_{r=1}^{N_{\rho}} r d_{s, q}^{(r)} \cos \left(r \pi \frac{\rho-r_{2}}{r_{1}-r_{2}}\right)\right) \Phi_{(\xi), s}(\phi) \hat{\boldsymbol{\phi}}
\end{aligned}
$$

donde $\Phi_{(\xi), s}^{\prime}(\phi)$ representa la derivada de la función $\Phi_{(\xi), s}(\phi)$ con respecto a la variable $\phi$. Finalmente, debemos normalizar las expresiones anteriores atendiendo a la siguiente condición de ortonormalización:

$$
\int_{S} \mathbf{e}_{t_{(\alpha), m}}^{\mathrm{TM}^{z}}(\rho, \phi) \cdot \mathbf{e}_{t_{(\beta), n}}^{\mathrm{TM}^{z}}(\rho, \phi) d S=\delta_{m, n} \delta_{(\alpha),(\beta)} \quad(\alpha),(\beta)=(c),(s)
$$

donde $m$ y $n$ indican el orden del modo; $(\alpha)$ y $(\beta)$ representan la paridad del modo; y $\delta_{m, n}$ es la delta de Kronecker.

Así pues, recordemos que $(s, q)$ representan los índices modales en las variables angular y radial, respectivamente, del modo $m$-ésimo. El factor de normalización para los modos $\mathrm{TM}^{z}$ puede calcularse como:

$$
\begin{aligned}
\mathcal{N}_{(\xi), m}^{\mathrm{TM}}= & \left(\int_{S} \widetilde{\mathbf{e}}_{t_{(\xi), m}}^{\mathrm{TM}^{z}}(\rho, \phi) \cdot \widetilde{\mathbf{e}}_{t_{(\xi), m}}^{\mathrm{TM}^{z}}(\rho, \phi) d S\right)^{-\frac{1}{2}} \\
= & {\left[\left(\frac{\pi}{r_{1}-r_{2}}\right)^{2} \int_{0}^{2 \pi} \Phi_{(\xi), s}^{2}(\phi) d \phi\right.} \\
& \cdot \sum_{r=1}^{N_{\rho}} \sum_{t=1}^{N_{\rho}} r t d_{s, q}^{(r)} d_{s, q}^{(t)} \int_{r_{2}}^{r_{1}} \rho \cos \left(r \pi \frac{\rho-r_{2}}{r_{1}-r_{2}}\right) \cos \left(t \pi \frac{\rho-r_{2}}{r_{1}-r_{2}}\right) d \rho \\
& +\int_{0}^{2 \pi}\left(\Phi_{(\xi), s}^{\prime}(\phi)\right)^{2} d \phi \\
& \left.\cdot \sum_{r=1}^{N_{\rho}} \sum_{t=1}^{N_{\rho}} d_{s, q}^{(r)} d_{s, q}^{(t)} \int_{r_{2}}^{r_{1}} \frac{1}{\rho} \sin \left(r \pi \frac{\rho-r_{2}}{r_{1}-r_{2}}\right) \sin \left(t \pi \frac{\rho-r_{2}}{r_{1}-r_{2}}\right) d \rho\right]^{-\frac{1}{2}}
\end{aligned}
$$


Las diferentes integrales que aparecen en la expresión anterior se encuentran resueltas en el Apéndice A. Finalmente, las expresiones de las funciones vectoriales modales normalizadas adoptan el siguiente aspecto:

$$
\begin{aligned}
& \mathbf{e}_{t_{(\xi), m}}^{\mathrm{TM}^{z}}(\rho, \phi)=\mathcal{N}_{(\xi), m}^{\mathrm{TM}^{z}} \widetilde{\mathbf{e}}_{t_{(\xi), m}}^{\mathrm{TM}^{z}}(\rho, \phi) \\
& \mathbf{h}_{t_{(\xi), m}}^{\mathrm{TM}^{z}}(\rho, \phi)=\mathcal{N}_{(\xi), m}^{\mathrm{TM}^{z}} \widetilde{\mathbf{h}}_{t_{(\xi), m}}^{\mathrm{TM}^{z}}(\rho, \phi)
\end{aligned}
$$

Para concluir este apartado, se han realizado diferentes estudios con el objetivo de validar la rigurosidad y la eficiencia de la herramienta implementada. Así, en primer lugar, en la tabla 2.2 se presentan los números de onda de corte para diferentes modos $\mathrm{TM}_{s, q}^{z}$ de una guía coaxial de radio externo $r_{1}=1,6 \mathrm{~m}$ y de radio interno $r_{2}=1,0 \mathrm{~m}\left(\varepsilon_{r}=1,0\right)$. Además, se consideran los siguientes valores para los índices modales: $(s, q)=(0,1),(2,1)$, $(0,2),(3,2),(0,3),(2,3),(0,4),(3,4)$. En la herramienta implementada se han considerado $N_{\rho}=25$ términos en la expansión de la función de variación radial de las soluciones modales. Los resultados obtenidos se comparan con los que se presentan en [28]. Tal y como se puede apreciar en la tabla, el error relativo en tanto por ciento con respecto a [28] es, en casi todos los casos, del orden de $10^{-3}$. Estos resultados validan completamente la rigurosidad del método propuesto. Por otro lado, es también muy importante destacar la eficiencia computacional del método. A este respecto, podemos decir que los primeros $500 \operatorname{modos}\left(N_{\rho}=50\right.$ e índice angular máximo igual a 10) de tipo $\mathrm{TM}^{z}$ de la guía coaxial (sin contar los modos degenerados) se calculan en 0,32 segundos en un PC Pentium IV@2.55 GHz con 512 MB de memoria RAM $^{9}$.

\begin{tabular}{|c|c|c|c|}
\hline \hline \multirow{2}{*}{$\begin{array}{c}\text { Índices modales } \\
(s, q)\end{array}$} & \multicolumn{3}{|c|}{ Número de onda de corte de los modos $\mathrm{TM}_{s, q}^{z}(\mathbf{r a d} / \mathbf{m})$} \\
\cline { 2 - 4 } & Ref. [28] & Software con $N_{\rho}=25$ & Error relativo ( \%) \\
\hline$(0,1)$ & 5,221667 & 5,221539 & $2 \cdot 10^{-3}$ \\
\hline$(2,1)$ & 5,45 & 5,447972 & $4 \cdot 10^{-2}$ \\
\hline$(0,2)$ & 10,465 & 10,464581 & $4 \cdot 10^{-3}$ \\
\hline$(3,2)$ & 10,728333 & 10,727760 & $5 \cdot 10^{-3}$ \\
\hline$(0,3)$ & 15,703333 & 15,703013 & $2 \cdot 10^{-3}$ \\
\hline$(2,3)$ & 15,781667 & 15,782105 & $3 \cdot 10^{-3}$ \\
\hline$(0,4)$ & 20,94 & 20,940233 & $1 \cdot 10^{-3}$ \\
\hline$(3,4)$ & 21,073333 & 21,073838 & $2 \cdot 10^{-3}$ \\
\hline \hline
\end{tabular}

Tabla 2.2: Comparación del número de onda de corte obtenido con la herramienta implementada con los resultados presentados en [28]. La guía coaxial analizada es de radio externo $r_{1}=1,6 \mathrm{~m}$ y de radio interno $r_{2}=1,0 \mathrm{~m}\left(\varepsilon_{r}=1,0\right)$. Los resultados presentados se refieren a los modos $\mathrm{TM}_{s, q}^{z}$ con $(s, q)=(0,1),(2,1),(0,2),(3,2),(0,3),(2,3),(0,4),(3,4)$.

Otro estudio comparativo se lleva a cabo en la tabla 2.3, donde se presentan los números de onda de corte para los modos $\mathrm{TM}_{s, 1}^{z}$ de una guía coaxial de radio externo $r_{1}=1,2222 \mathrm{~m}$

\footnotetext{
${ }^{9}$ Todos los tiempos de ejecución que se presentan en esta tesis doctoral se han obtenido utilizando un ordenador de las características mencionadas.
} 
y de radio interno $r_{2}=1,0 \mathrm{~m}\left(\varepsilon_{r}=1,0\right)$. Además, se consideran los siguientes valores para el índice modal angular: $s=1,2,5,10,200$, y se han utilizado $N_{\rho}=25$ términos en la expansión de la función de variación radial de las soluciones modales. Los resultados se comparan con éxito con los que se presentan en [40].

\begin{tabular}{|c|c|c|c|}
\hline \hline \multirow{2}{*}{$\begin{array}{c}\text { Índice modal } \\
\text { angular }(s)\end{array}$} & \multicolumn{3}{|c|}{ Número de onda de corte de los modos $\mathrm{TM}_{s, 1}^{z}(\mathbf{r a d} / \mathbf{m})$} \\
\cline { 2 - 4 } & Ref. $[\mathbf{4 0}]$ & Software con $N_{\rho}=25$ & Error relativo (\%) \\
\hline 1 & 14,158722 & 14,15872151 & $3 \cdot 10^{-6}$ \\
\hline 2 & 14,244609 & 14,24460931 & $2 \cdot 10^{-6}$ \\
\hline 5 & 14,831807 & 14,83180662 & $3 \cdot 10^{-6}$ \\
\hline 10 & 16,760613 & 16,76061319 & $1 \cdot 10^{-6}$ \\
\hline 100 & 89,048509 & 89,04850898 & $2 \cdot 10^{-8}$ \\
\hline 200 & 172,66023 & 172,66022728 & $2 \cdot 10^{-6}$ \\
\hline \hline
\end{tabular}

Tabla 2.3: Comparación del número de onda de corte obtenido con la herramienta implementada con los resultados presentados en [40]. La guía coaxial analizada es de radio externo $r_{1}=1,2222 \mathrm{~m}$ y de radio interno $r_{2}=1,0 \mathrm{~m}\left(\varepsilon_{r}=1,0\right)$. Los resultados presentados se refieren a los modos $\mathrm{TM}_{s, 1}^{z}$ con $s=1,2,5,10,100,200$.

Por otro lado, es también de especial importancia realizar un estudio de convergencia del método. A este respecto, en la tabla 2.4 se estudia la convergencia del número de onda de corte de diferentes modos $\mathrm{TM}_{s, q}^{z}$ en función del número de términos $N_{\rho}$ considerados en la expansión de la variación radial de las soluciones modales. De los resultados presentados se desprende que, incluso para el caso con $N_{\rho}=5$, el error relativo con respecto a los resultados extraídos de [28] sigue siendo muy pequeño. Por todo ello, la técnica propuesta puede considerarse rigurosa y muy eficiente desde un punto de vista computacional.

\begin{tabular}{|c|c|c|c|c|}
\hline \hline \multirow{2}{\text{Índicesmodales}}{$\begin{array}{c}\text { Número de términos radiales } \\
(s, q)\end{array}$} & $N_{\rho}=5$ & $N_{\rho}=25$ & $N_{\rho}=75$ & $N_{\rho}=150$ \\
\hline$(0,1)$ & 5,221681 & 5,221539 & 5,221537 & 5,221537 \\
\hline$(2,1)$ & 5,448104 & 5,447972 & 5,447971 & 5,447971 \\
\hline$(0,2)$ & 10,464789 & 10,464581 & 10,464579 & 10,464579 \\
\hline$(3,2)$ & 10,727951 & 10,727760 & 10,727759 & 10,727758 \\
\hline$(0,3)$ & 15,703809 & 15,703013 & 15,703009 & 15,703009 \\
\hline$(2,3)$ & 15,782873 & 15,782105 & 15,782102 & 15,782102 \\
\hline$(0,4)$ & 20,941177 & 20,940233 & 20,940229 & 20,940229 \\
\hline$(3,4)$ & 21,074694 & 21,073838 & 21,073834 & 21,073834 \\
\hline \hline
\end{tabular}

Tabla 2.4: Convergencia del número de onda de corte expresado en $\mathrm{rad} / \mathrm{m}$ de los modos $\mathrm{TM}_{s, q}^{z}$ para una guía coaxial de radio externo $r_{1}=1,6 \mathrm{~m}$ y de radio interno $r_{2}=1,0 \mathrm{~m}\left(\varepsilon_{r}=1,0\right)$ en función del número de términos $N_{\rho}$ considerados en la expansión radial de la solución modal. Los índices modales analizados son $(s, q)=(0,1),(2,1),(0,2),(3,2),(0,3),(2,3),(0,4),(3,4)$. 


\section{$\operatorname{Modos} \mathbf{T E}^{z}$}

Para los modos $\mathrm{TE}^{z}$ se cumple que la componente axial del campo eléctrico $e_{z}$ es nula. La obtención de los modos $\mathrm{TE}^{z}$ sigue el mismo procedimiento que el que se ha visto anteriormente para los modos $\mathrm{TM}^{z}$. Así, en primer lugar, se parte de la ecuación de Helmholtz planteada en (2.65) donde ahora $\psi=h_{z}$, y se resuelve dicha ecuación con respecto a la variable angular, obteniéndose la solución modal para dicha variación angular recogida en la expresión (2.71). Seguidamente, la ecuación diferencial en la variable radial se transforma en el siguiente problema de autovalores equivalente:

$$
\mathcal{L}_{s} R^{\mathrm{TE}^{z}}(\rho)=-k_{t}^{2} R^{\mathrm{TE}}(\rho)
$$

donde el operador $\mathcal{L}_{s}$ se ha definido en $(2.73), R^{\mathrm{TE}}(\rho)$ describe la variación radial de las soluciones modales y $k_{t}$ representa el número de onda de corte para los modos $\mathrm{TE}^{z}$.

Para el caso de los modos $\mathrm{TE}^{z}$, se escoge la siguiente función potencial:

$$
h_{z_{(\xi), m}}^{\mathrm{TE}^{z}}(\rho, \phi)=\left\{\sum_{r=0}^{\infty} l_{s, q}^{(r)} \cos \left(r \pi \frac{\rho-r_{2}}{r_{1}-r_{2}}\right)\right\} \Phi_{(\xi), s}(\phi)=R_{s, q}^{\mathrm{TE}^{z}}(\rho) \Phi_{(\xi), s}(\phi)
$$

En la expresión anterior, $m$ representa el orden del modo; $(s, q)$ es la pareja de índices modales asociados a las variables angular y radial, respectivamente; $l_{s, q}^{(r)}$ son los coeficientes de expansión desconocidos que representan los autovectores del problema; y $\Phi_{(\xi), s}(\phi)$ es la solución modal en la variable angular definida en (2.71). La función potencial (2.98) satisface las condiciones de contorno de Neumann:

$$
\left.\frac{\partial}{\partial \rho}\left(h_{z_{(\xi), m}}^{\mathrm{TE}^{z}}(\rho, \phi)\right)\right|_{\rho=r_{1}}=\left.\frac{\partial}{\partial \rho}\left(h_{z_{(\xi), m}}^{\mathrm{TE}^{z}}(\rho, \phi)\right)\right|_{\rho=r_{2}}=0
$$

Seguidamente, se debe resolver el problema de autovalores (2.97) teniendo en cuenta que ahora:

$$
R_{s, q}^{\mathrm{TE}^{z}}(\rho)=\sum_{r=0}^{\infty} l_{s, q}^{(r)} \cos \left(r \pi \frac{\rho-r_{2}}{r_{1}-r_{2}}\right)=\sum_{r=0}^{\infty} l_{s, q}^{(r)} \alpha_{r}^{\mathrm{TE}^{z}}(\rho)
$$

La resolución del problema de autovalores requiere la aplicación del método de los momentos (procedimiento de Galerkin) y, para ello, se emplea el producto interno definido en (2.78). En consecuencia, se tiene que:

$$
\left\langle\mathcal{L}_{s} R_{s, q}^{\mathrm{TE}^{z}}(\rho) \mid \alpha_{t}^{\mathrm{TE}}(\rho)\right\rangle=-k_{t, m}^{2}\left\langle R_{s, q}^{\mathrm{TE}^{z}}(\rho) \mid \alpha_{t}^{\mathrm{TE}^{z}}(\rho)\right\rangle
$$

siendo $\alpha_{t}^{\mathrm{TE}^{z}}(\rho)$ las funciones de test del procedimiento de Galerkin definidas en (2.100). En primer lugar, se calculará el producto interno del miembro de la derecha de la ecuación (2.101):

$$
\left\langle R_{s, q}^{\mathrm{TE}^{z}}(\rho) \mid \alpha_{t}^{\mathrm{TE}^{z}}(\rho)\right\rangle=\sum_{r=0}^{\infty} l_{s, q}^{(r)} \int_{r_{2}}^{r_{1}} \rho \cos \left(r \pi \frac{\rho-r_{2}}{r_{1}-r_{2}}\right) \cos \left(t \pi \frac{\rho-r_{2}}{r_{1}-r_{2}}\right) d \rho
$$


En cuanto al miembro de la izquierda de (2.101), podemos escribir inicialmente que:

$$
\begin{aligned}
\mathcal{L}_{s} R_{s, q}^{\mathrm{TE}^{z}}(\rho)= & \left(\frac{d^{2}}{d \rho^{2}}+\frac{1}{\rho} \frac{d}{d \rho}-\frac{s^{2}}{\rho^{2}}\right) R_{s, q}^{\mathrm{TE}^{z}}(\rho) \\
= & \sum_{r=0}^{\infty} l_{s, q}^{(r)}\left[\left(\frac{d^{2}}{d \rho^{2}}+\frac{1}{\rho} \frac{d}{d \rho}-\frac{s^{2}}{\rho^{2}}\right) \cos \left(r \pi \frac{\rho-r_{2}}{r_{1}-r_{2}}\right)\right] \\
= & \sum_{r=0}^{\infty} l_{s, q}^{(r)}\left[-\left(\left(\frac{r \pi}{r_{1}-r_{2}}\right)^{2}+\frac{s^{2}}{\rho^{2}}\right) \cos \left(r \pi \frac{\rho-r_{2}}{r_{1}-r_{2}}\right)\right. \\
& \left.-\frac{r \pi}{r_{1}-r_{2}} \frac{1}{\rho} \sin \left(r \pi \frac{\rho-r_{2}}{r_{1}-r_{2}}\right)\right]
\end{aligned}
$$

Gracias a este resultado se concluye que:

$$
\begin{aligned}
\left\langle\mathcal{L}_{s} R_{s, q}^{\mathrm{TE}^{z}}(\rho) \mid \alpha_{t}^{\mathrm{TE}}(\rho)\right\rangle= & -\sum_{r=0}^{\infty} l_{s, q}^{(r)} \int_{r_{2}}^{r_{1}}\left[\left(\rho\left(\frac{r \pi}{r_{1}-r_{2}}\right)^{2}+\frac{s^{2}}{\rho}\right) \cos \left(r \pi \frac{\rho-r_{2}}{r_{1}-r_{2}}\right)\right. \\
& \left.+\frac{r \pi}{r_{1}-r_{2}} \sin \left(r \pi \frac{\rho-r_{2}}{r_{1}-r_{2}}\right)\right] \cos \left(t \pi \frac{\rho-r_{2}}{r_{1}-r_{2}}\right) d \rho
\end{aligned}
$$

A continuación, se definen las siguientes matrices:

$$
\begin{aligned}
P_{r, t}^{\mathrm{TE}}= & \int_{r_{2}}^{r_{1}}\left[\left(\rho\left(\frac{r \pi}{r_{1}-r_{2}}\right)^{2}+\frac{s^{2}}{\rho}\right) \cos \left(r \pi \frac{\rho-r_{2}}{r_{1}-r_{2}}\right)\right. \\
& \left.+\frac{r \pi}{r_{1}-r_{2}} \sin \left(r \pi \frac{\rho-r_{2}}{r_{1}-r_{2}}\right)\right] \cos \left(t \pi \frac{\rho-r_{2}}{r_{1}-r_{2}}\right) d \rho \\
Q_{r, t}^{\mathrm{TE}^{z}}= & \int_{r_{2}}^{r_{1}} \rho \cos \left(r \pi \frac{\rho-r_{2}}{r_{1}-r_{2}}\right) \cos \left(t \pi \frac{\rho-r_{2}}{r_{1}-r_{2}}\right) d \rho
\end{aligned}
$$

con $r, t=0,1,2, \ldots, N_{\rho}-1$, siendo $N_{\rho}$ el número de términos efectivos que se consideran en la expansión (2.100). Estas nuevas definiciones nos permiten reescribir el problema de autovalores (2.97) de la siguiente manera:

$$
\sum_{r=0}^{N_{\rho}-1} P_{r, t}^{\mathrm{TE}^{z}} l_{s, q}^{(r)}=k_{t, m}^{2} \sum_{r=0}^{N_{\rho}-1} Q_{r, t}^{\mathrm{TE}^{z}} l_{s, q}^{(r)}
$$

De esta forma, se llega al siguiente problema matricial equivalente:

$$
\mathbf{P}^{\mathrm{TE}} \cdot \mathbf{L}_{m}=k_{t, m}^{2} \mathbf{Q}^{\mathrm{TE}} \cdot \mathbf{L}_{m}
$$


donde:

$$
\begin{aligned}
\mathbf{P}^{\mathrm{TE}}{ }^{z} & =\left(P_{r, t}^{\mathrm{TE}^{z}}\right) \\
\mathbf{Q}^{\mathrm{TE}^{z}} & =\left(Q_{r, t}^{\mathrm{TE}^{z}}\right) \\
\mathbf{L}_{m} & =\left(l_{s, q}^{(r)}\right)
\end{aligned}
$$

Las matrices $\mathbf{P}^{\mathrm{TE}}{ }^{z}$ y $\mathbf{Q}^{\mathrm{TE}}{ }^{z}$ son reales y simétricas, con lo que los autovalores obtenidos serán reales. El cálculo de los elementos de estas matrices se ha detallado en el Apéndice A. La resolución del problema matricial anterior nos permitirá obtener los autovalores del problema para los modos $\mathrm{TE}^{z}$ (números de onda de corte $k_{t, m}$ ) así como los coeficientes de expansión de la solución modal en la variable radial $l_{s, q}^{(r)}$.

A continuación, se procede al cálculo de las funciones vectoriales modales asociadas a los modos $\mathrm{TE}^{z}$ partiendo de las siguientes relaciones [39]:

$$
\begin{aligned}
\widetilde{\mathbf{h}}_{t_{(\xi), m}}^{\mathrm{TE}}(\rho, \phi) & =-\nabla_{t} h_{z_{(\xi), m}}^{\mathrm{TE}^{z}}(\rho, \phi) \\
\widetilde{\mathbf{e}}_{t_{(\xi), m}}^{\mathrm{TE}}(\rho, \phi) & =\widetilde{\mathbf{h}}_{t_{(\xi), m}}^{\mathrm{TE}^{z}}(\rho, \phi) \times \hat{\mathbf{z}}
\end{aligned}
$$

En esta expresión, $\widetilde{\mathbf{e}}_{t_{(\xi), m}}^{\mathrm{TE}}(\rho, \phi)$ y $\widetilde{\mathbf{h}}_{t_{(\xi), m}}^{\mathrm{TE}}(\rho, \phi)$ representan las funciones vectoriales modales no normalizadas de campo eléctrico y de campo magnético, respectivamente, asociadas al modo $m$-ésimo. Si ahora se desarrollan las expresiones (2.112) y (2.113) se obtendrá que:

$$
\begin{aligned}
\widetilde{\mathbf{e}}_{t_{(\xi), m}}^{\mathrm{TE}}(\rho, \phi)= & \frac{1}{\rho}\left(\sum_{r=0}^{N_{\rho}-1} l_{s, q}^{(r)} \cos \left(r \pi \frac{\rho-r_{2}}{r_{1}-r_{2}}\right)\right) \Phi_{(\xi), s}^{\prime}(\phi) \hat{\boldsymbol{\rho}} \\
& +\frac{\pi}{r_{1}-r_{2}}\left(\sum_{r=0}^{N_{\rho}-1} r l_{s, q}^{(r)} \sin \left(r \pi \frac{\rho-r_{2}}{r_{1}-r_{2}}\right)\right) \Phi_{(\xi), s}(\phi) \hat{\boldsymbol{\phi}} \\
\widetilde{\mathbf{h}}_{t_{(\xi), m}}^{\mathrm{TE}^{z}}(\rho, \phi)= & -\frac{\pi}{r_{1}-r_{2}}\left(\sum_{r=0}^{N_{\rho}-1} r l_{s, q}^{(r)} \sin \left(r \pi \frac{\rho-r_{2}}{r_{1}-r_{2}}\right)\right) \Phi_{(\xi), s}(\phi) \hat{\boldsymbol{\rho}} \\
& +\frac{1}{\rho}\left(\sum_{r=0}^{N_{\rho}-1} l_{s, q}^{(r)} \cos \left(r \pi \frac{\rho-r_{2}}{r_{1}-r_{2}}\right)\right) \Phi_{(\xi), s}^{\prime}(\phi) \hat{\boldsymbol{\phi}}
\end{aligned}
$$

Las expresiones anteriores deben normalizarse de acuerdo con la condición de ortonormalización que se recoge en (2.93) aplicada al caso de los modos $\mathrm{TE}^{z}$. Así, si recordamos que $(s, q)$ es la pareja de índices modales asociadas al $m$-ésimo modo de tipo $\mathrm{TE}^{z}$, el factor de 
normalización correspondiente puede obtenerse de la siguiente manera:

$$
\begin{aligned}
\mathcal{N}_{(\xi), m}^{\mathrm{TE} z}= & \left(\int_{S} \widetilde{\mathbf{e}}_{t_{(\xi), m}}^{\mathrm{TE}^{z}}(\rho, \phi) \cdot \widetilde{\mathbf{e}}_{t_{(\xi), m}}^{\mathrm{TE}^{z}}(\rho, \phi) d S\right)^{-\frac{1}{2}} \\
= & {\left[\left(\frac{\pi}{r_{1}-r_{2}}\right)^{2} \int_{0}^{2 \pi} \Phi_{(\xi), s}^{2}(\phi) d \phi\right.} \\
& \cdot \sum_{r=1}^{N_{\rho}} \sum_{t=1}^{N_{\rho}} r t l_{s, q}^{(r)} l_{s, q}^{(t)} \int_{r_{2}}^{r_{1}} \rho \sin \left(r \pi \frac{\rho-r_{2}}{r_{1}-r_{2}}\right) \sin \left(t \pi \frac{\rho-r_{2}}{r_{1}-r_{2}}\right) d \rho \\
& +\int_{0}^{2 \pi}\left(\Phi_{(\xi), s}^{\prime}(\phi)\right)^{2} d \phi \\
& \left.\cdot \sum_{r=0}^{N_{\rho}-1} \sum_{t=0}^{N_{\rho}-1} l_{s, q}^{(r)} l_{s, q}^{(t)} \int_{r_{2}}^{r_{1}} \frac{1}{\rho} \cos \left(r \pi \frac{\rho-r_{2}}{r_{1}-r_{2}}\right) \cos \left(t \pi \frac{\rho-r_{2}}{r_{1}-r_{2}}\right) d \rho\right]
\end{aligned}
$$

En consecuencia, las expresiones para las funciones vectoriales modales normalizadas de los modos $\mathrm{TE}^{z}$ son las siguientes:

$$
\begin{aligned}
& \mathbf{e}_{t_{(\xi), m}}^{\mathrm{TE}^{z}}(\rho, \phi)=\mathcal{N}_{(\xi), m}^{\mathrm{TE}^{z}} \widetilde{\mathbf{e}}_{t_{(\xi), m}}^{\mathrm{TE}^{z}}(\rho, \phi) \\
& \mathbf{h}_{t_{(\xi), m}}^{\mathrm{TE}^{z}}(\rho, \phi)=\mathcal{N}_{(\xi), m}^{\mathrm{TE}^{z}} \widetilde{\mathbf{h}}_{t_{(\xi), m}}^{\mathrm{TE}^{z}}(\rho, \phi)
\end{aligned}
$$

Las integrales en la variable radial que aparecen en la expresión del factor de normalización (2.116) se han calculado en el Apéndice A.

A continuación, se presentan diferentes resultados que validan la técnica propuesta para el caso de los modos $\mathrm{TE}^{z}$. Así pues, en la tabla 2.5 se comparan nuestros resultados para el número de onda de corte de diferentes modos $\mathrm{TE}_{s, q}^{z}$ con los datos extraídos de [28]. En este caso, la guía coaxial es de radio externo $r_{1}=1,6 \mathrm{~m}$ y de radio interno $r_{2}=1,0 \mathrm{~m}\left(\varepsilon_{r}=1,0\right)$, y se han considerado $N_{\rho}=25$ términos en la expansión (2.100). Los índices modales de los modos analizados son los siguientes: $(s, q)=(0,2),(2,2),(0,3),(3,3),(0,4),(2,4),(0,5)$, $(3,5)$. El análisis de los resultados obtenidos revela que la técnica propuesta es sumamente rigurosa y precisa.

Por otro lado, en la tabla 2.6 se realiza un nuevo estudio comparativo y se presentan los números de onda de corte de los modos $\mathrm{TE}_{s, 2}^{z}(s=1,2,5,10,100,200)$ para una guía coaxial de radio externo $r_{1}=1,0202 \mathrm{~m}$ y de radio interno $r_{2}=1,0 \mathrm{~m}\left(\varepsilon_{r}=1,0, N_{\rho}=25\right.$ términos en la expansión de la variación radial de la solución modal). Los resultados obtenidos, que se comparan con los proporcionados en [40], son de nuevo sumamente precisos.

Finalmente, en la tabla 2.7 se lleva a cabo un estudio de convergencia para el número de onda de corte de diferentes modos $\mathrm{TE}_{s, q}^{z}$ en función del número de términos radiales $N_{\rho}$ 


\begin{tabular}{|c|c|c|c|}
\hline \multirow{2}{*}{$\begin{array}{c}\text { Índices modales } \\
(s, q)\end{array}$} & \multicolumn{3}{|c|}{ Número de onda de corte de los modos $\mathrm{TE}_{s, q}^{z}(\mathrm{rad} / \mathrm{m})$} \\
\hline & Ref. [28] & Software con $N_{\rho}=25$ & Error relativo ( \%) \\
\hline$(0,2)$ & 5,278333 & 5,279092 & $1 \cdot 10^{-2}$ \\
\hline$(2,2)$ & 5,533333 & 5,523842 & $2 \cdot 10^{-1}$ \\
\hline$(0,3)$ & 10,493333 & 10,494135 & $8 \cdot 10^{-3}$ \\
\hline$(3,3)$ & 10,763333 & 10,763110 & $2 \cdot 10^{-3}$ \\
\hline$(0,4)$ & 15,723333 & 15,722817 & $3 \cdot 10^{-3}$ \\
\hline$(2,4)$ & 15,803333 & 15,802674 & $4 \cdot 10^{-3}$ \\
\hline$(0,5)$ & 20,955 & 20,955113 & $5 \cdot 10^{-4}$ \\
\hline$(3,5)$ & 21,09 & 21,089451 & $3 \cdot 10^{-3}$ \\
\hline
\end{tabular}

Tabla 2.5: Comparación del número de onda de corte obtenido con la herramienta implementada con los resultados presentados en [28]. La guía coaxial analizada es de radio externo $r_{1}=1,6 \mathrm{~m}$ y de radio interno $r_{2}=1,0 \mathrm{~m}\left(\varepsilon_{r}=1,0\right)$. Los resultados presentados se refieren a los modos $\mathrm{TE}_{s, q}^{z}$ con $(s, q)=(0,2),(2,2),(0,3),(3,3),(0,4),(2,4),(0,5),(3,5)$.

\begin{tabular}{|c|c|c|c|}
\hline \hline \multirow{2}{*}{$\begin{array}{c}\text { Índice modal } \\
\text { angular }(s)\end{array}$} & \multicolumn{3}{|c|}{ Número de onda de corte de los modos $\mathrm{TE}_{s, 2}^{z}(\mathbf{r a d} / \mathbf{m})$} \\
\cline { 2 - 4 } & Ref. [40] & Software con $N_{\rho}=25$ & Error relativo (\%) \\
\hline 1 & 155,51435 & 155,51435170 & $1 \cdot 10^{-6}$ \\
\hline 2 & 155,52380 & 155,52380679 & $4 \cdot 10^{-6}$ \\
\hline 5 & 155,58996 & 155,58997630 & $1 \cdot 10^{-5}$ \\
\hline 10 & 155,82606 & 155,82606670 & $4 \cdot 10^{-6}$ \\
\hline 100 & 184,35597 & 184,35596193 & $4 \cdot 10^{-6}$ \\
\hline 200 & 251,79803 & 251,79802942 & $2 \cdot 10^{-7}$ \\
\hline \hline
\end{tabular}

Tabla 2.6: Comparación del número de onda de corte obtenido con la herramienta implementada con los resultados presentados en [40]. La guía coaxial analizada es de radio externo $r_{1}=1,0202 \mathrm{~m}$ y de radio interno $r_{2}=1,0 \mathrm{~m}\left(\varepsilon_{r}=1,0\right)$. Los resultados presentados se refieren a los modos $\mathrm{TE}_{s, 2}^{z}$ con $s=1,2,5,10,100,200$.

considerados. De este estudio se concluye que, con tan sólo $N_{\rho}=5$ términos radiales, los resultados tienen una precisión muy aceptable. Por otro lado, la eficiencia computacional de la herramienta implementada es del mismo orden que la de los modos $\mathrm{TM}^{z}$ : los primeros 500 modos de tipo $\mathrm{TE}^{z}$ (sin contar los posibles modos degenerados) se calculan en unos 0,3 segundos.

\subsubsection{Análisis de la unión planar entre una guía rectangular y una guía coaxial}

En este apartado se analiza la unión planar entre una guía rectangular y una guía coaxial (ver figura 2.4) utilizando la técnica de la ecuación integral presentada en la sección 2.2. 


\begin{tabular}{|c|c|c|c|c|}
\hline \hline \multirow{2}{\text{Índicesmodales}}{$\begin{array}{c}\text { Número de términos radiales } \\
(s, q)\end{array}$} & $N_{\rho}=5$ & $N_{\rho}=25$ & $N_{\rho}=75$ & $N_{\rho}=150$ \\
\hline$(0,2)$ & 5,279096 & 5,279092 & 5,279092 & 5,279092 \\
\hline$(2,2)$ & 5,523844 & 5,523842 & 5,523842 & 5,523842 \\
\hline$(0,3)$ & 10,494239 & 10,494135 & 10,494135 & 10,494135 \\
\hline$(3,3)$ & 10,763177 & 10,763110 & 10,763110 & 10,763110 \\
\hline$(0,4)$ & 15,723041 & 15,722817 & 15,722817 & 15,722817 \\
\hline$(2,4)$ & 15,802868 & 15,802674 & 15,802674 & 15,802674 \\
\hline$(0,5)$ & 20,963390 & 20,955113 & 20,955113 & 20,955113 \\
\hline$(3,5)$ & 21,095870 & 21,089451 & 21,089451 & 21,089451 \\
\hline \hline
\end{tabular}

Tabla 2.7: Convergencia del número de onda de corte de los modos $\mathrm{TE}_{s, q}^{z}$ expresado en rad $/ \mathrm{m}$ para una guía coaxial de radio externo $r_{1}=1,6 \mathrm{~m}$ y de radio interno $r_{2}=1,0 \mathrm{~m}\left(\varepsilon_{r}=1,0\right)$ en función del número de términos $N_{\rho}$ considerados en la expansión radial de la solución modal. Los índices modales de los modos analizados son: $(s, q)=(0,2),(2,2),(0,3),(3,3),(0,4),(2,4),(0,5),(3,5)$.

Así, si se revisa la expresión de la matriz de impedancias generalizadas (2.49) asociada a una unión planar arbitraria, se comprobará que ésta depende de la denominada integral de acoplamiento $A_{u, v}$ entre las funciones vectoriales modales de las guías involucradas en la discontinuidad:

$$
A_{u, v}=\int_{S^{(2)}} \mathbf{h}_{u}^{(1)}\left(x^{\prime}, y^{\prime}\right) \cdot \mathbf{h}_{v}^{(2) *}\left(x^{\prime}, y^{\prime}\right) d S^{\prime}
$$

donde, para esta unión en particular, $\mathbf{h}_{u}^{(1)}\left(x^{\prime}, y^{\prime}\right)$ representa la función vectorial modal de tipo magnético asociada al $u$-ésimo modo de la guía rectangular; $\mathbf{h}_{v}^{(2)}\left(x^{\prime}, y^{\prime}\right)$ es la función vectorial modal de tipo magnético relativa al $v$-ésimo modo de la guía coaxial y $S^{(2)}$ representa la sección transversal de la guía coaxial. El cálculo de esta integral de acoplamiento es fundamental para caracterizar la unión planar entre las dos guías, y dicho cálculo es, precisamente, el primer objetivo de esta sección.

Consideremos entonces la figura 2.4, en la que se representa una unión planar entre una guía rectangular de dimensiones $a$ y $b$, y una guía coaxial de radio externo $r_{1}$ y de radio interno $r_{2}$. Los parámetros $\left(x_{s}, y_{s}\right)$ sitúan el centro de la guía coaxial con respecto a la esquina inferior derecha de la guía rectangular, de manera que, en un caso general, la guía coaxial puede estar descentrada con respecto a la guía rectangular. En el caso particular de que la guía coaxial esté en una posición centrada, se cumplirá que $x_{s}=a / 2, y_{s}=b / 2$. Las relaciones entre los diferentes sistemas de coordenadas representados en la figura 2.4 son las siguientes:

$$
\begin{aligned}
& x=x^{\prime}+x_{s}=\rho \cos \phi+x_{s} \\
& y=y^{\prime}+y_{s}=\rho \sin \phi+y_{s}
\end{aligned}
$$

Es importante observar que la integral de acoplamiento (2.119) es una integral de superficie cuyo cálculo se extiende sobre la sección transversal de la guía coaxial. Sin embargo, 


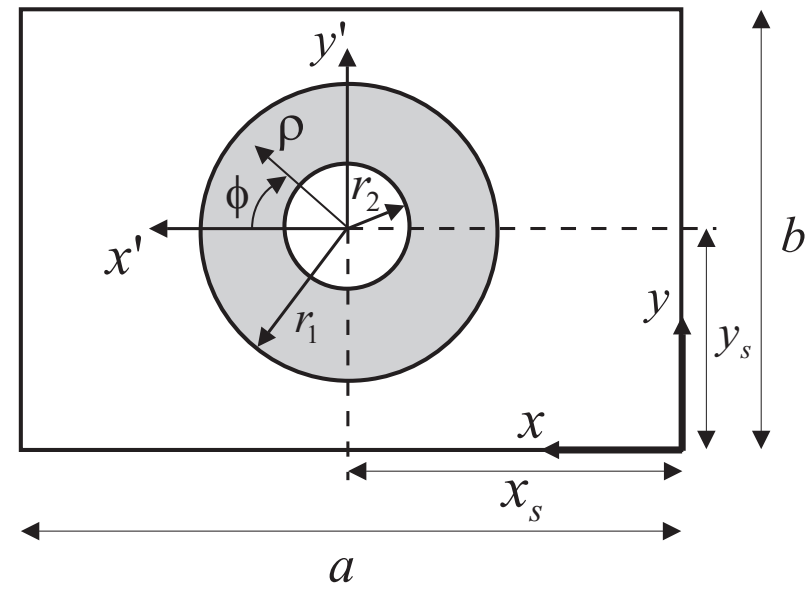

Figura 2.4: Unión planar entre una guía rectangular de dimensiones $a$ y $b$, y una guía coaxial de radio externo $r_{1}$ y radio interno $r_{2}$. Los parámetros $\left(x_{s}, y_{s}\right)$ sitúan el centro de la guía coaxial con respecto a la esquina inferior derecha de la guía rectangular.

según la teoría desarrollada en [41], esta integral de superficie puede transformarse en una integral de línea evaluada sobre el contorno de la guía coaxial ${ }^{10}$. La ventaja de esta transformación es muy importante desde un punto de vista computacional, ya que la integral de superficie se calculará utilizando técnicas numéricas de integración en una dimensión. En los resultados que se presentan en [41], la integral de acoplamiento $A_{u, v}$ adopta una expresión u otra dependiendo de si $u$ y $v$ son modos de tipo $\mathrm{TE}^{z}, \mathrm{TM}^{z}$ o $\mathrm{TEM}^{z}$. En concreto, gracias a los resultados de [41], [42], se puede afirmar que ${ }^{11}$ :

$$
\begin{aligned}
A_{u_{\mathrm{TE}}, v_{\mathrm{TE}}} & =\frac{k_{t, v}^{2}}{k_{t, v}^{2}-k_{t, u}^{2}} \oint_{c} \frac{\partial \psi_{u}^{\mathrm{TE}^{z}}(\rho, \phi)}{\partial \rho} \chi_{v}^{\mathrm{TE}^{z}}(\rho, \phi) \rho d \phi \\
A_{u_{\mathrm{TE}}, v_{\mathrm{TM}}} & =0 \\
A_{u_{\mathrm{TM}}, v_{\mathrm{TE}}} & =\oint_{c} \frac{\partial \psi_{u}^{\mathrm{TM}^{z}}(\rho, \phi)}{\partial \phi} \chi_{v}^{\mathrm{TE}^{z}}(\rho, \phi) d \phi \\
A_{u_{\mathrm{TM}}, v_{\mathrm{TM}}} & =-\frac{k_{t, u}^{2}}{k_{t, v}^{2}-k_{t, u}^{2}} \oint_{c} \psi_{u}^{\mathrm{TM}^{z}}(\rho, \phi) \frac{\partial \chi_{v}^{\mathrm{TM}^{z}}(\rho, \phi)}{\partial \rho} \rho d \phi
\end{aligned}
$$

\footnotetext{
${ }^{10}$ El contorno de la guía coaxial se define a partir de los radios externo e interno de dicha guía: $\rho=r_{1}$ y $\rho=r_{2}$. Asimismo, es muy importante definir el sentido de integración, el cual se debe escoger de manera que la superficie de interés se quede a la izquierda del sentido de integración. Según esta regla es fácil concluir que, en el contorno interno del coaxial, la integración seguirá un sentido horario y, en el contorno externo, el sentido será antihorario.

${ }^{11}$ En las expresiones (2.122) y (2.125) podría ocurrir que $k_{t, u}=k_{t, v}$. En ese caso, se tendría una indeterminación que se puede eliminar. Este tema se trata con rigor en [42], [43].
} 


$$
\begin{aligned}
& A_{u_{\mathrm{TE}}, v_{\mathrm{TEM}}}=0 \\
& A_{u_{\mathrm{TM}}, v_{\mathrm{TEM}}}=\oint_{c} \psi_{u}^{\mathrm{TM}^{z}}(\rho, \phi) \frac{\partial \chi_{v}^{\mathrm{TEM}^{z}}(\rho, \phi)}{\partial \rho} \rho d \phi
\end{aligned}
$$

En estas expresiones, $k_{t, u}$ denota el número de onda de corte asociado al $u$-ésimo modo de la guía rectangular; $k_{t, v}$ representa el número de onda de corte del $v$-ésimo modo de la guía coaxial; $\psi_{u}(\rho, \phi)$ es la función potencial modal normalizada relativa al $u$-ésimo modo de la guía rectangular (de tipo $\left.\mathrm{TE}^{z} \mathrm{o} \mathrm{TM}^{z}\right)$, y $\chi_{v}(\rho, \phi)$ denota la función potencial modal normalizada asociada al $v$-ésimo modo de la guía coaxial (de tipo $\mathrm{TE}^{z}, \mathrm{TM}^{z}$ o $\mathrm{TEM}^{z}$ ). Conviene recordar que, de acuerdo con la teoría expuesta anteriormente, las funciones potenciales modales normalizadas de una guía coaxial son las siguientes:

$$
\begin{aligned}
\chi_{v}^{\mathrm{TEM}^{z}}(\rho, \phi) & =\mathcal{N}_{\mathrm{TEM}^{z}} \ln \rho \\
\chi_{v}^{\mathrm{TE}^{z}}(\rho, \phi) & =\mathcal{N}_{(\xi), v}^{\mathrm{TE}^{z}}\left\{\sum_{r=0}^{N_{\rho}-1} l_{s, q}^{(r)} \cos \left(r \pi \frac{\rho-r_{2}}{r_{1}-r_{2}}\right)\right\} \Phi_{(\xi), s}(\phi) \\
\chi_{v}^{\mathrm{TM}^{z}}(\rho, \phi) & =\mathcal{N}_{(\xi), v}^{\mathrm{TM}^{z}}\left\{\sum_{r=1}^{N_{\rho}} d_{s, q}^{(r)} \sin \left(r \pi \frac{\rho-r_{2}}{r_{1}-r_{2}}\right)\right\} \Phi_{(\xi), s}(\phi)
\end{aligned}
$$

Por su parte, teniendo en cuenta las relaciones de transformación entre los sistemas de coordenadas rectangular y cartesiano definidas en (2.120)-(2.121), las funciones potenciales para la guía rectangular son las siguientes [39]:

$$
\begin{aligned}
\psi_{u}^{\mathrm{TE}^{z}}(\rho, \phi) & =\mathcal{N}_{u}^{\mathrm{TE}^{z}} \cos \left(\frac{m \pi}{a}\left(\rho \cos \phi+x_{s}\right)\right) \cos \left(\frac{n \pi}{b}\left(\rho \sin \phi+y_{s}\right)\right) \\
\psi_{u}^{\mathrm{TM}^{z}}(\rho, \phi) & =\mathcal{N}_{u}^{\mathrm{TM}^{z}} \sin \left(\frac{m \pi}{a}\left(\rho \cos \phi+x_{s}\right)\right) \sin \left(\frac{n \pi}{b}\left(\rho \sin \phi+y_{s}\right)\right)
\end{aligned}
$$

donde $(m, n)$ es la pareja de índices modales asociadas al $u$-ésimo modo de la guía rectangular correspondientes a las coordenadas $(x, y)$ representadas en la figura 2.4 ; y $\mathcal{N}_{u}$ denota el factor de normalización correspondiente y que se calcula como:

$$
\begin{aligned}
\mathcal{N}_{u}^{\mathrm{TE}} & =\sqrt{\frac{\epsilon_{m} \epsilon_{n}}{a b}}\left[\left(\frac{m \pi}{a}\right)+\left(\frac{n \pi}{b}\right)\right]^{-\frac{1}{2}} \\
\mathcal{N}_{u}^{\mathrm{TM}^{z}} & =\frac{2}{\sqrt{a b}}\left[\left(\frac{m \pi}{a}\right)+\left(\frac{n \pi}{b}\right)\right]^{-\frac{1}{2}}
\end{aligned}
$$

donde $\epsilon_{m}$ representa la constante de Neumann definida en (C.6).

Así pues, recordemos que $(s, q)$ representa la pareja de índices modales asociados al $v$-ésimo modo de la guía coaxial, correspondientes a las variables angular y radial, respectivamente; y consideremos, en primer lugar, que $u$ y $v$ son modos de tipo $\mathrm{TE}^{z}$. En este caso, 
la integral de acoplamiento adopta la siguiente expresión:

$$
\begin{aligned}
A_{u_{\mathrm{TE}}, v_{\mathrm{TE}}}= & -\mathcal{N}_{u}^{\mathrm{TE}^{z}} \mathcal{N}_{(\xi), v}^{\mathrm{TE}^{z}} \frac{k_{t, v}^{2}}{k_{t, v}^{2}-k_{t, u}^{2}}\left[\left.I_{\mathrm{TE}, \mathrm{TE}}(\rho, \phi)\right|_{\rho=r_{2}} \sum_{r=0}^{N_{\rho}-1} l_{s, q}^{(r)}\right. \\
& \left.-\left.I_{\mathrm{TE}, \mathrm{TE}}(\rho, \phi)\right|_{\rho=r_{1}} \sum_{r=0}^{N_{\rho}-1}(-1)^{r} l_{s, q}^{(r)}\right]
\end{aligned}
$$

donde hemos definido:

$$
I_{\mathrm{TE}, \mathrm{TE}}(\rho, \phi)=\left(\frac{m \pi}{a}\right) I_{\mathrm{TE}, \mathrm{TE}}^{(1)}(\rho, \phi)-\left(\frac{n \pi}{b}\right) I_{\mathrm{TE}, \mathrm{TE}}^{(2)}(\rho, \phi)
$$

siendo:

$$
\begin{aligned}
& I_{\mathrm{TE}, \mathrm{TE}}^{(1)}(\rho, \phi)=\oint_{c} \rho \sin \left(\frac{m \pi}{a}\left(\rho \cos \phi+x_{s}\right)\right) \cos \left(\frac{n \pi}{b}\left(\rho \sin \phi+y_{s}\right)\right) \cos \phi \Phi_{(\xi), s}(\phi) d \phi \\
& I_{\mathrm{TE}, \mathrm{TE}}^{(2)}(\rho, \phi)=\oint_{c} \rho \cos \left(\frac{m \pi}{a}\left(\rho \cos \phi+x_{s}\right)\right) \sin \left(\frac{n \pi}{b}\left(\rho \sin \phi+y_{s}\right)\right) \sin \phi \Phi_{(\xi), s}(\phi) d \phi
\end{aligned}
$$

donde el subíndice $c$ de las integrales representa el contorno de la guía coaxial. Estas últimas integrales se han resuelto empleando métodos numéricos.

A continuación, consideremos que $u$ es un modo $\mathrm{TM}^{z}$ de la guía rectangular y que $v$ es un modo de tipo $\mathrm{TE}^{z}$ de la guía coaxial. Teniendo en cuenta la expresión (2.124), se concluye que:

$$
\begin{aligned}
A_{u_{\mathrm{TM}}, v_{\mathrm{TE}}}= & -\mathcal{N}_{u}^{\mathrm{TM}^{z}} \mathcal{N}_{(\xi), v}^{\mathrm{TE}^{z}}\left[\left.I_{\mathrm{TM}, \mathrm{TE}}(\rho, \phi)\right|_{\rho=r_{2}} \sum_{r=0}^{N_{\rho}-1} l_{s, q}^{(r)}\right. \\
& \left.-\left.I_{\mathrm{TM}, \mathrm{TE}}(\rho, \phi)\right|_{\rho=r_{1}} \sum_{r=0}^{N_{\rho}-1}(-1)^{r} l_{s, q}^{(r)}\right]
\end{aligned}
$$

donde se ha definido la siguiente expresión:

$$
I_{\mathrm{TM}, \mathrm{TE}}(\rho, \phi)=-\left(\frac{m \pi}{a}\right) I_{\mathrm{TE}, \mathrm{TE}}^{(2)}(\rho, \phi)+\left(\frac{n \pi}{b}\right) I_{\mathrm{TE}, \mathrm{TE}}^{(1)}(\rho, \phi)
$$

siendo $I_{\mathrm{TE}, \mathrm{TE}}^{(1)}(\rho, \phi)$ y $I_{\mathrm{TE}, \mathrm{TE}}^{(2)}(\rho, \phi)$ las integrales definidas en $(2.137)$ y (2.138), respectiva- 
mente.

En el caso de que $u$ y $v$ sean modos de tipo $\mathrm{TM}^{z}$ tendremos que:

$$
\begin{aligned}
A_{u_{\mathrm{TM}}, v_{\mathrm{TM}}}= & -\frac{\pi}{r_{1}-r_{2}} \mathcal{N}_{u}^{\mathrm{TM}^{z}} \mathcal{N}_{(\xi), v}^{\mathrm{TM}^{z}} \frac{k_{t, u}^{2}}{k_{t, v}^{2}-k_{t, u}^{2}}\left[\left.I_{\mathrm{TM}, \mathrm{TM}}(\rho, \phi)\right|_{\rho=r_{2}} \sum_{r=1}^{N_{\rho}} r l_{s, q}^{(r)}\right. \\
& \left.-\left.I_{\mathrm{TM}, \mathrm{TM}}(\rho, \phi)\right|_{\rho=r_{1}} \sum_{r=1}^{N_{\rho}} r(-1)^{r} l_{s, q}^{(r)}\right]
\end{aligned}
$$

donde la integral $I_{\mathrm{TM}, \mathrm{TM}}(\rho, \phi)$, que se ha resuelto numéricamente, se define de la siguiente manera:

$$
I_{\mathrm{TM}, \mathrm{TM}}(\rho, \phi)=\oint_{c} \rho \sin \left(\frac{m \pi}{a}\left(\rho \cos \phi+x_{s}\right)\right) \sin \left(\frac{n \pi}{b}\left(\rho \sin \phi+y_{s}\right)\right) \Phi_{(\xi), s}(\phi) d \phi
$$

Finalmente, en el caso de que $u$ sea un modo $\mathrm{TM}^{z}$ de la guía rectangular y que $v$ sea un modo $\mathrm{TEM}^{z}$ de la guía coaxial se obtendrá que:

$$
A_{u_{\mathrm{TM}}, v_{\mathrm{TEM}}}=\mathcal{N}_{\mathrm{TEM}^{z}} \mathcal{N}_{u}^{\mathrm{TM}^{z}}\left(\left.I_{\mathrm{TM}, \mathrm{TEM}}(\rho, \phi)\right|_{\rho=r_{2}}-\left.I_{\mathrm{TM}, \mathrm{TEM}}(\rho, \phi)\right|_{\rho=r_{1}}\right)
$$

donde hemos definido la siguiente integral, la cual también se ha resuelto numéricamente:

$$
I_{\mathrm{TM}, \mathrm{TEM}}(\rho, \phi)=\oint_{c} \sin \left(\frac{m \pi}{a}\left(\rho \cos \phi+x_{s}\right)\right) \sin \left(\frac{n \pi}{b}\left(\rho \sin \phi+y_{s}\right)\right) d \phi
$$

Para el resto de casos (acoplamientos $\mathrm{TE}^{z}-\mathrm{TM}^{z}$ y $\mathrm{TE}^{z}-\mathrm{TEM}^{z}$ ), la integral de acoplamiento es nula en virtud de (2.123) y (2.126).

\subsubsection{Ejemplos de validación}

Una vez que se han calculado las integrales de acoplamiento para todos los casos posibles, el siguiente paso consiste en validar la teoría desarrollada en este apartado ${ }^{12}$. Para ello, a continuación se presentan los parámetros de dispersión asociados a diferentes uniones planares entre una guía rectangular y una guía coaxial. A este respecto es importante mencionar que, en todos los resultados presentados, se considera que el modo de excitación es el modo $\mathrm{TM}_{11}^{z}$ de la guía rectangular, y que el modo de respuesta es el modo fundamental de la guía coaxial (modo $\mathrm{TEM}^{z}$ ). La elección del modo de excitación anterior se basa en el hecho de que los modos $\mathrm{TE}^{z}$ de la guía rectangular no se acoplan con el modo $\mathrm{TEM}^{z}$ de la guía coaxial en virtud de (2.126). En consecuencia, se ha escogido como modo de excitación el

\footnotetext{
${ }^{12}$ Los resultados que se presentan en esta sección se comparan con los datos proporcionados por el programa Ansoft HFSS (High-Frequency Structure Simulator), ver. 9.0, Ansoft Corporation, Pittsburgh, PA, USA, 2005. Este programa se basa en el método de los elementos finitos.
} 
primer modo $\mathrm{TM}^{z}$ que se excita en la guía rectangular (modo $\mathrm{TM}_{11}^{z}$ ) con el fin de obtener unos resultados no nulos para el parámetro de dispersión $S_{21}$.

Así pues, en la figura 2.5 se analiza una unión planar entre una guía rectangular WR-75 ( $a=19,05 \mathrm{~mm}, b=9,525 \mathrm{~mm}$ ) y una guía coaxial de radio externo $r_{1}=1,9 \mathrm{~mm}$ y radio interno $r_{2}=0,6 \mathrm{~mm}$. Además, se ha considerado que la guía coaxial está rellena uniformemente de un material dieléctrico de permitividad dieléctrica relativa $\varepsilon_{r}=1,7^{13}$. Por otro lado, en este caso se considera que la guía coaxial está centrada con respecto a la guía rectangular $\left(x_{s}=a / 2, y_{s}=b / 2\right)$. Tal y como se aprecia en la figura 2.5 , los resultados obtenidos se comparan con éxito con los datos que proporciona HFSS ${ }^{14}$. Por otro lado, la

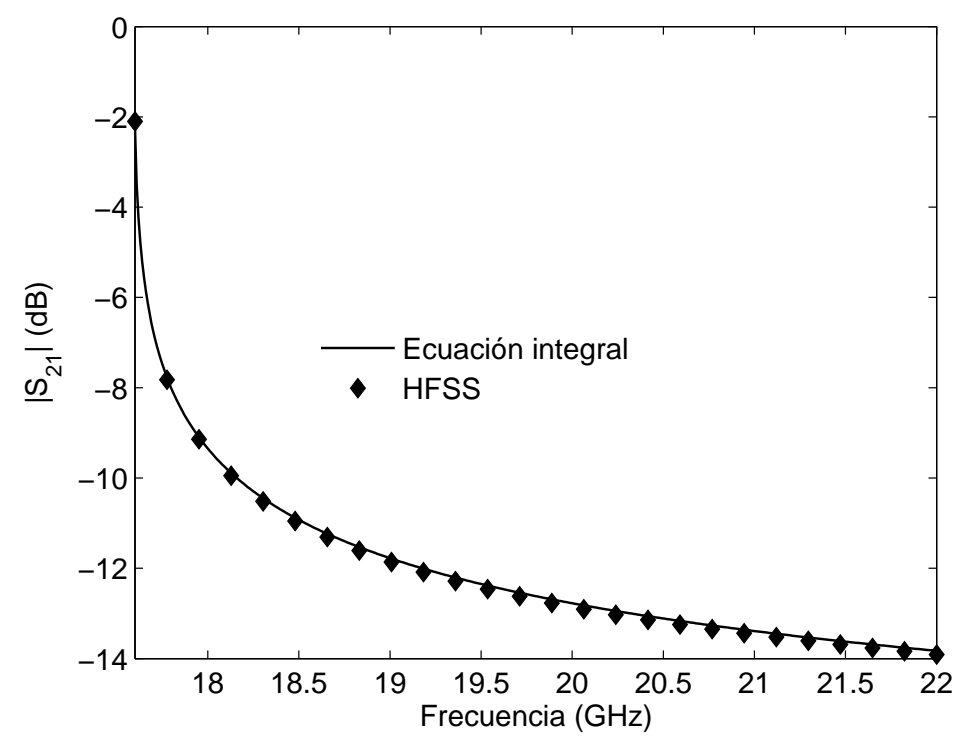

Figura 2.5: Módulo en dB del parámetro de transmisión $S_{21}$ relativo a una unión planar entre una guía rectangular WR-75 $(a=19,05 \mathrm{~mm}, b=9,525 \mathrm{~mm})$ y una guía coaxial $\left(r_{1}=1,9 \mathrm{~mm}\right.$, $r_{2}=0,6 \mathrm{~mm}, \varepsilon_{r}=1,7$ ). La guía coaxial se encuentra en una posición centrada con respecto a la guía rectangular. El modo de excitación es el modo $\mathrm{TM}_{11}^{z}$ de la guía rectangular WR-75 y el modo de respuesta es el modo $\mathrm{TEM}^{z}$ de la guía coaxial.

unión planar se ha analizado en 4,5 segundos, para un total de 440 puntos en frecuencia. Por su parte, la simulación realizada con HFSS empleó 15 minutos para 26 puntos en frecuencia, lo que demuestra la eficiencia computacional del método implementado.

Seguidamente, en la figura 2.6 se presentan los resultados del análisis correspondiente a una unión planar en la que la guía coaxial se encuentra descentrada con respecto a la guía rectangular $\left(x_{s}=4,25 \mathrm{~mm}, y_{s}=6,0 \mathrm{~mm}\right)$. Las dimensiones de las guías son las mismas que las del caso anterior. Nuevamente, los resultados obtenidos se comparan satisfactoriamente

\footnotetext{
${ }^{13}$ En todos los ejemplos de validación que se presentan en esta sección, se considera que en la guía rectangular se tiene $\varepsilon_{r}=1,0$.

${ }^{14} \mathrm{El}$ barrido en frecuencia considerado en la figura 2.5 comienza en $f=17,6 \mathrm{GHz}$, ya que éste es el valor de la frecuencia de corte del modo $\mathrm{TM}_{11}^{z}$ de la guía rectangular WR-75.
} 
con los que proporciona HFSS. Finalmente, en la figura 2.7 se analiza una unión entre una

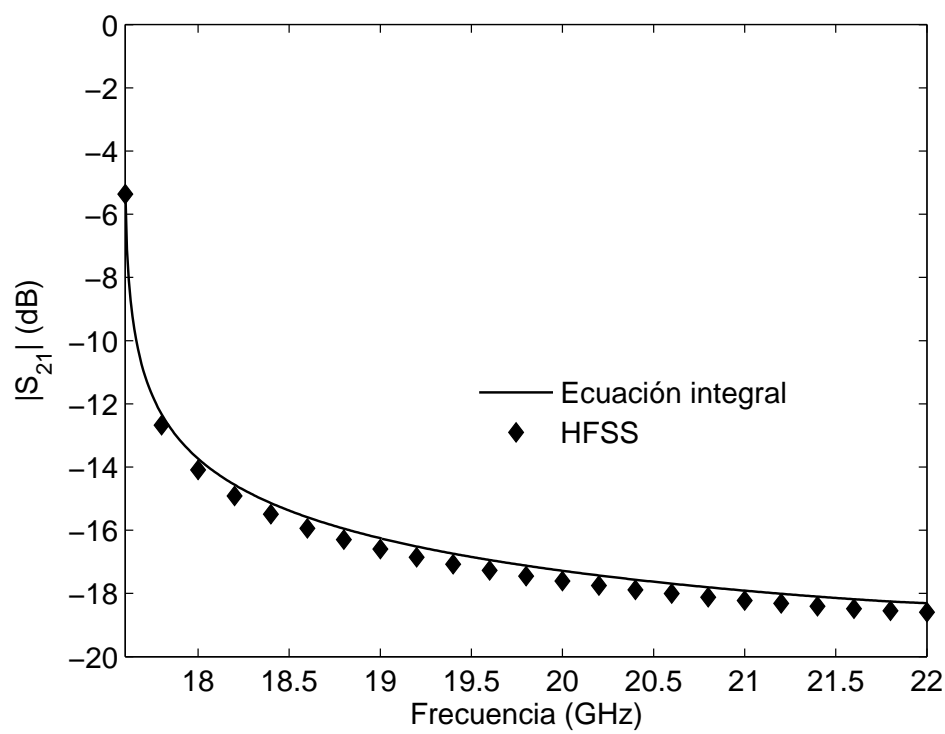

Figura 2.6: Módulo en dB del parámetro de transmisión $S_{21}$ relativo a una unión planar entre una guía rectangular WR-75 $(a=19,05 \mathrm{~mm}, b=9,525 \mathrm{~mm})$ y una guía coaxial $\left(r_{1}=1,9 \mathrm{~mm}\right.$, $\left.r_{2}=0,6 \mathrm{~mm}, \varepsilon_{r}=1,7\right)$. La guía coaxial se encuentra descentrada con respecto a la guía rectangular $\left(x_{s}=4,25 \mathrm{~mm}, y_{s}=6,0 \mathrm{~mm}\right)$. El modo de excitación es el modo $\mathrm{TM}_{11}^{z}$ de la guía rectangular WR-75 y el modo de respuesta es el modo $\mathrm{TEM}^{z}$ de la guía coaxial.

guía rectangular WR-90 ( $a=22,90 \mathrm{~mm}, b=10,20 \mathrm{~mm})$ y una guía coaxial de radio externo $r_{1}=3,2 \mathrm{~mm}$ y de radio interno $r_{2}=0,9 \mathrm{~mm}\left(\varepsilon_{r}=1,0\right)$. Además, la guía coaxial se encuentra centrada con respecto a la guía rectangular. Nuevamente, los resultados obtenidos concuerdan perfectamente con la simulación realizada con $\mathrm{HFSS}^{15}$.

Es importante mencionar que en todos los ejemplos de validación presentados en este capítulo se han utilizado 25 modos accesibles en las guías de onda involucradas en las discontinuidades planares analizadas y 60 funciones base en la expansión de la función vectorial incógnita $\mathbf{M}_{n}(x, y)$ presente en el método de la ecuación integral. Asimismo, el número de términos considerados en la expansión de la parte estática del núcleo de la ecuación integral es de 1200, y el número de términos considerados en la expansión de la parte dinámica es de 900 .

\footnotetext{
${ }^{15}$ En este caso, la simulación se inicia en $f=16,1 \mathrm{GHz}$, valor que coincide con la frecuencia de corte del modo $\mathrm{TM}_{11}^{z}$ de la guía rectangular WR-90.
} 


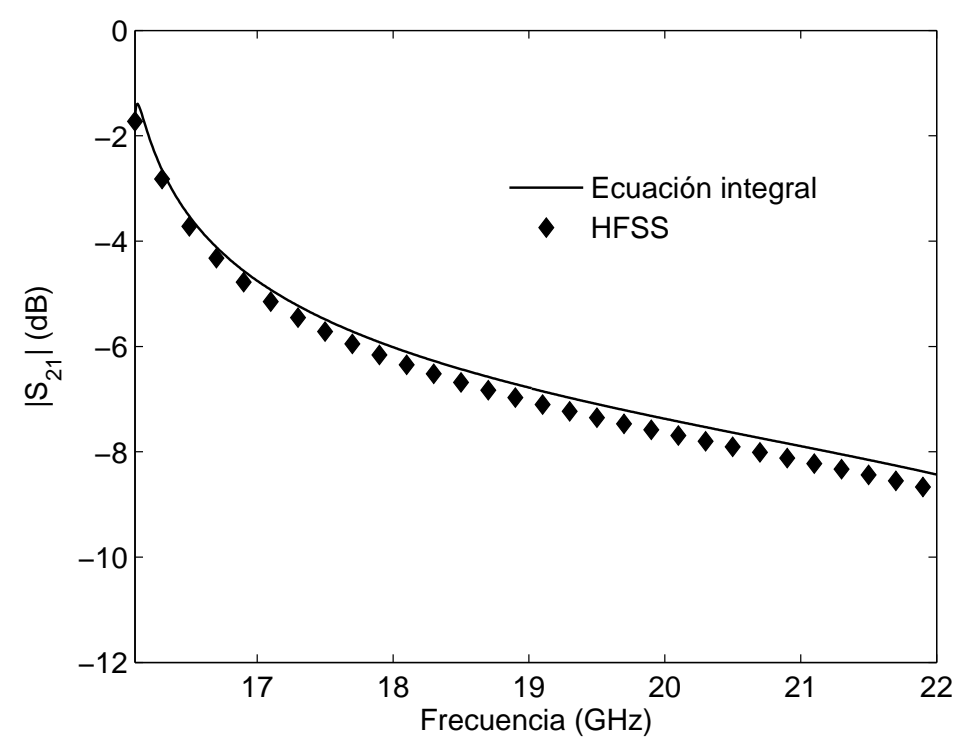

Figura 2.7: Módulo en dB del parámetro de transmisión $S_{21}$ relativo a una unión planar entre una guía rectangular WR-90 $(a=22,90 \mathrm{~mm}, b=10,20 \mathrm{~mm})$ y una guía coaxial $\left(r_{1}=3,2 \mathrm{~mm}\right.$, $\left.r_{2}=0,9 \mathrm{~mm}, \varepsilon_{r}=1,0\right)$. La guía coaxial se encuentra centrada con respecto a la guía rectangular. El modo de excitación es el modo $\mathrm{TM}_{11}^{z}$ de la guía rectangular WR-90 y el modo de respuesta es el modo $\mathrm{TEM}^{z}$ de la guía coaxial.

\subsection{Discontinuidades entre Guías Rectangulares y Guías Circulares}

El propósito de esta nueva sección es el análisis multimodal riguroso de la unión planar entre una guía rectangular y una guía circular utilizando la técnica de la ecuación integral. Tal y como ya se ha comentado en la sección anterior, el análisis de esta unión planar requerirá calcular las integrales de acoplamiento entre los modos de las guías involucradas en la unión. En consecuencia, se deberá obtener, previamente, la carta modal de una guía circular. A este respecto, es bien sabido que, según la solución modal clásica, las frecuencias de corte de una guía de sección transversal circular se obtienen calculando los ceros de las funciones de Bessel de primera especie y los de sus derivadas [39]. Con el objetivo de lograr un código eficiente desde un punto de vista computacional, en este apartado se propone evitar el uso de las funciones de Bessel en la descripción de la solución modal de la guía circular, utilizando en su lugar una formulación alternativa similar a la empleada en el apartado 2.3.1. En particular, la ecuación de Helmholtz se reescribirá en forma de un problema matricial lineal de autovalores equivalente y, posteriormente, se forzará a que la variación radial de las soluciones modales se exprese utilizando funciones sinusoidales que satisfagan las condiciones de contorno correspondientes. De esta manera, se acelerará la evaluación numérica de las integrales de acoplamiento obtenidas. 
La caracterización multimodal rigurosa de la unión planar entre una guía rectangular y una guía circular que se lleva a cabo en esta sección se utilizará en el capítulo 3, en el que se diseñarán uniones turnstile compensadas con postes cilíndricos. Las uniones turnstile son uniones de cinco puertos que constan de cuatro accesos coplanares de sección transversal rectangular, y de un quinto acceso de sección transversal circular que se sitúa en un plano perpendicular al que ocupan los puertos rectangulares [10]. Por tanto, en este tipo de dispositivos encontramos la unión planar objeto de estudio en este apartado. La unión turnstile se utiliza en el diseño de numerosos dispositivos pasivos de microondas, tal y como se refleja en el capítulo 3.

Por otro lado, en el capítulo 5 se presentará una herramienta destinada al análisis y diseño de filtros en configuración comb-line con excitación coaxial. Este tipo de filtros, que se caracterizan por ser muy compactos y que se utilizan en un gran número de aplicaciones, están constituidos por la asociación de diversas cavidades rectangulares cargadas con postes conductores de simetría cilíndrica. Las cavidades resonantes se comunican entre sí mediante irises de acoplamiento que pueden ser de sección transversal rectangular o circular. En consecuencia, el análisis multimodal de la unión planar que se estudia en esta sección será también de especial importancia para los objetivos del capítulo 5.

\subsubsection{Obtención de los modos de una guía circular utilizando el método de los momentos}

A continuación, se presenta un método alternativo para la obtención de las funciones vectoriales modales asociadas a una guía de sección transversal circular. La nueva técnica propuesta, cuyo desarrollo teórico es completamente paralelo al que se ha utilizado en la sección 2.3.1, expresa la variación radial de la soluciones modales utilizando funciones sinusoidales en lugar de emplear las clásicas funciones de Bessel. Por su parte, la ecuación de Helmholtz obtenida se transforma en un problema matricial lineal de autovalores equivalente que se resuelve mediante el conocido método de los momentos.

Así pues, sea una guía circular de radio $r_{1}$ como la representada en la figura 2.8. En la misma figura, se han representado los sistemas de referencia en cartesianas y en cilíndricas que utilizaremos posteriormente (la coordenada axial $z$ no se ha representado en la figura y se cumple $\hat{\mathbf{x}} \times \hat{\mathbf{y}}=\hat{\boldsymbol{\rho}} \times \hat{\boldsymbol{\phi}}=\hat{\mathbf{z}}$ ). Seguidamente, el análisis se particulariza para los dos tipos de modos que pueden existir en una guía circular: modos $\mathrm{TM}^{z}$ y modos $\mathrm{TE}^{z}$.

\section{$\operatorname{Modos}^{\mathbf{T}} \mathbf{M}^{z}$}

El cálculo de los modos $\mathrm{TM}^{z}$ de una guía circular sigue un procedimiento paralelo al que se desarrolló en la sección 2.3.1 para la obtención de los modos $\mathrm{TM}^{z}$ de una guía coaxial. Así pues, en primer lugar, se resuelve la ecuación de Helmholtz planteada en (2.65) con respecto a la variable angular, obteniéndose la solución de variación sinusoidal que se recoge en (2.71). Posteriormente, la ecuación diferencial con respecto a la variable radial se reescribe 


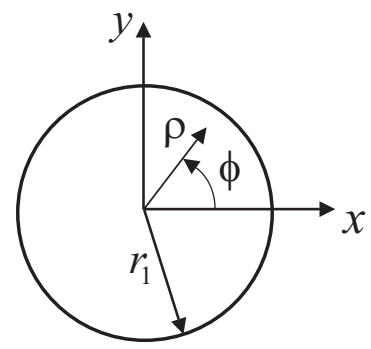

Figura 2.8: Sección transversal de una guía circular de radio $r_{1}$.

en términos del siguiente problema de autovalores equivalente:

$$
\mathcal{L}_{s} R^{\mathrm{TM}^{z}}(\rho)=-k_{t}^{2} R^{\mathrm{TM}^{z}}(\rho)
$$

donde el operador diferencial $\mathcal{L}_{s}$ se define en $(2.73), R^{\mathrm{TM}^{z}}(\rho)$ caracteriza la variación radial de las soluciones modales y $k_{t}$ denota el número de onda de corte de los modos $\mathrm{TM}^{z}$ de la guía circular.

Supongamos que $(s, q)$ es la pareja de índices modales relativos al $m$-ésimo modo de la guía circular, siendo $s$ el índice modal asociado a la variación angular y $q$ el índice modal asociado a la variación radial. La obtención de las funciones vectoriales modales de tipo $\mathrm{TM}^{z}$ parte de la elección de una función potencial adecuada. En este caso, la expresión que adopta dicha función potencial varía en función del valor que toma el índice modal angular $s$. Así, si $s \neq 0$ se tiene que:

$$
e_{z_{(\xi), m}}^{\mathrm{TM}^{z}}(\rho, \phi)=\left\{\sum_{r=1}^{\infty} d_{s, q}^{(r)} \sin \left(r \pi \frac{\rho}{r_{1}}\right)\right\} \Phi_{(\xi), s}(\phi)=R_{s, q}^{\mathrm{TM}^{z}}(\rho) \Phi_{(\xi), s}(\phi)
$$

donde $\Phi_{(\xi), s}(\phi)$ es la función que describe la variación angular de la solución modal y ya se ha definido en (2.71), y $d_{s, q}^{(r)}$ son unos coeficientes desconocidos. En caso de que el índice modal angular $s$ sea nulo se escoge la siguiente función potencial:

$$
e_{z_{m}(s=0)}^{\mathrm{TM}^{z}}(\rho, \phi)=\sum_{r=0}^{\infty} d_{0, q}^{(r)} \cos \left(\pi\left(r+\frac{1}{2}\right) \frac{\rho}{r_{1}}\right)=R_{0, q}^{\mathrm{TM}^{z}}(\rho)
$$

Es importante hacer notar que las funciones potenciales (2.146) y (2.147) satisfacen la condición de contorno de Dirichlet, es decir:

$$
\left.e_{z_{(\xi), m}}^{\mathrm{TM}^{z}}(\rho, \phi)\right|_{\rho=r_{1}}=\left.e_{z_{m}(s=0)}^{\mathrm{TM}^{z}}(\rho, \phi)\right|_{\rho=r_{1}}=0
$$

Por otro lado, es interesante observar que las funciones que rigen la variación radial de las funciones potenciales (2.146) y (2.147) tienen el mismo comportamiento que las funciones de Bessel de primera especie en el origen de coordenadas. En efecto, la función potencial que proporciona el método clásico para los modos $\mathrm{TM}^{z}$ es la siguiente [39]:

$$
e_{z}^{\mathrm{TM}^{z}}(\rho, \phi)=J_{s}\left(k_{t} \rho\right)\left\{\begin{array}{c}
\cos (s \phi) \\
\sin (s \phi)
\end{array}\right\}
$$


donde $J_{s}\left(k_{t} \rho\right)$ representa la función de Bessel de primera especie y de orden $s$. Así, para $s \neq 0$, obsérvese que $J_{s}(\rho=0)=0$. Es fácil comprobar que la función radial $R_{s, q}^{\mathrm{TM}^{z}}(\rho)$ que aparece en (2.146) tiene un comportamiento idéntico para $\rho=0$. Por otra parte, para $s=0$, se tiene que $J_{0}(\rho=0)=1$. Igualmente, la función radial $R_{0, q}^{\mathrm{TM}^{z}}(\rho)$ que se recoge en (2.147) es igual a un valor constante para $\rho=0$. De este modo se justifica la elección de dos funciones potenciales distintas dependiendo del valor que tome el índice angular $s$.

Seguidamente, se procede a la resolución del problema de autovalores (2.145) utilizando el procedimiento de Galerkin. Para ello, se utilizará el producto interno siguiente:

$$
\langle f(\rho) \mid g(\rho)\rangle=\int_{0}^{r_{1}} f(\rho) g^{*}(\rho) \rho d \rho
$$

Dado que la función $R_{s, q}^{\mathrm{TM}^{z}}(\rho)$ que rige la variación radial de la solución modal adopta dos expresiones diferentes en función del valor que tome el índice angular $s$, a continuación se tienen en cuenta estos dos casos.

Caso en que $s \neq 0$ :

En este caso, se tiene que:

$$
R_{s, q}^{\mathrm{TM}^{z}}(\rho)=\sum_{r=1}^{\infty} d_{s, q}^{(r)} \sin \left(r \pi \frac{\rho}{r_{1}}\right)=\sum_{r=1}^{\infty} d_{s, q}^{(r)} \alpha_{r}^{\mathrm{TM}^{z}}(\rho)
$$

Si ahora se aplica el producto interno definido en (2.150) en ambos miembros de la expresión (2.145) se tendrá que:

$$
\left\langle\mathcal{L}_{s} R_{s, q}^{\mathrm{TM}^{z}}(\rho) \mid \alpha_{t}^{\mathrm{TM}^{z}}(\rho)\right\rangle=-k_{t, m}^{2}\left\langle R_{s, q}^{\mathrm{TM}^{z}}(\rho) \mid \alpha_{t}^{\mathrm{TM}^{z}}(\rho)\right\rangle
$$

donde $\alpha_{t}^{\mathrm{TM}^{z}}(\rho)$ se corresponde con las funciones de test del método de los momentos cuya expresión puede deducirse de (2.151).

Primeramente, se calcula el producto interno que aparece en el miembro de la derecha de la expresión (2.152). Así, se tiene directamente que:

$$
\left\langle R_{s, q}^{\mathrm{TM}^{z}}(\rho) \mid \alpha_{t}^{\mathrm{TM}^{z}}(\rho)\right\rangle=\sum_{r=1}^{\infty} d_{s, q}^{(r)} \int_{0}^{r_{1}} \rho \sin \left(r \pi \frac{\rho}{r_{1}}\right) \sin \left(t \pi \frac{\rho}{r_{1}}\right) d \rho
$$

Posteriormente, en relación al miembro de la izquierda de (2.152), se aplica el operador diferencial $\mathcal{L}_{s}$ sobre la función de variación radial:

$$
\begin{aligned}
\mathcal{L}_{s} R_{s, q}^{\mathrm{TM}^{z}}(\rho) & =\left(\frac{d^{2}}{d \rho^{2}}+\frac{1}{\rho} \frac{d}{d \rho}-\frac{s^{2}}{\rho^{2}}\right) R_{s, q}^{\mathrm{TM}^{z}}(\rho) \\
& =\sum_{r=1}^{\infty} d_{s, q}^{(r)}\left[\left(\frac{d^{2}}{d \rho^{2}}+\frac{1}{\rho} \frac{d}{d \rho}-\frac{s^{2}}{\rho^{2}}\right) \sin \left(r \pi \frac{\rho}{r_{1}}\right)\right] \\
& =\sum_{r=1}^{\infty} d_{s, q}^{(r)}\left[-\left(\left(\frac{r \pi}{r_{1}}\right)^{2}+\frac{s^{2}}{\rho^{2}}\right) \sin \left(r \pi \frac{\rho}{r_{1}}\right)+\frac{r \pi}{r_{1}} \frac{1}{\rho} \cos \left(r \pi \frac{\rho}{r_{1}}\right)\right]
\end{aligned}
$$


De esta manera, se concluye que:

$$
\begin{aligned}
\left\langle\mathcal{L}_{s} R_{s, q}^{\mathrm{TM}^{z}}(\rho) \mid \alpha_{t}^{\mathrm{TM}^{z}}(\rho)\right\rangle= & -\sum_{r=1}^{\infty} d_{s, q}^{(r)} \int_{0}^{r_{1}}\left[\left(\rho\left(\frac{r \pi}{r_{1}}\right)^{2}+\frac{s^{2}}{\rho}\right) \sin \left(r \pi \frac{\rho}{r_{1}}\right)\right. \\
& \left.-\frac{r \pi}{r_{1}} \cos \left(r \pi \frac{\rho}{r_{1}}\right)\right] \sin \left(t \pi \frac{\rho}{r_{1}}\right) d \rho
\end{aligned}
$$

A continuación, se definen las siguientes matrices:

$$
\begin{aligned}
P_{r, t}^{\mathrm{TM}^{z}} & =\int_{0}^{r_{1}}\left[\left(\rho\left(\frac{r \pi}{r_{1}}\right)^{2}+\frac{s^{2}}{\rho}\right) \sin \left(r \pi \frac{\rho}{r_{1}}\right)-\frac{r \pi}{r_{1}} \cos \left(r \pi \frac{\rho}{r_{1}}\right)\right] \sin \left(t \pi \frac{\rho}{r_{1}}\right) d \rho \\
Q_{r, t}^{\mathrm{TM}^{z}} & =\int_{0}^{r_{1}} \rho \sin \left(r \pi \frac{\rho}{r_{1}}\right) \sin \left(t \pi \frac{\rho}{r_{1}}\right) d \rho
\end{aligned}
$$

donde $r, t=1,2, \ldots, N_{\rho}$, siendo $N_{\rho}$ el número de términos que se toman en el sumatorio de la expansión (2.151). Con estas nuevas definiciones, el problema de autovalores de partida queda expresado de la siguiente manera:

$$
\sum_{r=1}^{N_{\rho}} P_{r, t}^{\mathrm{TM}^{z}} d_{s, q}^{(r)}=k_{t, m}^{2} \sum_{r=1}^{N_{\rho}} Q_{r, t}^{\mathrm{TM}^{z}} d_{s, q}^{(r)}
$$

La ecuación anterior puede reescribirse en forma matricial de la siguiente manera ${ }^{16}$ :

$$
\mathbf{P}^{\mathrm{TM}} \cdot \mathbf{D}_{m}=k_{t, m}^{2} \mathbf{Q}^{\mathrm{TM}^{z}} \cdot \mathbf{D}_{m}
$$

donde $\mathbf{D}_{m}=\left(d_{s, q}^{(r)}\right)$, con $r, t=1,2, \ldots, N_{\rho}$. Los elementos de las matrices $\mathbf{P}^{\mathrm{TM}^{z}}$ y $\mathbf{Q}^{\mathrm{TM}^{z}}$ para el caso en que $s \neq 0$ se han recogido en el Apéndice B. Una vez resuelto el problema de autovalores (2.159), se obtendrán los números de onda de corte $k_{t, m}$ asociados a los modos $\mathrm{TM}^{z}$, así como los coeficientes de expansión $d_{s, q}^{(r)}$ utilizados en la función potencial de los modos $\mathrm{TM}^{z}$.

A continuación, la descripción de los modos de tipo $\mathrm{TM}^{z}$ se completa presentando las expresiones de las funciones vectoriales modales correspondientes al caso en el que $s \neq 0$. Así pues, aplicando las relaciones establecidas en (2.89)-(2.90), es posible obtener las si-

\footnotetext{
${ }^{16}$ Obsérvese que las matrices $\mathbf{P}^{\mathrm{TM}^{z}}$ y $\mathbf{Q}^{\mathrm{TM}^{z}}$ son reales y simétricas, por lo que los autovalores obtenidos serán también reales.
} 
guientes expresiones para las funciones vectoriales modales no normalizadas:

$$
\begin{aligned}
\widetilde{\mathbf{e}}_{t_{(\xi), m}}^{\mathrm{TM}^{z}}(\rho, \phi)= & \frac{\pi}{r_{1}}\left(\sum_{r=1}^{N_{\rho}} r d_{s, q}^{(r)} \cos \left(r \pi \frac{\rho}{r_{1}}\right)\right) \Phi_{(\xi), s}(\phi) \hat{\boldsymbol{\rho}} \\
& +\frac{1}{\rho}\left(\sum_{r=1}^{N_{\rho}} d_{s, q}^{(r)} \sin \left(r \pi \frac{\rho}{r_{1}}\right)\right) \Phi_{(\xi), s}^{\prime}(\phi) \hat{\boldsymbol{\phi}} \\
\widetilde{\mathbf{h}}_{t_{(\xi), m}}^{\mathrm{TM}^{z}}(\rho, \phi)= & -\frac{1}{\rho}\left(\sum_{r=1}^{N_{\rho}} d_{s, q}^{(r)} \sin \left(r \pi \frac{\rho}{r_{1}}\right)\right) \Phi_{(\xi), s}^{\prime}(\phi) \hat{\boldsymbol{\rho}} \\
& +\frac{\pi}{r_{1}}\left(\sum_{r=1}^{N_{\rho}} r d_{s, q}^{(r)} \cos \left(r \pi \frac{\rho}{r_{1}}\right)\right) \Phi_{(\xi), s}(\phi) \hat{\boldsymbol{\phi}}
\end{aligned}
$$

donde $\widetilde{\mathbf{e}}_{t_{(\xi), m}}^{\mathrm{TM}^{z}}(\rho, \phi)$ representa la función vectorial modal de campo eléctrico y $\widetilde{\mathbf{h}}_{t_{(\xi), m}}^{\mathrm{TM}^{z}}(\rho, \phi)$ denota la función vectorial modal de campo magnético. La ortonormalización de las funciones vectoriales anteriores se realiza atendiendo a la condición que se recoge en (2.93). Con ello, el factor de normalización puede calcularse como:

$$
\begin{aligned}
\mathcal{N}_{(\xi), m}^{\mathrm{TM}^{z}}= & \left(\int_{S} \widetilde{\mathbf{e}}_{t_{(\xi), m}}^{\mathrm{TM}^{z}}(\rho, \phi) \cdot \widetilde{\mathbf{e}}_{t_{(\xi), m}}^{\mathrm{TM}^{z}}(\rho, \phi) d S\right)^{-\frac{1}{2}} \\
= & {\left[\pi\left(\frac{\pi}{r_{1}}\right)^{2} \sum_{r=1}^{N_{\rho}} \sum_{t=1}^{N_{\rho}} r t d_{s, q}^{(r)} d_{s, q}^{(t)} \int_{0}^{r_{1}} \rho \cos \left(r \pi \frac{\rho}{r_{1}}\right) \cos \left(t \pi \frac{\rho}{r_{1}}\right) d \rho\right.} \\
& \left.+s^{2} \pi \sum_{r=1}^{N_{\rho}} \sum_{t=1}^{N_{\rho}} d_{s, q}^{(r)} d_{s, q}^{(t)} \int_{0}^{r_{1}} \frac{1}{\rho} \sin \left(r \pi \frac{\rho}{r_{1}}\right) \sin \left(t \pi \frac{\rho}{r_{1}}\right) d \rho\right]^{-\frac{1}{2}}
\end{aligned}
$$

Las integrales que aparecen en la expresión del factor de normalización anterior se calculan en el Apéndice B. De esta manera, las funciones vectoriales modales normalizadas para los modos $\mathrm{TM}^{z}$ cuyo índice angular $s$ sea no nulo son las siguientes:

$$
\begin{aligned}
\mathbf{e}_{t_{(\xi), m}}^{\mathrm{TM}^{z}}(\rho, \phi) & =\mathcal{N}_{(\xi), m}^{\mathrm{TM}^{z}} \widetilde{\mathbf{e}}_{t_{(\xi), m}}^{\mathrm{TM}^{z}}(\rho, \phi) \\
\mathbf{h}_{t_{(\xi), m}}^{\mathrm{TM}^{z}}(\rho, \phi) & =\mathcal{N}_{(\xi), m}^{\mathrm{TM}^{z}} \widetilde{\mathbf{h}}_{t_{(\xi), m}}^{\mathrm{TM}^{z}}(\rho, \phi)
\end{aligned}
$$

Caso en que $s=0$ :

Cuando el índice angular $s$ es nulo, la función que rige la variación radial de las soluciones modales es la siguiente:

$$
R_{0, q}^{\mathrm{TM}^{z}}(\rho)=\sum_{r=0}^{\infty} d_{0, q}^{(r)} \cos \left(\pi\left(r+\frac{1}{2}\right) \frac{\rho}{r_{1}}\right)
$$


donde el subíndice $q$ hace referencia al índice modal de variación radial. Siguiendo el mismo procedimiento que el utilizado para el caso en que $s \neq 0$, el problema de autovalores expresado en (2.145) a través de un operador diferencial, se puede transformar también en un problema de autovalores matricial equivalente como el que se recoge en (2.159) utilizando el método de los momentos. No obstante, hay que tener en cuenta que las matrices $\mathbf{P}^{\mathrm{TM}}{ }^{z} \mathrm{y}$ $\mathrm{Q}^{\mathrm{TM}}{ }^{z}$ adoptan ahora una nueva expresión, la cual se presenta a continuación:

$$
\begin{aligned}
P_{r, t_{(s=0)}}^{\mathrm{TM}^{z}}= & \frac{\pi^{2}}{r_{1}^{2}}\left(r+\frac{1}{2}\right)^{2} \int_{0}^{r_{1}} \cos \left(\pi\left(r+\frac{1}{2}\right) \frac{\rho}{r_{1}}\right) \cos \left(\pi\left(t+\frac{1}{2}\right) \frac{\rho}{r_{1}}\right) \rho d \rho \\
& +\frac{\pi}{r_{1}}\left(r+\frac{1}{2}\right) \int_{0}^{r_{1}} \sin \left(\pi\left(r+\frac{1}{2}\right) \frac{\rho}{r_{1}}\right) \cos \left(\pi\left(t+\frac{1}{2}\right) \frac{\rho}{r_{1}}\right) d \rho \\
Q_{r, t_{(s=0)}}^{\mathrm{TM}^{z}}= & \int_{0}^{r_{1}} \cos \left(\pi\left(r+\frac{1}{2}\right) \frac{\rho}{r_{1}}\right) \cos \left(\pi\left(t+\frac{1}{2}\right) \frac{\rho}{r_{1}}\right) \rho d \rho
\end{aligned}
$$

En estas expresiones hay que tener en cuenta que $r, t=0,1,2, \ldots, N_{\rho}-1$, siendo $N_{\rho}$ el número de términos efectivos considerados en el sumatorio de (2.165). Los elementos de estas matrices se han calculado analíticamente en el Apéndice B. Por otra parte, las funciones vectoriales modales normalizadas asociadas al $m$-ésimo modo $\mathrm{TM}^{z}$ con $s=0$, pueden calcularse fácilmente siguiendo la misma metodología presentada anteriormente, obteniéndose el siguiente resultado:

$$
\begin{aligned}
& \mathbf{e}_{t_{m}(s=0)}^{\mathrm{TM}^{z}}(\rho, \phi)=-\mathcal{N}_{m(s=0)}^{\mathrm{TM}^{z}} \frac{\pi}{r_{1}} \sum_{r=0}^{N_{\rho}-1} d_{0, q}^{(r)}\left(r+\frac{1}{2}\right) \sin \left(\pi\left(r+\frac{1}{2}\right) \frac{\rho}{r_{1}}\right) \hat{\boldsymbol{\rho}}(2.16 \\
& \mathbf{h}_{t_{m(s=0)}}^{\mathrm{TM}^{z}}(\rho, \phi)=-\mathcal{N}_{m(s=0)}^{\mathrm{TM}^{z}} \frac{\pi}{r_{1}} \sum_{r=0}^{N_{\rho}-1} d_{0, q}^{(r)}\left(r+\frac{1}{2}\right) \sin \left(\pi\left(r+\frac{1}{2}\right) \frac{\rho}{r_{1}}\right) \hat{\boldsymbol{\phi}}(2 .
\end{aligned}
$$

donde el factor de normalización $\mathcal{N}_{m(s=0)}^{\mathrm{TM}^{z}}$ se calcula ahora como:

$$
\begin{aligned}
\mathcal{N}_{m(s=0)}^{\mathrm{TM}^{z}}= & {\left[\frac{2 \pi^{3}}{r_{1}^{2}} \sum_{r=0}^{N_{\rho}-1} \sum_{t=0}^{N_{\rho}-1} d_{0, q}^{(r)} d_{0, q}^{(t)}\left(r+\frac{1}{2}\right)\left(t+\frac{1}{2}\right)\right.} \\
& \left.\cdot \int_{0}^{r_{1}} \rho \sin \left(\pi\left(r+\frac{1}{2}\right) \frac{\rho}{r_{1}}\right) \sin \left(\pi\left(t+\frac{1}{2}\right) \frac{\rho}{r_{1}}\right) d \rho\right]^{-\frac{1}{2}}
\end{aligned}
$$

La integral que aparece en esta última expresión se calcula también en el Apéndice B.

De esta manera se completa la formulación del problema correspondiente a la solución modal de tipo $\mathrm{TM}^{z}$ de una guía circular. Seguidamente, se procede a validar la teoría presentada y, para ello, se realizará una comparación de nuestros resultados con los que se proporcionan en [44]. Así pues, en la tabla 2.8 se presenta el número de onda de corte relativo a 
los modos $\mathrm{TM}_{s, 1}^{z}(s=0,1,2,5,6,7,8)$ de una guía circular de radio $r_{1}=1,0 \mathrm{~m}$, calculado con el código implementado tomando $N_{\rho}=75$ términos en la expansión de las funciones de variación radial presentadas en (2.151) y (2.165). Tal y como se puede comprobar, los resultados obtenidos son de una elevada precisión, ya que el error relativo en tanto por ciento es, al menos, del orden de $10^{-4}$ en todos los casos estudiados. Por otro lado, para finalizar esta

\begin{tabular}{|c|c|c|c|}
\hline \hline \multirow{2}{*}{$\begin{array}{c}\text { Índice modal } \\
\text { angular }(s)\end{array}$} & \multicolumn{3}{|c|}{ Número de onda de corte de los modos $\mathrm{TM}_{s, 1}^{z}(\mathbf{r a d} / \mathbf{m})$} \\
\cline { 2 - 4 } & Ref. [44] & Software con $N_{\rho}=75$ & Error relativo ( \%) \\
\hline 0 & 2,40482556 & 2,40482560 & $2 \cdot 10^{-6}$ \\
\hline 1 & 3,83171 & 3,83170603 & $1 \cdot 10^{-4}$ \\
\hline 2 & 5,13562 & 5,13562240 & $5 \cdot 10^{-5}$ \\
\hline 5 & 8,77148 & 8,77148395 & $5 \cdot 10^{-5}$ \\
\hline 6 & 9,93611 & 9,93610968 & $3 \cdot 10^{-6}$ \\
\hline 7 & 11,08637 & 11,08637019 & $2 \cdot 10^{-6}$ \\
\hline 8 & 12,22509 & 12,22509245 & $2 \cdot 10^{-5}$ \\
\hline \hline
\end{tabular}

Tabla 2.8: Número de onda de corte relativo a los modos $\operatorname{TM}_{s, 1}^{z}(s=0,1,2,5,6,7,8)$ de una guía circular de radio $r_{1}=1,0 \mathrm{~m}$. Los resultados obtenidos con la herramienta implementada se comparan con los datos que se recogen en [44].

sección, en la tabla 2.9 se presenta un estudio de convergencia para el número de onda de corte de los modos $\mathrm{TM}_{s, 1}^{z}(s=0,1,2,5,6,7,8)$ de una guía circular de radio $r_{1}=1,0 \mathrm{~m}$ en función del número de términos $N_{\rho}$ considerados en la expansión de la función de variación radial. Del estudio realizado se desprende que, con sólo $N_{\rho}=25$ términos, se obtienen unos resultados muy precisos. Finalmente, es importante resaltar la eficiencia computacional de la técnica propuesta. Así, los primeros 500 modos de tipo $\mathrm{TM}^{z}$ de una guía $\operatorname{circular}^{17}\left(N_{\rho}=50\right.$ e índice angular máximo igual a 10) se calculan en tan sólo 0,2 segundos.

\section{$\underline{\operatorname{Modos}}^{z}$}

A continuación, se presenta el cálculo de los modos de tipo $\mathrm{TE}^{z}$ de una guía circular de radio $r_{1}$ utilizando para ello la nueva técnica propuesta. Como es bien sabido de los apartados anteriores, el procedimiento se inicia escribiendo la ecuación de Helmholtz en la variable radial en la forma de un problema de autovalores equivalente como el que se recoge en (2.145), el cual se resuelve utilizando el método de los momentos. En lo que sigue, asumiremos que $(s, q)$ representan los índices modales relativos al $m$-ésimo modo de tipo $\mathrm{TE}^{z}$ de la guía circular, siendo el índice $s$ el asociado a la variación angular y el índice $q$ el correspondiente a la variación radial.

Para el caso de los modos $\mathrm{TE}^{z}$ con índice modal angular $s$ no nulo, se parte de la siguiente

\footnotetext{
${ }^{17} \mathrm{Si}$ se tienen en cuenta en este cómputo los modos $\mathrm{TM}^{z}$ de tipo degenerado, realmente el número total de modos calculado en el tiempo proporcionado es igual a 950.
} 


\begin{tabular}{|c|c|c|c|c|}
\hline \hline \multirow{2}{*}{$\begin{array}{c}\text { Índice modal } \\
\text { angular }(s)\end{array}$} & \multicolumn{4}{|c|}{ Número de términos radiales } \\
\cline { 2 - 5 } & $N_{\rho}=5$ & $N_{\rho}=25$ & $N_{\rho}=75$ & $N_{\rho}=150$ \\
\hline 0 & 2,40492940 & 2,40482654 & 2,40482560 & 2,40482556 \\
\hline 1 & 3,83183736 & 3,83170597 & 3,83170603 & 3,83170598 \\
\hline 2 & 5,13635845 & 5,13562578 & 5,13562240 & 5,13562231 \\
\hline 5 & 8,77175665 & 8,77148720 & 8,77148395 & 8,77148383 \\
\hline 6 & 9,93635556 & 9,93611334 & 9,93610968 & 9,93610954 \\
\hline 7 & 11,08658676 & 11,08637425 & 11,08637019 & 11,08637004 \\
\hline 8 & 12,22525089 & 12,22509690 & 12,22509245 & 12,22509229 \\
\hline \hline
\end{tabular}

Tabla 2.9: Estudio de convergencia para el número de onda de corte expresado en $\mathrm{rad} / \mathrm{m}$ de los modos $\mathrm{TM}_{s, 1}^{z}(s=0,1,2,5,6,7,8)$ de una guía circular de radio $r_{1}=1,0 \mathrm{~m}$ en función del número de términos $N_{\rho}$ considerados en la expansión radial de la solución modal.

función potencial:

$$
h_{z_{(\xi), m}}^{\mathrm{TE}^{z}}(\rho, \phi)=\left\{\sum_{r=0}^{\infty} l_{s, q}^{(r)} \sin \left(\pi\left(r+\frac{1}{2}\right) \frac{\rho}{r_{1}}\right)\right\} \Phi_{(\xi), s}(\phi)=R_{s, q}^{\mathrm{TE}^{z}}(\rho) \Phi_{(\xi), s}(\phi)
$$

En la expresión anterior, $l_{s, q}^{(r)}$ son unos coeficientes de expansión desconocidos, y $\Phi_{(\xi), s}(\phi)$ representa la solución de la ecuación de Helmholtz con respecto a la variable angular cuya expresión se recoge en (2.71). Para el caso en el que el índice modal angular $s$ sea nulo, se utilizará la siguiente función potencial:

$$
h_{z_{m}(s=0)}^{\mathrm{TE}^{z}}(\rho, \phi)=\sum_{r=0}^{\infty} l_{0, q}^{(r)} \cos \left(r \pi \frac{\rho}{r_{1}}\right)=R_{0, q}^{\mathrm{TE}^{z}}(\rho)
$$

Es importante hacer notar que las funciones potenciales recién definidas satisfacen automáticamente las condiciones de contorno de Neumann, es decir:

$$
\left.\frac{\partial}{\partial \rho}\left(h_{z_{(\xi), m}}^{\mathrm{TE}^{z}}(\rho, \phi)\right)\right|_{\rho=r_{1}}=\left.\frac{\partial}{\partial \rho}\left(h_{z_{m}(s=0)}^{\mathrm{TE}^{z}}(\rho, \phi)\right)\right|_{\rho=r_{1}}=0
$$

Por otro lado, obsérvese también que las funciones potenciales escogidas se comportan de la misma manera en $\rho=0$ que la función potencial de la solución modal clásica. La función potencial del método clásico para los modos de tipo $\mathrm{TE}^{z}$ es [39]:

$$
h_{z}^{\mathrm{TE} z}(\rho, \phi)=J_{s}\left(k_{t} \rho\right)\left\{\begin{array}{c}
\cos (s \phi) \\
\sin (s \phi)
\end{array}\right\}
$$

siendo $J_{s}\left(k_{t} \rho\right)$ la función de Bessel de primera especie de orden $s$. Es fácil comprobar que esta función toma en $\rho=0$ el mismo valor que adoptan las funciones potenciales (2.171) y 
(2.172) en el mismo punto. Este hecho justifica la elección de dichas funciones potenciales.

A continuación, se procede a resolver el problema de autovalores con respecto a la variable radial para los modos $\mathrm{TE}^{z}$ :

$$
\mathcal{L}_{s} R^{\mathrm{TE}^{z}}(\rho)=-k_{t}^{2} R^{\mathrm{TE}}(\rho)
$$

Para ello, se utilizará el producto interno definido en (2.150). Además, el análisis se particulariza para los casos en que $s \neq 0$ y $s=0$.

Caso en que $s \neq 0$ :

La función que rige la variación radial de las soluciones modales es la siguiente:

$$
R_{s, q}^{\mathrm{TE}^{z}}(\rho)=\sum_{r=0}^{\infty} l_{s, q}^{(r)} \sin \left(\pi\left(r+\frac{1}{2}\right) \frac{\rho}{r_{1}}\right)=\sum_{r=0}^{\infty} l_{s, q}^{(r)} \alpha_{r}^{\mathrm{TE}^{z}}(\rho)
$$

Aplicando el procedimiento de Galerkin sobre el problema (2.175) obtendremos:

$$
\left\langle\mathcal{L}_{s} R_{s, q}^{\mathrm{TE}^{z}}(\rho) \mid \alpha_{t}^{\mathrm{TE}^{z}}(\rho)\right\rangle=-k_{t, m}^{2}\left\langle R_{s, q}^{\mathrm{TE}^{z}}(\rho) \mid \alpha_{t}^{\mathrm{TE}^{z}}(\rho)\right\rangle
$$

donde las funciones de test $\alpha_{t}^{\mathrm{TE}^{z}}(\rho)$ tienen una expresión idéntica a las funciones de expansión $\alpha_{r}^{\mathrm{TE}^{z}}(\rho)$ definidas en (2.176). Como viene siendo habitual definimos:

$$
\begin{aligned}
\left\langle\mathcal{L}_{s} R_{s, q}^{\mathrm{TE}^{z}}(\rho) \mid \alpha_{t}^{\mathrm{TE}^{z}}(\rho)\right\rangle & =-\sum_{r=0}^{\infty} l_{s, q}^{(r)} P_{r, t}^{\mathrm{TE}^{z}} \\
\left\langle R_{s, q}^{\mathrm{TE}^{z}}(\rho) \mid \alpha_{t}^{\mathrm{TE}^{z}}(\rho)\right\rangle & =\sum_{r=0}^{\infty} l_{s, q}^{(r)} Q_{r, t}^{\mathrm{TE}^{z}}
\end{aligned}
$$

En la expresión anterior es fácil deducir que:

$$
\begin{aligned}
P_{r, t}^{\mathrm{TE}^{z}}= & \int_{0}^{r_{1}}\left[\left\{\rho\left(\frac{\pi}{r_{1}}\left(r+\frac{1}{2}\right)\right)^{2}+\frac{s^{2}}{\rho}\right\} \sin \left(\pi\left(r+\frac{1}{2}\right) \frac{\rho}{r_{1}}\right)\right. \\
& \left.-\frac{\pi}{r_{1}}\left(r+\frac{1}{2}\right) \cos \left(\pi\left(r+\frac{1}{2}\right) \frac{\rho}{r_{1}}\right)\right] \sin \left(\pi\left(t+\frac{1}{2}\right) \frac{\rho}{r_{1}}\right) d \rho \\
Q_{r, t}^{\mathrm{TE}^{z}}= & \int_{0}^{r_{1}} \rho \sin \left(\pi\left(r+\frac{1}{2}\right) \frac{\rho}{r_{1}}\right) \sin \left(\pi\left(t+\frac{1}{2}\right) \frac{\rho}{r_{1}}\right) d \rho
\end{aligned}
$$

donde, si $N_{\rho}$ es el número de términos efectivos considerados en la expansión (2.176), entonces se tiene que $r, t=0,1,2, \ldots, N_{\rho}-1$. Los elementos de estas matrices se calculan analíticamente en el Apéndice B. Con estas definiciones, el problema de autovalores (2.175) se reescribe de la siguiente manera:

$$
\sum_{r=0}^{N_{\rho}-1} P_{r, t}^{\mathrm{TE}^{z}} l_{s, q}^{(r)}=k_{t, m}^{2} \sum_{r=0}^{N_{\rho}-1} Q_{r, t}^{\mathrm{TE}^{z}} l_{s, q}^{(r)}
$$


Equivalentemente, y empleando una notación matricial, también podemos escribir:

$$
\mathbf{P}^{\mathrm{TE}} \cdot \mathbf{L}_{m}=k_{t, m}^{2} \mathbf{Q}^{\mathrm{TE}} \cdot \mathbf{L}_{m}
$$

donde $\mathbf{L}_{m}=\left(l_{s, q}^{(r)}\right)$, con $r=0,1, \ldots, N_{\rho}-1$. La resolución del problema de autovalores (2.183) permitirá la obtención de los números de onda de corte $k_{t, m}$ asociados a los modos de tipo $\mathrm{TE}^{z}$ de la guía circular, así como el cálculo de los coeficientes de expansión $l_{s, q}^{(r)}$.

Seguidamente, se calculan las funciones vectoriales modales normalizadas relativas a los modos $\mathrm{TE}^{z}$. Así pues, utilizando las relaciones (2.112)-(2.113), es posible establecer que:

$$
\begin{aligned}
\mathbf{e}_{t_{(\xi), m}}^{\mathrm{TE}^{z}}(\rho, \phi)= & \mathcal{N}_{(\xi), m}^{\mathrm{TE}}\left\{\frac{1}{\rho}\left[\sum_{r=0}^{N_{\rho}-1} l_{s, q}^{(r)} \sin \left(\pi\left(r+\frac{1}{2}\right) \frac{\rho}{r_{1}}\right)\right] \Phi_{(\xi), s}^{\prime}(\phi) \hat{\boldsymbol{\rho}}\right. \\
& \left.-\frac{\pi}{r_{1}}\left[\sum_{r=0}^{N_{\rho}-1} l_{s, q}^{(r)}\left(r+\frac{1}{2}\right) \cos \left(\pi\left(r+\frac{1}{2}\right) \frac{\rho}{r_{1}}\right)\right] \Phi_{(\xi), s}(\phi) \hat{\boldsymbol{\phi}}\right\} \\
\mathbf{h}_{t_{(\xi), m}}^{\mathrm{TE}^{z}}(\rho, \phi)= & \mathcal{N}_{(\xi), m}^{\mathrm{TE}^{z}}\left\{\frac{\pi}{r_{1}}\left[\sum_{r=0}^{N_{\rho}-1} l_{s, q}^{(r)}\left(r+\frac{1}{2}\right) \cos \left(\pi\left(r+\frac{1}{2}\right) \frac{\rho}{r_{1}}\right)\right] \Phi_{(\xi), s}(\phi) \hat{\boldsymbol{\rho}}\right. \\
& \left.+\frac{1}{\rho}\left[\sum_{r=0}^{N_{\rho}-1} l_{s, q}^{(r)} \sin \left(\pi\left(r+\frac{1}{2}\right) \frac{\rho}{r_{1}}\right)\right] \Phi_{(\xi), s}^{\prime}(\phi) \hat{\boldsymbol{\phi}}\right\}
\end{aligned}
$$

En las expresiones anteriores, $\mathcal{N}_{(\xi), m}^{\mathrm{TE}^{z}}$ representa el factor de normalización de los modos $\mathrm{TE}^{z}$ que se calcula utilizando la condición de ortonormalización que se recoge en (2.93) aplicada al caso de los modos $\mathrm{TE}^{z}$. Así pues, se puede concluir que:

$$
\begin{aligned}
\mathcal{N}_{(\xi), m}^{\mathrm{TE}^{z}}= & {\left[\frac{\pi^{3}}{r_{1}^{2}} \sum_{r=0}^{N_{\rho}-1} \sum_{t=0}^{N_{\rho}-1} l_{s, q}^{(r)} l_{s, q}^{(t)}\left(r+\frac{1}{2}\right)\left(t+\frac{1}{2}\right)\right.} \\
& \cdot \int_{0}^{r_{1}} \rho \cos \left(\pi\left(r+\frac{1}{2}\right) \frac{\rho}{r_{1}}\right) \cos \left(\pi\left(t+\frac{1}{2}\right) \frac{\rho}{r_{1}}\right) d \rho \\
& \left.+\pi s^{2} \sum_{r=0}^{N_{\rho}-1} \sum_{t=0}^{N_{\rho}-1} l_{s, q}^{(r)} l_{s, q}^{(t)} \int_{0}^{r_{1}} \frac{1}{\rho} \sin \left(\pi\left(r+\frac{1}{2}\right) \frac{\rho}{r_{1}}\right) \sin \left(\pi\left(t+\frac{1}{2}\right) \frac{\rho}{r_{1}}\right) d \rho\right]^{-\frac{1}{2}}
\end{aligned}
$$

Las integrales que encontramos en (2.186) se han calculado analíticamente en el Apéndice B.

Caso en que $s=0$ :

Si el índice angular $s$ es nulo, entonces la función elegida para describir la variación 
radial de las soluciones modales de tipo $\mathrm{TE}^{z}$ es la siguiente:

$$
R_{0, q}^{\mathrm{TE}^{z}}(\rho)=\sum_{r=0}^{\infty} l_{0, q}^{(r)} \cos \left(r \pi \frac{\rho}{r_{1}}\right)
$$

siendo $q$ el índice modal en la variable radial. En este caso, al igual que en los anteriores, el problema de autovalores inicial en la variable radial se transforma en un problema matricial equivalente como el mostrado en (2.183) gracias al método de los momentos. Las nuevas expresiones para los elementos de las matrices $\mathbf{P}^{\mathrm{TM}^{z}}$ y $\mathbf{Q}^{\mathrm{TM}^{z}}$ son las siguientes: :

$$
\begin{aligned}
P_{r, t_{(s=0)}}^{\mathrm{TE}^{z}}= & \left(\frac{\pi r}{r_{1}}\right)^{2} \int_{0}^{r_{1}} \rho \cos \left(r \pi \frac{\rho}{r_{1}}\right) \cos \left(t \pi \frac{\rho}{r_{1}}\right) d \rho \\
& +\left(\frac{\pi r}{r_{1}}\right) \int_{0}^{r_{1}} \sin \left(r \pi \frac{\rho}{r_{1}}\right) \cos \left(t \pi \frac{\rho}{r_{1}}\right) d \rho \\
Q_{r, t_{(s=0)}}^{\mathrm{TE}^{z}}= & \int_{0}^{r_{1}} \rho \cos \left(r \pi \frac{\rho}{r_{1}}\right) \cos \left(t \pi \frac{\rho}{r_{1}}\right) d \rho
\end{aligned}
$$

donde $r, t=0,1, \ldots, N_{\rho}-1$. Las integrales que aparecen en estas últimas expresiones se han resuelto analíticamente en el Apéndice B. Para completar la formulación de los modos $\mathrm{TE}^{z}$ de la guía circular, resta calcular las funciones vectoriales modales para el caso en que $s=0$. Así, haciendo uso de las relaciones (2.112)-(2.113) concluimos que:

$$
\begin{aligned}
\mathbf{e}_{t_{m(s=0)}}^{\mathrm{TE}^{z}}(\rho, \phi) & =\mathcal{N}_{m(s=0)}^{\mathrm{TE}^{z}} \frac{\pi}{r_{1}} \sum_{r=0}^{N_{\rho}-1} r l_{0, q}^{(r)} \sin \left(r \pi \frac{\rho}{r_{1}}\right) \hat{\boldsymbol{\phi}} \\
\mathbf{h}_{t_{m(s=0)}}^{\mathrm{TE} z}(\rho, \phi) & =-\mathcal{N}_{m(s=0)}^{\mathrm{TE}^{z}} \frac{\pi}{r_{1}} \sum_{r=0}^{N_{\rho}-1} r l_{0, q}^{(r)} \sin \left(r \pi \frac{\rho}{r_{1}}\right) \hat{\boldsymbol{\rho}}
\end{aligned}
$$

donde el factor de normalización $\mathcal{N}_{m(s=0)}^{\mathrm{TE}^{z}}$ se expresa esta vez como:

$$
\mathcal{N}_{m(s=0)}^{\mathrm{TE}^{z}}=\left[\frac{2 \pi^{3}}{r_{1}^{2}} \sum_{r=0}^{N_{\rho}-1} \sum_{t=0}^{N_{\rho}-1} r t l_{0, q}^{(r)} l_{0, q}^{(t)} \cdot \int_{0}^{r_{1}} \rho \sin \left(r \pi \frac{\rho}{r_{1}}\right) \sin \left(t \pi \frac{\rho}{r_{1}}\right) d \rho\right]^{-\frac{1}{2}}
$$

La integral que aparece en la expresión del factor de normalización anterior se calcula analíticamente en el Apéndice B.

De esta manera, se completa la teoría correspondiente a la obtención de las funciones vectoriales modales de tipo $\mathrm{TE}^{z}$ para una guía circular. Seguidamente, se procede a validar la teoría desarrollada presentando, en primer lugar, el número de onda de corte para los modos $\mathrm{TE}_{s, 3}^{z}(\operatorname{con} s=0,1,2,5,6,7,8)$ relativos a una guía circular de radio $r_{1}=1,0 \mathrm{~m}$. En los cálculos se han tomado $N_{\rho}=75$ términos en la expansión de las funciones de variación 


\begin{tabular}{|c|c|c|c|}
\hline \hline \multirow{2}{*}{$\begin{array}{c}\text { Índice modal } \\
\text { angular }(s)\end{array}$} & \multicolumn{3}{|c|}{ Número de onda de corte de los modos $\mathrm{TE}_{s, 3}^{z}(\mathbf{r a d} / \mathbf{m})$} \\
\cline { 2 - 4 } & Ref. [44] & Software con $N_{\rho}=75$ & Error relativo (\%) \\
\hline 0 & 10,17346814 & 10,17346814 & $1 \cdot 10^{-6}$ \\
\hline 1 & 8,53632 & 8,53631637 & $4 \cdot 10^{-5}$ \\
\hline 2 & 9,96947 & 9,96946814 & $2 \cdot 10^{-5}$ \\
\hline 5 & 13,98719 & 13,98718863 & $1 \cdot 10^{-5}$ \\
\hline 6 & 15,26818 & 15,26818146 & $1 \cdot 10^{-5}$ \\
\hline 7 & 16,52937 & 16,52936588 & $2 \cdot 10^{-5}$ \\
\hline 8 & 17,77401 & 17,77401237 & $1 \cdot 10^{-5}$ \\
\hline \hline
\end{tabular}

Tabla 2.10: Número de onda de corte relativo a los modos $\mathrm{TE}_{s, 3}^{z}(s=0,1,2,5,6,7,8)$ de una guía circular de radio $r_{1}=1,0 \mathrm{~m}$. Los resultados obtenidos con la herramienta implementada se comparan con los datos extraídos de [44].

radial de las soluciones modales. Los resultados se comparan con los datos que se recogen en [44] observándose una excelente concordancia. De los resultados presentados en la tabla 2.10 se desprende que la teoría desarrollada es sumamente rigurosa y precisa. Por otra parte, en la tabla 2.11 se presenta un estudio de convergencia para el número de onda de corte de los modos $\mathrm{TE}_{s, 3}^{z}(s=0,1,2,5,6,7,8)$ de una guía circular de radio $r_{1}=1,0 \mathrm{~m}$ en función del número de términos $N_{\rho}$ considerados en la expansión. Se observa en la mencionada tabla que, con sólo $N_{\rho}=25$ términos, los resultados son sumamente precisos ${ }^{18}$.

\begin{tabular}{|c|c|c|c|c|}
\hline \hline \multirow{2}{\text{Índicemodal}}{$\begin{array}{c}\text { Número de términos radiales } \\
\text { angular }(s)\end{array}$} & $N_{\rho}=5$ & $N_{\rho}=25$ & $N_{\rho}=75$ & $N_{\rho}=150$ \\
\hline 0 & 10,17394475 & 10,17346818 & 10,17346814 & 10,17346814 \\
\hline 1 & 8,53640686 & 8,53631639 & 8,53631637 & 8,53631637 \\
\hline 2 & 9,98931307 & 9,96949345 & 9,96946814 & 9,96946784 \\
\hline 5 & 13,98731706 & 13,98718868 & 13,98718863 & 13,98718863 \\
\hline 6 & 15,29377534 & 15,26818151 & 15,26818146 & 15,26818146 \\
\hline 7 & 16,66403183 & 16,52936593 & 16,52936588 & 16,52936588 \\
\hline 8 & 18,11693589 & 17,77401241 & 17,77401237 & 17,77401237 \\
\hline \hline
\end{tabular}

Tabla 2.11: Estudio de convergencia para el número de onda de corte expresado en $\mathrm{rad} / \mathrm{m}$ de los modos $\operatorname{TE}_{s, 3}^{z}(s=0,1,2,5,6,7,8)$ de una guía circular de radio $r_{1}=1,0 \mathrm{~m}$ en función del número de términos $N_{\rho}$ considerados en la expansión radial de la solución modal.

\footnotetext{
${ }^{18} \mathrm{El}$ tiempo de cálculo de los modos de tipo $\mathrm{TE}^{z}$ es del mismo orden que el de los modos $\mathrm{TM}^{z}$.
} 


\subsubsection{Análisis de la unión planar entre una guía rectangular y una guía circular}

El análisis de la unión planar entre una guía rectangular y una guía circular parte del cálculo de las integrales de acoplamiento entre las funciones vectoriales modales de tipo magnético asociadas a los modos de ambas guías. En particular, debe evaluarse la siguiente integral de superficie, la cual se extiende sobre la sección transversal de la guía circular:

$$
A_{u, v}=\int_{S^{(2)}} \mathbf{h}_{u}^{(1)}\left(x^{\prime}, y^{\prime}\right) \cdot \mathbf{h}_{v}^{(2) *}\left(x^{\prime}, y^{\prime}\right) d S^{\prime}
$$

Para la unión que nos ocupa, $\mathbf{h}_{u}^{(1)}\left(x^{\prime}, y^{\prime}\right)$ es la función vectorial modal de tipo magnético asociada al $u$-ésimo modo de la guía rectangular; $\mathbf{h}_{v}^{(2)}\left(x^{\prime}, y^{\prime}\right)$ representa la función vectorial modal de tipo magnético relativa al $v$-ésimo modo de la guía circular, y $S^{(2)}$ es la sección transversal de la guía circular.

Consideremos la figura 2.9, en la que hemos representado la sección transversal de una unión planar entre una guía rectangular de dimensiones $a$ y $b$, y una guía circular de radio $r_{1}$. Dado que la integral de acoplamiento debe evaluarse en la sección transversal de la guía

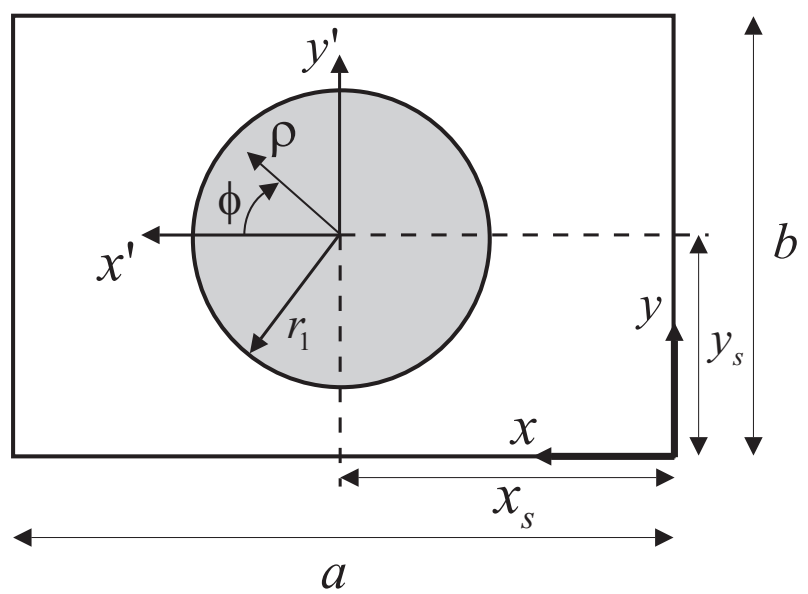

Figura 2.9: Unión planar entre una guía rectangular de dimensiones $a$ y $b$, y una guía circular de radio $r_{1}$. Los parámetros $\left(x_{s}, y_{s}\right)$ sitúan el centro de la guía circular con respecto a la esquina inferior derecha de la guía rectangular.

circular, los diferentes cálculos se van a realizar en coordenadas cilíndricas. Así pues, los sistemas de coordenadas cartesiano y cilíndrico representados en la figura 2.9 están relacionados entre sí a través de las siguientes ecuaciones:

$$
\begin{aligned}
& x=x^{\prime}+x_{s}=\rho \cos \phi+x_{s} \\
& y=y^{\prime}+y_{s}=\rho \sin \phi+y_{s}
\end{aligned}
$$


donde los parámetros $\left(x_{s}, y_{s}\right)$ se utilizan para situar el centro de la guía circular con respecto a la esquina inferior derecha de la guía rectangular. Por otro lado, tal y como ya se ha explicado en la sección 2.3.2 dedicada al análisis de la unión planar entre una guía rectangular y una guía coaxial, la integral de superficie (2.193) puede transformarse en una integral de línea equivalente en virtud de los resultados deducidos en [41]. De esta manera, las expresiones de las nuevas integrales de línea a resolver son las mismas que las que se recogen en (2.122)-(2.125). Asimismo, es importante recalcar que en dichas expresiones intervienen las funciones potenciales asociadas a los modos de las guías implicadas en la unión. Siguiendo la misma notación empleada en la sección 2.3.2, conviene recordar que, en las expresiones (2.122)-(2.125), $\psi_{u}(\rho, \phi)$ representa la función potencial relativa al $u$-ésimo modo de la guía rectangular, y $\chi_{v}(\rho, \phi)$ es la función potencial asociada al $v$-ésimo modo de la guía circular. Las funciones potenciales modales relativas a la guía rectangular expresadas en coordenadas cilíndricas se han definido en (2.131)-(2.132). Por otro lado, consideramos relevante recordar las expresiones asociadas a las funciones potenciales de los modos de una guía circular, las cuales fueron presentadas anteriormente:

$$
\begin{aligned}
& \left.\chi_{v}^{\mathrm{TE}^{z}}(\rho, \phi)\right|_{s \neq 0}=\mathcal{N}_{(\xi), v}^{\mathrm{TE}^{z}}\left\{\sum_{r=0}^{N_{\rho}-1} l_{s, q}^{(r)} \sin \left(\pi\left(r+\frac{1}{2}\right) \frac{\rho}{r_{1}}\right)\right\} \Phi_{(\xi), s}(\phi) \\
& \left.\chi_{v}^{\mathrm{TE}^{z}}(\rho, \phi)\right|_{s=0}=\mathcal{N}_{v(s=0)}^{\mathrm{TE}^{z}} \sum_{r=0}^{N_{\rho}-1} l_{0, q}^{(r)} \cos \left(r \pi \frac{\rho}{r_{1}}\right) \\
& \left.\chi_{v}^{\mathrm{TM}^{z}}(\rho, \phi)\right|_{s \neq 0}=\mathcal{N}_{(\xi), v}^{\mathrm{TM}^{z}}\left\{\sum_{r=1}^{N_{\rho}} d_{s, q}^{(r)} \sin \left(r \pi \frac{\rho}{r_{1}}\right)\right\} \Phi_{(\xi), s}(\phi) \\
& \chi_{v}^{\left.\mathrm{TM}^{z}(\rho, \phi)\right|_{s=0}}=\mathcal{N}_{v(s=0)}^{\mathrm{TM}^{z}} \sum_{r=0}^{N_{\rho}-1} d_{0, q}^{(r)} \cos \left(\pi\left(r+\frac{1}{2}\right) \frac{\rho}{r_{1}}\right)
\end{aligned}
$$

En estas expresiones, $(s, q)$ son los índices modales relativos al $v$-ésimo modo de la guía circular y están asociados a las variaciones angular y radial, respectivamente. Además, es interesante hacer notar que existen expresiones diferentes para las funciones potenciales del mismo tipo $\left(\mathrm{TE}^{z} \mathrm{o} \mathrm{TM}^{z}\right)$ en función de que el índice modal angular $s$ sea nulo o no.

Supongamos en primer lugar que $u$ y $v$ representan, ambos, modos de tipo $\mathrm{TE}^{z}$, siendo $(m, n)$ la pareja de índices modales relativos al $u$-ésimo modo de la guía rectangular. Además, asumiremos que el índice modal $s$ asociado al $v$-ésimo modo de la guía circular es no nulo. En este caso, es fácil concluir que la integral de acoplamiento (2.122) proporciona 
el siguiente resultado:

$$
\begin{aligned}
A_{u_{\mathrm{TE}}, v_{\mathrm{TE}}}= & \mathcal{N}_{u}^{\mathrm{TE}^{z}} \mathcal{N}_{(\xi), v}^{\mathrm{TE}^{z}} \frac{k_{t, v}^{2}}{k_{t, v}^{2}-k_{t, u}^{2}} \sum_{r=0}^{N_{\rho}-1}(-1)^{r} l_{s, q}^{(r)}\left\{\left.\left(\frac{m \pi}{a}\right) I_{\mathrm{TE}, \mathrm{TE}}^{(1)}(\rho, \phi)\right|_{\rho=r_{1}}\right. \\
& \left.+\left.\left(\frac{n \pi}{b}\right) I_{\mathrm{TE}, \mathrm{TE}}^{(2)}(\rho, \phi)\right|_{\rho=r_{1}}\right\}
\end{aligned}
$$

donde las integrales de contorno $I_{\mathrm{TE}, \mathrm{TE}}^{(1)}(\rho, \phi)$ y $I_{\mathrm{TE}, \mathrm{TE}}^{(2)}(\rho, \phi)$, que se han resuelto numéricamente, son las mismas que las definidas en (2.137)-(2.138). En caso de que el índice angular $s$ asociado al $v$-ésimo modo de la guía circular fuese nulo, la integral de acoplamiento anterior sigue siendo válida. La única precaución que debemos tener es la de tomar $s=0$ en las expresiones de las integrales de contorno anteriores.

Seguidamente, consideremos que $u$ es un modo de tipo $\mathrm{TM}^{z}$ y que $v$ es un modo de tipo $\mathrm{TE}^{z}$ cuyo índice modal angular $s$ asociado es no nulo. En virtud de (2.124) se concluye que:

$$
\begin{aligned}
A_{u_{\mathrm{TM}}, v_{\mathrm{TE}}}= & \mathcal{N}_{u}^{\mathrm{TM}^{z}} \mathcal{N}_{(\xi), v}^{\mathrm{TE}^{z}} \sum_{r=0}^{N_{\rho}-1}(-1)^{r} l_{s, q}^{(r)}\left\{\left.\left(\frac{m \pi}{a}\right) I_{\mathrm{TE}, \mathrm{TE}}^{(2)}(\rho, \phi)\right|_{\rho=r_{1}}\right. \\
& \left.-\left.\left(\frac{n \pi}{b}\right) I_{\mathrm{TE}, \mathrm{TE}}^{(1)}(\rho, \phi)\right|_{\rho=r_{1}}\right\}
\end{aligned}
$$

donde, nuevamente, las expresiones de las integrales de contorno $I_{\mathrm{TE}, \mathrm{TE}}^{(1)}(\rho, \phi)$ y $I_{\mathrm{TE}, \mathrm{TE}}^{(2)}(\rho, \phi)$ pueden encontrarse en (2.137)-(2.138). Asimismo, la expresión de la integral de acoplamiento (2.201) puede también emplearse en caso de que el índice angular $s$ sea nulo.

Finalmente, asumamos a continuación que $u$ representa un modo de tipo $\mathrm{TM}^{z}$ de la guía rectangular y que $v$ es un modo $\mathrm{TM}^{z}$ de la guía circular con índice angular $s$ no nulo. En este caso, utilizando la expresión (2.125), se obtiene:

$$
A_{u_{\mathrm{TM}}, v_{\mathrm{TM}}}=\left.\frac{\pi}{r_{1}} \mathcal{N}_{u}^{\mathrm{TM}^{z}} \mathcal{N}_{(\xi), v}^{\mathrm{TM}^{z}} \frac{k_{t, u}^{2}}{k_{t, v}^{2}-k_{t, u}^{2}} \sum_{r=1}^{N_{\rho}} r(-1)^{r} d_{s, q}^{(r)} I_{\mathrm{TM}, \mathrm{TM}}(\rho, \phi)\right|_{\rho=r_{1}}
$$

donde la expresión de la integral de contorno $I_{\mathrm{TM}, \mathrm{TM}}(\rho, \phi)$ se ha definido anteriormente en (2.142). En caso de que el índice angular $s$ relativo al $v$-ésimo modo de la guía circular fuese nulo, se obtendría la siguiente expresión para la integral de acoplamiento:

$$
A_{u_{\mathrm{TM}}, v_{\mathrm{TM}}(s=0)}=-\left.\frac{\pi}{r_{1}} \mathcal{N}_{u}^{\mathrm{TM}^{z}} \mathcal{N}_{v(s=0)}^{\mathrm{TM}^{z}} \frac{k_{t, u}^{2}}{k_{t, v}^{2}-k_{t, u}^{2}} \sum_{r=0}^{N_{\rho}-1}\left(r+\frac{1}{2}\right)(-1)^{r} d_{0, q}^{(r)} I_{\mathrm{TM}, \mathrm{TM}}(\rho, \phi)\right|_{\rho=r_{1}}
$$

En la expresión anterior, se debe tomar $s=0$ en el cálculo de la integral de contorno $I_{\mathrm{TM}, \mathrm{TM}}(\rho, \phi)$. Por último, en virtud de (2.123), en caso de que $u$ sea un modo de tipo $\mathrm{TE}^{z}$ de la guía rectangular, y $v$ un modo de tipo $\mathrm{TM}^{z}$ de la guía circular, la integral de acoplamiento es nula. 


\subsubsection{Ejemplos de validación}

Una vez concluido el análisis electromagnético de la unión, a continuación procedemos a la validación de la teoría desarrollada en esta sección. Así pues, en todos los ejemplos que se presentan seguidamente se asume que el modo incidente es el modo fundamental $\mathrm{TE}_{10}^{z}$ de la guía rectangular y que el modo de respuesta es el modo fundamental $\mathrm{TE}_{11}^{z}$ (coseno) de la guía circular. Por otro lado, se considera también que la guía circular se encuentra rellena uniformemente de un material de permitividad dieléctrica relativa $\varepsilon_{r}$. De esta forma, conseguimos reducir la frecuencia de corte efectiva del modo fundamental de la guía circular, permitiendo así la propagación de dicho modo a la región de la guía rectangular.

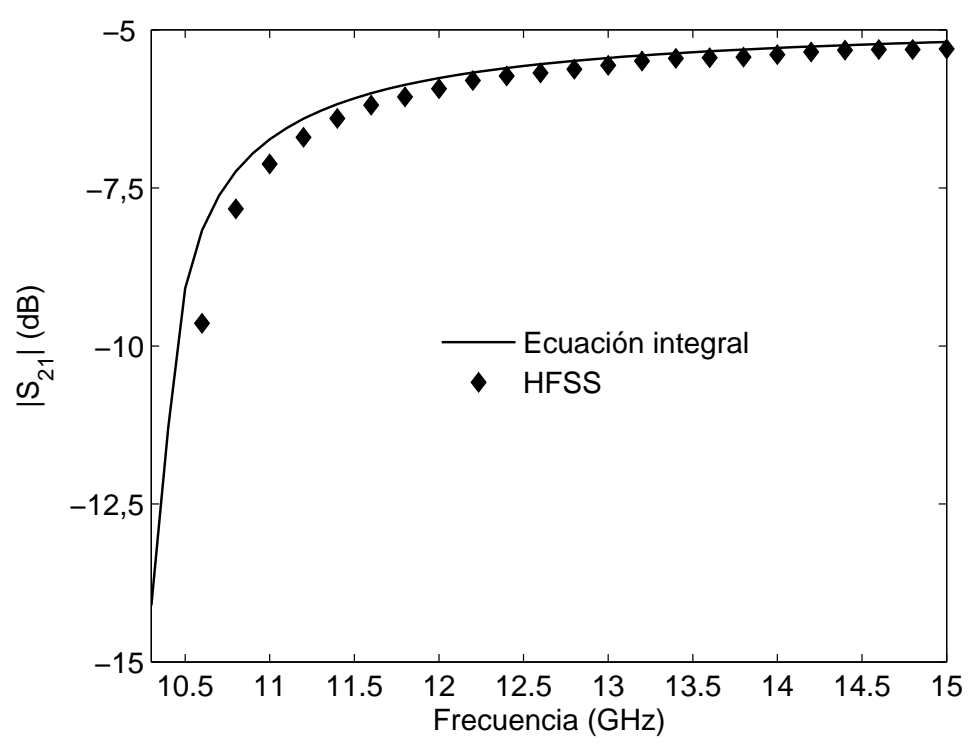

Figura 2.10: Módulo en dB del parámetro de transmisión $S_{21}$ relativo a una unión planar entre una guía rectangular WR-75 $(a=19,05 \mathrm{~mm}, b=9,525 \mathrm{~mm})$ y una guía circular de radio $r_{1}=3,0 \mathrm{~mm}$ $\left(\varepsilon_{r}=8,0\right)$. La guía circular se encuentra centrada con respecto a la guía rectangular.

El primero de los ejemplos de aplicación se muestra en la figura 2.10, en el cual se analiza una unión planar entre una guía circular de radio $r_{1}=3,0 \mathrm{~mm}\left(\varepsilon_{r}=8,0\right)$ y una guía rectangular WR-75 $(a=19,05 \mathrm{~mm}, b=9,525 \mathrm{~mm})^{19}$. La guía circular se encuentra centrada con respecto a la guía rectangular, con lo que refiriéndonos a la figura 2.9, se tiene que $x_{s}=a / 2$, $y_{s}=b / 2$. En la figura 2.10 comprobamos que los resultados de nuestra simulación se comparan satisfactoriamente con los proporcionados por HFSS. No obstante, a frecuencias bajas apreciamos una mínima discrepancia entre los datos presentados. A este respecto, es importante destacar que los resultados de nuestra simulación han sido también comparados con los

\footnotetext{
${ }^{19}$ Con el radio utilizado para la guía circular, la frecuencia de corte del modo fundamental de dicha guía resulta ser de $29,283 / \sqrt{\varepsilon_{r}}(\mathrm{GHz})$. Puesto que el rango de operación monomodo de la guía rectangular WR-75 es $10-15 \mathrm{GHz}$, precisamos que $\varepsilon_{r}<8,57$ para que se propague señal hacia la guía rectangular. De esta manera, para $\varepsilon_{r}=8,0$, se tiene que la frecuencia de corte del modo fundamental de la guía circular resulta ser de $10,35 \mathrm{GHz}$.
} 
proporcionados por la herramienta FEST 3D de la Agencia Espacial Europea ${ }^{20}$, observándose en este caso una perfecta concordancia entre ambos resultados (los datos proporcionados por FEST 3D no se han presentado, ya que se superponen a los obtenidos con la herramienta de simulación). Por ello, es bastante probable que la discrepancia observada se deba a un problema de convergencia de HFSS. Por otro lado, es importante mencionar que el análisis de la unión se ha realizado en 7,51 segundos para un total de 101 puntos en frecuencia. Por su parte, HFSS empleó unos 20 minutos para un total 23 de puntos en frecuencia.

En el siguiente ejemplo, se analiza una unión planar entre una guía circular de radio $r_{1}=2,7 \mathrm{~mm}\left(\varepsilon_{r}=14,0\right)$ y una guía rectangular WR-90 $(a=22,90 \mathrm{~mm}, b=10,20 \mathrm{~mm})$, estando la guía circular en una posición centrada con respecto a la guía rectangular ${ }^{21}$. Así, en la figura 2.11 se presenta el módulo del parámetro de transmisión $S_{21}$ para dicha unión y se compara el resultado obtenido con HFSS. Al igual que hemos comentado en el ejemplo anterior, encontramos también en este caso una pequeña discrepancia entre los resultados de la simulación y HFSS a frecuencias bajas, debidos muy probablemente a problemas de convergencia de esta última herramienta.

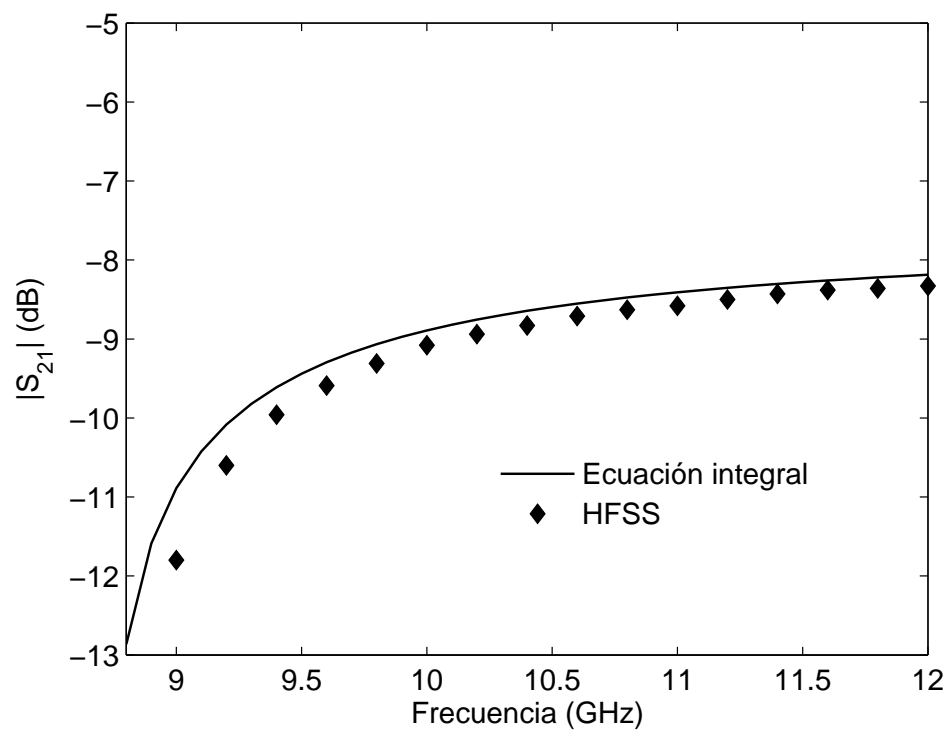

Figura 2.11: Módulo en dB del parámetro de transmisión $S_{21}$ relativo a una unión planar entre una guía rectangular WR-90 $(a=22,90 \mathrm{~mm}, b=10,20 \mathrm{~mm})$ y una guía circular de radio $r_{1}=2,7 \mathrm{~mm}$ $\left(\varepsilon_{r}=14,0\right)$. La guía circular se encuentra centrada con respecto a la guía rectangular.

Por último, se analiza una unión planar de las mismas dimensiones que la anterior, pe-

\footnotetext{
${ }^{20}$ La herramienta FEST 3D (Full-wave Electromagnetic Simulation Tool) utiliza también la técnica de la ecuación integral para caracterizar uniones planares entre guías de onda. Sin embargo, en esta herramienta se emplea la formulación clásica para la obtención de las soluciones modales de la guía circular.

${ }^{21}$ En este caso, la frecuencia de corte del modo fundamental de la guía circular es $32,537 / \sqrt{\varepsilon_{r}}(\mathrm{GHz})$. Dado que el rango frecuencial de operación de la guía rectangular WR-90 es $8-12 \mathrm{GHz}$, en este caso precisamos que $\varepsilon_{r}<16,54$.
} 
ro, en este caso, la guía circular se encuentra descentrada con respecto a la guía rectangular $\left(x_{s}=6,45 \mathrm{~mm}, y_{s}=3,1 \mathrm{~mm}\right)$. Los resultados obtenidos para este caso se han representado en la figura 2.12. Nuevamente, se detecta cierta discrepancia a frecuencias bajas entre nuestros resultados y HFSS. Por ello, se ha procedido a comparar nuestros resultados con los obtenidos con FEST 3D verificándose nuevamente la precisión de nuestros resultados. Los tiempos de simulación de los dos últimos casos prácticos presentados son similares a los indicados en el primero de los ejemplos, con lo que queda demostrada la eficiencia computacional de la herramienta implementada.

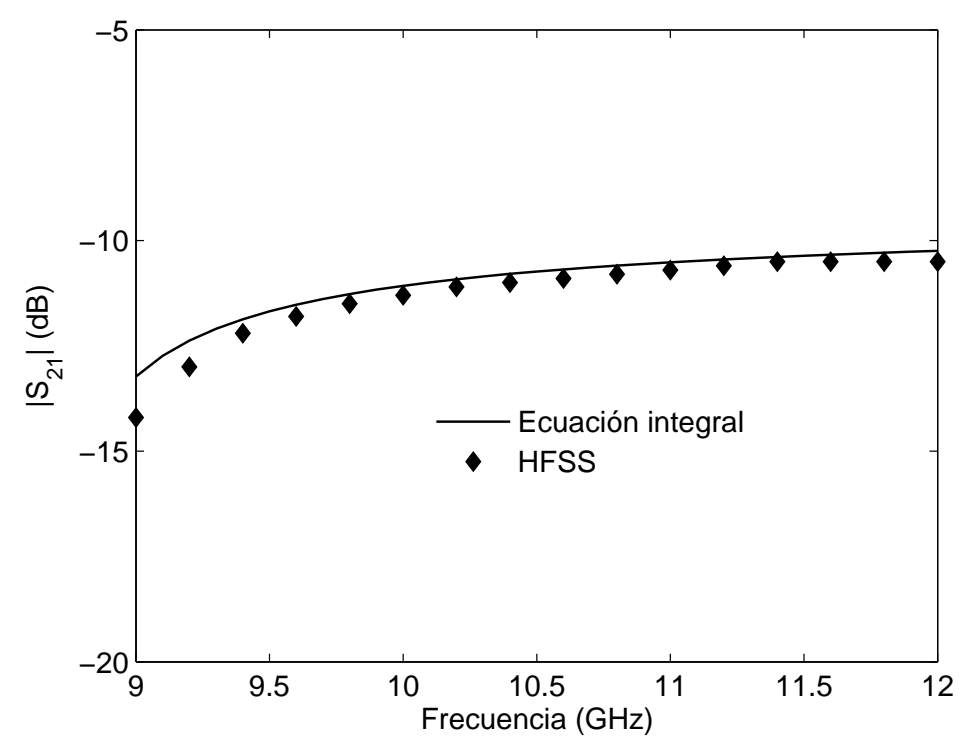

Figura 2.12: Módulo en dB del parámetro de transmisión $S_{21}$ relativo a una unión planar entre una guía rectangular WR-90 $(a=22,90 \mathrm{~mm}, b=10,20 \mathrm{~mm})$ y una guía circular de radio $r_{1}=2,7 \mathrm{~mm}\left(\varepsilon_{r}=14,0\right)$. La guía circular se encuentra descentrada con respecto a la guía rectangu$\operatorname{lar}\left(x_{s}=6,45 \mathrm{~mm}, y_{s}=3,1 \mathrm{~mm}\right)$.

\subsection{Discontinuidades entre Guías Coaxiales}

El objetivo de este apartado es el análisis multimodal riguroso de la unión planar entre dos guías coaxiales mediante la técnica de la ecuación integral. La caracterización multimodal de este tipo de uniones puede resultar muy interesante en una aplicación basada en la excitación coaxial de dispositivos que requiera una adaptación previa de la sonda coaxial de excitación. Aunque este tipo de uniones se ha analizado ya de forma precisa en la literatura técnica [36], en el mencionado trabajo la técnica de análisis multimodal empleada se basa en el método directo [3], el cual es menos eficiente que la técnica de la ecuación integral desde un punto de vista computacional, ya que precisa considerar un mayor número de modos en la red multimodal equivalente con el fin de lograr resultados convergentes. Por otro lado, en [36] no se realiza, estrictamente hablando, una caracterización modal completa de la unión 
planar, puesto que sólo se trabaja con la familia de modos que el modo fundamental $\mathrm{TEM}^{z}$ excita en la otra guía coaxial involucrada en la unión ${ }^{22}$. En cambio, la aportación realizada en esta sección se centra en el análisis multimodal riguroso y eficiente de la unión planar entre dos guías coaxiales considerando la totalidad de los modos en las dos guías de la unión planar, permitiendo así la utilización del código desarrollado en herramientas de simulación electromagnética que requieran un tratamiento multimodal del dispositivo considerado.

\subsubsection{Análisis de la unión planar entre dos guías coaxiales concéntricas}

Con el propósito de calcular las integrales de acoplamiento entre las guías involucradas en la unión, se utilizará la descripción modal para la guía coaxial desarrollada en la sección 2.3.1. A este respecto, es importante recordar que, en el análisis efectuado en la caracterización de las uniones planares anteriormente estudiadas, se utilizaba la teoría desarrollada en [41] con el objetivo de transformar las integrales de superficie de acoplamiento entre modos en integrales de contorno, reduciendo así el esfuerzo computacional asociado a la herramienta de simulación. En este caso, puesto que las dos guías de la unión bajo análisis poseen simetría cilíndrica y son concéntricas, la integral de acoplamiento podrá expresarse como el producto de dos integrales unidimensionales (una de ellas con respecto a la variable radial y la otra con respecto a la variable angular). Así, la integral unidimensional en la variable angular podrá resolverse analíticamente tal y como se va a comprobar a continuación, mientras que la integral unidimensional en la variable radial deberá, por lo general, resolverse numéricamente. Con todo ello, no será necesario aplicar la teoría desarrollada en [41] y podremos utilizar directamente la expresión para la integral de acoplamiento proporcionada en (2.119).

Sea entonces una unión planar entre dos guías coaxiales $(a)$ y $(b)$ como la representada en la figura 2.13. En dicha figura, $r_{1}^{(a)}$ y $r_{1}^{(b)}$ denotan los radios externos de las guías $(a)$ y (b), respectivamente, mientras que $r_{2}^{(a)}$ y $r_{2}^{(b)}$ son los correspondientes radios internos. Es importante mencionar que en nuestro análisis asumiremos que ambas guías son concéntricas y que se cumple que $r_{1}^{(a)} \geq r_{1}^{(b)}$ y $r_{2}^{(a)} \leq r_{2}^{(b)}$. De esta manera nos aseguramos de que la sección transversal de la guía coaxial $(b)$ siempre está contenida en la de la guía $(a)$. La

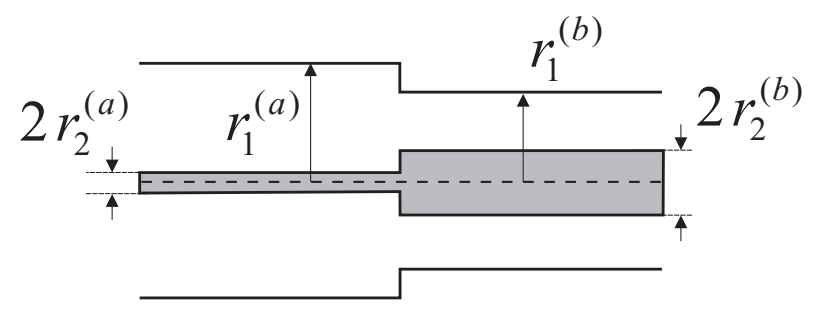

Figura 2.13: Unión planar entre dos guías coaxiales concéntricas $(a)$ y $(b)$ de radios externos $r_{1}^{(a)}$ y $r_{1}^{(b)}$, y de radios internos $r_{2}^{(a)}$ y $r_{2}^{(b)}$, respectivamente.

\footnotetext{
${ }^{22} \mathrm{Si}$ el modo incidente es el modo fundamental $\mathrm{TEM}^{z}$, en la otra guía coaxial involucrada en la unión se excitará la familia de modos $\mathrm{TM}^{z}$ con índice angular nulo así como el modo fundamental $\mathrm{TEM}^{z}$.
} 
integral de acoplamiento que se debe resolver es la siguiente:

$$
A_{u, v}=\int_{S^{(2)}} \mathbf{h}_{u}^{(1)}\left(\rho^{\prime}, \phi^{\prime}\right) \cdot \mathbf{h}_{v}^{(2) *}\left(\rho^{\prime}, \phi^{\prime}\right) d S^{\prime}
$$

donde $u$ es un modo de la guía coaxial $(a)$; $v$ es un modo de la guía coaxial $(b) ; S^{(2)}$ representa la sección transversal de la guía coaxial $(b) ; \mathrm{y} \mathbf{h}_{u}^{(1)}\left(\rho^{\prime}, \phi^{\prime}\right)$ y $\mathbf{h}_{v}^{(2)}\left(\rho^{\prime}, \phi^{\prime}\right)$ representan las funciones vectoriales modales de tipo magnético asociadas a los modos $u$ y $v$, respectivamente. Además, asumiremos que $\left(s_{u}, q_{u}\right)$ es la pareja de índices modales (angular y radial, respectivamente) asociados al $u$-ésimo modo de la guía coaxial $(a)$, y que $\left(s_{v}, q_{v}\right)$ son los índices modales relativos al $v$-ésimo modo de la guía coaxial $(b)$. Puesto que los modos $u$ y $v$ pueden ser de tipo $\mathrm{TEM}^{z}, \mathrm{TE}^{z}$ o $\mathrm{TM}^{z}$, la resolución de la integral (2.204) requiere contemplar todas las combinaciones de tipos de modos posibles. Así, la expresión de la función vectorial modal de tipo magnético $\mathrm{TEM}^{z}$ para una guía coaxial se recoge en (2.63), la correspondiente a los modos $\mathrm{TM}^{z}$ se presentó en (2.96) y la relativa a los modos $\mathrm{TE}^{z}$ se dedujo en (2.118).

Primeramente, supongamos que $u$ y $v$ son, ambos, modos de tipo $\mathrm{TEM}^{z}$. En este caso, es fácil concluir que el resultado de la integral de acoplamiento es el siguiente:

$$
A_{u_{\mathrm{TEM}}, v_{\mathrm{TEM}}}=\sqrt{\frac{\ln \left(r_{1}^{(b)} / r_{2}^{(b)}\right)}{\ln \left(r_{1}^{(a)} / r_{2}^{(a)}\right)}}
$$

A continuación, sea $u$ un modo de tipo $\mathrm{TE}^{z}$ y $v$ un modo de tipo $\mathrm{TEM}^{z}$. En virtud de las resultados extraídos de [41], cuyas conclusiones se resumen en las expresiones (2.122)(2.127), se tiene que para este caso la integral de acoplamiento es nula, es decir:

$$
A_{u_{\mathrm{TE}}, v_{\mathrm{TEM}}}=0
$$

Posteriormente, consideremos que $u$ es un modo de tipo $\mathrm{TM}^{z}$ y $v$ un modo de tipo $\mathrm{TEM}^{z}$. En este caso, se obtiene:

$$
\begin{aligned}
A_{u_{\mathrm{TM}}, v_{\mathrm{TEM}}}= & \sqrt{\frac{2 \pi}{\ln \left(r_{1}^{(b)} / r_{2}^{(b)}\right)}} \mathcal{N}_{(\xi), u}^{\mathrm{TM}^{z}} \sum_{r=1}^{N_{\rho}} d_{s_{u}, q_{u}}^{(r)}\left[\sin \left(r \pi \frac{r_{1}^{(b)}-r_{2}^{(a)}}{r_{1}^{(a)}-r_{2}^{(a)}}\right)\right. \\
& \left.-\sin \left(r \pi \frac{r_{2}^{(b)}-r_{2}^{(a)}}{r_{1}^{(a)}-r_{2}^{(a)}}\right)\right] \delta_{s_{u}, 0}
\end{aligned}
$$

donde $\delta_{s_{u}, 0}$ denota la delta de Kronecker. De este resultado se concluye que únicamente los modos $\mathrm{TM}^{z}$ con índice angular nulo (modos no degenerados) acoplan con el modo $\mathrm{TEM}^{z}$.

Seguidamente, sea $u$ un modo de tipo $\mathrm{TEM}^{z}$ y $v$ un modo de tipo $\mathrm{TE}^{z}$. Inspeccionando las expresiones de las funciones vectoriales modales correspondientes, se deduce que la integral de acoplamiento es proporcional a la siguiente integral:

$$
\int_{0}^{2 \pi} \Phi_{(\xi), s_{v}}^{\prime}(\phi) d \phi=0
$$


en la que $\Phi_{(\xi), s_{v}}^{\prime}(\phi)$ es la derivada de la función que rige la variación angular de los modos de la guía coaxial y cuya definición se recoge en (2.71). Como hemos indicado en (2.208), dicha integral es nula para cualquier valor de $s_{v}$. En consecuencia, podemos afirmar que:

$$
A_{u_{\mathrm{TEM}}, v_{\mathrm{TE}}}=0
$$

Sean ahora $u$ y $v$ modos de tipo $\mathrm{TE}^{z}$. En este caso, la integral de acoplamiento adopta la siguiente expresión:

$$
\begin{aligned}
A_{u_{\mathrm{TE}}, v_{\mathrm{TE}}}= & \frac{\mathcal{N}_{\left(\xi_{u}\right), u}^{\mathrm{TE}^{z}} \mathcal{N}_{\left(\xi_{v}\right), v}^{\mathrm{TE}^{z}} \pi^{2}}{\left(r_{1}^{(a)}-r_{2}^{(a)}\right)\left(r_{1}^{(b)}-r_{2}^{(b)}\right)} \int_{0}^{2 \pi} \Phi_{\left(\xi_{u}\right), s_{u}}(\phi) \Phi_{\left(\xi_{v}\right), s_{v}}(\phi) d \phi\left\{\sum_{r=0}^{N_{\rho}-1} \sum_{t=0}^{N_{\rho}-1} r t\right. \\
& \left.\cdot l_{s_{u}, q_{u}}^{(r)} l_{s_{v}, q_{v}}^{(t)} \int_{r_{2}^{(b)}}^{r_{1}^{(b)}} \rho \sin \left(r \pi \frac{\rho-r_{2}^{(a)}}{r_{1}^{(a)}-r_{2}^{(a)}}\right) \sin \left(t \pi \frac{\rho-r_{2}^{(b)}}{r_{1}^{(b)}-r_{2}^{(b)}}\right) d \rho\right\} \\
& +\mathcal{N}_{\left(\xi_{u}\right), u}^{\mathrm{TE}^{z}} \mathcal{N}_{\left(\xi_{v}\right), v}^{\mathrm{TE}^{z}} \int_{0}^{2 \pi} \Phi_{\left(\xi_{u}\right), s_{u}}^{\prime}(\phi) \Phi_{\left(\xi_{v}\right), s_{v}}^{\prime}(\phi) d \phi\left\{\sum_{r=0}^{N_{\rho}-1} \sum_{t=0}^{N_{\rho}-1} l_{s_{u}, q_{u}}^{(r)} l_{s_{v}, q_{v}}^{(t)}\right. \\
& \left.\cdot \int_{r_{2}^{(b)}}^{r_{1}^{(b)}} \frac{1}{\rho} \cos \left(r \pi \frac{\rho-r_{2}^{(a)}}{r_{1}^{(a)}-r_{2}^{(a)}}\right) \cos \left(t \pi \frac{\rho-r_{2}^{(b)}}{r_{1}^{(b)}-r_{2}^{(b)}}\right) d \rho\right\}
\end{aligned}
$$

En la expresión anterior, la primera de las integrales en la variable radial admite solución analítica, mientras que la segunda debe abordarse utilizando métodos numéricos ${ }^{23}$. Por otra parte, las integrales en la variable angular pueden calcularse como sigue:

$$
\begin{aligned}
\int_{0}^{2 \pi} \Phi_{\left(\xi_{u}\right), s_{u}}(\phi) \Phi_{\left(\xi_{v}\right), s_{v}}(\phi) d \phi & = \begin{cases}\frac{2 \pi}{\epsilon_{s_{u}}} \delta_{s_{u}, s_{v}} & \text { si }\left(\xi_{u}\right)=\left(\xi_{v}\right)=(c) \\
\pi\left(\epsilon_{s_{u}}-1\right) \delta_{s_{u}, s_{v}} & \text { si }\left(\xi_{u}\right)=\left(\xi_{v}\right)=(s) \\
0 & \text { resto de casos }\end{cases} \\
\int_{0}^{2 \pi} \Phi_{\left(\xi_{u}\right), s_{u}}^{\prime}(\phi) \Phi_{\left(\xi_{v}\right), s_{v}}^{\prime}(\phi) d \phi & = \begin{cases}s_{u} s_{v} \pi\left(\epsilon_{s_{u}}-1\right) \delta_{s_{u}, s_{v}} & \text { si }\left(\xi_{u}\right)=\left(\xi_{v}\right)=(c) \\
s_{u} s_{v} \frac{2 \pi}{\epsilon_{s_{u}}} \delta_{s_{u}, s_{v}} & \text { si }\left(\xi_{u}\right)=\left(\xi_{v}\right)=(s) \\
0 & \text { resto de casos }\end{cases}
\end{aligned}
$$

donde $\epsilon_{s_{u}}$ representa la constante de Neumann definida en (C.6) y $\delta_{s_{u}, s_{v}}$ denota la delta de Kronecker.

A continuación, se contempla el caso en que $u$ es un modo $\mathrm{TM}^{z}$ y $v$ es un modo $\mathrm{TE}^{z}$.

\footnotetext{
${ }^{23}$ Utilizando la librería de lenguaje simbólico que proporciona MATLAB, es posible resolver las integrales en la variable radial. El resultado final no se ha incluido aquí por motivos de brevedad.
} 
Esta vez, se tiene que:

$$
\begin{aligned}
A_{u_{\mathrm{TM}}, v_{\mathrm{TE}}}= & \mathcal{N}_{\left(\xi_{u}\right), u}^{\mathrm{TM}^{z}} \mathcal{N}_{\left(\xi_{v}\right), v}^{\mathrm{TE}^{z}} \frac{\pi}{r_{1}^{(b)}-r_{2}^{(b)}} \int_{0}^{2 \pi} \Phi_{\left(\xi_{u}\right), s_{u}}^{\prime}(\phi) \Phi_{\left(\xi_{v}\right), s_{v}}(\phi) d \phi\left\{\sum_{r=1}^{N_{\rho}} \sum_{t=0}^{N_{\rho}-1} t d_{s_{u}, q_{u}}^{(r)} l_{s_{v}, q_{v}}^{(t)}\right. \\
& \left.\cdot \int_{r_{2}^{(b)}}^{r_{1}^{(b)}} \sin \left(r \pi \frac{\rho-r_{2}^{(a)}}{r_{1}^{(a)}-r_{2}^{(a)}}\right) \sin \left(t \pi \frac{\rho-r_{2}^{(b)}}{r_{1}^{(b)}-r_{2}^{(b)}}\right) d \rho\right\} \\
& +\mathcal{N}_{\left(\xi_{u}\right), u}^{\mathrm{TM}^{z}} \mathcal{N}_{\left(\xi_{v}\right), v}^{\mathrm{TE}^{z}} \frac{\pi}{r_{1}^{(a)}-r_{2}^{(a)}} \int_{0}^{2 \pi} \Phi_{\left(\xi_{u}\right), s_{u}}(\phi) \Phi_{\left(\xi_{v}\right), s_{v}}^{\prime}(\phi) d \phi\left\{\sum_{r=1}^{N_{\rho}} \sum_{t=0}^{N_{\rho}-1} r d_{s_{u}, q_{u}}^{(r)} l_{s_{v}, q_{v}}^{(t)}\right. \\
& \left.\cdot \int_{r_{2}^{(b)}}^{r_{1}^{(b)}} \cos \left(r \pi \frac{\rho-r_{2}^{(a)}}{r_{1}^{(a)}-r_{2}^{(a)}}\right) \cos \left(t \pi \frac{\rho-r_{2}^{(b)}}{r_{1}^{(b)}-r_{2}^{(b)}}\right) d \rho\right\}
\end{aligned}
$$

Las dos integrales en la variable radial pueden resolverse analíticamente y su resultado no se incluye por brevedad. Por su parte, el resultado de la primera integral en la variable angular se expresa de la siguiente manera:

$$
\int_{0}^{2 \pi} \Phi_{\left(\xi_{u}\right), s_{u}}^{\prime}(\phi) \Phi_{\left(\xi_{v}\right), s_{v}}(\phi) d \phi= \begin{cases}s_{u} \frac{2 \pi}{\epsilon_{s_{u}}} \delta_{s_{u}, s_{v}} & \text { si }\left(\xi_{u}\right)=(s) \mathrm{y}\left(\xi_{v}\right)=(c) \\ s_{u} \pi\left(\epsilon_{s_{u}}-1\right) \delta_{s_{u}, s_{v}} & \text { si }\left(\xi_{u}\right)=(c) \mathrm{y}\left(\xi_{v}\right)=(s) \\ 0 & \text { si }\left(\xi_{u}\right)=\left(\xi_{v}\right)=(c) ; \\ & \text { o si }\left(\xi_{u}\right)=\left(\xi_{v}\right)=(s)\end{cases}
$$

El resultado de la segunda integral en la variable angular puede obtenerse a partir de esta última expresión, simplemente intercambiando $s_{u}$ por $s_{v}$.

Seguidamente, consideremos que $u$ es un modo de tipo $\mathrm{TEM}^{z}$ y que $v$ es de tipo $\mathrm{TM}^{z}$. En este caso, a partir de las funciones modales de tipo magnético correspondientes, es muy sencillo deducir que la integral de acoplamiento es nula por anularse la integral en la variable radial. Por tanto,

$$
A_{u_{\mathrm{TEM}}, v_{\mathrm{TM}}}=0
$$

Igualmente, en virtud de (2.123), podemos concluir que en caso de que $u$ sea de tipo $\mathrm{TE}^{z} \mathrm{y}$ $v$ de tipo $\mathrm{TM}^{z}$, la integral de acoplamiento será nula:

$$
A_{u_{\mathrm{TE}}, v_{\mathrm{TM}}}=0
$$


Finalmente, sean $u$ y $v$ modos de tipo $\mathrm{TM}^{z}$. En este caso podemos decir que:

$$
\begin{aligned}
A_{u_{\mathrm{TM}}, v_{\mathrm{TM}}}= & \mathcal{N}_{\left(\xi_{u}\right), u}^{\mathrm{TM}^{z}} \mathcal{N}_{\left(\xi_{v}\right), v}^{\mathrm{TM}^{z}} \int_{0}^{2 \pi} \Phi_{\left(\xi_{u}\right), s_{u}}^{\prime}(\phi) \Phi_{\left(\xi_{v}\right), s_{v}}^{\prime}(\phi) d \phi\left\{\sum_{r=1}^{N_{\rho}} \sum_{t=1}^{N_{\rho}} d_{s_{u}, q_{u}}^{(r)} d_{s_{v}, q_{v}}^{(t)}\right. \\
& \left.\cdot \int_{r_{2}^{(b)}}^{r_{1}^{(b)}} \frac{1}{\rho} \sin \left(r \pi \frac{\rho-r_{2}^{(a)}}{r_{1}^{(a)}-r_{2}^{(a)}}\right) \sin \left(t \pi \frac{\rho-r_{2}^{(b)}}{r_{1}^{(b)}-r_{2}^{(b)}}\right) d \rho\right\} \\
& +\mathcal{N}_{\left(\xi_{u}\right), u}^{\mathrm{TM}^{z}} \mathcal{N}_{\left(\xi_{v}\right), v}^{\mathrm{TM}^{z}} \frac{\pi^{2}}{\left(r_{1}^{(a)}-r_{2}^{(a)}\right)\left(r_{1}^{(b)}-r_{2}^{(b)}\right)} \int_{0}^{2 \pi} \Phi_{\left(\xi_{u}\right), s_{u}}(\phi) \Phi_{\left(\xi_{v}\right), s_{v}}(\phi) d \phi \\
& \cdot \sum_{r=1}^{N_{\rho}} \sum_{t=1}^{N_{\rho}} r t d_{s_{u}, q_{u}}^{(r)} d_{s_{v}, q_{v}}^{(t)} \int_{r_{2}^{(b)}}^{r_{1}^{(b)}} \rho \cos \left(r \pi \frac{\rho-r_{2}^{(a)}}{r_{1}^{(a)}-r_{2}^{(a)}}\right) \cos \left(t \pi \frac{\rho-r_{2}^{(b)}}{r_{1}^{(b)}-r_{2}^{(b)}}\right) d \rho
\end{aligned}
$$

Las integrales en la variable angular son las mismas que las obtenidas para el caso $A_{u_{\mathrm{TE}}, v_{\mathrm{TE}}}$. Por otro lado, la primera integral en la variable radial no admite solución analítica, mientras que la segunda de ellas sí la admite.

\subsubsection{Ejemplos de validación}

Una vez finalizado el análisis teórico de la unión planar entre dos guías coaxiales, a continuación se procede a la validación práctica de la herramienta implementada. Para ello, los resultados de nuestras simulaciones se compararán con los proporcionados por la herramienta utilizada en [36], la cual se basa en el método directo. Consideremos, en primer lugar, una unión planar entre dos guías coaxiales cuyas dimensiones se especifican a continuación: $r_{1}^{(a)}=6,0 \mathrm{~mm}, r_{2}^{(a)}=0,65 \mathrm{~mm}, r_{1}^{(b)}=5,0 \mathrm{~mm} \mathrm{y} r_{2}^{(b)}=2,65 \mathrm{~mm}$. Además, se asume que la permitividad dieléctrica relativa de las guías $(a)$ y $(b)$ es $\varepsilon_{r}^{(a)}=\varepsilon_{r}^{(b)}=1,0$. En la figura 2.14 se muestra el módulo del parámetro de reflexión para la unión bajo análisis, y se comprueba que los resultados de la simulación muestran una buena concordancia con los proporcionados por el método directo ${ }^{24}$.

Un nuevo ejemplo se recoge en la figura 2.15. En este caso, las dimensiones de la unión analizada son: $r_{1}^{(a)}=8,6 \mathrm{~mm}, r_{2}^{(a)}=0,65 \mathrm{~mm}, r_{1}^{(b)}=5,0 \mathrm{~mm}$ y $r_{2}^{(b)}=1,42 \mathrm{~mm}$. Por otro lado, la permitividad dieléctrica relativa de las guías es $\varepsilon_{r}^{(a)}=4,25$ y $\varepsilon_{r}^{(b)}=7,2$.

Finalmente, en la figura 2.16 se presenta el módulo del parámetro de transmisión $S_{21}$ para una nueva unión planar entre dos guías coaxiales. En este caso, las dimensiones de las guías son: $r_{1}^{(a)}=15,2 \mathrm{~mm}, r_{2}^{(a)}=7,41 \mathrm{~mm}, r_{1}^{(b)}=11,5 \mathrm{~mm} \mathrm{y} r_{2}^{(b)}=9,24 \mathrm{~mm}\left(\varepsilon_{r}^{(a)}=2,33\right.$, $\left.\varepsilon_{r}^{(b)}=15,1\right)$.

\footnotetext{
${ }^{24}$ En todos los ejemplos de aplicación de esta sección 2.5.2 se considera que el modo de excitación y de respuesta es el modo fundamental $\mathrm{TEM}^{z}$.
} 


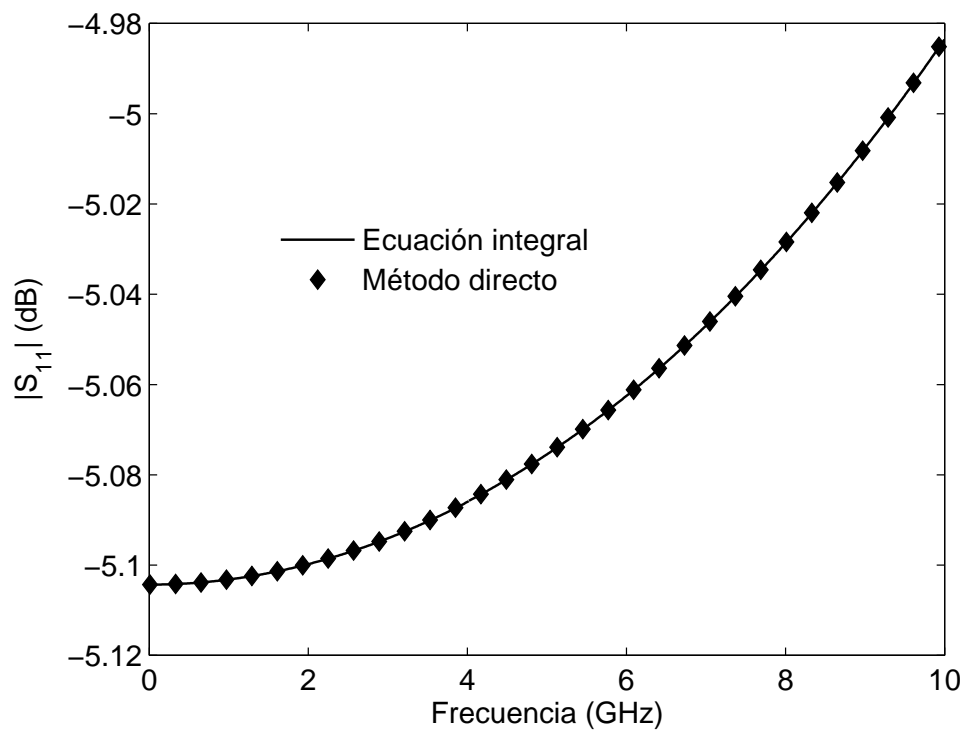

Figura 2.14: Módulo en $\mathrm{dB}$ del parámetro de reflexión $S_{11}$ relativo a una unión planar entre dos guías coaxiales $(a)$ y $(b)$. Las dimensiones de las guías son las siguientes: $r_{1}^{(a)}=6,0 \mathrm{~mm}$, $r_{2}^{(a)}=0,65 \mathrm{~mm}, r_{1}^{(b)}=5,0 \mathrm{~mm}$ y $r_{2}^{(b)}=2,65 \mathrm{~mm}\left(\varepsilon_{r}^{(a)}=\varepsilon_{r}^{(b)}=1,0\right)$. Los resultados de la simulación se comparan con los proporcionados por el método directo descrito en [36].

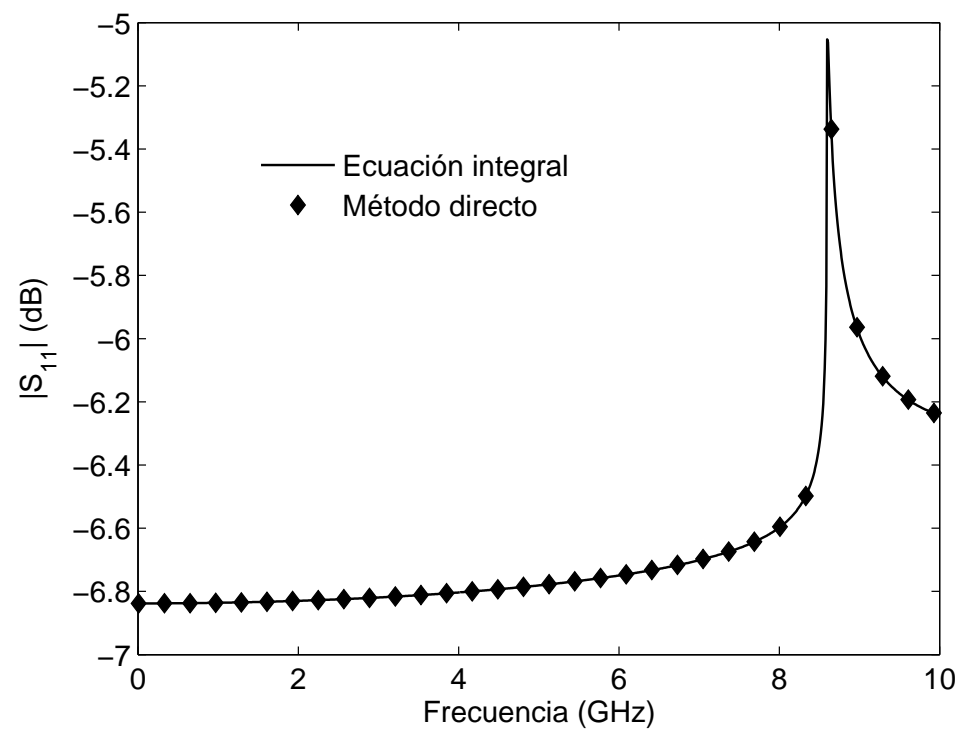

Figura 2.15: Módulo en $\mathrm{dB}$ del parámetro de reflexión $S_{11}$ relativo a una unión planar entre dos guías coaxiales $(a)$ y $(b)$. Las dimensiones de las guías son las siguientes: $r_{1}^{(a)}=8,6 \mathrm{~mm}$, $r_{2}^{(a)}=0,65 \mathrm{~mm}, r_{1}^{(b)}=5,0 \mathrm{~mm}$ y $r_{2}^{(b)}=1,42 \mathrm{~mm}\left(\varepsilon_{r}^{(a)}=4,25 \mathrm{y} \varepsilon_{r}^{(b)}=7,2\right)$. Los resultados de la simulación se comparan con los proporcionados por el método directo descrito en [36]. 


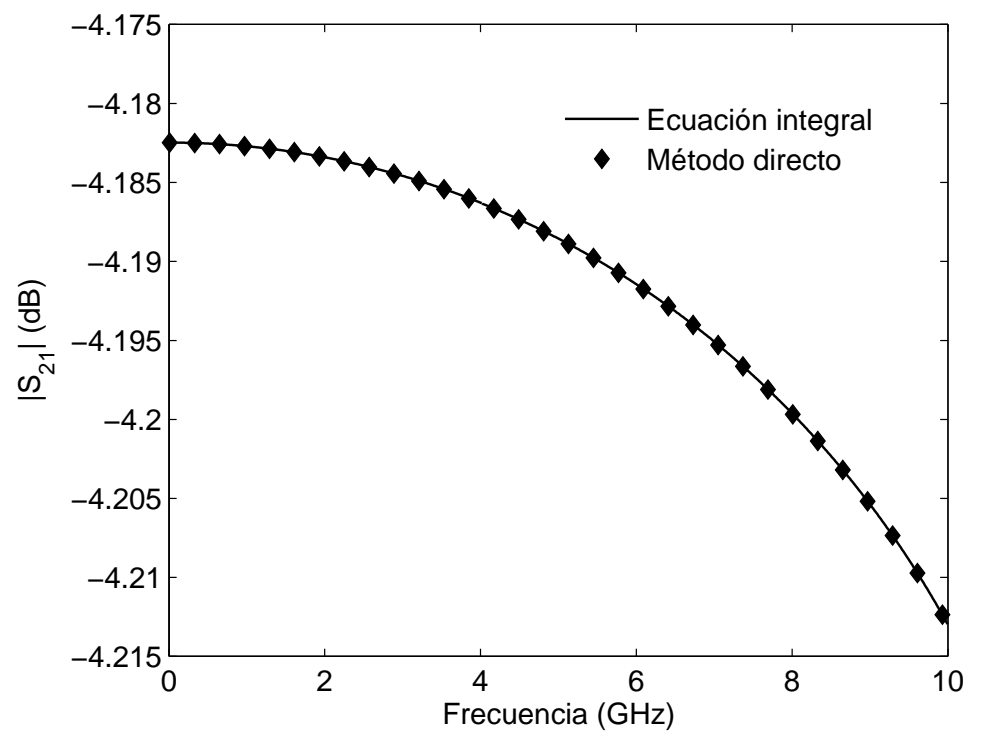

Figura 2.16: Módulo en dB del parámetro de transmisión $S_{21}$ relativo a una unión planar entre dos guías coaxiales $(a)$ y $(b)$. Las dimensiones de las guías son las siguientes: $r_{1}^{(a)}=15,2 \mathrm{~mm}$, $r_{2}^{(a)}=7,41 \mathrm{~mm}, r_{1}^{(b)}=11,5 \mathrm{~mm}$ y $r_{2}^{(b)}=9,24 \mathrm{~mm}\left(\varepsilon_{r}^{(a)}=2,33\right.$ y $\left.\varepsilon_{r}^{(b)}=15,1\right)$. Los resultados de la simulación se comparan con los proporcionados por el método directo descrito en [36].

En relación a la eficiencia computacional de la herramienta, conviene destacar que los resultados presentados se han calculado en 8,67 segundos para un total de 200 puntos en frecuencia.

\subsection{Discontinuidades Múltiples entre Guías Rectangulares}

Los dispositivos de microondas implementados en guía rectangular se utilizan frecuentemente en numerosas aplicaciones en las bandas de microondas y ondas milimétricas, tales como filtros en configuración comb-line, diplexores y multiplexores, transductores ortomodales, etc. Por este motivo, la caracterización rigurosa de las discontinuidades entre guías rectangulares ha sido objeto de investigación desde hace décadas (ver [28], por ejemplo). Un caso particular de este tipo de dispositivos son las denominadas estructuras inductivas o en plano $H$, las cuales se caracterizan por el hecho de que la altura de las diferentes guías que integran el dispositivo permanece constante. Así por ejemplo, un filtro inductivo en guía rectangular está formado por la asociación en cascada de un conjunto de guías de sección transversal rectangular cuya altura permanece constante a lo largo del dispositivo.

El objetivo de esta sección es la caracterización rigurosa y eficiente de discontinuidades múltiples entre guías rectangulares. El análisis multimodal de este tipo de discontinuidad puede utilizarse en la herramienta que se desarrolla en el capítulo 5 destinada al análisis de filtros en configuración comb-line. En concreto, las diferentes cavidades rectangulares que 
integran este tipo de filtros se comunican entre sí mediante ventanas o irises de acoplamiento constituidos por una discontinuidad simple entre dos guías de sección transversal rectangular. A este respecto, podría resultar de enorme interés investigar cómo afecta al diseño de este tipo de filtros el hecho de utilizar ventanas de acoplamiento múltiples entre las cavidades que integran el filtro comb-line. La figura 2.17 ilustra la sección transversal de una discontinuidad múltiple entre guías rectangulares. En dicha figura, $a \times b$ son las dimensiones de la guía rectangular grande; y $a_{1} \times b_{1}$ y $a_{2} \times b_{2}$ son las dimensiones de las dos guías rectangulares pequeñas. Por otro lado, los parámetros $\left(x_{s, 1}, y_{s, 1}\right)$ y $\left(x_{s, 2}, y_{s, 2}\right)$ se utilizan para situar la esquina inferior derecha de las guías rectangulares pequeñas con respecto a la esquina inferior derecha de la guía rectangular grande. Por otro lado, es importante mencionar que, a efec-

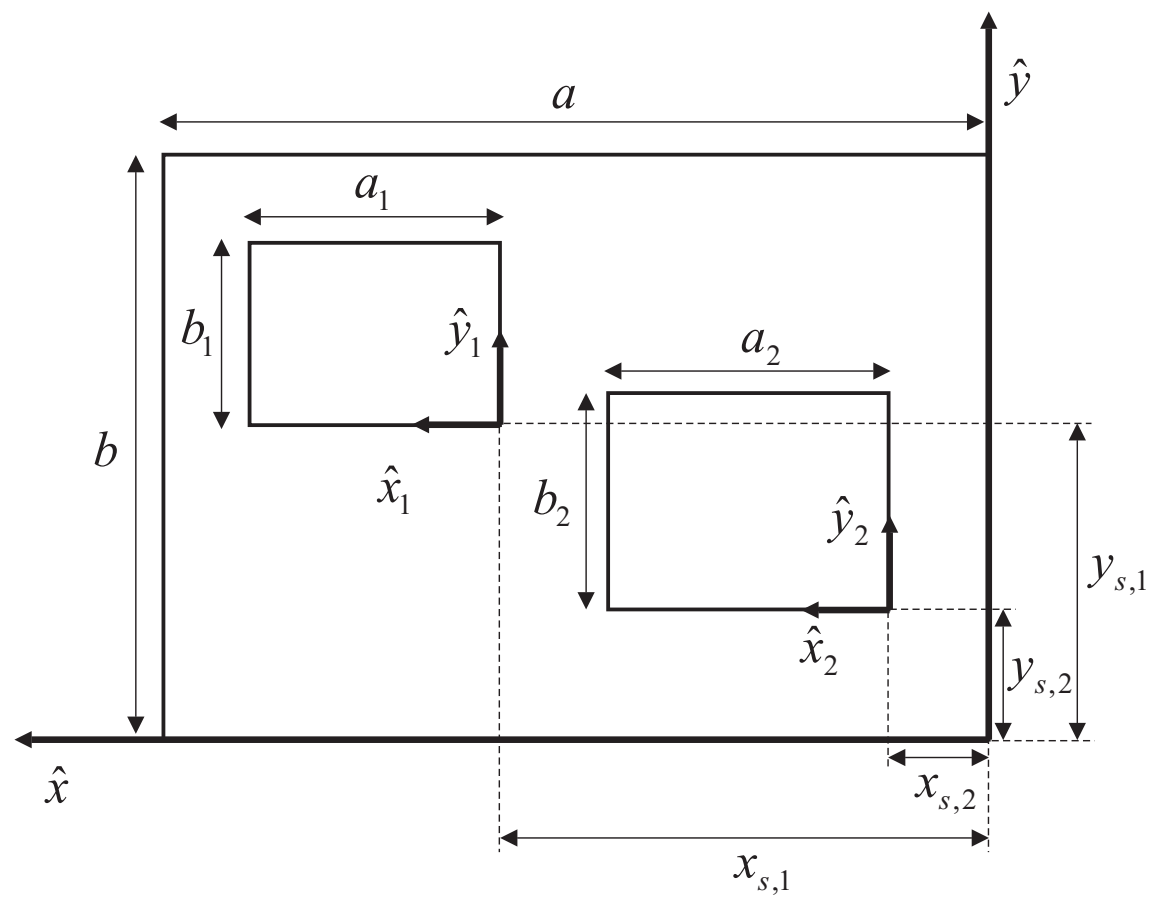

Figura 2.17: Discontinuidad múltiple entre guías rectangulares.

tos del análisis multimodal que se va a efectuar posteriormente, las dos guías rectangulares pequeñas pueden analizarse como si únicamente se tuviera una única guía rectangular cuya carta modal consistiera en la agrupación de las dos familias de modos que se excitan en las guías rectangulares pequeñas. Por otra parte, obsérvese que, desde este punto de vista, resulta sencillo extender la teoría expuesta en este apartado para el caso de una discontinuidad múltiple entre una guía rectangular grande y $N$ guías rectangulares pequeñas cuyas secciones transversales estuviesen completamente contenidas en la de la guía grande y no se solaparan entre sí.

Seguidamente, en esta sección se presenta el análisis multimodal de una discontinuidad múltiple entre guías rectangulares utilizando la técnica de ecuación integral. Para ello, se calcularán las expresiones de las correspondientes integrales de acoplamiento entre los modos 
que se excitan a ambos lados de la discontinuidad. Posteriormente, la teoría desarrollada se particularizará para el caso de estructuras en plano $H$.

\subsubsection{Análisis de una discontinuidad múltiple entre guías rectangula- res}

Sea la discontinuidad múltiple representada en la figura 2.17. La relación entre los diferentes sistemas de referencia que se recogen en dicha figura puede expresarse como sigue:

$$
\begin{aligned}
& x=x_{\delta}+x_{s, \delta} \\
& y=y_{\delta}+y_{s, \delta}
\end{aligned}
$$

donde $\delta=1,2$ denota cada una de las dos guías rectangulares pequeñas de la unión. Por otro lado, la integral de acoplamiento que se debe resolver es la siguiente:

$$
A_{u, v}^{(\delta)}=\int_{S^{(\delta)}} \mathbf{h}_{u}\left(x^{\prime}, y^{\prime}\right) \cdot \mathbf{h}_{v}^{(\delta) *}\left(x^{\prime}, y^{\prime}\right) d S^{\prime}
$$

donde $\mathbf{h}_{u}\left(x^{\prime}, y^{\prime}\right)$ es la función vectorial modal de tipo magnético asociada al $u$-ésimo modo de la guía rectangular grande; $\mathbf{h}_{v}^{(\delta)}\left(x^{\prime}, y^{\prime}\right)$ representa la función vectorial modal de tipo magnético del $v$-ésimo modo de la guía rectangular pequeña $(\delta)$; y $S^{(\delta)}$ denota la sección transversal de la guía rectangular pequeña $(\delta)$ ( $\operatorname{con} \delta=1,2)$. Las expresiones de las funciones vectoriales modales de tipo magnético relativas a las guías rectangulares implicadas en la unión pueden deducirse fácilmente a partir de la teoría desarrollada en la sección C.1 del Apéndice C. A este respecto, es importante mencionar que interesará expresar las funciones vectoriales modales asociadas a la guía rectangular grande en función de las coordenadas utilizadas para describir las soluciones modales de las guías rectangulares pequeñas, usando para ello las relaciones (2.218)-(2.219).

Así pues, supongamos a continuación que $u$ es un modo de tipo $\mathrm{TE}^{z}$ de la guía rectangular grande y que $v$ es un modo $\mathrm{TE}^{z}$ de la guía rectangular pequeña $(\delta)$. Asumamos también que $\left(m_{u}, n_{u}\right)$ es la pareja de índices modales asociados al $u$-ésimo modo de la guía rectangular grande, y que $\left(m_{v, \delta}, n_{v, \delta}\right)$ es la pareja de índices modales relativos al $v$-ésimo modo de la guía rectangular pequeña $(\delta)$. En este caso, podemos concluir fácilmente que la integral de acoplamiento adopta la siguiente expresión:

$$
\begin{aligned}
A_{u_{\mathrm{TE}}, v_{\mathrm{TE}}}^{(\delta)}= & \sqrt{\frac{\epsilon_{m_{u}} \epsilon_{n_{u}}}{a b}} \sqrt{\frac{\epsilon_{m_{v, \delta}} \epsilon_{n_{v, \delta}}}{a_{\delta} b_{\delta}}} \frac{1}{\sqrt{\left(\frac{m_{u} \pi}{a}\right)^{2}+\left(\frac{n_{u} \pi}{b}\right)^{2}}} \frac{1}{\sqrt{\left(\frac{m_{v, \delta} \pi}{a_{\delta}}\right)^{2}+\left(\frac{n_{v, \delta} \pi}{b_{\delta}}\right)^{2}}} \\
& \cdot\left\{\left(\frac{m_{u} \pi}{a}\right)\left(\frac{m_{v, \delta} \pi}{a_{\delta}}\right) I_{1}^{(\delta)}+\left(\frac{n_{u} \pi}{b}\right)\left(\frac{n_{v, \delta} \pi}{b_{\delta}}\right) I_{2}^{(\delta)}\right\}
\end{aligned}
$$


donde $\epsilon_{m_{u}}$ representa la constante de Neumann y $a_{\delta} \times b_{\delta}$ son las dimensiones de la guía pequeña $(\delta)$. Además, se han definido las siguientes integrales ${ }^{25}$ :

$$
\begin{aligned}
I_{1}^{(\delta)=} & \int_{0}^{a_{\delta}} \sin \left(\frac{m_{v, \delta} \pi x_{\delta}}{a_{\delta}}\right) \sin \left(\frac{m_{u} \pi\left(x_{\delta}+x_{s, \delta}\right)}{a}\right) d x_{\delta} \\
& \cdot \int_{0}^{b_{\delta}} \cos \left(\frac{n_{v, \delta} \pi y_{\delta}}{b_{\delta}}\right) \cos \left(\frac{n_{u} \pi\left(y_{\delta}+y_{s, \delta}\right)}{b}\right) d y_{\delta} \\
I_{2}^{(\delta)=} & \int_{0}^{a_{\delta}} \cos \left(\frac{m_{v, \delta} \pi x_{\delta}}{a_{\delta}}\right) \cos \left(\frac{m_{u} \pi\left(x_{\delta}+x_{s, \delta}\right)}{a}\right) d x_{\delta} \\
& \cdot \int_{0}^{b_{\delta}} \sin \left(\frac{n_{v, \delta} \pi y_{\delta}}{b_{\delta}}\right) \sin \left(\frac{n_{u} \pi\left(y_{\delta}+y_{s, \delta}\right)}{b}\right) d y_{\delta}
\end{aligned}
$$

Tal y como vamos a comprobar a continuación, el resto de las integrales de acoplamiento se expresarán en función de las integrales $I_{1}^{(\delta)}$ e $I_{2}^{(\delta)}$.

Seguidamente, sea $u$ un modo de tipo $\mathrm{TM}^{z}$ de la guía rectangular grande, y sea $v$ un modo de tipo $\mathrm{TE}^{z}$ de la guía rectangular pequeña $(\delta)$. En este caso, se tiene que:

$$
\begin{aligned}
A_{u_{\mathrm{TM}}, v_{\mathrm{TE}}}^{(\delta)}= & \sqrt{\frac{2}{a b}} \sqrt{\frac{\epsilon_{m_{v, \delta}} \epsilon_{n_{v, \delta}}}{a_{\delta} b_{\delta}}} \frac{1}{\sqrt{\left(\frac{m_{u} \pi}{a}\right)^{2}+\left(\frac{n_{u} \pi}{b}\right)^{2}}} \frac{1}{\sqrt{\left(\frac{m_{v, \delta} \pi}{a_{\delta}}\right)^{2}+\left(\frac{n_{v, \delta} \pi}{b_{\delta}}\right)^{2}}} \\
& \cdot\left\{\left(\frac{n_{u} \pi}{b}\right)\left(\frac{m_{v, \delta} \pi}{a_{\delta}}\right) I_{1}^{(\delta)}-\left(\frac{m_{u} \pi}{a}\right)\left(\frac{n_{v, \delta} \pi}{b_{\delta}}\right) I_{2}^{(\delta)}\right\}
\end{aligned}
$$

Supongamos ahora que $u$ y $v$ son modos de tipo $\mathrm{TM}^{z}$. Esta vez puede concluirse que:

$$
\begin{aligned}
A_{u_{\mathrm{TM}}, v_{\mathrm{TM}}}^{(\delta)}= & \sqrt{\frac{2}{a b}} \sqrt{\frac{2}{a_{\delta} b_{\delta}}} \frac{1}{\sqrt{\left(\frac{m_{u} \pi}{a}\right)^{2}+\left(\frac{n_{u} \pi}{b}\right)^{2}}} \frac{1}{\sqrt{\left(\frac{m_{v, \delta} \pi}{a_{\delta}}\right)^{2}+\left(\frac{n_{v, \delta} \pi}{b_{\delta}}\right)^{2}}} \\
& \cdot\left\{\left(\frac{n_{u} \pi}{b}\right)\left(\frac{n_{v, \delta} \pi}{b_{\delta}}\right) I_{1}^{(\delta)}+\left(\frac{m_{u} \pi}{a}\right)\left(\frac{m_{v, \delta} \pi}{a_{\delta}}\right) I_{2}^{(\delta)}\right\}
\end{aligned}
$$

Finalmente, en caso de que $u$ sea un modo $\mathrm{TE}^{z}$ de la guía rectangular grande y $v$ sea un modo $\mathrm{TM}^{z}$ de la guía rectangular pequeña $(\delta)$, la integral de acoplamiento será nula en virtud de los resultados extraídos de [41].

\footnotetext{
${ }^{25}$ Las integrales $I_{1}^{(\delta)}$ e $I_{2}^{(\delta)}$ admiten un cálculo analítico que puede efectuarse, por ejemplo, empleando la librería de cálculo simbólico que incorpora MATLAB.
} 


\subsubsection{Discontinuidades múltiples en estructuras inductivas implemen- tadas en guía rectangular}

Un caso particular de la teoría desarrollada en la sección anterior es aquél en el que las guías rectangulares involucradas en la unión poseen la misma altura. En este caso, se dice que la unión es de tipo inductivo. La figura 2.18 muestra la vista en planta de una unión planar múltiple de este tipo. En dicha figura, $a$ es la anchura de la guía rectangular grande; $a_{1}$ y $a_{2}$ son las anchuras correspondientes de las guías rectangulares pequeñas; y los parámetros $x_{s, 1}$ y $x_{s, 2}$ se utilizan para situar las guías rectangulares pequeñas con respecto a la guía rectangular grande. Por otra parte, la altura de todas las guías de la unión planar es $b$.

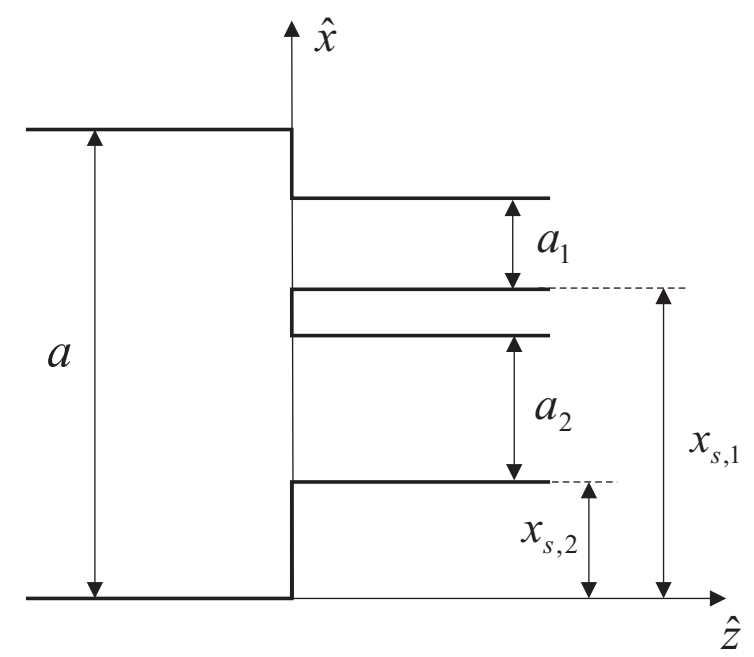

Figura 2.18: Vista en planta de una discontinuidad múltiple en una estructura inductiva implementada en guía rectangular.

Es bien conocido que en una estructura inductiva como la representada en la figura 2.18 únicamente se excita la familia de modos $\mathrm{TE}_{m, 0}^{z}$, puesto que la geometría no presenta variaciones en altura ${ }^{26}$. Por ese motivo, el cálculo de las integrales de acoplamiento se simplifica bastante con respecto al caso general estudiado anteriormente. Así pues, sea $u$ un modo de tipo $\mathrm{TE}_{m_{u}, 0}^{z}$ de la guía rectangular grande. La función vectorial modal de tipo magnético asociada a este modo puede deducirse a partir de las expresiones generales que se recogen en la sección C.1 del Apéndice C. En concreto se tiene que:

$$
\mathbf{h}_{u}(x)=\sqrt{\frac{2}{a b}} \sin \left(\frac{m_{u} \pi}{a} x\right) \hat{\mathbf{x}}
$$

Por otro lado, sea $v$ un modo de tipo $\mathrm{TE}_{m_{v, \delta}, 0}^{z}$ de la guía pequeña $(\delta)$ (con $\delta=1,2$ ). La función vectorial modal de tipo magnético correspondiente a este modo puede expresarse como sigue:

$$
\mathbf{h}_{v}^{(\delta)}(x)=\sqrt{\frac{2}{a^{(\delta)} b}} \sin \left(\frac{m_{v, \delta} \pi}{a}\left(x-x_{s, \delta}\right)\right) \hat{\mathbf{x}}
$$

\footnotetext{
${ }^{26} \mathrm{~A}$ este respecto, se asume que el modo incidente es el modo fundamental $\mathrm{TE}_{10}^{z}$.
} 
En estas dos últimas expresiones, $m_{u}$ y $m_{v, \delta}$ representan, respectivamente, los índices modales de los modos $u$ y $v$ asociados a la variación en la coordenada $x$.

La integral de acoplamiento que debe resolverse en este caso es la siguiente:

$$
\begin{aligned}
A_{u, v}^{(\delta)} & =\int_{x=x_{s, \delta}}^{x=a_{\delta}+x_{s, \delta}} \int_{y=0}^{y=b} \mathbf{h}_{u}(x) \cdot \mathbf{h}_{v}^{(\delta) *}(x) d x d y \\
& =\frac{2}{\sqrt{a a_{\delta}}} \int_{x_{s, \delta}}^{a_{\delta}+x_{s, \delta}} \sin \left(\frac{m_{u} \pi}{a} x\right) \sin \left(\frac{m_{v, \delta} \pi}{a_{\delta}}\left(x-x_{s, \delta}\right)\right) d x
\end{aligned}
$$

La integral unidimensional que aparece en esta última expresión admite una solución analítica que no se ha incluido por brevedad.

\subsubsection{Ejemplos de validación}

Con el objetivo de validar la teoría presentada en los apartados anteriores se ha desarrollado una herramienta capaz de analizar una asociación en cascada de $N$ guías rectangulares en una configuración inductiva. Esta herramienta puede ser utilizada para el análisis y diseño de filtros inductivos en guía rectangular (tanto de modo dual como con inserciones metálicas en plano $E$, [22], [45], [46], [47]). Las uniones planares que se pueden simular con la herramienta desarrollada pueden ser de dos tipos: uniones inductivas simples y uniones inductivas múltiples como la representada en la figura 2.18. La vista en planta correspondiente a la primera estructura que se ha escogido para validar la herramienta se ha representado en la figura 2.19. Como se puede apreciar, en la estructura inductiva encontramos cuatro dis-

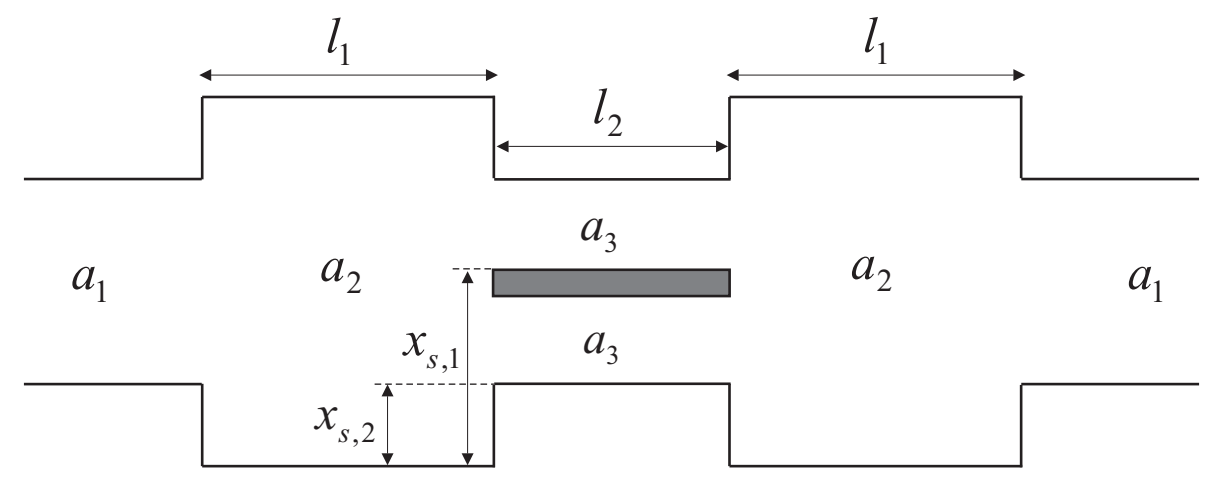

Figura 2.19: Vista en planta de una discontinuidad múltiple en una estructura inductiva implementada en guía rectangular.

continuidades planares, siendo dos de ellas de tipo múltiple. Con respecto a las dimensiones de la estructura inductiva, tenemos, en primer lugar, que las anchuras de las guías son las siguientes: $a_{1}=19,05 \mathrm{~mm}, a_{2}=22,30 \mathrm{~mm}$ y $a_{3}=9,075 \mathrm{~mm}$. Por otro lado, las longitudes de las guías son: $l_{1}=6,56 \mathrm{~mm}$ y $l_{2}=2,21 \mathrm{~mm}$. Finalmente, se tiene que $x_{s, 1}=11,6 \mathrm{~mm}$ y $x_{s, 2}=1,625 \mathrm{~mm}$. La respuesta de la estructura se muestra en la figura 2.20 , en la cual se 
ha representado el módulo de los parámetros de dispersión. Tal y como se puede comprobar, los resultados obtenidos concuerdan muy bien con los proporcionados por la herramienta HFSS. Por otro lado, es importante comentar que la estructura completa se simuló en 2,4 segundos para un total de 101 puntos en frecuencia. Por su parte, HFSS empleó 20 minutos, aproximadamente, para un total 26 de puntos en frecuencia.

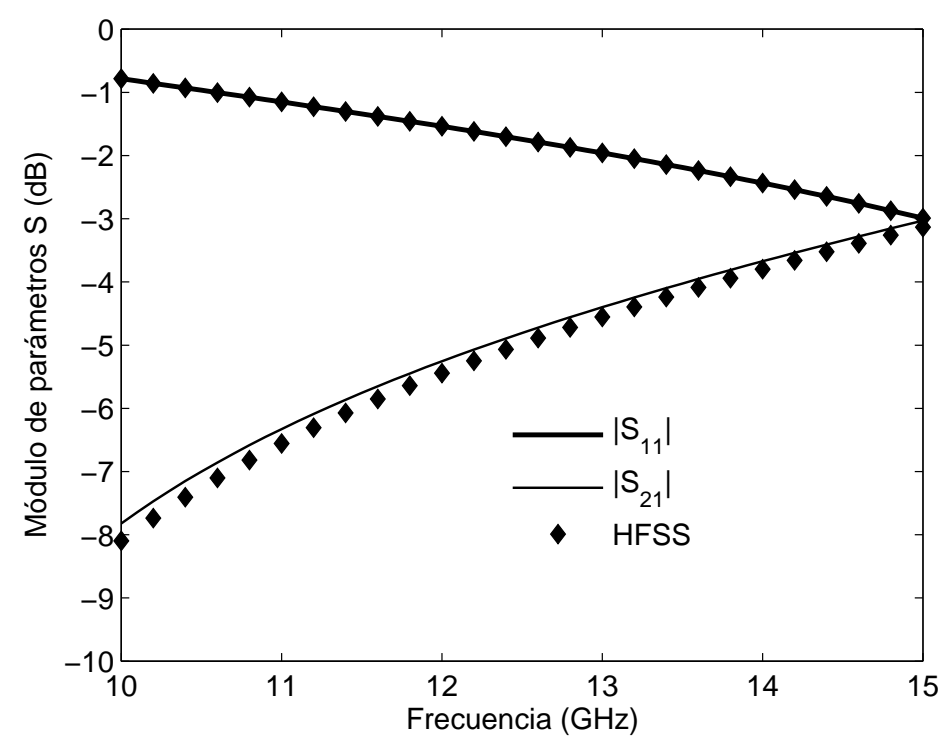

Figura 2.20: Módulo en dB de los parámetros de dispersión de la estructura inductiva implementada en guía rectangular cuya vista en planta se detalla en la figura 2.19. Comparación con los resultados proporcionados por HFSS.

Seguidamente, se analiza un filtro inductivo de tres resonadores implementado en la guía estándar WR-12 ( $a=7,112 \mathrm{~mm}, b=3,556 \mathrm{~mm})$ con inserciones metálicas en plano $E$. La vista en planta del filtro analizado, cuyas dimensiones se han extraído del diseño realizado en [45], se recoge en la figura 2.21. Las dimensiones del filtro, referidas a la figura 2.21, son las siguientes: $t=0,1 \mathrm{~mm}, a_{1}=3,301 \mathrm{~mm}, l_{1}=1,009 \mathrm{~mm}, l_{2}=4,778 \mathrm{~mm}, l_{3}=3,87 \mathrm{~mm}$, $l_{4}=4,79 \mathrm{~mm}$. Así, en la figura 2.22 se muestran las pérdidas de inserción del filtro calculadas con la técnica de la ecuación integral, y se comparan los resultados obtenidos con los datos experimentales que se presentan en [45]. A este respecto, es importante destacar que se observa una excelente concordancia entre los datos representados, lo que valida completamente la precisión de la herramienta implementada. Por otra parte, el filtro se ha analizado en 5,9 segundos para un total de 101 puntos en frecuencia.

Por último, nos gustaría añadir que el análisis electromagnético de estructuras inductivas en línea como las representadas en las figuras 2.19 y 2.21 implica, no sólo el cálculo de las matrices de impedancias generalizadas asociadas a las uniones planares de la estructura, sino también la obtención de las matrices multimodales correspondientes a los tramos de guías de onda uniforme presentes en el dispositivo. La expresión de la matriz multimodal relativa a los tramos de guía uniforme puede encontrarse, por ejemplo, en [48]. Por otra parte, la conexión 


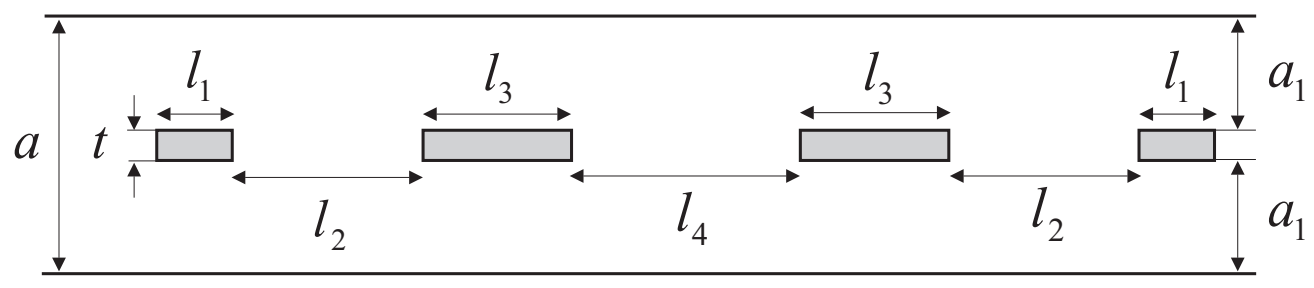

Figura 2.21: Vista en planta de un filtro inductivo de tres resonadores implementados en guía estándar WR-12 con inserciones metálicas en plano E. Las dimensiones del filtro se han extraído de [45].

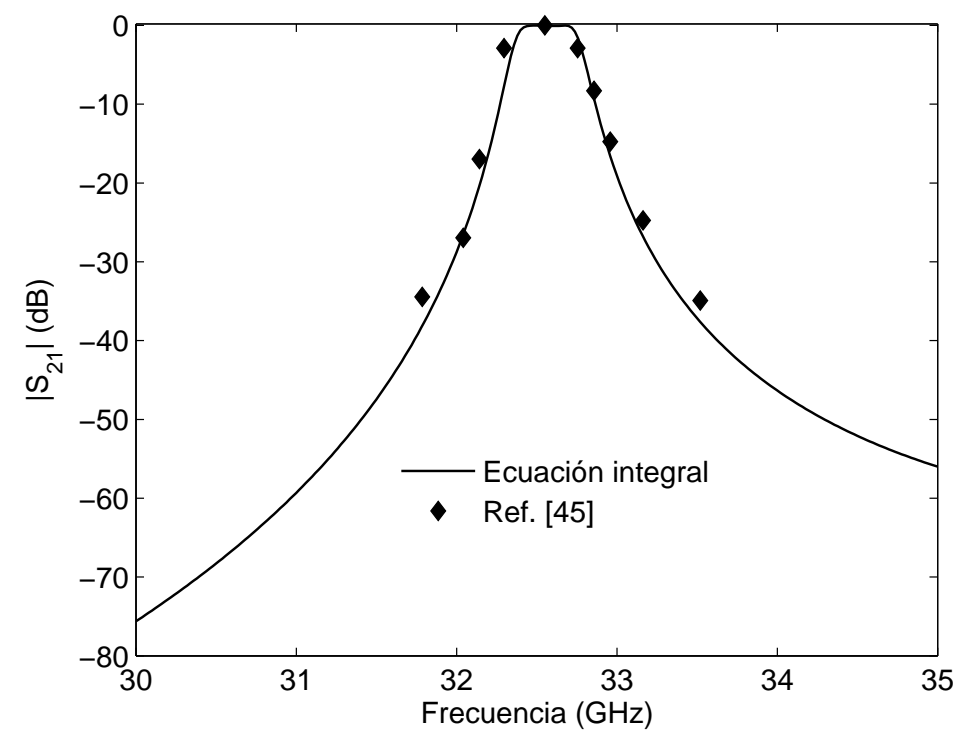

Figura 2.22: Módulo en dB del parámetro $S_{21}$ del filtro inductivo con inserciones metálicas en plano $E$ de la figura 2.21. Comparación de los resultados obtenidos utilizando la técnica de la ecuación integral con los datos experimentales extraídos de [45].

de las diferentes matrices multimodales calculadas se traduce en la obtención un sistema de ecuaciones lineales en banda, el cual puede resolverse de forma muy eficiente utilizando la técnica recursiva descrita en [49]. Esta técnica ha sido implementada en la herramienta desarrollada con el fin de incrementar la eficiencia computacional de la misma. 


\section{Capítulo 3}

\section{Análisis y Diseño de Uniones Multi-Puerto en Guía Rectangular Compensadas con Postes Cilíndricos de Geometría Arbitraria}

\subsection{Introducción}

El objetivo de este capítulo es desarrollar una formulación rigurosa y eficiente desde un punto de vista computacional para el análisis y diseño de uniones multi-puerto en guía rectangular compensadas con postes conductores de simetría cilíndrica. Las uniones multi-puerto implementadas en guía rectangular, tales como los codos rectos, las uniones en $\mathrm{T}$, las uniones en configuración de T-mágica, la unión turnstile o las uniones cúbicas, son elementos fundamentales en numerosos dispositivos pasivos en tecnología guiada (por ejemplo, transductores ortomodales, diplexores y multiplexores, acopladores, divisores de potencia, etc.) y, en consecuencia, se utilizan frecuentemente en numerosas aplicaciones prácticas en las bandas de microondas y ondas milimétricas [20], [50]. Por este motivo, durante los últimos años se ha observado un notable interés por parte de la comunidad científica en desarrollar técnicas de análisis eficientes desde el punto de vista computacional con el fin de caracterizar de manera rigurosa este tipo de uniones. A este respecto, en la literatura técnica se pueden encontrar numerosas aportaciones en las que se presentan diferentes métodos multimodales - basados en la obtención de una Matriz de Admitancias Generalizada (MAG) o de una Matriz de Dispersión Generalizada - con el fin de caracterizar eficientemente uniones en T en plano $H$ y en plano $E$ [8], [51] uniones en T-mágica [9], [52] uniones turnstile [10] y uniones cúbicas [53], [54]. Más recientemente, se han publicado diversas extensiones del método BI-RME (Boundary Integral - Resonant Mode Expansion) [21], conocidas como BI-RME 3D [12], que permiten la obtención eficiente de la MAG de cavidades de geometría arbitraria [11], así como de uniones cúbicas con puertos de acceso de geometría arbitraria [55].

Por otro lado, los sistemas de comunicaciones actuales requieren un ancho de banda de 
operación cada vez mayor, con lo que resulta indispensable ampliar el rango frecuencial de trabajo de las uniones en guía rectangular citadas previamente. Este objetivo, de enorme interés desde un punto de vista tecnológico, ha obligado a introducir nuevas variables de diseño en los dispositivos considerados, originándose así el concepto de uniones compensadas. Así por ejemplo, en 1991, Hirokawa [56] caracterizó una unión en T en plano $H$ cargada con un poste cilíndrico inductivo utilizando una formulación basada en las funciones de Green. En este caso, el radio del poste fue la nueva variable de diseño introducida. Posteriormente, Alessandri [57] estudió uniones compensadas en plano $E$ empleando una técnica de segmentación (en este trabajo se emplearon biseles de geometría triangular para compensar las diferentes estructuras) y Wang [58] investigó uniones en T implementadas en guías con doble reentrante (en inglés, guías ridge) utilizando una versión del método de adaptación modal conocida como técnica de los tres planos. En 2001, Wu realizó una extensión del trabajo de Hirokawa que publicó en [59]. En dicha contribución, se diseñaron uniones en T en plano $H$ compensadas con postes cilíndricos de altura arbitraria, añadiendo así una nueva variable de diseño: la altura del poste. Siguiendo esta misma técnica de diseño, Ritter [60] analizó uniones en T-mágica usando la técnica de diferencias finitas en el dominio del tiempo y Bunger [19] diseñó uniones en T en plano $E$ óptimas.

El propósito de este capítulo es, por un lado, generalizar el método BI-RME 3D para poder analizar de manera eficiente y rigurosa diferentes tipos de uniones multi-puerto implementadas en guía rectangular compensadas con postes conductores cilíndricos de altura arbitraria. Por otro lado, en este trabajo se propone la introducción de una nueva variable de diseño que no ha sido considerada previamente en la literatura técnica relativa a la compensación de uniones multi-puerto. Esta nueva variable de diseño es la posición relativa que ocupa el poste conductor en la estructura. En los trabajos mencionados anteriormente, el poste se encuentra siempre en una posición centrada. En este capítulo se demostrará que la posición relativa del poste es un parámetro de diseño que puede mejorar enormemente la respuesta en frecuencia de la unión considerada, logrando así una extensión considerable del ancho de banda de operación del dispositivo [61], [62].

El resto del capítulo se estructura de la siguiente manera: en primer lugar, se resume el método BI-RME 3D; posteriormente, se propone una extensión de dicho método con el objetivo de analizar uniones multi-puerto compensadas con postes conductores cilíndricos de altura y posición arbitrarias. Finalmente, se presentan distintos ejemplos de aplicación con el fin de validar la teoría desarrollada. En concreto, se analizan y diseñan de forma optimizada los siguientes tipos de uniones multi-puerto: codos rectos en plano $H$ y en plano $E$, uniones en $\mathrm{T}$ en plano $H$ y en plano $E$, uniones T-mágicas y uniones turnstile.

\subsection{Generalización del Método BI-RME 3D para el Análi- sis de Uniones Compensadas en Guía Rectangular}

Tal y como se ha comentado anteriormente, iniciaremos este apartado con un breve resumen del método BI-RME 3D. 


\subsubsection{Revisión del método BI-RME 3D}

El método BI-RME (Boundary Integral - Resonant Mode Expansion) 3D es una técnica rigurosa, y muy eficiente desde el punto de vista computacional, utilizada en el análisis multimodal de dispositivos de microondas sin pérdidas con geometría tridimensional arbitraria [12], [63]. El método, que se basa en la resolución de sendas ecuaciones integrales de campo eléctrico y de campo magnético, proporciona una caracterización electromagnética del dispositivo bajo análisis en términos de una Matriz de Admitancias Generalizada (MAG) válida en el rango frecuencial $\left[0, f_{\max }\right]$, siendo $f_{\max }$ la máxima frecuencia de análisis considerada. Por otra parte, una de las claves del método reside en el hecho de que se considera que la estructura bajo análisis se encuentra inmersa en un resonador externo de geometría canónica (rectangular, esférica, etc.) de manera que el problema queda formulado finalmente en términos de las funciones de Green escalares y diádicas de dicho resonador, evitando así el uso de funciones de Green de espacio libre ${ }^{1}$. Las funciones de Green clásicas de, por ejemplo, un resonador rectangular se expresan mediante series triples infinitas que convergen muy lentamente. Sin embargo, se puede acelerar la convergencia de dichas series utilizando el denominado método de Ewald, el cual descompone la serie original en dos series rápidamente convergentes (una de estas series se evalúa en el dominio espacial y la otra en el dominio espectral) [64], [65], [66]. De esta manera, se logra reducir enormemente el esfuerzo computacional asociado a la herramienta de simulación implementada.

Para obtener la MAG multimodal de la estructura, el método comienza expresando los campos eléctrico y magnético en el contorno de la geometría bajo análisis de la siguiente manera [12]:

$$
\begin{aligned}
\mathbf{E}_{\mathrm{tg}}(\mathbf{r})= & \frac{\eta}{j k} \nabla_{S} \int_{S} g^{\mathrm{e}}\left(\mathbf{r}, \mathbf{r}^{\prime}\right) \nabla_{S}^{\prime} \cdot \mathbf{J}\left(\mathbf{r}^{\prime}\right) d S^{\prime}-j k \eta \int_{S} \overline{\mathbf{G}}^{\mathrm{A}}\left(\mathbf{r}, \mathbf{r}^{\prime}\right) \cdot \mathbf{J}\left(\mathbf{r}^{\prime}\right) d S^{\prime} \\
& -\int_{S} \nabla \times \overline{\mathbf{G}}^{\mathrm{F}}\left(\mathbf{r}, \mathbf{r}^{\prime}\right) \cdot \mathbf{M}\left(\mathbf{r}^{\prime}\right) d S^{\prime}+\frac{1}{2} \mathbf{n} \times \mathbf{M}\left(\mathbf{r}^{\prime}\right) \\
\mathbf{H}_{\mathrm{tg}}(\mathbf{r})= & \frac{1}{j k \eta} \nabla_{S} \int_{S} g^{\mathrm{m}}\left(\mathbf{r}, \mathbf{r}^{\prime}\right) \nabla_{S}^{\prime} \cdot \mathbf{M}\left(\mathbf{r}^{\prime}\right) d S^{\prime}-\frac{j k}{\eta} \int_{S} \overline{\mathbf{G}}^{\mathrm{F}}\left(\mathbf{r}, \mathbf{r}^{\prime}\right) \cdot \mathbf{M}\left(\mathbf{r}^{\prime}\right) d S^{\prime} \\
& +\int_{S} \nabla \times \overline{\mathbf{G}}^{\mathrm{A}}\left(\mathbf{r}, \mathbf{r}^{\prime}\right) \cdot \mathbf{J}\left(\mathbf{r}^{\prime}\right) d S^{\prime}+\frac{1}{2} \mathbf{J}\left(\mathbf{r}^{\prime}\right) \times \mathbf{n}
\end{aligned}
$$

En estas expresiones, $\mathbf{r}$ y $\mathbf{r}^{\prime}$ representan posiciones arbitrarias sobre la superficie $S$ (en realidad, $\mathbf{r}$ es el punto de campo y $\mathbf{r}^{\prime}$ es el punto de fuente); $\mathbf{n}$ es un vector unitario normal a $S$ y definido en sentido entrante a la cavidad considerada; $g^{\mathrm{e}}\left(\mathbf{r}, \mathbf{r}^{\prime}\right)$ y $g^{\mathrm{m}}\left(\mathbf{r}, \mathbf{r}^{\prime}\right)$ son, respectivamente, las funciones de Green escalares de tipo eléctrico y de tipo magnético asociadas al

\footnotetext{
${ }^{1}$ Aunque la función de Green de espacio libre tiene una expresión analítica mucho más sencilla que la de un resonador rectangular o esférico, su utilización derivaría en la obtención de un problema no lineal de autovalores, cuya solución requeriría determinar los ceros de un determinante de una matriz compleja dependiente de la frecuencia.
} 
resonador externo considerado; $\overline{\mathbf{G}}^{\mathrm{A}}\left(\mathbf{r}, \mathbf{r}^{\prime}\right)$ y $\overline{\mathbf{G}}^{\mathrm{F}}\left(\mathbf{r}, \mathbf{r}^{\prime}\right)$ son las funciones de Green diádicas de tipo eléctrico y de tipo magnético del resonador externo, respectivamente; $\mathbf{J}\left(\mathbf{r}^{\prime}\right)$ constituye la incógnita del problema y representa la densidad de corriente eléctrica distribuida en el contorno de la superficie; y $\mathbf{M}\left(\mathbf{r}^{\prime}\right)$ es la densidad de corriente magnética impresa en los puertos de acceso del dispositivo (corriente de excitación). De hecho, esta densidad de corriente magnética puede expresarse en términos de las funciones vectoriales modales de campo magnético $\mathbf{h}_{n}\left(\mathbf{r}^{\prime}\right)$ relativas a los puertos de la estructura como sigue:

$$
\mathbf{M}\left(\mathbf{r}^{\prime}\right)=-\sum_{n=1}^{N} v_{n} \mathbf{h}_{n}\left(\mathbf{r}^{\prime}\right)
$$

donde $N$ es el número de modos considerados en los puertos de acceso, y $v_{n}$ son los voltajes modales equivalentes asociados a las funciones vectoriales modales de campo eléctrico. Además, las funciones de Green diádicas del resonador externo pueden escribirse mediante una expansión en serie utilizando los modos de dicho resonador [21]:

$$
\begin{aligned}
& \overline{\mathbf{G}}^{\mathrm{A}}\left(\mathbf{r}, \mathbf{r}^{\prime}\right) \approx \overline{\mathbf{G}}_{0}^{\mathrm{A}}\left(\mathbf{r}, \mathbf{r}^{\prime}\right)+k^{2} \sum_{i=1}^{M} \frac{\mathbf{E}_{i}(\mathbf{r}) \mathbf{E}_{i}\left(\mathbf{r}^{\prime}\right)}{\kappa_{i}^{2}\left(\kappa_{i}^{2}-k^{2}\right)} \\
& \overline{\mathbf{G}}^{\mathrm{F}}\left(\mathbf{r}, \mathbf{r}^{\prime}\right) \approx \overline{\mathbf{G}}_{0}^{\mathrm{F}}\left(\mathbf{r}, \mathbf{r}^{\prime}\right)+k^{2} \sum_{i=1}^{M} \frac{\mathbf{H}_{i}(\mathbf{r}) \mathbf{H}_{i}\left(\mathbf{r}^{\prime}\right)}{\kappa_{i}^{2}\left(\kappa_{i}^{2}-k^{2}\right)}
\end{aligned}
$$

donde $M$ es el número de términos considerados en la serie; $\overline{\mathbf{G}}_{0}^{\mathrm{A}}\left(\mathbf{r}, \mathbf{r}^{\prime}\right)$ y $\overline{\mathbf{G}}_{0}^{\mathrm{F}}\left(\mathbf{r}, \mathbf{r}^{\prime}\right)$ son diádicas estáticas o independientes de la frecuencia de tipo eléctrico y de tipo magnético, respectivamente $^{2} ; \mathbf{E}_{i}(\mathbf{r})$ y $\mathbf{H}_{i}(\mathbf{r})$ representan, respectivamente, el $i$-ésimo modo resonante de tipo eléctrico y de tipo magnético de la cavidad externa; $k \leq 2 \pi f_{\max } \sqrt{\mu \varepsilon}=k_{\max }$; y $\kappa_{i}$ es el número de onda de corte del $i$-ésimo modo del resonador externo ${ }^{3}$.

Seguidamente, teniendo en cuenta (3.4) y (3.5), e imponiendo la siguiente condición de contorno :

$$
\mathbf{E}_{\mathrm{tg}}(\mathbf{r})=\sum_{n=1}^{N} v_{n} \mathbf{e}_{n}(\mathbf{r})
$$

donde $\mathbf{e}_{n}(\mathbf{r})$ denota la $n$-ésima función vectorial modal de campo eléctrico de los puertos de acceso, se logra transformar las expresiones (3.1) y (3.2) en sendas ecuaciones integrales de campo eléctrico y de campo magnético cuyos núcleos son reales e independientes de la frecuencia [12]. Las ecuaciones integrales obtenidas pueden resolverse utilizando el Método de los Momentos (procedimiento de Galerkin) y, para ello, la densidad de corriente eléctrica

\footnotetext{
${ }^{2}$ Las expresiones analíticas de las funciones de Green escalares y de las funciones de Green diádicas independientes de la frecuencia se aceleran utilizando el método de Ewald citado con anterioridad.

${ }^{3} \mathrm{El}$ número de términos $M$ a considerar en el desarrollo en serie se escoge en base a la siguiente relación: $\kappa_{M} \leq \zeta k_{\max } \leq \kappa_{M+1}$. La constante $\zeta$ es un factor de precisión que controla el número de términos considerados en la expansión. Usualmente, un valor $\zeta=2$ suele proporcionar resultados convergentes, aunque para lograr una mayor precisión se recomienda $\zeta=4$.
} 
incógnita $\mathbf{J}(\mathbf{r})$ se expande utilizando un conjunto adecuado de funciones base definidas sobre la superficie $S$ [67]. En concreto:

$$
\mathbf{J}(\mathbf{r})=-\frac{j k}{\eta} \sum_{p=1}^{P} b_{p} \mathbf{V}_{p}(\mathbf{r})+\frac{1}{\eta} \sum_{q=1}^{Q} c_{q} \mathbf{W}_{q}(\mathbf{r})
$$

En esta expresión, $\mathbf{V}_{q}(\mathbf{r})$ y $\mathbf{W}_{p}(\mathbf{r})$ representan, respectivamente, un conjunto de funciones base no solenoidales y solenoidales; donde $b_{p}$ y $c_{q}$ se convierten en las nuevas incógnitas del problema. Las funciones base deben definirse sobre la superficie de la geometría bajo análisis y ello requiere, en un caso general, un mallado previo de dicha geometría. A este respecto, un conjunto de funciones base muy utilizadas para representar la corriente en la estructura son las de Rao-Wilton-Glisson [68].

Finalmente, tras aplicar el procedimiento de Galerkin, el problema queda formulado en términos de un problema generalizado de autovalores equivalente de la forma:

$$
\left(\mathbf{A}-k^{2} \mathbf{B}\right) \mathbf{x}=\mathbf{C} \mathbf{v}
$$

donde $\mathrm{x}$ es un vector que representa las variables de estado del problema y está relacionado con los coeficientes de expansión de la densidad de corriente eléctrica $b_{p}$ y $c_{q}$ utilizados en (3.7), y con las amplitudes de los modos resonantes de la cavidad externa considerados en la representación del campo (ver detalles en [12]); $\mathbf{y} \mathbf{v}=\left\{v_{i}\right\}$ es el voltaje modal equivalente de excitación. Por otra parte, las matrices A, B y C son reales, simétricas y definidas positivas, y sus expresiones son las siguientes [12]:

$$
\begin{aligned}
\mathbf{A}=\left[\begin{array}{rr}
\mathbf{K}^{4} & \mathbf{0} \\
\mathbf{0} & \mathbf{S}
\end{array}\right] \mathbf{B} & =\left[\begin{array}{cc}
\mathbf{K}^{2}-\mathbf{R}^{\prime \prime} \mathbf{W}^{-1} \mathbf{R}_{\mathrm{T}}^{\prime \prime} & \mathbf{R}^{\prime}-\mathbf{R}^{\prime \prime} \mathbf{W}^{-1} \mathbf{Q}_{\mathrm{T}} \\
\mathbf{R}_{\mathrm{T}}^{\prime}-\mathbf{Q} \mathbf{W}^{-1} \mathbf{R}_{\mathrm{T}}^{\prime \prime} & \mathbf{V}-\mathbf{Q} \mathbf{W}^{-1} \mathbf{Q}_{\mathrm{T}}
\end{array}\right] \\
\mathbf{C} & =\left[\begin{array}{c}
-\mathbf{K} \mathbf{F}+\mathbf{R}^{\prime \prime} \mathbf{W}^{-1} \mathbf{L}^{\prime \prime} \\
-\mathbf{L}^{\prime}+\mathbf{Q} \mathbf{W}^{-1} \mathbf{L}^{\prime \prime}
\end{array}\right]
\end{aligned}
$$

donde el subíndice T representa la operación de transposición. Además, las diferentes matrices que intervienen en las expresiones recogidas en (3.9) se han definido en la tabla 3.1.

La solución del problema de autovalores (3.8) permite determinar la MAG de la estructura utilizando la siguiente expansión polar [12]:

$$
\mathbf{Y}=\frac{1}{j k \eta} \mathbf{Y}^{\mathrm{A}}+\frac{j k}{\eta} \mathbf{Y}^{\mathrm{B}}+\frac{j k^{3}}{\eta} \sum_{i=1}^{M^{\prime}} \frac{\mathbf{y}^{(i)} \mathbf{y}_{\mathrm{T}}^{(i)}}{k_{i}^{2}-k^{2}} \quad k \leq k_{\max }
$$

donde las matrices $\mathbf{Y}^{\mathrm{A}}$ e $\mathbf{Y}^{\mathrm{B}}$, y el vector $\mathbf{y}^{(i)}$ se definen como sigue (consultar también la tabla 3.1):

$$
\begin{aligned}
\mathbf{Y}^{\mathrm{A}} & =\mathbf{G}+\mathbf{L}^{\prime \prime}{ }_{\mathrm{T}} \mathbf{W}^{-1} \mathbf{L}^{\prime \prime} \\
\mathbf{Y}^{\mathrm{B}} & =\mathbf{T}-\mathbf{F}_{\mathrm{T}} \mathbf{K}^{-2} \mathbf{F}+\mathbf{C}_{\mathrm{T}} \mathbf{A}^{-1} \mathbf{C} \\
\mathbf{y}^{(i)} & =k_{i}^{-1} \mathbf{C}_{\mathrm{T}} \mathbf{x}^{(i)}
\end{aligned}
$$




$$
\begin{gathered}
S_{r p}=\int_{S} \int_{S} \nabla_{S} \cdot \mathbf{V}_{r}(\mathbf{r}) g^{\mathrm{e}}\left(\mathbf{r}, \mathbf{r}^{\prime}\right) \nabla_{S}^{\prime} \cdot \mathbf{V}_{p}\left(\mathbf{r}^{\prime}\right) d S d S^{\prime} \quad V_{r p}=\int_{S} \int_{S} \mathbf{V}_{r}(\mathbf{r}) \cdot \overline{\mathbf{G}}_{0}^{\mathrm{A}}\left(\mathbf{r}, \mathbf{r}^{\prime}\right) \cdot \mathbf{V}_{p}\left(\mathbf{r}^{\prime}\right) d S d S^{\prime} \\
W_{s q}=\int_{S} \int_{S} \mathbf{W}_{s}(\mathbf{r}) \cdot \overline{\mathbf{G}}_{0}^{\mathrm{A}}\left(\mathbf{r}, \mathbf{r}^{\prime}\right) \cdot \mathbf{W}_{q}\left(\mathbf{r}^{\prime}\right) d S d S^{\prime} \quad Q_{r q}=\int_{S} \int_{S} \mathbf{V}_{r}(\mathbf{r}) \cdot \overline{\mathbf{G}}_{0}^{\mathrm{A}}\left(\mathbf{r}, \mathbf{r}^{\prime}\right) \cdot \mathbf{W}_{q}\left(\mathbf{r}^{\prime}\right) d S d S^{\prime} \\
G_{l n}=\int_{S} \int_{S} \nabla_{S} \cdot \mathbf{h}_{l}(\mathbf{r}) g^{\mathrm{m}}\left(\mathbf{r}, \mathbf{r}^{\prime}\right) \nabla_{S}^{\prime} \cdot \mathbf{h}_{n}\left(\mathbf{r}^{\prime}\right) d S d S^{\prime} \quad T_{l n}=\int_{S} \int_{S} \mathbf{h}_{l}(\mathbf{r}) \cdot \overline{\mathbf{G}}_{0}^{\mathrm{F}}\left(\mathbf{r}, \mathbf{r}^{\prime}\right) \cdot \mathbf{h}_{n}\left(\mathbf{r}^{\prime}\right) d S d S^{\prime} \\
R_{m p}^{\prime}=\int_{S} \mathbf{E}_{m}(\mathbf{r}) \cdot \mathbf{V}_{p}(\mathbf{r}) d S \quad R_{m p}^{\prime \prime}=\int_{S} \mathbf{E}_{m}(\mathbf{r}) \cdot \mathbf{W}_{p}(\mathbf{r}) d S \quad F_{m n}=\int_{S} \mathbf{H}_{m}(\mathbf{r}) \cdot \mathbf{h}_{n}(\mathbf{r}) d S \\
L_{r n}^{\prime}=\int_{S} \int_{S} \mathbf{V}_{r}(\mathbf{r}) \cdot \nabla \times \overline{\mathbf{G}}_{0}^{\mathrm{F}}\left(\mathbf{r}, \mathbf{r}^{\prime}\right) \cdot \mathbf{h}_{n}\left(\mathbf{r}^{\prime}\right) d S d S^{\prime}-\frac{1}{2} \int_{S} \mathbf{V}_{r}(\mathbf{r}) \cdot \mathbf{e}_{n}(\mathbf{r}) d S \\
L_{s n}^{\prime \prime}=\int_{S} \int_{S} \mathbf{W}_{s}(\mathbf{r}) \cdot \nabla \times \overline{\mathbf{G}}_{0}^{\mathrm{F}}\left(\mathbf{r}, \mathbf{r}^{\prime}\right) \cdot \mathbf{h}_{n}\left(\mathbf{r}^{\prime}\right) d S d S^{\prime}-\frac{1}{2} \int_{S} \mathbf{W}_{s}(\mathbf{r}) \cdot \mathbf{e}_{n}(\mathbf{r}) d S \\
\mathbf{K}=\operatorname{diag}\left\{\kappa_{1}, \kappa_{2}, \ldots, \kappa_{M}\right\} \\
\begin{array}{l}
l, n=1,2, \ldots, N \quad m=1,2, \ldots, M \\
r, p=1,2, \ldots, P \quad s, q=1,2, \ldots, Q
\end{array}
\end{gathered}
$$

Tabla 3.1: Definición de las matrices del método BI-RME 3D.

Las matrices $\mathrm{Y}^{\mathrm{A}}$ e $\mathrm{Y}^{\mathrm{B}}$ son reales, simétricas e independientes de la frecuencia [12]. Por otro lado, $k_{i}\left(i=1, \ldots, M^{\prime}\right)$ representa el $i$-ésimo número de onda de corte de la cavidad resonante obtenida tras cortocircuitar todos los puertos de acceso de la geometría bajo análisis ${ }^{4}$. A esta nueva cavidad la denominaremos, a partir de ahora, cavidad perturbada. Finalmente, el vector $\mathrm{x}^{(i)}$ representa el autovector asociado al $i$-ésimo autovalor $k_{i}$ de la cavidad perturbada [12].

Es importante resaltar que el cálculo de los elementos de la MAG requiere, en realidad, invertir la matriz $\left(\mathbf{A}-k^{2} \mathbf{B}\right)$ presente en (3.8), tal y como se concluye en [12] ${ }^{5}$. A este respecto, resulta sencillo demostrar que la inversa de esta matriz puede expresarse en función

\footnotetext{
${ }^{4}$ El número de modos resonantes $M^{\prime}$ de la cavidad con los accesos cortocircuitados debe satisfacer la relación $k_{M^{\prime}+1}>\zeta k_{\max }$.

${ }^{5}$ La expresión que se recoge en (3.10) es la expresión final para la MAG, que se obtiene en [12] tras expresar la inversa de la matriz $\left(\mathbf{A}-k^{2} \mathbf{B}\right)$ en términos de las frecuencias de resonancia de la cavidad perturbada y de los correspondientes autovectores asociados.
} 
de los autovalores y autovectores del problema (3.8) forzando la condición $\mathbf{v}=0$; es decir, se está considerando que los puertos de la geometría bajo análisis se han cortocircuitado. En consecuencia, la caracterización electromagnética de un dispositivo utilizando el método BI-RME 3D puede resolverse mediante los siguientes pasos:

- En primer lugar, se deben cortocircuitar los puertos de acceso de la estructura con el propósito de calcular las frecuencias de resonancia de la cavidad perturbada obtenida. Matemáticamente, este paso implica resolver el problema lineal de autovalores (3.8) tomando $\mathbf{v}=0$, es decir: $\mathbf{A} \mathbf{x}=k^{2} \mathbf{B} \mathbf{x}$.

- A continuación, deben considerarse los puertos de acceso de la estructura con el fin de obtener los elementos de la MAG de acuerdo con la expresión (3.10). Es importante observar que los autovalores (frecuencias de resonancia) y autovectores calculados en el paso anterior son necesarios para obtener los elementos de la MAG.

\subsubsection{Análisis multimodal eficiente de uniones multi-puerto compensa- das en guía rectangular}

Una vez que se ha presentado el método BI-RME 3D, esta sección se dedica a investigar su aplicación al análisis multimodal eficiente de uniones multi-puerto en guía rectangular compensadas con postes conductores cilíndricos de altura arbitraria ${ }^{6}$. La teoría que se desarrolla en este apartado puede interpretarse como una extensión del trabajo presentado en [67], donde la geometría básica bajo análisis es una unión compensada de dos puertos de acceso paralelos entre sí. En este capítulo se pretende generalizar la teoría anterior con el fin de analizar uniones multi-puerto (de hasta cinco puertos) compensadas en guía rectangular, permitiendo así la existencia de puertos de acceso ortogonales. Esta novedosa herramienta de diseño permitirá el análisis eficiente de una gran variedad de uniones compensadas en guía rectangular: codos rectos en plano $H$ y en plano $E$, uniones en $T$ en plano $H$ y en plano $E$, uniones en T-mágica y uniones turnstile (véase la figura 3.1). Tal y como ya se ha comentado con anterioridad, el objetivo será optimizar la respuesta en frecuencia de estas uniones considerando postes conductores cilíndricos de altura variable que se situarán en la base de la cavidad rectangular en una posición arbitraria.

En la sección anterior se indicó que el método BI-RME 3D asume que la estructura bajo análisis se encuentra inmersa en un resonador externo de geometría canónica. Por tanto, en primer lugar, se tiene que escoger el resonador externo que se va a utilizar. Puesto que, en nuestro caso, la geometría básica bajo análisis es una cavidad rectangular de dimensiones $a \times b \times c$ cargada con un poste conductor cilíndrico situado en la base de la cavidad, tal y como se recoge en la figura 3.2, la mejor opción será escoger un resonador externo rectangular cuyas dimensiones coincidan con las de la cavidad bajo análisis. De esta manera, la densidad de corriente eléctrica incógnita $\mathbf{J}(\mathbf{r})$ únicamente debe definirse en el contorno del poste, logrando así reducir enormemente el esfuerzo computacional de la herramienta implementada.

\footnotetext{
${ }^{6}$ Puesto que el poste conductor siempre estará situado en una posición arbitraria de la base de la cavidad rectangular, el número máximo de puertos a considerar en las uniones será de cinco.
} 


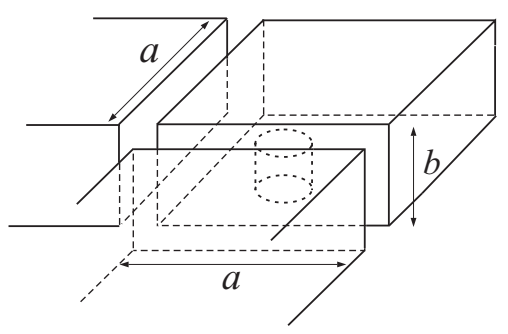

a)

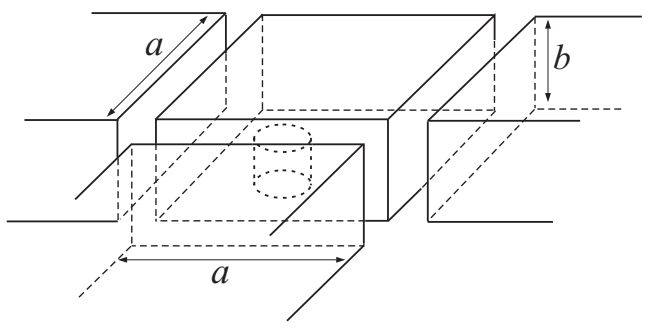

c)

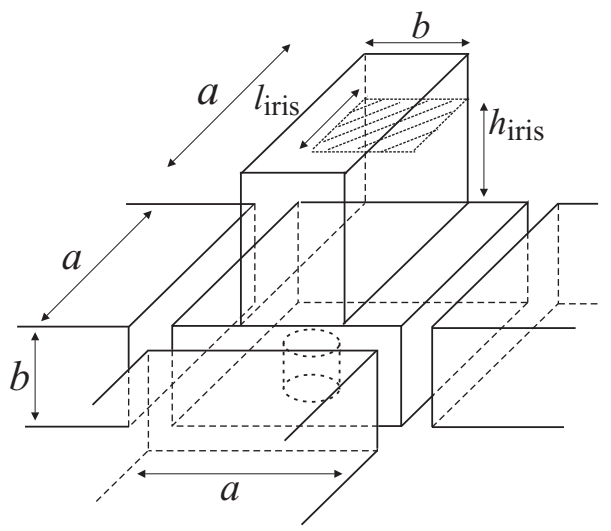

e)

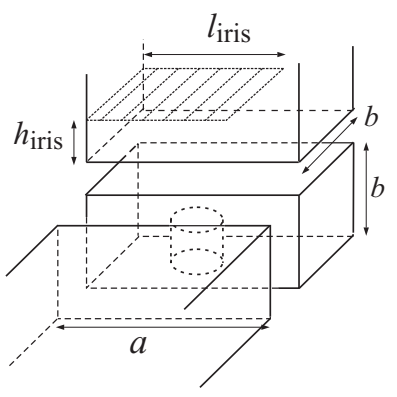

b)

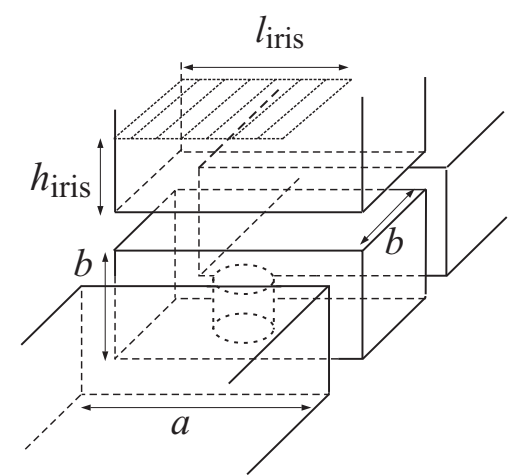

d)

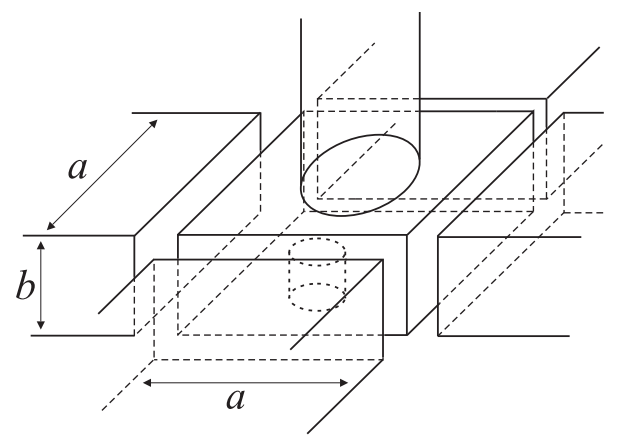

f)

Figura 3.1: Uniones multi-puerto en guía rectangular compensadas con un poste conductor cilíndrico de altura variable situado en una posición arbitraria. (a) Codo recto en plano $H$; (b) codo recto en plano $E$ con un iris de acoplo en uno de los puertos; (c) unión en T en plano $H$; (d) unión en $\mathrm{T}$ en plano $E$ con un iris de acoplo en uno de los puertos; (e) unión T-mágica con un iris de acoplo en uno de los puertos; (f) unión turnstile.

A continuación, de acuerdo con el método BI-RME 3D, deben cortocircuitarse todos los puertos de acceso de la estructura con el fin de calcular las frecuencias de resonancia de 
la cavidad perturbada obtenida, que en nuestro caso consistirá en un resonador rectangular cargado con un poste conductor. La resolución de este problema requiere evaluar las funciones de Green del resonador externo rectangular sobre los puntos de la superficie del poste cilíndrico y, para ello, como ya se ha comentado con anterioridad, se emplea el método de Ewald, el cual proporciona unas expresiones analíticas para dichas funciones que convergen muy rápidamente [65]. El cálculo de las frecuencias de resonancia de la cavidad perturbada se ha implementado siguiendo la teoría desarrollada en [66], [67] con lo que en este capítulo no se detallará la caracterización electromagnética de la cavidad resonante perturbada.

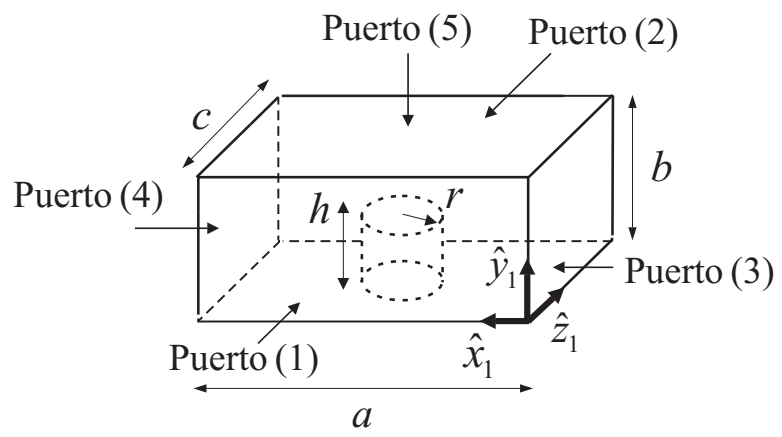

Figura 3.2: Geometría básica bajo análisis: unión en guía rectangular de 5 puertos cargada con un poste conductor cilíndrico de altura arbitraria situado en la base de la cavidad en la posición $\left(x_{0}, 0, z_{0}\right)$.

De acuerdo con el método BI-RME 3D, una vez calculadas las frecuencias de resonancia de la cavidad perturbada, se deben considerar los puertos de acceso de la estructura con el objetivo de determinar los elementos de la MAG de la geometría bajo análisis. A este respecto, conviene recordar que el cálculo de las matrices $\mathrm{Y}^{\mathrm{A}}$ e $\mathrm{Y}^{\mathrm{B}}$ introducidas en (3.10), requiere, a su vez, determinar las siguientes integrales dobles de superficie en las que intervienen las funciones vectoriales modales de campo magnético relativas a los puertos de acceso de la estructura:

$$
\begin{aligned}
G_{m n}^{(\gamma, \xi)} & =\int_{S} \int_{S^{\prime}} \nabla_{S} \cdot \mathbf{h}_{m}^{(\gamma)}(\mathbf{r}) g^{\mathrm{m}}\left(\mathbf{r}, \mathbf{r}^{\prime}\right) \nabla_{S}^{\prime} \cdot \mathbf{h}_{n}^{(\xi)}\left(\mathbf{r}^{\prime}\right) d S d S^{\prime} \\
T_{m n}^{(\gamma, \xi)} & =\int_{S} \int_{S^{\prime}} \mathbf{h}_{m}^{(\gamma)}(\mathbf{r}) \cdot \overline{\mathbf{G}}_{0}^{\mathrm{F}}\left(\mathbf{r}, \mathbf{r}^{\prime}\right) \cdot \mathbf{h}_{n}^{(\xi)}\left(\mathbf{r}^{\prime}\right) d S d S^{\prime} \\
L_{m n}^{(\xi)} & =\int_{S} \int_{S^{\prime}} \mathbf{N}_{m}(\mathbf{r}) \cdot \nabla \times \overline{\mathbf{G}}_{0}^{\mathrm{F}}\left(\mathbf{r}, \mathbf{r}^{\prime}\right) \cdot \mathbf{h}_{n}^{(\xi)}\left(\mathbf{r}^{\prime}\right) d S d S^{\prime} \\
F_{m n}^{(\xi)} & =\int_{S} \mathbf{H}_{m}(\mathbf{r}) \cdot \mathbf{h}_{n}^{(\xi)}(\mathbf{r}) d S
\end{aligned}
$$

En estas expresiones $\gamma, \xi=1, \ldots, 5$ representan los puertos de acceso; $m, n=1,2, \ldots, N$, siendo $N$ el número de modos considerados en los accesos; $\mathbf{h}_{n}^{(\xi)}(\mathbf{r})$ es la $n$-ésima función 
vectorial modal de campo magnético asociada al puerto $(\xi) ; g^{\mathrm{m}}\left(\mathbf{r}, \mathbf{r}^{\prime}\right)$ es la función de Green escalar magnética del resonador externo escogido; $\overline{\mathbf{G}}_{0}^{\mathrm{F}}\left(\mathbf{r}, \mathbf{r}^{\prime}\right)$ es la parte estática (independiente de la frecuencia) de la función de Green diádica de tipo magnético relativa al resonador externo; $\mathbf{H}_{m}(\mathbf{r})$ denota el $m$-ésimo modo resonante de tipo magnético de la cavidad externa, y $\mathbf{N}_{m}(\mathbf{r})$ representa la $m$-ésima función base utilizada en el desarrollo en serie de la densidad de corriente eléctrica incógnita sobre el poste (recordar que, en virtud de (3.7), la función base puede ser de tipo solenoidal o de tipo no solenoidal). Por otro lado, es importante observar que si se compara la expresión para la matriz $L_{m n}^{(\xi)}$ que se recoge en (3.16) con la que se proporciona en la tabla 3.1, se comprobará que en (3.16) se ha omitido el término $\frac{1}{2} \int_{S} \mathbf{N}_{m}(\mathbf{r}) \cdot \mathbf{e}_{n}(\mathbf{r}) d S$ por ser nula esta integral (las funciones base $\mathbf{N}_{m}(\mathbf{r})$ se definen únicamente sobre el poste conductor y las funciones vectoriales modales de campo eléctrico $\mathbf{e}_{n}(\mathbf{r})$ están definidas solamente en los accesos).

Tal y como ya se ha apuntado anteriormente, las matrices definidas en (3.14)-(3.17) son esenciales para calcular los elementos de la MAG, y la contribución teórica novedosa de este capítulo se centra, fundamentalmente, en el cálculo eficiente de las mismas.

\section{Cálculo de los Elementos de la Matriz de Admitancias Generalizada}

Considérese entonces la geometría básica bajo análisis de la figura 3.2. Es importante observar que cualquiera de las uniones multi-puerto consideradas en la figura 3.1 puede analizarse partiendo de la geometría presentada en la figura 3.2. En esta última figura, se ha representado una cavidad rectangular de dimensiones $a \times b \times c$ cargada con un poste conductor cilíndrico de radio $r$ y de altura $h$, situado en la posición arbitraria $\left(x_{0}, 0, z_{0}\right)$. Además, con respecto al sistema de referencia $\left(x_{1}, y_{1}, z_{1}\right)$, se considera que el puerto (1) de la estructura se encuentra en el plano $z_{1}=0$; el puerto (2) en el plano $z_{1}=c$; el puerto (3) en $x_{1}=0$; el puerto (4) en $x_{1}=a$, y el puerto (5) en el plano $y_{1}=b$.

El cálculo de los elementos de las matrices (3.14)-(3.17) requiere conocer las expresiones de las funciones vectoriales modales de campo magnético $\mathbf{h}_{n}^{(\xi)}(\mathbf{r})$ de los puertos de acceso de la geometría. Así pues, sean $\left(x_{\xi}, y_{\xi}\right)$ un par de coordenadas transversales definidas sobre el puerto $(\xi)$, y sea $z_{\xi}$ la correspondiente coordenada axial. Entonces, según [35], podemos escribir:

$$
\mathbf{h}_{n}^{(\xi)}(\mathbf{r})=\mathcal{N}_{x_{\xi}, n} F_{x_{\xi}, n}\left(x_{\xi}, y_{\xi}\right) \hat{\mathbf{x}}_{\xi}+\mathcal{N}_{y_{\xi}, n} F_{y_{\xi}, n}\left(x_{\xi}, y_{\xi}\right) \hat{\mathbf{y}}_{\xi}
$$

donde los factores de normalización $\mathcal{N}_{x_{\xi}, n}$ y $\mathcal{N}_{y_{\xi}, n}$, así como las variaciones funcionales $F_{x_{\xi}, n}\left(x_{\xi}, y_{\xi}\right)$ y $F_{y_{\xi}, n}\left(x_{\xi}, y_{\xi}\right)$ asociadas a cada puerto de acceso se han definido en la sección C. 1 del Apéndice $\mathrm{C}$.

En la figura 3.2 se ha representado el sistema de referencia $\left(x_{1}, y_{1}, z_{1}\right)$, el cual se utiliza para la descripción matemática de los modos de los puertos de acceso paralelos $\xi=1$ y $\xi=2$. A este respecto conviene observar que, tal y como se ha escogido el sistema de coordenadas, la dirección de propagación de los modos del puerto $\xi=1$ es entrante a la cavidad, pero la de los modos del puerto $\xi=2$ es saliente de la cavidad. Así pues, dado que las funciones vectoriales modales de campo magnético están relacionadas con las corrientes modales equivalentes que caracterizan a la MAG [28], y éstas se definen siempre en direc- 
ción entrante al acceso, se debe introducir un signo menos en la expresión de las funciones vectoriales modales del acceso $\xi=2$ para corregir el problema. Por otra parte, en la figura 3.3 se han representado los sistemas de coordenadas $\left(x_{3}, y_{3}, z_{3}\right)$ y $\left(x_{5}, y_{5}, z_{5}\right)$. El primero de ellos se utiliza para describir los modos de los accesos paralelos $\xi=3$ y $\xi=4$. Además, la expresión de las funciones vectoriales modales de campo magnético del acceso $\xi=4$ deben corregirse con un signo menos, por la misma razón expuesta anteriormente. El segundo de los sistemas se emplea para la descripción matemática de los modos del acceso $\xi=5$.

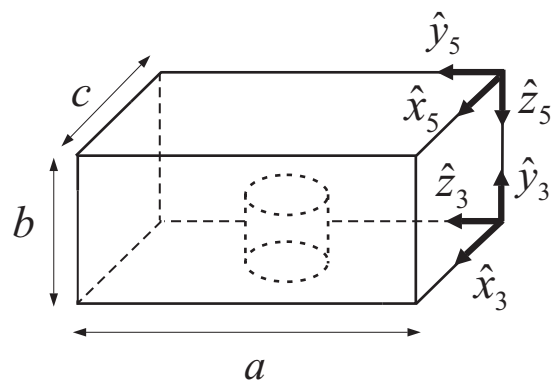

Figura 3.3: Representación de los sistemas de coordenadas utilizados para los puertos de acceso $\xi=3,4,5$.

Por otro lado, las dimensiones transversales $l_{x_{\xi}} \times l_{y_{\xi}}$ que definen el acceso rectangular $(\xi)$ se han escogido del siguiente modo:

$$
\begin{array}{lll}
l_{x_{1}}=l_{x_{2}}=a ; & l_{x_{3}}=l_{x_{4}}=c ; & l_{x_{5}}=c ; \\
l_{y_{1}}=l_{y_{2}}=b ; & l_{y_{3}}=l_{y_{4}}=b ; & l_{y_{5}}=a
\end{array}
$$

Asimismo, las dimensiones $l_{z_{\xi}}$ asociadas a la dirección de propagación considerada en cada uno de los puertos de acceso son:

$$
l_{z_{1}}=l_{z_{2}}=c ; \quad l_{z_{3}}=l_{z_{4}}=a ; \quad l_{z_{5}}=b
$$

\section{A. Cálculo de los elementos de la matriz $G_{m n}^{(\gamma, \xi)}$}

La expresión de la matriz $G_{m n}^{(\gamma, \xi)}$ es la siguiente:

$$
G_{m n}^{(\gamma, \xi)}=\int_{S} \int_{S^{\prime}} \nabla_{S} \cdot \mathbf{h}_{m}^{(\gamma)}(\mathbf{r}) g^{\mathrm{m}}\left(\mathbf{r}, \mathbf{r}^{\prime}\right) \nabla_{S}^{\prime} \cdot \mathbf{h}_{n}^{(\xi)}\left(\mathbf{r}^{\prime}\right) d S d S^{\prime}
$$

El cálculo de los elementos de dicha matriz se divide en dos partes. La primera de ellas consiste en evaluar la siguiente integral de superficie:

$$
\zeta_{n}^{(\xi)}(\mathbf{r})=\int_{S^{\prime}} g^{\mathrm{m}}\left(\mathbf{r}, \mathbf{r}^{\prime}\right) \nabla_{S}^{\prime} \cdot \mathbf{h}_{n}^{(\xi)}\left(\mathbf{r}^{\prime}\right) d S^{\prime}
$$


La función de Green escalar de tipo magnético de un resonador rectangular $g^{\mathrm{m}}\left(\mathbf{r}, \mathbf{r}^{\prime}\right)$ que se utiliza en la integral anterior puede encontrarse en la ecuación (C.14) del Apéndice C y, tal y como se puede comprobar, se trata de una expresión que converge muy lentamente. A pesar de ello, es importante destacar que resulta muy ventajoso utilizar este tipo de formulación. De hecho, como se comenta en la sección C. 2 del Apéndice C, puesto que la función de Green escalar utilizada se ha definido a partir de la función potencial escalar asociada a una guía de sección transversal rectangular, la integral (3.22) se puede resolver fácilmente de manera analítica. Un razonamiento completamente paralelo podría realizarse con respecto a la función de Green diádica de tipo magnético empleada en la expresión (3.15) de la matriz $T_{m n}^{(\gamma, \xi)}$, y también respecto al rotacional de dicha función utilizado en (3.16) para la matriz $L_{m n}^{(\xi)}$. En estos dos últimos casos, la función diádica de Green y su rotacional se han expresado en la sección C.2 del Apéndice C en términos de las funciones vectoriales modales de campo magnético asociadas a los puertos de acceso de la estructura. Todo ello facilitará enormemente el cálculo analítico de los elementos de todas estas matrices.

Volviendo al caso que nos ocupa, es fácil deducir que la divergencia de la función vectorial modal de campo magnético asociada al puerto de acceso $(\xi)$ se calcula como (ver expresiones (C.11) y (C.13) del Apéndice C):

$$
\nabla_{S}^{\prime} \cdot \mathbf{h}_{n}^{(\xi)}\left(\mathbf{r}^{\prime}\right)=\left\{\begin{array}{l}
\nu_{\xi} k_{t, n}^{(\xi)} \phi_{n_{x_{\xi}}, n_{y_{\xi}}}^{(\xi) \mathrm{TE}}\left(x_{\xi}, y_{\xi}\right), \text { si } n \text { es un modo } \mathrm{TE}^{z_{\xi}} \\
0, \text { si } n \text { es un modo } \mathrm{TM}^{z_{\xi}}
\end{array}\right.
$$

En esta última expresión, $\left(n_{x_{\xi}}, n_{y_{\xi}}\right)$ son los índices modales asociados al $n$-ésimo modo del puerto $(\xi)$ correspondientes a las coordenadas $x_{\xi}$ y $y_{\xi}$, respectivamente; $k_{t, n}^{(\xi)}$ representa el $n$-ésimo número de onda de corte en el mismo puerto; $\left.\phi_{n_{x_{\xi}}, n_{y_{\xi}}}^{(\xi) x_{\xi}}, y_{\xi}\right)$ es la función potencial utilizada para derivar las soluciones modales de tipo $\mathrm{TE}^{z_{\xi}}$ y se ha definido en la expresión (C.10) del Apéndice C; y $\nu_{\xi}$ es una constante que fuerza a que la dirección de propagación en cada uno de los puertos de acceso sea entrante a la cavidad. En particular:

$$
\nu_{\xi}=\left\{\begin{array}{l}
1, \quad \text { si } \xi=1,3,5 \\
-1, \text { si } \xi=2,4
\end{array}\right.
$$

Si ahora se utilizan (3.23) y (C.14) en (3.22), se obtiene que:

$$
\zeta_{n}^{(\xi)}(\mathbf{r})=\left\{\begin{array}{l}
\nu_{\xi} \frac{k_{t, n}^{(\xi)}}{l_{z_{\xi}}} \phi_{n_{x_{\xi}}, n_{y_{\xi}}}^{(\xi) \mathrm{TE}}\left(x_{\xi}, y_{\xi}\right) \sum_{n_{z_{\xi}}=0}^{\infty} \nu_{\xi}^{n_{z_{\xi}}} \epsilon_{n_{z_{\xi}}} \frac{\cos \left(k_{z_{\xi}} z_{\xi}\right)}{\left(k_{t, n}^{(\xi)}\right)^{2}+k_{z_{\xi}}^{2}}, \text { si } n \text { es un modo } \mathrm{TE}^{z_{\xi}} \\
0, \text { si } n \text { es un modo } \mathrm{TM}^{z_{\xi}}
\end{array}\right.
$$

donde $\epsilon_{n}$ es el factor de Neumann introducido en (C.6), y $k_{z_{\xi}}$ es el número de onda de la cavidad rectangular asociado a la coordenada $z_{\xi}$ cuya definición puede encontrarse en (C.16). Así pues, si $m$ es un modo del puerto de acceso $(\gamma)$ y $\left(m_{x_{\gamma}}, m_{y_{\gamma}}\right)$ son los índices 
modales correspondientes asociados a las coordenadas $x_{\gamma}$ e $y_{\gamma}$, respectivamente, la matriz $G_{m, n}^{(\gamma, \xi)}$ se calculará ahora como:

$$
\begin{aligned}
G_{m n}^{(\gamma, \xi)}= & \int_{S} \nabla_{S} \cdot \mathbf{h}_{m}^{(\gamma)}(\mathbf{r}) \zeta_{n}^{(\xi)}(\mathbf{r}) d S=\frac{1}{l_{z_{\xi}}} \nu_{\gamma} \nu_{\xi} k_{t, m}^{(\gamma)} k_{t, n}^{(\xi)} \sum_{n_{z_{\xi}}=0}^{\infty} \nu_{\xi}^{n_{z_{\xi}}} \frac{\epsilon_{n_{z_{\xi}}}}{\left(k_{t, n}^{(\xi)}\right)^{2}+k_{z_{\xi}}^{2}} \\
& \cdot \int_{S} \phi_{m_{x_{\gamma}}, m_{y_{\gamma}}}^{(\gamma) \mathrm{TE}}\left(x_{\gamma}, y_{\gamma}\right) \phi_{n_{x_{\xi}}, n_{y_{\xi}}}^{(\xi) \mathrm{TE}}\left(x_{\xi}, y_{\xi}\right) \cos \left(k_{z_{\xi}} z_{\xi}\right) d S
\end{aligned}
$$

donde $\phi_{m_{x_{\gamma}}, m_{y_{\gamma}}}^{(\gamma) \mathrm{TE}}\left(x_{\gamma}, y_{\gamma}\right)$ representa la función potencial del $m$-ésimo modo $\mathrm{TE}^{z_{\gamma}}$ del puerto $(\gamma)$; y $k_{t, m}^{(\gamma)}$ es el número de onda de corte de dicha solución modal. Además, hemos asumido que tanto $m$ como $n$ son modos de tipo TE. En caso de que alguno de los modos sea de tipo $\mathrm{TM}$, los elementos de la matriz serían nulos en virtud de (3.23).

La resolución de la integral (3.26) requiere que el integrando se exprese en un único sistema de coordenadas. Así pues, en primer lugar, la función potencial $\phi_{m_{x_{\gamma}}, m_{y_{\gamma}}}^{(\gamma) \mathrm{TE}}\left(x_{\gamma}, y_{\gamma}\right)$ deberá reescribirse en términos de las variables $\left(x_{\xi}, y_{\xi}, z_{\xi}\right)$. Por otro lado, conviene destacar que la integral se debe evaluar en la sección transversal rectangular que define el puerto $(\gamma)$ $\mathrm{y}$, matemáticamente, ello implica que el plano en el que se encuentra dicho puerto puede expresarse de la forma $\vartheta_{\xi}=$ cte, $\operatorname{con} \vartheta=(x, y, z)$. De esta forma, la integral de superficie anterior se reduce al producto de dos integrales unidimensionales cuyo integrando consiste, a su vez, en un producto de dos funciones cosenoidales ${ }^{7}$. En consecuencia, en la resolución de (3.26) siempre nos aparecen integrales de fácil solución analítica del tipo:

$$
\int_{\vartheta_{\xi}} \cos \left(k_{\vartheta_{\xi}, m} \vartheta_{\xi}\right) \cos \left(k_{\vartheta_{\xi}, n} \vartheta_{\xi}\right) d \vartheta_{\xi}=\frac{l_{\vartheta_{\xi}}}{\epsilon_{n_{\vartheta_{\xi}}}} \delta_{m_{\vartheta_{\xi}}, n_{\vartheta_{\xi}}}, \quad \operatorname{con} \vartheta=(x, y, z)
$$

donde $m_{\vartheta_{\xi}}$ es el índice modal de $m$ relativo a la coordenada $\vartheta_{\xi} ; k_{\vartheta_{\xi}, m}$ es el número de onda del modo $m$-ésimo correspondiente a la coordenada $\vartheta_{\xi}$ definido como $k_{\vartheta_{\xi}, m}=m_{\vartheta_{\xi}} \pi / l_{\vartheta_{\xi}} \mathrm{y}$ $\delta_{m, n}$ es la delta de Kronecker definida en (2.39). Asimismo, es importante hacer notar que en la expresión (3.26) intervienen unas series infinitas que admiten solución analítica, las cuales se detallan en la sección D.1 del Apéndice D.

Con todo ello, los elementos de la matriz $G_{m n}^{(\gamma, \xi)}$ que relacionan puertos de acceso paralelos, es decir, los elementos $G_{m n}^{(\xi, \xi)} \operatorname{con} \xi=1,2, \ldots, 5$ y los elementos $G_{m n}^{(\xi, \xi+1)} \operatorname{con} \xi=1,3$ pueden expresarse finalmente como:

$$
\begin{aligned}
G_{m n}^{(\xi, \xi)} & =k_{t, n}^{(\xi)} \operatorname{coth}\left(k_{t, n}^{(\xi)} l_{z_{\xi}}\right) \delta_{m, n} \\
G_{m n}^{(\xi, \xi+1)} & =-\frac{k_{t, n}^{(\xi)}}{\sinh \left(k_{t, n}^{(\xi)} l_{z_{\xi}}\right)} \delta_{m, n}
\end{aligned}
$$

\footnotetext{
${ }^{7}$ Recuerde de (C.17) que en la expresión del potencial $\phi(x, y)$ utilizado en (3.26) intervienen dos funciones cosenoidales.
} 
Finalmente, los elementos de la matriz que relacionan puertos de acceso ortogonales, es decir, los elementos $G_{m n}^{(\gamma, \xi)} \operatorname{con} \gamma=1,2$ y $\xi=3,4,5$, o con $\gamma=3,4$ y $\xi=5$, son:

$$
G_{m n}^{(\gamma, \xi)}=\frac{k_{t, m}^{(\gamma)} k_{t, n}^{(\xi)}}{\left(k_{t, n}^{(\xi)}\right)^{2}+k_{m_{z_{\xi}}}^{(\gamma) 2}} \frac{\nu_{\gamma} \nu_{\xi}}{\sqrt{l_{z_{\xi}} l_{z_{\gamma}}}} \rho_{m, n}^{(\gamma, \xi)} \psi_{m, n}^{(\gamma, \xi)}
$$

donde $k_{m_{z_{\xi}}}^{(\gamma)}$ representa el número de onda del $m$-ésimo modo del puerto $(\gamma)$ asociado a la coordenada $z_{\xi} ; \mathrm{y} \rho_{m, n}^{(\gamma, \xi)}$ y $\psi_{m, n}^{(\gamma, \xi)}$ son unas funciones auxiliares cuya definición puede encontrarse en la sección D.2 del Apéndice D.

Hay que observar que la matriz $G_{m n}^{(\gamma, \xi)}$ es simétrica, y por esta razón únicamente hemos presentado los resultados para el caso $\xi>\gamma$ (triángulo superior de la matriz). Además, es importante insistir en el hecho de que en las expresiones anteriores se asume que $m$ y $n$ son modos de tipo TE. En otro caso, los elementos de la matriz serían nulos.

\section{B. Cálculo de los elementos de la matriz $T_{m n}^{(\gamma, \xi)}$}

Seguidamente, se procede al cálculo de los elementos de la matriz $T_{m n}^{(\gamma, \xi)}$ :

$$
T_{m n}^{(\gamma, \xi)}=\int_{S} \int_{S^{\prime}} \mathbf{h}_{m}^{(\gamma)}(\mathbf{r}) \cdot \overline{\mathbf{G}}_{0}^{\mathrm{F}}\left(\mathbf{r}, \mathbf{r}^{\prime}\right) \cdot \mathbf{h}_{n}^{(\xi)}\left(\mathbf{r}^{\prime}\right) d S d S^{\prime}
$$

donde la expresión de la parte estática de la función de Green diádica de tipo magnético de un resonador rectangular $\overline{\mathbf{G}}_{0}^{\mathrm{F}}\left(\mathbf{r}, \mathbf{r}^{\prime}\right)$ puede encontrarse en la ecuación (C.18) del Apéndice $\mathrm{C}$. A lo largo de esta sección vamos a considerar que $m$ representa un modo del puerto $(\gamma)$ y que $\left(m_{x_{\gamma}}, m_{y_{\gamma}}\right)$ son los correspondientes índices modales asociados a las coordenadas $x_{\gamma} \mathrm{e}$ $y_{\gamma}$, respectivamente. Igualmente, asumiremos que $n$ es un modo del puerto $(\xi)$ de índices modales $\left(n_{x_{\xi}}, n_{y_{\xi}}\right)$ relativos a las coordenadas transversales $x_{\xi}$ e $y_{\xi}$, respectivamente.

En primer lugar, se evaluará la siguiente integral de superficie en la que interviene la parte estática de la función de Green diádica de tipo magnético $\overline{\mathbf{G}}_{0}^{\mathrm{F}}\left(\mathbf{r}, \mathbf{r}^{\prime}\right)$ :

$$
\boldsymbol{\Gamma}_{n}^{(\xi)}(\mathbf{r})=\int_{S^{\prime}} \overline{\mathbf{G}}_{0}^{\mathrm{F}}\left(\mathbf{r}, \mathbf{r}^{\prime}\right) \cdot \mathbf{h}_{n}^{(\xi)}\left(\mathbf{r}^{\prime}\right) d S^{\prime}=\Gamma_{x_{\xi}, n}^{(\xi)} \hat{\mathbf{x}}_{\xi}+\Gamma_{y_{\xi}, n}^{(\xi)} \hat{\mathbf{y}}_{\xi}+\Gamma_{z_{\xi}, n}^{(\xi)} \hat{\mathbf{z}}_{\xi}
$$

Las tres componentes vectoriales de $\Gamma_{n}^{(\xi)}(\mathbf{r})$ se pueden expresar en función de las tres componentes de $\mathbf{h}_{n}^{(\xi)}\left(\mathbf{r}^{\prime}\right)$ y de las nueve componentes de la diádica $\overline{\mathbf{G}}_{0}^{\mathrm{F}}\left(\mathbf{r}, \mathbf{r}^{\prime}\right)$ :

$$
\begin{aligned}
\Gamma_{x_{\xi}, n}^{(\xi)} & =\int_{S^{\prime}}\left(h_{x_{\xi}, n}^{(\xi)} G_{0, x_{\xi} x_{\xi}}^{\mathrm{F}}+h_{y_{\xi}, n}^{(\xi)} G_{0, x_{\xi} y_{\xi}}^{\mathrm{F}}+h_{z_{\xi}, n}^{(\xi)} G_{0, x_{\xi} z_{\xi}}^{\mathrm{F}}\right) d S^{\prime} \\
\Gamma_{y_{\xi}, n}^{(\xi)} & =\int_{S^{\prime}}\left(h_{x_{\xi}, n}^{(\xi)} G_{0, y_{\xi} x_{\xi}}^{\mathrm{F}}+h_{y_{\xi}, n}^{(\xi)} G_{0, y_{\xi} y_{\xi}}^{\mathrm{F}}+h_{z_{\xi}, n}^{(\xi)} G_{0, y_{\xi} z_{\xi}}^{\mathrm{F}}\right) d S^{\prime} \\
\Gamma_{z_{\xi}, n}^{(\xi)} & =\int_{S^{\prime}}\left(h_{x_{\xi}, n}^{(\xi)} G_{0, z_{\xi} x_{\xi}}^{\mathrm{F}}+h_{y_{\xi}, n}^{(\xi)} G_{0, z_{\xi} y_{\xi}}^{\mathrm{F}}+h_{z_{\xi}, n}^{(\xi)} G_{0, z_{\xi} z_{\xi}}^{\mathrm{F}}\right) d S^{\prime}
\end{aligned}
$$


Teniendo en cuenta las expresiones de las funciones vectoriales modales asociadas a los puertos de acceso rectangulares (véase Apéndice C), así como la de la diádica de tipo magnético, es posible concluir que en el caso de que $n$ sea un modo de tipo $\mathrm{TE}^{z_{\xi}}$ tendremos:

$$
\begin{aligned}
\Gamma_{x_{\xi}, n_{\mathrm{TE}}}^{(\xi)} & =\nu_{\xi} \frac{2}{l_{z_{\xi}}} N_{x_{\xi}, n}^{\mathrm{TE}} \sin \left(k_{x_{\xi}, n} x_{\xi}\right) \cos \left(k_{y_{\xi}, n} y_{\xi}\right) \Theta_{n_{\mathrm{TE}}}^{(\xi)}\left(z_{\xi}\right) \\
\Gamma_{y_{\xi}, n_{\mathrm{TE}}}^{(\xi)} & =\nu_{\xi} \frac{2}{l_{z_{\xi}}} N_{y_{\xi}, n}^{\mathrm{TE}} \cos \left(k_{x_{\xi}, n} x_{\xi}\right) \sin \left(k_{y_{\xi}, n} y_{\xi}\right) \Theta_{n_{\mathrm{TE}}}^{(\xi)}\left(z_{\xi}\right) \\
\Gamma_{z_{\xi}, n_{\mathrm{TE}}}^{(\xi) \mathrm{TE}} & =-\nu_{\xi} \frac{2}{l_{z_{\xi}}} N_{x_{\xi}, n}^{\mathrm{TE}} \frac{\left(k_{t, n}^{(\xi)}\right)^{2}}{k_{x_{\xi}, n}} \cos \left(k_{x_{\xi}, n} x_{\xi}\right) \cos \left(k_{y_{\xi}, n} y_{\xi}\right) \Phi_{n_{\mathrm{TE}}}^{(\xi)}\left(z_{\xi}\right)
\end{aligned}
$$

Por otro lado, en caso de que $n$ sea un modo de tipo $\mathrm{TM}^{z_{\xi}}$ se tiene que:

$$
\begin{aligned}
\Gamma_{x_{\xi}, n_{\mathrm{TM}}}^{(\xi)} & =\nu_{\xi} \frac{1}{l_{z_{\xi}}} N_{x_{\xi}, n}^{\mathrm{TM}} \sin \left(k_{x_{\xi}, n} x_{\xi}\right) \cos \left(k_{y_{\xi}, n} y_{\xi}\right) \Theta_{n_{\mathrm{TM}}}^{(\xi)}\left(z_{\xi}\right) \\
\Gamma_{y_{\xi}, n_{\mathrm{TM}}}^{(\xi)} & =-\nu_{\xi} \frac{1}{l_{z_{\xi}}} N_{y_{\xi}, n}^{\mathrm{TM}} \cos \left(k_{x_{\xi}, n} x_{\xi}\right) \sin \left(k_{y_{\xi}, n} y_{\xi}\right) \Theta_{n_{\mathrm{TM}}}^{(\xi)}\left(z_{\xi}\right) \\
\Gamma_{z_{\xi}, n_{\mathrm{TM}}}^{(\xi)} & =0
\end{aligned}
$$

Además, en las expresiones (3.36)-(3.41) se ha hecho uso de las siguientes series infinitas:

$$
\begin{aligned}
& \Theta_{n_{\mathrm{TE}}}^{(\xi)}\left(z_{\xi}\right)=\sum_{n_{z_{\xi}}=1}^{\infty} \frac{k_{z_{\xi}}^{2}}{\left(\left(k_{t, n}^{(\xi)}\right)^{2}+k_{z_{\xi}}^{2}\right)^{2}} \cos \left(k_{z_{\xi}} z_{\xi}\right) \nu_{\xi}^{n_{z_{\xi}}} \\
& \Theta_{n_{\mathrm{TM}}}^{(\xi)}\left(z_{\xi}\right)=\sum_{n_{z_{\xi}}=0}^{\infty} \frac{\epsilon_{n_{z_{\xi}}}}{\left(k_{t, n}^{(\xi)}\right)^{2}+k_{z_{\xi}}^{2}} \cos \left(k_{z_{\xi}} z_{\xi}\right) \nu_{\xi}^{n_{z_{\xi}}} \\
& \Phi_{n_{\mathrm{TE}}}^{(\xi)}\left(z_{\xi}\right)=\sum_{n_{z_{\xi}}=1}^{\infty} \frac{k_{z_{\xi}}}{\left(\left(k_{t, n}^{(\xi)}\right)^{2}+k_{z_{\xi}}^{2}\right)^{2}} \sin \left(k_{z_{\xi}} z_{\xi}\right) \nu_{\xi}^{n_{z_{\xi}}}
\end{aligned}
$$

siendo $k_{z_{\xi}}=n_{z_{\xi}} \pi / l_{z_{\xi}}$. En consecuencia, la matriz $T_{m n}^{(\gamma, \xi)}$ puede calcularse de la siguiente manera:

$$
T_{m n}^{(\gamma, \xi)}=\int_{S} \mathbf{h}_{m}^{(\gamma)}(\mathbf{r}) \cdot \Gamma_{n}^{(\xi)}(\mathbf{r}) d S=\int_{S}\left(h_{x_{\gamma}, m}^{(\gamma)} \Gamma_{x_{\xi}, n}^{(\xi)}+h_{y_{\gamma}, m}^{(\gamma)} \Gamma_{y_{\xi}, n}^{(\xi)}+h_{z_{\gamma}, m}^{(\gamma)} \Gamma_{z_{\xi}, n}^{(\xi)}\right) d S
$$

donde conviene hacer notar que el integrando de la expresión anterior está formado por funciones de variación sinusoidal.

Para poder evaluar la integral anterior, el integrando de la misma debe expresarse utilizando un sistema de coordenadas único. Por ello, el primer paso consiste en reescribir 
la expresión de la función vectorial modal $\mathbf{h}_{m}^{(\gamma)}(\mathbf{r})$ en términos del sistema de referencia $\left(x_{\xi}, y_{\xi}, z_{\xi}\right)$. Además, es importante tener en cuenta que la integral anterior se calcula en la sección transversal rectangular del puerto $(\gamma)$, con lo que la situación de este puerto fijará la condición $\vartheta_{\xi}=$ cte, $\operatorname{con} \vartheta=(x, y, z)$. Así pues, fácilmente se concluye que cada una de las integrales de superficie anteriores se transformará en un producto entre dos integrales unidimensionales cuyos integrandos serán de variación sinusoidal. En concreto, las integrales unidimensionales serán de dos tipos. El primero de ellos es el mismo que se indicó en (3.27), mientras que el segundo tipo de integral es el siguiente:

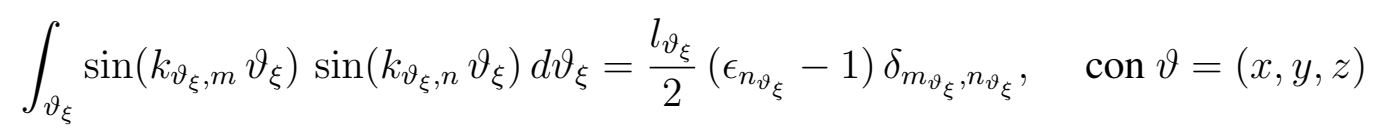

donde la explicación de los símbolos es la misma que se indicó con la ecuación (3.27). Por otro lado, en el cálculo de (3.45) aparecen diversas series infinitas cuya solución se detalla nuevamente en la sección D.1 del Apéndice D.

A continuación, presentamos los resultados para los bloques de la matriz que relacionan puertos de accesos paralelos, es decir, los elementos $T_{m n}^{(\xi, \xi)} \operatorname{con} \xi=1,2, \ldots, 5$ y los elementos $T_{m n}^{(\xi, \xi+1)} \operatorname{con} \xi=1,3$. Además, a partir de ahora, se tiene en cuenta que los modos $m$ y $n$ pueden ser de tipo TE o de tipo TM:

$$
\begin{aligned}
& T_{m_{\mathrm{TE}}, n_{\mathrm{TE}}}^{(\xi, \xi)}=\frac{1}{2 k_{t, n}^{(\xi)}}\left[\operatorname{coth}\left(k_{t, n}^{(\xi)} l_{z_{\xi}}\right)-\frac{k_{t, n}^{(\xi)} l_{z_{\xi}}}{\sinh ^{2}\left(k_{t, n}^{(\xi)} l_{z_{\xi}}\right)}\right] \delta_{m, n} \\
& T_{m_{\mathrm{TE}}, n_{\mathrm{TE}}}^{(\xi, \xi+1)}=-\frac{1}{2 k_{t, n}^{(\xi)}} \frac{1}{\sinh \left(k_{t, n}^{(\xi)} l_{z_{\xi}}\right)}\left(1-\frac{k_{t, n}^{(\xi)} l_{z_{\xi}}}{\tanh \left(k_{t, n}^{(\xi)} l_{z_{\xi}}\right)}\right) \delta_{m, n} \\
& T_{m_{\mathrm{TM}}, n_{\mathrm{TE}}}^{(\xi, \xi)}=T_{m_{\mathrm{TE}}, n_{\mathrm{TM}}}^{(\xi, \xi)}=T_{m_{\mathrm{TM}}, n_{\mathrm{TE}}}^{(\xi, \xi+1)}=T_{m_{\mathrm{TE}}, n_{\mathrm{TM}}}^{(\xi, \xi+1)}=0 \\
& T_{m_{\mathrm{TM}}, n_{\mathrm{TM}}}^{(\xi, \xi)}=\frac{1}{k_{t, n}^{(\xi)}} \operatorname{coth}\left(k_{t, n}^{(\xi)} l_{z_{\xi}}\right) \delta_{m, n} \\
& T_{m_{\mathrm{TM}}, n_{\mathrm{TM}}}^{(\xi, \xi+1)}=-\frac{1}{k_{t, n}^{(\xi)}} \frac{1}{\sinh \left(k_{t, n}^{(\xi)} l_{z_{\xi}}\right)} \delta_{m, n}
\end{aligned}
$$

Finalmente, se presentan los elementos de la matriz que relacionan puertos de acceso 
ortogonales, es decir, los elementos $T_{m n}^{(\gamma, \delta)} \operatorname{con} \gamma=1,2$ y $\xi=3,4,5$; o con $\gamma=3,4$ y $\xi=5$ :

$$
\begin{aligned}
& T_{m, \mathrm{TE}, n_{\mathrm{TE}}}^{(\gamma, \xi)}=\nu_{\gamma} \nu_{\xi} \frac{k_{m_{x_{\gamma}}}^{2}}{k_{t, m}^{(\gamma)}} \frac{\rho_{m, n}^{(\gamma, \xi)} \psi_{m, n}^{(\gamma, \xi)}}{\left(\left(k_{t, n}^{(\xi)}\right)^{2}+k_{m_{z_{\xi}}}^{2}\right)^{2}} \frac{1}{\sqrt{l_{z_{\gamma}} l_{z_{\xi}}}}\left[\frac{1}{k_{t, n}^{(\xi)}} \chi_{m, n}^{(\gamma, \xi)}-k_{t, n}^{(\xi)} \varphi_{m, n}^{(\gamma, \xi)}\right] \\
& T_{m_{\mathrm{TM}}, n_{\mathrm{TE}}}^{(\gamma, \xi)}=-\nu_{\gamma} \nu_{\xi} \frac{k_{m_{x_{\gamma}}}}{k_{t, m}^{(\gamma)}} \frac{\zeta_{m, n}^{(\gamma, \xi)} \tau^{(\gamma, \xi)}}{\left(\left(k_{t, n}^{(\xi)}\right)^{2}+k_{m_{z_{\xi}}}^{2}\right)^{2}} \sqrt{\frac{2}{l_{z_{\gamma}} l_{z_{\xi}}}}\left[k_{t, n}^{(\xi)}+\frac{k_{m_{x_{\gamma}}}^{2}}{k_{t, n}^{(\xi)}}\right] \psi_{m, n}^{(\gamma, \xi)} \\
& T_{m_{\mathrm{TE}}, n_{\mathrm{TM}}}^{(\gamma, \xi)}=-\frac{k_{n_{x_{\xi}}} k_{n_{y_{\xi}}}}{k_{t, m}^{(\gamma)} k_{t, n}^{(\xi)}} \frac{\nu_{\gamma} \nu_{\xi} \theta_{m, n}^{(\gamma, \xi)}}{\left(k_{t, n}^{(\xi)}\right)^{2}+k_{m_{z_{\xi}}}^{2}} \sqrt{\frac{2}{l_{z_{\gamma}} l_{z_{\xi}}}} \psi_{m, n}^{(\gamma, \xi)} \\
& T_{m_{\mathrm{TM}}, n_{\mathrm{TM}}}^{(\gamma, \xi)}=\frac{k_{m_{x_{\gamma}}}}{k_{t, m}^{(\gamma)} k_{t, n}^{(\xi)}} \frac{\nu_{\gamma} \nu_{\xi} \lambda_{m, n}^{(\gamma, \xi)}}{\left(k_{t, n}^{(\xi)}\right)^{2}+k_{m_{z_{\xi}}}^{2}} \frac{2}{\sqrt{l_{z_{\gamma}} l_{z_{\xi}}}} \tau^{(\gamma, \xi)} \psi_{m, n}^{(\gamma, \xi)}
\end{aligned}
$$

donde las nuevas funciones auxiliares $\chi_{m, n}^{(\gamma, \xi)}, \varphi_{m, n}^{(\gamma, \xi)}, \zeta_{m, n}^{(\gamma, \xi)}, \tau^{(\gamma, \xi)}, \theta_{m, n}^{(\gamma, \xi)}$ y $\lambda_{m, n}^{(\gamma, \xi)}$ se han definido en la sección D.2 del Apéndice D. Para concluir, es importante observar que la matriz $T_{m n}^{(\gamma, \xi)}$ es también simétrica. Por ello, sólo se han proporcionado las expresiones para el caso $\xi>\gamma$ (triángulo superior de la matriz).

\section{Cálculo de los elementos de la matriz $L_{m n}^{(\xi)}$}

La matriz $L_{m n}^{(\xi)}$ se definió de la siguiente manera:

$$
L_{m n}^{(\xi)}=\int_{S} \int_{S^{\prime}} \mathbf{N}_{m}(\mathbf{r}) \cdot \nabla \times \overline{\mathbf{G}}_{0}^{\mathrm{F}}\left(\mathbf{r}, \mathbf{r}^{\prime}\right) \cdot \mathbf{h}_{n}^{(\xi)}\left(\mathbf{r}^{\prime}\right) d S d S^{\prime}
$$

donde la expresión del rotacional de la función diádica de Green de tipo magnético se recoge en la ecuación (C.21) del Apéndice C. La resolución de la integral anterior comienza con el cálculo analítico de la integral de superficie ligada a las variables primadas. De esta forma, definimos en primer lugar:

$$
\Psi_{n}^{(\xi)}(\mathbf{r})=\int_{S^{\prime}} \nabla \times \overline{\mathbf{G}}_{0}^{\mathrm{F}}\left(\mathbf{r}, \mathbf{r}^{\prime}\right) \cdot \mathbf{h}_{n}^{(\xi)}\left(\mathbf{r}^{\prime}\right) d S^{\prime}
$$

Si ahora se sustituyen las ecuaciones (C.19)-(C.20) y (C.22)-(C.23) en (C.21) con el fin de obtener la expresión del rotacional de la función diádica de Green de tipo magnético, y se tiene también en cuenta la función vectorial modal de campo magnético asociada a los puertos de acceso, podemos obtener el siguiente resultado expresado en función de que $n$ 
sea un modo de tipo $\mathrm{TE}^{z_{\xi}}$ o de tipo $\mathrm{TM}^{z_{\xi}}$ :

$$
\begin{aligned}
& \Psi_{n_{\mathrm{TE}}}^{(\xi)}(\mathbf{r})=\frac{\epsilon_{n_{x_{\xi}}} \epsilon_{n_{y_{\xi}}}}{\sqrt{l_{x_{\xi}} l_{y_{\xi}}}} \frac{1}{k_{t, n}^{(\xi)}} \frac{\sinh \left(k_{t, n}^{(\xi)} \Upsilon^{(\xi)}\left(z_{\xi}\right)\right)}{\sinh \left(k_{t, n}^{(\xi)} l_{z_{\xi}}\right)}\left\{k_{y_{\xi}, n} \cos \left(k_{x_{\xi}, n} x_{\xi}\right) \sin \left(k_{y_{\xi}, n} y_{\xi}\right) \hat{\mathbf{x}}_{\xi}\right. \\
& \left.-k_{x_{\xi}, n} \sin \left(k_{x_{\xi}, n} x_{\xi}\right) \cos \left(k_{y_{\xi}, n} y_{\xi}\right) \hat{\mathbf{y}}_{\xi}\right\} \\
& \Psi_{n_{\mathrm{TM}}}^{(\xi)}(\mathbf{r})=-\frac{2}{\sqrt{l_{x_{\xi}} l_{y_{\xi}}}} \frac{1}{k_{t, n}^{(\xi)}} \frac{\sinh \left(k_{t, n}^{(\xi)} \Upsilon^{(\xi)}\left(z_{\xi}\right)\right)}{\sinh \left(k_{t, n}^{(\xi)} l_{z_{\xi}}\right)}\left\{k_{x_{\xi}, n} \cos \left(k_{x_{\xi}, n} x_{\xi}\right) \sin \left(k_{y_{\xi}, n} y_{\xi}\right) \hat{\mathbf{x}}_{\xi}\right. \\
& \left.+k_{y_{\xi}, n} \sin \left(k_{x_{\xi}, n} x_{\xi}\right) \cos \left(k_{y_{\xi}, n} y_{\xi}\right) \hat{\mathbf{y}}_{\xi}\right\} \\
& +\nu_{\xi} \frac{2}{\sqrt{l_{x_{\xi}} l_{y_{\xi}}}} \sin \left(k_{x_{\xi}, n} x_{\xi}\right) \sin \left(k_{y_{\xi}, n} y_{\xi}\right) \frac{\cosh \left(k_{t, n}^{(\xi)} \Upsilon^{(\xi)}\left(z_{\xi}\right)\right)}{\sinh \left(k_{t, n}^{(\xi)} l_{z_{\xi}}\right)} \hat{\mathbf{z}}_{\xi}
\end{aligned}
$$

donde hemos definido la siguiente función auxiliar:

$$
\Upsilon^{(\xi)}\left(z_{\xi}\right)=\left\{\begin{array}{l}
l_{z_{\xi}}-z_{\xi}, \text { si } \xi=1,3,5 \\
z_{\xi}, \text { si } \xi=2,4
\end{array}\right.
$$

De esta manera, los elementos de la matriz $L_{m n}^{(\xi)}$ pueden calcularse finalmente como:

$$
L_{m n}^{(\xi)}=\int_{S} \mathbf{N}_{m}(\mathbf{r}) \cdot \Psi_{n}^{(\xi)}(\mathbf{r}) d S
$$

Esta integral se debe resolver de forma numérica, ya que las funciones base $\mathbf{N}_{m}(\mathbf{r})$ utilizadas en el simulador para describir la densidad de corriente eléctrica existente sobre el poste conductor están definidas por segmentos y, en consecuencia, no existe solución analítica para dicha integral (ver detalles en [66], [67]).

\section{Cálculo de los elementos de la matriz $F_{m n}^{(\xi)}$}

La matriz $F_{m n}^{(\xi)}$ se calcula del siguiente modo:

$$
F_{m n}^{(\xi)}=\int_{S} \mathbf{H}_{m}(\mathbf{r}) \cdot \mathbf{h}_{n}^{(\xi)}(\mathbf{r}) d S
$$

donde $\mathbf{H}_{m}(\mathbf{r})$ representa el campo magnético asociado al $m$-ésimo modo resonante de una cavidad rectangular. Para obtener la expresión de los elementos de la matriz $F_{m n}^{(\xi)}$ debemos escoger un sistema de referencia de trabajo y realizar todos los cálculos con respecto a dicho 
sistema. En este sentido, se ha optado por fijar el sistema de coordenadas $\left(x_{1}, y_{1}, z_{1}\right)$ representado en la figura 3.2, por lo que la expresión de cada función vectorial modal $\mathbf{h}_{n}^{(\xi)}(\mathbf{r})$ debe reescribirse en función de dicho sistema de referencia.

Así pues, consideremos a continuación que $\left(m_{x_{1}}, m_{y_{1}}, m_{z_{1}}\right)$ representan los índices modales relativos al $m$-ésimo modo resonante de la cavidad rectangular, correspondientes a las coordenadas $\left(x_{1}, y_{1}, z_{1}\right)$, respectivamente; y que $\left(n_{x_{\xi}}, n_{y_{\xi}}\right)$ son los índices modales del $n$-ésimo modo del puerto $(\xi)$, correspondientes a las coordenadas $x_{\xi}$ e $y_{\xi}$, respectivamente. Utilizando ahora (C.19) y (C.20) del Apéndice C, que recogen las expresiones del campo magnético de tipo $\mathrm{TE}^{z_{\xi}}$ y $\mathrm{TM}^{z_{\xi}}$ de una cavidad rectangular, es posible concluir que para el caso $\xi=1,2$ :

$$
\begin{aligned}
F_{m_{\mathrm{TE}}, n_{\mathrm{TE}}}^{(\xi)} & =\nu_{\xi} \sqrt{\frac{2}{l_{z_{1}}}} \frac{k_{z_{1}, m}}{\kappa_{m}} \nu_{\xi}^{m_{z_{1}}} \delta_{m, n} \\
F_{m_{\mathrm{TM}}, n_{\mathrm{TE}}}^{(\xi)} & =F_{m_{\mathrm{TE}}, n_{\mathrm{TM}}}^{(\xi)}=0 \\
F_{m_{\mathrm{TM}}, n_{\mathrm{TM}}}^{(\xi)} & =\nu_{\xi} \sqrt{\frac{\epsilon_{m_{z_{1}}}}{l_{z_{1}}}} \nu_{\xi}^{m_{z_{1}}} \delta_{m, n}
\end{aligned}
$$

donde $\kappa_{m}$ representa el $m$-ésimo número de onda de resonancia de la cavidad rectangular (véase su definición en (C.15) del Apéndice C); y $k_{z_{1}, m}$ es el número de onda de la cavidad rectangular relativo a la coordenada $z_{1}$ asociado con el $m$-ésimo modo resonante (consultar su definición en (C.16) del Apéndice C). Por otro lado, si $\xi=3,4$ se obtiene fácilmente que:

$$
\begin{aligned}
F_{m_{\mathrm{TE}}, n_{\mathrm{TE}}}^{(\xi)}= & \nu_{\xi} \frac{1}{\sqrt{2 l_{x_{1}}}} \epsilon_{n_{y_{\xi}}} \sqrt{\epsilon_{n_{x_{\xi}}}} \epsilon_{m_{x_{1}}} \frac{k_{x_{\xi}, n}}{k_{t, n}^{(\xi)} \kappa_{m}}\left[\frac{k_{y_{\xi}, n}^{2}}{k_{t, m}} \frac{\epsilon_{n_{y_{\xi}}}-1}{\epsilon_{n_{x_{\xi}}}}\right. \\
& \left.-k_{t, m} \frac{\epsilon_{n_{x_{\xi}}}-1}{\epsilon_{n_{y_{\xi}}}}\right](-1)^{n_{x_{\xi}}} \nu_{\xi}^{m_{x_{1}}} \delta_{m_{y_{1}}, n_{y_{\xi}}} \delta_{m_{z_{1}}, n_{x_{\xi}}} \\
F_{m_{\mathrm{TM}}, n_{\mathrm{TE}}}^{(\xi)}= & -\nu_{\xi} \sqrt{\frac{\epsilon_{n_{y_{\xi}}}}{l_{x_{1}}}}\left(\epsilon_{n_{y_{\xi}}}-1\right) \frac{k_{y_{\xi}, n} k_{x_{1}, m}}{k_{t, n}^{(\xi)} k_{t, m}}(-1)^{n_{x_{\xi}}} \nu_{\xi}^{m_{x_{1}}} \delta_{m_{y_{1}}, n_{y_{\xi}}} \delta_{m_{z_{1}}, n_{x_{\xi}}} \\
F_{m_{\mathrm{TE}}, n_{\mathrm{TM}}}^{(\xi)}= & -\nu_{\xi} \sqrt{\frac{\epsilon_{m_{x_{1}}}}{l_{x_{1}}}} \frac{k_{y_{\xi}, n}}{k_{t, n}^{(\xi)} \kappa_{m}}\left[k_{t, m}+\frac{k_{x_{\xi}, n}^{2}}{k_{t, m}}\right] \\
& \cdot(-1)^{n_{x_{\xi}}} \nu_{\xi}^{m_{x_{1}}} \delta_{m_{y_{1}}, n_{y_{\xi}}} \delta_{m_{z_{1}}, n_{x_{\xi}}} \\
F_{m_{\mathrm{TM}}, n_{\mathrm{TM}}}^{(\xi)}= & \nu_{\xi} \sqrt{\frac{2}{l_{x_{1}}}} \frac{k_{x_{1}, m} k_{x_{\xi}, n}}{k_{t, n}^{(\xi)} k_{t, m}}(-1)^{n_{x_{\xi}}} \nu_{\xi}^{m_{x_{1}}} \delta_{m_{y_{1}}, n_{y_{\xi}}} \delta_{m_{z_{1}}, n_{x_{\xi}}}
\end{aligned}
$$


Finalmente, si $\xi=5$ se tiene que:

$$
\begin{aligned}
F_{m_{\mathrm{TE}}, n_{\mathrm{TE}}}^{(\xi)}= & \frac{1}{\sqrt{2 l_{y_{1}}}} \epsilon_{n_{y_{\xi}}} \sqrt{\epsilon_{n_{x_{\xi}}}} \epsilon_{m_{y_{1}}} \frac{k_{x_{\xi}, n}}{k_{t, n}^{(\xi)} \kappa_{m}}\left[\frac{k_{y_{\xi}, n}^{2}}{k_{t, m}} \frac{\epsilon_{n_{y_{\xi}}}-1}{\epsilon_{n_{x_{\xi}}}}\right. \\
& \left.-k_{t, m} \frac{\epsilon_{n_{x_{\xi}}}-1}{\epsilon_{n_{y_{\xi}}}}\right](-1)^{n_{x_{\xi}}}(-1)^{m_{y_{1}}} \delta_{m_{z_{1}}, n_{x_{\xi}}} \delta_{m_{x_{1}}, n_{y_{\xi}}} \\
F_{m_{\mathrm{TM}}, n_{\mathrm{TE}}}^{(\xi)}= & \sqrt{\frac{\epsilon_{n_{y_{\xi}}}}{l_{y_{1}}}\left(\epsilon_{n_{y_{\xi}}}-1\right) \frac{k_{y_{\xi}, n} k_{y_{1}, m}}{k_{t, n}^{(\xi)} k_{t, m}}(-1)^{n_{x_{\xi}}}(-1)^{m_{y_{1}}} \delta_{m_{z_{1}}, n_{x_{\xi}}} \delta_{m_{x_{1}}, n_{y_{\xi}}}} \\
F_{m_{\mathrm{TE}}, n_{\mathrm{TM}}}^{(\xi)}= & -\sqrt{\frac{\epsilon_{m_{y_{1}}}}{l_{y_{1}}}} \frac{k_{y_{\xi}, n}}{k_{t, n}^{(\xi)} \kappa_{m}}\left[k_{t, m}+\frac{k_{x_{\xi}, n}^{2}}{k_{t, m}}\right] \\
& \cdot(-1)^{n_{x_{\xi}}}(-1)^{m_{y_{1}}} \delta_{m_{z_{1}}, n_{x_{\xi}}} \delta_{m_{x_{1}}, n_{y_{\xi}}} \\
F_{m_{\mathrm{TM}}, n_{\mathrm{TM}}}^{(\xi)}= & -\sqrt{\frac{2}{l_{y_{1}}}} \frac{k_{y_{1}, m} k_{x_{\xi}, n}}{k_{t, n}^{(\xi)} k_{t, m}}(-1)^{n_{x_{\xi}}}(-1)^{m_{y_{1}}} \delta_{m_{z_{1}}, n_{x_{\xi}}} \delta_{m_{x_{1}}, n_{y_{\xi}}}
\end{aligned}
$$

En las expresiones anteriores $k_{t, m}=\sqrt{k_{x_{1}, m}^{2}+k_{y_{1}, m}^{2}}$ representa el número de onda transversal del $m$-ésimo modo resonante de la cavidad rectangular.

\subsection{Ejemplos de Aplicación}

El objetivo de esta sección es validar la teoría desarrollada en los apartados anteriores presentando diferentes ejemplos de aplicación. Así pues, se pretende analizar y diseñar diferentes uniones multi-puerto en guía rectangular compensadas con postes conductores cilíndricos de altura variable. A este respecto, se introducirá una nueva variable de diseño que, hasta la fecha, no se ha tenido en cuenta en la literatura técnica dedicada a la investigación de uniones compensadas. Este nuevo parámetro de diseño será la posición relativa que ocupa el poste conductor en la estructura, y se utilizará para optimizar la respuesta en frecuencia de las diferentes uniones consideradas. En esta sección se analizarán y diseñarán los siguientes tipos de uniones multi-puerto compensadas (véase la figura 3.1): codos rectos en plano $H$ y en plano $E$ (uniones de dos puertos), uniones en $\mathrm{T}$ en plano $H$ y en plano $E$ (uniones de tres puertos), uniones T mágica (unión de cuatro puertos) y uniones turnstile (unión de cinco puertos).

Los resultados obtenidos con la herramienta de simulación implementada se compararán, bien con datos numéricos extraídos de la literatura técnica, o bien con los resultados proporcionados por el programa HFSS. Además, todos los resultados que se presentan en este apartado se han obtenido utilizando un ordenador PC Pentium IV@2.55 GHz con 512 MB 
de memoria RAM.

Por otro lado, con el objetivo de asegurar la convergencia de los resultados presentados, se han considerado 25 modos en cada uno de los puertos de acceso de la estructura bajo análisis, 300 modos en el resonador rectangular externo y 250 funciones base en la descripción de la densidad de corriente eléctrica sobre el poste conductor. Los modos de excitación y de respuesta considerados en los diferentes ejemplos de validación presentados se corresponden con los modos fundamentales de cada tipo de guía.

\subsubsection{Codos rectos en plano $H$ y en plano $E$}

A continuación, se presenta el análisis y diseño de codos rectos en plano $H$ y en plano $E$ compensados con postes conductores cilíndricos de altura variable (véase las figuras 3.1a y 3.1b). Partiendo de la geometría básica bajo análisis de la figura 3.2, obsérvese que el codo recto en plano $H$ se obtiene, por ejemplo, abriendo los puertos (1) y (4). En primer lugar, en la figura 3.4 se representan las pérdidas de retorno de un codo recto en plano $H$ implementado en la guía rectangular WR-90 ( $a=22,90 \mathrm{~mm}, b=10,20 \mathrm{~mm})$. El codo se ha compensado de forma óptima con un poste conductor cilíndrico de radio $r=0,5 \mathrm{~mm}$ y altura $h=9,0 \mathrm{~mm}$ situado en una posición descentrada. Con respecto al sistema de referencia de la figura 3.2, las coordenadas del centro de la base del poste conductor óptimo son $x_{0}=9,45 \mathrm{~mm}$ y $z_{0}=17,45 \mathrm{~mm}$ (obsérvese que el caso centrado se correspondería con $x_{0}=z_{0}=a / 2=11,45 \mathrm{~mm}$ ). Además, en la figura 3.4 se han representado los resultados para el caso en el que el codo se compensa con el mismo poste conductor situado en una posición centrada, y también para el caso de un codo sin compensar. Todos los resultados se han comparado satisfactoriamente con los datos proporcionados por HFSS.

Del análisis de los resultados obtenidos se desprende que la respuesta en frecuencia óptima se consigue para el caso en el que el poste está descentrado. Además, la respuesta del codo compensado con un poste situado en una posición centrada apenas mejora la del caso sin compensar. En consecuencia, la posición relativa del poste en esta estructura desempeña un papel fundamental en la optimización de su respuesta en frecuencia.

Con el propósito de poder apreciar la influencia que tiene la posición del poste en la respuesta en frecuencia del dispositivo, en la figura 3.5 hemos representado las pérdidas de retorno del codo en plano $H$ para diferentes valores de la posición relativa del poste, manteniendo los valores del radio y de la altura anteriores. En concreto, los valores considerados en la figura son los siguientes: $\left(x_{0,1}, y_{0,1}\right)=(a / 4, a / 4),\left(x_{0,2}, y_{0,2}\right)=(3 a / 4, a / 4)$, $\left(x_{0,3}, y_{0,3}\right)=(a / 4,3 a / 4)$ y $\left(x_{0,4}, y_{0,4}\right)=(3 a / 4,3 a / 4)$ (este último valor proporciona una respuesta en frecuencia idéntica a la del caso $\left(x_{0,1}, y_{0,1}\right)$ y por ello no se ha representado en la figura). Además, en la figura 3.5 se representa también la respuesta en frecuencia del caso óptimo ( $x_{0}=9,45 \mathrm{~mm}$ y $z_{0}=17,45 \mathrm{~mm}$ ). Tal y como podemos apreciar, la posición relativa del poste es una variable muy importante que debe tenerse en cuenta en el proceso de diseño del dispositivo.

Otro estudio interesante es el que se lleva a cabo en la figura 3.6. En dicha figura, se investiga la influencia del radio del poste en la respuesta en frecuencia del dispositivo. En 


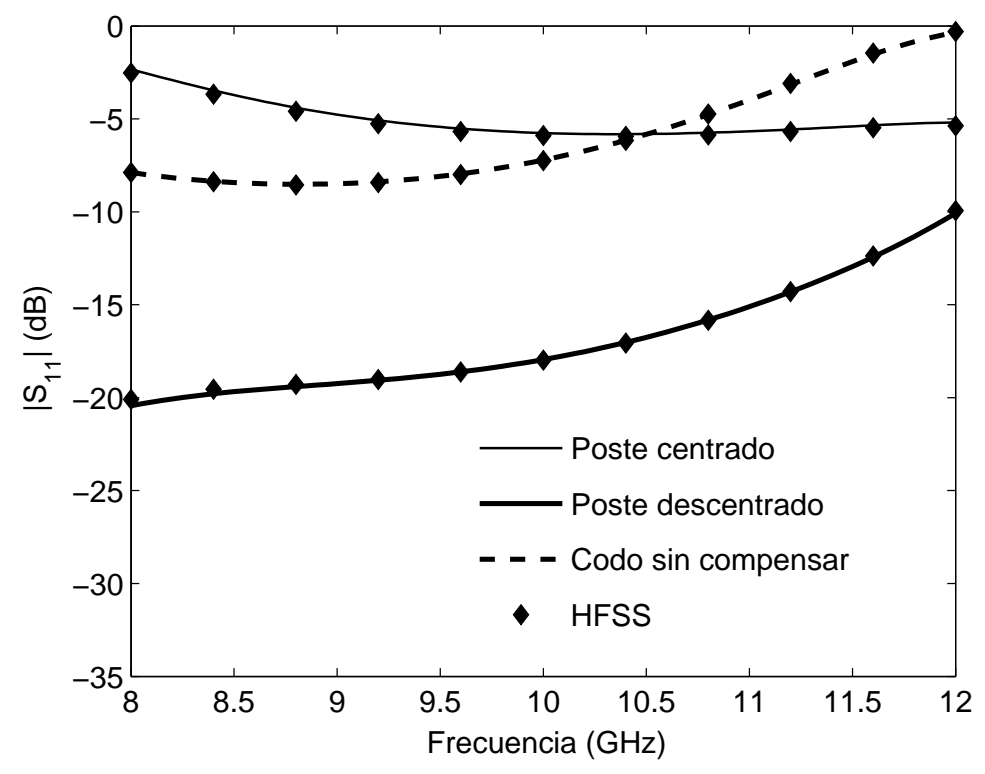

Figura 3.4: Pérdidas de retorno de un codo recto en plano $H$ implementado en guía rectangular WR-90 compensado con un poste conductor cilíndrico $(r=0,5 \mathrm{~mm}, h=9,0 \mathrm{~mm})$. Comparación del caso en el que el poste está en una posición descentrada $\left(x_{0}=9,45 \mathrm{~mm}, z_{0}=17,45 \mathrm{~mm}\right)$ con el caso centrado.

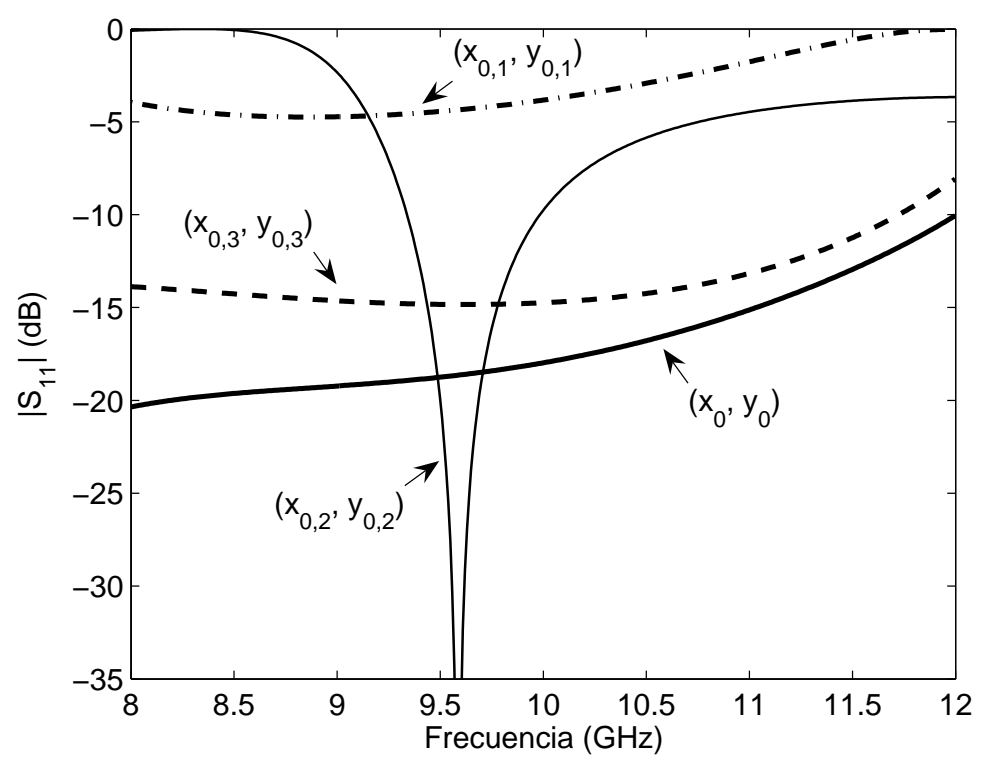

Figura 3.5: Pérdidas de retorno de un codo recto en plano $H$ implementado en guía rectangular WR-90 compensado con un poste conductor cilíndrico $(r=0,5 \mathrm{~mm}, h=9,0 \mathrm{~mm})$. Influencia de la posición relativa del poste en la respuesta en frecuencia de la estructura. 
este estudio se mantiene el poste en la posición descentrada óptima, y se fija la altura a $h=9 \mathrm{~mm}$. En la figura se han calculado las pérdidas de retorno del dispositivo para los siguientes casos: $r=0,3 \mathrm{~mm}, r=0,5 \mathrm{~mm}, r=1,5 \mathrm{~mm}, r=3,0 \mathrm{~mm}$ y $r=4,5 \mathrm{~mm}$. A la vista de los resultados se deduce que al aumentar el radio del poste empeoran las pérdidas de retorno.

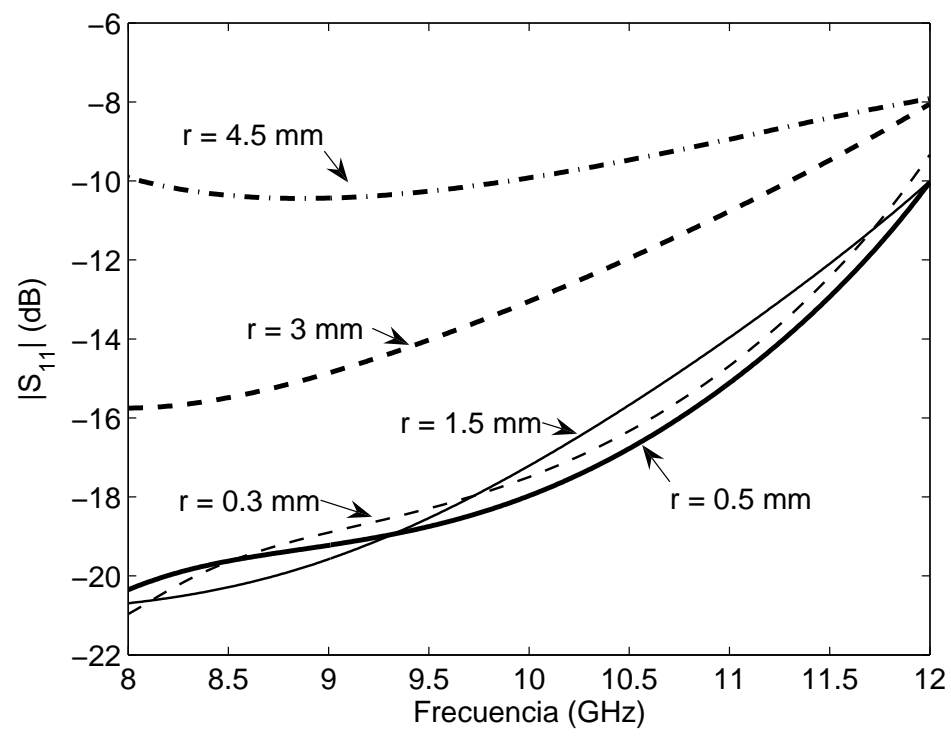

Figura 3.6: Pérdidas de retorno de un codo recto en plano $H$ implementado en guía rectangular WR-90 compensado con un poste conductor cilíndrico $(h=9,0 \mathrm{~mm})$ situado en una posición descentrada $\left(x_{0}=9,45 \mathrm{~mm}, z_{0}=17,45 \mathrm{~mm}\right)$. Influencia del radio del poste en la respuesta en frecuencia de la estructura.

Finalmente, en la figura 3.7 se estudia la influencia de la altura del poste. En este caso, se fija el radio al valor $r=0,5 \mathrm{~mm}$ y se mantiene el poste en la posición descentrada óptima. Los casos estudiados son: $h=1,0 \mathrm{~mm}, h=3,0 \mathrm{~mm}, h=5,0 \mathrm{~mm}, h=9,0 \mathrm{~mm}$ y $h=10,0 \mathrm{~mm}$. Del estudio de los resultados obtenidos es posible concluir que, en el caso de estructuras en plano $H$, los postes de elevada altura y de radios pequeños son los que proporcionan una respuesta en frecuencia óptima. Con respecto a la eficiencia computacional de la herramienta implementada, es importante destacar que todos los resultados presentados se han calculado en 5,17 segundos (101 puntos en frecuencia). En cambio, la simulaciones con HFSS han consumido 15 minutos (11 puntos en frecuencia). Estos datos suponen que el software implementado es, por punto en frecuencia, 1600 veces más rápido que HFSS.

Seguidamente, procedemos al análisis y diseño de codos rectos en plano $E$. Partiendo del bloque básico de la figura 3.2, el codo en plano $E$ puede obtenerse abriendo, por ejemplo, los puertos (2) y (5). Así, en la figura 3.8 se presenta el diseño de un codo en plano $E$ implementado en la guía rectangular WR-90. El codo se ha compensado con un poste conductor cilíndrico de radio $r=3,75 \mathrm{~mm}$ y de altura $h=1,5 \mathrm{~mm}$ situado en una posición descentrada óptima (coordenadas del centro de la base del poste relativas al sistema de referencia 


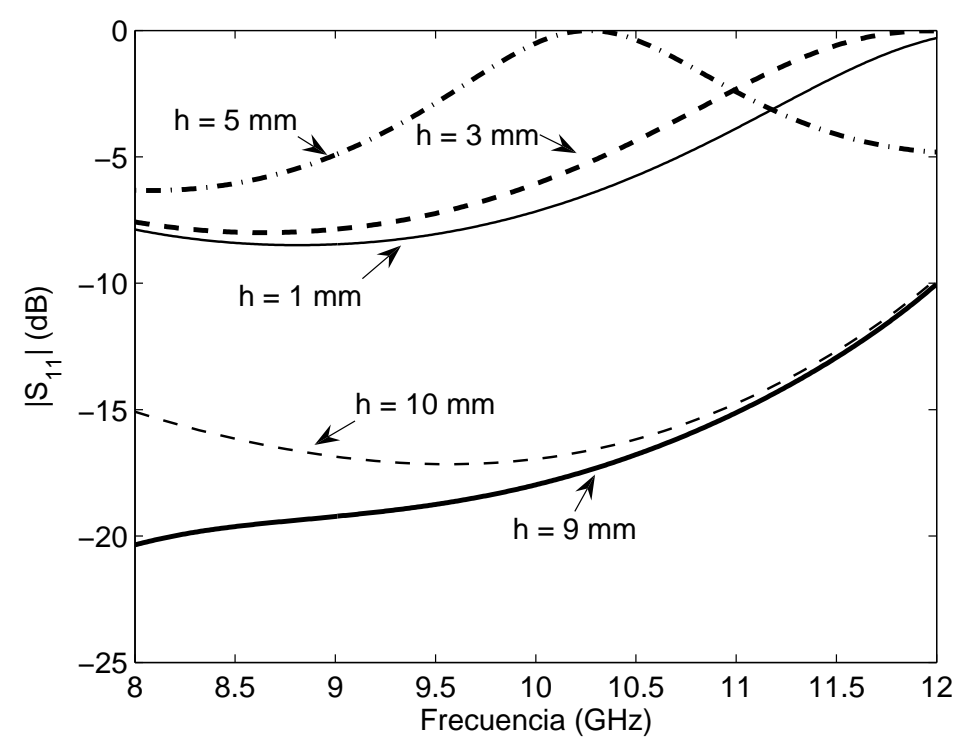

Figura 3.7: Pérdidas de retorno de un codo recto en plano $H$ implementado en guía rectangular WR-90 compensado con un poste conductor cilíndrico $(r=0,5 \mathrm{~mm})$ situado en una posición descentrada $\left(x_{0}=9,45 \mathrm{~mm}, z_{0}=17,45 \mathrm{~mm}\right)$. Influencia de la altura del poste en la respuesta en frecuencia de la estructura.

de la figura 3.2: $x_{0}=11,45 \mathrm{~mm}, z_{0}=4,10 \mathrm{~mm}$ ). Además, en este caso, para minimizar las pérdidas de retorno de la estructura ha sido necesario recurrir a la introducción de un iris de adaptación en el puerto (5) del codo (ver la figura 3.1b). El iris se ha situado a una altura $h_{\text {iris }}=7,0 \mathrm{~mm}$, su longitud es de $l_{\text {iris }}=20,5 \mathrm{~mm}$ y su espesor es de $t_{\text {iris }}=1,5 \mathrm{~mm}^{8}$. La discontinuidad planar que se produce entre la guía rectangular del puerto (5) del codo y el iris introducido se ha caracterizado implementando la técnica de la ecuación integral presentada en el capítulo 2. Por otro lado, hay que recordar que la técnica de la ecuación integral proporciona una matriz de impedancias generalizada, mientras que el método BI-RME 3D origina una matriz de admitancias generalizada. Con el objetivo de que la caracterización del codo en plano $E$ sea eficiente desde un punto de vista computacional, en el Apéndice E se describe un procedimiento para la conexión de ambos tipos de matrices generalizadas ${ }^{9}$.

Con respecto al codo en plano $E$ diseñado, en la figura 3.8 se presentan las pérdidas de retorno del puerto (5) para los siguientes casos:

- Codo en plano $E$ sin compensar.

- Codo en plano $E$ compensado con un poste situado en posición centrada y sin iris de adaptación.

\footnotetext{
${ }^{8}$ En una aplicación práctica, interesa que el espesor del iris sea pequeño para minimizar las pérdidas.

${ }^{9}$ Aunque en el Apéndice E la MAG utilizada es de 2 puertos, la teoría presentada puede extenderse fácilmente para el caso de una MAG de 3, 4 y 5 puertos. Esta extensión será necesaria en el análisis de uniones en T, uniones T-mágica y uniones turnstile.
} 
- Codo en plano $E$ compensado con un poste situado en posición centrada y con un iris de adaptación en el puerto (5).

- Codo en plano $E$ compensado con un poste situado en posición descentrada y con un iris de adaptación en el puerto (5).

Puede apreciarse que, en este caso, la posición relativa del poste no juega un papel tan importante como en el caso de la estructura en plano $H$. De hecho, la mejora conseguida al descentrar el poste es mínima. No obstante, la introducción del iris de acoplo en el puerto (5) ha resultado crucial para minimizar las pérdidas de retorno del dispositivo. Por otro lado, los resultados obtenidos con HFSS validan completamente la herramienta implementada.

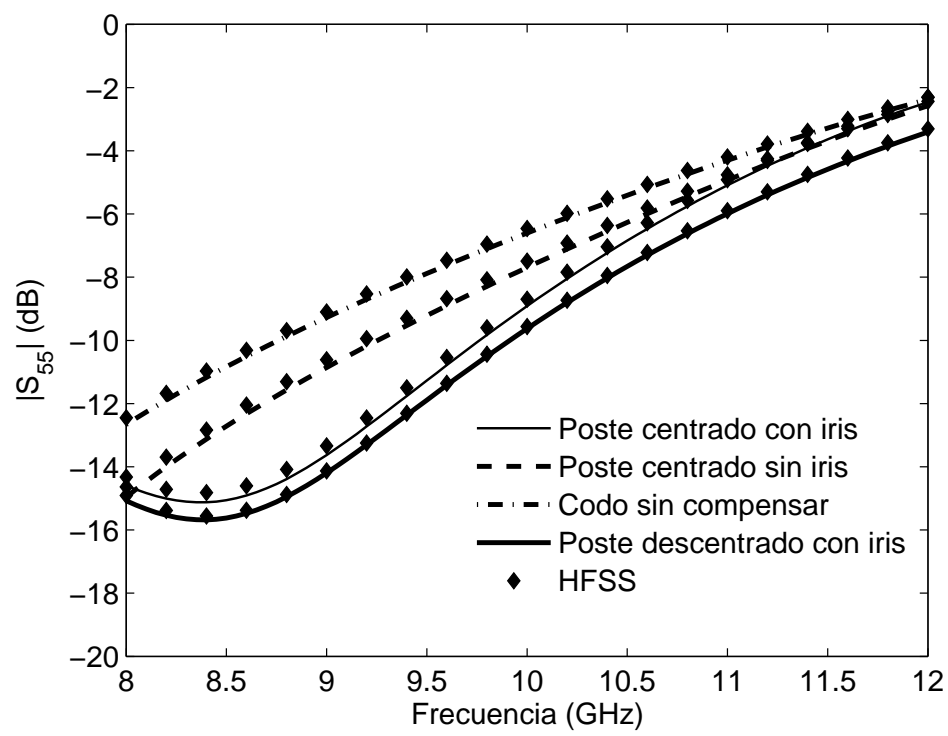

Figura 3.8: Pérdidas de retorno de un codo recto en plano $E$ implementado en guía rectangular WR-90 compensado con un poste conductor cilíndrico ( $r=3,75 \mathrm{~mm}, h=1,5 \mathrm{~mm}$ ) en posición descentrada $\left(x_{0}=11,45 \mathrm{~mm}, z_{0}=4,10 \mathrm{~mm}\right)$. En el puerto (5) del codo se ha introducido un iris de adaptación $\left(h_{\text {iris }}=7,0 \mathrm{~mm}, l_{\text {iris }}=20,5 \mathrm{~mm}, t_{\text {iris }}=1,5 \mathrm{~mm}\right)$.

Un estudio adicional es el que se representa en la figura 3.9, donde se investiga la influencia del radio del poste en estructuras en plano $E$. Así, en dicha figura se mantiene el poste en posición descentrada $\left(x_{0}=11,45 \mathrm{~mm}, z_{0}=4,10 \mathrm{~mm}\right)$, se fija la altura al valor $h=1,5 \mathrm{~mm}$, y se dejan constantes las dimensiones del iris proporcionadas anteriormente. El estudio se realiza para los siguientes valores del radio del poste: $r=0,5 \mathrm{~mm}, r=1,5 \mathrm{~mm}$, $r=2,5 \mathrm{~mm}$ y $r=3,75 \mathrm{~mm}^{10}$. Puede comprobarse que, a medida que el radio del poste disminuye, empeoran las pérdidas de retorno del codo. Por otro lado, en la figura 3.10 se estudia la influencia de la altura del poste en la respuesta en frecuencia del dispositivo. Así, en este caso se fija el radio al valor $r=3,75 \mathrm{~mm}$, se mantiene el poste en posición descentrada

\footnotetext{
${ }^{10}$ Cuando $r>3,75 \mathrm{~mm}$ (el máximo radio permitido en este caso teniendo en cuenta la posición del poste es de 4,1 mm) los resultados obtenidos son, prácticamente, iguales a los del caso óptimo en que $r=3,75 \mathrm{~mm}$.
} 


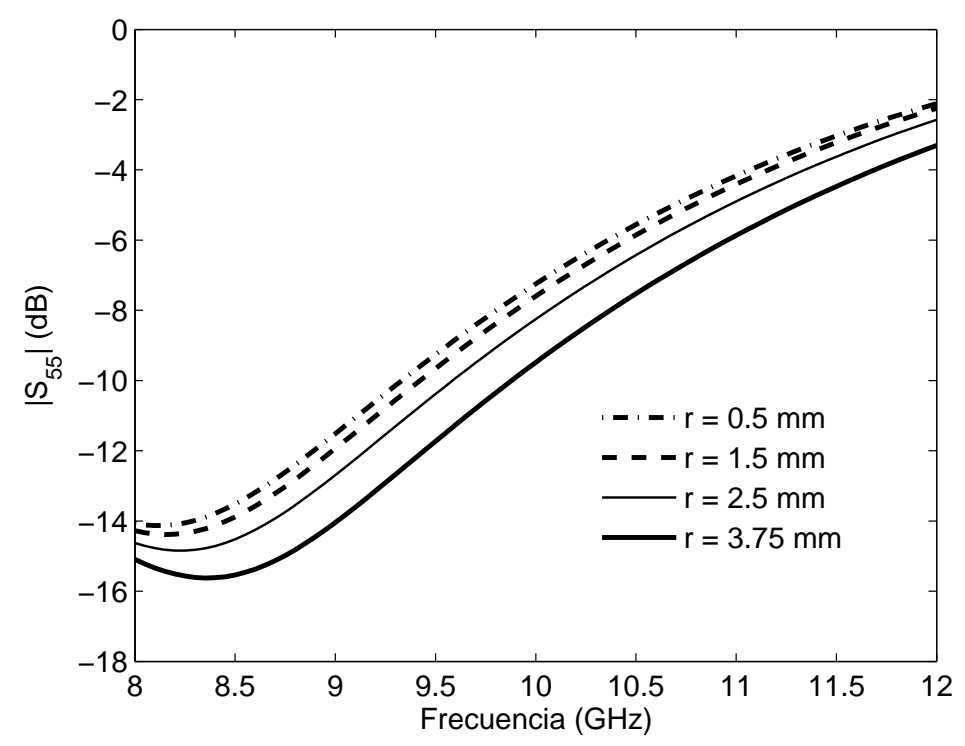

Figura 3.9: Pérdidas de retorno de un codo recto en plano $E$ implementado en guía rectangular WR-90 compensado con un poste conductor cilíndrico $(h=1,5 \mathrm{~mm})$ situado en una posición descentrada. En el puerto (5) del codo se ha introducido un iris de adaptación. Influencia del radio del poste en la respuesta en frecuencia de la estructura.

y se dejan constantes el resto de dimensiones. El estudio se lleva a cabo para los valores: $h=0,5 \mathrm{~mm}, h=1,5 \mathrm{~mm}, h=4,0 \mathrm{~mm}$ y $h=8,0 \mathrm{~mm}$. Puede comprobarse que, para el codo en plano $E$, los postes de baja altura y de radios elevados son los que proporcionan mejores resultados.

Finalmente, puesto que el iris de acoplo ha resultado ser un elemento importante en esta estructura en plano $E$, en las figuras 3.11 y 3.12 se realiza un estudio sobre la influencia de la altura a la que se sitúa el iris $\left(h_{\text {iris }}\right)$ y de su longitud $\left(l_{\text {iris }}\right)$. En ambas figuras se mantiene constante el espesor del iris $\left(t_{\text {iris }}=1,5 \mathrm{~mm}\right)$, y el poste se deja en posición descentrada (dimensiones del poste iguales a las consideradas en la figura 3.8).

La simulación de las estructuras en plano $E$ presentadas anteriormente se ha realizado en 11,67 segundos (101 puntos en frecuencia). Por su parte, los resultados proporcionados por HFSS se han obtenido en 16 minutos (21 puntos en frecuencia).

\subsubsection{Uniones en $\mathbf{T}$ en plano $H$ y en plano $E$}

El diseño de divisores de potencia es una de las aplicaciones más importantes de las uniones de tres puertos, como las uniones en $\mathrm{T}$ en plano $H$ y en plano $E$ representadas en las figuras 3.1c y 3.1d, respectivamente [69]. En este tipo de aplicaciones, se desea que la potencia introducida por el puerto común del dispositivo se reparta por igual hacia los otros dos puertos. A este respecto, $\mathrm{Wu}$ [59] realiza un estudio muy interesante sobre divisores de potencia implementados a partir de uniones en $\mathrm{T}$ en plano $H$. En particular, en dicho 


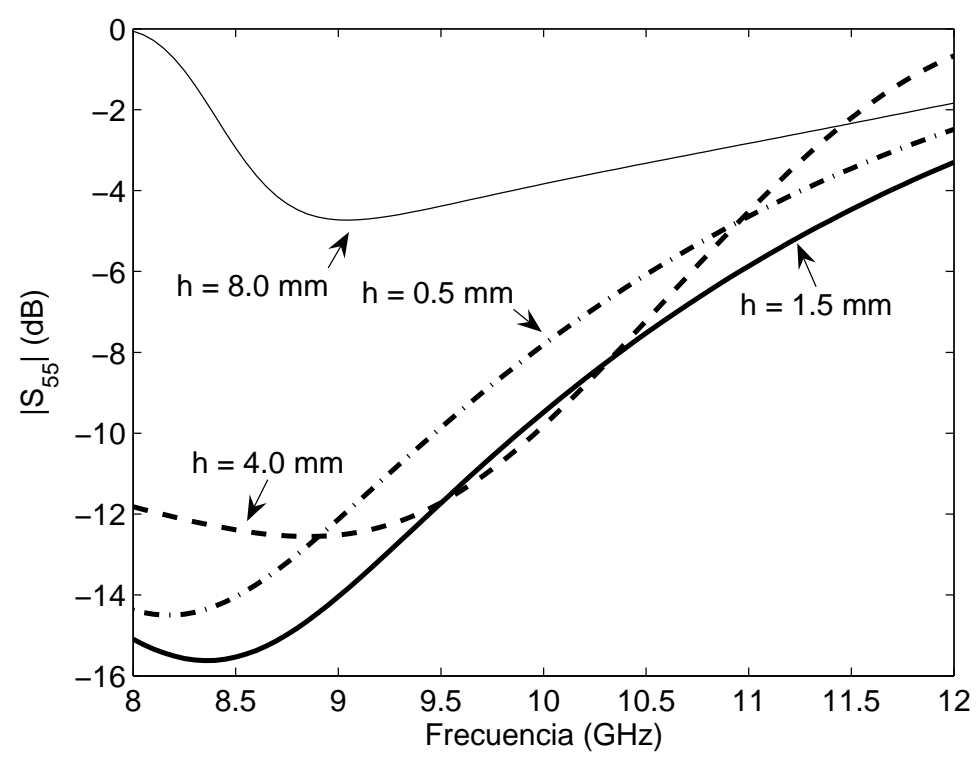

Figura 3.10: Pérdidas de retorno de un codo recto en plano $E$ implementado en guía rectangular WR-90 compensado con un poste conductor cilíndrico $(r=3,75 \mathrm{~mm})$ situado en una posición descentrada $\left(x_{0}=11,45 \mathrm{~mm}, z_{0}=4,10 \mathrm{~mm}\right)$. En el puerto (5) del codo se ha introducido un iris de adaptación. Influencia de la altura del poste en la respuesta en frecuencia de la estructura.

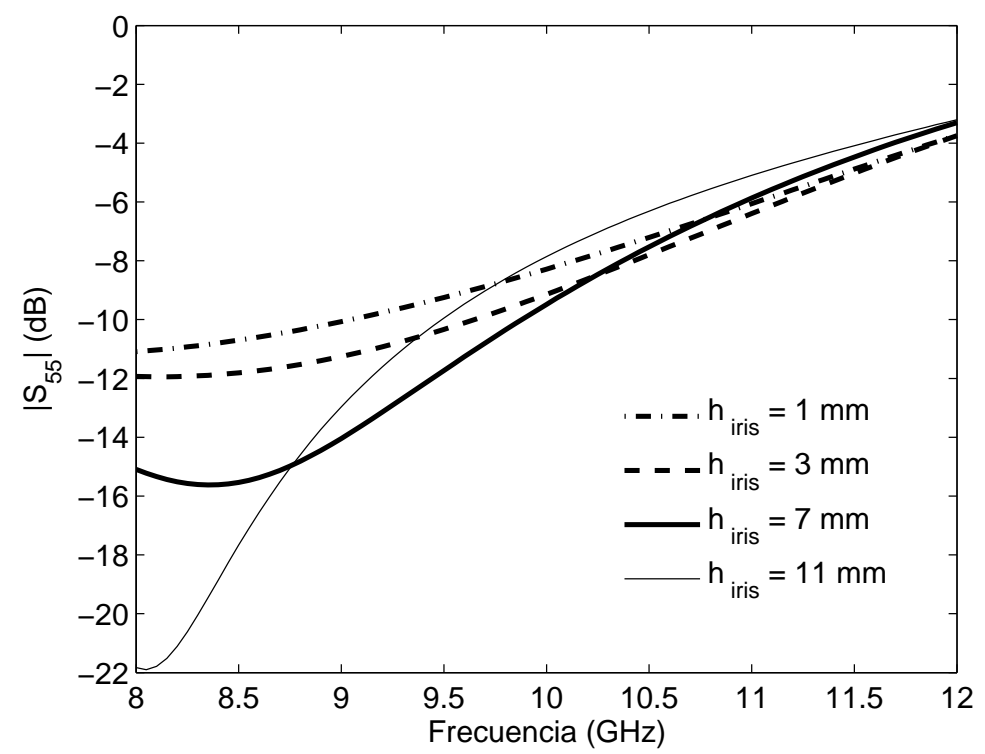

Figura 3.11: Pérdidas de retorno de un codo recto en plano $E$ implementado en guía rectangular WR-90 compensado con un poste situado en una posición descentrada. Influencia de la altura a la que se sitúa el iris $\left(h_{\text {iris }}\right)$ en la respuesta en frecuencia de la estructura. 


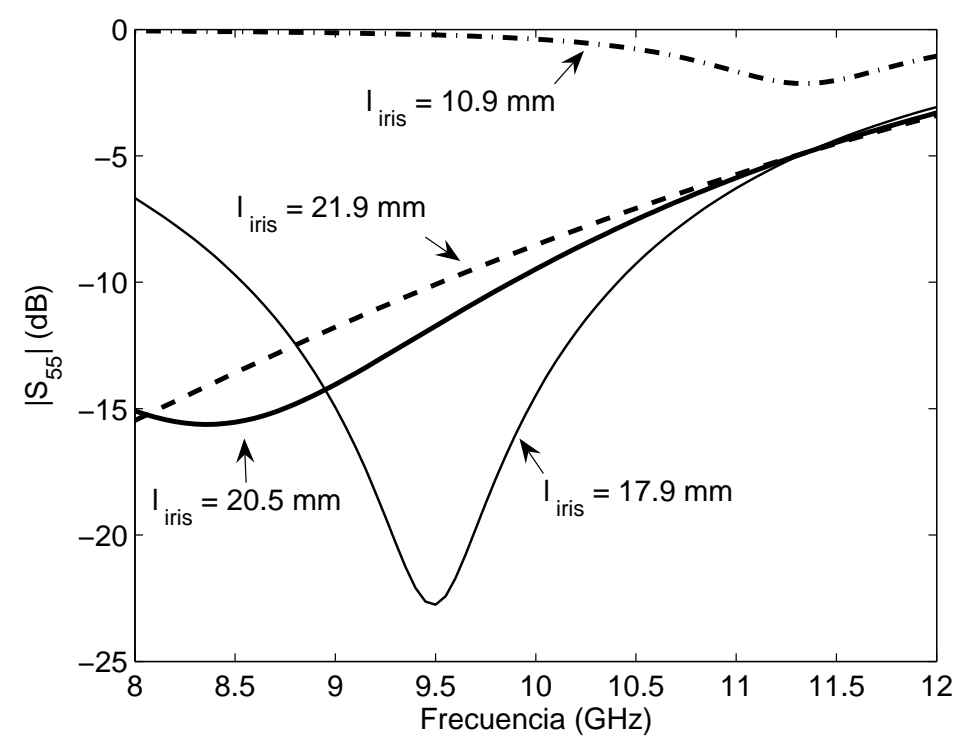

Figura 3.12: Pérdidas de retorno de un codo recto en plano $E$ implementado en guía rectangular WR-90 compensado con un poste situado en una posición descentrada. Influencia de la longitud del iris $\left(l_{\text {iris }}\right)$ en la respuesta en frecuencia de la estructura.

trabajo se demuestra que la respuesta en frecuencia del divisor de potencia puede mejorarse enormemente si la unión en T se carga con un poste conductor cilíndrico de altura variable, y se concluye que la altura del poste es un parámetro de diseño muy importante. Sin embargo, en la investigación llevada a cabo en [59], el poste está siempre situado en una posición centrada y no se estudia cómo se modificaría la respuesta en frecuencia del divisor si se descentrara dicho poste.

Así pues, aprovechando la gran flexibilidad de la herramienta de simulación implementada, en este trabajo de tesis se han investigado las consecuencias de situar el poste en una posición descentrada en la estructura. En primer lugar, hemos partido del mejor diseño presentado en [59, pág. 898], y hemos considerado la posibilidad de descentrar el poste con el objetivo de optimizar la respuesta en frecuencia del divisor. Para poder interpretar correctamente los resultados que se presentan a continuación es importante comentar que, si se parte del bloque básico de la figura 3.2, la unión en T en plano $H$ puede obtenerse abriendo los puertos (1), (3) y (4), siendo el puerto (1) el denominado puerto común. Así, en la figura 3.13, en primer lugar se valida nuestra herramienta de simulación comparando nuestros resultados con los que se presentan en [59] para una unión en T en plano $H$ implementada en la guía rectangular WR-75 ( $a=19,05 \mathrm{~mm}, b=9,525 \mathrm{~mm}$ ), y compensada con un poste de radio $r=0,254 \mathrm{~mm}$ y de altura $h=7,62 \mathrm{~mm}$ situado en posición centrada.

Posteriormente, en la misma figura 3.13, se demuestra que si el poste se descentra a lo largo del plano de simetría de la estructura (coordenadas del centro de la base del poste referidas al sistema de referencia de la figura 3.2: $x_{0}=a / 2, z_{0}=12,525 \mathrm{~mm}$ ), la respuesta en frecuencia del divisor mejora notablemente. Así, se aprecia que el módulo de los coeficientes 
de transmisión $S_{31}$ y $S_{41}$ (este último no aparece en la figura ya que la estructura es simétrica y entonces $S_{31}=S_{41}$ ) es prácticamente igual a $-3 \mathrm{~dB}$ en, prácticamente, la totalidad de la banda de frecuencia de operación de la guía WR-75. Igualmente, se observa que las pérdidas de retorno están por debajo de $-12 \mathrm{~dB}$ en toda la banda.

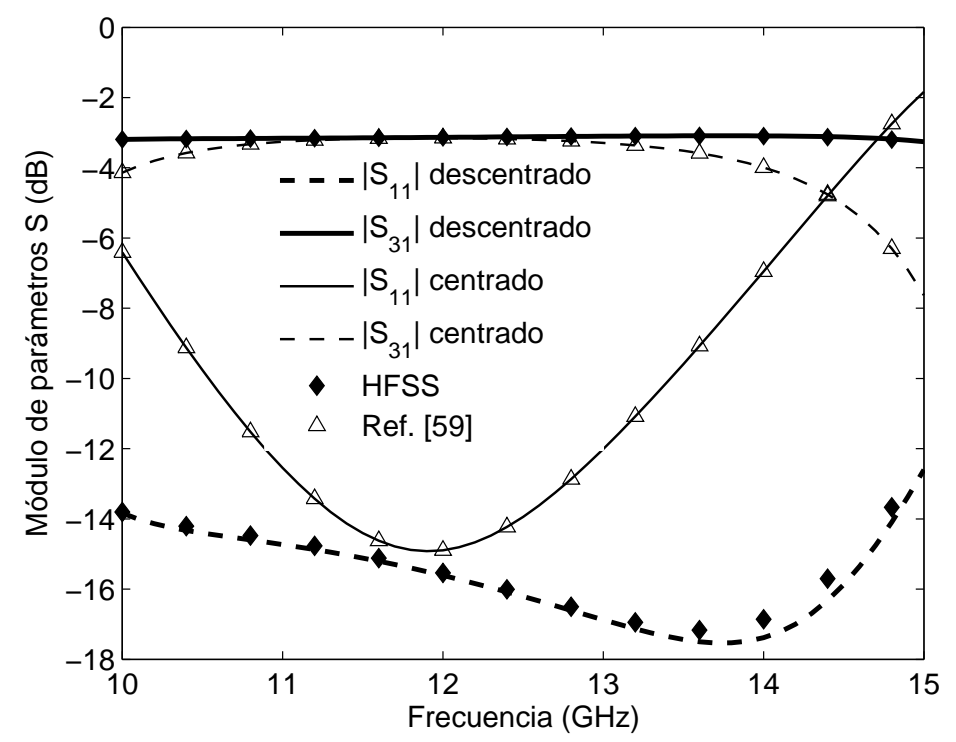

Figura 3.13: Parámetros de dispersión de una unión en T en plano $H$ en guía WR-75 compensada con un poste cilíndrico ( $r=0,254 \mathrm{~mm}, h=7,62 \mathrm{~mm}$ ). Comparación entre el caso en que el poste está centrado con el caso en que está descentrado $\left(x_{0}=9,525 \mathrm{~mm}, z_{0}=12,525 \mathrm{~mm}\right)$. Los resultados obtenidos para el caso en que el poste está centrado se comparan con los datos presentados en [59].

Por otro lado, con el propósito de poner de manifiesto que la posición del poste en la estructura es una variable crucial en el diseño de uniones en $\mathrm{T}$ en plano $H$, en la figura 3.14 se han representado las pérdidas de retorno del divisor de potencia para diferentes valores de la coordenada $z_{0}$ que define la posición del poste (la coordenada $x_{0}$ se mantiene en el valor $x_{0}=a / 2$ para asegurar la simetría física del divisor).

Otro ejemplo de diseño de uniones en $\mathrm{T}$ en plano $H$ compensadas se presenta en la figura 3.15. De nuevo, partimos de uno de los diseños presentados en [59, pág. 897] con el objetivo de optimizar la respuesta en frecuencia del divisor. En este caso, se trata de una unión en T en plano $H$ implementada en la guía rectangular WR-28 ( $a=7,112 \mathrm{~mm}, b=3,556 \mathrm{~mm})$. El divisor se carga con un poste de radio $r=0,635 \mathrm{~mm}$ y de altura $h=3,048 \mathrm{~mm}$ situado en una posición centrada. En la figura 3.15, primeramente, se comparan con éxito los resultados de nuestra simulación con los datos numéricos extraídos de [59]. Después, el poste se descentra manteniendo la simetría física de la estructura y se sitúa en la posición $x_{0}=a / 2$, $z_{0}=5,362 \mathrm{~mm}$ (coordenadas del centro de la base del poste referidas al sistema de referencia de la figura 3.2). Tal y como se puede observar, la respuesta en frecuencia del divisor mejora de manera significativa. Además, los datos obtenidos en nuestra simulación concuerdan perfectamente con los proporcionados por HFSS. Con respecto a la eficiencia computacional de 


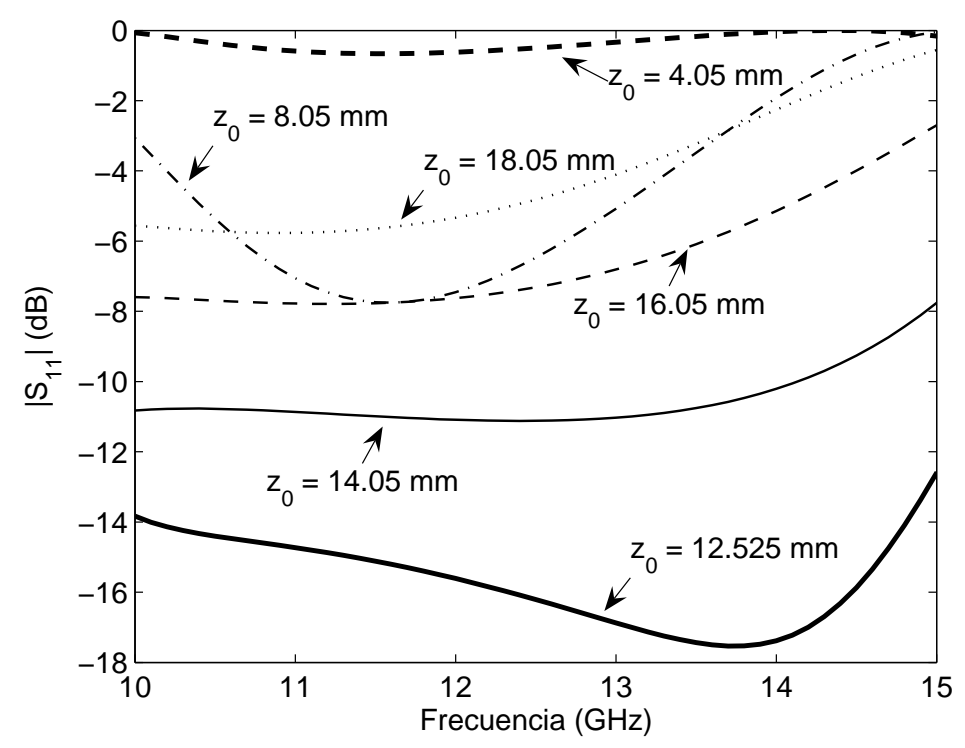

Figura 3.14: Pérdidas de retorno de una unión en T en plano $H$ en guía WR-75 compensada con un poste cilíndrico $(r=0,254 \mathrm{~mm}, h=7,62 \mathrm{~mm})$ situado en la posición $\left(x_{0}=a / 2, z_{0}\right)$. Influencia de la posición relativa del poste en la respuesta en frecuencia del divisor.

la herramienta implementada para uniones de tres puertos en plano $H$, es importante destacar que, por ejemplo, los resultados presentados en la figura 3.13 han consumido 8,33 segundos de CPU para un total de 101 puntos en frecuencia. En ese mismo ejemplo, la simulación con HFSS se realizó en unos 22 minutos para un total de 14 puntos en frecuencia.

Para terminar esta sección, resta presentar las uniones en $\mathrm{T}$ en plano $E$ compensadas e investigar su aplicación como divisores de potencia (ver la figura 3.1d). En primer lugar, se observa que la unión en $T$ en plano $E$ puede analizarse partiendo de la geometría básica de la figura 3.2 si se abren los puertos (1), (2) y (5) (este último puerto es el puerto común del divisor). En el apartado 3.3.1, cuando se estudiaron los codos rectos en plano $E$, ya se apuntó que en este tipo de estructuras la posición relativa del poste no desempeña un papel significante en el proceso de diseño. De hecho, en ese caso tuvimos que introducir un iris de adaptación en uno de los puertos para mejorar la respuesta en frecuencia del dispositivo. En el caso de las uniones en T en plano $E$ hemos observado el mismo comportamiento; es decir, la posición relativa del poste en la estructura no es un parámetro de diseño importante. Además, al igual que se hizo con los codos rectos, ha sido también necesario introducir un iris de adaptación en el puerto (5) de la unión para minimizar las pérdidas de retorno en dicho puerto. El análisis multimodal de las uniones planares que surgen en la estructura tras introducir el iris se ha realizado de nuevo empleando la técnica de la ecuación integral estudiada en el capítulo 2.

Con todo ello, en la figura 3.16 se presenta el diseño de una unión en T en plano $E$ implementada en la guía rectangular WR-75. La unión se ha compensado con un poste de radio $r=2,9 \mathrm{~mm}$ y de altura $h=4,65 \mathrm{~mm}$ situado en una posición centrada. Además, 


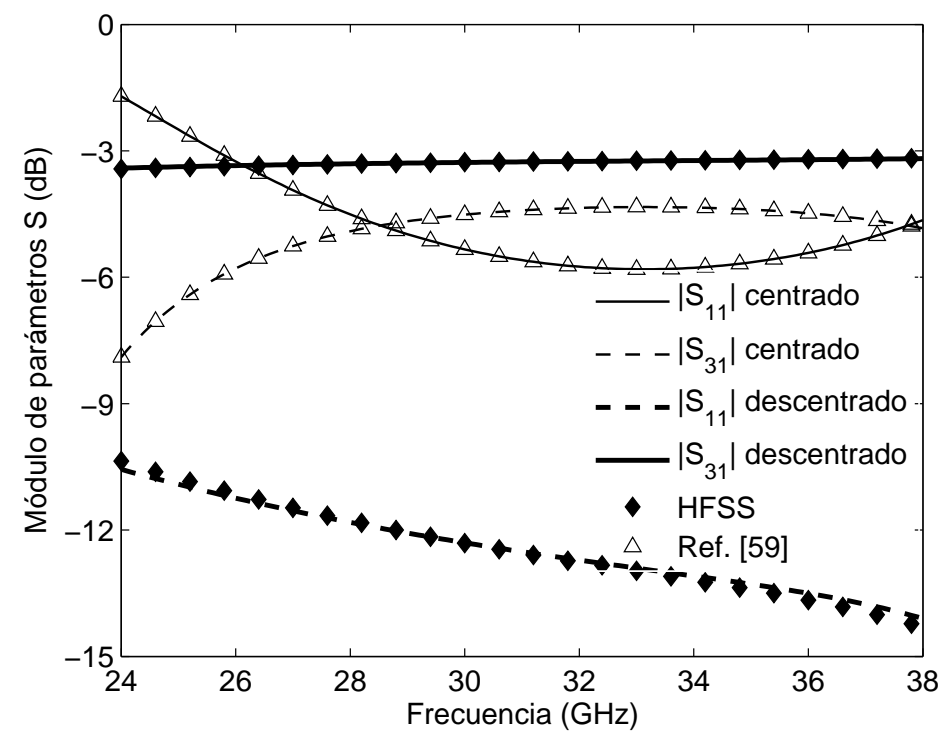

Figura 3.15: Parámetros de dispersión de una unión en T en plano $H$ en guía WR-28 compensada con un poste cilíndrico $(r=0,635 \mathrm{~mm}, h=3,048 \mathrm{~mm})$. Comparación entre el caso en que el poste está centrado con el caso en que está descentrado $\left(x_{0}=9,525 \mathrm{~mm}, z_{0}=5,362 \mathrm{~mm}\right)$. Los resultados obtenidos para el caso en que el poste está centrado se comparan con los datos presentados en [59].

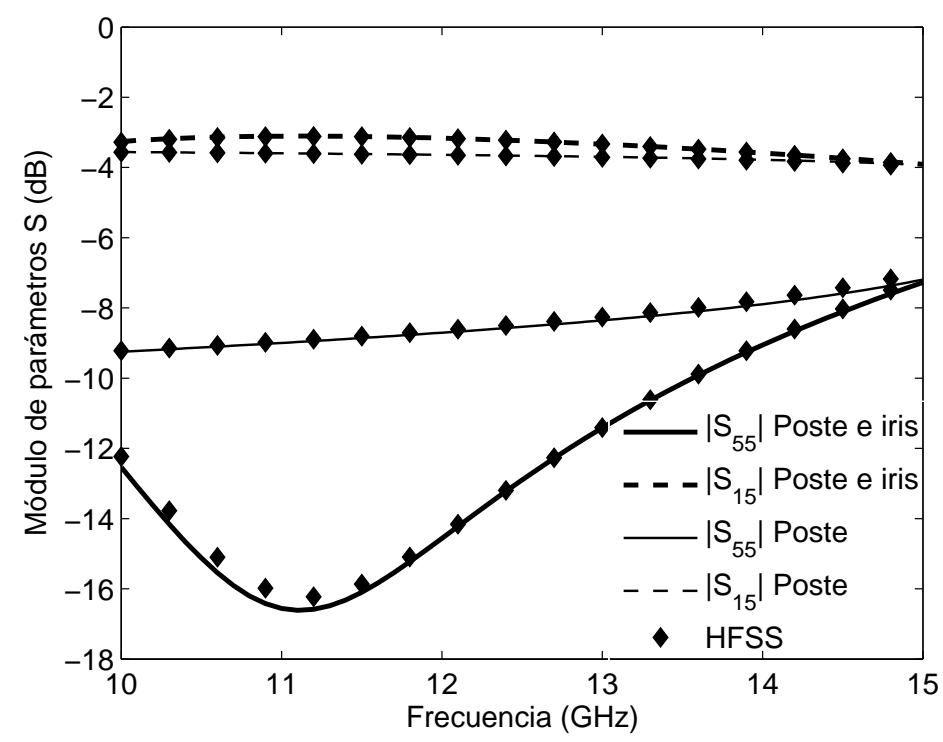

Figura 3.16: Parámetros de dispersión de una unión en T en plano $E$ en guía WR-75 compensada con un poste cilíndrico $(r=2,9 \mathrm{~mm}, h=4,65 \mathrm{~mm})$ situado en una posición centrada. En el puerto (5) se ha introducido un iris de adaptación $\left(h_{\text {iris }}=1,5 \mathrm{~mm}, l_{\text {iris }}=15,3 \mathrm{~mm}, t_{\text {iris }}=1,25 \mathrm{~mm}\right)$.

en el puerto (5) de la estructura, a una altura $h_{\text {iris }}=1,5 \mathrm{~mm}$, se ha introducido un iris de longitud $l_{\text {iris }}=15,3 \mathrm{~mm}$ y de espesor $t_{\text {iris }}=1,25 \mathrm{~mm}$. En la figura se observa que, cuando 
se introduce el iris en la unión, la respuesta en frecuencia mejora de forma significativa con respecto al caso en que únicamente se introduce el poste. La eficiencia computacional de la herramienta implementada se pone de manifiesto en este nuevo tipo de aplicación: los resultados presentados se han obtenido en 11,68 segundos (101 puntos en frecuencia). Por su parte, la simulación con HFSS se completó en 21 minutos (17 puntos en frecuencia).

\subsubsection{Uniones en $\mathbf{T}$ mágica}

La unión en T mágica es una unión híbrida de cuatro puertos (véase la figura 3.1e) que se utiliza muy frecuentemente en numerosos dispositivos de microondas, tales como acopladores direccionales, mezcladores, multiplexores, discriminadores de frecuencia y transductores ortomodales [9]. Sus características más relevantes son las de actuar como divisor de potencia, proporcionar aislamiento entre puertos y generar salidas desfasadas $180^{\circ}$. En esta sección se va a demostrar que la respuesta en frecuencia de una unión en T mágica puede optimizarse introduciendo un poste en la estructura en una posición descentrada.

Así pues, en primer lugar, es importante comentar que si se parte de la geometría básica de la figura 3.2, la unión en T mágica puede obtenerse abriendo los puertos (1), (3), (4) y $(5)^{11}$. Con el propósito de validar la herramienta de simulación para el caso de uniones de cuatro puertos, inicialmente, en la figura 3.17 se analiza una unión T mágica sin compensar implementada en la guía rectangular WR-90 $(a=22,90 \mathrm{~mm}, b=10,20 \mathrm{~mm})$ donde los resultados obtenidos se comparan satisfactoriamente con las medidas experimentales extraídas de [9].

A continuación, la unión anterior se compensa con un poste de radio $r=0,65 \mathrm{~mm}$ y de altura $h=9,5 \mathrm{~mm}$ situado en una posición centrada. Además, con el fin de mejorar la adaptación de la estructura, en el puerto (5) se introduce un iris $\left(h_{\text {iris }}=4,0 \mathrm{~mm}, l_{\text {iris }}=16,11 \mathrm{~mm}\right)$ de espesor $t_{\text {iris }}=1,5 \mathrm{~mm}$. Los parámetros de dispersión de esta nueva estructura se presentan en la figura 3.18, donde se comparan con los datos proporcionados por HFSS. Es importante hacer notar que la respuesta en frecuencia ha mejorado con respecto a la unión sin compensar.

Seguidamente, se procede a situar el poste en una posición descentrada en la estructura con el objetivo de investigar si se produce alguna mejora en la respuesta en frecuencia. En concreto, el poste se sitúa en las coordenadas $x_{0}=a / 2, z_{0}=14,45 \mathrm{~mm}$, referidas al sistema de referencia de la figura 3.2 (obsérvese que se mantiene la simetría de la estructura). En la figura 3.19 se muestran los resultados obtenidos y se puede apreciar que la respuesta en frecuencia de la unión ha mejorado drásticamente. En primer lugar, es importante destacar que todos los parámetros de reflexión se han optimizado alrededor de la frecuencia central de la banda de operación del dispositivo $(f=10 \mathrm{GHz}$ ). Por otro lado, el módulo de los parámetros de transmisión $S_{31}$ y $S_{35}$ es igual a $-3 \mathrm{~dB}$ en un amplio rango frecuencial, consiguiendo así que la unión se comporte como divisor de potencia con respecto a los puertos

\footnotetext{
${ }^{11}$ Según esta numeración, los puertos (3)-(4) y (1)-(5) son las parejas de puertos desacoplados o aislados. Así, por ejemplo, cuando se excita la unión desde el puerto (3), se desea que la potencia incidente se distribuya por igual hacia los puertos (1) y (5), y que el puerto (4) se quede desacoplado.
} 


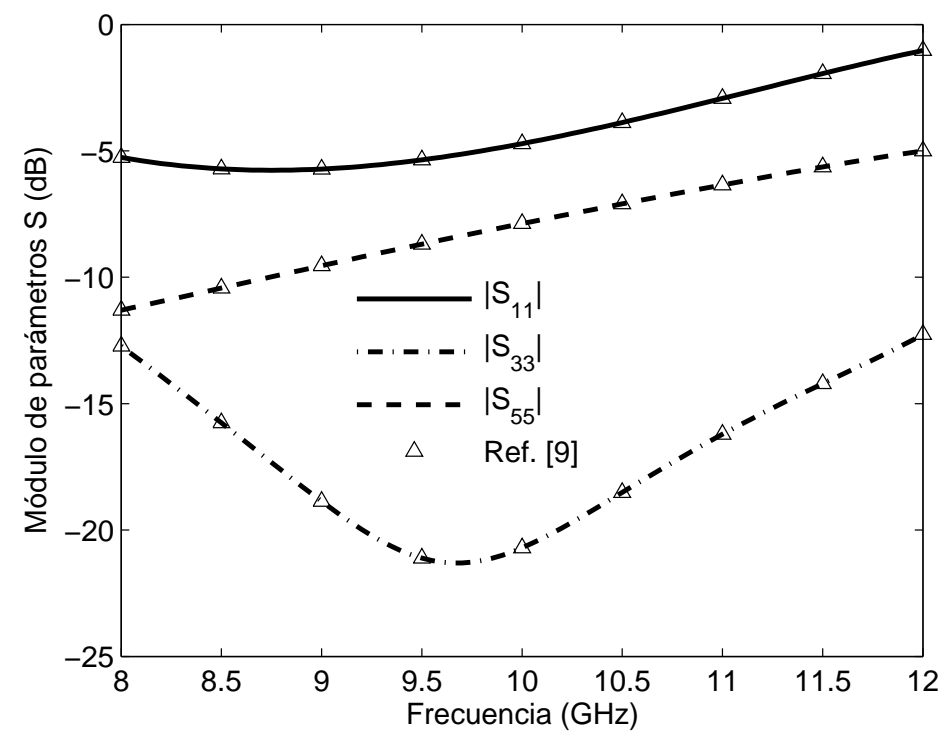

a)

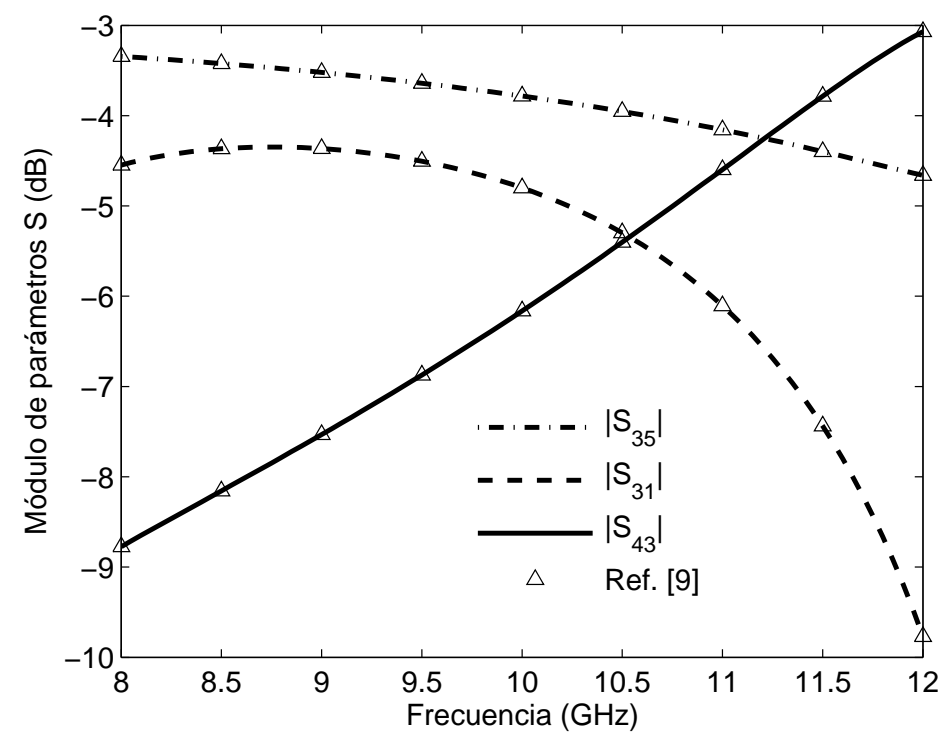

b)

Figura 3.17: Parámetros de dispersión de una unión en $T$ mágica sin compensar implementada en guía rectangular WR-90. Comparación con las medidas experimentales extraídas de [9]. (a) Parámetros de reflexión. (b) Parámetros de transmisión.

no desacoplados. Además, el módulo del parámetro de aislamiento $S_{43}$ es muy bajo en el entorno de la frecuencia central de la banda. En consecuencia, la posición relativa del poste es una variable de diseño crucial en este tipo de uniones. Finalmente, es importante destacar que las simulaciones se han realizado en 16,5 segundos (81 puntos en frecuencia), mientras que HFSS consumió 23 minutos de CPU (21 puntos en frecuencia). 


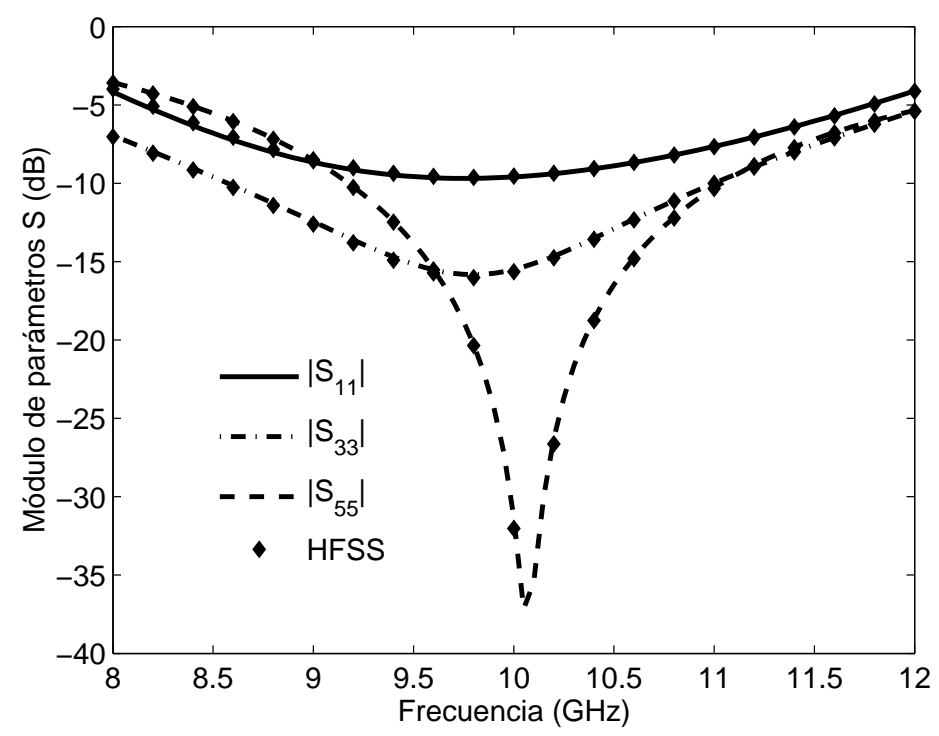

a)

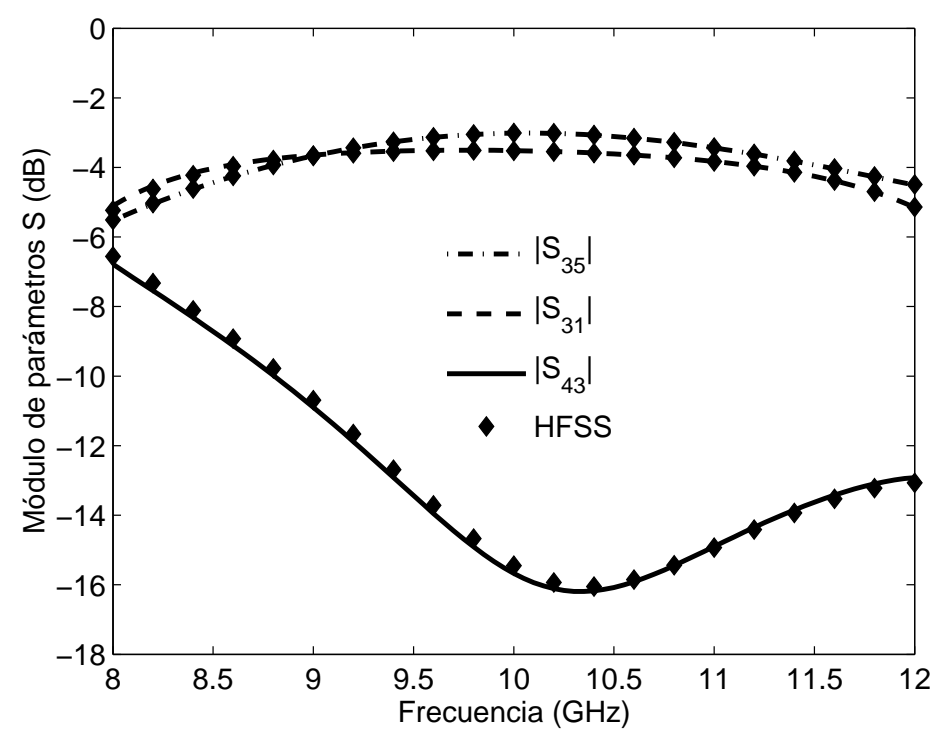

b)

Figura 3.18: Parámetros de dispersión de una unión en T mágica implementada en la guía rectangular WR-90 compensada con un poste situado en posición centrada $(r=0,65 \mathrm{~mm}, h=9,5 \mathrm{~mm})$. En el puerto (5) de la estructura se ha introducido un iris de adaptación $\left(h_{\text {iris }}=4,0 \mathrm{~mm}, l_{\text {iris }}=16,11 \mathrm{~mm}\right.$, $t_{\text {iris }}=1,5 \mathrm{~mm}$ ). (a) Parámetros de reflexión. (b) Parámetros de transmisión.

\subsubsection{Uniones turnstile}

La unión turnstile es una unión de cinco puertos que consta de cuatro guías rectangulares coplanares y de una guía circular situada en un plano perpendicular al ocupado por las guías 


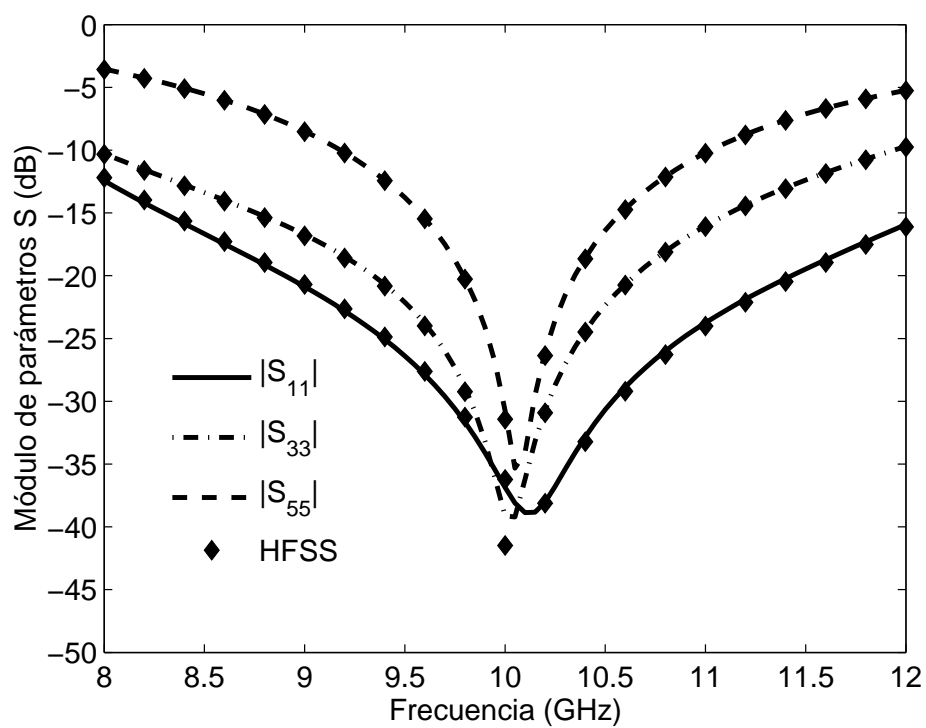

a)

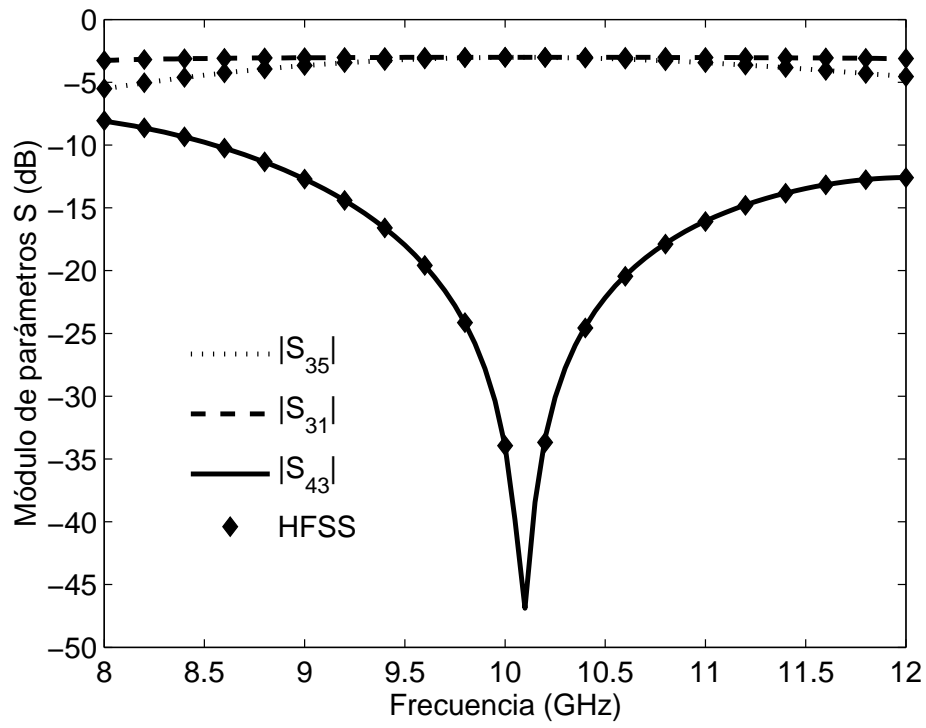

b)

Figura 3.19: Parámetros de dispersión de una unión en T mágica implementada en la guía rectangular WR-90 compensada con un poste $(r=0,65 \mathrm{~mm}, h=9,5 \mathrm{~mm})$ situado en posición descentrada $\left(x_{0}=a / 2, z_{0}=14,45 \mathrm{~mm}\right)$. En el puerto (5) de la estructura se ha introducido un iris de adaptación ( $h_{\text {iris }}=4,0 \mathrm{~mm}, l_{\text {iris }}=16,11 \mathrm{~mm}, t_{\text {iris }}=1,5 \mathrm{~mm}$ ). (a) Parámetros de reflexión. (b) Parámetros de transmisión.

rectangulares (véase la figura 3.1f). La estructura se excita con el modo fundamental $\mathrm{TE}_{11}$ de la guía circular, el cual, es bien sabido que se trata de un modo degenerado. En particular, la solución modal $\mathrm{TE}_{11}$ está compuesta por dos modos de la misma frecuencia de corte pero 
de polarizaciones ortogonales. Estos dos modos ortogonales se denominan $\mathrm{TE}_{11, \mathrm{~s}}$ (seno) y $\mathrm{TE}_{11, \mathrm{c}}$ (coseno) según la variación que presentan en la variable acimutal. Siguiendo la numeración de puertos establecida en la figura 3.2, el principio de funcionamiento de la unión turnstile es el siguiente: la potencia asociada al modo de la guía circular polarizado en la dirección paralela al brazo formado por los puertos (1) y (2) se distribuye equitativamente hacia dichos puertos, quedando aislados los puertos (3) y (4). Además, las señales de salida en los puertos (1) y (2) están desfasadas $180^{\circ}$. Asimismo, la potencia asociada al modo de la guía circular polarizado en la dirección paralela al brazo formado por los puertos (3) y (4), se reparte hacia dichos puertos, quedando ahora desacoplados los puertos (1) y (2). Además, al igual que antes, las salidas de los puertos (3) y (4) presentan un desfase de $180^{\circ}$. Estas características le confieren a la unión turnstile un gran interés práctico y, de ahí, que sea frecuentemente utilizada en numerosos dispositivos pasivos de microondas [70]. Así, las principales aplicaciones son las de divisor de potencia de cuatro vías, transductor ortomodal [71], generador de polarizaciones elípticas y circulador [72].

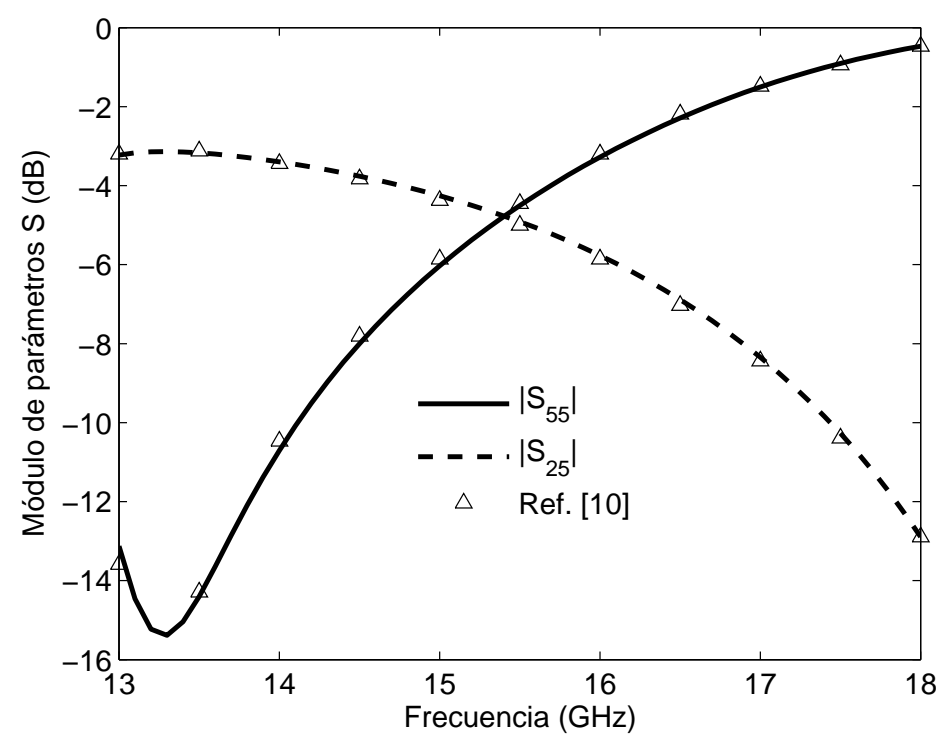

Figura 3.20: Parámetros de dispersión de una unión turnstile sin compensar implementada en la guía rectangular WR-62. La guía circular es de radio $\rho=6,99 \mathrm{~mm}$. Comparación con los resultados presentados en [10].

El análisis electromagnético de esta unión requiere, en primer lugar, disponer de la carta modal de la guía circular. Para ello, se ha utilizado la teoría desarrollada en el capítulo 2. Por otro lado, la unión planar entre las guías circular y rectangular de la unión turnstile se ha caracterizado siguiendo la formulación de la técnica de la ecuación integral presentada en el capítulo 2. Con el propósito de validar la herramienta implementada, en la figura 3.20 se presenta la respuesta en frecuencia para una unión turnstile sin compensar implementada en la guía rectangular WR-62 ( $a=15,799 \mathrm{~mm}, b=7,8995 \mathrm{~mm})$, siendo el radio de la guía circular $\rho=6,99 \mathrm{~mm}$. Los resultados obtenidos se comparan con éxito con los datos 


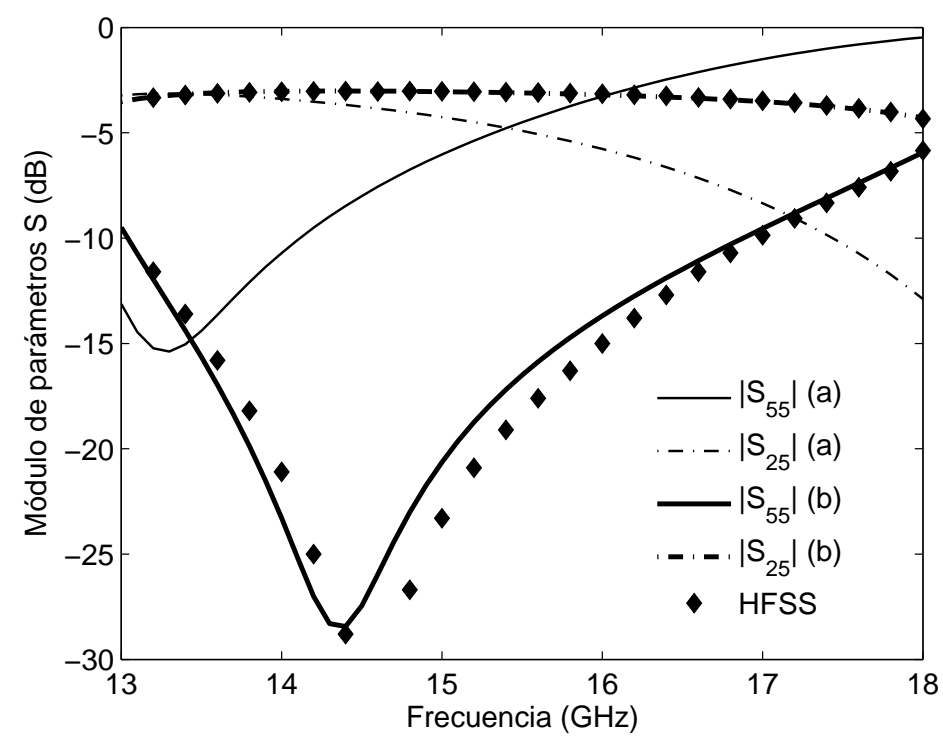

Figura 3.21: Parámetros de dispersión de una unión turnstile compensada (poste centrado; $r=2,8 \mathrm{~mm}, h=3,7 \mathrm{~mm}$ ) implementada en la guía rectangular WR-62. La guía circular es de radio $\rho=6,99 \mathrm{~mm}$. El caso (a) se corresponde con la unión sin compensar, mientras que el caso (b) se corresponde con la unión compensada. Los resultados de la simulación se comparan con HFSS.

presentados en $[10]^{12}$.

A continuación, se persigue optimizar la respuesta en frecuencia de la unión introduciendo un poste en la estructura. Es importante comentar que, en este caso, el poste debe permanecer obligatoriamente en una posición centrada para no romper la simetría de la unión. Así, en la figura 3.21 se han representado los parámetros de dispersión para la unión turnstile compensada con un poste de radio $r=2,8 \mathrm{~mm}$ y de altura $h=3,7 \mathrm{~mm}$. La excitación se realiza utilizando el modo $\mathrm{TE}_{11, \mathrm{~s}}$ de la guía circular, el cual se encuentra polarizado en la dirección paralela al brazo formado por los puertos (1) y (2). En la figura, se han incluido también los resultados para el caso no compensado con el fin de resaltar la mejora conseguida. Es importante destacar que las pérdidas de retorno con respecto al puerto (5) se han minimizado y que la transmisión hacia los puertos (1) y (2) es de casi $-3 \mathrm{~dB}$ en una banda de unos $3 \mathrm{GHz}$. Además, el nivel de transmisión a los puertos desacoplados es inferior a $-170 \mathrm{~dB}$ en toda la banda, tal y como cabía esperar. Por otro lado, no hemos considerado oportuno presentar la simulación correspondiente a la excitación con el modo $\mathrm{TE}_{11, \mathrm{c}}$ de la guía circular, ya que es idéntica completamente a la de la figura 3.21 con la salvedad de que, en ese caso, los puertos desacoplados serían el (1) y el (2).

Finalmente, las simulaciones presentadas se han realizado en 25,23 segundos para un total de 51 puntos en frecuencia. La simulación con HFSS utilizó 25 minutos para 24 puntos en frecuencia.

\footnotetext{
${ }^{12}$ Aunque el rango estándar de operación de la guía rectangular WR-62 es 12-18 GHz, en este caso se ha optado por iniciar las simulaciones a partir de $13 \mathrm{GHz}$ ya que la frecuencia de corte del modo fundamental de la guía circular utilizada es de $12,57 \mathrm{GHz}$.
} 


\section{Capítulo 4}

\section{Análisis Riguroso de la Excitación Coaxial de Dispositivos en Guía de Ondas}

\subsection{Introducción}

El análisis multimodal de la excitación coaxial de dispositivos de microondas implementados en tecnología guiada constituye un relevante tema de investigación, que ha sido ampliamente estudiado durante las últimas décadas por parte de numerosos autores. La caracterización rigurosa de este tipo de excitación resulta esencial desde un punto de vista electromagnético, pues las guías coaxiales se utilizan muy frecuentemente en la alimentación e interconexión de los diferentes subsistemas que integran numerosos dispositivos de microondas. Aunque en la literatura técnica dedicada a este tema es posible encontrar un elevado número de aportaciones que investigan el problema, la mayor parte de las mismas, tal y como se comprobará posteriormente, se centra en el cálculo de la impedancia de entrada de la estructura bajo análisis vista desde la guía coaxial con el fin de lograr el mejor grado de adaptación posible. Sin embargo, estos estudios no realizan ninguna investigación sobre la caracterización multimodal de la transición que se produce entre la guía coaxial y el subsistema al que ésta alimenta.

Por otra parte, actualmente, gran parte de los subsistemas que se emplean en numerosos dispositivos pasivos de microondas se diseñan considerando, exclusivamente, transiciones de guíaonda de tipo estándar y es posteriormente, tras finalizar el proceso de diseño, cuando se integran las distintas transiciones en las que intervienen excitaciones e interconexiones mediante guía coaxial. Este procedimiento de diseño puede ocasionar que la respuesta experimental del dispositivo final no coincida exactamente con la respuesta esperada y que, en consecuencia, el diseñador tenga que recurrir a la introducción de elementos de sintonía que ajusten a posteriori la respuesta experimental, con los consiguientes inconvenientes que dicha técnica ocasiona. Si se dispusiese de una herramienta de diseño asistido por ordenador (en inglés, Computer Aided Design, CAD) que, de forma rigurosa y eficiente, integrase en la herramienta de simulación la caracterización multimodal de la excitación mediante sonda coaxial, se conseguiría no sólo un importante ahorro en el tiempo invertido en el proceso de diseño, sino también una importante reducción en masa y volumen en el subsistema final- 
mente diseñado. La implementación de una herramienta CAD como la mencionada anteriormente constituye pues una de las motivaciones fundamentales de este capítulo. En concreto, se pretende caracterizar de manera rigurosa la excitación coaxial de dispositivos pasivos de microondas implementados en guía rectangular, con el propósito de implementar una herramienta de simulación multimodal que pueda ser utilizada en el análisis y diseño eficiente de dichas estructuras (por ejemplo, filtros en tecnología guiada). Para ello, se empleará la técnica de análisis multimodal basada en el método BI-RME 3D presentada en el capítulo 3.

En el presente capítulo se caracterizarán dos tipos diferentes de excitación coaxial. El primero de ellos está basado en la configuración clásica en la que la alimentación del dispositivo se consigue situando la sonda coaxial en la tapa superior o inferior de la guía rectangular, logrando así fácilmente la excitación del modo fundamental de la guía rectangular. Este tipo de configuración se ha representado en la figura 4.1. Es importante observar en dicha figura que a la sonda coaxial se le ha añadido en su extremo un disco conductor, con el propósito de investigar si esta nueva estructura puede mejorar las pérdidas de retorno con respecto a la configuración estándar ${ }^{1}$.

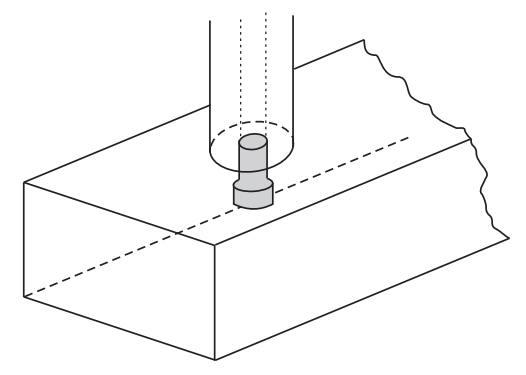

Figura 4.1: Excitación clásica de dispositivos implementados en guía rectangular mediante sonda coaxial terminada en disco.

En el segundo tipo de excitación coaxial analizada en este capítulo, la sonda coaxial se mantiene colineal con la guía rectangular a la que excita. En este tipo de configuración, la cual se representa en la figura 4.2, es usual situar un poste conductor de geometría cilíndrica en una posición perpendicular a la sonda coaxial de excitación. Además, la sonda puede contactar o no con el poste conductor. En caso de que la sonda coaxial esté en contacto con el poste, la excitación del dispositivo se consigue con la creación de un lazo de corriente que se cierra gracias a la introducción del poste. Por otro lado, en el caso de que la sonda coaxial no llegue a contactar con el poste, es la existencia de dicho poste conductor la que permite la aparición de un modo en la estructura que origina la excitación del dispositivo. En este capítulo se analizará de una manera rigurosa y eficiente la configuración en la que la sonda coaxial no contacta con el poste conductor, y se dejará el otro tipo de configuración como futura línea de investigación. La excitación de dispositivos mediante sonda coaxial en configuración colineal suele emplearse frecuentemente en el diseño de filtros en configuración comb-line $e^{2}$ [73], [74], en el diseño de diplexores [75], y en el de filtros en línea

\footnotetext{
${ }^{1}$ A este tipo de configuración en la que a la sonda coaxial se le añade en su extremo un disco conductor se le suele denominar comúnmente excitación mediante sonda de tipo "champiñón".

${ }^{2} \mathrm{El}$ análisis de filtros en configuración comb-line se tratará en profundidad en el capítulo 5.
} 


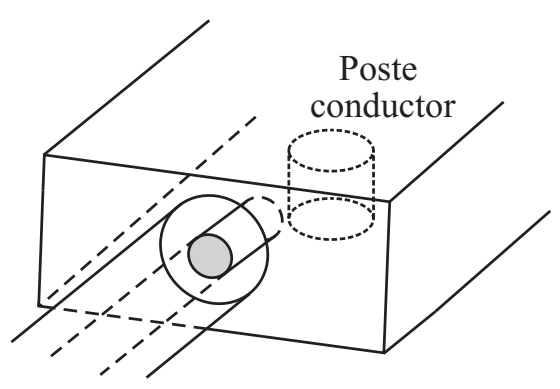

Figura 4.2: Excitación de dispositivos implementados en guía rectangular mediante sonda coaxial en configuración colineal.

implementados en guía rectangular [76], [77], [78].

Seguidamente, se presenta en esta sección un estudio sobre el estado del arte relativo a la caracterización electromagnética de la excitación coaxial de dispositivos. Tal y como ya se ha apuntado anteriormente, el análisis electromagnético de la excitación coaxial de dispositivos en guía rectangular es un problema muy importante, puesto que un gran número de dispositivos pasivos de microondas de tecnología guiada utilizan frecuentemente este tipo de alimentación. Por ello, durante las últimas décadas, numerosos autores se han dedicado a investigar el problema proponiendo una gran variedad de configuraciones y utilizando diferentes métodos de análisis. Sin embargo, la gran mayoría de estas contribuciones se centran en la obtención de la impedancia de entrada de la estructura vista desde la guía coaxial, y en ellas no se realiza ningún tipo de caracterización multimodal de esta estructura. En consecuencia, desde el punto de vista de los propósitos que se han fijado para el presente capítulo, los resultados que ofrecen dichos estudios apenas son aprovechables. Así por ejemplo, en 1972 Williamson [13] estudió la radiación de antenas cilíndricas en guía rectangular alimentadas mediante sonda coaxial, obteniendo expresiones aproximadas para el valor de la impedancia de entrada vista desde el coaxial en función de series infinitas en las que intervenían funciones de Bessel. Collin [35], por su parte, realizó un estudio de tipo variacional con el fin de calcular la impedancia de entrada de una guía rectangular excitada mediante sonda coaxial. Sin embargo, los resultados obtenidos eran bastante imprecisos, puesto que para calcular la corriente inducida en la sonda coaxial se utilizaba una aproximación que resultaba ser válida únicamente para radios pequeños del conductor interno del coaxial. Tanto Collin como Williamson asumieron en sus investigaciones que la corriente de superficie que fluía por la sonda coaxial estaba uniformemente distribuida (no asumieron una posible variación acimutal).

Por otro lado, Leviatan [14], [79] estudió en 1983-1984 el problema de la caracterización de postes cilíndricos inductivos en guía rectangular usando una representación en términos de hilos de corriente para describir la corriente inducida en el poste. En su trabajo, se calculaba la matriz de dispersión monomodal de la estructura, se deducían los parámetros de un circuito equivalente, y se demostraba que la corriente de superficie inducida en el poste inductivo sí que poseía variación acimutal. Más tarde, en 1987 Jarem [80] calculó la impedancia de entrada de una guía rectangular semi-infinita alimentada mediante una sonda 
coaxial. La precisión de las expresiones deducidas mejoraba notablemente los resultados anteriores, gracias a que no sólo se consideraron distribuciones de corriente no uniformes en la sonda coaxial, sino que también se usó una representación para la corriente inducida basada en la consideración de ocho hilos de corriente angularmente equiespaciados.

Posteriormente, en 1991, Jarem [81] estudió cómo mejorar el grado de adaptación conseguido para el caso de una guía rectangular alimentada mediante sonda coaxial introduciendo elementos parásitos (postes cilíndricos conductores) en la región de la guía rectangular. En su trabajo, en el que se empleaba una formulación que combinaba el método de los momentos y la técnica de diferencias finitas en el dominio del tiempo, se demostró que la presencia del elemento parásito (en inglés, sleeve probe) afectaba de forma sustancial al valor de la impedancia de entrada, con lo que se ofrecía al diseñador un mayor número de grados de libertad para mejorar la adaptación. Otros estudios interesantes son los que ha realizado Bialkowski. Así, en 1991 [82], se analizan transiciones de guía coaxial a guía rectangular con la particularidad de que el conductor interno del coaxial penetra en una zona rodeada de material dieléctrico (en inglés, dielectric coated probe) y, en 1995 [16], se investigan nuevos tipos de adaptadores de guía coaxial a guía rectangular: a la sonda coaxial se le incorpora un disco conductor en su extremo superior (en inglés, disc-ended probe) y se añade, además, en la región de la guía rectangular, un poste cilíndrico conductor parásito que actúa como una segunda sonda coaxial cortocircuitada. El trabajo demuestra que esta nueva configuración mejora las pérdidas de retorno que ofrecen los adaptadores convencionales. Por otro lado, en 1994 Keam [15] también investigó la configuración en la que la sonda coaxial está rodeada de material dieléctrico, demostrando que de esa forma es posible aumentar el ancho de banda en la que se produce condición de adaptación.

Ya más recientemente, en 1996 Lee [83] investiga el grado de adaptación que se logra cuando una sonda coaxial radia en el interior de una guía de placas plano-paralelas mediante un método de análisis que combina el uso de la transformada de Hankel y una técnica de adaptación modal. En ese mismo año, Leong [84] calcula la impedancia de entrada vista desde una sonda coaxial que excita una cavidad rectangular utilizando una formulación basada en la consideración de fuentes de tipo eléctrico y de tipo magnético y, en 1995-1997, Li [85], [86] estudia la adaptación que se logra en una transición de guía coaxial a guía rectangular semi-infinita, considerando que esta última puede tener múltiples cargas. La técnica de análisis empleada combina el método de los momentos y la consideración de fuentes de tipo eléctrico y de tipo magnético, proporcionando nuevas expresiones aceleradas para el cálculo de las funciones diádicas de Green relativas a una guía rectangular cargada. En el año 1999, Leung [87] investiga resonadores dieléctricos alimentados mediante sonda coaxial situados en guías de placas plano-paralelas; en 2000 Bialkowski [88] compara el grado de adaptación conseguido por varias estructuras en guías de placas plano-paralelas alimentadas mediante sonda coaxial, y en este mismo año Chen [89] investiga un nuevo tipo de monopolo integrado en una guía de placas plano-paralelas para lograr un buen grado de adaptación en un amplio ancho de banda.

En todos los trabajos comentados hasta este momento, la excitación coaxial de la estructura se realiza alimentando la guía rectangular desde su tapa superior o inferior, con 
el propósito de que la corriente inducida en el conductor interno del coaxial pueda excitar fácilmente el modo fundamental $\mathrm{TE}_{10}$ de la guía rectangular. Pero existen otras posibles configuraciones, como la que Saad presentó en 1990 [90], y que ya ha sido comentada anteriormente (configuración colineal). En concreto, analizó un tipo particular de transición entre guía coaxial y guía rectangular en la que el conductor interno del coaxial se mantiene colineal con la guía rectangular que alimenta (en inglés, configuración end-launcher), para finalmente ser cortocircuitado mediante el uso de un lazo de corriente que lo conecta a la base de la guía rectangular. Este tipo de configuración puede resultar interesante en aquellas aplicaciones en las que las restricciones de fabricación que imponga el dispositivo de microondas considerado obligue a este tipo de alimentación. Otro tipo de investigaciones son, por ejemplo, las realizadas por Lee [91], quien analiza cavidades cilíndricas excitadas mediante guía coaxial, o las que lleva a cabo Yung [92], que estudia la alimentación de una guía coaxial mediante sonda coaxial. En ambos casos, el objetivo es la determinación de la impedancia de entrada vista por el coaxial.

Las aportaciones técnicas presentadas hasta este momento se centran, fundamentalmente, en el estudio del grado de adaptación que se puede lograr cuando una estructura implementada en guía rectangular se alimenta mediante sonda coaxial. En ninguno de estos trabajos se efectúa un análisis multimodal de la transición que se produce entre ambos medios guiados. Sin embargo, éste es precisamente, como ya hemos comentado, el objetivo fundamental de este capítulo. En la literatura técnica relativa a la excitación coaxial de dispositivos en configuración clásica (ver figura 4.1), sólo es posible encontrar un reducido número de estudios que abordan este objetivo y, entre ellos, cabe destacar la investigación realizada por Liang [17] en 1992. En este trabajo se consideran estructuras en guía rectangular alimentadas mediante sonda coaxial, caracterizando la transición entre la guía coaxial y la guía rectangular en términos de una matriz de dispersión. Para ello, se emplea un método de análisis basado en la consideración de fuentes de tipo eléctrico y de tipo magnético. No obstante, la matriz de dispersión calculada es de tipo monomodal, dificultándose de este modo la integración del método desarrollado en herramientas de análisis y diseño de dispositivos pasivos de microondas ya existentes que requieran una caracterización electromagnética de tipo multimodal (por ejemplo, cuando la excitación coaxial está muy próxima a una discontinuidad entre guías de onda de diferente sección transversal). Otra valiosa aportación es la que realiza Yao [18] en 1995. En su trabajo, se modela de forma rigurosa la transición entre una guía coaxial y una guía rectangular empleando el método de expansión ortogonal, obteniendo una matriz de dispersión multimodal de la estructura. Sin embargo, la matriz de dispersión calculada es multimodal sólo con respecto a la guía rectangular; es decir, a pesar de que se tienen en cuenta la totalidad de los modos en la región de la guía rectangular, en la región del coaxial se considera únicamente el modo fundamental TEM. Por tanto, no se puede considerar que, estrictamente, se haya caracterizado la transición empleando una formulación multimodal completa. Más recientemente, en 2000 Bunger [19] presenta un trabajo en el que se analiza la excitación coaxial de dispositivos empleando una técnica multimodal híbrida. Sin embargo, la herramienta desarrollada precisa mallar completamente la estructura bajo análisis, por lo que una limitación potencial de este método reside en el hecho de que, a medida que aumenta la complejidad de la estructura, es de esperar que empeore la eficiencia 
computacional de la herramienta de simulación.

Por otra parte, en la literatura técnica podemos encontrar también diversas aportaciones en las que se estudia de forma multimodal la excitación coaxial de dispositivos en configuración colineal estando el vivo del coaxial en contacto con el poste conductor (configuración en lazo cerrado de corriente). Así, en 1999 Boria [76] implementa una herramienta destinada al análisis multimodal y diseño de filtros comb-line en línea implementados en guía rectangular considerando puertos de entrada y de salida de tipo coaxial (la herramienta permite también simular la inserción de tornillos de sintonía de sección transversal cuadrada en la estructura). El método de análisis empleado combina el uso de la técnica BI-RME 2D para el cálculo de los modos de las guías con sección transversal arbitraria, y la utilización de matrices de admitancias generalizadas para caracterizar las diferentes discontinuidades. En dicho trabajo, las guías coaxiales de entrada son colineales con las guías rectangulares que constituyen el filtro, empleándose un lazo cerrado de corriente para excitar la estructura. Este mismo tipo de alimentación colineal fue también empleado en 2001 por Gerini [93]. En su trabajo, destinado a la implementación de una herramienta de análisis de filtros en guía rectangular que integre la excitación coaxial, se presenta un estudio en el que se caracteriza de forma multimodal la transición entre una guía coaxial y una guía rectangular utilizando el método BI-RME $2 \mathrm{D}^{3}$. Por otra parte, este mismo tipo de alimentación colineal se emplea muy frecuentemente para la excitación de filtros en configuración comb-line. Así por ejemplo, en [73], [74] se presenta un método multimodal basado en la técnica de adaptación modal para el análisis y diseño de filtros en configuración comb-line de respuesta elíptica.

En relación con el análisis multimodal de la excitación coaxial de dispositivos en configuración colineal, para el caso en el que la sonda coaxial no llega a contactar con el poste conductor, en la literatura técnica apenas podemos encontrar aportaciones relevantes. Así por ejemplo, aunque en [78] se diseñan filtros en línea implementados en guía rectangular para aplicaciones WiMAX ${ }^{4}$ que emplean este tipo de excitación, el análisis multimodal de la estructura se lleva a cabo utilizando el programa HFSS, el cual es ineficiente desde un punto de vista computacional para el diseño de este tipo de dispositivos.

En el presente capítulo, se utiliza el método BI-RME 3D para llevar a cabo un análisis multimodal riguroso y eficiente de la excitación coaxial de dispositivos pasivos de microondas implementados en guía rectangular. En particular, se pretende desarrollar una herramienta de simulación capaz de caracterizar de manera multimodal las dos configuraciones de excitación coaxial comentadas anteriormente: la configuración clásica representada en la figura 4.1, y la configuración colineal representada en la figura 4.2 en la que la sonda del coaxial no llega a contactar con el poste conductor.

\footnotetext{
${ }^{3}$ En [76] y [93] se tiene la limitación de que los postes conductores que se emplean en las guías rectangulares deben ser de sección transversal rectangular para poder caracterizar la estructura empleando el método BI-RME 2D. Por ello, con este tipo de formulación no se podrían caracterizar los filtros clásicos en configuración comb-line objeto de estudio en el capítulo 5.

${ }^{4}$ WiMAX (Worldwide Interoperability for Microwave Access) es un estándar para la transmisión inalámbrica de datos basado en la norma IEEE 802.16.
} 


\subsection{Análisis Multimodal de la Excitación Coaxial de Dis- positivos en Configuración Clásica mediante el Méto- do BI-RME 3D}

El objetivo de este apartado es la caracterización multimodal rigurosa de la excitación coaxial de dispositivos pasivos de microondas y ondas milimétricas implementados en guía rectangular utilizando el método BI-RME 3D presentado en el capítulo 3, lo que permitirá considerar cualquier poste presente en la estructura con sección transversal circular. En particular, esta sección se dedica al análisis de la configuración clásica para la excitación coaxial de dispositivos (ver figura 4.1), en la que la sonda coaxial se sitúa en la tapa superior de la guía rectangular a la que alimenta. Las vistas frontal y lateral de este tipo de excitación coaxial se han representado en la figura 4.3. En dicha figura, es importante hacer notar que a la sonda coaxial se le ha añadido en su extremo un disco conductor con simetría cilíndrica con el objetivo de investigar si, con esta nueva configuración, se consigue minimizar las pérdidas de retorno (o maximizar el ancho de banda útil) con respecto a la configuración estándar en la que no existe dicho disco conductor. En relación a las dimensiones representadas en la figura 4.3 se tiene que $a$ y $b$ son la anchura y la altura de la guía rectangular, respectivamente; $r_{1}$ y $r_{2}$ representan, respectivamente, los radios externo e interno de la guía coaxial; asumiremos también que la permitividad dieléctrica relativa de dicha guía es $\varepsilon_{r} ; h_{p}$ es la profundidad de penetración de la sonda coaxial en el interior de la guía rectangular; $r_{d}$ y $h_{d}$ son el radio y la altura del disco conductor, respectivamente; $d_{x}$ se utiliza para situar el centro de la sonda coaxial en la dirección del eje $\hat{\mathbf{x}} ; \mathrm{y} d_{z}$ representa la distancia desde el centro de la sonda coaxial hasta la pared cortocircuitada de la guía rectangular ${ }^{5}$.
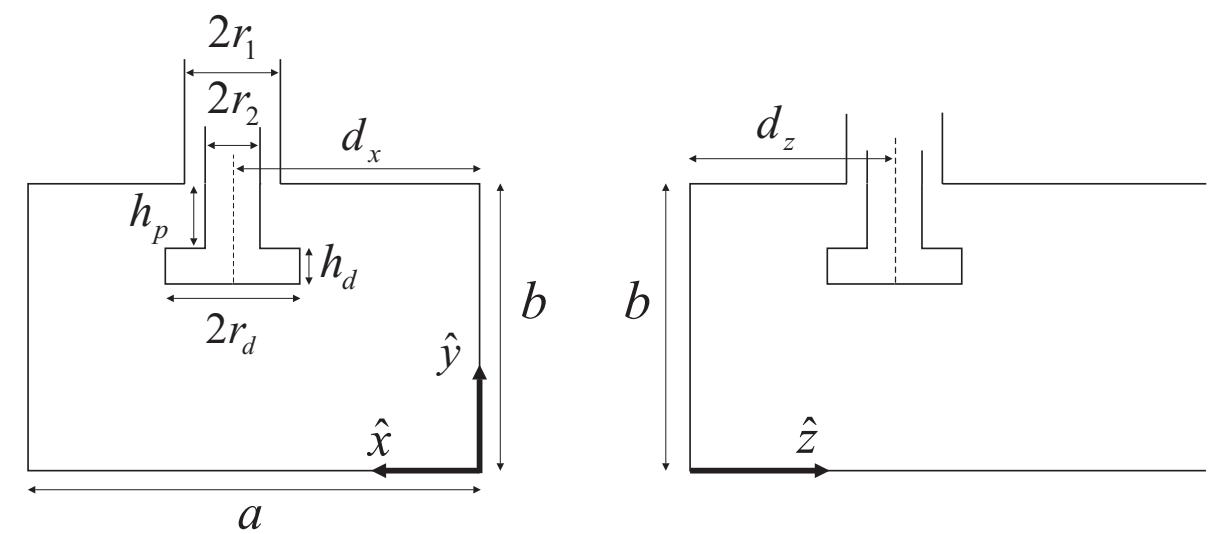

Figura 4.3: Vistas frontal y lateral de la excitación coaxial clásica de una guía rectangular semiinfinita mediante sonda terminada en disco.

Por otra parte, en la figura 4.4 se presenta una vista superior de la estructura ${ }^{6}$ y se re-

\footnotetext{
${ }^{5}$ Es importante hacer notar que si en la figura 4.3 se fuerza $r_{d}=h_{d}=0$, la sonda terminada en disco se convierte en la sonda coaxial estándar.

${ }^{6}$ Aunque en la vista lateral de la figura 4.3 se ha representado una guía rectangular semi-infinita, el análisis
} 
coge el sistema de referencia en coordenadas cilíndricas que se va a utilizar en los cálculos posteriores. Asimismo, a partir de la información representada en dicha figura, es posible establecer la siguiente relación entre los sistemas de referencia en coordenadas cartesianas $(x, y, z)$ y en coordenadas cilíndricas $(\rho, \phi, y)$ :

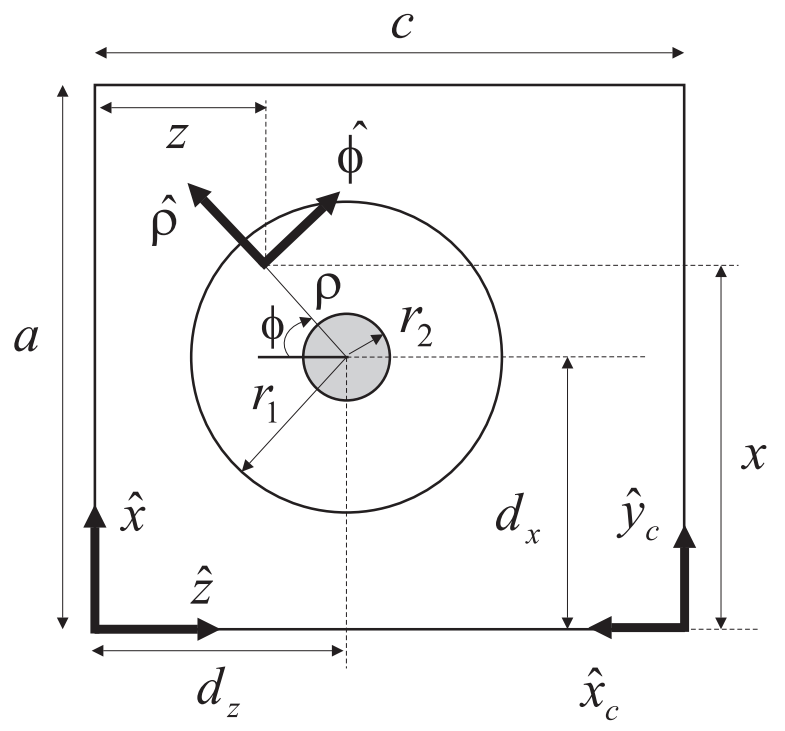

Figura 4.4: Vista superior de la excitación clásica de dispositivos implementados en guía rectangular. Representación del sistema de referencia en coordenadas cilíndricas utilizado.

$$
\begin{aligned}
x & =d_{x}+\rho \sin \phi \\
z & =d_{z}-\rho \cos \phi \\
\hat{\mathbf{x}} & =\sin \phi \hat{\boldsymbol{\rho}}+\cos \phi \hat{\boldsymbol{\phi}} \\
\hat{\mathbf{z}} & =\sin \phi \hat{\boldsymbol{\phi}}-\cos \phi \hat{\boldsymbol{\rho}}
\end{aligned}
$$

A continuación, se procede al análisis electromagnético de la excitación coaxial clásica de dispositivos implementados en guía rectangular utilizando el método BI-RME 3D. En primer lugar, conviene hacer notar que la estructura bajo análisis (ver figura 4.3) posee dos puertos de acceso, siendo uno de ellos de tipo coaxial y el otro de tipo rectangular. A efectos de cálculo, consideraremos a partir de ahora que el acceso coaxial es el puerto (1) de la estructura y que el acceso de tipo rectangular es el puerto (2). Además, según los sistemas de referencia en coordenadas cartesianas de las figuras 4.3 y 4.4 , se tiene que el puerto (1) está situado en el plano $y=b$, mientras que el puerto (2) se encuentra en el plano $z=c$.

En virtud de la teoría del método BI-RME 3D presentada en el capítulo 3, la caracterización de la estructura bajo análisis requiere considerar, en primer lugar, que dicha estructura

electromagnético que se realizará más adelante en esta sección requiere que la guía rectangular sea de una longitud dada. Por este motivo, en la figura 4.4 se asume que la guía rectangular es de longitud $c$ (en el eje êz). 
se encuentra inmersa en un resonador externo de geometría canónica. En este sentido, puesto que se está ante un dispositivo de dos puertos implementado en guía rectangular, la mejor opción será escoger, como resonador externo, una cavidad rectangular cuyas dimensiones coincidan con las de la guía rectangular de la estructura (ver figuras 4.3 y 4.4). Así pues, el resonador externo será una cavidad rectangular de dimensiones $a \times b \times c$. Gracias a esta elección, la densidad de corriente eléctrica incógnita del método sólo debe definirse en la superficie de la sonda coaxial terminada en disco que penetra en el interior de la cavidad.

Seguidamente, de acuerdo con la técnica BI-RME 3D, se deben cortocircuitar todos los accesos de la estructura con el propósito de calcular las frecuencias de resonancia de la cavidad perturbada obtenida. En este caso, tras cortocircuitar los accesos coaxial y rectangular de la figura 4.3, es fácil concluir que la cavidad perturbada que se obtiene es un resonador rectangular de dimensiones $a \times b \times c$ cargado con un poste conductor cilíndrico terminado en disco situado en $y=b$, es decir, en la tapa superior de la cavidad. El cálculo de las frecuencias de resonancia de esta nueva cavidad perturbada puede realizarse utilizando la misma herramienta que se empleó para resolver las estructuras consideradas en el capítulo 3, ya que las funciones base utilizadas para describir la densidad de corriente eléctrica incógnita son válidas siempre que el poste de la cavidad perturbada posea simetría cilíndrica ${ }^{7}$ [66]. En la figura 4.5 se ha representado la vista frontal de la cavidad perturbada obtenida.

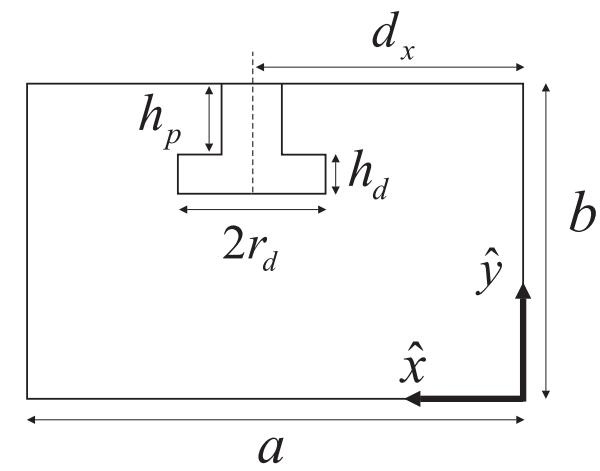

Figura 4.5: Vista frontal de la cavidad perturbada obtenida tras cortocircuitar los puertos de acceso de la estructura bajo análisis. La cavidad rectangular es de longitud $c$ (en el eje $\hat{\mathbf{z}}$ ).

El cálculo de las frecuencias de resonancia de la cavidad perturbada se necesita, tal y como se explicó en la sección 3.2.1 del capítulo 3, para la obtención de la matriz de admitancias generalizada (MAG) relativa a la estructura bajo análisis. La caracterización electromagnética de la cavidad perturbada de la figura 4.5 se realiza utilizando la teoría desarrollada en [66], donde se ha resuelto este problema de forma rigurosa.

Posteriormente, según el método BI-RME 3D, el siguiente paso consiste en considerar los puertos de acceso de la estructura con el fin de calcular la MAG correspondiente. Para ello, es

\footnotetext{
${ }^{7}$ Además, se permite la existencia en la estructura de más de un poste con simetría cilíndrica. Esta enorme flexibilidad de la herramienta implementada se aprovechará en el capítulo 5 para el análisis de filtros en configuración comb-line.
} 
importante recordar (consultar ecuación (3.10)) que se deben calcular las matrices G, T, L y $\mathbf{F}$, ya que en todas ellas intervienen las funciones vectoriales modales de campo magnético asociadas a los puertos de la estructura (las expresiones de estas matrices se pueden consultar en la tabla 3.1 del capítulo 3).

\subsubsection{Cálculo de los elementos de la matriz $G$}

La expresión de los elementos de la matriz $\mathbf{G}$ es la siguiente:

$$
G_{m n}^{(\gamma, \xi)}=\int_{S} \int_{S^{\prime}} \nabla_{S} \cdot \mathbf{h}_{m}^{(\gamma)}(\mathbf{r}) g^{\mathrm{m}}\left(\mathbf{r}, \mathbf{r}^{\prime}\right) \nabla_{S}^{\prime} \cdot \mathbf{h}_{n}^{(\xi)}\left(\mathbf{r}^{\prime}\right) d S d S^{\prime}
$$

donde $m, n=1,2, \ldots, N$, siendo $N$ el número de modos escogido en los accesos de la estructura; $g^{\mathrm{m}}\left(\mathbf{r}, \mathbf{r}^{\prime}\right)$ representa la función de Green escalar de tipo magnético asociada al resonador externo; $\gamma, \xi=1,2$ denotan cada uno de los accesos de la estructura; y $\mathbf{h}_{m}^{(\gamma)}(\mathbf{r})$ es la función vectorial modal de tipo magnético asociada al $m$-ésimo modo del acceso $(\gamma)$. En primer lugar, es importante comentar que en la ecuación (C.13) del Apéndice C, se demuestra que la divergencia de toda función vectorial modal de campo magnético de tipo $\mathrm{TM}^{z}$ es nula ${ }^{8}$. Así pues, cuando $m$ o $n$ hagan referencia a un modo de tipo $\mathrm{TM}^{z}$ en la expresión (4.5), se tendrá directamente que $G_{m n}^{(\gamma, \xi)}=0$. Igualmente, la divergencia de la función vectorial modal de campo magnético relativa al modo fundamental $\mathrm{TEM}^{z}$ de la guía coaxial es nula, por lo que, en definitiva, en la expresión (4.5) sólo se considerará el caso en que $m$ y $n$ sean ambos modos de tipo $\mathrm{TE}^{z}$. Por otra parte, se sabe que la matriz $\mathbf{G}$ es simétrica, por lo que $G_{m n}^{(\gamma, \xi)}=G_{n m}^{(\xi, \gamma)}$ cuando $\gamma \neq \xi$. Así pues, de los cuatro bloques que componen la matriz $\mathbf{G}$, en esta sección sólo se calcularán dos: $G_{m n}^{(1,2)}$ y $G_{m n}^{(1,1)}$. La expresión para el bloque $G_{m n}^{(2,1)}$ se obtendrá por simetría a partir de $G_{m n}^{(1,2)}$, mientras que el cálculo del bloque $G_{m n}^{(2,2)}$, puede realizarse a partir de la expresión (3.28) deducida en el capítulo 3.

\section{A. Cálculo de los elementos del bloque $G_{m n}^{(1,2)}$}

A continuación, se procede al cálculo del bloque $G_{m n}^{(1,2)}$; es decir, se asume que $m$ es un modo del puerto (1) (acceso coaxial) y que $n$ representa un modo del puerto (2) (acceso rectangular). Además, basándonos en los comentarios realizados en el párrafo anterior, asumiremos también que $m$ y $n$ son modos de tipo $\mathrm{TE}^{z}$. Por otro lado, sean $\left(n_{x}, n_{y}\right)$ los índices modales del $n$-ésimo modo del acceso (2) referidos al sistema de referencia en coordenadas cartesianas representado en la figura 4.3. Gracias a los resultados deducidos en la ecuación (3.25) del capítulo 3, podemos calcular directamente la integral de superficie presente en

\footnotetext{
${ }^{8}$ Aunque el Apéndice C está dedicado a las guías de onda rectangulares, el resultado obtenido en (C.13) es independiente del tipo de guía considerado.
} 
(4.5) con respecto a las variables primadas:

$$
\begin{aligned}
\int_{S^{\prime}} g^{\mathrm{m}}\left(\mathbf{r}, \mathbf{r}^{\prime}\right) \nabla_{S}^{\prime} \cdot \mathbf{h}_{n}^{\mathrm{TE}(2)}\left(\mathbf{r}^{\prime}\right) d S^{\prime}= & -\frac{k_{t, n}^{(2)}}{c} \sqrt{\frac{\epsilon_{n_{x}} \epsilon_{n_{y}}}{a b}} \cos \left(\frac{n_{x} \pi}{a} x\right) \cos \left(\frac{n_{y} \pi}{b} y\right) \\
& \cdot \sum_{n_{z}=0}^{\infty}(-1)^{n_{z}} \frac{\epsilon_{n_{z}}}{\left(k_{t, n}^{(2)}\right)^{2}+k_{z}^{2}} \cos \left(\frac{n_{z} \pi}{c} z\right) \\
= & -\sqrt{\frac{\epsilon_{n_{x}} \epsilon_{n_{y}}}{a b}} \cos \left(\frac{n_{x} \pi}{a} x\right) \cos \left(\frac{n_{y} \pi}{b} y\right) \frac{\cosh \left(k_{t, n}^{(2)} z\right)}{\sinh \left(k_{t, n}^{(2)} c\right)}
\end{aligned}
$$

En esta última expresión, $k_{t, n}^{(2)}$ es el número de onda de corte del $n$-ésimo modo de tipo $\mathrm{TE}^{z}$ del acceso (2); $\epsilon_{n_{x}}$ representa el factor de Neumann, y $k_{z}=n_{z} \pi / c$. Además, en la última igualdad se ha utilizado el resultado (D.2) del Apéndice D para calcular analíticamente el sumatorio obtenido en la expresión.

Por otro lado, en virtud de los resultados del capítulo 2, para los modos de tipo $\mathrm{TE}^{z}$ de la guía coaxial se cumple?

$$
\nabla_{S} \cdot \mathbf{h}_{m}^{\mathrm{TE}(1)}(\mathbf{r})=-\left(k_{t, m}^{(1)}\right)^{2} \mathcal{N}_{(\xi), m}^{\mathrm{TE}^{z}}\left\{\sum_{r=0}^{N_{\rho}-1} l_{s, q}^{(r)} \cos \left(r \pi \frac{\rho-r_{2}}{r_{1}-r_{2}}\right)\right\} \Phi_{(\xi), s}(\phi)
$$

donde $k_{t, m}^{(1)}$ representa el número de onda de corte del $m$-ésimo modo de tipo $\mathrm{TE}^{z}$ de la guía coaxial; $\mathcal{N}_{(\xi), m}^{\mathrm{TE}^{z}}$ es el factor de normalización de dicho modo; $(s, q)$ son los índices modales asociados a las variaciones angular y radial, respectivamente; y $\Phi_{(\xi), s}(\phi)$ es la función potencial que rige la variación angular de las soluciones modales, cuya expresión puede encontrarse en (2.71).

Posteriormente, se debe expresar (4.6) en coordenadas cilíndricas utilizando las relaciones establecidas en (4.1)-(4.2), y se debe particularizar el resultado obtenido en $y=b$, ya que es en este plano donde se encuentra situado el puerto de acceso (1). Con todo ello, se puede escribir finalmente:

$$
G_{m n}^{(1,2)}=\left(k_{t, m}^{(1)}\right)^{2} \mathcal{N}_{(\xi), m}^{\mathrm{TE}^{z}} \sqrt{\frac{\epsilon_{n_{x}} \epsilon_{n_{y}}}{a b}}(-1)^{n_{y}} \frac{1}{\sinh \left(k_{t, n}^{(2)} c\right)} \sum_{r=0}^{N_{\rho}-1} l_{s, q}^{(r)} I^{(r)}
$$

En esta última expresión, $I^{(r)}$ denota la siguiente integral de superficie, la cual se ha resuelto

\footnotetext{
${ }^{9}$ Partiendo de la ecuación de Helmholtz, es sencillo concluir que $\nabla \cdot \mathbf{h}_{m}^{\mathrm{TE}^{z}}=-k_{t, m}^{2} h_{z_{(\xi), m}}^{\mathrm{TE}^{z}}(\rho, \phi)$, siendo $h_{z_{(\xi), m}}^{\mathrm{TE}^{z}}(\rho, \phi)$ la función potencial asociada a los modos $\mathrm{TE}^{z}$ de la guía coaxial, cuya definición se recoge en (2.98).
} 
mediante métodos numéricos ${ }^{10}$ :

$I^{(r)}=\int_{S} \cos \left(\frac{n_{x} \pi}{a}\left(d_{x}+\rho \sin \phi\right)\right) \cosh \left(k_{t, n}^{(2)}\left(d_{z}-\rho \cos \phi\right)\right) \cos \left(r \pi \frac{\rho-r_{2}}{r_{1}-r_{2}}\right) \Phi_{(\xi), s}(\phi) d S$

donde $S$ representa la sección transversal de la guía coaxial y $d S=\rho d \rho d \phi$.

\section{B. Cálculo de los elementos del bloque $G_{m n}^{(1,1)}$}

Seguidamente, se presenta el cálculo del bloque $G_{m n}^{(1,1)}$. En este caso, $m$ y $n$ son modos de tipo $\mathrm{TE}^{z}$ relativos a la guía coaxial, por lo que el punto de campo $\mathbf{r}$ y el punto de fuente $\mathbf{r}^{\prime}$ pueden coincidir al evaluar la integral (4.5). Este hecho ocasiona que la función de Green escalar de tipo magnético $g^{\mathrm{m}}\left(\mathbf{r}, \mathbf{r}^{\prime}\right)$ que interviene en el cálculo de la matriz $\mathbf{G}$ diverja, tal y como se explica en [94]. De hecho, la singularidad de la función de Green escalar de tipo magnético ${ }^{11}$ puede escribirse en la forma [95]:

$$
g_{(\mathrm{s})}^{\mathrm{m}}=\frac{1}{4 \pi\left|\mathbf{r}-\mathbf{r}^{\prime}\right|}
$$

En consecuencia, el bloque $G_{m n}^{(1,1)}$ de la matriz $\mathbf{G}$ es también singular cuando $\mathbf{r}=\mathbf{r}^{\prime}$, siendo dicha singularidad de la forma:

$$
G_{(\mathrm{s}), m n}^{(1,1)}=\int_{S} \int_{S^{\prime}} \nabla_{S} \cdot \mathbf{h}_{m}^{\mathrm{TE}(1)}(\mathbf{r}) \frac{1}{4 \pi\left|\mathbf{r}-\mathbf{r}^{\prime}\right|} \nabla_{S}^{\prime} \cdot \mathbf{h}_{n}^{\mathrm{TE}(1)}\left(\mathbf{r}^{\prime}\right) d S d S^{\prime}
$$

Así pues, la obtención de los elementos del bloque $G_{m n}^{(1,1)}$ requiere evaluar la integral singular anterior. Este cálculo supone un enorme esfuerzo desde un punto de vista teórico, que se va a tratar de evitar.

A este respecto, es muy importante destacar que, aunque no se haya mencionado de manera explícita durante el desarrollo del capítulo 3, en el cálculo del bloque $G_{m n}^{(2,2)}$ también existe el mismo problema de singularidad que el descrito para el bloque $G_{m n}^{(1,1)}$, pues $m$ y $n$ son, en aquel caso, modos del mismo puerto rectangular y debe considerarse el caso en que $\mathbf{r}=\mathbf{r}^{\prime 12}$. Sin embargo, es fundamental observar que, en el caso del bloque $G_{m n}^{(2,2)}$, los modos $m$ y $n$ están referidos a un puerto de tipo rectangular cuyas dimensiones coinciden exactamente con las dimensiones de uno de los accesos laterales rectangulares del resonador externo de geometría canónica que se ha escogido siguiendo el método BI-RME 3D (en el caso que nos ocupa, el resonador externo escogido es de dimensiones $a \times b \times c$, y el puerto $(2)$ es de dimensiones $a \times b$ ). Esta particularidad es crucial desde un punto de vista

\footnotetext{
${ }^{10} \mathrm{La}$ resolución numérica de las integrales deducidas en este capítulo se ha llevado a cabo utilizando el método de cuadratura de Gauss-Legendre.

${ }^{11}$ Esta singularidad está presente también, aunque no de forma explícita, en la expresión clásica para la función de Green escalar magnética que se recoge en (C.14).

${ }^{12}$ En el cálculo del bloque $G_{m n}^{(1,2)}$ no encontramos problemas de singularidad, puesto que $m$ y $n$ pertenecen a puertos de acceso diferentes $\mathrm{y}$, por tanto, $\mathbf{r}$ y $\mathbf{r}^{\prime}$ no pueden coincidir.
} 
matemático, ya que, como se explica a continuación, permite evitar la integración explícita de la singularidad de la función de Green. En concreto, dado que la función de Green escalar de tipo magnético relativa al resonador rectangular externo se puede formular a partir de las funciones potenciales modales asociadas a uno de los accesos laterales de dicha cavidad (consulte los resultados del Apéndice C), el cálculo de la integral de superficie que define los elementos del bloque $G_{m n}^{(2,2)}$ puede abordarse directamente sin preocuparse de extraer previamente el término singular de la función de Green. De hecho, el cálculo de la matriz G para las uniones compensadas analizadas en el capítulo 3 se abordó exactamente de esta manera.

Esta propiedad, que resulta muy ventajosa desde un punto de vista matemático, se va a aprovechar para resolver el cálculo de los elementos del bloque $G_{m n}^{(1,1)}$. En particular, la estrategia que se propone consiste en expresar las funciones vectoriales modales de la guía coaxial como una combinación lineal de las soluciones modales de la guía rectangular a la que la sonda coaxial alimenta ${ }^{13}$. De esta manera, al expresar los modos de la guía coaxial en función de un conjunto de soluciones modales asociadas a uno de los accesos laterales rectangulares del resonador externo escogido, se conseguirá un tratamiento matemático para el cálculo del bloque $G_{m n}^{(1,1)}$ completamente equivalente al desarrollado con los elementos del bloque $G_{m n}^{(2,2)}$, evitándose así el cálculo de la integral singular (4.11).

En el caso que nos ocupa, se ha optado por que las soluciones modales de la guía coaxial se expresen en términos de una combinación lineal de modos de la guía rectangular de dimensiones $c \times a$ (ver figura 4.3), la cual se corresponde con uno de los accesos laterales rectangulares del resonador externo escogido. Además, de aquí en adelante, a la guía rectangular de dimensiones $c \times a$ la denominaremos guía rectangular de expansión. Por otro lado, en términos matemáticos, si $\mathbf{h}_{i}^{(\mathrm{R})}(\mathbf{r})$ denota la función vectorial modal de tipo magnético relativa al $i$-ésimo modo de la guía rectangular de dimensiones $c \times a$, la función vectorial modal de campo magnético asociada al $m$-ésimo modo de la guía coaxial $\mathbf{h}_{m}^{(1)}(\mathbf{r})$ se podrá expresar de la siguiente manera:

$$
\mathbf{h}_{m}^{(1)}(\mathbf{r})=\sum_{i=1}^{Q} \alpha_{i}^{(m)} \mathbf{h}_{i}^{(\mathrm{R})}(\mathbf{r})
$$

donde $Q$ representa el número de términos considerados en la expansión modal, y $\alpha_{i}^{(m)}$ son unos coeficientes de expansión desconocidos que pueden calcularse proyectando el $m$-ésimo modo de la guía coaxial sobre el $i$-ésimo modo de la guía rectangular de expansión:

$$
\alpha_{i}^{(m)}=\int_{S} \mathbf{h}_{m}^{(1)}(\mathbf{r}) \cdot \mathbf{h}_{i}^{(\mathrm{R})}(\mathbf{r}) d S
$$

siendo $S$ la sección transversal de la guía coaxial. Además, es importante observar que los coeficientes de expansión $\alpha_{i}^{(m)}$ se corresponden con los coeficientes de acoplamiento entre una guía rectangular y una guía coaxial. Así, dichos coeficientes se han calculado en la sección 2.3.2, en la que se estudia la discontinuidad entre una guía rectangular y una guía

\footnotetext{
${ }^{13}$ La misma estrategia se utilizará en el cálculo del resto de matrices BI-RME 3D.
} 
coaxial. En concreto, el cálculo que se plantea en (4.13) ya se ha resuelto en el capítulo 2 al evaluar la integral de acoplamiento (2.119).

Por otra parte, del estudio de las integrales de acoplamiento (2.122)-(2.127) relativas a la unión planar entre una guía coaxial y una guía rectangular, se pueden extraer las siguientes conclusiones:

- El modo $\mathrm{TEM}^{z}$ de la guía coaxial únicamente acopla con los modos de tipo $\mathrm{TM}^{z}$ de la guía rectangular.

- Los modos de tipo $\mathrm{TE}^{z}$ de la guía coaxial acoplan con los modos $\mathrm{TE}^{z}$ y $\mathrm{TM}^{z}$ de la guía rectangular.

- Los modos de tipo $\mathrm{TM}^{z}$ de la guía coaxial solamente acoplan con los modos de tipo $\mathrm{TM}^{z}$ de la guía rectangular.

En consecuencia, la expansión modal efectuada en (4.12) se puede particularizar para cada tipo de modo $\left(\mathrm{TEM}^{z}, \mathrm{TE}^{z}\right.$ y $\left.\mathrm{TM}^{z}\right)$ de la guía coaxial. En concreto:

$$
\begin{aligned}
\mathbf{h}^{\mathrm{TEM}(1)}(\mathbf{r}) & =\sum_{i=1}^{Q^{\mathrm{TM}}} v_{i}^{(\mathrm{TEM})} \mathbf{h}_{i}^{\mathrm{TM}(\mathrm{R})}(\mathbf{r}) \\
\mathbf{h}_{m}^{\mathrm{TE}(1)}(\mathbf{r}) & =\sum_{i=1}^{Q^{\mathrm{TE}}} \sigma_{i}^{(m)} \mathbf{h}_{i}^{\mathrm{TE}(\mathrm{R})}(\mathbf{r})+\sum_{j=1}^{Q^{\mathrm{TM}}} \tau_{j}^{(m)} \mathbf{h}_{j}^{\mathrm{TM}(\mathrm{R})}(\mathbf{r}) \\
\mathbf{h}_{m}^{\mathrm{TM}(1)}(\mathbf{r}) & =\sum_{i=1}^{Q^{\mathrm{TM}}} \nu_{i}^{(m)} \mathbf{h}_{i}^{\mathrm{TM}(\mathrm{R})}(\mathbf{r})
\end{aligned}
$$

En estas expresiones, $Q^{\mathrm{TE}}$ y $Q^{\mathrm{TM}}$ representan, respectivamente, el número de modos de tipo $\mathrm{TE}^{z}$ y $\mathrm{TM}^{z}$ considerados en la guía rectangular de expansión; $\mathbf{h}_{i}^{\mathrm{TE}(\mathrm{R})}(\mathbf{r})$ y $\mathbf{h}_{i}^{\mathrm{TM}(\mathrm{R})}(\mathbf{r})$ son, respectivamente, las funciones vectoriales modales normalizadas de campo magnético de tipo $\mathrm{TE}^{z}$ y $\mathrm{TM}^{z}$ asociadas al $i$-ésimo modo de la guía rectangular de expansión; $v_{i}^{\text {(TEM) }}$ es el coeficiente de acoplamiento entre el $i$-ésimo modo de tipo $\mathrm{TM}^{z}$ de la guía rectangular de expansión y el modo $\mathrm{TEM}^{z}$ de la guía coaxial; $\sigma_{i}^{(m)}$ representa el acoplamiento entre el $i$-ésimo modo de tipo $\mathrm{TE}^{z}$ de la guía rectangular de expansión y el $m$-ésimo modo de tipo $\mathrm{TE}^{z}$ de la guía coaxial; $\tau_{j}^{(m)}$ es el coeficiente de acoplamiento entre el $j$-ésimo modo de tipo $\mathrm{TM}^{z}$ de la guía rectangular de expansión y el $m$-ésimo modo de tipo $\mathrm{TE}^{z}$ de la guía coaxial; y $\nu_{i}^{(m)}$ representa el acoplamiento entre el $i$-ésimo modo de tipo $\mathrm{TM}^{z}$ de la guía rectangular de expansión y el $m$-ésimo modo de tipo $\mathrm{TM}^{z}$ de la guía coaxial. De acuerdo con (4.14)-(4.16), 
estos coeficientes de acoplamiento pueden calcularse de la siguiente manera:

$$
\begin{aligned}
v_{i}^{(\mathrm{TEM})} & =\int_{S} \mathbf{h}^{\mathrm{TEM}(1)}(\mathbf{r}) \cdot \mathbf{h}_{i}^{\mathrm{TM}(\mathrm{R})}(\mathbf{r}) d S \\
\sigma_{i}^{(m)} & =\int_{S} \mathbf{h}_{m}^{\mathrm{TE}(1)}(\mathbf{r}) \cdot \mathbf{h}_{i}^{\mathrm{TE}(\mathrm{R})}(\mathbf{r}) d S \\
\tau_{j}^{(m)} & =\int_{S} \mathbf{h}_{m}^{\mathrm{TE}(1)}(\mathbf{r}) \cdot \mathbf{h}_{j}^{\mathrm{TM}(\mathrm{R})}(\mathbf{r}) d S \\
\nu_{i}^{(m)} & =\int_{S} \mathbf{h}_{m}^{\mathrm{TM}(1)}(\mathbf{r}) \cdot \mathbf{h}_{i}^{\mathrm{TM}(\mathrm{R})}(\mathbf{r}) d S
\end{aligned}
$$

En primer lugar, es importante comentar que, puesto que los modos de la guía coaxial se van a expresar en función de las soluciones modales de la guía rectangular de expansión, en esta sección interesará trabajar con un nuevo sistema de referencia. En concreto, se utilizará el sistema de referencia representado en la figura 3.3 del capítulo 3 relativo al puerto $(\xi)=(5)^{14}$. Además, con este cambio en el sistema de referencia, el acceso coaxial queda situado en el plano $z=0$. Así pues, según la expansión (4.15), la divergencia de la función vectorial modal de campo magnético relativa al $n$-ésimo modo de tipo $\mathrm{TE}^{z}$ de la guía coaxial puede calcularse como:

$$
\begin{aligned}
\nabla_{S} \cdot \mathbf{h}_{n}^{\mathrm{TE}(1)}(\mathbf{r}) & =\sum_{i=1}^{Q^{\mathrm{TE}}} \sigma_{i}^{(n)} \nabla_{S} \cdot \mathbf{h}_{i}^{\mathrm{TE}(\mathrm{R})}(\mathbf{r})+\sum_{j=1}^{Q^{\mathrm{TM}}} \tau_{j}^{(n)} \nabla_{S} \cdot \mathbf{h}_{j}^{\mathrm{TM}(\mathrm{R})}(\mathbf{r}) \\
& =\sum_{i=1}^{Q^{\mathrm{TE}}} \sigma_{i}^{(n)} \nabla_{S} \cdot \mathbf{h}_{i}^{\mathrm{TE}(\mathrm{R})}(\mathbf{r})
\end{aligned}
$$

ya que $\nabla_{S} \cdot \mathbf{h}_{j}^{\mathrm{TM}(\mathrm{R})}(\mathbf{r})=0$ (ver sección C.1 del Apéndice C). En consecuencia, la integral con respecto a las variables primadas de la expresión (4.5) para el bloque $G_{m n}^{(1,1)}$ queda de la siguiente manera:

$$
\zeta_{n}(\mathbf{r})=\int_{S^{\prime}} g^{\mathrm{m}}\left(\mathbf{r}, \mathbf{r}^{\prime}\right) \nabla_{S}^{\prime} \cdot \mathbf{h}_{n}^{\mathrm{TE}(1)}\left(\mathbf{r}^{\prime}\right) d S^{\prime}=\sum_{i=1}^{Q^{\mathrm{TE}}} \sigma_{i}^{(n)} \int_{S_{\mathrm{R}}^{\prime}} g^{\mathrm{m}}\left(\mathbf{r}, \mathbf{r}^{\prime}\right) \nabla_{S}^{\prime} \cdot \mathbf{h}_{i}^{\mathrm{TE}(\mathrm{R})}\left(\mathbf{r}^{\prime}\right) d S^{\prime}
$$

donde $S_{\mathrm{R}}$ denota la superficie de la sección transversal de la guía rectangular de expansión. Por otro lado, utilizando ahora el resultado deducido en la expresión (3.25) del capítulo 3, se

\footnotetext{
${ }^{14} \mathrm{De}$ acuerdo con este nuevo sistema de coordenadas, que se ha representado en la figura 4.4 usando la notación $\left(\hat{\mathbf{x}}_{c}, \hat{\mathbf{y}}_{c}\right)$, los modos de la guía rectangular de expansión se propagan en sentido entrante a la estructura.
} 
puede afirmar:

$$
\begin{aligned}
\int_{S_{\mathrm{R}}^{\prime}} g^{\mathrm{m}}\left(\mathbf{r}, \mathbf{r}^{\prime}\right) \nabla_{S}^{\prime} \cdot \mathbf{h}_{i}^{\mathrm{TE}(\mathrm{R})}\left(\mathbf{r}^{\prime}\right) d S^{\prime}= & \frac{k_{t, i}^{(\mathrm{R})}}{b} \sqrt{\frac{\epsilon_{i_{x}} \epsilon_{i_{y}}}{c a}} \cos \left(\frac{i_{x} \pi}{c} x\right) \cos \left(\frac{i_{y} \pi}{a} y\right) \\
& \cdot \sum_{n_{z}=0}^{\infty} \frac{\epsilon_{n_{z}}}{\left(k_{t, i}^{(\mathrm{R})}\right)^{2}+k_{z}^{2}} \cos \left(\frac{n_{z} \pi}{b} z\right)
\end{aligned}
$$

donde $\left(i_{x}, i_{y}\right)$ son los índices modales del $i$-ésimo modo de tipo $\mathrm{TE}^{z}$ de la guía rectangular de expansión relativos a las coordenadas $\hat{\mathbf{x}}$ e $\hat{\mathbf{y}}$ del sistema de referencia anteriormente indicado; $k_{t, i}^{(\mathrm{R})}$ representa el número de onda de corte del $i$-ésimo modo de la guía rectangular de expansión; y $k_{z}=n_{z} \pi / b$. Así pues, finalmente se tiene que:

$$
\begin{aligned}
\zeta_{n}(\mathbf{r})= & \frac{1}{b \sqrt{c a}} \sum_{i=1}^{Q^{\mathrm{TE}}}\left\{\sigma_{i}^{(n)} k_{t, i}^{(\mathrm{R})} \sqrt{\epsilon_{i_{x}} \epsilon_{i_{y}}} \cos \left(\frac{i_{x} \pi}{c} x\right) \cos \left(\frac{i_{y} \pi}{a} y\right)\right. \\
& \left.\cdot \sum_{n_{z}=0}^{\infty} \frac{\epsilon_{n_{z}}}{\left(k_{t, i}^{(\mathrm{R})}\right)^{2}+k_{z}^{2}} \cos \left(\frac{n_{z} \pi}{b} z\right)\right\}
\end{aligned}
$$

Con este resultado, y teniendo en cuenta que, según el nuevo sistema de referencia, el puerto (1) se encuentra en el plano $z=0$, los elementos del bloque $G_{m n}^{(1,1)}$ se pueden expresar de la siguiente manera:

$$
G_{m n}^{(1,1)}=\left.\int_{S} \zeta_{n}(\mathbf{r})\right|_{z=0} \nabla_{S} \cdot \mathbf{h}_{m}^{\mathrm{TE}(1)}(\mathbf{r}) d S
$$

Por otro lado, si $\left(j_{x}, j_{y}\right)$ son los índices modales del $j$-ésimo modo de tipo $\mathrm{TE}^{z}$ de la guía rectangular de expansión relativos a las coordenadas $\hat{\mathbf{x}}$ e $\hat{\mathbf{y}}$ del nuevo sistema de referencia, respectivamente, podemos escribir gracias a los resultados del Apéndice C:

$$
\nabla_{S} \cdot \mathbf{h}_{m}^{\mathrm{TE}(1)}(\mathbf{r})=\sum_{j=1}^{Q^{\mathrm{TE}}} \sigma_{j}^{(m)} \nabla_{S} \cdot \mathbf{h}_{j}^{\mathrm{TE}(\mathrm{R})}(\mathbf{r})=\sum_{j=1}^{Q^{\mathrm{TE}}} \sigma_{j}^{(m)} k_{t, j}^{(\mathrm{R})} \sqrt{\frac{\epsilon_{j_{x}} \epsilon_{j_{y}}}{c a}} \cos \left(\frac{j_{x} \pi}{c} x\right) \cos \left(\frac{j_{y} \pi}{a} y\right)
$$

Así pues, introduciendo (4.24) y (4.26) en (4.25) nos queda:

$$
\begin{aligned}
G_{m n}^{(1,1)}= & \frac{1}{a b c} \sum_{i=1}^{Q^{\mathrm{TE}}} \sum_{j=1}^{Q^{\mathrm{TE}}} \sigma_{j}^{(m)} \sigma_{i}^{(n)} k_{t, i}^{(\mathrm{R})} k_{t, j}^{(\mathrm{R})} \sqrt{\epsilon_{i_{x}} \epsilon_{i_{y}}} \sqrt{\epsilon_{j_{x}} \epsilon_{j_{y}}}\left(\sum_{n_{z}=0}^{\infty} \frac{\epsilon_{n_{z}}}{\left(k_{t, i}^{(\mathrm{R})}\right)^{2}+k_{z}^{2}}\right) \\
& \cdot \int_{S_{\mathrm{R}}} \cos \left(\frac{i_{x} \pi}{c} x\right) \cos \left(\frac{j_{x} \pi}{c} x\right) \cos \left(\frac{i_{y} \pi}{a} y\right) \cos \left(\frac{j_{y} \pi}{a} y\right) d S
\end{aligned}
$$

La integral de superficie de la expresión (4.27) puede resolverse fácilmente atendiendo al resultado obtenido en (3.27). Efectivamente, se tiene que:

$$
\int_{S_{\mathrm{R}}} \cos \left(\frac{i_{x} \pi}{c} x\right) \cos \left(\frac{j_{x} \pi}{c} x\right) \cos \left(\frac{i_{y} \pi}{a} y\right) \cos \left(\frac{j_{y} \pi}{a} y\right) d S=\frac{c}{\epsilon_{i_{x}}} \frac{a}{\epsilon_{i_{y}}} \delta_{i_{x}, j_{x}} \delta_{i_{y}, j_{y}}
$$


donde $\delta_{i_{x}, j_{x}}$ representa la delta de Kronecker. Igualmente, el sumatorio infinito en la variable $n_{z}$ presente en (4.27) puede resolverse analíticamente utilizando el resultado (D.1) del Apéndice $\mathrm{D}^{15}$. Con todo ello, se puede concluir fácilmente:

$$
G_{m n}^{(1,1)}=\sum_{i=1}^{Q^{\mathrm{TE}}} \sigma_{i}^{(m)} \sigma_{i}^{(n)} k_{t, i}^{(\mathrm{R})} \operatorname{coth}\left(k_{t, i}^{(\mathrm{R})} b\right)
$$

\subsubsection{Cálculo de los elementos de la matriz $\mathrm{T}$}

Los elementos de la matriz $\mathbf{T}$ se calculan de la siguiente forma:

$$
T_{m n}^{(\gamma, \xi)}=\int_{S} \int_{S^{\prime}} \mathbf{h}_{m}^{(\gamma)}(\mathbf{r}) \cdot \overline{\mathbf{G}}_{0}^{\mathrm{F}}\left(\mathbf{r}, \mathbf{r}^{\prime}\right) \cdot \mathbf{h}_{n}^{(\xi)}\left(\mathbf{r}^{\prime}\right) d S d S^{\prime}
$$

donde $m, n=1,2, \ldots, N ; \gamma, \xi=1,2$ representan los accesos de la estructura, y $\overline{\mathbf{G}}_{0}^{\mathrm{F}}\left(\mathbf{r}, \mathbf{r}^{\prime}\right)$ es la función diádica de Green de tipo magnético relativa al resonador externo escogido. Al igual que la matriz $\mathbf{G}$, la matriz $\mathbf{T}$ también es simétrica, por lo que $T_{m n}^{(\gamma, \xi)}=T_{n m}^{(\xi, \gamma)}(\gamma \neq \xi)$. En consecuencia, en esta sección se calcularán dos de los cuatro bloques que componen la matriz T. En primer lugar, se obtendrán los elementos del bloque $T_{m n}^{(1,2)}$, mientras que el bloque $T_{m n}^{(2,1)}$ se obtendrá por simetría a partir de $T_{m n}^{(1,2)}$. Posteriormente, se calcularán los elementos del bloque $T_{m n}^{(1,1)}$. En este último caso, puesto que este bloque también presenta problemas de singularidad, se empleará la misma estrategia que la que se ha utilizado en la sección anterior para el cálculo de los elementos del bloque $G_{m n}^{(1,1)}$. Finalmente, el cálculo de los elementos del bloque $T_{m n}^{(2,2)}$ puede llevarse a cabo a partir de las expresiones (3.47)-(3.51) deducidas en el capítulo 3.

\section{A. Cálculo de los elementos del bloque $T_{m n}^{(1,2)}$}

En primer lugar, se procede al cálculo de los elementos del bloque $T_{m n}^{(1,2)}$. En este caso, $m$ es un modo del puerto (1) (acceso coaxial) y $n$ representa un modo del puerto (2) (acceso rectangular). Además, se asume que $\left(n_{x}, n_{y}\right)$ son los índices modales del $n$-ésimo modo del puerto (2) referidos al sistema de referencia en coordenadas cartesianas representado en la figura 4.3. Teniendo en cuenta que $n$ puede ser un modo de tipo $\mathrm{TE}^{z}$ o $\mathrm{TM}^{z}$, y que $m$ puede ser de tipo $\mathrm{TEM}^{z}, \mathrm{TE}^{z}$ o $\mathrm{TM}^{z}$, el resultado final deberá particularizarse para cada uno de estos casos.

Caso 1: $n$ es un modo de tipo $\mathrm{TE}^{z}$

En virtud de los resultados obtenidos en las expresiones (3.36)-(3.38) del capítulo 3,

\footnotetext{
${ }^{15}$ En concreto, si se toma $z=0$ en la expresión (D.1), se obtiene la serie infinita de la expresión (4.27).
} 
podemos escribir en primer lugar:

$$
\begin{aligned}
\boldsymbol{\Gamma}_{n_{\mathrm{TE}}}(\mathbf{r})=\int_{S^{\prime}} \overline{\mathbf{G}}_{0}^{\mathrm{F}}\left(\mathbf{r}, \mathbf{r}^{\prime}\right) \cdot \mathbf{h}_{n}^{\mathrm{TE}(2)}\left(\mathbf{r}^{\prime}\right) d S^{\prime}= & -\frac{k_{x, n}^{(2)}}{k_{t, n}^{(2)}} \frac{2}{c} \sqrt{\frac{\epsilon_{n_{x}} \epsilon_{n_{y}}}{a b}} \sin \left(k_{x, n}^{(2)} x\right) \cos \left(k_{y, n}^{(2)} y\right) S_{n}^{(1)}(z) \hat{\mathbf{x}} \\
& -\frac{k_{y, n}^{(2)}}{k_{t, n}^{(2)}} \frac{2}{c} \sqrt{\frac{\epsilon_{n_{x}} \epsilon_{n_{y}}}{a b}} \cos \left(k_{x, n}^{(2)} x\right) \sin \left(k_{y, n}^{(2)} y\right) S_{n}^{(1)}(z) \hat{\mathbf{y}} \\
& +k_{t, n}^{(2)} \frac{2}{c} \sqrt{\frac{\epsilon_{n_{x}} \epsilon_{n_{y}}}{a b}} \cos \left(k_{x, n}^{(2)} x\right) \cos \left(k_{y, n}^{(2)} y\right) S_{n}^{(2)}(z) \hat{\mathbf{z}}
\end{aligned}
$$

donde $k_{x, n}^{(2)}=n_{x} \pi / a ; k_{y, n}^{(2)}=n_{y} \pi / b ; \mathrm{y} k_{t, n}^{(2)}$ representa el número de onda de corte del $n$-ésimo modo de tipo $\mathrm{TE}^{z}$ del acceso (2). Además, según los resultados deducidos en el Apéndice $\mathrm{F}$, las series $S_{n}^{(1)}(z)$ y $S_{n}^{(2)}(z)$ de (4.31) se pueden sumar analíticamente obteniéndose el siguiente resultado:

$$
\begin{aligned}
S_{n}^{(1)}(z) & =\sum_{n_{z}=1}^{\infty}(-1)^{n_{z}} \frac{k_{z}^{2}}{\left(\left(k_{t, n}^{(2)}\right)^{2}+k_{z}^{2}\right)^{2}} \cos \left(k_{z} z\right) \\
& =\frac{c}{4 \sinh \left(k_{t, n}^{(2)} c\right)}\left\{z \sinh \left(k_{t, n}^{(2)} z\right)+\left[\frac{1}{k_{t, n}^{(2)}}-c \operatorname{coth}\left(k_{t, n}^{(2)} c\right)\right] \cosh \left(k_{t, n}^{(2)} z\right)\right\} \\
S_{n}^{(2)}(z) & =\sum_{n_{z}=1}^{\infty}(-1)^{n_{z}} \frac{k_{z}}{\left(\left(k_{t, n}^{(2)}\right)^{2}+k_{z}^{2}\right)^{2}} \sin \left(k_{z} z\right) \\
& =\frac{c}{4 k_{t, n}^{(2)} \sinh \left(k_{t, n}^{(2)} c\right)}\left[z \operatorname{coth}\left(k_{t, n}^{(2)} z\right)-\frac{c \cosh \left(k_{t, n}^{(2)} c\right)}{\sinh \left(k_{t, n}^{(2)} c\right)} \sinh \left(k_{t, n}^{(2)} z\right)\right]
\end{aligned}
$$

donde $k_{z}=n_{z} \pi / c$. Para finalizar, se debe expresar el resultado (4.31) en coordenadas cilíndricas, y para ello se emplean las relaciones deducidas en (4.1)-(4.4). Así, a partir de ahora, se utilizará la notación $\Gamma_{n_{\mathrm{TE}}}(\rho, \phi)$ para representar el resultado de la ecuación (4.31) expresado en coordenadas cilíndricas. Asimismo, se empleará $S_{n}^{(1)}(\rho, \phi)$ y $S_{n}^{(2)}(\rho, \phi)$ para referirnos a las series (4.32) y (4.33), respectivamente, expresadas en coordenadas cilíndricas.

Supongamos ahora que $m$ es un modo de tipo $\mathrm{TEM}^{z}$ del puerto (1) (acceso coaxial). En este caso, según el resultado (2.63) de la sección 2.3.1, la función vectorial modal normalizada de campo magnético es de la forma:

$$
\mathbf{h}_{m}^{\operatorname{TEM}(1)}(\rho)=\frac{1}{\sqrt{2 \pi \ln \left(r_{1} / r_{2}\right)}} \frac{1}{\rho} \hat{\boldsymbol{\phi}}
$$


En consecuencia, tras particularizar el resultado (4.31) expresado en coordenadas cilíndricas en el plano $y=b$ (plano en el que se encuentra el acceso coaxial), se concluye:

$$
\begin{aligned}
T_{m_{\mathrm{TEM},}, n_{\mathrm{TE}}}^{(1,2)} & =\int_{S} \mathbf{h}_{m}^{\mathrm{TEM}(1)}(\rho) \cdot \boldsymbol{\Gamma}_{n_{\mathrm{TE}}}(\rho, \phi) d S \\
& =\frac{1}{\sqrt{2 \pi \ln \left(r_{1} / r_{2}\right)}} \frac{2}{c} \sqrt{\frac{\epsilon_{n_{x}} \epsilon_{n_{y}}}{a b}}(-1)^{n_{y}}\left\{k_{t, n}^{(2)} I_{m_{\mathrm{TEM}}, n_{\mathrm{TE}}}^{(2)}-\frac{k_{x, n}^{(2)}}{k_{t, n}^{(2)}} I_{m_{\mathrm{TEM}}, n_{\mathrm{TE}}}^{(1)}\right\}
\end{aligned}
$$

donde las integrales $I_{m_{\mathrm{TEM}}, n_{\mathrm{TE}}}^{(\alpha)}(\alpha=1,2)$ se definen en la sección G.1 del Apéndice G.

A continuación, supongamos que $m$ es un modo de tipo $\mathrm{TE}^{z}$ del acceso coaxial, y que $(s, q)$ son los correspondientes índices modales relativos a las variaciones angular y radial, respectivamente. La función vectorial modal normalizada de campo magnético de tipo $\mathrm{TE}^{z}$ es (ver expresión (2.118) de la sección 2.3.1):

$$
\begin{aligned}
\mathbf{h}_{m_{(\zeta)}}^{\mathrm{TE}(1)}(\rho, \phi)= & -\mathcal{N}_{(\zeta), m}^{\mathrm{TE}} \frac{\pi}{r_{1}-r_{2}}\left(\sum_{r=0}^{N_{\rho}-1} r l_{s, q}^{(r)} \sin \left(r \pi \frac{\rho-r_{2}}{r_{1}-r_{2}}\right)\right) \Phi_{(\zeta), s}(\phi) \hat{\boldsymbol{\rho}} \\
& +\mathcal{N}_{(\zeta), m}^{\mathrm{TE}} \frac{1}{\rho}\left(\sum_{r=0}^{N_{\rho}-1} l_{s, q}^{(r)} \cos \left(r \pi \frac{\rho-r_{2}}{r_{1}-r_{2}}\right)\right) \Phi_{(\zeta), s}^{\prime}(\phi) \hat{\boldsymbol{\phi}}
\end{aligned}
$$

donde $(\zeta)=(c),(s)$ denota la paridad del modo, y $\Phi_{(\zeta), s}(\phi)$, cuya expresión se recoge en (2.71), representa la variación angular de las soluciones modales para la guía coaxial. Así pues, se puede concluir:

$$
\begin{aligned}
T_{m_{\mathrm{TE}}, n_{\mathrm{TE}}}^{(1,2)}= & \int_{S} \mathbf{h}_{m_{(\zeta)}}^{\mathrm{TE}(1)}(\rho, \phi) \cdot \boldsymbol{\Gamma}_{n_{\mathrm{TE}}}(\rho, \phi) d S \\
= & \mathcal{N}_{(\zeta), m}^{\mathrm{TE}} \frac{2}{c} \sqrt{\frac{\epsilon_{n_{x}} \epsilon_{n_{y}}}{a b}}(-1)^{n_{y}}\left\{\frac{\pi}{r_{1}-r_{2}} \frac{k_{x, n}^{(2)}}{k_{t, n}^{(2)}} \sum_{r=0}^{N_{\rho}-1} r l_{s, q}^{(r)} I_{m_{\mathrm{TE}}, n_{\mathrm{TE}}}^{(r),(1)}\right. \\
& +\frac{\pi}{r_{1}-r_{2}} k_{t, n}^{(2)} \sum_{r=0}^{N_{\rho}-1} r l_{s, q}^{(r)} I_{m_{\mathrm{TE}}, n_{\mathrm{TE}}}^{(r),(2)}-\frac{k_{x, n}^{(2)}}{k_{t, n}^{(2)}} \sum_{r=0}^{N_{\rho}-1} l_{s, q}^{(r)} I_{m_{\mathrm{TE}}, n_{\mathrm{TE}}}^{(r),(3)} \\
& \left.+k_{t, n}^{(2)} \sum_{r=0}^{N_{\rho}-1} l_{s, q}^{(r)} I_{m_{\mathrm{TE}}, n_{\mathrm{TE}}}^{(r),(4)}\right\}
\end{aligned}
$$

La definición de las integrales de superficie $I_{m \mathrm{TE}}^{(r), n_{\mathrm{TE}}}(\alpha=1,2,3,4)$ que aparecen en esta última expresión puede encontrarse en la sección G.1 del Apéndice G. 
Finalmente, supongamos que $m$ es un modo del acceso coaxial de tipo $\mathrm{TM}^{z}$ y que $(s, q)$ siguen siendo los índices modales correspondientes a las variaciones angular y radial, respectivamente. En este caso, la función vectorial modal normalizada de campo magnético es la siguiente (ver expresión (2.96) de la sección 2.3.1):

$$
\begin{aligned}
\mathbf{h}_{m_{(\zeta)}}^{\mathrm{TM}(1)}(\rho, \phi)= & -\mathcal{N}_{(\zeta), m}^{\mathrm{TM}} \frac{1}{\rho}\left(\sum_{r=1}^{N_{\rho}} d_{s, q}^{(r)} \sin \left(r \pi \frac{\rho-r_{2}}{r_{1}-r_{2}}\right)\right) \Phi_{(\zeta), s}^{\prime}(\phi) \hat{\boldsymbol{\rho}} \\
& +\mathcal{N}_{(\zeta), m}^{\mathrm{TM}} \frac{\pi}{r_{1}-r_{2}}\left(\sum_{r=1}^{N_{\rho}} r d_{s, q}^{(r)} \cos \left(r \pi \frac{\rho-r_{2}}{r_{1}-r_{2}}\right)\right) \Phi_{(\zeta), s}(\phi) \hat{\boldsymbol{\phi}}(4.3
\end{aligned}
$$

En este caso, se puede afirmar que:

$$
\begin{aligned}
T_{m_{\mathrm{TM}}, n_{\mathrm{TE}}}^{(1,2)}= & \int_{S} \mathbf{h}_{m_{(\zeta)}}^{\mathrm{TM}(1)}(\rho, \phi) \cdot \boldsymbol{\Gamma}_{n_{\mathrm{TE}}}(\rho, \phi) d S \\
= & \mathcal{N}_{(\zeta), m}^{\mathrm{TM}} \frac{2}{c} \sqrt{\frac{\epsilon_{n_{x}} \epsilon_{n_{y}}}{a b}}(-1)^{n_{y}}\left\{\frac{k_{x, n}^{(2)}}{k_{t, n}^{(2)}} \sum_{r=1}^{N_{\rho}} d_{s, q}^{(r)} I_{m_{\mathrm{TM}}, n_{\mathrm{TE}}}^{(r),(1)}\right. \\
& +k_{t, n}^{(2)} \sum_{r=1}^{N_{\rho}} d_{s, q}^{(r)} I_{m_{\mathrm{TM}}, n_{\mathrm{TE}}}^{(r),(2)}-\frac{\pi}{r_{1}-r_{2}} \frac{k_{x, n}^{(2)}}{k_{t, n}^{(2)}} \sum_{r=1}^{N_{\rho}} r d_{s, q}^{(r)} I_{m_{\mathrm{TM}}, n_{\mathrm{TE}}}^{(r),(3)} \\
& \left.+\frac{\pi}{r_{1}-r_{2}} k_{t, n}^{(2)} \sum_{r=1}^{N_{\rho}} r d_{s, q}^{(r)} I_{m_{\mathrm{TM}}, n_{\mathrm{TE}}}^{(r),(4)}\right\}
\end{aligned}
$$

donde las integrales $I_{m_{\mathrm{TM}}, n_{\mathrm{TE}}}^{(r),(\alpha)}(\alpha=1,2,3,4)$ se han definido en la sección G.1 del Apéndice $\mathrm{G}$.

Caso 2: n es un modo de tipo $\mathrm{TM}^{z}$

Si recuperamos las expresiones (3.39)-(3.41) deducidas en el capítulo 3, podemos escribir que:

$$
\begin{aligned}
\boldsymbol{\Gamma}_{n_{\mathrm{TM}}}(\mathbf{r})=\int_{S^{\prime}} \overline{\mathbf{G}}_{0}^{\mathrm{F}}\left(\mathbf{r}, \mathbf{r}^{\prime}\right) \cdot \mathbf{h}_{n}^{\mathrm{TM}(2)}\left(\mathbf{r}^{\prime}\right) d S^{\prime}= & -\frac{k_{y, n}^{(2)}}{k_{t, n}^{(2)}} \frac{2}{c \sqrt{a b}} \sin \left(k_{x, n}^{(2)} x\right) \cos \left(k_{y, n}^{(2)} y\right) S_{n}(z) \hat{\mathbf{x}} \\
& +\frac{k_{x, n}^{(2)}}{k_{t, n}^{(2)}} \frac{2}{c \sqrt{a b}} \cos \left(k_{x, n}^{(2)} x\right) \sin \left(k_{y, n}^{(2)} y\right) S_{n}(z) \hat{\mathbf{y}}
\end{aligned}
$$

En esta última expresión, $k_{x, n}^{(2)}=n_{x} \pi / a ; k_{y, n}^{(2)}=n_{y} \pi / b ; k_{t, n}^{(2)}$ es el número de onda de corte del $n$-ésimo modo de tipo $\mathrm{TM}^{z}$ del acceso $(2)$; y $S_{n}(z)$ representa el siguiente sumatorio 
recogido en el Apéndice D:

$$
S_{n}(z)=\sum_{n_{z}=0}^{\infty}(-1)^{n_{z}} \frac{\epsilon_{n_{z}}}{\left(k_{t, n}^{(2)}\right)^{2}+k_{z}^{2}} \cos \left(k_{z} z\right)=\frac{c}{k_{t, n}^{(2)}} \frac{\cosh \left(k_{t, n}^{(2)} z\right)}{\sinh \left(k_{t, n}^{(2)} c\right)}
$$

donde $k_{z}=n_{z} \pi / c$. A continuación, el resultado obtenido en (4.40) debe expresarse en coordenadas cilíndricas utilizando las relaciones (4.1)-(4.4). Por ello, a partir de ahora, emplearemos la notación $\boldsymbol{\Gamma}_{n_{\mathrm{TM}}}(\rho, \phi)$ para referirnos al resultado (4.40) expresado en coordenadas cilíndricas, y $S_{n}(\rho, \phi)$ para representar la serie (4.41) en coordenadas cilíndricas.

Seguidamente, supongamos en primer lugar que $m$ representa al modo de tipo $\mathrm{TEM}^{z}$ del puerto (1) (acceso coaxial). En este caso, si utilizamos la función vectorial modal (4.34), y particularizamos la función vectorial $\Gamma_{n_{\mathrm{TM}}}(\rho, \phi)$ en el plano $y=b$, se deduce fácilmente que:

$$
\begin{aligned}
T_{m_{\mathrm{TEM}}, n_{\mathrm{TM}}}^{(1,2)} & =\int_{S} \mathbf{h}_{m}^{\mathrm{TEM}(1)}(\rho) \cdot \boldsymbol{\Gamma}_{n_{\mathrm{TM}}}(\rho, \phi) d S \\
& =-\frac{1}{\sqrt{2 \pi \ln \left(r_{1} / r_{2}\right)}} \frac{2}{\sqrt{a b}} \frac{k_{y, n}^{(2)}}{\left(k_{t, n}^{(2)}\right)^{2}}(-1)^{n_{y}} \frac{1}{\sinh \left(k_{t, n}^{(2)} c\right)} I_{m_{\mathrm{TEM}}, n_{\mathrm{TM}}}
\end{aligned}
$$

donde la integral $I_{m_{\mathrm{TEM}}, n_{\mathrm{TM}}}$ se encuentra definida en la sección G.1 del Apéndice G.

Sea ahora $m$ un modo de tipo $\mathrm{TE}^{z}$ de la guía coaxial, y sean $(s, q)$ los índices modales asociados a las variaciones angular y radial, respectivamente. Si seguidamente se emplea la función vectorial modal normalizada de campo magnético de tipo $\mathrm{TE}^{z}$ definida en (4.36), se obtiene que:

$$
\begin{aligned}
T_{m_{\mathrm{TE}}, n_{\mathrm{TM}}}^{(1,2)}= & \int_{S} \mathbf{h}_{m_{(\zeta)}^{\mathrm{TE}(1)}}(\rho, \phi) \cdot \boldsymbol{\Gamma}_{n_{\mathrm{TM}}}(\rho, \phi) d S \\
= & \mathcal{N}_{(\zeta), m}^{\mathrm{TE}} \frac{2}{c \sqrt{a b}} \frac{k_{y, n}^{(2)}}{k_{t, n}^{(2)}}(-1)^{n_{y}}\left\{\frac{\pi}{r_{1}-r_{2}} \sum_{r=0}^{N_{\rho}-1} r l_{s, q}^{(r)} I_{m_{\mathrm{TE}}, n_{\mathrm{TM}}}^{(r),(1)}\right. \\
& \left.-\sum_{r=0}^{N_{\rho}-1} l_{s, q}^{(r)} I_{m_{\mathrm{TE}}, n_{\mathrm{TM}}}^{(r),(2)}\right\}
\end{aligned}
$$

donde, nuevamente, la definición de las integrales $I_{m_{\mathrm{TE}}, n_{\mathrm{TM}}}^{(r),(\alpha)}(\alpha=1,2)$ puede hallarse en el Apéndice G. Finalmente, si $m$ es un modo de tipo $\mathrm{TM}^{z}$ de la guía coaxial, y $(s, q)$ es la 
pareja de índices modales correspondiente, podemos escribir utilizando (4.38) y (4.40) que:

$$
\begin{aligned}
T_{m_{\mathrm{TM}}, n_{\mathrm{TM}}}^{(1,2)}= & \int_{S} \mathbf{h}_{m_{(\zeta)}}^{\mathrm{TM}(1)}(\rho, \phi) \cdot \boldsymbol{\Gamma}_{n_{\mathrm{TM}}}(\rho, \phi) d S \\
= & \mathcal{N}_{(\zeta), m}^{\mathrm{TM}} \frac{2}{c \sqrt{a b}} \frac{k_{y, n}^{(2)}}{k_{t, n}^{(2)}}(-1)^{n_{y}}\left\{\sum_{r=1}^{N_{\rho}} d_{s, q}^{(r)} I_{m_{\mathrm{TM}}, n_{\mathrm{TM}}}^{(r),(1)}\right. \\
& \left.-\frac{\pi}{r_{1}-r_{2}} \sum_{r=1}^{N_{\rho}} r d_{s, q}^{(r)} I_{m_{\mathrm{TM}}, n_{\mathrm{TM}}}^{(r),(2)}\right\}
\end{aligned}
$$

donde las integrales $I_{m_{\mathrm{TM}}, n_{\mathrm{TM}}}^{(r),(\alpha)}(\alpha=1,2)$ se han definido en el Apéndice G.

\section{B. Cálculo de los elementos del bloque $T_{m n}^{(1,1)}$}

En este caso, $m$ y $n$ son modos del acceso de tipo coaxial, por lo que, al evaluar la integral (4.30), se tiene una singularidad en $\mathbf{r}=\mathbf{r}^{\prime} \mathrm{y}$, en consecuencia, la función diádica de Green de tipo magnético diverge en dicho punto [94]. En concreto, la singularidad de la función diádica de Green de tipo magnético es de la forma [95]:

$$
\overline{\mathbf{G}}_{0(\mathrm{~s})}^{\mathrm{F}}\left(\mathbf{r}, \mathbf{r}^{\prime}\right)=\frac{1}{8 \pi\left|\mathbf{r}-\mathbf{r}^{\prime}\right|}\left(\overline{\mathbf{I}}+\frac{\left(\mathbf{r}-\mathbf{r}^{\prime}\right)\left(\mathbf{r}-\mathbf{r}^{\prime}\right)}{\left|\mathbf{r}-\mathbf{r}^{\prime}\right|^{2}}\right)
$$

donde $\overline{\mathbf{I}}$ representa la diádica unidad [96]. Por tanto, la parte singular del bloque $T_{m n}^{(1,1)}$ de la matriz $\mathbf{T}$ puede expresarse de la siguiente manera:

$$
T_{(\mathrm{s}), m n}^{(1,1)}=\int_{S} \int_{S^{\prime}} \mathbf{h}_{m}^{(1)}(\mathbf{r}) \cdot \frac{1}{8 \pi\left|\mathbf{r}-\mathbf{r}^{\prime}\right|}\left(\overline{\mathbf{I}}+\frac{\left(\mathbf{r}-\mathbf{r}^{\prime}\right)\left(\mathbf{r}-\mathbf{r}^{\prime}\right)}{\left|\mathbf{r}-\mathbf{r}^{\prime}\right|^{2}}\right) \cdot \mathbf{h}_{n}^{(1)}\left(\mathbf{r}^{\prime}\right) d S d S^{\prime}
$$

Con el objetivo de evitar el cálculo de la singularidad presente en este bloque de la matriz $\mathbf{T}$ se propone utilizar la misma estrategia que la que se empleó para la matriz G. En particular, las soluciones modales del acceso coaxial se expresarán en términos de una combinación lineal de las funciones vectoriales modales de la guía rectangular a la que la sonda coaxial alimenta (guía rectangular de expansión de dimensiones $c \times a$ ). De esta manera, puesto que la función diádica de Green de tipo magnético del resonador externo puede expresarse en términos de las funciones vectoriales modales correspondientes a la guía rectangular de expansión, el cálculo de los elementos del bloque $T_{m n}^{(1,1)}$ puede efectuarse directamente sin extraer previamente el término singular (4.46). A continuación, dado que $m$ y $n$ pueden referirse a un modo de tipo $\mathrm{TEM}^{z}, \mathrm{TE}^{z}$ o $\mathrm{TM}^{z}$, el cálculo debe particularizarse para cada uno de estos casos.

\section{Caso 1: $n$ es un modo de tipo $\mathrm{TEM}^{z}$}

En primer lugar, es importante comentar que, ya que los modos de la guía coaxial se van a expresar en función de las soluciones modales de la guía rectangular de expansión, en esta 
sección interesará trabajar con un nuevo sistema de referencia, tal y como se planteó en el cálculo del bloque $G_{m n}^{(1,1)}$. Así pues, se utilizará el sistema de referencia representado en la figura 3.3 del capítulo 3 relativo al puerto $(\xi)=(5)$. Es importante hacer notar que el acceso coaxial queda ahora situado en el plano $z=0$. Si recuperamos las expresiones presentadas en (4.14)-(4.16) sabemos que, en caso de que $n$ sea un modo de tipo $\mathrm{TEM}^{z}$ de la guía coaxial, la expansión de la función vectorial modal correspondiente en términos de las soluciones modales de la guía rectangular de dimensiones $c \times a$ es de la forma:

$$
\mathbf{h}_{n}^{\mathrm{TEM}(1)}(\mathbf{r})=\sum_{i=1}^{Q^{\mathrm{TM}}} v_{i}^{(\mathrm{TEM})} \mathbf{h}_{i}^{\mathrm{TM}(\mathrm{R})}(\mathbf{r})
$$

donde la expresión de la función vectorial modal normalizada de campo magnético $\mathbf{h}_{i}^{\mathrm{TM}(\mathrm{R})}(\mathbf{r})$ puede deducirse fácilmente a partir de la teoría desarrollada en el Apéndice C. En consecuencia, utilizando (4.47), y gracias a los resultados deducidos en (3.39)-(3.41), podemos escribir directamente que:

$$
\begin{aligned}
\int_{S^{\prime}} \overline{\mathbf{G}}_{0}^{\mathrm{F}}\left(\mathbf{r}, \mathbf{r}^{\prime}\right) \cdot \mathbf{h}_{n}^{\mathrm{TEM}(1)}\left(\mathbf{r}^{\prime}\right) d S^{\prime}= & \sum_{i=1}^{Q^{\mathrm{TM}}} v_{i}^{(\mathrm{TEM})} \int_{S_{\mathrm{R}}^{\prime}} \overline{\mathbf{G}}_{0}^{\mathrm{F}}\left(\mathbf{r}, \mathbf{r}^{\prime}\right) \cdot \mathbf{h}_{i}^{\mathrm{TM}(\mathrm{R})}\left(\mathbf{r}^{\prime}\right) d S^{\prime} \\
= & \frac{2}{b \sqrt{c a}}\left\{\sum_{i=1}^{Q^{\mathrm{TM}}} v_{i}^{(\mathrm{TEM})}\left(\frac{k_{y, i}^{(\mathrm{R})}}{k_{t, i}^{(\mathrm{R})}}\right) \sin \left(k_{x, i}^{(\mathrm{R})} x\right) \cos \left(k_{y, i}^{(\mathrm{R})} y\right) S^{(i)}(z) \hat{\mathbf{x}}\right. \\
& \left.-\sum_{i=1}^{Q^{\mathrm{TM}}} v_{i}^{(\mathrm{TEM})}\left(\frac{k_{x, i}^{(\mathrm{R})}}{k_{t, i}^{(\mathrm{R})}}\right) \cos \left(k_{x, i}^{(\mathrm{R})} x\right) \sin \left(k_{y, i}^{(\mathrm{R})} y\right) S^{(i)}(z) \hat{\mathbf{y}}\right\}
\end{aligned}
$$

donde $\left(k_{t, i}^{(\mathrm{R})}\right)^{2}=\left(k_{x, i}^{(\mathrm{R})}\right)^{2}+\left(k_{y, i}^{(\mathrm{R})}\right)^{2}$ es el cuadrado del número de onda de corte relativo al $i$-ésimo modo de tipo $\mathrm{TM}^{z}$ de la guía rectangular de expansión, y además se ha definido la siguiente serie (ver Apéndice D):

$$
S^{(i)}(z)=\sum_{n_{z}=1}^{\infty} \frac{\epsilon_{n_{z}}}{\left(k_{t, i}^{(\mathrm{R})}\right)^{2}+k_{z}^{2}} \cos \left(k_{z} z\right)=\frac{b}{k_{t, i}^{(\mathrm{R})}} \frac{\cosh \left[k_{t, i}^{(\mathrm{R})}(b-z)\right]}{\sinh \left(k_{t, i}^{(\mathrm{R})} b\right)}
$$

siendo $k_{z}=n_{z} \pi / b$.

Seguidamente, consideremos que $m$ es un modo de tipo $\mathrm{TEM}^{z}$ del acceso coaxial (realmente, $m$ y $n$ representan el mismo modo $\mathrm{TEM}^{z}$, por lo que el modo $m$ se expandirá también 
utilizando una expresión idéntica a (4.47)). En este caso, resulta sencillo concluir que:

$$
\begin{aligned}
T_{m_{\mathrm{TEM}}, n_{\mathrm{TEM}}}^{(1,1)}= & \int_{S} \int_{S^{\prime}} \mathbf{h}_{m}^{\mathrm{TEM}(1)}(\mathbf{r}) \cdot \overline{\mathbf{G}}_{0}^{\mathrm{F}}\left(\mathbf{r}, \mathbf{r}^{\prime}\right) \cdot \mathbf{h}_{n}^{\mathrm{TEM}(1)}\left(\mathbf{r}^{\prime}\right) d S d S^{\prime} \\
= & \frac{4}{a b c}\left\{\sum_{i=1}^{Q^{\mathrm{TM}}} \sum_{j=1}^{Q^{\mathrm{TM}}} v_{i}^{(\mathrm{TEM})} v_{j}^{(\mathrm{TEM})} \frac{k_{y, i}^{(\mathrm{R})} k_{y, j}^{(\mathrm{R})}}{k_{t, i}^{(\mathrm{R})} k_{t, j}^{(\mathrm{R})}} S^{(i)}(z=0) I_{i, j}^{(1)}\right. \\
& \left.+\sum_{i=1}^{Q^{\mathrm{TM}}} \sum_{j=1}^{Q^{\mathrm{TM}}} v_{i}^{(\mathrm{TEM})} v_{j}^{(\mathrm{TEM})} \frac{k_{x, i}^{(\mathrm{R})} k_{x, j}^{(\mathrm{R})}}{k_{t, i}^{(\mathrm{R})} k_{t, j}^{(\mathrm{R})}} S^{(i)}(z=0) I_{i, j}^{(2)}\right\}
\end{aligned}
$$

donde hemos definido:

$$
\begin{aligned}
I_{i, j}^{(1)} & =\int_{S_{\mathrm{R}}} \sin \left(k_{x, i}^{(\mathrm{R})} x\right) \sin \left(k_{x, j}^{(\mathrm{R})} x\right) \cos \left(k_{y, i}^{(\mathrm{R})} y\right) \cos \left(k_{y, j}^{(\mathrm{R})} y\right) d x d y=\frac{a}{\epsilon_{i_{y}}} \frac{c}{2}\left(\epsilon_{i_{x}}-1\right) \delta_{i_{x}, j_{x}} \delta_{i_{y}, j_{y}} \\
I_{i, j}^{(2)} & =\int_{S_{\mathrm{R}}} \cos \left(k_{x, i}^{(\mathrm{R})} x\right) \cos \left(k_{x, j}^{(\mathrm{R})} x\right) \sin \left(k_{y, i}^{(\mathrm{R})} y\right) \sin \left(k_{y, j}^{(\mathrm{R})} y\right) d x d y=\frac{a}{2} \frac{c}{\epsilon_{i_{x}}}\left(\epsilon_{i_{y}}-1\right) \delta_{i_{x}, j_{x}} \delta_{i_{y}, j_{y}}
\end{aligned}
$$

En esta última expresión, $S_{\mathrm{R}}$ representa la sección transversal de la guía rectangular de expansión; $\left(i_{x}, i_{y}\right)$ son los índices modales del $i$-ésimo modo de tipo $\mathrm{TM}^{z}$ de la guía rectangular de expansión, referidos a las variaciones en las coordenadas $\hat{\mathbf{x}}$ e $\hat{\mathbf{y}}$, respectivamente; y $\left(j_{x}, j_{y}\right)$ representan los índices modales del $j$-ésimo modo de tipo $\mathrm{TM}^{z}$ de la misma guía. Además, $k_{x, \alpha}=\alpha_{x} \pi / c$ y $k_{y, \alpha}=\alpha_{y} \pi / a$, siendo $\alpha=i, j$. Finalmente, se puede deducir la siguiente expresión:

$$
T_{m_{\mathrm{TEM}}, n_{\mathrm{TEM}}}^{(1,1)}=\sum_{i=1}^{Q^{\mathrm{TM}}}\left(v_{i}^{(\mathrm{TEM})}\right)^{2} \frac{1}{k_{t, i}^{(\mathrm{R})}} \operatorname{coth}\left(k_{t, i}^{(\mathrm{R})} b\right)
$$

A continuación, asumimos que $m$ es un modo de tipo $\mathrm{TE}^{z}$ del acceso coaxial. En este caso, la función vectorial modal correspondiente se puede expresar en función de las soluciones modales de la guía rectangular de expansión en la forma:

$$
\mathbf{h}_{m}^{\mathrm{TE}(1)}(\mathbf{r})=\sum_{i=1}^{Q^{\mathrm{TE}}} \sigma_{i}^{(m)} \mathbf{h}_{i}^{\mathrm{TE}(\mathrm{R})}(\mathbf{r})+\sum_{j=1}^{Q^{\mathrm{TM}}} \tau_{j}^{(m)} \mathbf{h}_{j}^{\mathrm{TM}(\mathrm{R})}(\mathbf{r})
$$


Por tanto, se puede afirmar que:

$$
\begin{aligned}
T_{m \mathrm{TE}, n_{\mathrm{TEM}}}^{(1,1)}= & \int_{S} \int_{S^{\prime}} \mathbf{h}_{m}^{\mathrm{TE}(1)}(\mathbf{r}) \cdot \overline{\mathbf{G}}_{0}^{\mathrm{F}}\left(\mathbf{r}, \mathbf{r}^{\prime}\right) \cdot \mathbf{h}_{n}^{\mathrm{TEM}(1)}\left(\mathbf{r}^{\prime}\right) d S d S^{\prime} \\
= & \sum_{i=1}^{Q^{\mathrm{TM}}} \sum_{j=1}^{Q^{\mathrm{TE}}} v_{i}^{(\mathrm{TEM})} \sigma_{j}^{(m)} \int_{S_{\mathrm{R}}} \int_{S_{\mathrm{R}}^{\prime}} \mathbf{h}_{j}^{\mathrm{TE}(\mathrm{R})}(\mathbf{r}) \cdot \overline{\mathbf{G}}_{0}^{\mathrm{F}}\left(\mathbf{r}, \mathbf{r}^{\prime}\right) \cdot \mathbf{h}_{i}^{\mathrm{TM}(\mathrm{R})}\left(\mathbf{r}^{\prime}\right) d S d S^{\prime} \\
& +\sum_{i=1}^{Q^{\mathrm{TM}}} \sum_{j=1}^{Q^{\mathrm{TM}}} v_{i}^{(\mathrm{TEM})} \tau_{j}^{(m)} \int_{S_{\mathrm{R}}} \int_{S_{\mathrm{R}}^{\prime}} \mathbf{h}_{j}^{\mathrm{TM}(\mathrm{R})}(\mathbf{r}) \cdot \overline{\mathbf{G}}_{0}^{\mathrm{F}}\left(\mathbf{r}, \mathbf{r}^{\prime}\right) \cdot \mathbf{h}_{i}^{\mathrm{TM}(\mathrm{R})}\left(\mathbf{r}^{\prime}\right) d S d S^{\prime}
\end{aligned}
$$

De las dos integrales dobles de superficie presentes en la última igualdad, se tiene que la primera de ellas es nula. La justificación de esta afirmación se basa en el hecho de que la función vectorial modal $\mathbf{h}_{n}^{\text {TEM(1) }}\left(\mathbf{r}^{\prime}\right)$ del acceso coaxial se ha expresado en función de una combinación lineal de modos de tipo $\mathrm{TM}^{z}$ de la guía rectangular de expansión y, en virtud del resultado que se recoge en (3.49) del capítulo 3, se puede concluir que la primera integral doble de superficie en $S_{\mathrm{R}}$ presente en (4.55) debe ser nula. Por tanto, teniendo en cuenta el desarrollo efectuado anteriormente para el caso en que $m$ es un modo de tipo $\mathrm{TEM}^{z}$, se puede deducir directamente que:

$$
T_{m_{\mathrm{TE}}, n_{\mathrm{TEM}}}^{(1,1)}=\sum_{i=1}^{Q^{\mathrm{TM}}} v_{i}^{(\mathrm{TEM})} \tau_{i}^{(m)} \frac{1}{k_{t, i}^{(\mathrm{R})}} \operatorname{coth}\left(k_{t, i}^{(\mathrm{R})} b\right)
$$

Finalmente, sea $m$ un modo de tipo $\mathrm{TM}^{z}$ del acceso coaxial. En este caso, la expansión que utilizamos es la siguiente:

$$
\mathbf{h}_{m}^{\mathrm{TM}(1)}(\mathbf{r})=\sum_{i=1}^{Q^{\mathrm{TM}}} \nu_{i}^{(m)} \mathbf{h}_{i}^{\mathrm{TM}(\mathrm{R})}(\mathbf{r})
$$

Gracias al desarrollo efectuado para los dos casos anteriores, concluimos que:

$$
T_{m_{\mathrm{TM}}, n_{\mathrm{TEM}}}^{(1,1)}=\sum_{i=1}^{Q^{\mathrm{TM}}} v_{i}^{(\mathrm{TEM})} \nu_{i}^{(m)} \frac{1}{k_{t, i}^{(\mathrm{R})}} \operatorname{coth}\left(k_{t, i}^{(\mathrm{R})} b\right)
$$

Caso 2: n es un modo de tipo $\mathrm{TE}^{z}$

En este caso, partimos del hecho de que $n$ es un modo de tipo $\mathrm{TE}^{z}$ del acceso coaxial. Por ello, la función vectorial modal de tipo magnético puede expresarse de la siguiente manera:

$$
\mathbf{h}_{n}^{\mathrm{TE}(1)}(\mathbf{r})=\sum_{i=1}^{Q^{\mathrm{TE}}} \sigma_{i}^{(n)} \mathbf{h}_{i}^{\mathrm{TE}(\mathrm{R})}(\mathbf{r})+\sum_{j=1}^{Q^{\mathrm{TM}}} \tau_{j}^{(n)} \mathbf{h}_{j}^{\mathrm{TM}(\mathrm{R})}(\mathbf{r})
$$


Así pues, podemos evaluar inicialmente la siguiente integral de superficie con respecto a las variables primadas:

$$
\begin{aligned}
\int_{S^{\prime}} \overline{\mathbf{G}}_{0}^{\mathrm{F}}\left(\mathbf{r}, \mathbf{r}^{\prime}\right) \cdot \mathbf{h}_{n}^{\mathrm{TE}(1)}\left(\mathbf{r}^{\prime}\right) d S^{\prime}= & \sum_{i=1}^{Q^{\mathrm{TE}}} \sigma_{i}^{(n)} \int_{S_{\mathrm{R}}^{\prime}} \overline{\mathbf{G}}_{0}^{\mathrm{F}}\left(\mathbf{r}, \mathbf{r}^{\prime}\right) \cdot \mathbf{h}_{i}^{\mathrm{TE}(\mathrm{R})}(\mathbf{r}) d S^{\prime} \\
& +\sum_{j=1}^{Q^{\mathrm{TM}}} \tau_{j}^{(n)} \int_{S_{\mathrm{R}}^{\prime}} \overline{\mathbf{G}}_{0}^{\mathrm{F}}\left(\mathbf{r}, \mathbf{r}^{\prime}\right) \cdot \mathbf{h}_{j}^{\mathrm{TM}(\mathrm{R})}(\mathbf{r}) d S^{\prime}
\end{aligned}
$$

Si ahora se consideran las expresiones (3.36)-(3.38) (cálculo de la contribución de los modos de tipo $\mathrm{TE}^{z}$ ) y (3.39)-(3.41) (cálculo de la contribución de los modos de tipo $\mathrm{TM}^{z}$ ), se puede obtener el siguiente resultado:

$$
\begin{aligned}
\int_{S^{\prime}} \overline{\mathbf{G}}_{0}^{\mathrm{F}}\left(\mathbf{r}, \mathbf{r}^{\prime}\right) \cdot \mathbf{h}_{n}^{\mathrm{TE}(1)}\left(\mathbf{r}^{\prime}\right) d S^{\prime}= & \frac{2}{b \sqrt{c a}}\left[\left\{\sum_{i=1}^{Q^{\mathrm{TE}}} \sigma_{i}^{(n)} \frac{k_{x, i}^{(\mathrm{R})}}{k_{t, i}^{(\mathrm{R})}} \sqrt{\epsilon_{i_{x}} \epsilon_{i_{y}}} \sin \left(k_{x, i}^{(\mathrm{R})} x\right) \cos \left(k_{y, i}^{(\mathrm{R})} y\right) S_{1}^{(i) \mathrm{TE}}(z)\right.\right. \\
& \left.+\sum_{j=1}^{Q^{\mathrm{TM}}} \tau_{j}^{(n)} \frac{k_{y, j}^{(\mathrm{R})}}{k_{t, j}^{(\mathrm{R})}} \sin \left(k_{x, j}^{(\mathrm{R})} x\right) \cos \left(k_{y, j}^{(\mathrm{R})} y\right) S^{(j) \mathrm{TM}}(z)\right\} \hat{\mathbf{x}} \\
& +\left\{\sum_{i=1}^{Q^{\mathrm{TE}}} \sigma_{i}^{(n)} \frac{k_{y, i}^{(\mathrm{R})}}{k_{t, i}^{(\mathrm{R})}} \sqrt{\epsilon_{i_{x}} \epsilon_{i_{y}}} \cos \left(k_{x, i}^{(\mathrm{R})} x\right) \sin \left(k_{y, i}^{(\mathrm{R})} y\right) S_{1}^{(i) \mathrm{TE}}(z)\right. \\
& \left.-\sum_{j=1}^{Q^{\mathrm{TM}}} \tau_{j}^{(n)} \frac{k_{x, j}^{(\mathrm{R})}}{k_{t, j}^{(\mathrm{R})}} \cos \left(k_{x, j}^{(\mathrm{R})} x\right) \sin \left(k_{y, j}^{(\mathrm{R})} y\right) S^{(j) \mathrm{TM}}(z)\right\} \hat{\mathbf{y}} \\
& \left.-\left\{\sum_{i=1}^{Q^{\mathrm{TE}}} \sigma_{i}^{(n)} k_{t, i}^{(\mathrm{R})} \sqrt{\epsilon_{i_{x}} \epsilon_{i_{y}}} \cos \left(k_{x, i}^{(\mathrm{R})} x\right) \cos \left(k_{y, i}^{(\mathrm{R})} y\right) S_{2}^{(i) \mathrm{TE}}(z)\right\} \hat{\mathbf{z}}\right]
\end{aligned}
$$

donde la definición para la serie $S^{(j) \mathrm{TM}}(z)$ se puede encontrar en (4.49). Además, se han definido los siguientes sumatorios:

$$
\begin{aligned}
& S_{1}^{(i) \mathrm{TE}}(z)=\sum_{n_{z}=1}^{\infty} \frac{k_{z}^{2}}{\left(\left(k_{t, i}^{(\mathrm{R})}\right)^{2}+k_{z}^{2}\right)^{2}} \cos \left(k_{z} z\right) \\
& S_{2}^{(i) \mathrm{TE}}(z)=\sum_{n_{z}=1}^{\infty} \frac{k_{z}}{\left(\left(k_{t, i}^{(\mathrm{R})}\right)^{2}+k_{z}^{2}\right)^{2}} \sin \left(k_{z} z\right)
\end{aligned}
$$


siendo $k_{z}=n_{z} \pi / b$. A continuación, el cálculo de los elementos del bloque $T_{m n}^{(1,1)}$ requiere particularizar el resultado obtenido en (4.61) en el plano en el que se encuentra el acceso (1), es decir, en $z=0$. Esto provoca que se anule la componente en $\hat{\mathbf{z}}$ de (4.61), ya que según (4.63), $S_{2}^{(i) \mathrm{TE}}(z=0)=0$.

En primer lugar, sea $m$ un modo de tipo $\mathrm{TEM}^{z}$ del acceso coaxial. Puesto que, en este caso, este modo se podrá expresar en función de las soluciones modales de tipo $\mathrm{TM}^{z}$ de la guía rectangular de expansión, la primera integral de superficie presente en (4.60) no contribuirá en el resultado final, ya que en ella intervienen las soluciones modales de tipo $\mathrm{TE}^{z}$ de la guía rectangular de expansión. En efecto:

$$
\begin{aligned}
T_{m_{\mathrm{TEM}}, n_{\mathrm{TE}}}^{(1,1)} & =\int_{S} \int_{S^{\prime}} \mathbf{h}_{m}^{\mathrm{TEM}(1)}(\mathbf{r}) \cdot \overline{\mathbf{G}}_{0}^{\mathrm{F}}\left(\mathbf{r}, \mathbf{r}^{\prime}\right) \cdot \mathbf{h}_{n}^{\mathrm{TE}(1)}\left(\mathbf{r}^{\prime}\right) d S d S^{\prime} \\
& =\sum_{i=1}^{Q^{\mathrm{TM}}} \sum_{j=1}^{Q^{\mathrm{TM}}} \tau_{i}^{(n)} v_{j}^{(\mathrm{TEM})} \int_{S_{\mathrm{R}}} \int_{S_{\mathrm{R}}^{\prime}} \mathbf{h}_{j}^{\mathrm{TM}(\mathrm{R})}(\mathbf{r}) \cdot \overline{\mathbf{G}}_{0}^{\mathrm{F}}\left(\mathbf{r}, \mathbf{r}^{\prime}\right) \cdot \mathbf{h}_{i}^{\mathrm{TM}(\mathrm{R})}\left(\mathbf{r}^{\prime}\right) d S d S^{\prime} \\
& =\sum_{i=1}^{Q^{\mathrm{TM}}} \tau_{i}^{(n)} v_{i}^{(\mathrm{TEM})} \frac{1}{k_{t, i}^{(\mathrm{R})}} \operatorname{coth}\left(k_{t, i}^{(\mathrm{R})} b\right)
\end{aligned}
$$

Consideremos ahora que $m$ es un modo $\mathrm{TE}^{z}$ del acceso coaxial, por lo que podemos escribir:

$$
\mathbf{h}_{m}^{\mathrm{TE}(1)}(\mathbf{r})=\sum_{i=1}^{Q^{\mathrm{TE}}} \sigma_{i}^{(m)} \mathbf{h}_{i}^{\mathrm{TE}(\mathrm{R})}(\mathbf{r})+\sum_{j=1}^{Q^{\mathrm{TM}}} \tau_{j}^{(m)} \mathbf{h}_{j}^{\mathrm{TM}(\mathrm{R})}(\mathbf{r})
$$

En consecuencia, teniendo en cuenta (4.59), se tiene que:

$$
\begin{aligned}
T_{m_{\mathrm{TE}}, n_{\mathrm{TE}}}^{(1,1)} & \int_{S} \int_{S^{\prime}} \mathbf{h}_{m}^{\mathrm{TE}(1)}(\mathbf{r}) \cdot \overline{\mathbf{G}}_{0}^{\mathrm{F}}\left(\mathbf{r}, \mathbf{r}^{\prime}\right) \cdot \mathbf{h}_{n}^{\mathrm{TE}(1)}\left(\mathbf{r}^{\prime}\right) d S d S^{\prime} \\
= & \sum_{i=1}^{Q^{\mathrm{TE}}} \sum_{j=1}^{Q^{\mathrm{TE}}} \sigma_{i}^{(n)} \sigma_{j}^{(m)} \int_{S_{\mathrm{R}}} \int_{S_{\mathrm{R}}^{\prime}} \mathbf{h}_{j}^{\mathrm{TE}(\mathrm{R})}(\mathbf{r}) \cdot \overline{\mathbf{G}}_{0}^{\mathrm{F}}\left(\mathbf{r}, \mathbf{r}^{\prime}\right) \cdot \mathbf{h}_{i}^{\mathrm{TE}(\mathrm{R})}\left(\mathbf{r}^{\prime}\right) d S d S^{\prime} \\
& +\sum_{i=1}^{Q^{\mathrm{TM}}} \sum_{j=1}^{Q^{\mathrm{TE}}} \tau_{i}^{(n)} \sigma_{j}^{(m)} \int_{S_{\mathrm{R}}} \int_{S_{\mathrm{R}}^{\prime}} \mathbf{h}_{j}^{\mathrm{TE}(\mathrm{R})}(\mathbf{r}) \cdot \overline{\mathbf{G}}_{0}^{\mathrm{F}}\left(\mathbf{r}, \mathbf{r}^{\prime}\right) \cdot \mathbf{h}_{i}^{\mathrm{TM}(\mathrm{R})}\left(\mathbf{r}^{\prime}\right) d S d S^{\prime} \\
& +\sum_{i=1}^{Q^{\mathrm{TE}}} \sum_{j=1}^{Q^{\mathrm{TM}}} \sigma_{i}^{(n)} \tau_{j}^{(m)} \int_{S_{\mathrm{R}}} \int_{S_{\mathrm{R}}^{\prime}} \mathbf{h}_{j}^{\mathrm{TM}(\mathrm{R})}(\mathbf{r}) \cdot \overline{\mathbf{G}}_{0}^{\mathrm{F}}\left(\mathbf{r}, \mathbf{r}^{\prime}\right) \cdot \mathbf{h}_{i}^{\mathrm{TE}(\mathrm{R})}\left(\mathbf{r}^{\prime}\right) d S d S^{\prime} \\
& +\sum_{i=1}^{Q^{\mathrm{TM}}} \sum_{j=1}^{Q^{\mathrm{TM}}} \tau_{i}^{(n)} \tau_{j}^{(m)} \int_{S_{\mathrm{R}}} \int_{S_{\mathrm{R}}^{\prime}} \mathbf{h}_{j}^{\mathrm{TM}(\mathrm{R})}(\mathbf{r}) \cdot \overline{\mathbf{G}}_{0}^{\mathrm{F}}\left(\mathbf{r}, \mathbf{r}^{\prime}\right) \cdot \mathbf{h}_{i}^{\mathrm{TM}(\mathrm{R})}\left(\mathbf{r}^{\prime}\right) d S d S^{\prime}
\end{aligned}
$$


donde, en virtud de (3.49), se tiene que la segunda y la tercera integral doble de superficie en $S_{\mathrm{R}}$ son nulas. Así pues, partiendo de (4.61), se obtiene el siguiente resultado:

$T_{m_{\mathrm{TE}}, n_{\mathrm{TE}}}^{(1,1)}=\frac{1}{2} \sum_{i=1}^{Q^{\mathrm{TE}}} \sigma_{i}^{(n)} \sigma_{j}^{(m)} \frac{1}{k_{t, i}^{(\mathrm{R})}}\left[\operatorname{coth}\left(k_{t, i}^{(\mathrm{R})} b\right)-\frac{k_{t, i}^{(\mathrm{R})} b}{\sinh ^{2}\left(k_{t, i}^{(\mathrm{R})} b\right)}\right]+\sum_{i=1}^{Q^{\mathrm{TM}}} \tau_{i}^{(n)} \tau_{i}^{(m)} \frac{\operatorname{coth}\left(k_{t, i}^{(\mathrm{R})} b\right)}{k_{t, i}^{(\mathrm{R})}}$

Para finalizar, sea $m$ un modo de tipo $\mathrm{TM}^{z}$ del acceso coaxial:

$$
\mathbf{h}_{m}^{\mathrm{TM}(1)}(\mathbf{r})=\sum_{i=1}^{Q^{\mathrm{TM}}} \nu_{i}^{(m)} \mathbf{h}_{i}^{\mathrm{TM}(\mathrm{R})}(\mathbf{r})
$$

Así pues, tenemos que:

$$
\begin{aligned}
T_{m \mathrm{TM}, n_{\mathrm{TE}}}^{(1,1)}= & \int_{S} \int_{S^{\prime}} \mathbf{h}_{m}^{\mathrm{TM}(1)}(\mathbf{r}) \cdot \overline{\mathbf{G}}_{0}^{\mathrm{F}}\left(\mathbf{r}, \mathbf{r}^{\prime}\right) \cdot \mathbf{h}_{n}^{\mathrm{TE}(1)}\left(\mathbf{r}^{\prime}\right) d S d S^{\prime} \\
= & \sum_{i=1}^{Q^{\mathrm{TE}}} \sum_{j=1}^{Q^{\mathrm{TM}}} \sigma_{i}^{(n)} \nu_{j}^{(m)} \int_{S_{\mathrm{R}}} \int_{S_{\mathrm{R}}^{\prime}} \mathbf{h}_{j}^{\mathrm{TM}(\mathrm{R})}(\mathbf{r}) \cdot \overline{\mathbf{G}}_{0}^{\mathrm{F}}\left(\mathbf{r}, \mathbf{r}^{\prime}\right) \cdot \mathbf{h}_{i}^{\mathrm{TE}(\mathrm{R})}\left(\mathbf{r}^{\prime}\right) d S d S^{\prime} \\
& +\sum_{i=1}^{Q^{\mathrm{TM}}} \sum_{j=1}^{Q^{\mathrm{TM}}} \tau_{i}^{(n)} \nu_{j}^{(m)} \int_{S_{\mathrm{R}}} \int_{S_{\mathrm{R}}^{\prime}} \mathbf{h}_{j}^{\mathrm{TM}(\mathrm{R})}(\mathbf{r}) \cdot \overline{\mathbf{G}}_{0}^{\mathrm{F}}\left(\mathbf{r}, \mathbf{r}^{\prime}\right) \cdot \mathbf{h}_{i}^{\mathrm{TM}(\mathrm{R})}\left(\mathbf{r}^{\prime}\right) d S d S^{\prime}
\end{aligned}
$$

donde ya sabemos que la primera integral doble de superficie en $S_{\mathrm{R}}$ va a ser nula. Por tanto, gracias al desarrollo teórico efectuado en las secciones anteriores se concluye:

$$
T_{m_{\mathrm{TM}}, n_{\mathrm{TE}}}^{(1,1)}=\sum_{i=1}^{Q^{\mathrm{TM}}} \tau_{i}^{(n)} \nu_{i}^{(m)} \frac{1}{k_{t, i}^{(\mathrm{R})}} \operatorname{coth}\left(k_{t, i}^{(\mathrm{R})} b\right)
$$

Caso 3: n es un modo de tipo $\mathrm{TM}^{z}$

Si $n$ es un modo de tipo $\mathrm{TM}^{z}$ del acceso coaxial, la función vectorial modal de campo magnético correspondiente se puede expandir de la siguiente manera:

$$
\mathbf{h}_{n}^{\mathrm{TM}(1)}(\mathbf{r})=\sum_{i=1}^{Q^{\mathrm{TM}}} \nu_{i}^{(n)} \mathbf{h}_{i}^{\mathrm{TM}(\mathrm{R})}(\mathbf{r})
$$

Con ello, la integral de superficie con respecto a las variables primadas presente en (4.30) se puede expresar como:

$$
\int_{S^{\prime}} \overline{\mathbf{G}}_{0}^{\mathrm{F}}\left(\mathbf{r}, \mathbf{r}^{\prime}\right) \cdot \mathbf{h}_{n}^{\mathrm{TM}(1)}\left(\mathbf{r}^{\prime}\right) d S^{\prime}=\sum_{i=1}^{Q^{\mathrm{TM}}} \nu_{i}^{(n)} \int_{S_{\mathrm{R}}^{\prime}} \overline{\mathbf{G}}_{0}^{\mathrm{F}}\left(\mathbf{r}, \mathbf{r}^{\prime}\right) \cdot \mathbf{h}_{i}^{\mathrm{TM}(\mathrm{R})}(\mathbf{r}) d S^{\prime}
$$


donde el resultado de la integral de superficie en $S_{\mathrm{R}}^{\prime}$ puede deducirse fácilmente a partir de (4.48).

Sea $m$ un modo de tipo $\mathrm{TEM}^{z}$ del acceso coaxial. En este caso, dicho modo se puede expresar en términos de una combinación lineal de las soluciones modales de tipo $\mathrm{TM}^{z}$ de la guía rectangular de expansión:

$$
\mathbf{h}_{m}^{\mathrm{TEM}(1)}(\mathbf{r})=\sum_{i=1}^{Q^{\mathrm{TM}}} v_{i}^{(\mathrm{TEM})} \mathbf{h}_{i}^{\mathrm{TM}(\mathrm{R})}(\mathbf{r})
$$

Así pues, podemos deducir que:

$$
\begin{aligned}
T_{m_{\mathrm{TEM}}, n_{\mathrm{TM}}}^{(1,1)} & =\int_{S} \int_{S^{\prime}} \mathbf{h}_{m}^{\mathrm{TEM}(1)}(\mathbf{r}) \cdot \overline{\mathbf{G}}_{0}^{\mathrm{F}}\left(\mathbf{r}, \mathbf{r}^{\prime}\right) \cdot \mathbf{h}_{n}^{\mathrm{TM}(1)}\left(\mathbf{r}^{\prime}\right) d S d S^{\prime} \\
& =\sum_{i=1}^{Q^{\mathrm{TM}}} \sum_{j=1}^{Q^{\mathrm{TM}}} \nu_{i}^{(n)} v_{j}^{(\mathrm{TEM})} \int_{S_{\mathrm{R}}} \int_{S_{\mathrm{R}}^{\prime}} \mathbf{h}_{j}^{\mathrm{TM}(\mathrm{R})}(\mathbf{r}) \cdot \overline{\mathbf{G}}_{0}^{\mathrm{F}}\left(\mathbf{r}, \mathbf{r}^{\prime}\right) \cdot \mathbf{h}_{i}^{\mathrm{TM}(\mathrm{R})}\left(\mathbf{r}^{\prime}\right) d S d S^{\prime} \\
& =\sum_{i=1}^{Q^{\mathrm{TM}}} \nu_{i}^{(n)} v_{i}^{(\mathrm{TEM})} \frac{1}{k_{t, i}^{(\mathrm{R})}} \operatorname{coth}\left(k_{t, i}^{(\mathrm{R})} b\right)
\end{aligned}
$$

Si ahora $m$ es un modo de tipo $\mathrm{TE}^{z}$ del acceso coaxial, la función vectorial modal de campo magnético de dicho modo se podrá expresar como:

$$
\mathbf{h}_{m}^{\mathrm{TE}(1)}(\mathbf{r})=\sum_{i=1}^{Q^{\mathrm{TE}}} \sigma_{i}^{(m)} \mathbf{h}_{i}^{\mathrm{TE}(\mathrm{R})}(\mathbf{r})+\sum_{j=1}^{Q^{\mathrm{TM}}} \tau_{j}^{(m)} \mathbf{h}_{j}^{\mathrm{TM}(\mathrm{R})}(\mathbf{r})
$$

En consecuencia, se obtendrá que:

$$
\begin{aligned}
T_{m_{\mathrm{TE}}, n_{\mathrm{TM}}}^{(1,1)}= & \int_{S} \int_{S^{\prime}} \mathbf{h}_{m}^{\mathrm{TE}(1)}(\mathbf{r}) \cdot \overline{\mathbf{G}}_{0}^{\mathrm{F}}\left(\mathbf{r}, \mathbf{r}^{\prime}\right) \cdot \mathbf{h}_{n}^{\mathrm{TM}(1)}\left(\mathbf{r}^{\prime}\right) d S d S^{\prime} \\
= & \sum_{i=1}^{Q^{\mathrm{TM}}} \sum_{j=1}^{Q^{\mathrm{TE}}} \nu_{i}^{(n)} \sigma_{j}^{(m)} \int_{S_{\mathrm{R}}} \int_{S_{\mathrm{R}}^{\prime}} \mathbf{h}_{j}^{\mathrm{TE}(\mathrm{R})}(\mathbf{r}) \cdot \overline{\mathbf{G}}_{0}^{\mathrm{F}}\left(\mathbf{r}, \mathbf{r}^{\prime}\right) \cdot \mathbf{h}_{i}^{\mathrm{TM}(\mathrm{R})}\left(\mathbf{r}^{\prime}\right) d S d S^{\prime} \\
& +\sum_{i=1}^{Q^{\mathrm{TM}}} \sum_{j=1}^{Q^{\mathrm{TM}}} \nu_{i}^{(n)} \tau_{j}^{(m)} \int_{S_{\mathrm{R}}} \int_{S_{\mathrm{R}}^{\prime}} \mathbf{h}_{j}^{\mathrm{TM}(\mathrm{R})}(\mathbf{r}) \cdot \overline{\mathbf{G}}_{0}^{\mathrm{F}}\left(\mathbf{r}, \mathbf{r}^{\prime}\right) \cdot \mathbf{h}_{i}^{\mathrm{TM}(\mathrm{R})}\left(\mathbf{r}^{\prime}\right) d S d S^{\prime} \\
= & \sum_{i=1}^{Q^{\mathrm{TM}}} \nu_{i}^{(n)} \tau_{i}^{(m)} \frac{1}{k_{t, i}^{(\mathrm{R})}} \operatorname{coth}\left(k_{t, i}^{(\mathrm{R})} b\right)
\end{aligned}
$$


ya que la primera integral doble de superficie en $S_{\mathrm{R}}$ es nula.

Finalmente, consideremos que $m$ es un modo $\mathrm{TM}^{z}$ del acceso coaxial. Esta vez, se tiene que:

$$
\mathbf{h}_{m}^{\mathrm{TM}(1)}(\mathbf{r})=\sum_{i=1}^{Q^{\mathrm{TM}}} \nu_{i}^{(m)} \mathbf{h}_{i}^{\mathrm{TM}(\mathrm{R})}(\mathbf{r})
$$

Por lo tanto, se obtiene:

$$
\begin{aligned}
T_{m_{\mathrm{TM}}, n_{\mathrm{TM}}}^{(1,1)} & =\int_{S} \int_{S^{\prime}} \mathbf{h}_{m}^{\mathrm{TM}(1)}(\mathbf{r}) \cdot \overline{\mathbf{G}}_{0}^{\mathrm{F}}\left(\mathbf{r}, \mathbf{r}^{\prime}\right) \cdot \mathbf{h}_{n}^{\mathrm{TM}(1)}\left(\mathbf{r}^{\prime}\right) d S d S^{\prime} \\
& =\sum_{i=1}^{Q^{\mathrm{TM}}} \sum_{j=1}^{Q^{\mathrm{TM}}} \nu_{i}^{(n)} \nu_{j}^{(m)} \int_{S_{\mathrm{R}}} \int_{S_{\mathrm{R}}^{\prime}} \mathbf{h}_{j}^{\mathrm{TM}(\mathrm{R})}(\mathbf{r}) \cdot \overline{\mathbf{G}}_{0}^{\mathrm{F}}\left(\mathbf{r}, \mathbf{r}^{\prime}\right) \cdot \mathbf{h}_{i}^{\mathrm{TM}(\mathrm{R})}\left(\mathbf{r}^{\prime}\right) d S d S^{\prime} \\
& =\sum_{i=1}^{Q^{\mathrm{TM}}} \nu_{i}^{(n)} \nu_{i}^{(m)} \frac{1}{k_{t, i}^{(\mathrm{R})}} \operatorname{coth}\left(k_{t, i}^{(\mathrm{R})} b\right)
\end{aligned}
$$

\subsubsection{Cálculo de los elementos de la matriz $\mathrm{L}$}

Los elementos de la matriz $\mathbf{L}$ se definen de la siguiente manera:

$$
L_{m n}^{(\xi)}=\int_{S} \int_{S^{\prime}} \mathbf{N}_{m}(\mathbf{r}) \cdot \nabla \times \overline{\mathbf{G}}_{0}^{\mathrm{F}}\left(\mathbf{r}, \mathbf{r}^{\prime}\right) \cdot \mathbf{h}_{n}^{(\xi)}\left(\mathbf{r}^{\prime}\right) d S d S^{\prime}
$$

donde $\xi=1,2$ representa los dos accesos de la estructura, y $\mathbf{N}_{m}(\mathbf{r})$ es la $m$-ésima función base utilizada para describir la densidad de corriente eléctrica sobre la superficie del poste conductor de simetría cilíndrica presente en la cavidad perturbada de la figura 4.5 (este poste representa, realmente, la sonda de la guía coaxial). En este caso, también existen problemas de singularidad en el cálculo de los elementos de la matriz, ya que $\mathbf{r}$ y $\mathbf{r}^{\prime}$ pueden coincidir en el contorno de la superficie de la base del poste conductor de simetría cilíndrica ${ }^{16}$, con lo cual aparecerán problemas de divergencia en el rotacional de la función diádica de Green de tipo magnético. Por este motivo, cuando $n$ haga referencia a un modo del acceso coaxial $(\xi)=(1)$ en la expresión (4.79), los elementos de la matriz L se calcularán empleando la misma estrategia utilizada anteriormente con las matrices G y T. Es decir, la funciones vectoriales modales del acceso coaxial se expresarán en términos de una combinación lineal de las soluciones modales de la guía rectangular de expansión de dimensiones $c \times a$. Por otro lado, en caso de que $n$ sea un modo del acceso rectangular $(\xi)=(2)$, las expresiones correspondientes que permiten calcular los elementos $L_{m n}^{(2)}$ se recogen en las ecuaciones

\footnotetext{
${ }^{16} \mathrm{Si}$ nos referimos a la figura 4.4, se tiene que las funciones vectoriales modales de campo magnético de la guía coaxial tienen componente acimutal no nula en $\rho=r_{2}$. Asimismo, las funciones base $\mathbf{N}_{m}(\mathbf{r})$ utilizadas para describir la densidad de corriente eléctrica sobre el poste son no nulas sobre dicho contorno $\rho=r_{2}$ de la guía coaxial.
} 
(3.57)-(3.61) obtenidas en la sección 3.2.2 del capítulo 3. Así pues, seguidamente se detalla el cálculo de los elementos $L_{m n}^{(1)}$.

Consideremos, en primer lugar, que $n$ es un modo de tipo $\mathrm{TEM}^{z}$ del acceso coaxial. En este caso, la función vectorial modal de campo magnético correspondiente puede expresarse de la siguiente manera:

$$
\mathbf{h}_{n}^{\mathrm{TEM}(1)}(\mathbf{r})=\sum_{i=1}^{Q^{\mathrm{TM}}} v_{i}^{(\mathrm{TEM})} \mathbf{h}_{i}^{\mathrm{TM}(\mathrm{R})}(\mathbf{r})
$$

donde $\mathbf{h}_{i}^{\mathrm{TM}(\mathrm{R})}(\mathbf{r})$ se corresponde con la función vectorial modal de campo magnético del $i$-ésimo modo de tipo $\mathrm{TM}^{z}$ de la guía rectangular de expansión. Así pues, si calculamos, en primer lugar, la integral de superficie con respecto a las variables primadas de la expresión (4.79) tendremos que:

$$
\boldsymbol{\Psi}_{n_{\mathrm{TEM}}}(\mathbf{r})=\int_{S^{\prime}} \nabla \times \overline{\mathbf{G}}_{0}^{\mathrm{F}}\left(\mathbf{r}, \mathbf{r}^{\prime}\right) \cdot \mathbf{h}_{n}^{\mathrm{TEM}(1)}\left(\mathbf{r}^{\prime}\right) d S^{\prime}=\sum_{i=1}^{Q^{\mathrm{TM}}} v_{i}^{(\mathrm{TEM})} \boldsymbol{\Psi}_{i_{\mathrm{TM}}}^{(\mathrm{R})}(\mathbf{r})
$$

donde se ha definido la siguiente función vectorial auxiliar:

$$
\Psi_{i_{\mathrm{TM}}}^{(\mathrm{R})}(\mathbf{r})=\int_{S_{\mathrm{R}}^{\prime}} \nabla \times \overline{\mathbf{G}}_{0}^{\mathrm{F}}\left(\mathbf{r}, \mathbf{r}^{\prime}\right) \cdot \mathbf{h}_{i}^{\mathrm{TM}(\mathrm{R})}\left(\mathbf{r}^{\prime}\right) d S^{\prime}
$$

La expresión para la función vectorial $\Psi_{i_{\mathrm{TM}}}^{(\mathrm{R})}(\mathbf{r})$ puede deducirse fácilmente a partir del resultado (3.59) obtenido en el capítulo 3. En efecto:

$$
\begin{aligned}
\Psi_{i_{\mathrm{TM}}}^{(\mathrm{R})}(\mathbf{r})= & -\frac{2}{\sqrt{c a}} \frac{1}{k_{t, i}^{(\mathrm{R})}} \frac{\sinh \left(k_{t, i}^{(\mathrm{R})}(b-z)\right)}{\sinh \left(k_{t, i}^{(\mathrm{R})} b\right)}\left\{k_{x, i}^{(\mathrm{R})} \cos \left(k_{x, i}^{(\mathrm{R})} x\right) \sin \left(k_{y, i}^{(\mathrm{R})} y\right) \hat{\mathbf{x}}\right. \\
& \left.+k_{y, i}^{(\mathrm{R})} \sin \left(k_{x, i}^{(\mathrm{R})} x\right) \cos \left(k_{y, i}^{(\mathrm{R})} y\right) \hat{\mathbf{y}}\right\} \\
& +\frac{2}{\sqrt{c a}} \sin \left(k_{x, i}^{(\mathrm{R})} x\right) \sin \left(k_{y, i}^{(\mathrm{R})} y\right) \frac{\cosh \left(k_{t, i}^{(\mathrm{R})}(b-z)\right)}{\sinh \left(k_{t, i}^{(\mathrm{R})} b\right)} \hat{\mathbf{z}}
\end{aligned}
$$

La definición de las diferentes variables que intervienen en esta última expresión puede encontrarse tras la ecuación (4.52). Por otra parte, es importante destacar que el sistema de referencia utilizado en (4.83) se corresponde con el sistema de coordenadas representado en la figura 3.3 del capítulo 3 para $(\xi)=5$ (el resto de resultados que se presentan en esta sección también se refieren a dicho sistema de referencia). Finalmente, los elementos del bloque $L_{m, n_{\text {TEM }}}^{(1)}$ se podrán calcular mediante la evaluación de la siguiente integral de superficie utilizando métodos numéricos:

$$
L_{m, n_{\mathrm{TEM}}}^{(1)}=\int_{S} \mathbf{N}_{m}(\mathbf{r}) \cdot \mathbf{\Psi}_{n_{\mathrm{TEM}}}(\mathbf{r}) d S=\sum_{i=1}^{Q^{\mathrm{TM}}} v_{i}^{(\mathrm{TEM})} \int_{S} \mathbf{N}_{m}(\mathbf{r}) \cdot \mathbf{\Psi}_{i_{\mathrm{TM}}}^{(\mathrm{R})}(\mathbf{r}) d S
$$


En caso de que $n$ sea un modo de tipo $\mathrm{TE}^{z}$ del acceso coaxial, se podrá expresar como sigue:

$$
\mathbf{h}_{n}^{\mathrm{TE}(1)}(\mathbf{r})=\sum_{i=1}^{Q^{\mathrm{TE}}} \sigma_{i}^{(n)} \mathbf{h}_{i}^{\mathrm{TE}(\mathrm{R})}(\mathbf{r})+\sum_{j=1}^{Q^{\mathrm{TM}}} \tau_{j}^{(n)} \mathbf{h}_{j}^{\mathrm{TM}(\mathrm{R})}(\mathbf{r})
$$

De esta manera, podemos calcular en primer lugar la integral de superficie siguiente con respecto a las variables primadas:

$$
\boldsymbol{\Psi}_{n_{\mathrm{TE}}}(\mathbf{r})=\int_{S^{\prime}} \nabla \times \overline{\mathbf{G}}_{0}^{\mathrm{F}}\left(\mathbf{r}, \mathbf{r}^{\prime}\right) \cdot \mathbf{h}_{n}^{\mathrm{TE}(1)}\left(\mathbf{r}^{\prime}\right) d S^{\prime}=\sum_{i=1}^{Q^{\mathrm{TE}}} \sigma_{i}^{(n)} \Psi_{i_{\mathrm{TE}}}^{(\mathrm{R})}(\mathbf{r})+\sum_{j=1}^{Q^{\mathrm{TM}}} \tau_{j}^{(n)} \boldsymbol{\Psi}_{j_{\mathrm{TM}}}^{(\mathrm{R})}(\mathbf{r})
$$

donde la expresión para la función vectorial $\Psi_{j_{\mathrm{TM}}}^{(\mathrm{R})}(\mathbf{r})$ se recoge en (4.82)-(4.83), y donde hemos definido:

$$
\Psi_{i_{\mathrm{TE}}}^{(\mathrm{R})}(\mathbf{r})=\int_{S_{\mathrm{R}}^{\prime}} \nabla \times \overline{\mathbf{G}}_{0}^{\mathrm{F}}\left(\mathbf{r}, \mathbf{r}^{\prime}\right) \cdot \mathbf{h}_{i}^{\mathrm{TE}(\mathrm{R})}\left(\mathbf{r}^{\prime}\right) d S^{\prime}
$$

La expresión para la función vectorial auxiliar $\Psi_{i_{\mathrm{TE}}}^{(\mathrm{R})}(\mathbf{r})$ puede deducirse, esta vez, a partir de la ecuación (3.58). En particular, se obtiene el siguiente resultado:

$$
\begin{aligned}
\Psi_{i_{\mathrm{TE}}}^{(\mathrm{R})}(\mathbf{r})= & \frac{\epsilon_{i_{x}} \epsilon_{i_{y}}}{\sqrt{c a}} \frac{1}{k_{t, i}^{(\mathrm{R})}} \frac{\sinh \left(k_{t, i}^{(\mathrm{R})}(b-z)\right)}{\sinh \left(k_{t, i}^{(\mathrm{R})} b\right)}\left\{k_{y, i}^{(\mathrm{R})} \cos \left(k_{x, i}^{(\mathrm{R})} x\right) \sin \left(k_{y, i}^{(\mathrm{R})} y\right) \hat{\mathbf{x}}\right. \\
& \left.-k_{x, i}^{(\mathrm{R})} \sin \left(k_{x, i}^{(\mathrm{R})} x\right) \cos \left(k_{y, i}^{(\mathrm{R})} y\right) \hat{\mathbf{y}}\right\}
\end{aligned}
$$

donde, nuevamente, la definición de las diferentes variables utilizadas en esta expresión puede encontrarse tras la ecuación (4.52). Finalmente, los elementos del bloque $L_{m, n_{\mathrm{TE}}}^{(1)}$ se calculan como:

$$
\begin{aligned}
L_{m, n_{\mathrm{TE}}}^{(1)}= & \int_{S} \mathbf{N}_{m}(\mathbf{r}) \cdot \boldsymbol{\Psi}_{n_{\mathrm{TE}}}(\mathbf{r}) d S \\
= & \sum_{i=1}^{Q^{\mathrm{TE}}} \sigma_{i}^{(n)} \int_{S} \mathbf{N}_{m}(\mathbf{r}) \cdot \mathbf{\Psi}_{i_{\mathrm{TE}}}^{(\mathrm{R})}(\mathbf{r}) d S+\sum_{j=1}^{Q^{\mathrm{TM}}} \tau_{j}^{(n)} \int_{S} \mathbf{N}_{m}(\mathbf{r}) \cdot \mathbf{\Psi}_{j_{\mathrm{TM}}}^{(\mathrm{R})}(\mathbf{r}) d S
\end{aligned}
$$

donde estas últimas integrales de superficie se calcularán utilizando métodos numéricos.

Por último, supongamos que $n$ sea un modo de tipo $\mathrm{TM}^{z}$ del acceso coaxial. En esta ocasión, se puede afirmar:

$$
\mathbf{h}_{n}^{\mathrm{TM}(1)}(\mathbf{r})=\sum_{i=1}^{Q^{\mathrm{TM}}} \nu_{i}^{(n)} \mathbf{h}_{i}^{\mathrm{TM}(\mathrm{R})}(\mathbf{r})
$$


En consecuencia, se tiene que:

$$
\mathbf{\Psi}_{n_{\mathrm{TM}}}(\mathbf{r})=\int_{S^{\prime}} \nabla \times \overline{\mathbf{G}}_{0}^{\mathrm{F}}\left(\mathbf{r}, \mathbf{r}^{\prime}\right) \cdot \mathbf{h}_{n}^{\mathrm{TM}(1)}\left(\mathbf{r}^{\prime}\right) d S^{\prime}=\sum_{i=1}^{Q^{\mathrm{TM}}} \nu_{i}^{(n)} \mathbf{\Psi}_{i_{\mathrm{TM}}}^{(\mathrm{R})}(\mathbf{r})
$$

donde la función vectorial $\Psi_{i_{\mathrm{TM}}}^{(\mathrm{R})}(\mathbf{r})$ ya se ha definido en (4.82)-(4.83). Así pues, los elementos del bloque $L_{m, n_{\mathrm{TM}}}^{(1)}$ se calcularán como:

$$
L_{m, n_{\mathrm{TM}}}^{(1)}=\int_{S} \mathbf{N}_{m}(\mathbf{r}) \cdot \Psi_{n_{\mathrm{TM}}}(\mathbf{r}) d S=\sum_{i=1}^{Q^{\mathrm{TM}}} \nu_{i}^{(n)} \int_{S} \mathbf{N}_{m}(\mathbf{r}) \cdot \Psi_{i_{\mathrm{TM}}}^{(\mathrm{R})}(\mathbf{r}) d S
$$

donde la integral de superficie debe resolverse mediante métodos numéricos.

\subsubsection{Cálculo de los elementos de la matriz $\mathrm{F}$}

La caracterización electromagnética de la excitación coaxial de dispositivos en configuración clásica concluye con el cálculo de la matriz $\mathbf{F}$ del método BI-RME 3D. Los elementos de dicha matriz se calculan de la siguiente manera:

$$
F_{m n}^{(\xi)}=\int_{S} \mathbf{H}_{m}(\mathbf{r}) \cdot \mathbf{h}_{n}^{(\xi)}(\mathbf{r}) d S
$$

donde $\xi=1,2$ denota los accesos de la estructura; y $\mathbf{H}_{m}(\mathbf{r})$ (con $m=1,2, \ldots, M$, siendo $M$ el parámetro de truncamiento de las series de las expresiones (3.4) y (3.5)) representa el campo magnético asociado al $m$-ésimo modo resonante de una cavidad rectangular de dimensiones $a \times b \times c$ (resonador externo que se ha escogido siguiendo el método BI-RME 3D). Por otro lado, es importante resaltar que los elementos del bloque $F_{m n}^{(2)}$, relacionados con el acceso rectangular $(\xi)=(2)$ pueden obtenerse partiendo de los resultados deducidos en las expresiones (3.63)-(3.65) del capítulo 3. Por tanto, esta sección se dedicará al cálculo de los elementos $F_{m n}^{(1)}$, relacionados con el acceso coaxial. Además, puesto que el modo $m$-ésimo de la cavidad resonante puede ser de tipo $\mathrm{TE}^{z}$ o $\mathrm{TM}^{z}$, y el $n$-ésimo modo del acceso coaxial puede ser de tipo $\mathrm{TEM}^{z}, \mathrm{TE}^{z} \mathrm{o} \mathrm{TM}^{z}$, el cálculo se debe particularizar para cada uno de estos casos.

En los siguientes apartados se asume que $\left(m_{x}, m_{y}, m_{z}\right)$ son los índices modales del $m$-ésimo modo resonante de la cavidad rectangular relativos a las coordenadas $(x, y, z)$ representadas en la figura 4.3. Además, $(s, q)$ representan los índices modales del $n$-ésimo modo en la guía coaxial relativos a las variaciones angular y radial, respectivamente.

Caso 1: m es un modo de tipo $\mathrm{TE}^{z}$

En caso de que el $m$-ésimo modo de la cavidad rectangular sea de tipo $\mathrm{TE}^{z}$, la expresión para el campo magnético $\mathbf{H}_{m}^{\mathrm{TE}}(\mathbf{r})$ asociado a dicho modo puede deducirse a partir de la 
ecuación (C.19) que se recoge en el Apéndice $\mathrm{C}^{17}$. Además, dicho resultado puede expresarse fácilmente en coordenadas cilíndricas utilizando las relaciones (4.1)-(4.4) ${ }^{18}$. Así pues, sea $\mathbf{H}_{m}^{\mathrm{TE}}(\rho, \phi)$ la expresión en coordenadas cilíndricas correspondiente. Por otro lado, en este apartado se van a necesitar las expresiones de las funciones vectoriales modales normalizadas de campo magnético de tipo $\mathrm{TEM}^{z}, \mathrm{TM}^{z}$ y $\mathrm{TE}^{z}$, relativas a los modos del acceso coaxial. Estas expresiones pueden encontrase en (2.63), (2.96) y (2.118), respectivamente.

Así pues, en caso de que $n$ sea un modo de tipo $\mathrm{TEM}^{z}$ se puede obtener la siguiente expresión:

$$
F_{m_{\mathrm{TE}}, n_{\mathrm{TEM}}}^{(1)}=\frac{1}{\kappa_{m}} \sqrt{\frac{2 \epsilon_{m_{x}} \epsilon_{m_{y}}}{a b c}} \frac{1}{\sqrt{2 \pi \ln \left(r_{1} / r_{2}\right)}}(-1)^{m_{y}}\left[\frac{k_{x, m} k_{z, m}}{k_{t, m}} I_{m_{\mathrm{TE}}, n_{\mathrm{TEM}}}^{(1)}+k_{t, m} I_{m_{\mathrm{TE}}, n_{\mathrm{TEM}}}^{(2)}\right]
$$

donde $k_{x, m}=m_{x} \pi / a ; k_{y, m}=m_{y} \pi / b ; k_{z, m}=m_{z} \pi / c ; k_{t, m}^{2}=k_{x, m}^{2}+k_{y, m}^{2} ; \kappa_{m}^{2}=k_{t, m}^{2}+k_{z, m}^{2}$ representa el número de onda de resonancia del modo, y $r_{1}$ y $r_{2}$ son, respectivamente, los radios externo e interno de la guía coaxial. Además, se han definido las siguientes integrales de superficie:

$$
\begin{aligned}
I_{m_{\mathrm{TE}}, n_{\mathrm{TEM}}}^{(1)} & =\int_{\rho} \int_{\phi} \sin \left(k_{x, m}\left(d_{x}+\rho \sin \phi\right)\right) \cos \left(k_{z, m}\left(d_{z}-\rho \cos \phi\right)\right) \cos \phi d \rho d \phi \\
I_{m_{\mathrm{TE}}, n_{\mathrm{TEM}}}^{(2)} & =\int_{\rho} \int_{\phi} \cos \left(k_{x, m}\left(d_{x}+\rho \sin \phi\right)\right) \sin \left(k_{z, m}\left(d_{z}-\rho \cos \phi\right)\right) \sin \phi d \rho d \phi
\end{aligned}
$$

Si $n$ es un modo de tipo $\mathrm{TE}^{z}$ del acceso coaxial, la expresión resultante para los elementos de la matriz $\mathbf{F}$ es la siguiente:

$$
\begin{aligned}
F_{m_{\mathrm{TE}}, n_{\mathrm{TE}}}^{(1)}= & \frac{1}{\kappa_{m}} \sqrt{\frac{2 \epsilon_{m_{x}} \epsilon_{m_{y}}}{a b c}} \mathcal{N}_{(\zeta), m}^{\mathrm{TE}}(-1)^{m_{y}}\left[-\frac{\pi}{r_{1}-r_{2}} \frac{k_{x, m} k_{z, m}}{k_{t, m}} \sum_{r=0}^{N_{\rho}-1} r l_{s, q}^{(r)} I_{m_{\mathrm{TE}}, n_{\mathrm{TE}}}^{(r),()}\right. \\
& -k_{t, m} \frac{\pi}{a-b} \sum_{r=0}^{N_{\rho}-1} r l_{s, q}^{(r)} I_{m_{\mathrm{TE}}, n_{\mathrm{TE}}}^{(r),(2)}+\frac{k_{x, m} k_{z, m}}{k_{t, m}} \sum_{r=0}^{N_{\rho}-1} l_{s, q}^{(r)} I_{m_{\mathrm{TE}}, n_{\mathrm{TE}}}^{(r),(3)} \\
& \left.-k_{t, m} \sum_{r=0}^{N_{\rho}-1} l_{s, q}^{(r)} I_{m_{\mathrm{TE}}, n_{\mathrm{TE}}}^{(r),(4)}\right]
\end{aligned}
$$

\footnotetext{
${ }^{17}$ La expresión de este campo magnético está referida al sistema de ejes coordenados en cartesianas de la figura 4.3.

${ }^{18}$ El uso del sistema de referencia en coordenadas cilíndricas representado en la figura 4.4 implica que los modos de la guía coaxial se propagan en dirección entrante.
} 
donde se han definido las siguientes integrales:

$I_{m_{\mathrm{TE}}, n_{\mathrm{TE}}}^{(r),(1)}=\int_{\rho} \int_{\phi} \rho \sin \left(k_{x, m}\left(d_{x}+\rho \sin \phi\right)\right) \cos \left(k_{z, m}\left(d_{z}-\rho \cos \phi\right)\right) s_{r}(\rho) \Phi_{(\zeta), s}(\phi) \sin \phi d \rho d \phi$

$I_{m_{\mathrm{TE}}, n_{\mathrm{TE}}}^{(r),(2)}=\int_{\rho} \int_{\phi} \rho \cos \left(k_{x, m}\left(d_{x}+\rho \sin \phi\right)\right) \sin \left(k_{z, m}\left(d_{z}-\rho \cos \phi\right)\right) s_{r}(\rho) \Phi_{(\zeta), s}(\phi) \cos \phi d \rho d \phi$

$I_{m_{\mathrm{TE}}, n_{\mathrm{TE}}}^{(r),(3)}=\int_{\rho} \int_{\phi} \sin \left(k_{x, m}\left(d_{x}+\rho \sin \phi\right)\right) \cos \left(k_{z, m}\left(d_{z}-\rho \cos \phi\right)\right) c_{r}(\rho) \Phi_{(\zeta), s}^{\prime}(\phi) \cos \phi d \rho d \phi$

$I_{m_{\mathrm{TE}}, n_{\mathrm{TE}}}^{(r),(4)}=\int_{\rho} \int_{\phi} \cos \left(k_{x, m}\left(d_{x}+\rho \sin \phi\right)\right) \sin \left(k_{z, m}\left(d_{z}-\rho \cos \phi\right)\right) c_{r}(\rho) \Phi_{(\zeta), s}^{\prime}(\phi) \sin \phi d \rho d \phi$

siendo

$$
\begin{aligned}
& s_{r}(\rho)=\sin \left(r \pi \frac{\rho-r_{2}}{r_{1}-r_{2}}\right) \\
& c_{r}(\rho)=\cos \left(r \pi \frac{\rho-r_{2}}{r_{1}-r_{2}}\right)
\end{aligned}
$$

Finalmente, si $n$ es un modo de tipo $\mathrm{TM}^{z}$ del acceso coaxial se puede concluir:

$$
\begin{aligned}
F_{m_{\mathrm{TE}}, n_{\mathrm{TM}}}^{(1)}= & \frac{1}{\kappa_{m}} \sqrt{\frac{2 \epsilon_{m_{x}} \epsilon_{m_{y}}}{a b c}} \mathcal{N}_{(\zeta), m}^{\mathrm{TM}}(-1)^{m_{y}}\left[-\frac{k_{x, m} k_{z, m}}{k_{t, m}} \sum_{r=1}^{N_{\rho}} d_{s, q}^{(r)} I_{m_{\mathrm{TE}}, n_{\mathrm{TM}}}^{(r),(1)}\right. \\
& -k_{t, m} \sum_{r=1}^{N_{\rho}} d_{s, q}^{(r)} I_{m_{\mathrm{TE}}, n_{\mathrm{TM}}}^{(r),(2)}+\frac{\pi}{r_{1}-r_{2}} \frac{k_{x, m} k_{z, m}}{k_{t, m}} \sum_{r=1}^{N_{\rho}} r d_{s, q}^{(r)} I_{m_{\mathrm{TE}}, n_{\mathrm{TM}}}^{(r),(3)} \\
& \left.-\frac{\pi}{r_{1}-r_{2}} k_{t, m} \sum_{r=1}^{N_{\rho}} r d_{s, q}^{(r)} I_{m_{\mathrm{TE}}, n_{\mathrm{TM}}}^{(r),(4)}\right]
\end{aligned}
$$

donde se han definido las siguientes integrales de superficie:

$$
\begin{aligned}
& I_{m_{\mathrm{TE}}, n_{\mathrm{TM}}}^{(r),(1)}=\int_{\rho} \int_{\phi} \sin \left(k_{x, m}\left(d_{x}+\rho \sin \phi\right)\right) \cos \left(k_{z, m}\left(d_{z}-\rho \cos \phi\right)\right) s_{r}(\rho) \Phi_{(\zeta), s}^{\prime}(\phi) \sin \phi d \rho d \phi \\
& I_{m \mathrm{TE}, n_{\mathrm{TM}}}^{(r),(2)}=\int_{\rho} \int_{\phi} \cos \left(k_{x, m}\left(d_{x}+\rho \sin \phi\right)\right) \sin \left(k_{z, m}\left(d_{z}-\rho \cos \phi\right)\right) s_{r}(\rho) \Phi_{(\zeta), s}^{\prime}(\phi) \cos \phi d \rho d \phi
\end{aligned}
$$


$I_{m_{\mathrm{TE}}, n_{\mathrm{TM}}}^{(r),(3)}=\int_{\rho} \int_{\phi} \rho \sin \left(k_{x, m}\left(d_{x}+\rho \sin \phi\right)\right) \cos \left(k_{z, m}\left(d_{z}-\rho \cos \phi\right)\right) c_{r}(\rho) \Phi_{(\zeta), s}(\phi) \cos \phi d \rho d \phi$

$I_{m_{\mathrm{TE}}, n_{\mathrm{TM}}}^{(r),(4)}=\int_{\rho} \int_{\phi} \rho \cos \left(k_{x, m}\left(d_{x}+\rho \sin \phi\right)\right) \sin \left(k_{z, m}\left(d_{z}-\rho \cos \phi\right)\right) c_{r}(\rho) \Phi_{(\zeta), s}(\phi) \sin \phi d \rho d \phi$

Caso 2: m es un modo de tipo $\mathrm{TM}^{z}$

Sea ahora $m$ un modo de la cavidad rectangular de tipo $\mathrm{TM}^{z}$. En este caso, la expresión para el campo magnético $\mathbf{H}_{m}^{\mathrm{TM}}(\mathbf{r})$ asociado a este modo puede deducirse a partir de la ecuación (C.20) que se recoge en el Apéndice C. Por otra parte, el campo magnético $\mathbf{H}_{m}^{\mathrm{TM}}(\mathbf{r})$ deberá expresarse en coordenadas cilíndricas utilizando las relaciones (4.1)-(4.4). Así pues, sea $\mathbf{H}_{m}^{\mathrm{TM}}(\rho, \phi)$ la expresión en coordenadas cilíndricas correspondiente.

En primer lugar, supongamos que $n$ es un modo de tipo $\mathrm{TEM}^{z}$ del acceso coaxial. En este caso, se tiene que:

$$
F_{m_{\mathrm{TM}}, n_{\mathrm{TEM}}}^{(1)}=2 \sqrt{\frac{\epsilon_{m_{z}}}{a b c}} \frac{1}{\sqrt{2 \pi \ln \left(r_{1} / r_{2}\right)}} \frac{k_{y, m}}{k_{t, m}}(-1)^{m_{y}} I_{m_{\mathrm{TM},}, n_{\mathrm{TEM}}}
$$

donde la integral $I_{m_{\mathrm{TM}}, n_{\mathrm{TEM}}}$ tiene una expresión idéntica a la integral $I_{m_{\mathrm{TE}}, n_{\mathrm{TEM}}}^{(1)}$ definida en (4.95).

En caso de que $n$ sea un modo de tipo $\mathrm{TE}^{z}$ del acceso coaxial, se obtiene:

$F_{m_{\mathrm{TM}}, n_{\mathrm{TE}}}^{(1)}=2 \sqrt{\frac{\epsilon_{m_{z}}}{a b c}} \mathcal{N}_{(\zeta), m}^{\mathrm{TE}}(-1)^{m_{y}} \frac{k_{y, m}}{k_{t, m}}\left[-\frac{\pi}{r_{1}-r_{2}} \sum_{r=0}^{N_{\rho}-1} r l_{s, q}^{(r)} I_{m_{\mathrm{TM}}, n_{\mathrm{TE}}}^{(r),(1)}+\sum_{r=0}^{N_{\rho}-1} l_{s, q}^{(r)} I_{m_{\mathrm{TM}}, n_{\mathrm{TE}}}^{(r),(2)}\right]$

donde las integrales $I_{m_{\mathrm{TM}}, n_{\mathrm{TE}}}^{(r),(1)}$ y $I_{m_{\mathrm{TM}}, n_{\mathrm{TE}}}^{(r),(2)}$ tienen una expresión idéntica a las integrales $I_{m_{\mathrm{TE}}, n_{\mathrm{TE}}}^{(r),(1)}$ y $I_{m_{\mathrm{TE}}, n_{\mathrm{TE}}}^{(r),(3)}$ definidas en (4.98) y (4.100), respectivamente.

Finalmente, si $n$ es un modo de tipo $\mathrm{TM}^{z}$ del acceso coaxial, se concluye que:

$F_{m_{\mathrm{TM}}, n_{\mathrm{TM}}}^{(1)}=2 \sqrt{\frac{\epsilon_{m_{z}}}{a b c}} \mathcal{N}_{(\zeta), m}^{\mathrm{TM}}(-1)^{m_{y}} \frac{k_{y, m}}{k_{t, m}}\left[-\sum_{r=1}^{N_{\rho}} d_{s, q}^{(r)} I_{m_{\mathrm{TM}}, n_{\mathrm{TM}}}^{(r),(1)}+\frac{\pi}{r_{1}-r_{2}} \sum_{r=1}^{N_{\rho}} r d_{s, q}^{(r)} I_{m_{\mathrm{TM}}, n_{\mathrm{TM}}}^{(r),(2)}\right]$

donde, esta vez, las expresiones de las integrales $I_{m_{\mathrm{TM}}, n_{\mathrm{TM}}}^{(r),(1)}$ y $I_{m_{\mathrm{TM}}, n_{\mathrm{TM}}}^{(r),(2)}$ son iguales a las de las integrales $I_{m_{\mathrm{TE}}, n_{\mathrm{TM}}}^{(r),(1)}$ y $I_{m_{\mathrm{TE}}, n_{\mathrm{TM}}}^{(r),(3)}$ definidas en (4.105) y (4.107), respectivamente. Es importante mencionar que todas las integrales de superficie definidas en esta sección se han resuelto utilizando métodos numéricos.

Una vez programado el cálculo de los elementos $F_{m n}^{(1)}$ de la matriz $\mathbf{F}$ se ha observado que el esfuerzo computacional asociado a dicho cálculo es, en general, elevado para la mayoría de las estructuras analizadas. La razón de ello es que las integrales de superficie definidas en esta sección (ver (4.98), por ejemplo) dependen simultáneamente de los índices $m$ (el valor 
máximo del índice $m$ es igual al valor que tome el parámetro $M$ que se haya utilizado para truncar las series que aparecen en las expresiones (3.4) y (3.5) del capítulo 3) y $r$ (el valor máximo del índice $r$ depende del número de funciones base $N_{\rho}$ utilizadas para describir la variación radial de las soluciones modales de la guía coaxial). Un valor típico para el parámetro $M$ suele ser de varios centenares de modos resonantes, mientras que un valor típico para el parámetro $N_{\rho}$ puede ser de 100 términos. Todo esto implica que las integrales de superficie definidas en esta sección deben evaluarse miles de veces con el fin de calcular los elementos $F_{m n}^{(1)}$. Por este motivo, el esfuerzo computacional asociado a este cálculo es elevado.

Una posible alternativa consiste, nuevamente, en expresar las funciones vectoriales modales $\mathbf{h}_{n}^{(\xi)}(\mathbf{r})$ de la guía coaxial en función de las soluciones modales de la guía rectangular de expansión de dimensiones $c \times a$. Aunque la matriz $\mathbf{F}$ no presenta problemas de singularidades, pues en su expresión no intervienen las funciones de Green del resonador externo, se ha comprobado que la utilización de este procedimiento reduce notablemente el esfuerzo computacional asociado al cálculo de los elementos $F_{m n}^{(1)}$. Así pues, teniendo en cuenta las expansiones modales presentadas en (4.14)-(4.16), se puede obtener el siguiente resultado:

$$
\begin{aligned}
& F_{m_{\mathrm{TE}}, n_{\mathrm{TEM}}}^{(1)}=\sum_{i=1}^{Q^{\mathrm{TM}}} v_{i}^{(\mathrm{TEM})} \int_{S_{\mathrm{R}}} \mathbf{H}_{m}^{\mathrm{TE}}(\mathbf{r}) \cdot \mathbf{h}_{i}^{\mathrm{TM}(\mathrm{R})}(\mathbf{r}) d S \\
& F_{m_{\mathrm{TE}}, n_{\mathrm{TE}}}^{(1)}=\sum_{i=1}^{Q^{\mathrm{TE}}} \sigma_{i}^{(n)} \int_{S_{\mathrm{R}}} \mathbf{H}_{m}^{\mathrm{TE}}(\mathbf{r}) \cdot \mathbf{h}_{i}^{\mathrm{TE}(\mathrm{R})}(\mathbf{r}) d S+\sum_{j=1}^{Q^{\mathrm{TM}}} \tau_{j}^{(n)} \int_{S_{\mathrm{R}}} \mathbf{H}_{m}^{\mathrm{TE}}(\mathbf{r}) \cdot \mathbf{h}_{j}^{\mathrm{TM}(\mathrm{R})}(\mathbf{r}) d S \\
& F_{m_{\mathrm{TE}}, n_{\mathrm{TM}}}^{(1)}=\sum_{i=1}^{Q^{\mathrm{TM}}} \nu_{i}^{(n)} \int_{S_{\mathrm{R}}} \mathbf{H}_{m}^{\mathrm{TE}}(\mathbf{r}) \cdot \mathbf{h}_{i}^{\mathrm{TM}(\mathrm{R})}(\mathbf{r}) d S \\
& F_{m_{\mathrm{TM}}, n_{\mathrm{TEM}}}^{(1)}=\sum_{i=1}^{Q^{\mathrm{TM}}} v_{i}^{(\mathrm{TEM})} \int_{S_{\mathrm{R}}} \mathbf{H}_{m}^{\mathrm{TM}}(\mathbf{r}) \cdot \mathbf{h}_{i}^{\mathrm{TM}(\mathrm{R})}(\mathbf{r}) d S \\
& F_{m_{\mathrm{TM}}, n_{\mathrm{TE}}}^{(1)}=\sum_{i=1}^{Q^{\mathrm{TE}}} \sigma_{i}^{(n)} \int_{S_{\mathrm{R}}} \mathbf{H}_{m}^{\mathrm{TM}}(\mathbf{r}) \cdot \mathbf{h}_{i}^{\mathrm{TE}(\mathrm{R})}(\mathbf{r}) d S+\sum_{j=1}^{Q^{\mathrm{TM}}} \tau_{j}^{(n)} \int_{S_{\mathrm{R}}} \mathbf{H}_{m}^{\mathrm{TM}}(\mathbf{r}) \cdot \mathbf{h}_{j}^{\mathrm{TM}(\mathrm{R})}(\mathbf{r}) d S \\
& F_{m_{\mathrm{TM}}, n_{\mathrm{TM}}}^{(1)}=\sum_{i=1}^{Q^{\mathrm{TM}}} \nu_{i}^{(n)} \int_{S_{\mathrm{R}}} \mathbf{H}_{m}^{\mathrm{TM}}(\mathbf{r}) \cdot \mathbf{h}_{i}^{\mathrm{TM}(\mathrm{R})}(\mathbf{r}) d S
\end{aligned}
$$

En relación con estas últimas expresiones, es importante destacar que las integrales de superficie que involucran a las funciones vectoriales modales de la guía rectangular de expansión ya han sido calculadas en el capítulo 3 . En concreto, el resultado para dichas integrales puede deducirse a partir de las expresiones (3.70)-(3.73) del mencionado capítulo, tomando $l_{y_{1}}=b$, 
y considerando que los modos de la guía rectangular de expansión se expresan esta vez utilizando el sistema de referencia representado en la figura 3.3 del capítulo 3, relativo al puerto $(\xi)=(5)$. Tal y como se puede comprobar, con esta estrategia no se debe resolver ninguna integral utilizando métodos numéricos, por lo que la eficiencia computacional del método mejora enormemente.

\subsubsection{Ejemplos de validación}

En esta sección se presentan diferentes ejemplos de aplicación que validan por completo la rigurosidad del desarrollo teórico presentado anteriormente. En primer lugar, se analiza una guía rectangular semi-infinita implementada en la guía estándar WR-62 ( $a=15,799$ mm, $b=7,8995 \mathrm{~mm}$ ) excitada mediante una sonda coaxial convencional ${ }^{19}$. Las dimensiones que caracterizan a la guía coaxial son las siguientes (ver figura 4.3): los radios externo e interno son $r_{1}=1,6764 \mathrm{~mm}$ y $r_{2}=0,635 \mathrm{~mm}$, respectivamente; $r_{d}=h_{d}=0$ por tratarse de una sonda coaxial convencional; la distancia a la pared cortocircuitada de la guía rectangular es $d_{z}=5,0 \mathrm{~mm}$, la impedancia característica del coaxial es $Z_{0}=50 \Omega$, y $d_{x}=a / 2$ (sonda coaxial centrada respecto al eje $\hat{\mathbf{x}}$ ). En la figura 4.6, se presentan las pérdidas de retorno de la estructura y se comparan los resultados obtenidos con los datos experimentales extraídos de [17]. Además, en la citada figura se presentan los resultados para dos valores diferentes de la profundidad de penetración de la sonda: $h_{p}=1,2954 \mathrm{~mm}$ y $h_{p}=1,9812 \mathrm{~mm}$. Tal y como se puede comprobar, los resultados de la herramienta implementada presentan una excelente concordancia con los datos experimentales presentados en [17]. Para lograr estos resultados, se han considerado $N=15$ modos en los accesos coaxial y rectangular. Además, se han empleado $N_{\rho}=85$ términos para expresar las soluciones modales en la variable radial de la guía coaxial, y se han utilizado 80 puntos en la variable radial y 15 en la variable angular para la integración numérica de las diferentes integrales que aparecen en el cálculo de las matrices G y T. Por otro lado, según el método BI-RME 3D, se ha empleado un factor de precisión $\zeta=5$, lo que implica, en este caso, que el número de modos resonantes considerados en las series (3.4) y (3.5) es de $M=269$. Finalmente, se han tomado $Q^{\mathrm{TE}}=Q^{\mathrm{TM}}=15000$ modos de la guía rectangular de expansión (es decir, 30000 modos en total) para expresar las funciones vectoriales modales de la guía coaxial en términos de las soluciones modales de la guía rectangular de dimensiones $c \times a$. Por último, en relación con la eficiencia computacional del método, hay que comentar que los resultados presentados se han obtenido en 42,9 segundos para un total de 41 puntos en frecuencia.

De los parámetros de simulación presentados, merece especial mención el número de mo$\operatorname{dos} Q^{\mathrm{TE}}$ y $Q^{\mathrm{TM}}$ utilizado para expresar las soluciones modales de la guía coaxial en términos de las funciones vectoriales modales de la guía rectangular de expansión. Así, atendiendo a los resultados del correspondiente estudio de convergencia que se presenta a continuación, se concluye que, en general, se debe utilizar un número elevado de modos de la guía rectangular de expansión con el fin de alcanzar resultados convergentes. A este respecto, en la figura 4.7

\footnotetext{
${ }^{19}$ En todos los resultados presentados en este capítulo se considera que los modos de excitación y de respuesta se corresponden con los modos fundamentales de cada tipo de acceso considerado.
} 


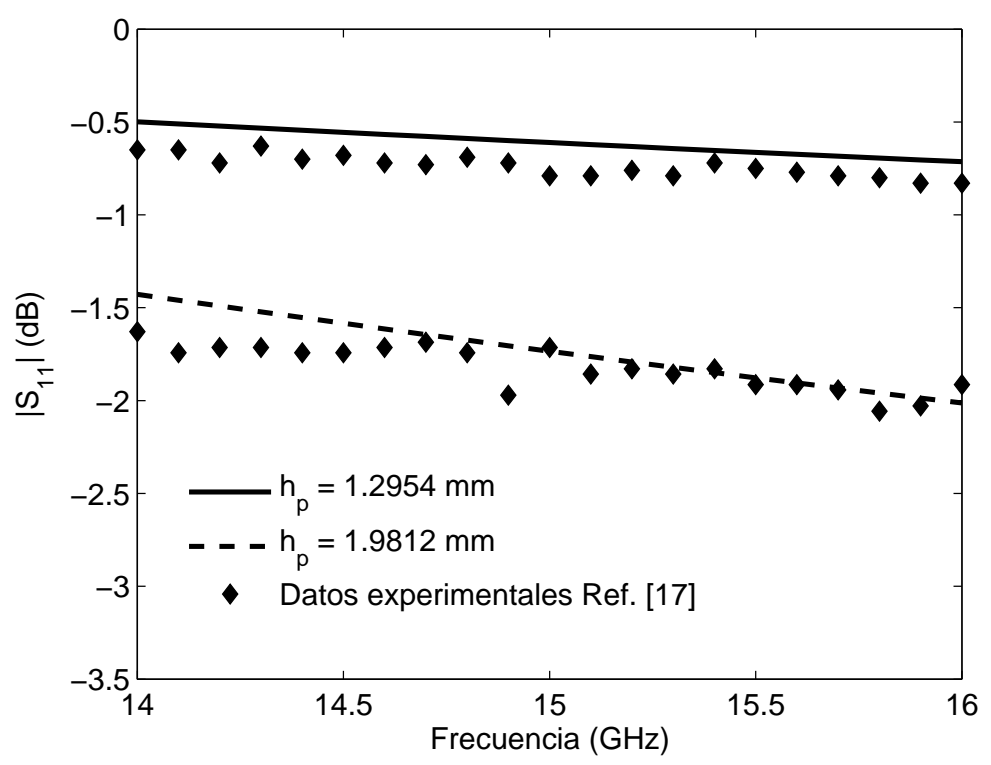

Figura 4.6: Pérdidas de retorno para una guía rectangular semi-infinita implementada en la guía estándar WR-62 excitada mediante sonda coaxial convencional $\left(r_{1}=1,6764 \mathrm{~mm}, r_{2}=0,635 \mathrm{~mm}\right.$, $\left.Z_{0}=50 \Omega, d_{z}=5,0 \mathrm{~mm}, d_{x}=a / 2\right)$. En la figura se estudian dos casos en función del valor de la profundidad de penetración de la sonda $h_{p}$. Los resultados de la simulación se comparan con los datos experimentales presentados en [17].

se presenta un estudio en el que se investiga la convergencia del módulo del parámetro de reflexión $S_{11}$ para el ejemplo de validación presentado en la figura 4.6 con $h_{p}=1,2954 \mathrm{~mm}$. Además, en el estudio se ha fijado la frecuencia de análisis en el valor $f=15 \mathrm{GHz}$. En la figura 4.7 se puede comprobar que, para alcanzar resultados convergentes, se precisan cerca de $Q^{\mathrm{TE}}=Q^{\mathrm{TM}}=15000$ modos de la guía rectangular de expansión (el error relativo de este caso con respecto al caso en que $Q^{\mathrm{TE}}=Q^{\mathrm{TM}}=60000$ es inferior al $1 \%$ ). A pesar de este número de modos tan elevado, la eficiencia computacional del método es buena.

A continuación, se presentan varios ejemplos de validación más y, en estos casos, la solución obtenida se compara con los resultados que proporciona el programa HFSS. En los ejemplos siguientes relacionados con la excitación mediante sonda coaxial convencional, se han utilizado los mismos parámetros de simulación comentados anteriormente, por lo que los tiempos de simulación son muy similares a los del ejemplo de la figura 4.6. En primer lugar, se simula una guía rectangular semi-infinita implementada en la guía estándar WR-75 de dimensiones $a=19,05 \mathrm{~mm}$ y $b=9,525 \mathrm{~mm}$, excitada mediante una sonda coaxial convencional $\left(r_{d}=h_{d}=0\right)$ de radio externo $r_{1}=3,18 \mathrm{~mm}$ y radio interno $r_{2}=0,3 \mathrm{~mm}$, siendo la permitividad dieléctrica relativa igual a $\varepsilon_{r}=2,09$. Además, la profundidad de penetración es $h_{p}=4,7625 \mathrm{~mm}$, la distancia a la pared cortocircuitada es $d_{z}=6,8 \mathrm{~mm}$ y $d_{x}=a / 2$. En la figura 4.8 se presentan los resultados para el módulo del parámetro de reflexión $S_{11}$ y se comparan con éxito con los datos proporcionados por HFSS (la simulación con HFSS se efectuó en, aproximadamente, 15 minutos para un total de 26 puntos en frecuencia). 


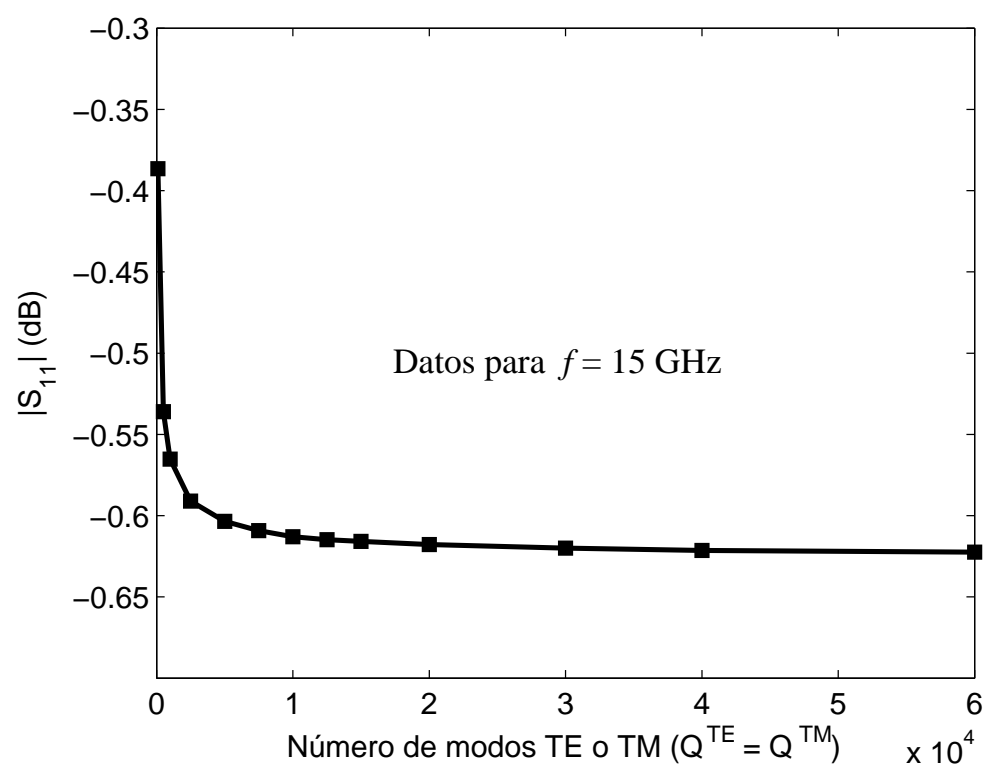

Figura 4.7: Estudio de convergencia para el módulo del parámetro $S_{11}(f=15 \mathrm{GHz})$ relativo a la estructura simulada en la figura 4.6 con $h_{p}=1,2954 \mathrm{~mm}$. El estudio se realiza en función del número de modos $Q^{\mathrm{TE}}=Q^{\mathrm{TM}}$ utilizados en la guía rectangular de expansión.

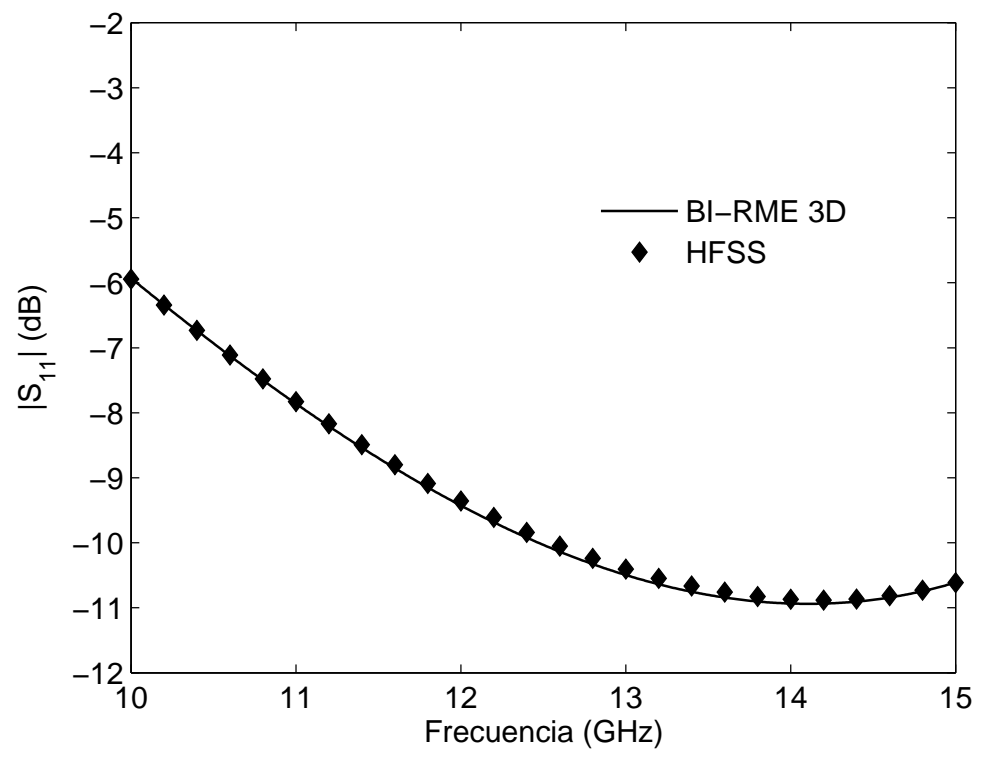

Figura 4.8: Pérdidas de retorno para una guía rectangular semi-infinita implementada en la guía estándar WR-75 excitada mediante sonda coaxial convencional $\left(r_{1}=3,18 \mathrm{~mm}, r_{2}=0,3 \mathrm{~mm}\right.$, $\left.\varepsilon_{r}=2,09, h_{p}=4,7625 \mathrm{~mm}, d_{z}=6,8 \mathrm{~mm}, d_{x}=a / 2\right)$. Los resultados de la simulación se comparan con los datos proporcionados por HFSS. 
Un nuevo ejemplo se presenta en la figura 4.9, en la que se presentan las pérdidas de retorno para una guía rectangular semi-infinita ( $a=57,04 \mathrm{~mm}, b=28,8 \mathrm{~mm}$ ) excitada mediante sonda coaxial convencional $\left(r_{1}=2,12 \mathrm{~mm}, r_{2}=0,635 \mathrm{~mm}, \varepsilon_{r}=2,09\right.$, $\left.h_{p}=14,4 \mathrm{~mm}, d_{z}=19,2 \mathrm{~mm}, d_{x}=a / 2\right)$. Nuevamente, los resultados obtenidos muestran una excelente similitud con los proporcionados por HFSS. Finalmente, en la figura $4.10 \mathrm{se}$

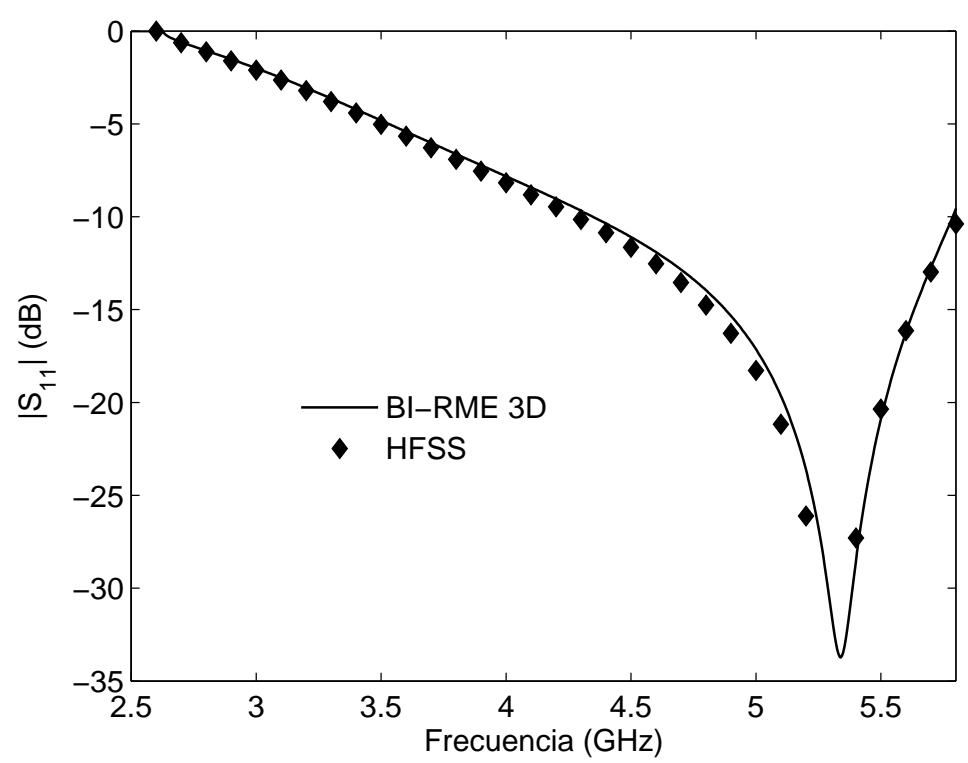

Figura 4.9: Pérdidas de retorno para una guía rectangular semi-infinita ( $a=57,04 \mathrm{~mm}$, $b=28,8 \mathrm{~mm})$ excitada mediante sonda coaxial convencional $\left(r_{1}=2,12 \mathrm{~mm}, r_{2}=0,635 \mathrm{~mm}\right.$, $\left.\varepsilon_{r}=2,09, h_{p}=14,4 \mathrm{~mm}, d_{z}=19,2 \mathrm{~mm}, d_{x}=a / 2\right)$. Los resultados de la simulación se comparan con los datos proporcionados por HFSS.

presenta el último ejemplo que utiliza sondas coaxiales de tipo convencional. En dicha figura, se analiza la excitación mediante sonda coaxial convencional $\left(r_{1}=3,45 \mathrm{~mm}, r_{2}=0,55 \mathrm{~mm}\right.$, $\left.\varepsilon_{r}=2,09, h_{p}=5,5 \mathrm{~mm}, d_{z}=8,6 \mathrm{~mm}, d_{x}=a / 2\right)$ de una guía rectangular semi-infinita implementada en la guía estándar WR-90 ( $a=22,9 \mathrm{~mm}, b=10,2 \mathrm{~mm})$. Los resultados de la simulación muestran una concordancia muy buena con los datos que proporciona HFSS.

Seguidamente, se presentan varios ejemplos de validación en los que la sonda coaxial está terminada en disco (sonda de tipo “champiñón"). Así, en primer lugar, en la figura 4.11 se muestran las pérdidas de retorno para una guía rectangular semi-infinita implementada en la guía estándar WR-75 $(a=19,05 \mathrm{~mm}, b=9,525 \mathrm{~mm})$ excitada por una guía coaxial cuya sonda está terminada en disco. Las dimensiones de la guía coaxial utilizada son las siguientes (ver figura 4.3): radio externo $r_{1}=3,18 \mathrm{~mm}$, radio interno $r_{2}=0,3 \mathrm{~mm}$, permitividad dieléctrica relativa $\varepsilon_{r}=2,09$, profundidad de penetración $h_{p}=4,7625 \mathrm{~mm}$, distancia a la pared cortocircuitada $d_{z}=6,8 \mathrm{~mm}$ y $d_{x}=a / 2$. El disco de la sonda es de altura $h_{d}=1,5 \mathrm{~mm}$ y de radio $r_{d}$ (en la figura 4.11 se analizan los casos para $r_{d}=0,5 \mathrm{~mm}$ y $r_{d}=1,0 \mathrm{~mm}$ ). Tal y como se puede comprobar, los resultados obtenidos presentan una excelente concordancia con los datos proporcionados por HFSS. Por otro lado, la estructura se simuló en 151,6 


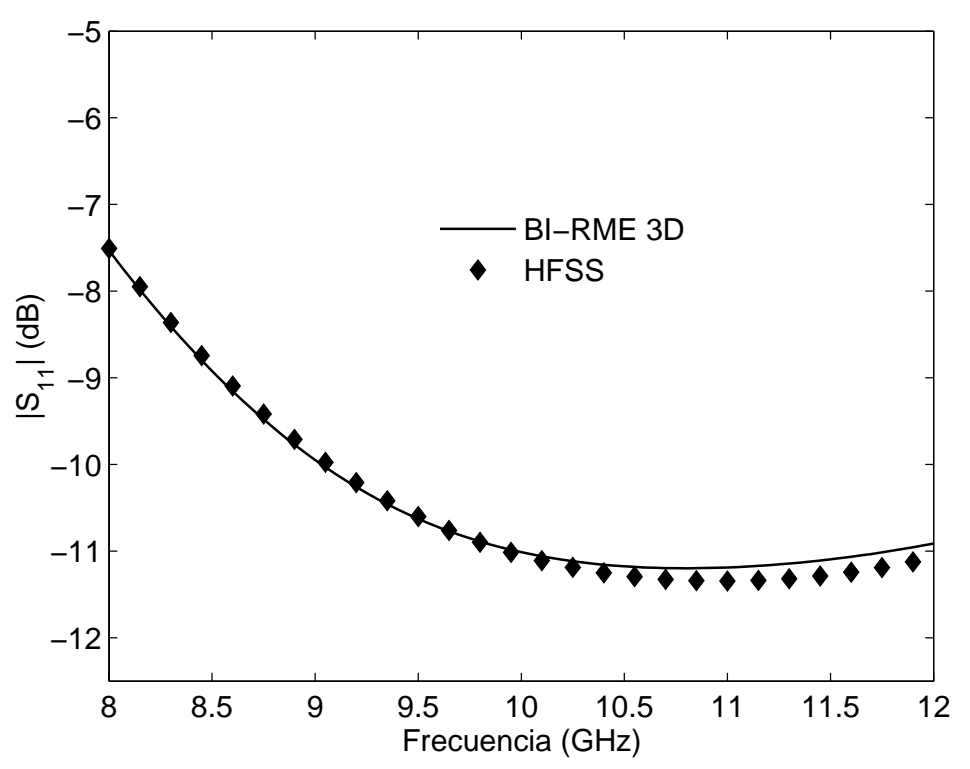

Figura 4.10: Pérdidas de retorno para una guía rectangular semi-infinita implementada en la guía estándar WR-90 excitada mediante sonda coaxial convencional $\left(r_{1}=3,45 \mathrm{~mm}, r_{2}=0,55 \mathrm{~mm}\right.$, $\left.\varepsilon_{r}=2,09, h_{p}=5,5 \mathrm{~mm}, d_{z}=8,6 \mathrm{~mm}, d_{x}=a / 2\right)$. Los resultados de la simulación se comparan con los datos proporcionados por HFSS.

segundos para un total de 501 puntos en frecuencia. Por su parte, la simulación con HFSS empleó 16 minutos para 26 puntos en frecuencia, lo que demuestra nuevamente la eficiencia computacional del método propuesto.

Seguidamente, se presenta en la figura 4.12 la respuesta eléctrica de una nueva estructura excitada mediante sonda coaxial terminada en disco. En este caso, la guía rectangular semiinfinita está implementada en la guía estándar WR-137 ( $a=34,8488 \mathrm{~mm}, b=15,7988 \mathrm{~mm})$, mientras que las dimensiones de la sonda coaxial son las siguientes: $r_{1}=4,8768 \mathrm{~mm}$, $r_{2}=1,524 \mathrm{~mm}, r_{d}=4,0 \mathrm{~mm}$ (radios de la sonda); $h_{p}=3,4 \mathrm{~mm}, h_{d}=4,5 \mathrm{~mm}$ (longitudes de penetración de la sonda); $Z_{0}=50 \Omega$ (impedancia característica de la guía coaxial); $d_{z}=11,6 \mathrm{~mm}$ (distancia de la sonda a la pared de la guía cortocircuitada) y $d_{x}=a / 2$. Así, en la figura 4.12 se presentan en trazo continuo las pérdidas de retorno de la estructura, y se comparan con éxito, tanto con los resultados obtenidos con HFSS, como con los datos extraídos de las simulaciones realizadas en [19]. Por otro lado, la estructura se ha simulado en 177,8 segundos para un total de 301 puntos en frecuencia, mientras que HFSS empleó unos 10 minutos para un total de 11 puntos en frecuencia.

Por otro lado, en la misma figura 4.12 se presenta en trazo punteado la respuesta de la misma estructura pero considerando ahora que la sonda coaxial es de tipo convencional $\left(r_{d}=0\right)$. Así, se han mantenido todas las dimensiones de la estructura excepto que, ahora, para la profundidad de penetración de la sonda se considera $h_{p}^{\prime}=h_{p}+h_{d}=7,9 \mathrm{~mm}$. De los resultados obtenidos se concluye que la sonda coaxial terminada en disco mejora notable- 


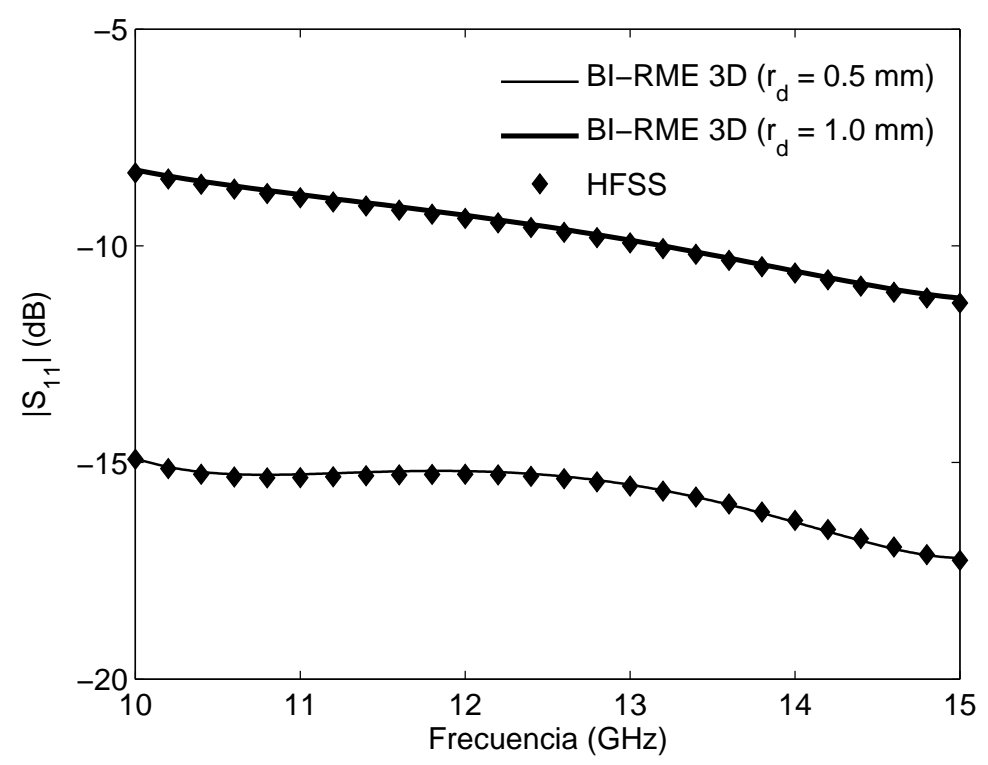

Figura 4.11: Pérdidas de retorno para una guía rectangular semi-infinita implementada en la guía estándar WR-75 excitada mediante una sonda coaxial terminada en disco $\left(r_{1}=3,18 \mathrm{~mm}\right.$, $\left.r_{2}=0,3 \mathrm{~mm}, \varepsilon_{r}=2,09, h_{p}=4,7625 \mathrm{~mm}, h_{d}=1,5 \mathrm{~mm}, d_{z}=6,8 \mathrm{~mm}, d_{x}=a / 2\right)$. Los resultados de la simulación se comparan con los datos proporcionados por HFSS.

mente la respuesta eléctrica de la estructura en relación con la sonda coaxial convencional.

Si se recuerdan los tiempos empleados por la herramienta de análisis en el caso de sondas convencionales (alrededor de 43 segundos), se observará que cuando la sonda coaxial está terminada en disco, la herramienta emplea, por lo general, un tiempo de simulación mayor. La razón de este incremento se debe, por un lado, a que la sonda terminada en disco es una estructura más compleja que la sonda coaxial convencional, por lo que el cálculo de las frecuencias de resonancia de la cavidad perturbada obtenida emplea un tiempo de simulación mayor ${ }^{20}$. Por otro lado, si nos remitimos a la ecuación (4.79) que define la expresión de los elementos de la matriz $\mathbf{L}$, comprobaremos que dicha expresión depende de las funciones base $\mathbf{N}_{m}(\mathbf{r})$ definidas sobre la sonda coaxial. Nuevamente, por ser la sonda coaxial terminada en disco una estructura más compleja que la sonda coaxial convencional, el tiempo empleado para evaluar los elementos de la matriz $\mathbf{L}$ aumenta significativamente. Además, este último hecho influye también de manera notable en la convergencia de los resultados. Así, en la figura 4.13 se presenta un estudio de convergencia para el módulo del parámetro de reflexión $S_{11}(f=7,5 \mathrm{GHz})$ para la estructura de la figura 4.12 en la que la sonda está terminada en disco. El estudio se realiza en función del número de modos $Q^{\mathrm{TE}}=Q^{\mathrm{TM}}$ utilizados en la guía rectangular de expansión. Así pues, de los resultados presentados se desprende que se precisan alrededor de $Q^{\mathrm{TE}}=Q^{\mathrm{TM}}=30000$ modos (en total, 60000 modos) de la guía

\footnotetext{
${ }^{20}$ La sonda coaxial convencional consta de una superficie cilíndrica de tipo base y de otra de tipo lado. En cambio, la sonda terminada en disco está formada por dos superficies cilíndricas de tipo base y por otras dos de tipo lado.
} 


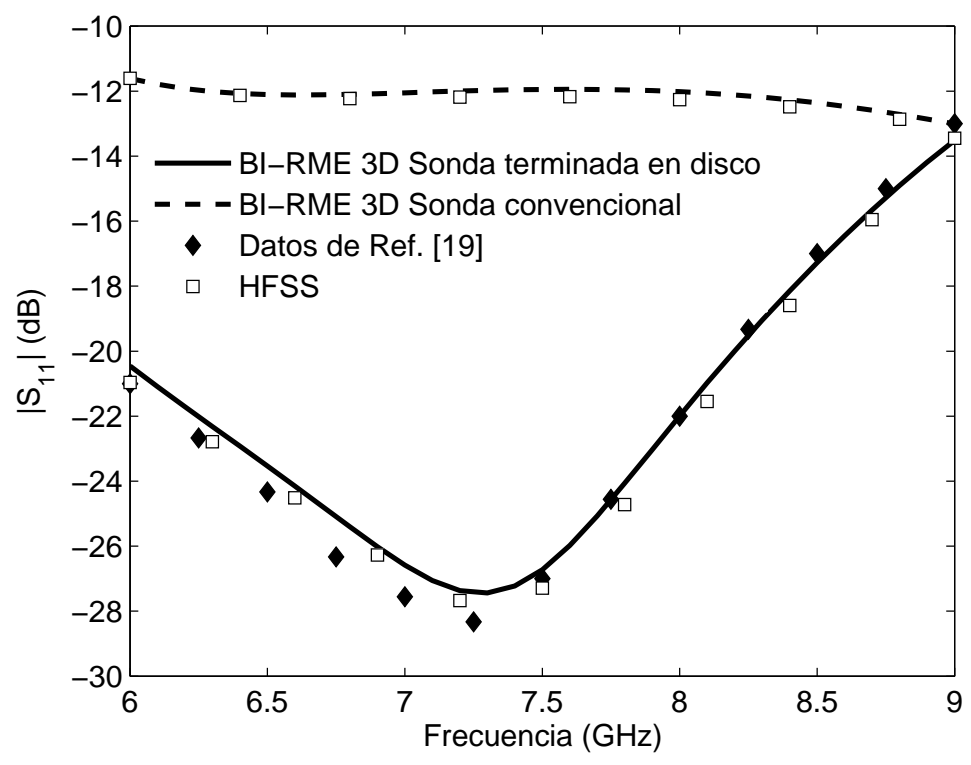

Figura 4.12: Pérdidas de retorno para una guía rectangular semi-infinita implementada en la guía estándar WR-137 excitada mediante una sonda coaxial terminada en disco $\left(r_{1}=4,8768 \mathrm{~mm}\right.$, $\left.r_{2}=1,524 \mathrm{~mm}, r_{d}=4,0 \mathrm{~mm}, h_{p}=3,4 \mathrm{~mm}, h_{d}=4,5 \mathrm{~mm}, Z_{0}=50 \Omega, d_{z}=11,6 \mathrm{~mm}, d_{x}=a / 2\right)$. Los resultados de la simulación se comparan con los datos proporcionados por HFSS y con los datos extraídos de [19]. En la misma figura se presenta en trazo punteado la respuesta de una sonda coaxial convencional con $h_{p}^{\prime}=7,9 \mathrm{~mm}$.

rectangular de expansión para lograr resultados convergentes (para sondas coaxiales de tipo convencional se requiere, en general, un número menor de modos).

Un parámetro de simulación que influye notablemente en la respuesta eléctrica de las estructuras analizadas en esta sección es la distancia $d_{z}$ a la pared cortocircuitada de la guía rectangular. En la figura 4.14 se han representado las pérdidas de retorno de la estructura excitada mediante sonda terminada en disco de la figura 4.12 en función de la distancia normalizada $d_{z} /\left(\lambda_{g} / 4\right)$ a la pared cortocircuitada de la guía $\left(\lambda_{g}\right.$ representa la longitud de onda en la guía para el modo fundamental a la frecuencia $f=7,5 \mathrm{GHz}$, es decir, la frecuencia central de operación de la guía estándar WR-137). Los casos analizados son $d_{z}=0,7 \lambda_{g} / 4$, $d_{z}=0,95 \lambda_{g} / 4$ (éste se corresponde con el caso estudiado en la figura 4.12) y $d_{z}=1,2 \lambda_{g} / 4$. Del análisis de los resultados presentados, los cuales se comparan con éxito con los obtenidos con HFSS, se deduce que el parámetro $d_{z}$ es una variable de diseño muy importante y se desprende, tal y como cabía esperar, que el resultado óptimo se obtiene para $d_{z} \approx \lambda_{g} / 4$.

Finalmente, se presenta el diseño de un filtro inductivo de 7 polos implementado en guía rectangular y excitado mediante sonda coaxial convencional. En la figura 4.15 se ha representado el filtro inductivo, el cual consta de 7 cavidades, 8 irises y 2 puertos de acceso (el filtro está formado por la conexión en cascada de 17 guías rectangulares). El puerto de acceso (1) es de tipo coaxial y el puerto de acceso (2) es de tipo rectangular, los cuales se conectan a la primera y última cavidad del filtro, respectivamente. Los radios externo e 


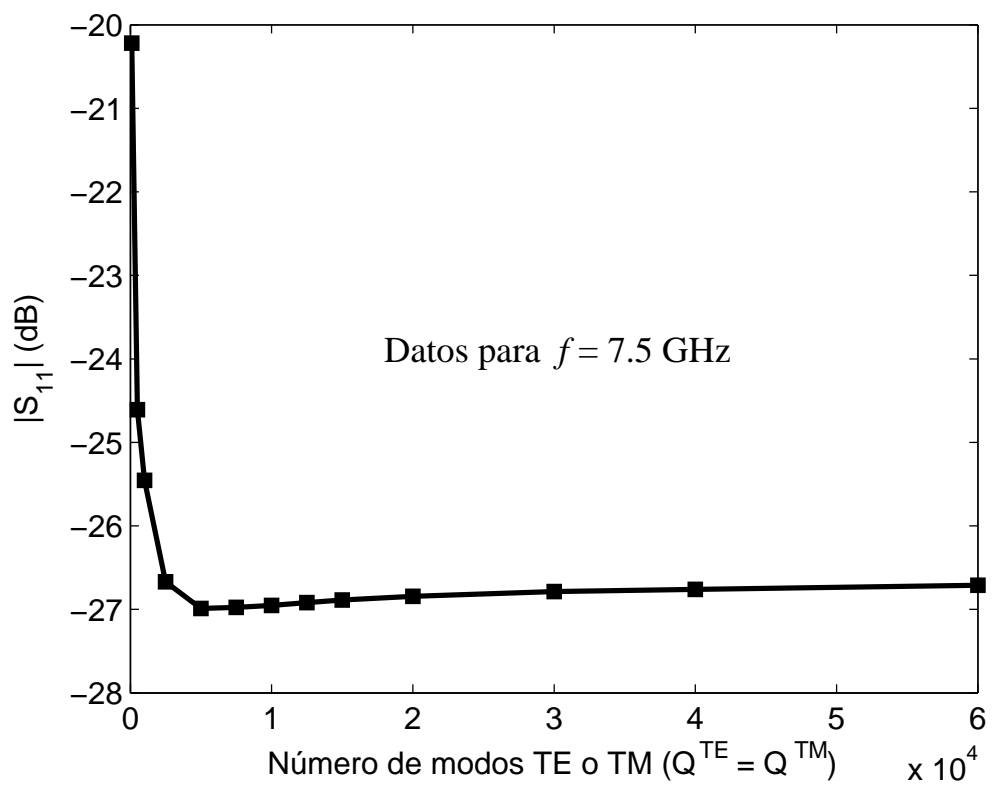

Figura 4.13: Estudio de convergencia para el módulo del parámetro $S_{11}(f=7,5 \mathrm{GHz})$ relativo a la estructura simulada en la figura 4.12. El estudio se realiza en función del número de modos $Q^{\mathrm{TE}}=Q^{\mathrm{TM}}$ utilizados en la guía rectangular de expansión.

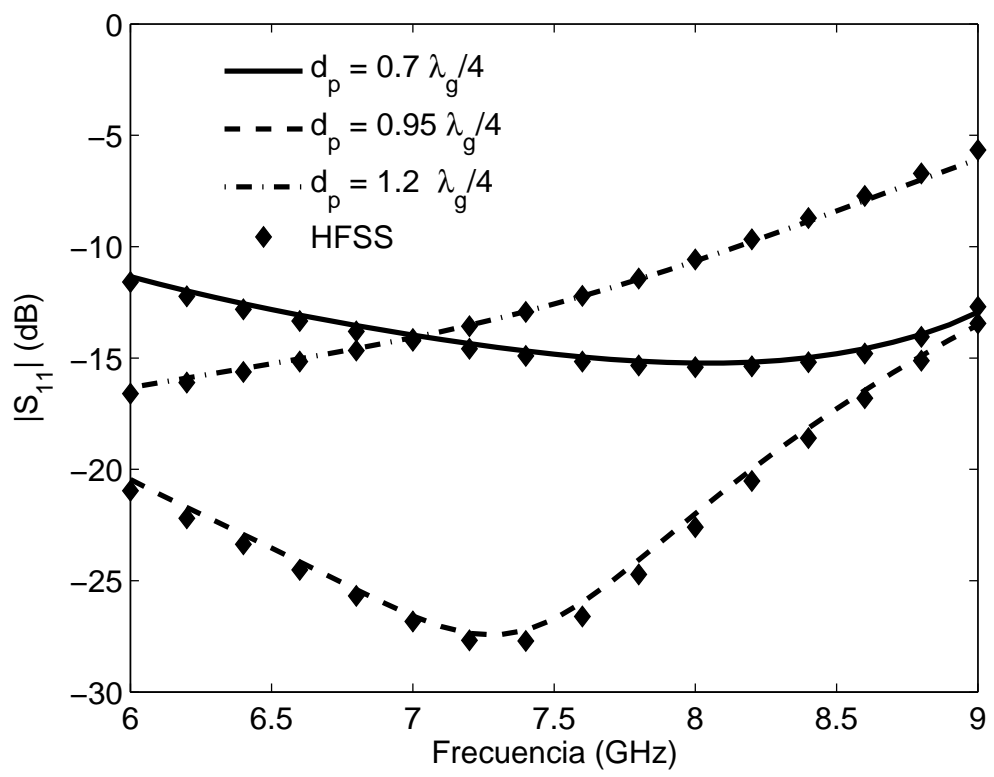

Figura 4.14: Pérdidas de retorno de la estructura analizada en la figura 4.12 en función de la distancia normalizada $d_{z} /\left(\lambda_{g} / 4\right)$ a la pared cortocircuitada de la guía rectangular. Los resultados se comparan con los datos proporcionados por HFSS. 
interno de la sonda coaxial son $r_{1}=2,11 \mathrm{~mm}$ y $r_{2}=0,635 \mathrm{~mm}$, respectivamente, y la permitividad dieléctrica relativa de la guía es $\varepsilon_{r}=2,08$. Por otro lado, si nos referimos a la notación utilizada en la figura 4.3, se tiene que la profundidad de penetración de la sonda coaxial es $h_{p}=4,07 \mathrm{~mm}, d_{x}=15,25 \mathrm{~mm} \mathrm{y} d_{z}=10,6065 \mathrm{~mm}$. Por otra parte, en la tabla 4.1 se encuentran las anchuras y las longitudes de las 17 guías rectangulares que integran el filtro numeradas de izquierda a derecha (se considera que la altura de las guías es constante e igual a $b=12,624 \mathrm{~mm})^{21}$.

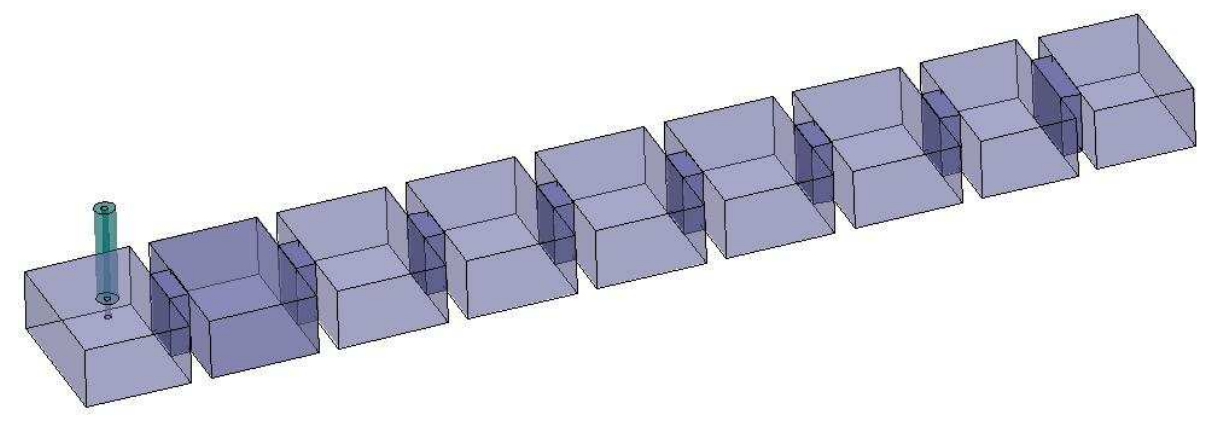

Figura 4.15: Filtro inductivo de 7 cavidades excitado mediante sonda coaxial convencional.

\begin{tabular}{|c|c|c|}
\hline \hline Número de guía & Anchura $(\mathrm{mm})$ & Longitud $(\mathrm{mm})$ \\
\hline 1 & 30,5 & 21,213 \\
\hline 2 y 14 & 9,594 & 4,04 \\
\hline 3 y 13 & 30,5 & 21,68 \\
\hline 4 y 12 & 8,684 & 4,04 \\
\hline 5 y 11 & 30,5 & 21,964 \\
\hline 6 y 10 & 8,498 & 4,04 \\
\hline 7 y 9 & 30,5 & 22,018 \\
\hline 8 & 8,458 & 4,04 \\
\hline 15 & 30,5 & 19,348 \\
\hline 16 & 14,626 & 4,04 \\
\hline 17 & 28,5 & 10,0 \\
\hline \hline
\end{tabular}

Tabla 4.1: Valores de las anchuras y de las longitudes de las 17 guías rectangulares que integran el filtro inductivo excitado mediante sonda coaxial convencional representado en la figura 4.15 (se asume que las guías se numeran de izquierda a derecha). La altura de todas las guías que integran la estructura se mantiene constante e igual a $b=12,624 \mathrm{~mm}$.

El análisis multimodal del filtro inductivo de la figura 4.15 combina el uso del método BI-RME 3D para caracterizar la excitación mediante sonda coaxial del filtro, y de la técnica de la ecuación integral para caracterizar las diversas uniones planares entre las guías rectangulares que integran el filtro. La respuesta eléctrica del filtro se representa en la figura 4.16,

\footnotetext{
${ }^{21}$ Las dimensiones del filtro inductivo excitado mediante sonda coaxial han sido proporcionadas por la empresa Thales Alenia Space (Tres Cantos, Madrid).
} 
donde se presenta el módulo del coeficiente de reflexión $S_{11}$ del dispositivo, y se compara con éxito la respuesta obtenida con los datos proporcionados por HFSS. En relación con la eficiencia computacional de la herramienta implementada es importante comentar que la simulación con BI-RME 3D utilizó 256 segundos para un total de 301 puntos en frecuencia. En cambio, el programa HFSS empleó 6,5 horas para un total de 212 puntos en frecuencia.

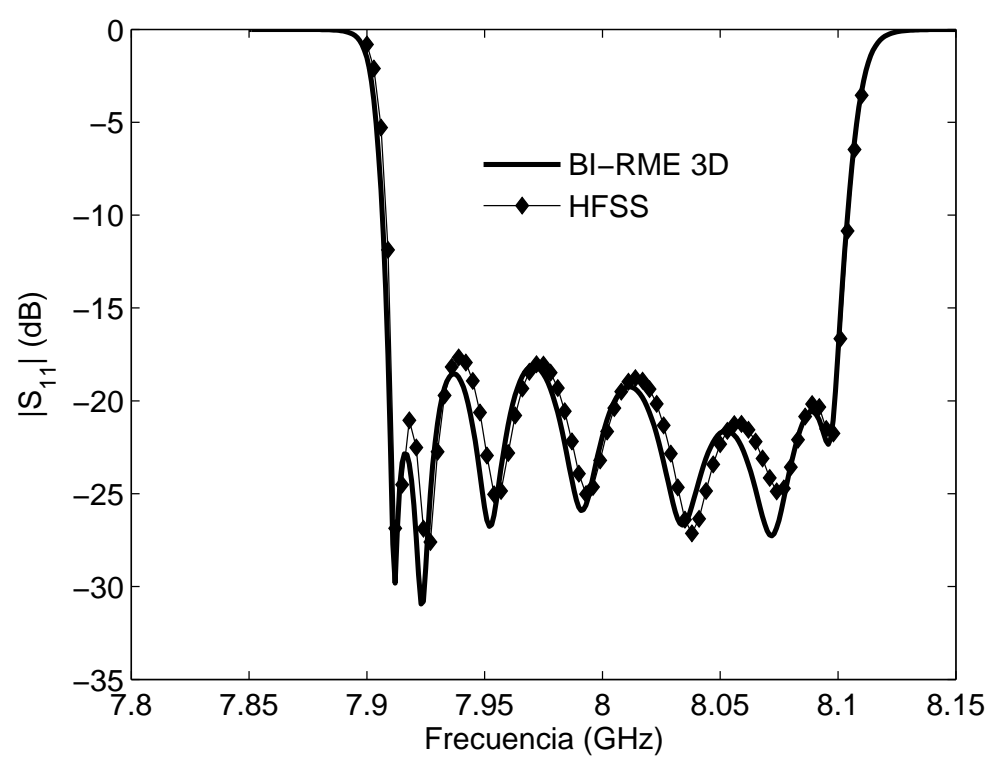

Figura 4.16: Pérdidas de retorno asociadas al filtro inductivo excitado mediante sonda coaxial representado en la figura 4.15 .

\subsection{Análisis Multimodal de la Excitación Coaxial de Dis- positivos en Configuración Colineal mediante el Méto- do BI-RME 3D}

Este nuevo apartado se dedica al análisis multimodal riguroso de estructuras implementadas en guía rectangular que estén excitadas mediante sonda coaxial en configuración colineal. En este tipo de configuración, la sonda coaxial se mantiene colineal a la guía rectangular que alimenta, tal y como se recoge en la figura 4.2. Además, es bastante usual introducir un poste conductor en la región de la guía rectangular con el propósito de facilitar la aparición de un modo en la estructura que logre la excitación del dispositivo. A este respecto, como ya se ha comentado en el apartado de introducción, la teoría que se desarrolla en esta sección considera que la sonda coaxial no llega a contactar con el poste conductor. La técnica de análisis que se utilizará se basa de nuevo en el método BI-RME 3D.

Por otro lado, el objetivo fundamental de este apartado consiste en desarrollar una herramienta de simulación que permita la caracterización electromagnética del bloque básico 
de excitación de los denominados filtros en configuración comb-line. Este tipo de filtros, que usualmente se excitan mediante sonda coaxial en configuración colineal, está constituido por la asociación de un conjunto de resonadores rectangulares cargados con postes conductores [73]. Los resonadores se acoplan entre sí mediante los correspondientes irises de acoplamiento cuya geometría, generalmente, es de tipo rectangular. Además, según la posición en la que se sitúan los irises, se habla de acoplamiento de tipo eléctrico o de acoplamiento de tipo magnético. Por otro lado, los filtros en configuración comb-line suelen tratarse como líneas de transmisión acopladas mediante modos cuasi-estáticos [97].

El bloque básico de excitación de los filtros en configuración comb-line se representa en la figura 4.17. Se trata de una cavidad rectangular cargada con un poste conductor de geometría cilíndrica que se excita mediante una sonda coaxial en configuración colineal, la cual no llega a contactar con el poste conductor. Por otro lado, la estructura consta de cuatro puertos de acceso, siendo uno de los puertos de acceso de tipo coaxial y los restantes de tipo rectangular (la numeración que utilizaremos para los diferentes puertos de acceso es la que aparece en la figura 4.17).

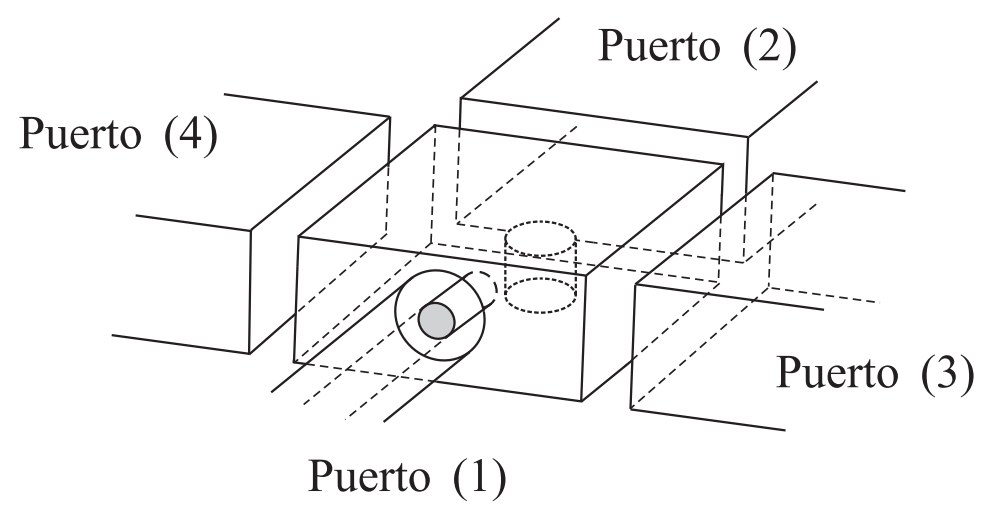

Figura 4.17: Excitación coaxial en configuración colineal de dispositivos implementados en guía rectangular.

Las vistas lateral y frontal del bloque básico de excitación se han representado en la figura 4.18. La cavidad rectangular es de dimensiones $a \times b \times c$; los radios externo e interno de la guía coaxial son $r_{1}$ y $r_{2}$, respectivamente; la profundidad de penetración de la sonda coaxial es $h_{p}$; y el poste conductor es de radio $r_{c}$ y de altura $h_{c}$. Además, el centro de la sonda coaxial está situado en la posición $(x, y, z)=\left(d_{x}, d_{y}, 0\right)$, mientras que el centro de la base inferior del poste conductor está situado en $(x, y, z)=\left(d_{x, c}, 0, d_{z, c}\right)$. Por otro lado, recordando la numeración de los puertos presentada en la figura 4.17, el acceso (1) (acceso coaxial) se sitúa en el plano $z=0$, el acceso (2) se encuentra en el plano $z=c$, el acceso (3) está en el plano $x=0$ y el acceso (4) se sitúa en el plano $x=a$. Por otra parte, en la figura 4.19 se recoge una nueva vista frontal de la estructura ${ }^{22}$ en la que se presenta el sistema de referencia en coordenadas cilíndricas que se empleará posteriormente. Según dicha figura,

\footnotetext{
${ }^{22}$ En la figura 4.19 no se ha representado el poste conductor ya que no es relevante para establecer las relaciones entre los sistemas de referencia en coordenadas cartesianas y en coordenadas cilíndricas.
} 

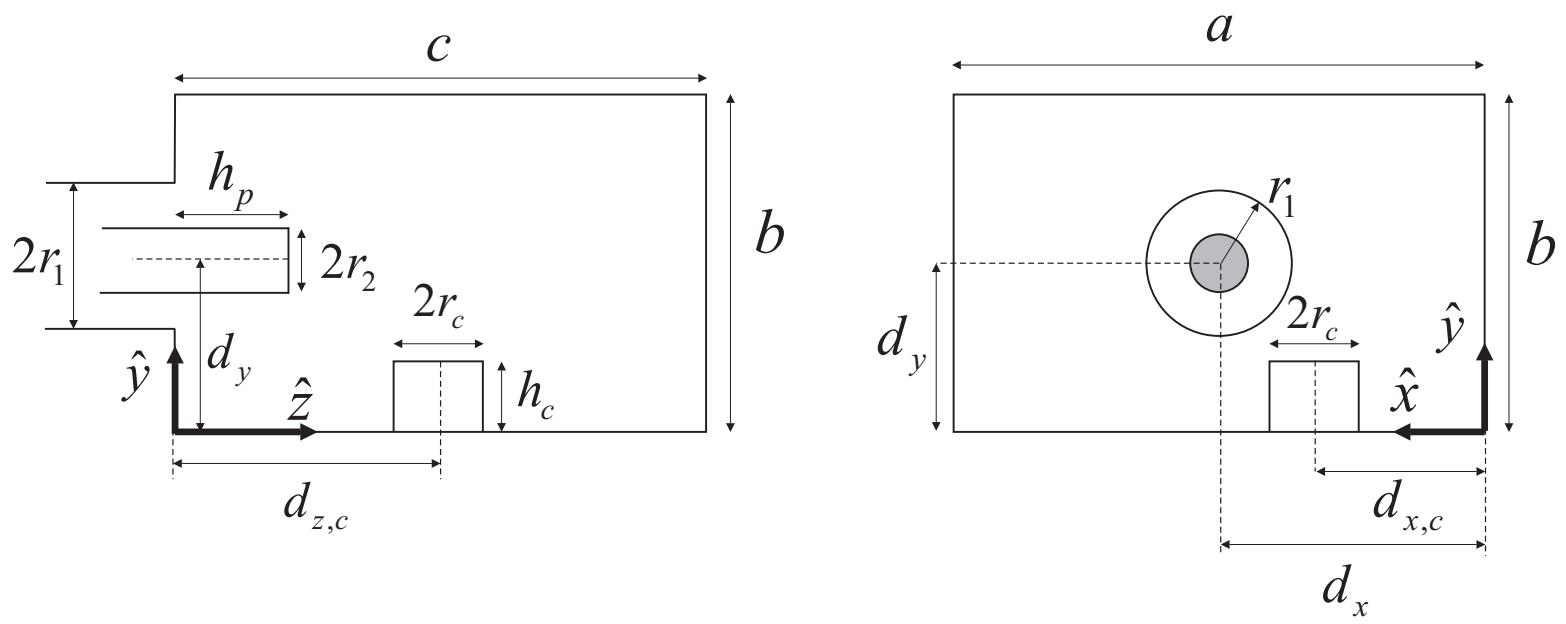

Figura 4.18: Vistas lateral y frontal del bloque básico relativo a la excitación coaxial en configuración colineal de dispositivos implementados en guía rectangular.

se pueden establecer las siguientes relaciones entre los sistemas de referencia en coordenadas cartesianas y en coordenadas cilíndricas:

$$
\begin{aligned}
x & =d_{x}+\rho \cos \phi \\
y & =d_{y}+\rho \sin \phi \\
z & =z \\
\hat{\mathbf{x}} & =\cos \phi \hat{\boldsymbol{\rho}}-\sin \phi \hat{\boldsymbol{\phi}} \\
\hat{\mathbf{y}} & =\sin \phi \hat{\boldsymbol{\rho}}+\cos \phi \hat{\boldsymbol{\phi}} \\
\hat{\mathbf{z}} & =\hat{\mathbf{z}}
\end{aligned}
$$

Seguidamente, se presenta el análisis electromagnético del bloque básico de excitación de la figura 4.17 utilizando el método BI-RME 3D. Según esta técnica, en primer lugar, se debe considerar que la estructura bajo análisis se encuentra inmersa en un resonador externo de geometría canónica. Nuevamente, la mejor elección en este caso consiste en escoger como resonador externo una cavidad rectangular de dimensiones $a \times b \times c$. De esta manera, la densidad superficial de corriente eléctrica incógnita sólo ha de definirse en la superficie de la sonda coaxial y en la del poste conductor. Posteriormente, siguiendo con el método BI-RME 3D, se deben cortocircuitar todos los puertos de acceso de la estructura y calcular las frecuencias de resonancia de la cavidad perturbada obtenida ${ }^{23}$ siguiendo la teoría desarrollada en [67]. Finalmente, se deben considerar los puertos de acceso de la estructura con

\footnotetext{
${ }^{23}$ En este caso, la cavidad perturbada consiste en un resonador rectangular cargado con dos postes conductores cilíndricos. Uno de los postes (sonda coaxial) es de radio $r_{2}$ y de altura $h_{p}$, y su base está situada en la posición $(x, y, z)=\left(d_{x}, d_{y}, 0\right)$. El otro poste es de radio $r_{c} \mathrm{y}$ de altura $h_{c}, \mathrm{y}$ su base está situada en $(x, y, z)=\left(d_{x, c}, 0, d_{z, c}\right)$.
} 


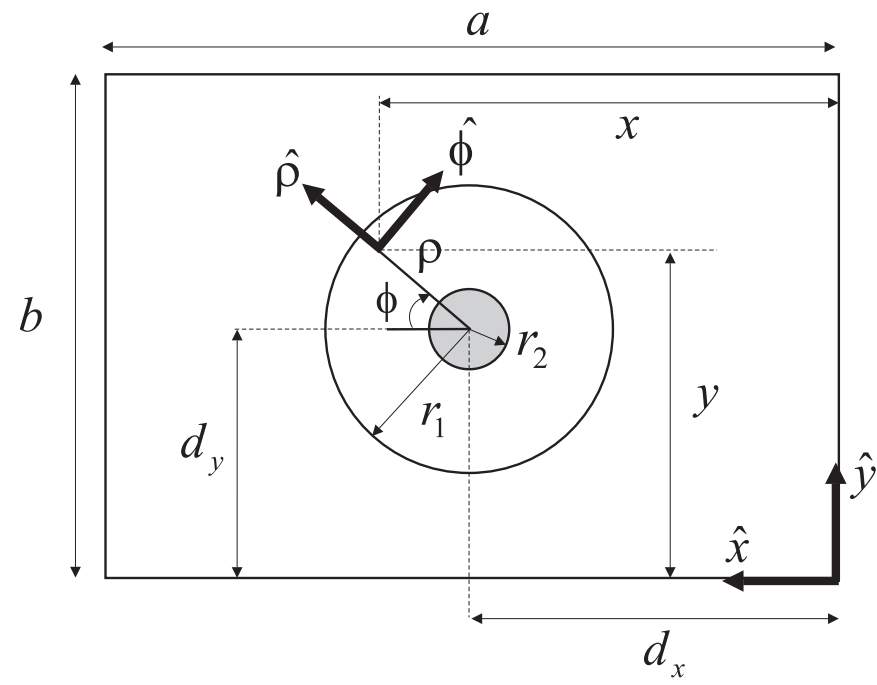

Figura 4.19: Sistema de referencia en coordenadas cilíndricas utilizado en el análisis de la excitación coaxial en configuración colineal de dispositivos implementados en guía rectangular.

el objetivo de calcular la correspondiente matriz de admitancias generalizada. Para ello, se deben calcular las matrices G, T, L y F, en cuyas expresiones intervienen las funciones vectoriales modales de campo magnético de los diferentes accesos (ver tabla 3.1). La contribución teórica de este apartado consiste, precisamente, en la evaluación eficiente de los elementos de dichas matrices BI-RME 3D.

\subsubsection{Cálculo de los elementos de la matriz $G$}

En primer lugar, se recuerda que la expresión de los elementos de la matriz $\mathbf{G}$ es la siguiente:

$$
G_{m n}^{(\gamma, \xi)}=\int_{S} \int_{S^{\prime}} \nabla_{S} \cdot \mathbf{h}_{m}^{(\gamma)}(\mathbf{r}) g^{\mathrm{m}}\left(\mathbf{r}, \mathbf{r}^{\prime}\right) \nabla_{S}^{\prime} \cdot \mathbf{h}_{n}^{(\xi)}\left(\mathbf{r}^{\prime}\right) d S d S^{\prime}
$$

donde, en este caso, $\gamma, \xi=1,2,3,4$ representan los puertos de acceso de la estructura (recordar la numeración de los puertos de la figura 4.17). Dado que la estructura bajo análisis tiene 4 accesos, la matriz G constará de 16 bloques. Por otro lado, como la matriz G es simétrica, únicamente se precisará calcular 10 de estos 16 bloques. Además, no será necesario calcular los bloques que relacionen puertos de acceso rectangulares (bloques $G_{m n}^{(\gamma, \xi)} \operatorname{con} \gamma, \xi \neq 1$ ) puesto que ese análisis ya se ha efectuado en el capítulo 3. Así pues, en esta sección se presenta el cálculo de los bloques $G_{m n}^{(1, \xi)}$, con $\xi=1,2,3,4$. Finalmente, es importante comentar que, según las conclusiones presentadas en la sección 4.2.1, cuando $m$ o $n$ representen a un modo de tipo $\mathrm{TM}^{z}$, se tendrá directamente que $G_{m n}^{(\gamma, \xi)}=0$. En lo que sigue, se asume que $m$ es un modo de tipo $\mathrm{TE}^{z}$ del acceso coaxial $(1)$, y que $(s, q)$ representa la pareja de índices 
modales relativos a las variaciones en las coordenadas angular y radial, respectivamente.

\section{A. Cálculo de los elementos del bloque $G_{m n}^{(1,2)}$}

Sea $n$ un modo de tipo $\mathrm{TE}^{z}$ del acceso rectangular (2), siendo $\left(n_{x}, n_{y}\right)$ la pareja de índices modales asociadas a las variaciones en las coordenadas $\hat{\mathbf{x}}$ e $\hat{\mathbf{y}}$, respectivamente (ver sistema de referencia de la figura 4.18). Del estudio efectuado en el capítulo 3 podemos escribir directamente que:

$$
\int_{S^{\prime}} g^{\mathrm{m}}\left(\mathbf{r}, \mathbf{r}^{\prime}\right) \nabla_{S}^{\prime} \cdot \mathbf{h}_{n}^{\mathrm{TE}(2)}\left(\mathbf{r}^{\prime}\right) d S^{\prime}=-\sqrt{\frac{\epsilon_{n_{x} \epsilon_{n_{y}}}}{a b}} \cos \left(\frac{n_{x} \pi}{a} x\right) \cos \left(\frac{n_{y} \pi}{b} y\right) \frac{\cosh \left(k_{t, n}^{(2)} z\right)}{\sinh \left(k_{t, n}^{(2)} c\right)}
$$

donde $k_{t, n}^{(2)}$ es el número de onda de corte del $n$-ésimo modo de tipo TE ${ }^{z}$ del acceso rectangular (2). Si ahora se expresa el resultado anterior en coordenadas cilíndricas utilizando las relaciones (4.118)-(4.120), y se recupera la expresión (4.7) en la que se calcula la divergencia de la función vectorial modal de campo magnético de los modos de tipo $\mathrm{TE}^{z}$ del acceso coaxial, se concluye fácilmente que:

$$
G_{m n}^{(1,2)}=\left(k_{t, m}^{(1)}\right)^{2} \mathcal{N}_{(\xi), m}^{\mathrm{TE}^{z}} \sqrt{\frac{\epsilon_{n_{x}} \epsilon_{n_{y}}}{a b}} \frac{1}{\sinh \left(k_{t, n}^{(2)} c\right)} \sum_{r=0}^{N_{\rho}-1} l_{s, q}^{(r)} I_{1}^{(r)}
$$

donde se ha definido la siguiente integral:

$$
I_{1}^{(r)}=\int_{S} \cos \left(\frac{n_{x} \pi}{a}\left(d_{x}+\rho \cos \phi\right)\right) \cos \left(\frac{n_{y} \pi}{b}\left(d_{y}+\rho \sin \phi\right)\right) \cos \left(r \pi \frac{\rho-r_{2}}{r_{1}-r_{2}}\right) \Phi_{(\xi), s}(\phi) d S
$$

siendo $S$ la sección transversal de la guía coaxial.

\section{B. Cálculo de los elementos del bloque $G_{m n}^{(1,3)}$}

En caso de que $n$ sea un modo $\mathrm{TE}^{z}$ del acceso rectangular (3), interesará trabajar con un nuevo sistema de referencia. En concreto, utilizaremos el sistema de coordenadas cartesianas representado en la figura 3.3 del capítulo 3 para $(\xi)=(3)$. La relación entre el sistema de coordenadas cilíndricas representado en la figura 4.19 y el sistema de coordenadas cartesianas del nuevo sistema de referencia es la siguiente:

$$
\begin{aligned}
& z=d_{x}+\rho \cos \phi \\
& y=d_{y}+\rho \sin \phi
\end{aligned}
$$

Nuevamente, utilizando los resultados del capítulo 3, se tiene:

$$
\int_{S^{\prime}} g^{\mathrm{m}}\left(\mathbf{r}, \mathbf{r}^{\prime}\right) \nabla_{S}^{\prime} \cdot \mathbf{h}_{n}^{\mathrm{TE}(3)}\left(\mathbf{r}^{\prime}\right) d S^{\prime}=\sqrt{\frac{\epsilon_{n_{x}} \epsilon_{n_{y}}}{c b}} \cos \left(\frac{n_{x} \pi}{c} x\right) \cos \left(\frac{n_{y} \pi}{b} y\right) \frac{\cosh \left(k_{t, n}^{(3)}(a-z)\right)}{\sinh \left(k_{t, n}^{(3)} a\right)}
$$


donde $\left(n_{x}, n_{y}\right)$ es la pareja de índices modales del $n$-ésimo modo $\mathrm{TE}^{z}$ del acceso (3) referidos a las coordenadas $\hat{\mathbf{x}}$ e $\hat{\mathbf{y}}$, respectivamente, del nuevo sistema de referencia. Seguidamente, se debe expresar el resultado (4.130) en coordenadas cilíndricas utilizando las relaciones (4.128)-(4.129). Además, se debe tener en cuenta que, según el nuevo sistema de referencia definido, el acceso coaxial se sitúa ahora en el plano $x=c$. De esta manera, teniendo en cuenta (4.7), se puede concluir fácilmente que:

$$
G_{m n}^{(1,3)}=-\left(k_{t, m}^{(1)}\right)^{2} \mathcal{N}_{(\xi), m}^{\mathrm{TE}^{z}} \sqrt{\frac{\epsilon_{n_{x}} \epsilon_{n_{y}}}{c b}} \frac{(-1)^{n_{x}}}{\sinh \left(k_{t, n}^{(3)} a\right)} \sum_{r=0}^{N_{\rho}-1} l_{s, q}^{(r)} I_{2}^{(r)}
$$

donde se ha definido:

$$
I_{2}^{(r)}=\int_{S} \cos \left(\frac{n_{y} \pi}{b}\left(d_{y}+\rho \sin \phi\right)\right) \cosh \left(k_{t, n}^{(3)}\left(a-d_{x}-\rho \cos \phi\right)\right) \cos \left(r \pi \frac{\rho-r_{2}}{r_{1}-r_{2}}\right) \Phi_{(\xi), s}(\phi) d S
$$

\section{Cálculo de los elementos del bloque $G_{m n}^{(1,4)}$}

Si $n$ es un modo $\mathrm{TE}^{z}$ del acceso rectangular (4), también se debe utilizar el nuevo sistema de referencia comentado en el apartado anterior. Gracias a los resultados obtenidos en el capítulo 3, se puede afirmar:

$$
\int_{S^{\prime}} g^{\mathrm{m}}\left(\mathbf{r}, \mathbf{r}^{\prime}\right) \nabla_{S}^{\prime} \cdot \mathbf{h}_{n}^{\mathrm{TE}(4)}\left(\mathbf{r}^{\prime}\right) d S^{\prime}=-\sqrt{\frac{\epsilon_{n_{x}} \epsilon_{n_{y}}}{c b}} \cos \left(\frac{n_{x} \pi}{c} x\right) \cos \left(\frac{n_{y} \pi}{b} y\right) \frac{\cosh \left(k_{t, n}^{(4)} z\right)}{\sinh \left(k_{t, n}^{(3)} a\right)}
$$

Si ahora se expresa la ecuación anterior en coordenadas cilíndricas y se particulariza el resultado obtenido para $x=c$ (plano de situación del acceso coaxial), se puede deducir finalmente:

$$
G_{m n}^{(1,4)}=\left(k_{t, m}^{(1)}\right)^{2} \mathcal{N}_{(\xi), m}^{\mathrm{TE}^{z}} \sqrt{\frac{\epsilon_{n_{x}} \epsilon_{n_{y}}}{c b}} \frac{(-1)^{n_{x}}}{\sinh \left(k_{t, n}^{(3)} a\right)} \sum_{r=0}^{N_{\rho}-1} l_{s, q}^{(r)} I_{3}^{(r)}
$$

donde hemos definido la siguiente integral de superficie:

$I_{3}^{(r)}=\int_{S} \cos \left(\frac{n_{y} \pi}{b}\left(d_{y}+\rho \sin \phi\right)\right) \cosh \left(k_{t, n}^{(3)}\left(d_{x}+\rho \cos \phi\right)\right) \cos \left(r \pi \frac{\rho-r_{2}}{r_{1}-r_{2}}\right) \Phi_{(\xi), s}(\phi) d S$

Es importante comentar que las integrales definidas en esta sección se han resuelto mediante métodos numéricos.

\section{Cálculo de los elementos del bloque $G_{m n}^{(1,1)}$}

En este caso, dado que los modos $m$ y $n$ pertenecen al mismo acceso, se tendrán problemas de singularidad asociados a la función escalar de Green de tipo magnético. La manera de abordar el problema es la misma que la que se utilizó en la sección 4.2.1; es decir, se propone expresar las soluciones modales de la guía coaxial en términos de las funciones vectoriales modales de la guía rectangular a la que alimenta la sonda coaxial. En este caso, dicha guía rectangular, a la que a partir de ahora denominaremos guía rectangular de expansión, es de 
dimensiones $a \times b$. Con todo ello, el desarrollo teórico que se debe realizar para obtener la expresión de los elementos del bloque $G_{m n}^{(1,1)}$ es completamente paralelo al efectuado en la sección 4.2.1. En consecuencia, partiendo de la expresión (4.29), se puede escribir:

$$
G_{m n}^{(1,1)}=\sum_{i=1}^{Q^{\mathrm{TE}}} \sigma_{i}^{(m)} \sigma_{i}^{(n)} k_{t, i}^{(\mathrm{R})} \operatorname{coth}\left(k_{t, i}^{(\mathrm{R})} c\right)
$$

donde $k_{t, i}^{(\mathrm{R})}$ es el número de onda de corte relativo al $i$-ésimo modo de tipo $\mathrm{TE}^{z}$ de la guía rectangular de expansión; $Q^{\mathrm{TE}}$ es el número de modos $\mathrm{TE}^{z}$ considerados en la expansión; y $\sigma_{i}^{(m)}$ representa el coeficiente de acoplamiento entre el $m$-ésimo modo $\mathrm{TE}^{z}$ del acceso coaxial y el $i$-ésimo modo $\mathrm{TE}^{z}$ de la guía rectangular de expansión.

\subsubsection{Cálculo de los elementos de la matriz $\mathrm{T}$}

Los elementos de la matriz $\mathbf{T}$ se calculan de la siguiente manera:

$$
T_{m n}^{(\gamma, \xi)}=\int_{S} \int_{S^{\prime}} \mathbf{h}_{m}^{(\gamma)}(\mathbf{r}) \cdot \overline{\mathbf{G}}_{0}^{\mathrm{F}}\left(\mathbf{r}, \mathbf{r}^{\prime}\right) \cdot \mathbf{h}_{n}^{(\xi)}\left(\mathbf{r}^{\prime}\right) d S d S^{\prime}
$$

donde $\gamma, \xi=1,2,3,4$ denotan los accesos de la estructura. Al igual que en el caso de la matriz $\mathbf{G}$, en esta sección únicamente se calculan los bloques $T_{m, n}^{(1, \xi)}(\xi=1,2,3,4)$. El resto de bloques de la matriz pueden obtenerse, o bien por simetría, o bien consultando los resultados obtenidos en el capítulo 3 (bloques referidos a los accesos en guía rectangular). Por otra parte, en esta sección se van a necesitar las expresiones para las funciones vectoriales modales normalizadas de campo magnético relativas a los modos $\mathrm{TEM}^{z}, \mathrm{TM}^{z}$ y $\mathrm{TE}^{z}$ de la guía coaxial. Estas expresiones pueden encontrarse, respectivamente, en (2.63), (2.96) y (2.118). Además, se asume que $(s, q)$ son los índices modales asociados al $m$-ésimo modo del acceso coaxial con respecto a las variaciones angular y radial, respectivamente.

\section{A. Cálculo de los elementos del bloque $T_{m n}^{(1,2)}$}

En primer lugar, se calculan los elementos del bloque $T_{m n}^{(1,2)}$. Así pues, consideremos que $m$ es un modo del acceso coaxial (1) y que $n$ es un modo del acceso rectangular (2). Por otro lado, se asume que $\left(n_{x}, n_{y}\right)$ son los índices modales del $n$-ésimo modo del acceso (2) referidos al sistema de referencia en coordenadas cartesianas representado en la figura 4.19.

Caso 1: n es un modo de tipo $\mathrm{TE}^{z}$

En caso de que $n$ sea un modo de tipo $\mathrm{TE}^{z}$ del acceso rectangular (2), se debe calcular en primer lugar la siguiente integral con respecto a las variables primadas:

$$
\boldsymbol{\Gamma}_{n \mathrm{TE}}^{(2)}(\mathbf{r})=\int_{S^{\prime}} \overline{\mathbf{G}}_{0}^{\mathrm{F}}\left(\mathbf{r}, \mathbf{r}^{\prime}\right) \cdot \mathbf{h}_{n}^{\mathrm{TE}(2)}\left(\mathbf{r}^{\prime}\right) d S^{\prime}
$$

El resultado de esta integral, que puede consultarse en la expresión (4.31), debe expresarse en coordenadas cilíndricas usando las transformaciones (4.118)-(4.123). Posteriormente, el 
resultado obtenido se debe particularizar en el plano $z=0$ en el que se sitúa el acceso coaxial. Con todo ello, en caso de que $m$ sea un modo $\mathrm{TEM}^{z}$ del acceso coaxial, se obtiene:

$$
\begin{aligned}
T_{m_{\mathrm{TEM}}, n_{\mathrm{TE}}}^{(1,2)} & =\int_{S} \mathbf{h}_{m}^{\mathrm{TEM}(1)}(\rho) \cdot \boldsymbol{\Gamma}_{n_{\mathrm{TE}}}^{(2)}(\rho, \phi) d S \\
& =\frac{1}{\sqrt{2 \pi \ln \left(r_{1} / r_{2}\right)}} \frac{2}{c} \sqrt{\frac{\epsilon_{n_{x}} \epsilon_{n_{y}}}{a b}} \frac{S_{n}^{(1)}(z=0)}{k_{t, n}^{(2)}}\left\{k_{x, n}^{(2)} I_{m_{\mathrm{TEM}}, n_{\mathrm{TE}}}^{(1,2)(a)} k_{y, n}^{(2)} I_{m_{\mathrm{TEM}}, n_{\mathrm{TE}}}^{(1,2)(b)}\right\}
\end{aligned}
$$

donde la expresión para la serie $S_{n}^{(1)}(z)$ se encuentra en (4.32). Además, las expresiones de las integrales $I_{m_{\mathrm{TEM}}, n_{\mathrm{TE}}}^{(1,2)(\alpha)}(\alpha=a, b)$ se han definido en la sección G.2 del Apéndice G.

En caso de que $m$ sea un modo de tipo $\mathrm{TE}^{z}$ del puerto coaxial, se puede concluir:

$$
\begin{aligned}
T_{m_{\mathrm{TE}}, n_{\mathrm{TE}}}^{(1,2)}= & \int_{S} \mathbf{h}_{m_{(\zeta)}}^{\mathrm{TE}(1)}(\rho, \phi) \cdot \boldsymbol{\Gamma}_{n_{\mathrm{TE}}}^{(2)}(\rho, \phi) d S \\
= & \mathcal{N}_{(\zeta), m}^{\mathrm{TE}} \frac{2}{c} \sqrt{\frac{\epsilon_{n_{x}} \epsilon_{n_{y}}}{a b}} \frac{S_{n}^{(1)}(z=0)}{k_{t, n}^{(2)}}\left\{\frac{\pi}{r_{1}-r_{2}} k_{x, n}^{(2)} \sum_{r=0}^{N_{\rho}-1} r l_{s, q}^{(r)} I_{m_{\mathrm{TE}}, n_{\mathrm{TE}}}^{(r),(1,2)^{(a)}}\right. \\
& +k_{x, n}^{(2)} \sum_{r=0}^{N_{\rho}-1} l_{s, q}^{(r)} I_{m_{\mathrm{TE}}, n_{\mathrm{TE}}}^{(r),(1,2)}+\frac{\pi}{r_{1}-r_{2}} k_{y, n}^{(2)} \sum_{r=0}^{N_{\rho}-1} r l_{s, q}^{(r)} I_{m_{\mathrm{TE}}, n_{\mathrm{TE}}^{(r),(1,2)}}^{(c)} \\
& \left.-k_{y, n}^{(2)} \sum_{r=0}^{N_{\rho}-1} l_{s, q}^{(r)} I_{m_{\mathrm{TE}}, n_{\mathrm{TE}}^{(r),(1,2)}}^{(d)}\right\}
\end{aligned}
$$

donde las integrales $I_{m_{\mathrm{TE}}, n_{\mathrm{TE}}}^{(r),(1,2)^{(\alpha)}}(\alpha=a, b, c, d)$ se han definido en la sección G.2 del Apéndice G. Finalmente, si $m$ es un modo de tipo $\mathrm{TM}^{z}$ del acceso coaxial, se tiene que:

$$
\begin{aligned}
& T_{m_{\mathrm{TM}}, n_{\mathrm{TE}}}^{(1,2)}=\int_{S} \mathbf{h}_{m_{(\zeta)}}^{\mathrm{TM}(1)}(\rho, \phi) \cdot \boldsymbol{\Gamma}_{n_{\mathrm{TE}}}^{(2)}(\rho, \phi) d S \\
& =\mathcal{N}_{(\zeta), m}^{\mathrm{TM}} \frac{2}{c} \sqrt{\frac{\epsilon_{n_{x}} \epsilon_{n_{y}}}{a b}} \frac{S_{n}^{(1)}(z=0)}{k_{t, n}^{(2)}}\left\{k_{x, n}^{(2)} \sum_{r=1}^{N_{\rho}} d_{s, q}^{(r)} I_{m_{\mathrm{TM}}, n_{\mathrm{TE}}}^{(r),(1,2)^{(a)}}\right. \\
& +k_{x, n}^{(2)} \frac{\pi}{r_{1}-r_{2}} \sum_{r=1}^{N_{\rho}} r d_{s, q}^{(r)} I_{m_{\mathrm{TM}}, n_{\mathrm{TE}}}^{(r),(1,2)^{(b)}}+k_{y, n}^{(2)} \sum_{r=1}^{N_{\rho}} d_{s, q}^{(r)} I_{m_{\mathrm{TM}}, n_{\mathrm{TE}}}^{(r),(1,2)^{(c)}} \\
& \left.-k_{y, n}^{(2)} \frac{\pi}{r_{1}-r_{2}} \sum_{r=1}^{N_{\rho}} r d_{s, q}^{(r)} I_{m_{\mathrm{TM}}, n_{\mathrm{TE}}}^{(r),(1),(d)}\right\}
\end{aligned}
$$


Las expresiones para las integrales $I_{m_{\mathrm{TM}}, n_{\mathrm{TE}}}^{(r),(1,2)}(\alpha=a, b, c, d)$ pueden encontrarse en el Apéndice G.

Caso 2: $n$ es un modo de tipo $\mathrm{TM}^{z}$

En lo que sigue, se asume que $n$ es un modo de tipo $\mathrm{TM}^{z}$ del acceso rectangular (2). En primer lugar, se calcula la siguiente integral cuyo resultado puede consultarse en (4.40):

$$
\boldsymbol{\Gamma}_{n_{\mathrm{TM}}}^{(2)}(\mathbf{r})=\int_{S^{\prime}} \overline{\mathbf{G}}_{0}^{\mathrm{F}}\left(\mathbf{r}, \mathbf{r}^{\prime}\right) \cdot \mathbf{h}_{n}^{\mathrm{TM}(2)}\left(\mathbf{r}^{\prime}\right) d S^{\prime}
$$

Después, se debe expresar el resultado de la integral en coordenadas cilíndricas utilizando (4.118)-(4.123) y se debe particularizar el resultado obtenido en el plano $z=0$ (situación del acceso coaxial). Así, en caso de que $m$ sea un modo de tipo TEM ${ }^{z}$ del acceso coaxial, se obtiene:

$$
\begin{aligned}
T_{m_{\mathrm{TEM}}, n_{\mathrm{TM}}}^{(1,2)} & =\int_{S} \mathbf{h}_{m}^{\mathrm{TEM}(1)}(\rho) \cdot \boldsymbol{\Gamma}_{n_{\mathrm{TM}}}^{(2)}(\rho, \phi) d S \\
& =\frac{2}{\sqrt{2 \pi a b \ln \left(r_{1} / r_{2}\right)}} \frac{1}{\left(k_{t, n}^{(2)}\right)^{2} \sinh \left(k_{t, n}^{(2)} c\right)}\left\{k_{y, n}^{(2)} I_{m_{\mathrm{TEM}}(1,2) n_{\mathrm{TE}}^{(a)}}+k_{x, n}^{(2)} I_{m_{\mathrm{TEM}}, n_{\mathrm{TE}}}^{(1,2)^{(b)}}\right\}
\end{aligned}
$$

donde las expresiones para las integrales $I_{m_{\mathrm{TEM}}, n_{\mathrm{TE}}}^{(1,2)^{(a)}}$ y $I_{m_{\mathrm{TEM}}, n_{\mathrm{TE}}}^{(1,2)^{(b)}}$ pueden encontrarse en el Apéndice $\mathrm{G}$ (nótese que, aunque $n$ es un modo de tipo $\mathrm{TM}^{z}$, son válidas las expresiones de las integrales deducidas cuando $n$ es un modo de tipo $\mathrm{TE}^{z}$ ).

Si $m$ es un modo $\mathrm{TE}^{z}$ del acceso coaxial se concluye:

$$
\begin{aligned}
T_{m_{\mathrm{TE}}, n_{\mathrm{TM}}}^{(1,2)}= & \int_{S} \mathbf{h}_{m_{(\zeta)}^{\mathrm{TE}}(1)}(\rho, \phi) \cdot \boldsymbol{\Gamma}_{n_{\mathrm{TM}}}^{(2)}(\rho, \phi) d S \\
= & \mathcal{N}_{(\zeta), m}^{\mathrm{TE}} \frac{2}{\sqrt{a b}} \frac{1}{\left(k_{t, n}^{(2)}\right)^{2} \sinh \left(k_{t, n}^{(2)} c\right)}\left\{\frac{\pi}{r_{1}-r_{2}} k_{y, n}^{(2)} \sum_{r=0}^{N_{\rho}-1} r l_{s, q}^{(r)} I_{m_{\mathrm{TE}}, n_{\mathrm{TE}}^{(r),(1,2)(a)}}^{(a)}\right. \\
& +k_{y, n}^{(2)} \sum_{r=0}^{N_{\rho}-1} l_{s, q}^{(r)} I_{m_{\mathrm{TE}}, n_{\mathrm{TE}}}^{(r),(1,2)}-\frac{\pi}{r_{1}-r_{2}} k_{x, n}^{(2)} \sum_{r=0}^{N_{\rho}-1} r l_{s, q}^{(r)} I_{m_{\mathrm{TE}}, n_{\mathrm{TE}}}^{(r),(1,)^{(c)}} \\
& \left.+k_{x, n}^{(2)} \sum_{r=0}^{N_{\rho}-1} l_{s, q}^{(r)} I_{m_{\mathrm{TE}}, n_{\mathrm{TE}}^{(r),(1,2)(d)}}^{(d)}\right\}
\end{aligned}
$$

donde las integrales $I_{m \mathrm{TE}, n_{\mathrm{TE}}}^{(r),(1,2)^{(\alpha)}}(\alpha=a, b, c, d)$ se han definido en el Apéndice G. Para termi- 
nar, si $m$ es un modo de tipo $\mathrm{TM}^{z}$ del acceso coaxial, se tendrá que:

$$
\begin{aligned}
T_{m_{\mathrm{TM}}, n_{\mathrm{TM}}}^{(1,2)}= & \int_{S} \mathbf{h}_{m_{(\zeta)}^{\mathrm{TM}(1)}(\rho, \phi) \cdot \boldsymbol{\Gamma}_{n_{\mathrm{TM}}}^{(2)}(\rho, \phi) d S} \\
= & \mathcal{N}_{(\zeta), m}^{\mathrm{TM}} \frac{2}{\sqrt{a b}} \frac{1}{\left(k_{t, n}^{(2)}\right)^{2} \sinh \left(k_{t, n}^{(2)} c\right)}\left\{k_{y, n}^{(2)} \sum_{r=1}^{N_{\rho}} d_{s, q}^{(r)} I_{m_{\mathrm{TM}}, n_{\mathrm{TE}}^{(r),(1,2)(a)}}\right. \\
& +k_{y, n}^{(2)} \frac{\pi}{r_{1}-r_{2}} \sum_{r=1}^{N_{\rho}} r d_{s, q}^{(r)} I_{m_{\mathrm{TM}}, n_{\mathrm{TE}}}^{(r),(1,2)}-k_{x, n}^{(2)} \sum_{r=1}^{N_{\rho}} d_{s, q}^{(r)} I_{m_{\mathrm{TM}}, n_{\mathrm{TE}}^{(r),(1,2)(())}}^{(2)} \\
& \left.+k_{x, n}^{(2)} \frac{\pi}{r_{1}-r_{2}} \sum_{r=1}^{N_{\rho}} r d_{s, q}^{(r)} I_{m_{\mathrm{TM}}, n_{\mathrm{TE}}}^{(r),(1,2)(d)}\right\}
\end{aligned}
$$

donde las integrales $I_{m_{\mathrm{TM}}, n_{\mathrm{TE}}}^{(r),(1,2)^{(\alpha)}}(\alpha=a, b, c, d)$ pueden encontrarse en (G.22)-(G.25).

\section{B. Cálculo de los elementos del bloque $T_{m n}^{(1,3)}$}

A continuación, se asume que $m$ es un modo del acceso coaxial y que $n$ es un modo del acceso rectangular (3). En este caso, como ya hicimos para el bloque $G_{m n}^{(1,3)}$ de la matriz $\mathbf{G}$, interesará trabajar con el sistema de referencia representado en la figura 3.3 del capítulo 3 para $(\xi)=(3)$, con lo que el acceso coaxial queda situado en el plano $x=c$. Por otra parte, la relación entre el nuevo sistema de coordenadas cartesianas y el sistema en coordenadas cilíndricas de la figura 4.19 viene dado por las expresiones (4.128)-(4.129). Además, también se necesitarán las siguientes relaciones:

$$
\begin{aligned}
\hat{\mathbf{z}} & =\cos \phi \hat{\boldsymbol{\rho}}-\sin \phi \hat{\boldsymbol{\phi}} \\
\hat{\mathbf{y}} & =\sin \phi \hat{\boldsymbol{\rho}}+\cos \phi \hat{\boldsymbol{\phi}}
\end{aligned}
$$

En lo que sigue, se considera que $\left(n_{x}, n_{y}\right)$ son los índices modales asociados al $n$-ésimo modo del acceso (3) relativo a las coordenadas $\hat{\mathbf{x}}$ e $\hat{\mathbf{y}}$ del nuevo sistema de referencia.

\section{Caso 1: n es un modo de tipo $\mathrm{TE}^{z}$}

Sea $n$ un modo de tipo $\mathrm{TE}^{z}$ del acceso rectangular (3). En primer lugar, se calcula la siguiente integral con respecto a las variables primadas cuyo resultado se ha deducido a 
partir de (3.36)-(3.38):

$$
\begin{aligned}
\boldsymbol{\Gamma}_{n_{\mathrm{TE}}}^{(3)}(\mathbf{r})= & \int_{S^{\prime}} \overline{\mathbf{G}}_{0}^{\mathrm{F}}\left(\mathbf{r}, \mathbf{r}^{\prime}\right) \cdot \mathbf{h}_{n}^{\mathrm{TE}(3)}\left(\mathbf{r}^{\prime}\right) d S^{\prime} \\
= & \frac{2}{a} \sqrt{\frac{\epsilon_{n_{x}} \epsilon_{n_{y}}}{c b}}\left\{\frac{k_{x, n}^{(3)}}{k_{t, n}^{(3)}} \sin \left(k_{x, n}^{(3)} x\right) \cos \left(k_{y, n}^{(3)} y\right) S_{n}^{(3)}(z) \hat{\mathbf{x}}\right. \\
& +\frac{k_{y, n}^{(3)}}{k_{t, n}^{(3)}} \cos \left(k_{x, n}^{(3)} x\right) \sin \left(k_{y, n}^{(3)} y\right) S_{n}^{(3)}(z) \hat{\mathbf{y}} \\
& \left.-k_{t, n}^{(3)} \cos \left(k_{x, n}^{(3)} x\right) \cos \left(k_{y, n}^{(3)} y\right) S_{n}^{(4)}(z) \hat{\mathbf{z}}\right\}
\end{aligned}
$$

donde $k_{x, n}^{(3)}=n_{x} \pi / c ; k_{y, n}^{(3)}=n_{y} \pi / b ; k_{t, n}^{(3)}$ es el número de onda de corte asociado al $n$-ésimo modo $\mathrm{TE}^{z}$ del acceso (3); y donde se han definido las siguientes series:

$$
\begin{aligned}
S_{n}^{(3)}(z) & =\sum_{n_{z}=1}^{\infty} \frac{k_{z}^{2}}{\left(\left(k_{t, n}^{(3)}\right)^{2}+k_{z}^{2}\right)^{2}} \cos \left(k_{z} z\right) \\
S_{n}^{(4)}(z) & =\sum_{n_{z}=1}^{\infty} \frac{k_{z}}{\left(\left(k_{t, n}^{(3)}\right)^{2}+k_{z}^{2}\right)^{2}} \sin \left(k_{z} z\right)
\end{aligned}
$$

siendo $k_{z}=n_{z} \pi / a$. Las series anteriores pueden sumarse analíticamente y su resultado puede deducirse a partir de las series $S_{n}^{(1)}(z)$ y $S_{n}^{(2)}(z)$, cuyas expresiones pueden encontrarse en (4.32)-(4.33), respectivamente. En efecto, si en (4.32)-(4.33) se considera que $k_{z}=n_{z} \pi / a$, resulta sencillo comprobar que:

$$
\begin{aligned}
& S_{n}^{(3)}(z)=S_{n}^{(1)}(z-a) \\
& S_{n}^{(4)}(z)=S_{n}^{(2)}(z-a)
\end{aligned}
$$

A continuación, el resultado de la integral (4.148) y el de las series $S_{n}^{(3)}(z)$ y $S_{n}^{(4)}(z)$ se debe expresar en coordenadas cilíndricas empleando las relaciones (4.128)-(4.129) y (4.146)(4.147). Posteriormente, las expresiones obtenidas se deben particularizar en el plano $x=c$, en el cual se sitúa el acceso coaxial. Así pues, en caso de que $m$ sea un modo de tipo TEM $^{z}$ del acceso coaxial se deduce que:

$$
\begin{aligned}
T_{m_{\mathrm{TEM}}, n_{\mathrm{TE}}}^{(1,3)} & =\int_{S} \mathbf{h}_{m}^{\mathrm{TEM}(1)}(\rho) \cdot \boldsymbol{\Gamma}_{n_{\mathrm{TE}}}^{(3)}(\rho, \phi) d S \\
& =\frac{1}{\sqrt{2 \pi \ln \left(r_{1} / r_{2}\right)}} \frac{2}{a} \sqrt{\frac{\epsilon_{n_{x}} \epsilon_{n_{y}}}{c b}}(-1)^{n_{x}}\left\{\frac{k_{y, n}^{(3)}}{k_{t, n}^{(3)}} I_{m_{\mathrm{TEM}}^{(1,3)} n_{\mathrm{TE}}^{(a)}}-k_{t, n}^{(3)} I_{m_{\mathrm{TEM}}^{(1,3)} n_{\mathrm{TE}}^{(b)}}\right\}
\end{aligned}
$$


Las expresiones para las integrales $I_{m_{\mathrm{TEM}}, n_{\mathrm{TE}}}^{(1,3)^{(\alpha)}}(\alpha=a, b)$ pueden encontrarse en el Apéndice G. Por otro lado, en caso de que $m$ sea un modo $\mathrm{TE}^{z}$ del acceso coaxial se obtiene que:

$$
\begin{aligned}
T_{m_{\mathrm{TE}}, n_{\mathrm{TE}}}^{(1,3)}= & \int_{S} \mathbf{h}_{m_{(\zeta)}^{\mathrm{TE}}(1)}(\rho, \phi) \cdot \boldsymbol{\Gamma}_{n_{\mathrm{TE}}}^{(3)}(\rho, \phi) d S \\
= & \mathcal{N}_{(\zeta), m}^{\mathrm{TE}} \frac{2}{a} \sqrt{\frac{\epsilon_{n_{x}} \epsilon_{n_{y}}}{c b}}(-1)^{n_{x}}\left\{-\frac{\pi}{r_{1}-r_{2}} \frac{k_{y, n}^{(3)}}{k_{t, n}^{(3)}} \sum_{r=0}^{N_{\rho}-1} r l_{s, q}^{(r)} I_{m_{\mathrm{TE}}, n_{\mathrm{TE}}^{(r),(1,3)^{(a)}}}\right. \\
& +\frac{k_{y, n}^{(3)}}{k_{t, n}^{(3)}} \sum_{r=0}^{N_{\rho}-1} l_{s, q}^{(r)} I_{m_{\mathrm{TE}}, n_{\mathrm{TE}}}^{(r),(1,3)}+\frac{\pi}{r_{1}-r_{2}} k_{t, n}^{(3)} \sum_{r=0}^{N_{\rho}-1} r l_{s, q}^{(r)} I_{m_{\mathrm{TE}}, n_{\mathrm{TE}}^{(r),(1,3)}}^{(c)} \\
& \left.+k_{t, n}^{(3)} \sum_{r=0}^{N_{\rho}-1} l_{s, q}^{(r)} I_{m_{\mathrm{TE}}, n_{\mathrm{TE}}}^{(r),(1,3)^{(d)}}\right\}
\end{aligned}
$$

donde las integrales $I_{m_{\mathrm{TE}}, n_{\mathrm{TE}}}^{(r),(1,3)^{(\alpha)}}(\alpha=a, b, c, d)$ se han definido en el Apéndice G. Finalmente, si $m$ es un modo $\mathrm{TM}^{z}$ del acceso coaxial, se cumple que:

$$
\begin{aligned}
& T_{m_{\mathrm{TM}}, n_{\mathrm{TE}}}^{(1,3)}=\int_{S} \mathbf{h}_{m_{(\zeta)}}^{\mathrm{TM}(1)}(\rho, \phi) \cdot \boldsymbol{\Gamma}_{n_{\mathrm{TE}}}^{(3)}(\rho, \phi) d S \\
& =\mathcal{N}_{(\zeta), m}^{\mathrm{TM}} \frac{2}{a} \sqrt{\frac{\epsilon_{n_{x}} \epsilon_{n_{y}}}{c b}}(-1)^{n_{x}}\left\{-\frac{k_{y, n}^{(3)}}{k_{t, n}^{(3)}} \sum_{r=1}^{N_{\rho}} d_{s, q}^{(r)} I_{m_{\mathrm{TM}}, n_{\mathrm{TE}}}^{(r),(1,3)^{(a)}}\right. \\
& +\frac{\pi}{r_{1}-r_{2}} \frac{k_{y, n}^{(3)}}{k_{t, n}^{(3)}} \sum_{r=1}^{N_{\rho}} r d_{s, q}^{(r)} I_{m_{\mathrm{TM}}, n_{\mathrm{TE}}}^{(r),(1,3)^{(b)}}+k_{t, n}^{(3)} \sum_{r=1}^{N_{\rho}} d_{s, q}^{(r)} I_{m_{\mathrm{TM}}, n_{\mathrm{TE}}}^{(r),(1,3)^{(c)}} \\
& \left.+\frac{\pi}{r_{1}-r_{2}} k_{t, n}^{(3)} \sum_{r=1}^{N_{\rho}} r d_{s, q}^{(r)} I_{m_{\mathrm{TM}}, n_{\mathrm{TE}}}^{(r),(1),(d)}\right\}
\end{aligned}
$$

donde, nuevamente, las integrales $I_{m_{\mathrm{TM}}, n_{\mathrm{TE}}}^{(r),(1,3)^{(\alpha)}}(\alpha=a, b, c, d)$ pueden encontrarse en el Apéndice G.

\section{Caso 2: nes un modo de tipo $\mathrm{TM}^{z}$}

Si $n$ es un modo $\mathrm{TM}^{z}$ del acceso rectangular (3), se puede deducir a partir de (3.39)- 
(3.41):

$$
\begin{aligned}
\boldsymbol{\Gamma}_{n_{\mathrm{TM}}}^{(3)}(\mathbf{r})= & \int_{S^{\prime}} \overline{\mathbf{G}}_{0}^{\mathrm{F}}\left(\mathbf{r}, \mathbf{r}^{\prime}\right) \cdot \mathbf{h}_{n}^{\mathrm{TM}(3)}\left(\mathbf{r}^{\prime}\right) d S^{\prime} \\
= & \frac{2}{\sqrt{c b}} \frac{1}{\left(k_{t, n}^{(3)}\right)^{2}} \frac{\cosh \left(k_{t, n}^{(3)}(a-z)\right)}{\sinh \left(k_{t, n}^{(3)} a\right)}\left\{k_{y, n}^{(3)} \sin \left(k_{x, n}^{(3)} x\right) \cos \left(k_{y, n}^{(3)} y\right) \hat{\mathbf{x}}\right. \\
& \left.-k_{x, n}^{(3)} \cos \left(k_{x, n}^{(3)} x\right) \sin \left(k_{y, n}^{(3)} y\right) \hat{\mathbf{y}}\right\}
\end{aligned}
$$

Seguidamente, se debe expresar el resultado anterior en coordenadas cilíndricas y particularizar la expresión obtenida en el plano $x=c$ (plano donde se sitúa el acceso coaxial). Después de realizar estas transformaciones, y en caso de que $m$ sea un modo $\mathrm{TEM}^{z}$ del acceso coaxial, se concluye:

$$
\begin{aligned}
T_{m_{\mathrm{TEM}}, n_{\mathrm{TM}}}^{(1,3)} & =\int_{S} \mathbf{h}_{m}^{\mathrm{TEM}(1)}(\rho) \cdot \boldsymbol{\Gamma}_{n_{\mathrm{TM}}}^{(3)}(\rho, \phi) d S \\
& =-\frac{2}{\sqrt{2 \pi c b \ln \left(r_{1} / r_{2}\right)}} \frac{k_{x, n}^{(3)}}{\left(k_{t, n}^{(3)}\right)^{2}} \frac{1}{\sinh \left(k_{t, n}^{(3)} a\right)}(-1)^{n_{x}} I_{m_{\mathrm{TEM}}, n_{\mathrm{TM}}}^{(1,3)}
\end{aligned}
$$

donde la integral $I_{m \text { TEM }, n_{\mathrm{TM}}}^{(1,3)}$ se ha definido en el Apéndice G.

Si $m$ es un modo de tipo $\mathrm{TE}^{z}$ del acceso coaxial, se deduce:

$$
\begin{aligned}
T_{m_{\mathrm{TE}}, n_{\mathrm{TM}}}^{(1,3)}= & \int_{S} \mathbf{h}_{m_{(\zeta)}^{\mathrm{TE}}(1)}^{\mathrm{T}}(\rho, \phi) \cdot \boldsymbol{\Gamma}_{n_{\mathrm{TM}}}^{(3)}(\rho, \phi) d S \\
= & \mathcal{N}_{(\zeta), m}^{\mathrm{TE}} \frac{2}{\sqrt{c b}} \frac{k_{x, n}^{(3)}}{\left(k_{t, n}^{(3)}\right)^{2}} \frac{1}{\sinh \left(k_{t, n}^{(3)} a\right)}(-1)^{n_{x}}\left\{\frac{\pi}{r_{1}-r_{2}} \sum_{r=0}^{N_{\rho}-1} r l_{s, q}^{(r)} I_{m_{\mathrm{TE}}, n_{\mathrm{TM}}^{(r),(1,3)(a)}}^{()^{(a)}}\right. \\
& \left.-\sum_{r=0}^{N_{\rho}-1} l_{s, q}^{(r)} I_{m_{\mathrm{TE}}, n_{\mathrm{TM}}}^{(r),(1,3)}\right\}
\end{aligned}
$$

Las definiciones para las integrales $I_{m_{\mathrm{TE}}, n_{\mathrm{TM}}}^{(r),(1,3)^{(\alpha)}}(\alpha=a, b)$ se pueden encontrar en el Apéndice G. Finalmente, en caso de que $m$ sea un modo $\mathrm{TM}^{z}$ del acceso coaxial, se tiene que:

$$
\begin{aligned}
T_{m_{\mathrm{TM}}, n_{\mathrm{TM}}}^{(1,3)}= & \int_{S} \mathbf{h}_{m_{(\zeta)}}^{\mathrm{TM}(1)}(\rho, \phi) \cdot \boldsymbol{\Gamma}_{n_{\mathrm{TM}}}^{(3)}(\rho, \phi) d S \\
= & \mathcal{N}_{(\zeta), m}^{\mathrm{TM}} \frac{2}{\sqrt{c b}} \frac{k_{x, n}^{(3)}}{\left(k_{t, n}^{(3)}\right)^{2}} \frac{1}{\sinh \left(k_{t, n}^{(3)} a\right)}(-1)^{n_{x}}\left\{\sum_{r=1}^{N_{\rho}} d_{s, q}^{(r)} I_{m_{\mathrm{TM}}, n_{\mathrm{TM}}}^{(r),(1,3)^{(a)}}\right. \\
& \left.-\sum_{r=1}^{N_{\rho}} r d_{s, q}^{(r)} I_{m_{\mathrm{TM}}, n_{\mathrm{TM}}}^{(r),(1,3)}\right\}
\end{aligned}
$$


donde las integrales $I_{m_{\mathrm{TM}}, n_{\mathrm{TM}}}^{(r),(1,3)^{(\alpha)}}(\alpha=a, b)$ se han definido en el Apéndice G.

\section{Cálculo de los elementos del bloque $T_{m n}^{(1,4)}$}

En este último caso, se considera que $n$ es un modo del acceso rectangular (4). Además, al igual que en el cálculo de los elementos del bloque $T_{m n}^{(1,3)}$, utilizaremos en los cálculos el sistema de referencia de la figura 3.3 para $(\xi)=(3)$. Por tanto, seguirán siendo válidas las relaciones de transformación entre sistemas de coordenadas establecidas en (4.128)-(4.129) y (4.146)-(4.147). A continuación, se asume que $\left(n_{x}, n_{y}\right)$ son los índices modales relativos al $n$-ésimo modo del acceso rectangular (4).

\section{Caso 1: n es un modo de tipo $\mathrm{TE}^{z}$}

Supongamos que $n$ es un modo $\mathrm{TE}^{z}$ del acceso rectangular (4). La integral de superficie que se calcula en primer lugar es la siguiente:

$$
\boldsymbol{\Gamma}_{n_{\mathrm{TE}}}^{(4)}(\mathbf{r})=\int_{S^{\prime}} \overline{\mathbf{G}}_{0}^{\mathrm{F}}\left(\mathbf{r}, \mathbf{r}^{\prime}\right) \cdot \mathbf{h}_{n}^{\mathrm{TE}(4)}\left(\mathbf{r}^{\prime}\right) d S^{\prime}
$$

El resultado de esta integral puede obtenerse directamente a partir de la expresión (4.31), si en dicha expresión se permuta la dimensión $c$ por la dimensión $a$, y se cambian los superíndices iguales a (2) por el superíndice (4). Posteriormente, el resultado obtenido se debe expresar en coordenadas cilíndricas, y se debe particularizar después la expresión resultante en el plano $x=c$ (plano de situación del acceso coaxial). Así pues, si $m$ es un modo $\mathrm{TEM}^{z}$ del acceso coaxial, se tiene que:

$$
\begin{aligned}
T_{m_{\mathrm{TEM}}, n_{\mathrm{TE}}}^{(1,4)} & =\int_{S} \mathbf{h}_{m}^{\mathrm{TEM}(1)}(\rho) \cdot \boldsymbol{\Gamma}_{n_{\mathrm{TE}}}^{(4)}(\rho, \phi) d S \\
& =-\frac{1}{\sqrt{2 \pi \ln \left(r_{1} / r_{2}\right)}} \frac{2}{a} \sqrt{\frac{\epsilon_{n_{x}} \epsilon_{n_{y}}}{c b}}(-1)^{n_{x}}\left\{\frac{k_{y, n}^{(4)}}{k_{t, n}^{(4)}} I_{m_{\mathrm{TEM}}, n_{\mathrm{TE}}}^{(1,4)(a)}+k_{t, n}^{(4)} I_{m_{\mathrm{TEM}}, n_{\mathrm{TE}}}^{(1,4)}\right\}
\end{aligned}
$$

donde $k_{y, n}^{(4)}=n_{y} \pi / b$, y $k_{t, n}^{(4)}$ es el número de onda de corte asociado al $n$-ésimo modo del acceso (4). Además, las expresiones de las integrales $I_{m_{\mathrm{TEM}}, n_{\mathrm{TE}}}^{(1,4)^{(\alpha)}}(\alpha=a, b)$ pueden encontrarse en la sección G.2 del Apéndice G. En caso de que $m$ sea un modo de tipo TE ${ }^{z}$ del acceso 
coaxial, entonces se deduce:

$$
\begin{aligned}
T_{m_{\mathrm{TE}}, n_{\mathrm{TE}}}^{(1,4)}= & \int_{S} \mathbf{h}_{m_{(\zeta)}}^{\mathrm{TE}(1)}(\rho, \phi) \cdot \boldsymbol{\Gamma}_{n_{\mathrm{TE}}}^{(4)}(\rho, \phi) d S \\
= & \mathcal{N}_{(\zeta), m}^{\mathrm{TE}} \frac{2}{a} \sqrt{\frac{\epsilon_{n_{x}} \epsilon_{n_{y}}}{c b}}(-1)^{n_{x}}\left\{\frac{\pi}{r_{1}-r_{2}} \frac{k_{y, n}^{(4)} \sum_{t, n}^{(4)}}{k_{r=0}^{N_{\rho}-1}} r l_{s, q}^{(r)} I_{m_{\mathrm{TE}}, n_{\mathrm{TE}}}^{(r),(1)}\right. \\
& -\frac{k_{y, n}^{(4)}}{k_{t, n}^{(4)}} \sum_{r=0}^{N_{\rho}-1} l_{s, q}^{(r)} I_{m_{\mathrm{TE}}, n_{\mathrm{TE}}}^{(r),(2)}-k_{t, n}^{(4)} \frac{\pi}{r_{1}-r_{2}} \sum_{r=0}^{N_{\rho}-1} r l_{s, q}^{(r)} I_{m_{\mathrm{TE}}, n_{\mathrm{TE}}}^{(r),(3)} \\
& \left.-k_{t, n}^{(4)} \sum_{r=0}^{N_{\rho}-1} l_{s, q}^{(r)} I_{m_{\mathrm{TE}}, n_{\mathrm{TE}}}^{(r),(4)}\right\}
\end{aligned}
$$

donde las integrales $I_{m_{\mathrm{TE}}, n_{\mathrm{TE}}}^{(r),(\alpha)}(\alpha=1,2,3,4)$ se han definido en la sección G.2 del Apéndice G. Finalmente, si $m$ es un modo $\mathrm{TM}^{z}$ del acceso coaxial, se obtiene:

$$
\begin{aligned}
T_{m_{\mathrm{TM}}, n_{\mathrm{TE}}}^{(1,4)}= & \int_{S} \mathbf{h}_{m_{(\zeta)}^{\mathrm{TM}(1)}}^{\mathrm{TM}}(\rho, \phi) \cdot \boldsymbol{\Gamma}_{n_{\mathrm{TE}}}^{(4)}(\rho, \phi) d S \\
= & \mathcal{N}_{(\zeta), m}^{\mathrm{TM}} \frac{2}{a} \sqrt{\frac{\epsilon_{n_{x}} \epsilon_{n_{y}}}{c b}}(-1)^{n_{x}}\left\{\frac{k_{y, n}^{(4)}}{k_{t, n}^{(4)}} \sum_{r=1}^{N_{\rho}} d_{s, q}^{(r)} I_{m_{\mathrm{TM}}, n_{\mathrm{TE}}}^{(r),(1)}\right. \\
& -\frac{\pi}{r_{1}-r_{2}} \frac{k_{y, n}^{(4)}}{k_{t, n}^{(4)}} \sum_{r=1}^{N_{\rho}} r d_{s, q}^{(r)} I_{m_{\mathrm{TM}}, n_{\mathrm{TE}}}^{(r),(2)}-k_{t, n}^{(4)} \sum_{r=1}^{N_{\rho}} d_{s, q}^{(r)} I_{m_{\mathrm{TM}}, n_{\mathrm{TE}}}^{(r),(3)} \\
& \left.-\frac{\pi}{r_{1}-r_{2}} k_{t, n}^{(4)} \sum_{r=1}^{N_{\rho}} r d_{s, q}^{(r)} I_{m_{\mathrm{TM}}, n_{\mathrm{TE}}}^{(r),(4)}\right\}
\end{aligned}
$$

Nuevamente, la definición de las integrales $I_{m_{\mathrm{TM}}, n_{\mathrm{TE}}}^{(r),(\alpha)}(\alpha=1,2,3,4)$ puede encontrarse en la sección G.2 del Apéndice G.

Caso 2: n es un modo de tipo $\mathrm{TM}^{z}$

Sea ahora $n$ un modo de tipo $\mathrm{TM}^{z}$ del acceso rectangular (4). En primer lugar, calculamos la siguiente integral con respecto a las variables primadas:

$$
\boldsymbol{\Gamma}_{n_{\mathrm{TM}}}^{(4)}(\mathbf{r})=\int_{S^{\prime}} \overline{\mathbf{G}}_{0}^{\mathrm{F}}\left(\mathbf{r}, \mathbf{r}^{\prime}\right) \cdot \mathbf{h}_{n}^{\mathrm{TM}(4)}\left(\mathbf{r}^{\prime}\right) d S^{\prime}
$$

El resultado de la integral anterior puede deducirse a partir de la expresión (4.40), si en dicha expresión se permuta la dimensión $c$ por la dimensión $a$, y se cambian los superíndices 
iguales a (2) por el subíndice (4). Después, se debe expresar el resultado $\boldsymbol{\Gamma}_{n_{\mathrm{TM}}}^{(4)}(\mathbf{r})$ en coordenadas cilíndricas y particularizar la expresión obtenida en $x=c$ (plano de situación del acceso coaxial). Así pues, en caso de que $m$ sea un modo $\mathrm{TEM}^{z}$ del acceso coaxial se tiene que:

$$
\begin{aligned}
T_{m_{\mathrm{TEM}}, n_{\mathrm{TM}}}^{(1,4)} & =\int_{S} \mathbf{h}_{m}^{\mathrm{TEM}(1)}(\rho) \cdot \boldsymbol{\Gamma}_{n_{\mathrm{TM}}}^{(4)}(\rho, \phi) d S \\
& =\frac{1}{\sqrt{2 \pi \ln \left(r_{1} / r_{2}\right)}} \frac{2}{\sqrt{c b}} \frac{k_{x, n}^{(4)}}{\left(k_{t, n}^{(4)}\right)^{2}}(-1)^{n_{x}} \frac{1}{\sinh \left(k_{t, n}^{(4)} a\right)} I_{m_{\mathrm{TEM}}, n_{\mathrm{TM}}}
\end{aligned}
$$

donde $k_{x, n}^{(4)}=n_{x} \pi / c$. Por otro lado, la integral $I_{m_{\mathrm{TEM}}, n_{\mathrm{TM}}}$ se ha definido en el Apéndice G. En caso de que $m$ sea un modo $\mathrm{TE}^{z}$ del acceso coaxial, se obtiene:

$$
\begin{aligned}
T_{m_{\mathrm{TE}}, n_{\mathrm{TM}}}^{(1,4)} & \int_{S} \mathbf{h}_{m_{(\zeta)}}^{\mathrm{TE}(1)}(\rho, \phi) \cdot \boldsymbol{\Gamma}_{n_{\mathrm{TM}}}^{(4)}(\rho, \phi) d S \\
= & \mathcal{N}_{(\zeta), m}^{\mathrm{TE}} \frac{2}{\sqrt{c b}} \frac{k_{x, n}^{(4)}}{\left(k_{t, n}^{(4)}\right)^{2}}(-1)^{n_{x}} \frac{1}{\sinh \left(k_{t, n}^{(4)} a\right)}\left\{-\frac{\pi}{r_{1}-r_{2}} \sum_{r=0}^{N_{\rho}-1} r l_{s, q}^{(r)} I_{m_{\mathrm{TE}}, n_{\mathrm{TM}}}^{(r),(1)}\right. \\
& \left.+\sum_{r=0}^{N_{\rho}-1} l_{s, q}^{(r)} I_{m_{\mathrm{TE}}, n_{\mathrm{TM}}}^{(r),(2)}\right\}
\end{aligned}
$$

donde, de nuevo, la definición de las integrales $I_{m_{\mathrm{TE}}, n_{\mathrm{TM}}}^{(r),(\alpha)}(\alpha=1,2)$ puede hallarse en el Apéndice G. Finalmente, sea $m$ un modo de tipo $\mathrm{TM}^{z}$ del acceso coaxial. En este caso se concluye:

$$
\begin{aligned}
T_{m_{\mathrm{TM}}, n_{\mathrm{TM}}}^{(1,4)}= & \int_{S} \mathbf{h}_{m_{(\zeta)}}^{\mathrm{TM}(1)}(\rho, \phi) \cdot \boldsymbol{\Gamma}_{n_{\mathrm{TM}}}^{(4)}(\rho, \phi) d S \\
= & \mathcal{N}_{(\zeta), m}^{\mathrm{TM}} \frac{2}{\sqrt{c b}} \frac{k_{x, n}^{(4)}}{\left(k_{t, n}^{(4)}\right)^{2}}(-1)^{n_{x}} \frac{1}{\sinh \left(k_{t, n}^{(4)} a\right)}\left\{-\sum_{r=1}^{N_{\rho}} d_{s, q}^{(r)} I_{m_{\mathrm{TM}}, n_{\mathrm{TM}}}^{(r),(1)}\right. \\
& \left.+\sum_{r=1}^{N_{\rho}} r d_{s, q}^{(r)} I_{m_{\mathrm{TM}}, n_{\mathrm{TM}}}^{(r),(2)}\right\}
\end{aligned}
$$

Las expresiones de las integrales $I_{m_{\mathrm{TM}}, n_{\mathrm{TM}}}^{(r),(\alpha)}(\alpha=1,2)$ se pueden encontrar en el Apéndice G.

\section{Cálculo de los elementos del bloque $T_{m n}^{(1,1)}$}

En este caso, sabemos que existen problemas de singularidad en la función diádica de Green, por lo que la estrategia a seguir consiste en expresar las soluciones modales de la 
guía coaxial en términos de las funciones vectoriales modales de la guía rectangular a la que alimenta el coaxial. En este caso, dicha guía rectangular (guía rectangular de expansión) es de dimensiones $a \times b$ (ver figura 4.18). Así, el desarrollo teórico que se debe realizar es completamente equivalente al que se ha realizado en la sección 4.2.2 para el caso de la excitación coaxial en configuración clásica. Por este motivo, los elementos del bloque $T_{m n}^{(1,1)}$ bajo análisis se pueden calcular a partir de los resultados obtenidos en la sección 4.2.2 cambiando en dichas expresiones la dimensión $b$ por la dimensión $c$. Además, si se revisan las expresiones obtenidas en dicha sección, $k_{t, i}^{(\mathrm{R})}$ ahora hará referencia al número de onda de corte del $i$-ésimo modo de la guía rectangular de expansión de dimensiones $a \times b$.

\subsubsection{Cálculo de los elementos de la matriz $L$}

La expresión de los elementos de la matriz L se recuerda a continuación:

$$
L_{m n}^{(\xi)}=\int_{S} \int_{S^{\prime}} \mathbf{N}_{m}(\mathbf{r}) \cdot \nabla \times \overline{\mathbf{G}}_{0}^{\mathrm{F}}\left(\mathbf{r}, \mathbf{r}^{\prime}\right) \cdot \mathbf{h}_{n}^{(\xi)}\left(\mathbf{r}^{\prime}\right) d S d S^{\prime}
$$

donde $\xi=1,2,3,4$ hace referencia a los puertos de acceso de la estructura. Es importante observar que los elementos de esta matriz para los bloques $\xi=2,3,4$ (puertos de acceso en guía rectangular) se han calculado en las expresiones (3.58)-(3.61) obtenidas en la sección 3.2.2 del capítulo 3. Para el caso en que $\xi=1$ (bloque relativo al acceso coaxial), puesto que, nuevamente, existen problemas de singularidad asociados con el rotacional de la función diádica de Green, se emplea la estrategia de expresar las funciones vectoriales modales de la guía coaxial en términos de las soluciones modales relativas a la guía rectangular de expansión de dimensiones $a \times b$. Así pues, las expresiones de los elementos del bloque $L_{m n}^{(1)}$ pueden deducirse a partir de los resultados obtenidos en la sección 4.2.3, en la que se estudiaba la matriz L para el caso de la excitación coaxial en configuración clásica. En particular, los elementos del bloque $L_{m n}^{(1)}$ bajo análisis pueden obtenerse a partir de las expresiones (4.84), (4.89) y (4.92) de la sección 4.2.3. Para ello, en dichas expresiones, simplemente se debe cambiar la dimensión $b$ por la dimensión $c$, y viceversa.

\subsubsection{Cálculo de los elementos de la matriz $\mathrm{F}$}

La expresión de los elementos de la matriz $\mathbf{F}$ es la siguiente:

$$
F_{m n}^{(\xi)}=\int_{S} \mathbf{H}_{m}(\mathbf{r}) \cdot \mathbf{h}_{n}^{(\xi)}(\mathbf{r}) d S
$$

donde $\xi=1,2,3,4$ representa los accesos de la estructura. Los elementos de los bloques $F_{m n}^{(\xi)} \operatorname{con} \xi=2,3,4$ relativos a los accesos en guía rectangular ya se han calculado en las expresiones (3.63)-(3.69) obtenidas en la sección 3.2.2 del capítulo 3. Para calcular los elementos del bloque $F_{m n}^{(1)}$ relacionados con el acceso coaxial se recurre nuevamente a expresar las soluciones modales de la guía coaxial en términos de las funciones vectoriales modales de la guía rectangular de expansión. Tal y como se ha comentado en la sección 4.2.4, aunque 
la matriz $\mathbf{F}$ no presenta problemas de singularidad, interesa utilizar esta técnica por motivos de eficiencia computacional. Siguiendo esta estrategia, los elementos del bloque $F_{m n}^{(1)}$ pueden obtenerse a partir de las expresiones (4.112)-(4.117). Si se revisan dichas expresiones, se observará que se encuentran en función de unas integrales de superficie en las que intervienen las funciones vectoriales modales de la guía rectangular de expansión. En este caso, los resultados de dichas integrales de superficie pueden obtenerse a partir de las expresiones (3.63)-(3.65), considerando en dichas ecuaciones que $l_{z_{1}}=c$.

En los apartados anteriores se ha presentado la teoría necesaria para caracterizar el bloque básico de excitación representado en la figura 4.17 en términos de una matriz de admitancias generalizada (MAG) utilizando el método BI-RME 3D. A este respecto, es importante recordar que la estructura bajo análisis consta de un único acceso de tipo coaxial y de tres accesos en guía rectangular. Además, hay que observar que, según la numeración de puertos establecida en la figura 4.17, el acceso coaxial se sitúa en el puerto (1) de la estructura y que en la teoría que se ha desarrollado en las secciones anteriores se ha asumido que el acceso coaxial está en dicho puerto. Sin embargo, en un caso práctico, el acceso coaxial puede estar situado en cualquiera de los cuatro puertos representados en la figura 4.17, por lo que es necesario comentar cómo se debe proceder en tales $\operatorname{casos}^{24}$. Supongamos entonces que el acceso coaxial esté situado, por ejemplo, en el puerto (3) de la figura 4.17. En ese caso, se procedería a renumerar los puertos de la estructura con el objetivo de que el acceso coaxial volviese a ser el puerto número $\left(1^{\prime}\right)$. Para ello, el acceso rectangular situado enfrente del acceso coaxial sería el nuevo puerto $\left(2^{\prime}\right)$ de la estructura, el acceso rectangular situado a la derecha del acceso coaxial sería el nuevo puerto $\left(3^{\prime}\right)$, mientras que el acceso rectangular situado a la izquierda del acceso coaxial sería el nuevo puerto $\left(4^{\prime}\right)$ (observar que de esta manera se está siendo coherente con la numeración original de los puertos $)^{25}$. Seguidamente, se analizaría la estructura siguiendo el método BI-RME 3D y, una vez obtenida la MAG correspondiente, se procedería a reordenar los bloques de la misma con el propósito de obtener una nueva MAG cuyos bloques estén referidos al sistema de numeración de puertos de la figura 4.17. En caso de que el acceso coaxial estuviese en cualquier otro puerto de acceso, se procedería de la misma manera.

\subsubsection{Ejemplos de validación}

En esta sección se van a presentar diferentes ejemplos de validación que servirán para comprobar la precisión y la eficiencia computacional de la técnica BI-RME 3D aplicada al análisis de la excitación coaxial de dispositivos en configuración colineal. En todas las estructuras analizadas se utiliza la numeración de puertos establecida en la figura 4.17. Además, en todos los ejemplos que se presentan en esta sección se han utilizado los parámetros de simulación que se indican a continuación. En todos los puertos de acceso se han considerado

\footnotetext{
${ }^{24}$ En el capítulo 5, dedicado al análisis de filtros en configuración comb-line, se deberán conectar una serie de matrices de admitancias generalizadas relativas a diferentes bloques básicos como el de la figura 4.17. Por este motivo, interesa fijar una numeración para los cuatro puertos de acceso del bloque básico que, posteriormente, permita organizar la conexión de las diferentes matrices multimodales.

${ }^{25}$ Adicionalmente, se debe considerar que las nuevas dimensiones de la cavidad rectangular son $c \times b \times a$.
} 
$N=15$ modos; se han utilizado $N_{\rho}=85$ términos para expresar las soluciones modales en la variable radial de la guía coaxial; se han empleado 80 puntos en la variable radial y 15 puntos en la variable angular para la integración numérica de las diferentes integrales que aparecen en el cálculo de las matrices $\mathbf{G}$ y T; se ha tomado un factor de precisión BI-RME 3D de $\zeta=5$; y se han utilizado $Q^{\mathrm{TE}}=Q^{\mathrm{TM}}=15000$ modos de la guía rectangular de expansión (es decir, un total de 30000 modos). A este respecto, es importante recalcar que se ha realizado un estudio de convergencia del método en función de los parámetros $Q^{\mathrm{TE}}$ y $Q^{\mathrm{TM}}$ y, como era de esperar, los resultados obtenidos son completamente similares a los presentados en la sección 4.2.5.

La vista en planta de la primera estructura bajo análisis se ha representado en la figura 4.20, donde se aprecia que se trata de un dispositivo de dos puertos. En concreto, se han abierto los puertos (1) y (2) del bloque básico de la figura 4.17. Si se consulta la figura 4.18

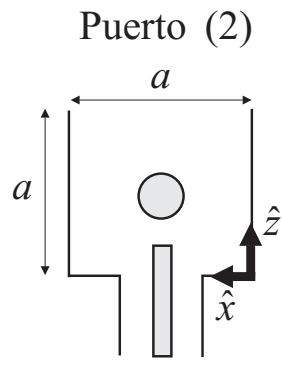

Puerto (1)

Figura 4.20: Estructura utilizada para validar la herramienta de análisis de la excitación coaxial de dispositivos en configuración colineal. En este primer ejemplo, se abren los accesos (1) y (2) del bloque básico.

para recordar la nomenclatura utilizada para representar las diferentes variables que definen las dimensiones del bloque básico, se tiene que las dimensiones de la cavidad rectangular son $a=22,9 \mathrm{~mm}, b=10,2 \mathrm{~mm}$ y $c=a$ (la cavidad se ha implementado en la guía rectangular estándar WR-90). Por otro lado, el poste conductor es de radio $r_{c}=2,0 \mathrm{~mm}$, su altura es $h_{c}=5,1 \mathrm{~mm}$ y su posición viene determinada por los parámetros $d_{x, c}=a / 2 \mathrm{y}$ $d_{z, c}=10,0 \mathrm{~mm}$. Además, el radio externo de la guía coaxial es $r_{1}=2,4 \mathrm{~mm}$, se asume que $\varepsilon_{r}=4,6$, y se considera que dicha guía está situada en una posición centrada, es decir, $d_{x}=a / 2$ y $d_{y}=b / 2$. Por otra parte, la longitud de penetración de la sonda es $h_{p}=5,0 \mathrm{~mm}$. En la figura 4.21 se ha representado el módulo de los parámetros de dispersión $S_{11}$ y $S_{12}$ obtenidos para diferentes valores del radio interno $r_{2}$ de la guía coaxial. En particular, se han considerado los casos en que $r_{2}=0,8 \mathrm{~mm}$ y $r_{2}=1,5 \mathrm{~mm}$. Además, los resultados obtenidos con la herramienta de simulación implementada se comparan satisfactoriamente con los resultados proporcionados por el programa HFSS. En relación con la eficiencia computacional del método, conviene destacar que la estructura se ha analizado en 155 segundos para un total de 401 puntos en frecuencia. Por su parte, HFSS empleó 23 minutos para 41 puntos en frecuencia.

En la figura 4.22 se presenta la vista en planta de la segunda estructura de validación. 


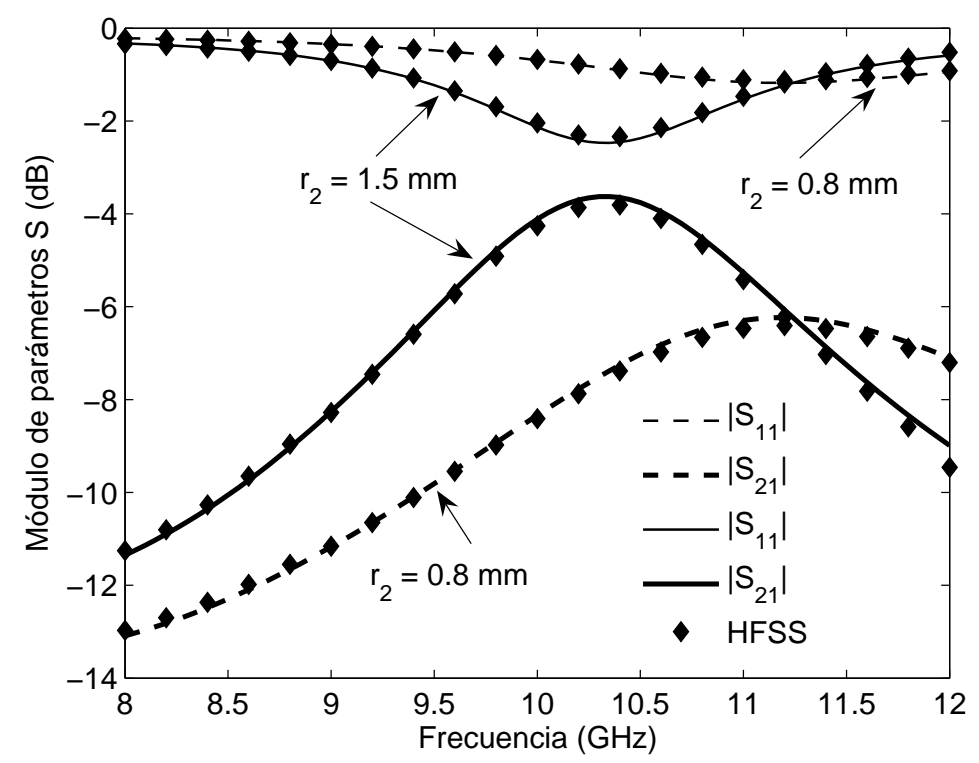

Figura 4.21: Parámetros de dispersión asociados a la estructura representada en la figura 4.20. Los datos obtenidos se comparan con los que proporciona HFSS.

Esta vez, se abren los accesos (1) y (3) del bloque básico. Las dimensiones de la cavidad rectangular, de la guía coaxial y del poste conductor son las mismas que las del ejemplo anterior. Por otro lado, la guía coaxial continúa en una posición centrada, mientras que para el poste conductor se consideran las siguientes posiciones relativas: $\left(d_{x, c}^{(1)}=a / 2, d_{z, c}^{(1)}=10 \mathrm{~mm}\right)$ $\mathrm{y}\left(d_{x, c}^{(2)}=16,45 \mathrm{~mm}, d_{z, c}^{(2)}=14,0 \mathrm{~mm}\right)$. En la figura 4.23 se muestran los resultados para los

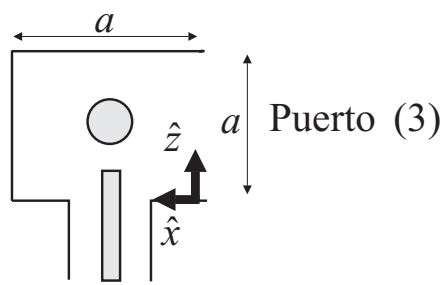

Puerto (1)

Figura 4.22: Estructura utilizada para validar la herramienta de análisis de la excitación coaxial de dispositivos en configuración colineal. En este segundo ejemplo, se abren los accesos (1) y (3) del bloque básico.

parámetros de dispersión de la estructura y se consideran dos valores diferentes para el radio interno del coaxial: $r_{2}=0,8 \mathrm{~mm}$ y $r_{2}=1,5 \mathrm{~mm}$. Las curvas de trazo continuo se refieren al caso en que la posición relativa del poste es $\left(d_{x, c}^{(1)}, d_{z, c}^{(1)}\right)$, mientras que las curvas de trazo punteado son las relativas al caso en que la posición relativa del poste es $\left(d_{x, c}^{(2)}, d_{z, c}^{(2)}\right)$ y el radio interno de coaxial es $r_{2}=1,5 \mathrm{~mm}$. Nuevamente, los resultados obtenidos concuerdan 
perfectamente con los proporcionados por HFSS. En este caso, la estructura se simuló en 178 segundos para un total de 401 puntos en frecuencia, mientras que la simulación con HFSS empleó 23 minutos para un total de 41 puntos en frecuencia.

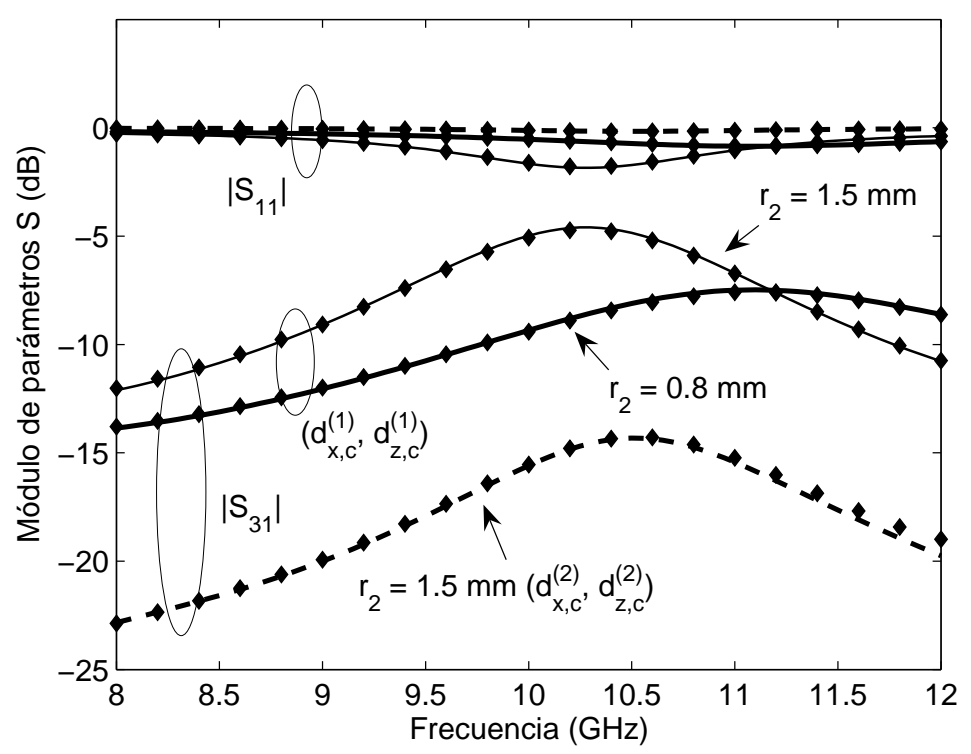

Figura 4.23: Parámetros de dispersión asociados a la estructura representada en la figura 4.22. Los datos obtenidos se comparan con los que proporciona HFSS.

A continuación se analiza la estructura cuya vista en planta se representa en la figura 4.24. Se puede apreciar que es una estructura en la que se han abierto los puertos (1), (2) y (3) del bloque básico. Las dimensiones de los diferentes elementos se mantienen constantes con respecto a los ejemplos de validación anteriores. Los parámetros de dispersión

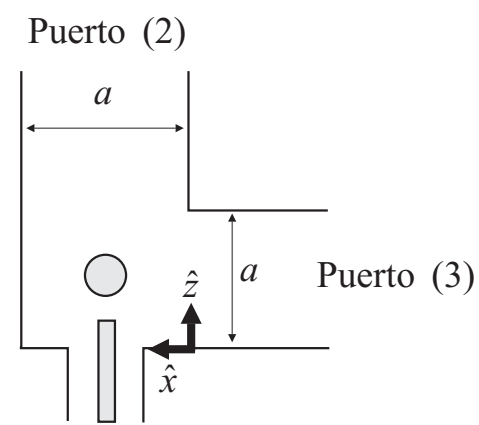

Puerto (1)

Figura 4.24: Estructura utilizada para validar la herramienta de análisis de la excitación coaxial de dispositivos en configuración colineal. En este tercer ejemplo, se abren los accesos (1), (2) y (3) del bloque básico.

de esta nueva estructura se presentan en la figura 4.25 , donde las curvas de trazo continuo 
grueso se refieren al parámetro $S_{21}$, mientras que las curvas de trazo continuo más fino son las relativas al parámetro $S_{31}$. Además, se han considerado dos casos diferentes para el radio interno del coaxial $r_{2}$, y para la posición relativa $\left(d_{x, c}, d_{z, c}\right)$ del poste conductor (los valores considerados para la posición relativa del poste son los mismos que los del ejemplo de validación anterior). En este caso, donde nuevamente los resultados obtenidos presentan una excelente concordancia con HFSS, la simulación se realizó en 187 segundos (401 puntos en frecuencia). Por su parte, HFSS tardó 26 minutos (41 puntos en frecuencia).

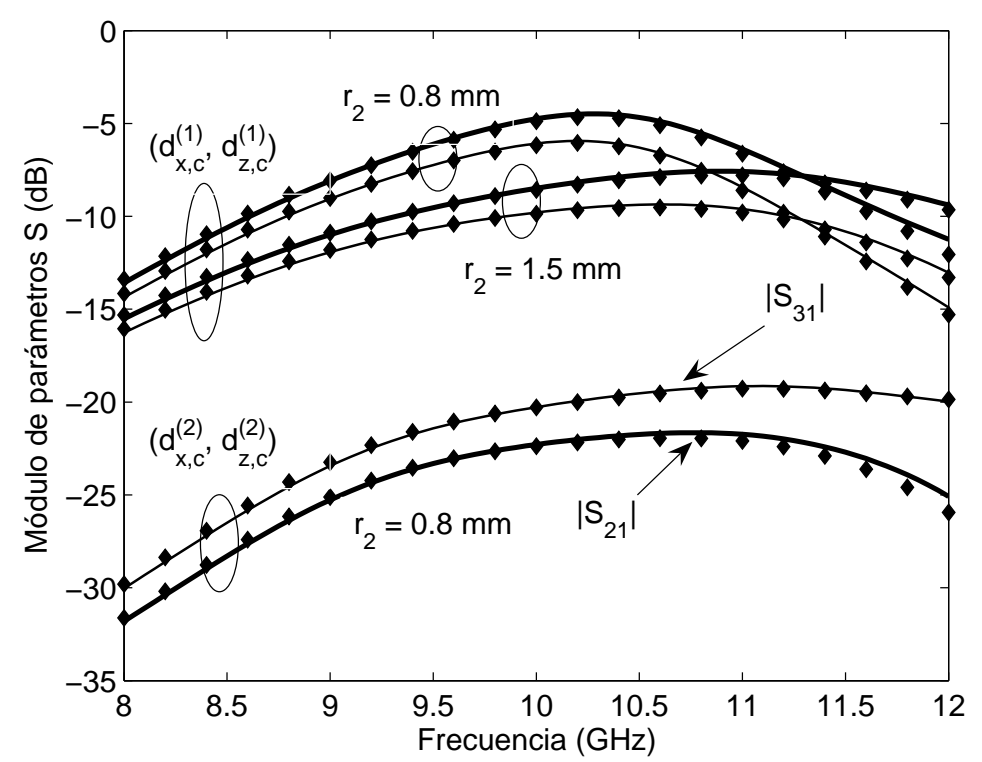

Figura 4.25: Parámetros de dispersión asociados a la estructura representada en la figura 4.24. Los datos obtenidos se comparan con los que proporciona HFSS.

Seguidamente, se analiza la estructura representada en la figura 4.26, donde, esta vez, se observa que se abren los puertos (1), (3) y (4) del bloque básico. Las dimensiones de los diferentes elementos son las mismas que las consideradas en los ejemplos anteriores (se toma $\left.r_{2}=0,8 \mathrm{~mm}\right)$. Sin embargo, en este nuevo ejemplo el poste se encuentra en una posición centrada, es decir, $\left(d_{x, c}, d_{z, c}\right)=(a / 2, c / 2)$. Además, se considera que el coaxial está en una posición descentrada con respecto a la guía rectangular que alimenta. En concreto, se analizan dos casos diferentes. En el primero de ellos, se toma $\left(d_{x}^{(1)}=a / 2, d_{y}^{(1)}=b / 2+2,0 \mathrm{~mm}\right)$ y en el segundo se caso se considera $\left(d_{x}^{(2)}=a / 2-4,0 \mathrm{~mm}, d_{y}^{(2)}=b / 2\right)$ (ver figura 4.19). El módulo del parámetro $S_{31}$ de este ejemplo de validación se muestra en la figura 4.27, donde se aprecia que la comparación con HFSS es muy satisfactoria. En este caso, los tiempos de simulación son muy similares a los del último caso analizado.

A continuación, se analiza la estructura representada en la figura 4.28, en la que se abren los 4 accesos del bloque básico. Las dimensiones de la guía coaxial, que en este caso se encuentra en una posición centrada, son las mismas que en los ejemplos anteriores. Por otro lado, el poste conductor, cuyas dimensiones también se mantienen, se sitúa en la posición re- 


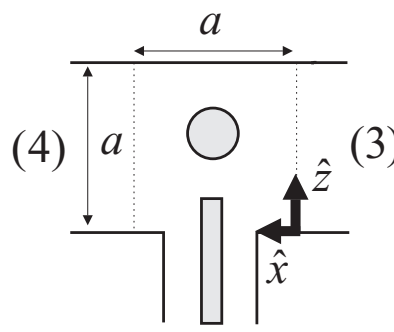

(1)

Figura 4.26: Estructura utilizada para validar la herramienta de análisis de la excitación coaxial de dispositivos en configuración colineal. En este cuarto ejemplo, se abren los accesos (1), (3) y (4) del bloque básico.

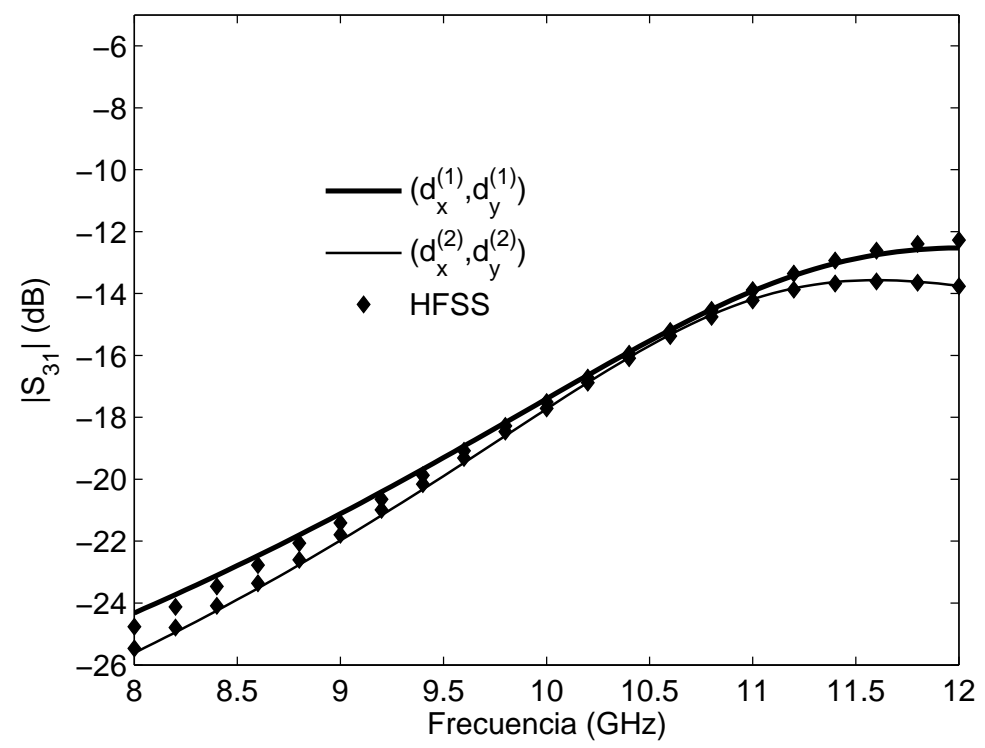

Figura 4.27: Parámetros de dispersión asociados a la estructura representada en la figura 4.26. Los datos obtenidos se comparan con los que proporciona HFSS.

lativa $\left(d_{x, c}=16,45 \mathrm{~mm}, d_{z, c}=14,0 \mathrm{~mm}\right)$. Los resultados para los parámetros de dispersión de esta nueva estructura se presentan en la figura 4.29, donde, nuevamente, apreciamos una excelente concordancia con respecto a HFSS. En este caso, la estructura se ha analizado en 229 segundos para un total de 401 puntos en frecuencia. La simulación con HFSS utilizó 28 minutos para 41 puntos en frecuencia.

Finalmente, en la figura 4.30 se representa la geometría del último ejemplo de validación que se presenta en esta sección. Se observa que la estructura posee dos puertos de acceso, siendo uno de ellos de tipo coaxial. Por otro lado, al puerto (2) del bloque básico se le conecta una guía rectangular de dimensiones $a^{(2)} \times b^{(2)}$ a través de un iris de sección transversal rectangular de dimensiones $a^{(1)} \times b^{(1)}$. Las dimensiones de la cavidad rectangular son $a=22,15 \mathrm{~mm}, b=47,55 \mathrm{~mm}$ y $c=25,4 \mathrm{~mm}$. Por su parte, los radios externo e interno 
(2)

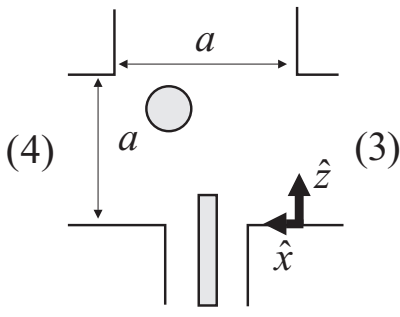

(1)

Figura 4.28: Estructura utilizada para validar la herramienta de análisis de la excitación coaxial de dispositivos en configuración colineal. En este quinto ejemplo, se abren los 4 accesos del bloque básico.

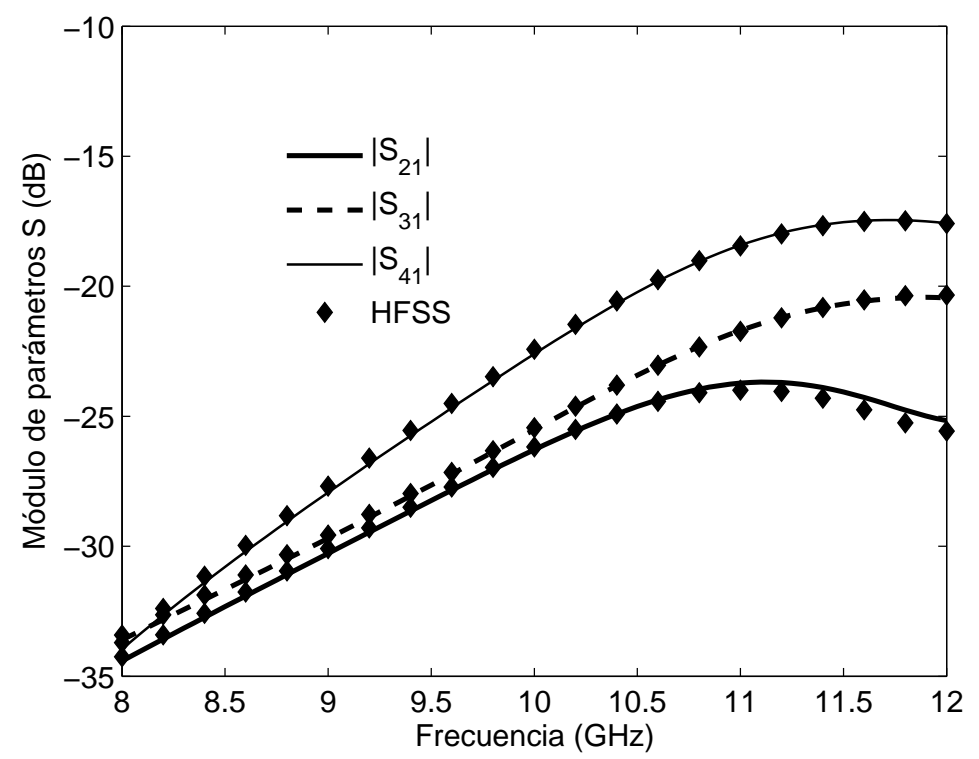

Figura 4.29: Parámetros de dispersión asociados a la estructura representada en la figura 4.28. Los datos obtenidos se comparan con los que proporciona HFSS.

de la guía coaxial son $r_{1}=2,4 \mathrm{~mm}$ y $r_{2}=0,8 \mathrm{~mm}$, respectivamente; se considera que $\varepsilon_{r}=4,6$; y la profundidad de penetración de la sonda es $h_{p}=5,0 \mathrm{~mm}$. Por otro lado, la posición relativa de la guía coaxial es $d_{x}=a / 2$ y $d_{y}=38,68 \mathrm{~mm}$. El poste conductor es de radio $r_{c}=3,302 \mathrm{~mm}$, su altura es $h_{c}=33,28 \mathrm{~mm}$ y está situado en una posición centrada. Con respecto al iris de acoplamiento, se tiene que sus dimensiones son $a^{(1)}=19,93 \mathrm{~mm}$, $b^{(1)}=9,97 \mathrm{~mm}$ y $l_{\text {iris }}=2,0 \mathrm{~mm}$, y está situado a una altura $d=18,8 \mathrm{~mm}$ (el iris se encuentra centrado con respecto a la otra dimensión). Finalmente, las dimensiones del puerto rectangular (2) de salida son $a^{(2)}=109,22 \mathrm{~mm}$ y $b^{(2)}=54,61 \mathrm{~mm}$.

El módulo del parámetro de transmisión $S_{21}$ asociado a esta estructura se presenta en la figura 4.31, y los resultados obtenidos se comparan con éxito con los datos proporcionados 

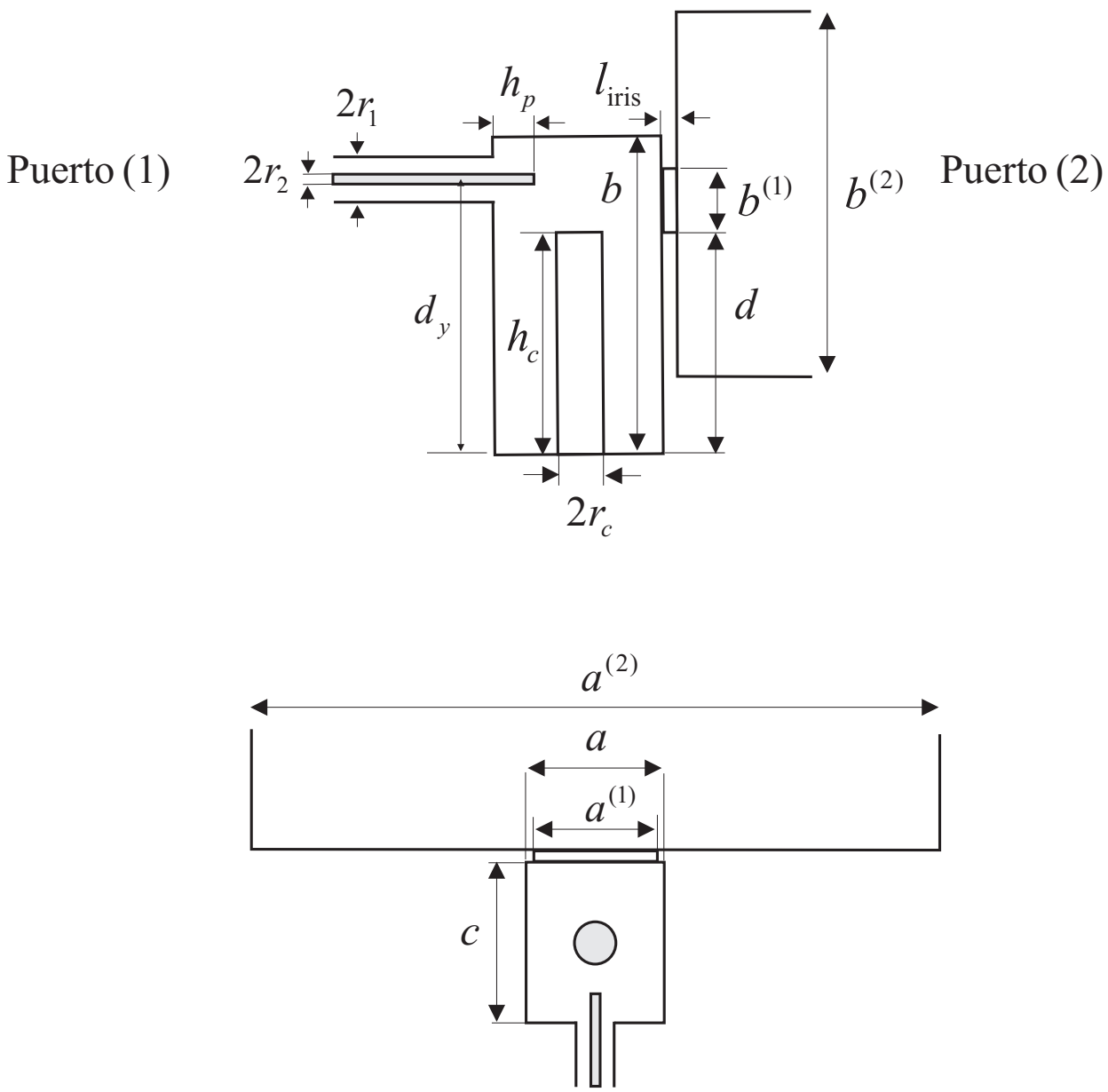

Figura 4.30: Vistas lateral y superior de la conexión del bloque básico de la figura 4.17 con una guía rectangular de dimensiones $a^{(2)} \times b^{(2)}$ a través de un iris rectangular de sección transversal $a^{(1)} \times b^{(1)}$ y longitud $l_{\text {iris }}$.

por HFSS. La estructura se simuló en un tiempo de 181 segundos para un total de 401 puntos en frecuencia. Por su parte, HFSS empleó un total de 22 minutos para 41 puntos en frecuencia. Por último, es importante comentar que las diferentes discontinuidades entre las guías de sección transversal rectangular presentes en la estructura analizada se han caracterizado utilizando la técnica de la ecuación integral presentada en el capítulo 2. 


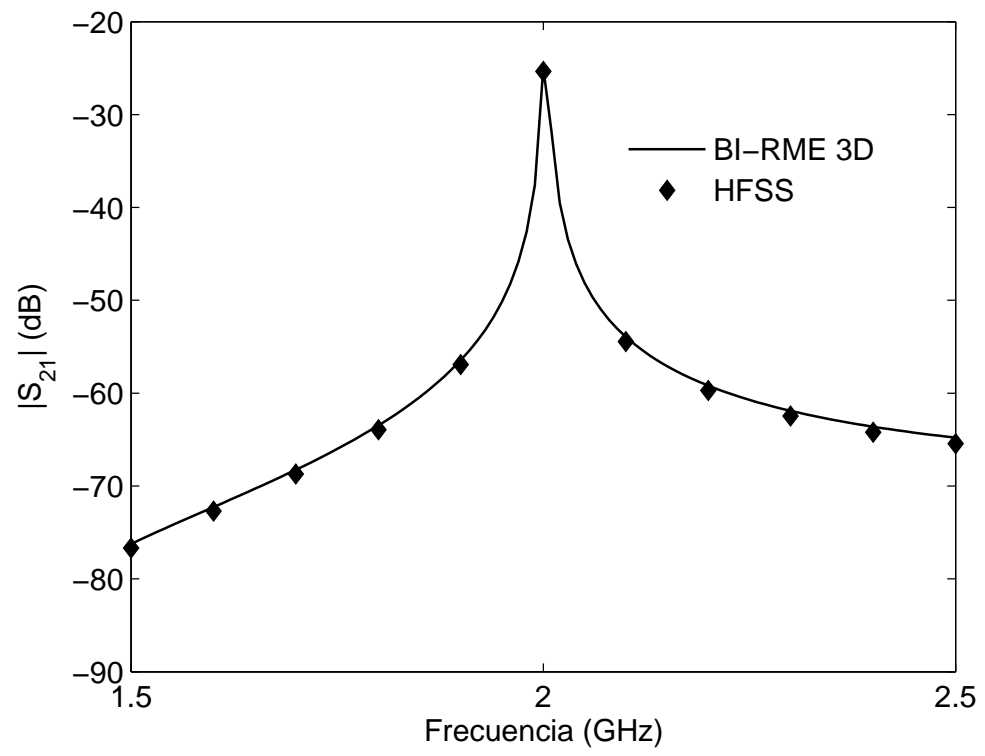

Figura 4.31: Parámetro de transmisión asociado a la estructura representada en la figura 4.30. Los datos obtenidos se comparan con los que proporciona HFSS. 


\section{Capítulo 5}

\section{Herramienta de Análisis de Filtros y Dispositivos en Configuración Comb-Line}

\subsection{Introducción}

El objetivo fundamental de este capítulo es presentar una herramienta de simulación destinada al análisis multimodal riguroso y eficiente de los denominados filtros en configuración comb-line. Este tipo de filtros está constituido por la asociación de diferentes cavidades rectangulares cargadas con postes conductores de altura variable que poseen geometría cilíndrica [73], [97] (ver figura 5.1). El acoplamiento electromagnético entre las diferentes cavidades rectangulares que integran el filtro se logra mediante un conjunto de irises que, tradicionalmente, son de geometría rectangular. Por otro lado, este tipo de filtros suele alimentarse mediante una guía coaxial en configuración colineal, como las estudiadas en el capítulo anterior. Además, la sonda coaxial puede contactar o no con el poste conductor de la cavidad rectangular a la que se conecta la guía coaxial. La herramienta de análisis que se describe en el presente capítulo considera que la sonda coaxial no contacta con dicho poste conductor, considerando el otro tipo de configuración como una posible futura línea de investigación.

Los filtros en configuración comb-line se emplean frecuentemente en sistemas de comunicaciones espaciales debido, fundamentalmente, a que presentan un diseño compacto, el coste de fabricación es relativamente bajo, ofrecen una gran facilidad para ajustar la frecuencia central de la banda de paso del filtro en un amplio rango frecuencial, y presentan una respuesta en frecuencia libre de espúreos [98], [99], [100]. Por el contrario, la principal desventaja de este tipo de filtros reside en el hecho de que tienen pérdidas elevadas, como consecuencia de que el factor de calidad $Q$ que se puede lograr es relativamente bajo [100]. En la literatura técnica se pueden encontrar numerosas aportaciones dedicadas al análisis y diseño de este tipo de filtros, de entre las cuales merece destacar las investigaciones desarrolladas por Yao (1995) [97] y El-Sabbagh (2001) [73]. En estos trabajos se efectúa una caracterización multimodal rigurosa de este tipo de estructuras utilizando la técnica de adaptación modal, y se estudia en profundidad el acoplamiento que se produce entre las diferentes cavidades del filtro con el fin de optimizar la respuesta en frecuencia de los diferentes diseños presentados. Por otro lado, otra contribución interesante es la que presenta Wang 


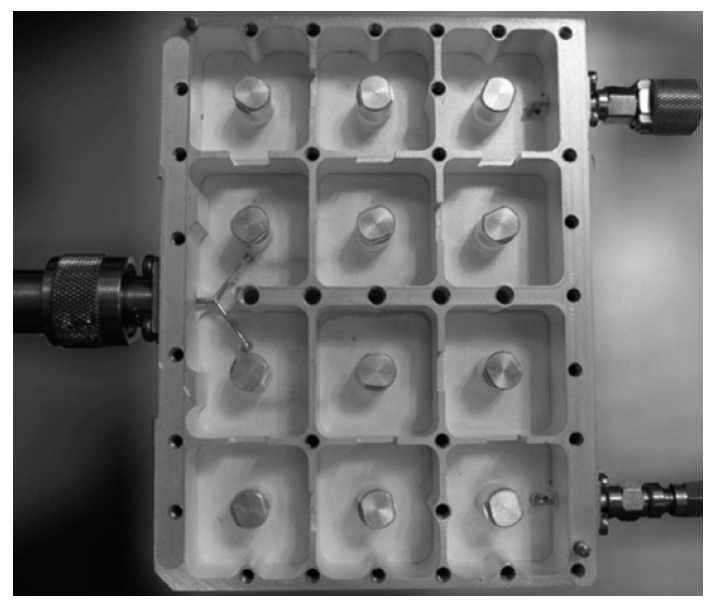

Figura 5.1: Diplexor en configuración comb-line implementado en tecnología guiada. El dispositivo consta de 12 resonadores rectangulares cargados con postes conductores de geometría cilíndrica (realmente, se trata de 2 filtros, cada uno con 6 resonadores rectangulares).

(1998) [100]. En este trabajo, se investiga cómo minimizar las pérdidas de los filtros en configuración comb-line y, para ello, se propone sustituir los postes conductores clásicos de este tipo de filtros por postes dieléctricos. El estudio demuestra que, efectivamente, con esta nueva estructura es posible reducir sustancialmente las pérdidas del dispositivo. Sin embargo, con esta nueva configuración, las frecuencias espúreas están más cercanas a la frecuencia central de la banda de paso del filtro, en comparación con la respuesta eléctrica del filtro comb-line clásico que utiliza postes conductores.

En el presente capítulo se muestra una herramienta de simulación muy eficiente desde un punto de vista computacional, destinada al análisis y diseño de filtros en guía de onda en configuración comb-line. La estrategia utilizada para lograr una caracterización multimodal de las estructuras bajo análisis combina el uso de la técnica de la ecuación integral desarrollada en el capítulo 2 y el método BI-RME 3D presentado en los capítulos 3 y 4 . Además de la elevada eficiencia computacional ya comentada, es importante destacar la enorme flexibilidad de la herramienta implementada. Así por ejemplo, tal y como se detalla en secciones posteriores, la herramienta permite considerar, además de los clásicos postes cilíndricos, postes conductores de geometría cilíndrica arbitraria en las diferentes cavidades rectangulares que integran el filtro, como son postes en forma de vaso o postes en forma de champiñón. La herramienta permite, asimismo, la introducción de postes de sintonía en las cavidades, la consideración de sondas coaxiales de excitación terminadas en disco e, incluso, introducir irises de acoplamiento de geometría circular entre las cavidades. Todas estas modificaciones con respecto a la geometría canónica de los filtros en configuración comb-line, las cuales no han sido investigadas hasta ahora en la literatura técnica relativa al análisis de este tipo de filtros, posibilitarán un diseño sumamente optimizado de los diferentes dispositivos bajo análisis.

Por otro lado, es importante comentar que todo el trabajo desarrollado en este capítulo se enmarca en un contrato de colaboración entre la Universidad Politécnica de Valencia, la Uni- 
versidad de Valencia, la Universidad Miguel Hernández de Elche y la empresa Thales Alenia Space (Ref. AAS-UPV/0F221/05.011). El objetivo fundamental del contrato de investigación es el desarrollo de una herramienta de análisis y diseño de filtros en tecnología guiada con resonadores en configuración comb-line. Además, se considera crucial que la nueva herramienta reduzca sustancialmente el tiempo de análisis de dichos dispositivos con respecto al que se consume con otros programas comerciales de propósito general, como por ejemplo HFSS. A continuación, se describe en primer lugar la herramienta de análisis implementada; posteriormente se presenta la base teórica que se ha utilizado para conseguir la caracterización multimodal de los filtros bajo análisis y, finalmente, se procede a validar la herramienta presentando diferentes ejemplos de filtros y dispositivos en configuración comb-line.

\subsection{Descripción de la Herramienta Implementada}

La herramienta de análisis de filtros en configuración comb-line debe satisfacer los siguientes requisitos:

- La herramienta debe poder simular filtros de tecnología guiada en configuración combline con un número arbitrario de resonadores. La definición de las topologías se realizará mediante una matriz de $M \times N$ cavidades, mediante la cual se indicará el esquema de interconexión.

- Los resonadores estarán formados por una cavidad de geometría rectangular cargada con un poste conductor de altura variable. El poste conductor puede presentar otras dos posibles formas, aparte de la geometría cilíndrica clásica, a saber: poste en forma de champiñón y poste en forma de vaso (poste parcialmente hueco). También existirá la posibilidad de simular un tornillo metálico de sintonía con cualquiera de los postes descritos. Este tornillo de sintonía podrá estar situado en una posición arbitraria de la tapa de la cavidad.

- Los resonadores se acoplarán mediante irises de geometría rectangular o circular y de dimensiones arbitrarias. Los irises se practicarán en cualquiera de las caras verticales del resonador y, en principio, su posición será arbitraria.

- Las interfaces de entrada y de salida serán mediante guía coaxial de dimensión arbitraria situadas en cualquiera de las caras verticales del resonador (excitación coaxial en configuración colineal). También se debe contemplar la posibilidad de que las interfaces de entrada y de salida se implementen mediante guía rectangular de las mismas o de diferentes dimensiones que las caras laterales del resonador.

- Para el caso de las interfaces de entrada y salida de tipo coaxial, los acoplamientos se realizarán mediante sondas convencionales o mediante sondas coaxiales terminadas en disco.

Así pues, siguiendo estos requerimientos, en la figura 5.2 se ha representado la vista en planta de un filtro en configuración comb-line genérico con 10 resonadores y 2 puertos de 
acceso en guía coaxial. Uno de los puertos de acceso se ha implementado mediante sonda coaxial estándar, mientras que en el otro puerto de acceso la sonda coaxial se encuentra terminada en disco. En la estructura, se aprecian los postes conductores de diferentes radios y alturas, los irises de acoplamiento entre cavidades, así como los tornillos de sintonía presentes en 3 resonadores (en la figura 5.2, los tornillos de sintonía se han representado utilizando círculos más oscuros y de un radio menor que el de los postes conductores $)^{1}$.

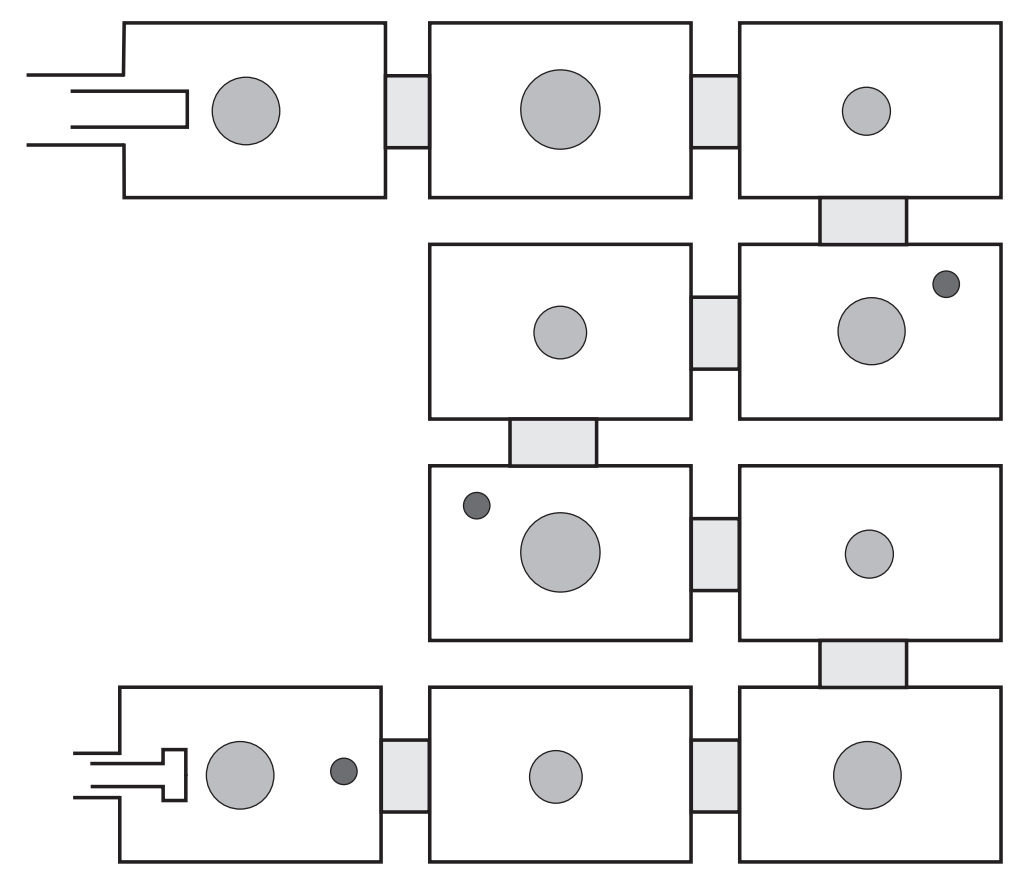

Figura 5.2: Filtro genérico en configuración comb-line constituido por 10 resonadores y 2 puertos de acceso en guía coaxial. En tres de los resonadores se ha incluido un tornillo de sintonía.

La implementación de la herramienta de simulación se ha estructurado en 5 etapas de complejidad creciente. El objetivo de cada uno de estas etapas se detalla a continuación:

- Etapa 1. Análisis de la cavidad rectangular básica de la estructura. El objetivo de esta etapa es la implementación de un módulo de simulación que permita la caracterización multimodal de una cavidad rectangular cargada con un poste conductor cilíndrico de altura variable y situado en una posición arbitraria en la base de la cavidad. Se considerará que la cavidad puede tener hasta un máximo de 4 puertos de acceso de tipo rectangular de dimensiones arbitrarias, situados en cada una de las 4 paredes laterales del resonador.

- Etapa 2. Análisis del bloque de excitación de la estructura. El objetivo de esta segunda etapa es generar una herramienta de simulación que caracterice de forma efi-

\footnotetext{
${ }^{1}$ La topología del filtro de la figura 5.2 puede definirse mediante una matriz de dimensiones $M \times N(M=4$ filas, $N=3$ columnas), estando vacías las posiciones $(2,1)$ y $(3,1)$ de dicha matriz. Siguiendo esta notación, se observa que los tornillos de sintonía se encuentran en los resonadores $(2,3),(3,2)$ y $(4,1)$. Por otro lado, los puertos de acceso se encuentran situados en los resonadores $(1,1)$ y $(4,1)$.
} 
ciente y rigurosa la excitación de la estructura mediante sonda coaxial en configuración colineal. La sonda coaxial será de tipo estándar y no estará soldada al poste conductor. En otras palabras, el bloque básico de excitación es una cavidad rectangular cargada con un poste conductor que puede tener hasta un máximo de 4 puertos de acceso situados en las paredes laterales del resonador. Uno de estos puertos de acceso debe ser de tipo coaxial en configuración colineal, y el resto de puertos de acceso se implementarán en guía rectangular.

En este punto es importante comentar que las etapas 1 y 2 ya se han resuelto y validado en capítulos anteriores. En particular, la estructura básica de la etapa 1 se resolvió en el capítulo 3 dedicado al análisis y diseño de uniones compensadas en guía rectangular. Por otro lado, la etapa 2 se ha resuelto en la sección 4.3 del capítulo anterior dedicado al análisis de la excitación coaxial de dispositivos en configuración colineal.

- Etapa 3. Herramienta preliminar de análisis de la estructura. El objetivo de esta tercera etapa es implementar una herramienta de simulación que, mediante la conexión de los bloques generados en las dos etapas anteriores, permita analizar una primera solución básica de las estructuras propuestas. La estructura básica consiste en un filtro de cavidades rectangulares de tipo comb-line que incluya acoplamientos entre cavidades mediante irises de geometría rectangular. Las excitaciones de entrada y salida serán mediante sonda coaxial estándar o terminada en disco. También se incluirá la posibilidad de que la excitación se realice mediante guía rectangular.

- Etapa 4. Herramienta básica de análisis de filtros. El objetivo de esta cuarta etapa consiste en ampliar la herramienta básica generada en la etapa anterior para que incluya postes conductores en forma de champiñón y de vaso como elemento resonante, así como la presencia de tornillos metálicos de sintonía de forma cilíndrica.

- Etapa 5. Ampliación de la herramienta de análisis. El objetivo de esta etapa es ampliar la herramienta de la etapa anterior para que incluya irises de interconexión entre cavidades de geometría circular.

A continuación se presenta una descripción detallada de la herramienta de análisis implementada. En particular, se describen las características de los diferentes elementos que integran los filtros en configuración comb-line: resonadores, postes conductores, irises de acoplamiento, tornillos metálicos de sintonía y puertos de acceso.

\section{Resonadores}

Los resonadores que integran el filtro son de geometría rectangular y sus dimensiones son arbitrarias. Las dimensiones de los resonadores se denotarán como $a \times b \times c$, tal y como se presenta en la figura 5.3. Los resonadores están cargados con postes conductores de simetría cilíndrica, los cuales están en contacto con la tapa inferior del resonador. Por otra parte, en la figura 5.3 se ha indicado la numeración que se va a utilizar para identificar cada una de las cuatro caras verticales de los resonadores. En cada una de estas caras verticales se puede 
conectar un iris de acoplamiento, o bien un puerto de acceso de cualquiera de los tipos que soporta la herramienta (los tipos de puerto de acceso soportados se detallan posteriormente). Así pues, según el sistema de referencia de la figura 5.3, el puerto (1) se sitúa en $z=-c / 2$, el puerto (2) en $z=c / 2$, el puerto (3) se encuentra en $x=-a / 2$ y el puerto (4) en $x=a / 2$.

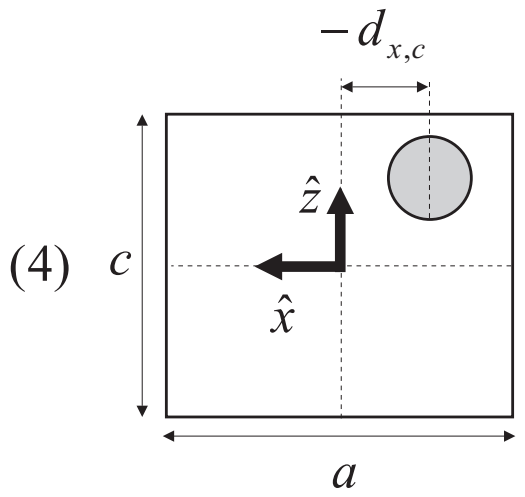

(3)

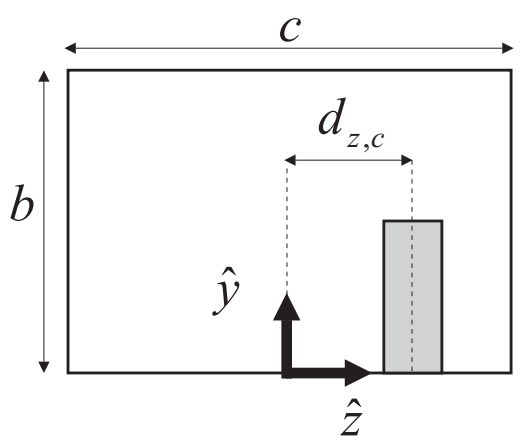

(1)

Figura 5.3: Vista en planta y vista lateral de un resonador comb-line de dimensiones $a \times b \times c$ cargado con un poste conductor cilíndrico situado en la posición relativa $\left(-d_{x, c}, 0, d_{z, c}\right)$.

\section{Postes conductores}

Los postes conductores que soporta la herramienta implementada son de tres tipos (todos ellos poseen simetría cilíndrica):

- Poste conductor de forma cilíndrica. Este tipo de poste está definido a partir del radio $r_{c}$ y la altura $h_{c}$ (ver figura 5.4).

- Poste conductor en forma de champiñón. Este tipo de poste se define a partir de los radios $r_{c h, 1} \mathrm{y} r_{c h, 2}$, y las alturas $h_{c h, 1} \mathrm{y} h_{c h, 2}$ (ver figura 5.4).

- Poste conductor en forma de vaso (poste parcialmente hueco). El poste en forma de vaso se define a partir de los radios $r_{v, 1}$ y $r_{v, 2}$, y las alturas $h_{v, 1}$ y $h_{v, 2}$ (ver figura 5.4).

Las dimensiones de los diferentes tipos de postes se han representado en la figura 5.4. Por otro lado, para definir la posición relativa de los postes conductores se usa el sistema de referencia representado en la figura 5.3, el cual se encuentra centrado en la base inferior del resonador comb-line. Así por ejemplo, la posición relativa del poste conductor de la figura 5.3 vendría dada por los parámetros $\left(-d_{x, c}, 0, d_{z, c}\right)$. Por otra parte, en caso de que el poste se encuentre centrado en el resonador, se tendría $d_{x, c}=d_{z, c}=0$. 

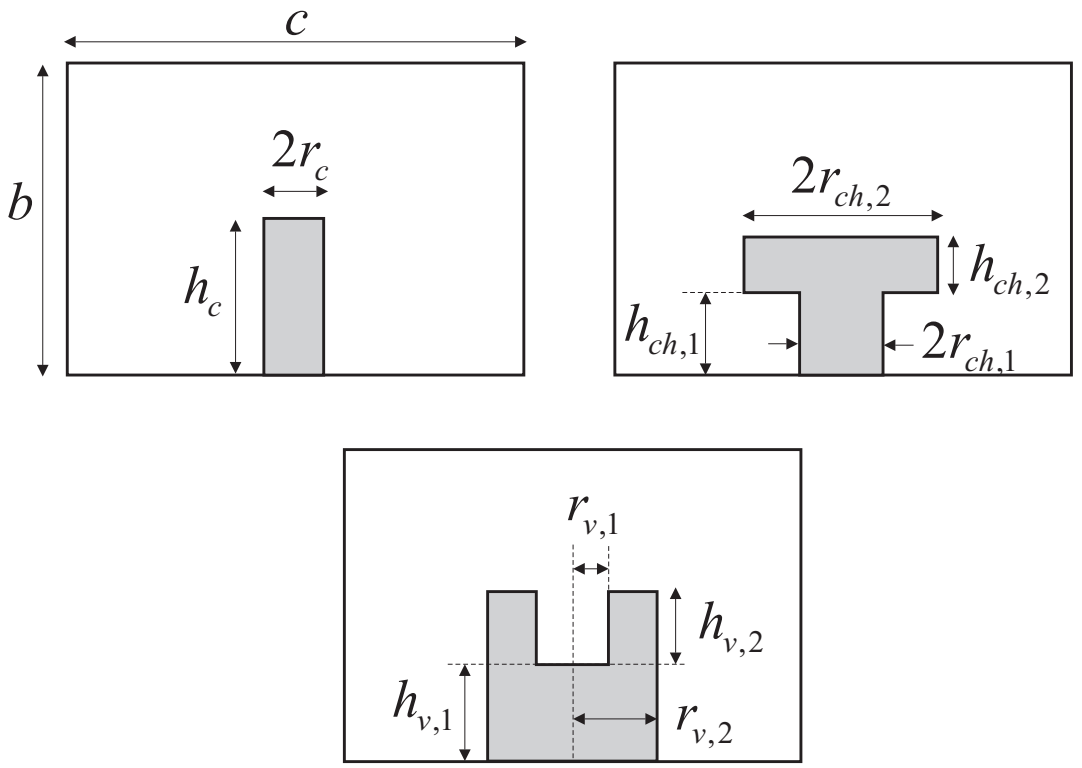

Figura 5.4: Vistas laterales de los diferentes tipos de postes conductores soportados por la herramienta de simulación: poste cilíndrico (arriba izquierda); poste en forma de champiñón (arriba derecha); y poste en forma de vaso (abajo).

\section{Irises de acoplamiento}

Los resonadores comb-line se acoplan entre sí mediante irises de interconexión. Los irises de acoplamiento pueden estar en cualquiera de las cuatro caras verticales de los resonadores comb-line. Para definir la posición relativa del iris se emplea un sistema de referencia centrado en cada una de las caras verticales del resonador. En la figura 5.5 se tiene un ejemplo para un iris situado en la cara vertical del resonador de dimensiones $a \times b$. La posición relativa del iris viene dada por los parámetros $\left(d_{x, i r}, d_{y, i r}\right)$ (en caso de que el iris esté en una posición centrada se tendrá $\left.d_{x, i r}=d_{y, i r}=0\right)$. Por otra parte, la herramienta soporta dos tipos de irises: rectangulares y circulares. Los primeros de ellos están definidos a partir de sus dimensiones transversales $a_{i r} \times b_{i r}$, mientras que los irises circulares se definen a partir del radio $r_{i r}{ }^{2}$. La longitud de los irises se define con el parámetro $l_{i r}$ (esta longitud no se representa en la figura 5.5).

\section{Tornillos de sintonía}

La herramienta implementada permite la posibilidad de incluir tornillos metálicos de sintonía de geometría cilíndrica en los resonadores rectangulares que integran el filtro comb-line ${ }^{3}$. Estos tornillos, que se sitúan en la tapa superior de los resonadores, se definen a partir del radio $r_{t}$ y la altura $h_{t}$ (ver figura 5.6). Por otro lado, para definir la posición relativa de los

\footnotetext{
${ }^{2}$ En caso de que el iris sea de tipo circular, la definición de la posición relativa de dicho iris se realiza de la misma manera que la explicada en la figura 5.5 para el caso del iris rectangular.

${ }^{3}$ La versión actual del simulador sólo permite incluir un tornillo metálico de sintonía por resonador.
} 


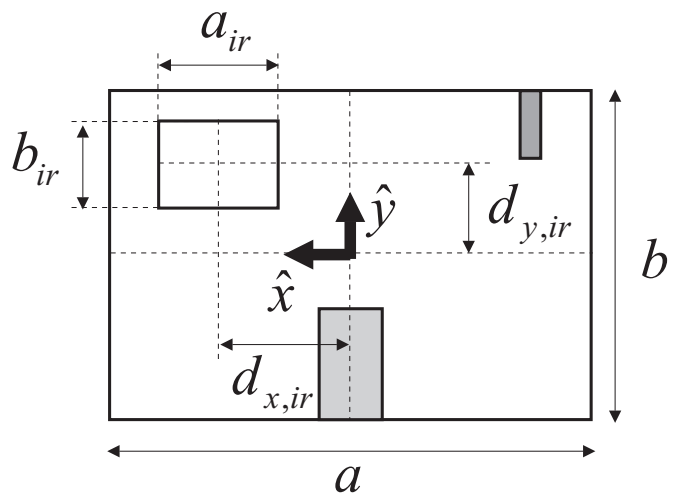

Figura 5.5: Sistema de referencia utilizado para definir la posición relativa de los irises de acoplamiento entre resonadores comb-line.

tornillos de sintonía se utiliza el mismo sistema de referencia que el utilizado para definir la posición de los postes conductores; es decir, un sistema de coordenadas centrado en la base inferior del resonador comb-line. Así por ejemplo, la posición relativa del tornillo de sintonía de la figura 5.6 se definiría a partir los parámetros $\left(d_{x, t}, b, d_{z, t}\right)$.
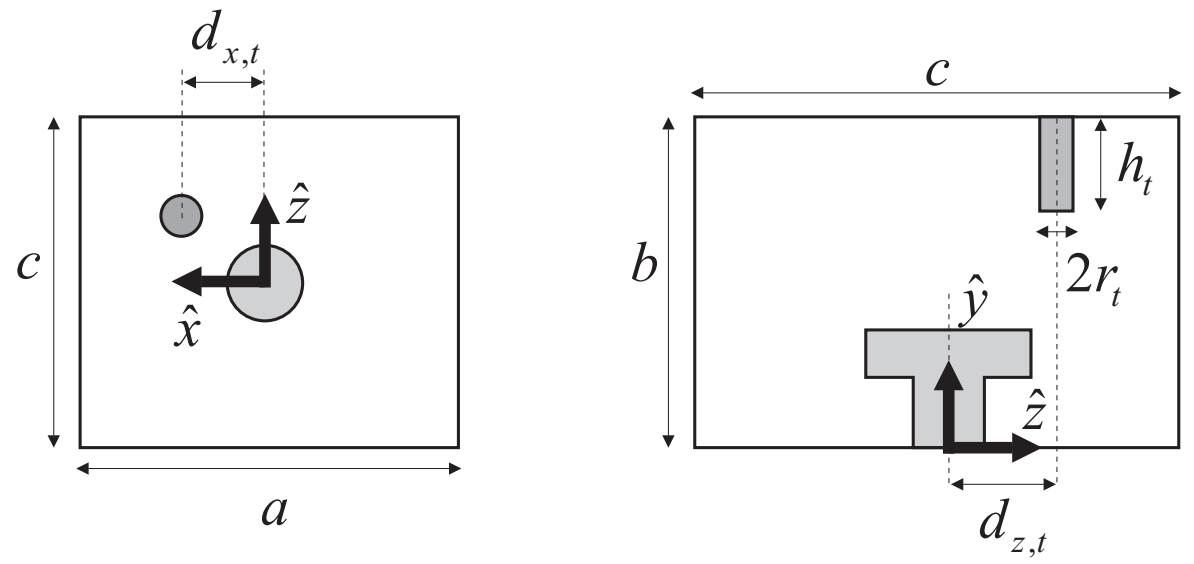

Figura 5.6: Vista en planta y vista lateral de un resonador comb-line con un tornillo de sintonía de radio $r_{t}$ y altura $h_{t}$ situado en la posición relativa $\left(d_{x, t}, b, d_{z, t}\right)$.

\section{Puertos de acceso}

La herramienta implementada permite considerar cinco tipos de puertos de acceso diferentes. Los puertos de acceso, que pueden estar situados en cualquier cara vertical del resonador comb-line, se describen a continuación ${ }^{4}$ :

\footnotetext{
${ }^{4}$ En cada resonador comb-line solamente se puede definir un único puerto de acceso.
} 
- Puerto de acceso de sección transversal rectangular cuyas dimensiones coinciden con las de la cara lateral del resonador en que se sitúa el acceso.

- Puerto de acceso de sección transversal rectangular cuyas dimensiones no coinciden con las de la cara lateral del resonador en que se sitúa el acceso. Este tipo de puerto se define a partir de las dimensiones transversales $a_{p} \times b_{p}$. En la figura 5.7, se ha representado un ejemplo en el que un puerto de acceso de este tipo está centrado con respecto a la cara vertical del resonador de dimensiones $a \times b$. Por otro lado, para definir la posición relativa del puerto con respecto al resonador, se emplea un sistema de referencia idéntico al representado en la figura 5.5. En este caso, la posición relativa del puerto con respecto a la cara vertical del resonador se define con los parámetros $\left(d_{x, p}, d_{y, p}\right)$.

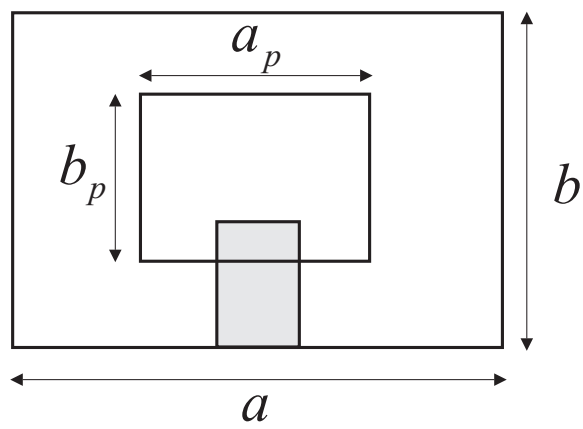

Figura 5.7: Puerto de acceso de sección transversal rectangular $\left(a_{p} \times b_{p}\right)$ cuyas dimensiones no coinciden con las de la cara lateral del resonador $(a \times b)$.

- Puerto de acceso de sección transversal rectangular a través de iris de tipo rectangular. En este caso, el puerto de acceso es también de sección transversal rectangular. Sin embargo, a diferencia de los dos casos anteriores, esta vez se sitúa un iris rectangular entre la cara vertical del resonador y el puerto de acceso, tal y como se recoge en la figura 5.8. En este caso, las dimensiones transversales del iris son $a_{i r, p} \times b_{i r, p}$, y su longitud es $l_{i r, p}$. Además, las dimensiones transversales del puerto de acceso rectangular son $a_{p}^{\prime} \times b_{p}^{\prime}$. Por otro lado, para definir la posición relativa entre las diferentes guías rectangulares implicadas en este tipo de acceso, se utiliza un sistema de referencia de tipo centrado como el utilizado en la figura 5.5.

- Puerto de acceso mediante sonda coaxial convencional en configuración colineal. Este tipo de puerto se ha representado en la figura 5.9 si se toma $r_{d}=h_{d}=0$. Además, en dicha figura se considera el caso en que el acceso coaxial se sitúa en la cara vertical del resonador comb-line de dimensiones $a \times b$. El radio externo de la guía coaxial es $r_{1}$, el radio interno es $r_{2}, h_{p}$ representa la profundidad de penetración de la sonda y se asume que la permitividad dieléctrica relativa de la guía es $\varepsilon_{r}$. Para definir la posición relativa 


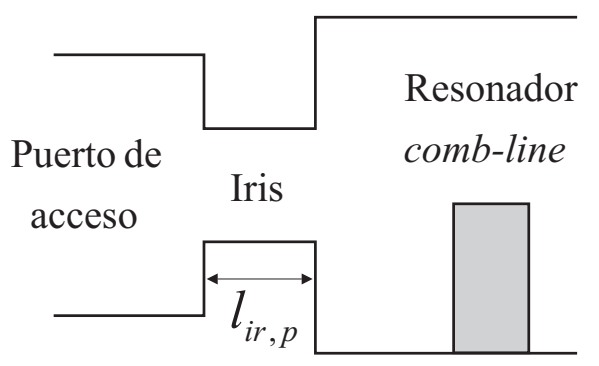

Figura 5.8: Puerto de acceso de sección transversal rectangular de dimensiones $a_{p}^{\prime} \times b_{p}^{\prime}$ a través de un iris de tipo rectangular de sección transversal $a_{i r, p} \times b_{i r, p}$ y longitud $l_{i r, p}$.

de la sonda coaxial se emplea el sistema de referencia mostrado en la figura 5.9, el cual se encuentra centrado en la cara lateral del resonador comb-line en que se sitúa el acceso coaxial. La posición relativa del coaxial se establece a partir de los parámetros $\left(d_{x}, d_{y}\right)$ (en caso de estar en una posición centrada se tiene $\left.d_{x}=d_{y}=0\right)$.

- Puerto de acceso mediante sonda coaxial terminada en disco en configuración colineal. En este caso, tal y como se recoge en la figura 5.9, la sonda coaxial se encuentra terminada en disco de radio $r_{d}$ y altura $h_{d}$. La definición de la posición relativa del puerto se realiza de la misma manera que en el puerto coaxial con sonda convencional.

Una vez definidas las características básicas de la herramienta implementada, seguidamente se procede a explicar la base teórica en la que se fundamenta el simulador.

\subsection{Fundamentos Teóricos}

El análisis multimodal de los filtros en configuración comb-line se fundamenta en el método BI-RME 3D desarrollado en los capítulos 3 y 4, y en la técnica de la ecuación integral presentada en el capítulo 2. En particular, el método BI-RME 3D se utiliza para caracterizar los resonadores comb-line que integran el filtro (cavidades rectangulares cargadas con postes conductores de simetría cilíndrica incluyendo también los posibles tornillos de sintonía) en términos de una matriz de admitancias generalizada (MAG). Por su parte, la técnica de la ecuación integral se utiliza para caracterizar las discontinuidades planares que surgen al interconectar los resonadores comb-line mediante irises de acoplamiento en términos de matrices de impedancias generalizadas (MIG).

Con el fin de aclarar los conceptos, supongamos que cierto resonador comb-line posee cuatro irises de acoplamiento, situados en cada una de las cuatro caras verticales del resonador (estos irises pueden ser de sección transversal rectangular o circular). La herramienta está implementada de manera que la MAG que proporciona el método BI-RME 3D está siempre referida a puertos de acceso de sección transversal rectangular cuyas dimensiones coinciden con las de las caras verticales del resonador ${ }^{5}$. En consecuencia, con el objeto

\footnotetext{
${ }^{5}$ En el caso particular de que los resonadores comb-line incluyan un puerto de acceso de excitación en guía coaxial, se utilizará la teoría desarrollada en el capítulo 4 para caracterizar este tipo de resonador.
} 

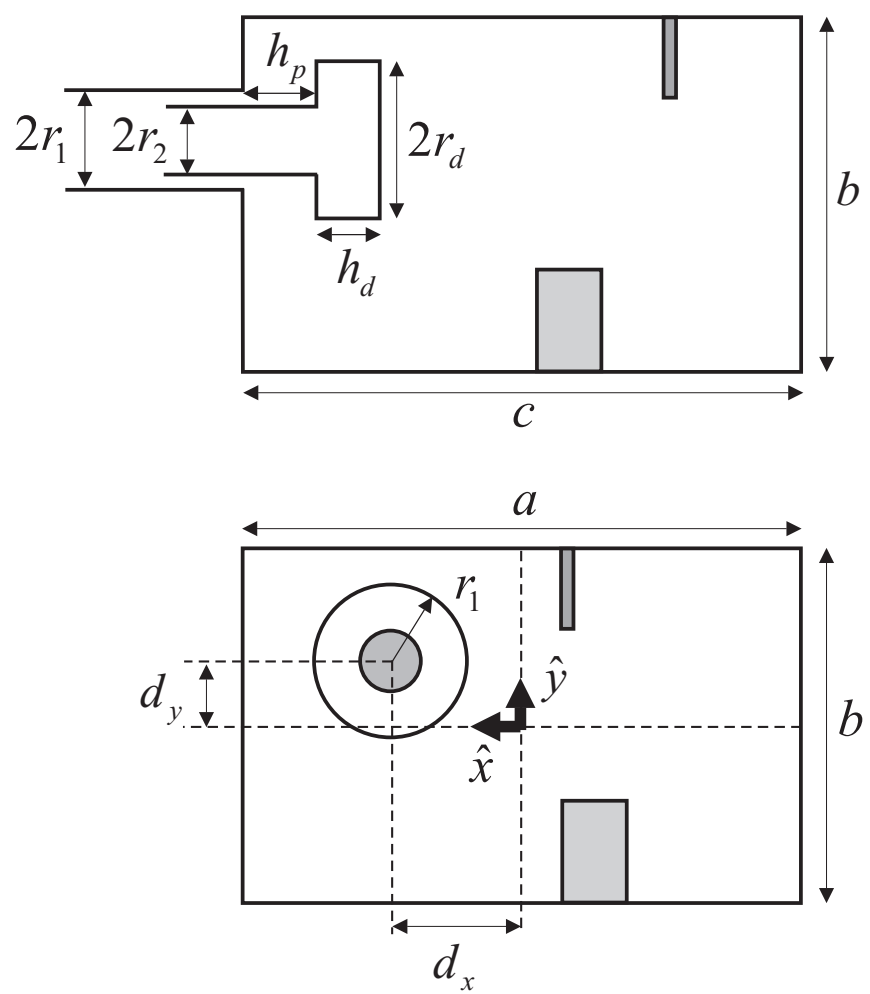

Figura 5.9: Puerto de acceso mediante sonda coaxial terminada en disco. En caso de que $r_{d}=h_{d}=0$ se obtiene la sonda coaxial convencional.

de incluir el efecto de los irises, a continuación se debe conectar en cada uno de los puertos del resonador comb-line la MIG correspondiente a la unión planar que se produce entre las dos guías de sección transversal rectangular presentes en la discontinuidad: una de ellas se corresponde con una de las caras laterales del resonador, y la otra con la guía rectangular (o circular) que define el iris. Con el objetivo de caracterizar dicha unión planar en términos de una MIG se empleará la técnica de la ecuación integral. Además, puesto que el iris es un tramo de guía uniforme de cierta longitud $l$, posteriormente también se debe conectar a las matrices multimodales anteriores, la MIG correspondiente a un tramo de guía uniforme de sección transversal rectangular (o circular).

A continuación, se describe el procedimiento que utiliza la herramienta implementada para analizar un filtro genérico en configuración comb-line. Sea, por ejemplo, el filtro comb-line de 10 resonadores representado en la figura 5.2. Si se utiliza la notación matricial comentada anteriormente para denotar cada uno de los resonadores del filtro, se observa que, por ejemplo, el resonador $(2,2)$ posee dos irises de acoplamiento de cierta longitud $l$. La caracterización multimodal de dicho resonador implica, en primer lugar, la obtención de la MAG asociada a una cavidad rectangular cargada con un poste conductor de radio $r$ y de altura $h$ situado en una posición arbitraria. Además, se considera que la MAG de dicho resonador está calculada con respecto a dos puertos de acceso de sección transversal rectangular cuyas dimensiones coinciden con las de las caras laterales de la cavidad rectangular. 
Seguidamente, se deben calcular las MIG asociadas a las dos uniones planares que se crean al conectar los dos irises de acoplamiento en las dos caras laterales del resonador comb-line. Ya finalmente, se deben obtener las MIG correspondientes a los dos tramos de guía uniforme de longitud $l$ definidos por los irises de acoplamiento. La conexión de todas las matrices multimodales implicadas en la caracterización multimodal de este resonador se ha representado en la figura 5.10. En dicha figura, $Y$ hace referencia a la MAG correspondiente al resonador comb-line; $Z_{i r 1}$ y $Z_{i r 2}$ son las MIG asociadas a las uniones planares que se producen al insertar los irises de acoplamiento; y $Z_{g u 1}$ y $Z_{g u 2}$ representan las MIG relativas a los dos tramos de guía uniforme que definen los irises. Si se sigue este procedimiento para cada uno

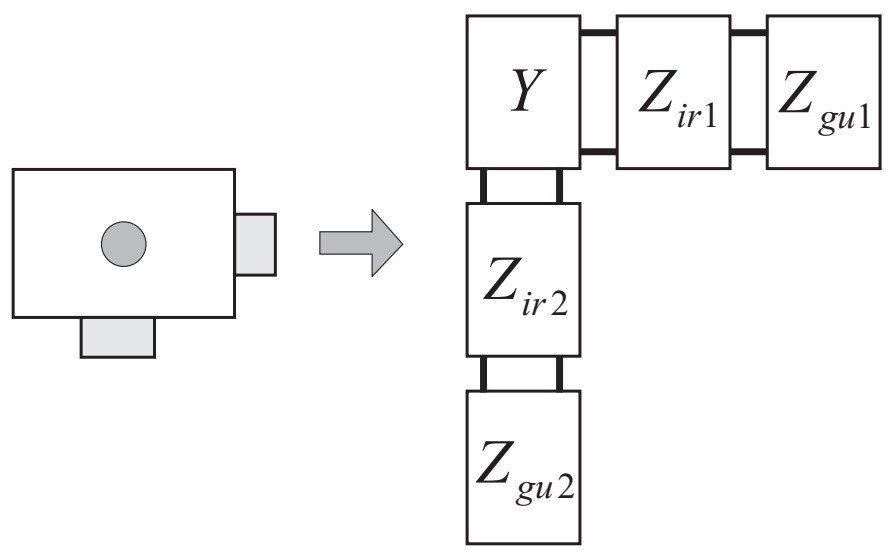

Figura 5.10: Conexión de todas las matrices multimodales implicadas en la caracterización del resonador $(2,2)$ del filtro comb-line genérico de la figura 5.2.

de los 10 resonadores comb-line que integran el filtro, se consigue una representación multimodal equivalente para cada uno de los resonadores en términos de un conjunto de matrices multimodales de admitancias e impedancias generalizadas.

Por otro lado, es importante observar que, dado que cada iris de acoplamiento interconecta dos resonadores del filtro, surge la necesidad de definir una regla que establezca a qué resonador de los dos se debe asociar las matrices de impedancias generalizadas que surgen al caracterizar los tramos de guía uniforme que definen los irises de acoplamiento. La estrategia que se utiliza consiste en sumar los índices matriciales que definen la posición de cada resonador del filtro. Si para cierto resonador dicha suma proporciona un número par, entonces las MIG asociadas a los tramos de guía uniforme definidos por los irises de acoplamiento que se conectan a dicho resonador sí deben asociarse a la MAG que lo caracteriza (este es precisamente el caso del resonador $(2,2)$ analizado en la figura 5.10). Si, por el contrario, dicha suma origina un número impar para un resonador dado, ello significa que a la MAG que caracteriza dicho resonador no se le deben asociar las MIG correspondientes a los tramos de guía uniforme que aportan los irises de acoplamiento que se conecten al resonador. A este respecto, consideremos el resonador $(2,3)$ del filtro de la figura 5.2. En este caso, la suma de los índices matriciales que definen su posición en el filtro proporciona un número impar, por lo que a la MAG de este resonador no se le deben asociar las MIG correspondientes a los tramos de guía uniforme asociados a los irises de acoplamiento que se conecten a 
dicho resonador. En la figura 5.11 se ha representado la caracterización multimodal de este resonador, y se observa que está definido a partir de una MAG de dos puertos asociada al propio resonador comb-line y de las dos MIG relativas a las uniones planares que surgen al insertar los irises (las MIG asociadas a los dos tramos de guía uniforme que se conectan al resonador $(2,3)$ se considerarán en las representaciones multimodales equivalentes de los resonadores $(1,3)$ y $(2,2)$, cuyos índices matriciales de posición suman números pares).

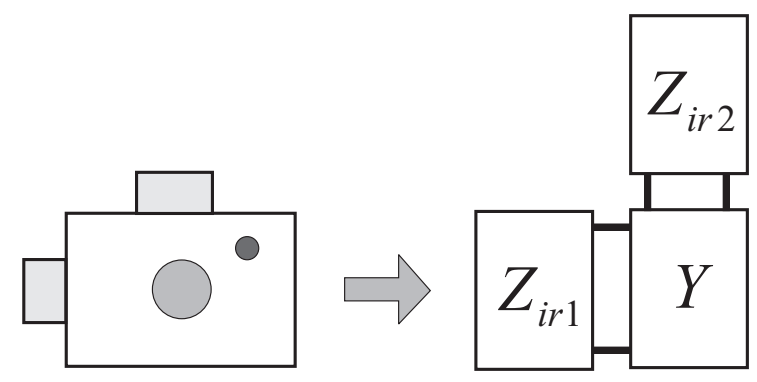

Figura 5.11: Matrices multimodales implicadas en la caracterización del resonador $(2,3)$ del filtro comb-line genérico de la figura 5.2.

Posteriormente, se debe partir de la representación multimodal obtenida para cada resonador con el fin de obtener una MIG equivalente (la teoría relativa a este tema se presenta al final de esta sección). Así, por ejemplo, en la figura 5.12 se representa el objetivo que se persigue para el caso particular del resonador $(2,2)$ del filtro de la figura 5.2: se parte de la representación multimodal obtenida para el resonador (ver figura 5.10) y se calcula una MIG equivalente. Es importante destacar que el número de puertos de acceso de la MIG equiva-
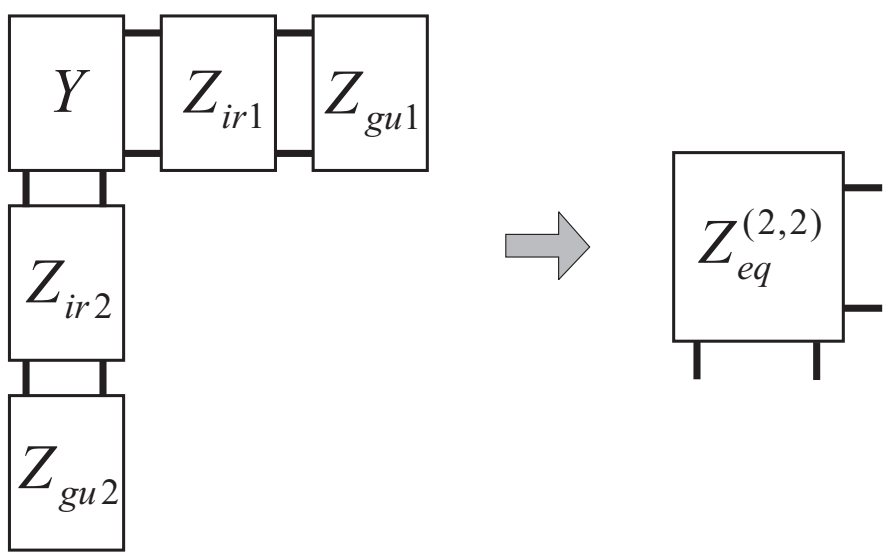

Figura 5.12: Obtención de una MIG equivalente para la representación multimodal del resonador $(2,2)$ del filtro de la figura 5.2.

lente obtenida coincide con el número de puertos de acceso de la MAG asociada al resonador en cuestión. Así por ejemplo, observamos que la MIG equivalente obtenida en la figura 5.12 es una matriz multimodal de dos puertos de acceso, al igual que la MAG presente en dicha figura.

Una vez que se han calculado las MIG equivalentes para cada una de las representaciones 
multimodales asociadas a los resonadores que integran el filtro, se deben conectar todas entre sí adecuadamente, de acuerdo con la configuración del filtro comb-line. La interconexión de las MIG equivalentes para el caso particular del filtro de la figura 5.2 se ha representado en la figura 5.13. En esta figura, las MIG $Z_{e q}^{(i, j)}$ representan las matrices multimodales equivalentes asociadas a cada uno de los resonadores del filtro. A partir de la interconexión de las diferentes MIG equivalentes, se puede fácilmente plantear un sistema de ecuaciones lineales cuya resolución permite caracterizar el filtro en términos de los correspondientes parámetros de dispersión.

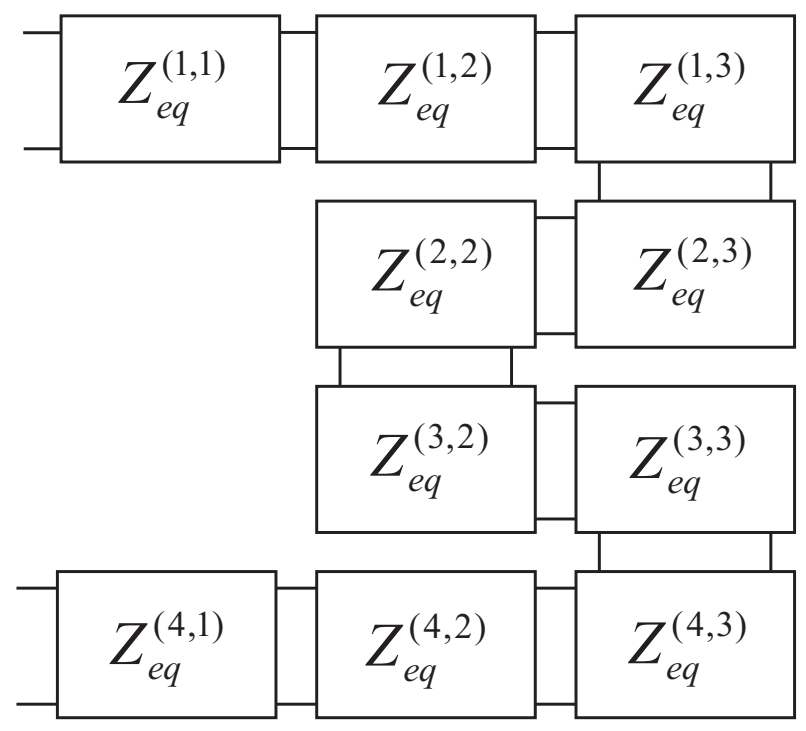

Figura 5.13: Interconexión de las MIG equivalentes para el filtro comb-line genérico de la figura 5.2.

Para finalizar esta sección, a continuación se presenta la teoría que permite obtener la MIG equivalente correspondiente a la representación multimodal de cada resonador (ver figura 5.12 para el caso del resonador $(2,2)$ del filtro de la figura 5.2). Para ello, consideremos la figura 5.14, en la que se tiene la conexión entre una MIG de 4 accesos asociada a un resonador comb-line y $^{6}$ una MIG de 2 accesos relativa a la unión planar correspondiente a la discontinuidad que se produce al conectar un iris de acoplamiento a una de las caras laterales del resonador comb-line ${ }^{7}$. En la figura 5.14, $V_{c}^{(i)}$ e $I_{c}^{(i)}(i=1, \ldots, 4)$ se corresponden con las tensiones y corrientes modales en los puertos de acceso de la MIG $Z_{c}$; y $V_{i r}^{(i)}$ e $I_{i r}^{(i)}$ $(i=1,2)$ son las tensiones y corrientes modales asociadas a los puertos de la MIG $Z_{i r}$. Por otro lado, se observa que en el puerto (1) de la MIG de dos accesos se han tenido en cuenta las admitancias asintóticas $\hat{Y}_{i r}^{(1)}$ que introduce la técnica de la ecuación integral. Asimismo,

\footnotetext{
${ }^{6}$ En la herramienta implementada, la MIG de cada resonador comb-line se obtiene mediante inversión directa de la MAG obtenida tras aplicar el método BI-RME 3D.

${ }^{7}$ En la figura 5.14, el iris se conecta al puerto (2) del resonador; sin embargo, en un caso general, el iris se puede conectar en cualquier puerto. Así, la teoría que se desarrolla en esta sección puede extenderse fácilmente para el caso en que el puerto de conexión sea diferente del puerto (2).
} 
es importante destacar que, a efectos de cálculo, se consideran $N$ modos en todos los puertos de acceso implicados en la interconexión.
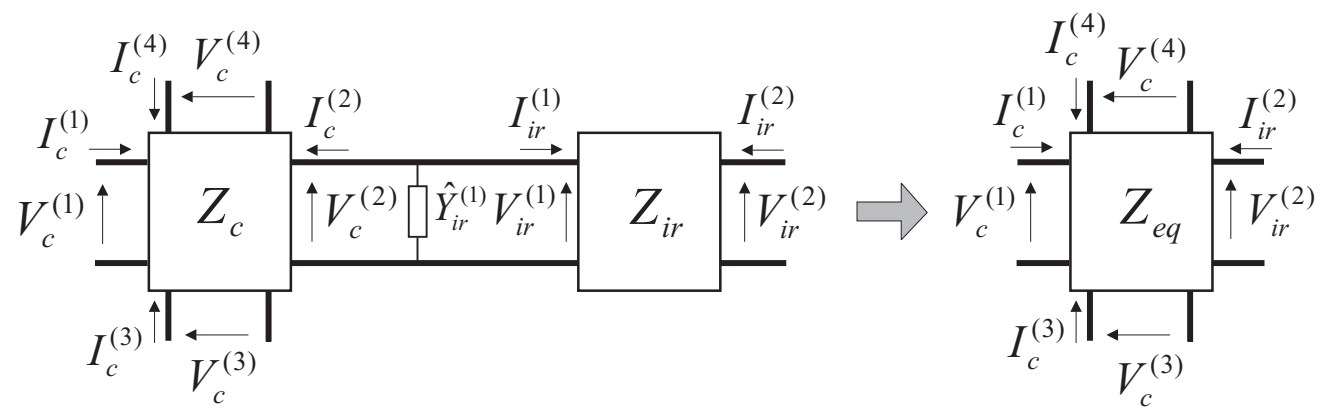

Figura 5.14: Conexión de la MIG correspondiente a un resonador comb-line de 4 accesos con la MIG asociada a la unión planar que se produce al introducir el iris de acoplamiento.

En el plano de unión de las dos matrices multimodales se tiene continuidad para la tensión modal, es decir, $V_{c}^{(2)}=V_{i r}^{(1)}$, por lo que podemos escribir:

$$
\begin{aligned}
& V_{c}^{(2)}=Z_{i r}^{(1,1)} I_{i r}^{(1)}+Z_{i r}^{(1,2)} I_{i r}^{(2)} \\
& V_{c}^{(2)}=Z_{c}^{(2,1)} I_{c}^{(1)}+Z_{c}^{(2,2)} I_{c}^{(2)}+Z_{c}^{(2,3)} I_{c}^{(3)}+Z_{c}^{(2,4)} I_{c}^{(4)}
\end{aligned}
$$

Por otro lado, para la corriente $I_{c}^{(2)}$ se cumple:

$$
I_{c}^{(2)}=-I_{i r}^{(1)}-\hat{Y}_{i r}^{(1)} V_{c}^{(2)}
$$

Si ahora se introduce (5.3) en (5.2) se deduce:

$$
\left(U+Z_{c}^{(2,2)} \hat{Y}_{i r}^{(1)}\right) V_{c}^{(2)}=-Z_{c}^{(2,2)} I_{i r}^{(1)}+Z_{c}^{(2,1)} I_{c}^{(1)}+Z_{c}^{(2,3)} I_{c}^{(3)}+Z_{c}^{(2,4)} I_{c}^{(4)}
$$

donde $U$ es una matriz identidad de dimensiones $N \times N$. Si, a continuación, se inserta (5.1) en (5.4) se obtiene:

$$
\begin{aligned}
\left(Z_{i r}^{(1,1)}+Z_{c}^{(2,2)} \hat{Y}_{i r}^{(1)} Z_{i r}^{(1,1)}+Z_{c}^{(2,2)}\right) I_{i r}^{(1)}= & -\left(Z_{i r}^{(1,2)}+Z_{c}^{(2,2)} \hat{Y}_{i r}^{(1)} Z_{i r}^{(1,2)}\right) I_{i r}^{(2)}+Z_{c}^{(2,1)} I_{c}^{(1)} \\
& +Z_{c}^{(2,3)} I_{c}^{(3)}+Z_{c}^{(2,4)} I_{c}^{(4)}
\end{aligned}
$$

Seguidamente, se define la siguiente matriz:

$$
Y_{a}=\left(Z_{i r}^{(1,1)}+Z_{c}^{(2,2)} \hat{Y}_{i r}^{(1)} Z_{i r}^{(1,1)}+Z_{c}^{(2,2)}\right)^{-1}
$$

Partiendo de (5.5) y utilizando (5.6), es posible expresar $I_{i r}^{(1)}$ de la forma:

$$
I_{i r}^{(1)}=-Y_{a}\left(Z_{i r}^{(1,2)}+Z_{c}^{(2,2)} \hat{Y}_{i r}^{(1)} Z_{i r}^{(1,2)}\right) I_{i r}^{(2)}+Y_{a} Z_{c}^{(2,1)} I_{c}^{(1)}+Y_{a} Z_{c}^{(2,3)} I_{c}^{(3)}+Y_{a} Z_{c}^{(2,4)} I_{c}^{(4)}
$$


Por otro lado, la tensión $V_{i r}^{(2)}$ satisface:

$$
V_{i r}^{(2)}=Z_{i r}^{(2,1)} I_{i r}^{(1)}+Z_{i r}^{(2,2)} I_{i r}^{(2)}
$$

Si definimos la matriz $M_{a}=Z_{i r}^{(2,1)} Y_{a}$, y se introduce (5.7) en (5.8), se obtiene:

$$
\begin{aligned}
V_{i r}^{(2)}= & \left(-M_{a}\left[Z_{i r}^{(1,2)}+Z_{c}^{(2,2)} \hat{Y}_{i r}^{(1)} Z_{i r}^{(1,2)}\right]+Z_{i r}^{(2,2)}\right) I_{i r}^{(2)}+M_{a} Z_{c}^{(2,1)} I_{c}^{(1)} \\
& +M_{a} Z_{c}^{(2,3)} I_{c}^{(3)}+M_{a} Z_{c}^{(2,4)} I_{c}^{(4)}
\end{aligned}
$$

Esta es la primera ecuación que caracteriza a la MIG equivalente $Z_{e q}$ presente en la figura 5.14. Para las tensiones modales en los otros tres puertos, podemos hacer uso de (5.1) y (5.3) para obtener:

$V_{c}^{(i)}=Z_{c}^{(i, 1)} I_{c}^{(1)}-Z_{c}^{(i, 2)}\left(U+\hat{Y}_{i r}^{(1)} Z_{i r}^{(1,1)}\right) I_{i r}^{(1)}-Z_{c}^{(i, 2)} \hat{Y}_{i r}^{(1)} Z_{i r}^{(1,2)} I_{i r}^{(2)}+Z_{c}^{(i, 3)} I_{c}^{(3)}+Z_{c}^{(i, 4)} I_{c}^{(4)}$

donde $i=1,3,4$. Si ahora se define la matriz:

$$
M_{b}^{(i)}=Z_{c}^{(i, 2)}\left(U+\hat{Y}_{i r}^{(1)} Z_{i r}^{(1,1)}\right) Y_{a} \quad i=1,3,4
$$

y se introduce (5.7) en (5.10) se deduce finalmente:

$$
\begin{aligned}
V_{c}^{(i)}= & \left(M_{b}^{(i)}\left[Z_{i r}^{(1,2)}+Z_{c}^{(2,2)} \hat{Y}_{i r}^{(1)} Z_{i r}^{(1,2)}\right]-Z_{c}^{(i, 2)} \hat{Y}_{i r}^{(1)} Z_{i r}^{(1,2)}\right) I_{i r}^{(2)}+\left(Z_{c}^{(i, 1)}-M_{b}^{(i)} Z_{c}^{(2,1)}\right) I_{c}^{(1)} \\
& +\left(Z_{c}^{(i, 3)}-M_{b}^{(i)} Z_{c}^{(2,3)}\right) I_{c}^{(3)}+\left(Z_{c}^{(i, 4)}-M_{b}^{(i)} Z_{c}^{(2,4)}\right) I_{c}^{(4)} \quad i=1,3,4 \quad(5.12)
\end{aligned}
$$

Con esta última ecuación se concluye la formulación teórica del problema. De hecho, este algoritmo puede aplicarse tantas veces como se precise hasta conseguir una representación multimodal equivalente de cada resonador comb-line. Así por ejemplo, para el caso de la figura 5.12, este algoritmo se debería aplicar un total de 4 veces (la matriz multimodal asociada al resonador debe absorber las MIG asociadas a dos uniones planares y las MIG asociadas a dos tramos de guía uniforme).

En el caso particular de que la MIG a absorber esté asociada a un tramo de guía uniforme, no se tendrían las admitancias asintóticas en paralelo que se han representado en la figura $5.14^{8}$. En este caso, si $Z_{g u}$ denota la MIG relativa a un tramo de guía uniforme, y $V_{g u}^{(2)}$ e $I_{g u}^{(2)}$ representan las tensiones y corrientes multimodales equivalentes en el puerto (2) de la

\footnotetext{
${ }^{8}$ Para obtener la MIG asociada a un tramo de guía uniforme se parte, para cada modo, de una red circuital en pi de dos accesos, y se considera que, en dichos accesos, se tienen en paralelo las admitancias asintóticas que surgen al caracterizar las uniones planares asociadas a los irises de acoplamiento. Por ello, si la MIG a absorber es la relativa a un tramo de guía uniforme, no se deben considerar las admitancias asintóticas mencionadas, ya que su contribución se ha integrado en la caracterización del tramo de guía uniforme.
} 
MIG $Z_{g u}$, se tiene que la primera ecuación que define la nueva matriz multimodal equivalente $Z_{e q}$ es la siguiente ${ }^{9}$ :

$$
V_{g u}^{(2)}=\left(Z_{g u}^{(2,2)}-M_{c} Z_{g u}^{(1,2)}\right) I_{g u}^{(2)}+M_{c} Z_{c}^{(2,1)} I_{c}^{(1)}+M_{c} Z_{c}^{(2,3)} I_{c}^{(3)}+M_{c} Z_{c}^{(2,4)} I_{c}^{(4)}
$$

donde hemos definido la siguiente matriz auxiliar:

$$
M_{c}=Z_{g u}^{(2,1)}\left(Z_{g u}^{(1,1)}+Z_{c}^{(2,2)}\right)^{-1}
$$

Las otras tres ecuaciones que definen la MIG $Z_{e q}$ son las siguientes:

$$
\begin{aligned}
V_{c}^{(i)}= & M_{d}^{(i)} Z_{g u}^{(1,2)} I_{g u}^{(2)}+\left(Z_{c}^{(i, 1)}-M_{d}^{(i)} Z_{c}^{(2,1)}\right) I_{c}^{(1)}+\left(Z_{c}^{(i, 3)}-M_{d}^{(i)} Z_{c}^{(2,3)}\right) I_{c}^{(3)} \\
& +\left(Z_{c}^{(i, 4)}-M_{d}^{(i)} Z_{c}^{(2,4)}\right) I_{c}^{(4)} \quad i=1,3,4
\end{aligned}
$$

donde se ha definido:

$$
M_{d}^{(i)}=Z_{c}^{(i, 2)}\left(Z_{g u}^{(1,1)}+Z_{c}^{(2,2)}\right)^{-1} \quad i=1,3,4
$$

\subsection{Ejemplos de Aplicación}

En esta sección se presentan diferentes ejemplos de aplicación que servirán para validar la precisión y la eficiencia computacional de la técnica utilizada para analizar filtros en configuración comb-line implementados en tecnología guiada. En primer lugar, es importante mencionar los parámetros de simulación que se han utilizado en todos los ejemplos que se presentan a continuación. Con el fin de obtener los modos de las guías coaxiales, se han utilizado $N_{\rho}=85$ términos para representar la variación radial de las soluciones modales. Por otro lado, en relación con el método BI-RME 3D, se han empleado $Q^{\mathrm{TE}}=Q^{\mathrm{TM}}=15000$ modos de las correspondientes guías rectangulares de expansión para calcular los elementos singulares de las matrices G, T y L. Asimismo, se han utilizado 80 puntos de integración en la variable radial y 10 puntos en la variable angular, para resolver con precisión las diferentes integrales numéricas que surgen al aplicar el método BI-RME 3D en el análisis de la excitación coaxial. Con respecto a la técnica de la ecuación integral, la cual se ha utilizado para caracterizar las distintas uniones planares, se han empleado 20 modos accesibles en todas las guías (rectangulares o circulares), 80 funciones base en la expansión de la función vectorial incógnita $\mathbf{M}_{n}(x, y), 1200$ términos en la expansión de la parte estática del núcleo de la ecuación integral, y 900 términos en la expansión de la parte dinámica del núcleo de la ecuación integral. Por otro lado, los modos de excitación y de respuesta son siempre los modos fundamentales de cada tipo de acceso considerado.

\footnotetext{
${ }^{9}$ En este desarrollo se parte de la situación de la figura 5.14, considerando que no se tienen las admitancias asintóticas $\hat{Y}_{i r}^{(1)}$ (es decir, $\hat{Y}_{i r}^{(1)}=0$ ). Por otro lado, se asume también que la MIG $Z_{i r}$ (que en (5.13)-(5.16) se denota mediante $Z_{g u}$ ) está ahora referida a un tramo de guía uniforme.
} 


\subsubsection{Validación de la herramienta de la etapa 3}

A continuación, se procede a la validación de la herramienta de simulación relativa a la etapa 3 (ver sección 5.2). Así, en la figura 5.15 se presenta el primer ejemplo de validación consistente en un filtro comb-line de 4 cavidades y de dos puertos de acceso. Uno de los puertos es de tipo coaxial (puerto 1) y el otro puerto (puerto 2) es de tipo rectangular, y sus dimensiones coinciden con las de la cara lateral de la cavidad. Por otro lado, a efectos de notación, asumiremos que el resonador en el que se encuentra el puerto (1) constituye la cavidad $(1,1)$ del filtro, y que el puerto coaxial se encuentra situado en el acceso local número (1) de dicho resonador $(1,1)$ (recordar la numeración local que se estableció en la figura 5.3 para los cuatro puertos laterales de los resonadores comb-line).

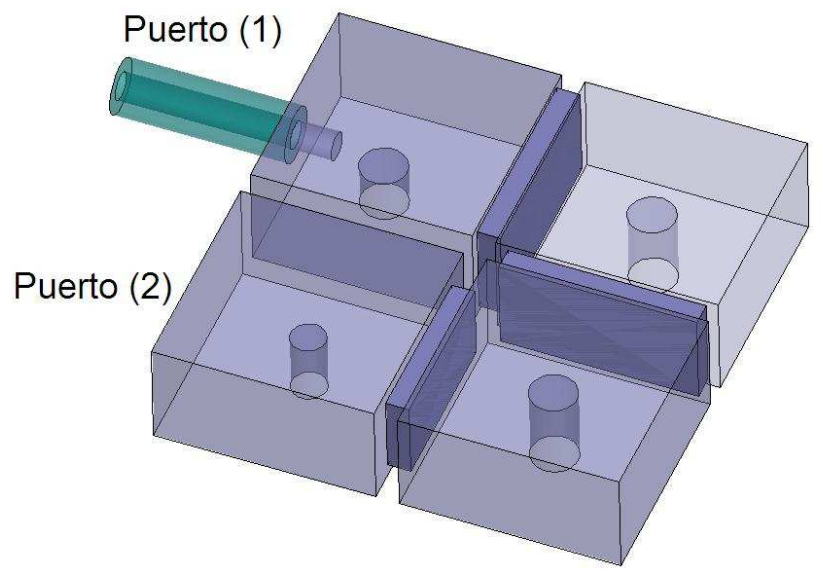

Figura 5.15: Filtro comb-line de 4 cavidades con dos puertos de acceso, siendo uno de ellos de tipo coaxial y el otro de tipo rectangular. Esta estructura se utiliza para validar la herramienta de simulación relativa a la etapa 3 .

Los cuatro resonadores del filtro son de las mismas dimensiones; en concreto se tiene que $a=19,05 \mathrm{~mm}, b=9,525 \mathrm{~mm}$ y $c=a$ (resonadores implementados en la guía estándar WR-75). Por otro lado, las dimensiones y la posición relativa de los postes conductores de cada resonador (ver figura 5.4) se recogen en la tabla 5.1 (los postes conductores son todos de geometría cilíndrica).

\begin{tabular}{|c|c|c|c|}
\hline \hline \multirow{2}{*}{$\begin{array}{c}\text { Resonador } \\
(i, j)\end{array}$} & \multicolumn{3}{|c|}{ Dimensiones de los postes conductores (mm) } \\
\cline { 2 - 4 } & Radio $r_{c}$ & Altura $h_{c}$ & Posición $\left(d_{x, c}, d_{z, c}\right)$ \\
\hline$(1,1)$ & 2,0 & 4,0 & $(0,-2)$ \\
\hline$(1,2)$ & 2,0 & 7,0 & $(0,0)$ \\
\hline$(2,1)$ & 1,5 & 5,5 & $(0,0)$ \\
\hline$(2,2)$ & 2,0 & 7,0 & $(0,0)$ \\
\hline \hline
\end{tabular}

Tabla 5.1: Dimensiones y posición relativa $(\mathrm{mm})$ de los postes conductores de geometría cilíndrica del filtro comb-line de 4 resonadores de la figura 5.15. 
Asimismo, las dimensiones y la posición relativa de los tres irises de acoplamiento de geometría rectangular que integran el filtro (ver figura 5.5) se presentan en la tabla 5.2 (la longitud de los tres irises es $l_{i r}=2,0 \mathrm{~mm}$ ). Finalmente, en relación a las dimensiones del puerto coaxial (ver figura 5.9), se tiene que el radio externo es $r_{1}=2,4 \mathrm{~mm}$; el radio interno es $r_{2}=1,2 \mathrm{~mm}$; la longitud de penetración de la sonda es $h_{p}=3,5 \mathrm{~mm}$, y la permitividad dieléctrica relativa de la guía coaxial es $\varepsilon_{r}=4,6$ (el coaxial se encuentra centrado en la cara lateral del resonador). En la figura 5.16 se presenta el módulo del parámetro de dispersión

\begin{tabular}{|c|c|c|c|}
\hline \hline \multirow{2}{*}{$\begin{array}{c}\text { Resonadores que conecta } \\
(i, j)-(k, l)\end{array}$} & \multicolumn{3}{|c|}{ Dimensiones de los irises de acoplamiento (mm) } \\
\cline { 2 - 4 } & Anchura $a_{i r}$ & Altura $b_{i r}$ & Posición $\left(d_{x, i r}, d_{y, i r}\right)$ \\
\hline$(1,1)-(1,2)$ & 17,0 & 8,5 & $(0,0)$ \\
\hline$(1,2)-(2,2)$ & 17,0 & 8,5 & $(0,0)$ \\
\hline$(2,1)-(2,2)$ & 14,0 & 7,0 & $(0,0)$ \\
\hline \hline
\end{tabular}

Tabla 5.2: Dimensiones y posición relativa $(\mathrm{mm})$ de los irises de acoplamiento del filtro comb-line de 4 resonadores de la figura 5.15 .

$S_{21}$ del filtro de la figura 5.15, y se comparan con éxito los resultados obtenidos con los proporcionados por HFSS. En relación con la eficiencia computacional de la herramienta implementada, es importante comentar que los resultados presentados se han calculado en 188 segundos para un total de 121 puntos en frecuencia. Por su parte, HFSS necesitó un total de 50 minutos para 25 puntos en frecuencia, lo que demuestra la excelente eficiencia computacional de la herramienta desarrollada.

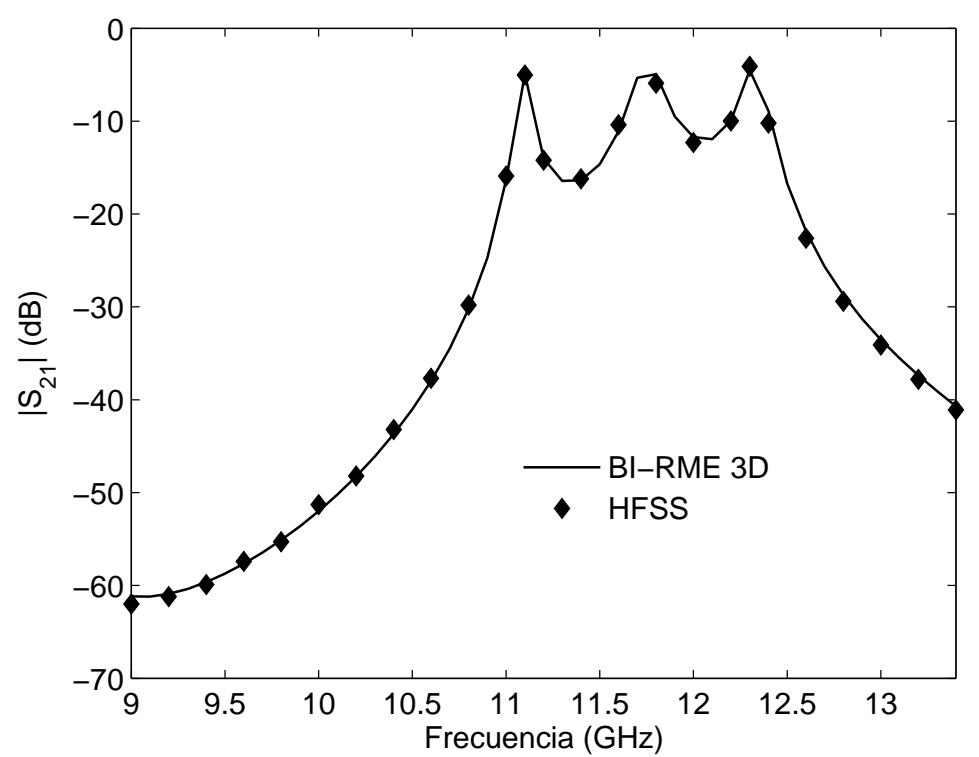

Figura 5.16: Módulo del parámetro $S_{21}$ para el filtro comb-line de la figura 5.15. Los resultados obtenidos con la herramienta de simulación se comparan con los datos proporcionados por HFSS. 
El segundo ejemplo de validación de la herramienta relativa a la etapa 3 se presenta en la figura 5.17. Se trata de una estructura en configuración comb-line de 7 resonadores y con 3 puertos de acceso, siendo dos de ellos de tipo coaxial (puertos (1) y (3)) y uno de tipo rectangular (puerto (2)) cuyas dimensiones coinciden con las de la cara lateral del resonador. En este ejemplo asumiremos que la cavidad donde está situado el puerto coaxial (1) se corresponde con el resonador $(1,1)$ de la estructura ${ }^{10}$. Además, supondremos que el puerto coaxial (1) está situado en el acceso local número (1) del resonador $(1,1)$. En este caso, las dimensiones de todos los resonadores del dispositivo son iguales y de valores $a=19,05 \mathrm{~mm}, b=9,525 \mathrm{~mm}$ y $c=a$. Por otra parte, las dimensiones de los postes conductores presentes en la estructura se encuentran en la tabla 5.3, y las de los irises de acoplamiento se recogen en la tabla 5.4 (la longitud de todos los irises es $l_{i r}=2,0 \mathrm{~mm}$ ). Las dimensiones de los dos puertos coaxiales son las siguientes: el radio externo es $r_{1}=2,4 \mathrm{~mm}$; el radio interno es $r_{2}=1,2 \mathrm{~mm}$; la longitud de penetración de la sonda es $h_{p}=3,5 \mathrm{~mm}$ y la permitividad dieléctrica relativa es $\varepsilon_{r}=4,6$. Además, los puertos coaxiales se encuentran en una posición centrada en la cara lateral del resonador donde se conectan.

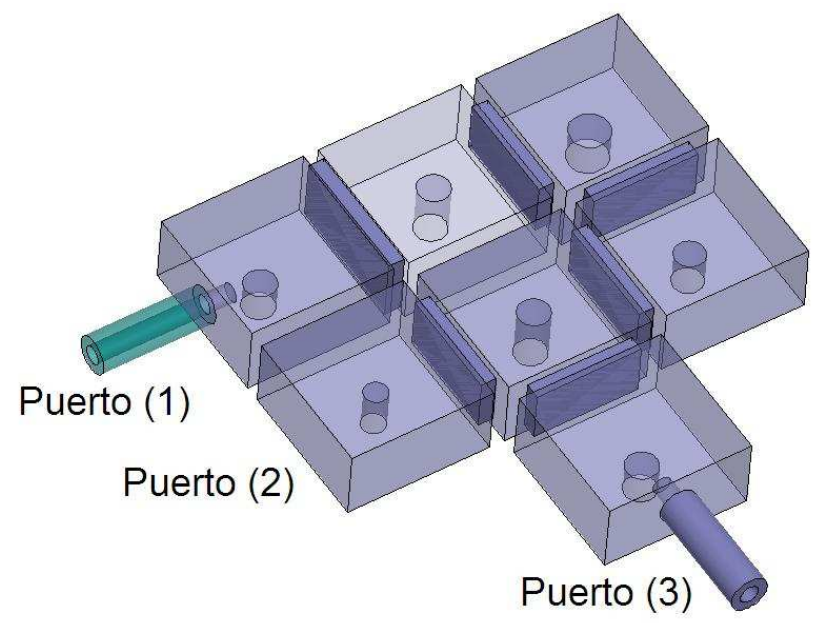

Figura 5.17: Dispositivo comb-line de 7 cavidades con tres puertos de acceso, siendo dos de ellos de tipo coaxial y el otro de tipo rectangular. Esta estructura se utiliza para validar la herramienta de simulación relativa a la etapa 3 .

La respuesta de este dispositivo se ha representado en la figura 5.18, donde se muestra el módulo de los parámetros de dispersión $S_{21}$ y $S_{31}$. Por otro lado, se observa que los resultados obtenidos coinciden perfectamente con los que proporciona HFSS. Además, los resultados se han obtenido en 6 minutos y 23 segundos para un total de 241 puntos en frecuencia. Por su parte, HFSS utilizó 75 minutos en un total de 11 puntos en frecuencia.

Finalmente, en la figura 5.19 se representa el último dispositivo que se ha escogido para validar la herramienta relativa a la etapa 3 . En este caso, se trata de una estructura comb-line de 11 resonadores y 5 puertos de acceso. El puerto (1) es un puerto de tipo coaxial cuya

\footnotetext{
${ }^{10}$ De esta forma, el puerto de sección transversal rectangular $(2)$ está situado en el resonador $(2,1)$, y el puerto coaxial $(3)$ se encuentra en el resonador $(3,2)$ del dispositivo.
} 


\begin{tabular}{|c|c|c|c|}
\hline \hline \multirow{2}{*}{$\begin{array}{c}\text { Resonador } \\
(i, j)\end{array}$} & \multicolumn{3}{|c|}{ Dimensiones de los postes conductores (mm) } \\
\cline { 2 - 4 } & Radio $r_{c}$ & Altura $h_{c}$ & Posición $\left(d_{x, c}, d_{z, c}\right)$ \\
\hline$(1,1)$ & 2,0 & 4,0 & $(0,-2)$ \\
\hline$(1,2)$ & 2,0 & 7,0 & $(0,0)$ \\
\hline$(1,3)$ & 2,5 & 4,5 & $(0,0)$ \\
\hline$(2,1)$ & 1,5 & 5,5 & $(0,0)$ \\
\hline$(2,2)$ & 2,0 & 7,0 & $(0,0)$ \\
\hline$(2,3)$ & 1,9 & 4,7 & $(0,0)$ \\
\hline$(3,2)$ & 2,0 & 4,0 & $(-2,0)$ \\
\hline \hline
\end{tabular}

Tabla 5.3: Dimensiones y posición relativa $(\mathrm{mm})$ de los postes conductores de geometría cilíndrica del dispositivo comb-line de 7 resonadores de la figura 5.17.

\begin{tabular}{|c|c|c|c|}
\hline \hline Resonadores que conecta & \multicolumn{3}{|c|}{ Dimensiones de los irises de acoplamiento (mm) } \\
\cline { 2 - 4 }$(i, j)-(k, l)$ & Anchura $a_{i r}$ & Altura $b_{i r}$ & Posición $\left(d_{x, i r}, d_{y, i r}\right)$ \\
\hline$(1,1)-(1,2)$ & 17,0 & 8,5 & $(0,0)$ \\
\hline$(1,2)-(1,3)$ & 14,0 & 7,0 & $(0,0)$ \\
\hline$(1,3)-(2,3)$ & 14,0 & 7,0 & $(0,0)$ \\
\hline$(2,1)-(2,2)$ & 14,0 & 7,0 & $(0,0)$ \\
\hline$(2,2)-(2,3)$ & 14,0 & 7,0 & $(0,0)$ \\
\hline$(2,2)-(3,2)$ & 14,0 & 7,0 & $(0,0)$ \\
\hline \hline
\end{tabular}

Tabla 5.4: Dimensiones y posición relativa $(\mathrm{mm})$ de los irises de acoplamiento del dispositivo comb-line de 7 resonadores de la figura 5.17.

sonda se encuentra terminada en disco (este puerto se encuentra en una posición centrada); los puertos (2) y (5) son puertos rectangulares cuyas dimensiones coinciden con las de las caras laterales de los resonadores en que están situados; el puerto (3) es un puerto de sección transversal rectangular cuyas dimensiones no coinciden con las de la cara lateral del resonador en que se sitúa el acceso; y el puerto (4) es un puerto de sección transversal rectangular a través de un iris rectangular ${ }^{11}$ (ver la descripción de los diferentes puertos de acceso en la sección 5.2). Con respecto a las dimensiones de la estructura, se tiene en primer lugar que las dimensiones de todos los resonadores son $a=109,22 \mathrm{~mm}, b=54,61 \mathrm{~mm}$ y $c=a$. Por su parte, el radio externo del puerto coaxial es $r_{1}=2,4 \mathrm{~mm}$; el radio interno es $r_{2}=0,8 \mathrm{~mm}$; la profundidad de penetración de la sonda es $h_{p}=3,5 \mathrm{~mm}$; las dimensiones del disco de la sonda son $r_{d}=2,0 \mathrm{~mm}$ y $h_{d}=4,0 \mathrm{~mm}$; y se considera que la permitividad dieléctrica relativa de la guía coaxial es $\varepsilon_{r}=4,6$. Por otro lado, las dimensiones del puerto (3) son $a_{p}=82,0 \mathrm{~mm}$ y $b_{p}=41,0 \mathrm{~mm}$ (en posición centrada con respecto a la cara lateral del

\footnotetext{
${ }^{11}$ Para el dispositivo de la figura 5.19 se asume que el puerto (1) está situado en el acceso local (1) del resonador $(1,1)$ de la estructura. De esta manera, el puerto (2) está situado en el resonador $(1,4)$; el puerto (3) en el resonador $(2,4)$; el puerto $(4)$ en el resonador $(3,2)$, y el puerto $(5)$ en el resonador $(5,4)$ del dispositivo.
} 


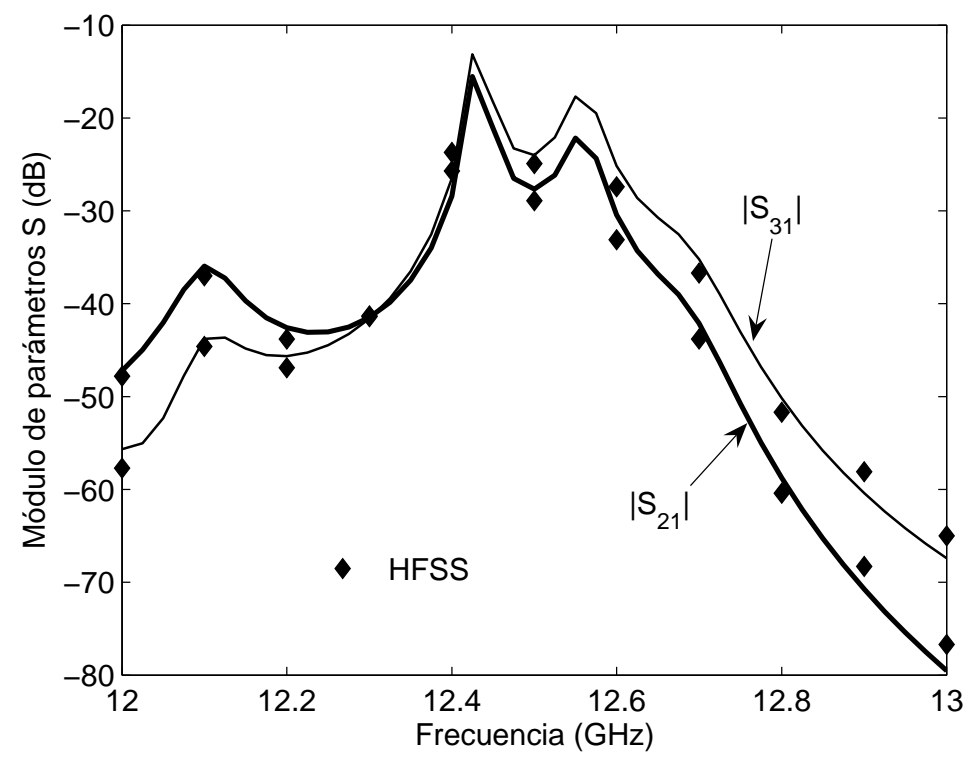

Figura 5.18: Módulo de los parámetros $S_{21}$ y $S_{31}$ (líneas continuas) para el dispositivo comb-line de la figura 5.17. Los resultados obtenidos con la herramienta de simulación se comparan con los datos proporcionados por HFSS.

resonador). Además, las dimensiones del puerto (5) son $a_{p}^{\prime}=109,22 \mathrm{~mm}$ y $b_{p}^{\prime}=54,61 \mathrm{~mm}$, siendo las dimensiones transversales del iris $a_{i r, p}=82,0 \mathrm{~mm}$ y $b_{i r, p}=41,0 \mathrm{~mm}$, y su longitud $l_{i r, p}=2,0 \mathrm{~mm}$ (el iris se encuentra en una posición centrada). Las dimensiones de los postes conductores y de los irises de acoplamiento (estos últimos, todos ellos de longitud $l_{i r}=2,0 \mathrm{~mm}$ ) se han recogido en las tablas 5.5 y 5.6 , respectivamente.

Los resultados de la simulación relativos al módulo de los parámetros de dispersión se han representado en la figura 5.20, mientras que en la figura 5.21 se recogen las fases de dichos parámetros de dispersión. Además, en ambas figuras se presenta una comparación con los resultados que proporciona HFSS. Como se observa, se aprecia una excelente concordancia entre ambos resultados. Por otro lado, la estructura fue simulada en 7 minutos y 23 segundos para un total de 151 puntos en frecuencia. Por su parte, HFSS empleó, aproximadamente, 100 minutos para un total de 18 puntos en frecuencia. Finalmente, es importante destacar que la precisión de los resultados presentados validan por completo la herramienta de simulación relativa a la etapa 3 .

\subsubsection{Validación de la herramienta de la etapa 4}

Seguidamente, se presentan varios resultados con el objetivo de validar la herramienta de simulación relativa a la etapa 4 . En esta etapa, se permite la introducción de postes conductores con forma de vaso y de champiñón, así como la presencia de tornillos de sintonía en los resonadores comb-line. El primer ejemplo se representa en la figura 5.22, y se trata de un filtro comb-line en línea con 2 resonadores y 2 puertos de acceso. El puerto (1) es 


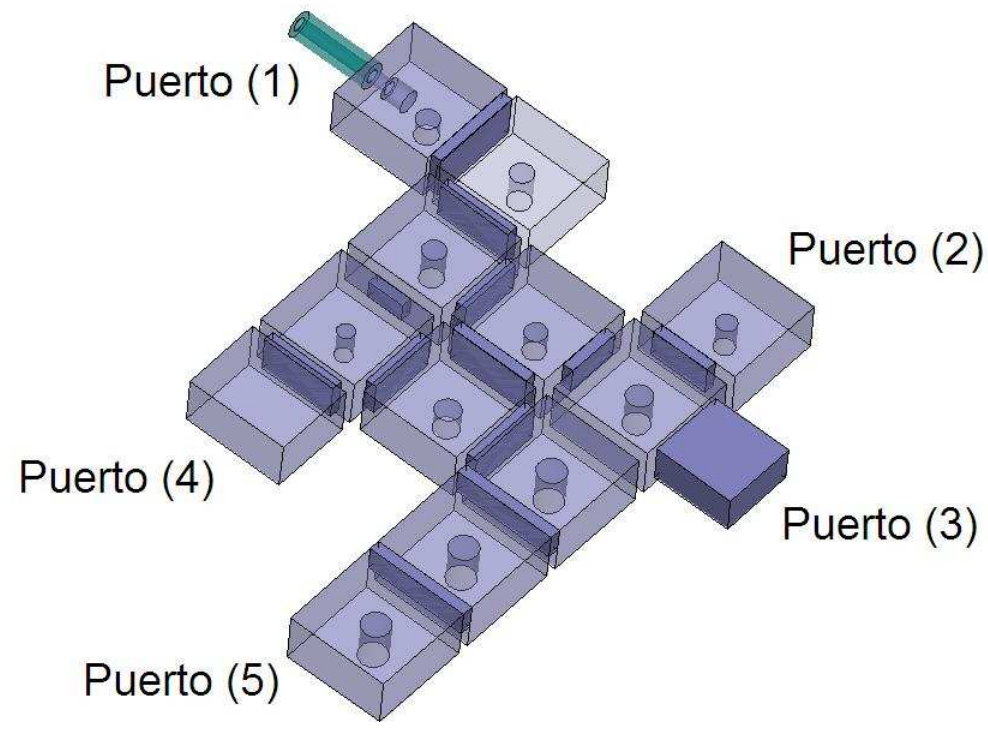

Figura 5.19: Dispositivo comb-line de 11 cavidades y 5 puertos de acceso. Esta estructura se utiliza para validar la herramienta de simulación relativa a la etapa 3 .

\begin{tabular}{|c|c|c|c|}
\hline \hline \multirow{2}{*}{$\begin{array}{c}\text { Resonador } \\
(i, j)\end{array}$} & \multicolumn{3}{|c|}{ Dimensiones de los postes conductores (mm) } \\
\cline { 2 - 4 } & Radio $r_{c}$ & Altura $h_{c}$ & Posición $\left(d_{x, c}, d_{z, c}\right)$ \\
\hline$(1,1)$ & 3,3 & 20,0 & $(0,-39,61)$ \\
\hline$(1,2)$ & 2,0 & 25,5 & $(0,0)$ \\
\hline$(1,4)$ & 1,75 & 18,0 & $(0,0)$ \\
\hline$(2,2)$ & 2,0 & 24,5 & $(0,0)$ \\
\hline$(2,3)$ & 1,9 & 32,7 & $(0,0)$ \\
\hline$(2,4)$ & 2,25 & 15,0 & $(0,0)$ \\
\hline$(3,2)$ & 1,5 & 22,2 & $(0,0)$ \\
\hline$(3,3)$ & 2,2 & 34,5 & $(0,0)$ \\
\hline$(3,4)$ & 2,5 & 21,5 & $(0,0)$ \\
\hline$(4,4)$ & 2,5 & 16,5 & $(0,0)$ \\
\hline$(5,4)$ & 2,5 & 37,5 & $(0,0)$ \\
\hline \hline
\end{tabular}

Tabla 5.5: Dimensiones y posición relativa $(\mathrm{mm})$ de los postes conductores de geometría cilíndrica del dispositivo comb-line de 11 resonadores de la figura 5.19.

de tipo coaxial y asumiremos que se encuentra en el acceso local (1) del resonador $(1,1)$; mientras que el puerto (2) es de tipo rectangular, se encuentra en el acceso local (2) del resonador $(1,2)$, y sus dimensiones son iguales a las de la cara lateral del resonador. Tal y como se aprecia en la figura 5.22, en el resonador $(1,1)$ del filtro se tiene un poste conductor en forma de vaso y un tornillo de sintonía, mientras que en el resonador $(1,2)$ hay un poste conductor en forma de champiñón y un tornillo de sintonía. Las dimensiones de los 


\begin{tabular}{|c|c|c|c|}
\hline \hline Resonadores que conecta & \multicolumn{3}{|c|}{ Dimensiones de los irises de acoplamiento (mm) } \\
\cline { 2 - 4 }$(i, j)-(k, l)$ & Anchura $a_{i r}$ & Altura $b_{i r}$ & Posición $\left(d_{x, i r}, d_{y, i r}\right)$ \\
\hline$(1,1)-(1,2)$ & 100,0 & 50,0 & $(0,2,305)$ \\
\hline$(1,2)-(2,2)$ & 85,0 & 42,5 & $(0,0)$ \\
\hline$(1,4)-(2,4)$ & 94,0 & 47,0 & $(0,3,805)$ \\
\hline$(2,2)-(2,3)$ & 78,0 & 39,0 & $(0,0)$ \\
\hline$(2,3)-(2,4)$ & 80,0 & 40,0 & $(0,7,305)$ \\
\hline$(2,2)-(3,2)$ & 68,0 & 34,0 & $(0,0)$ \\
\hline$(2,3)-(3,3)$ & 92,0 & 46,0 & $(0,4,305)$ \\
\hline$(3,2)-(3,3)$ & 78,0 & 39,0 & $(0,0)$ \\
\hline$(3,3)-(3,4)$ & 81,0 & 40,5 & $(0,0)$ \\
\hline$(3,4)-(4,4)$ & 109,22 & 27,305 & $(0,13,6525)$ \\
\hline$(4,4)-(5,4)$ & 109,22 & 27,305 & $(0,13,6525)$ \\
\hline \hline
\end{tabular}

Tabla 5.6: Dimensiones y posición relativa $(\mathrm{mm})$ de los irises de acoplamiento del dispositivo comb-line de 11 resonadores de la figura 5.19.

dos resonadores son $a=19,05 \mathrm{~mm}, b=9,525 \mathrm{~mm} \mathrm{y} c=a$. Por otro lado, con respecto a las dimensiones del puerto coaxial (en posición centrada), se tiene que el radio externo es $r_{1}=2,4 \mathrm{~mm}$; el radio interno es $r_{2}=1,2 \mathrm{~mm}$; la longitud de penetración de la sonda es igual a $h_{p}=3,5 \mathrm{~mm}$, y la permitividad dieléctrica relativa de la guía coaxial es $\varepsilon_{r}=4,6$. En relación con las dimensiones de los postes conductores, se tiene que los radios que definen el poste en forma de vaso son $r_{v, 1}=1,2 \mathrm{~mm}$ y $r_{v, 2}=2,0 \mathrm{~mm}$, mientras que las alturas son $h_{v, 1}=h_{v, 2}=2,0 \mathrm{~mm}$ (ver figura 5.4). Además, la posición relativa de este poste en el resonador viene dada por los parámetros $(0,0,-2,0) \mathrm{mm}$ (ver sistema de referencia de la figura 5.3). Por otra parte, los radios que definen el poste conductor en forma de champiñón, el cual se sitúa en una posición centrada, son $r_{c h, 1}=1,5 \mathrm{~mm}$ y $r_{c h, 2}=2,5 \mathrm{~mm}$, mientras que las alturas son $h_{c h, 1}=2,2 \mathrm{~mm}$ y $h_{c h, 2}=1,5 \mathrm{~mm}$ (ver figura 5.4). En relación con el tornillo de sintonía del resonador $(1,1)$, su radio es $r_{t}=2,0 \mathrm{~mm}$ y su altura es $h_{t}=3,0 \mathrm{~mm}$. Por otro lado, el tornillo de sintonía del resonador $(1,2)$ es de radio $r_{t}=1,5 \mathrm{~mm}$ y de altura $h_{t}=4,0 \mathrm{~mm}$. La posición relativa de los dos tornillos de sintonía es $(0, b, 2,0) \mathrm{mm}$ (ver sistema de referencia de la figura 5.6). Finalmente, las dimensiones del iris de acoplamiento (en posición centrada) son $a_{i r}=17,0 \mathrm{~mm}, b_{i r}=8,5 \mathrm{~mm}$ y $l_{i r}=2,0 \mathrm{~mm}$.

En la figura 5.23 se presentan los resultados de la simulación para el módulo del parámetro $S_{21}$ del filtro comb-line de la figura 5.22, y se comparan con éxito los datos obtenidos con los que proporciona HFSS. Este filtro se analizó en 6 minutos y 42 segundos para un total de 121 puntos en frecuencia, mientras que HFSS utilizó 38 minutos para 19 puntos en frecuencia.

El segundo ejemplo que se ha escogido para validar la herramienta de la etapa 4 se presenta en la figura 5.24. Se trata de un dispositivo con 8 resonadores y 3 puertos de acceso, cuyos postes conductores son de los tres tipos posibles: postes cilíndricos, postes en forma 


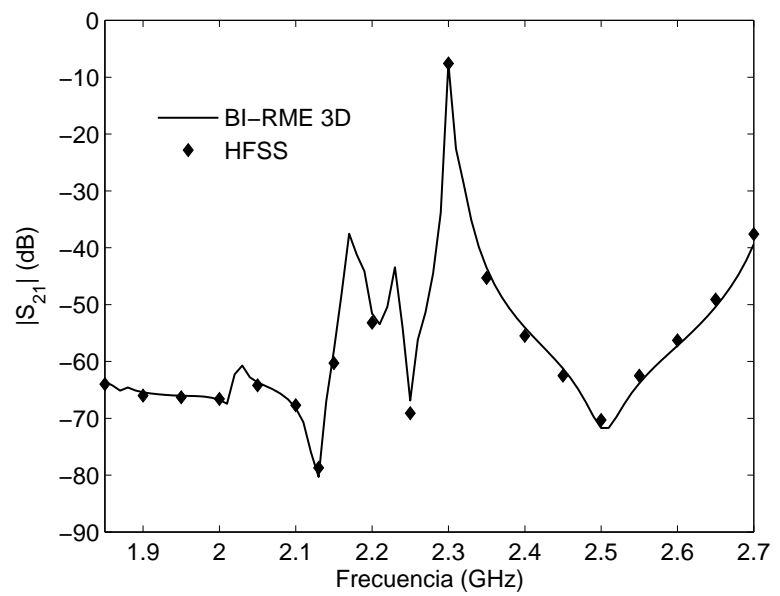

a)

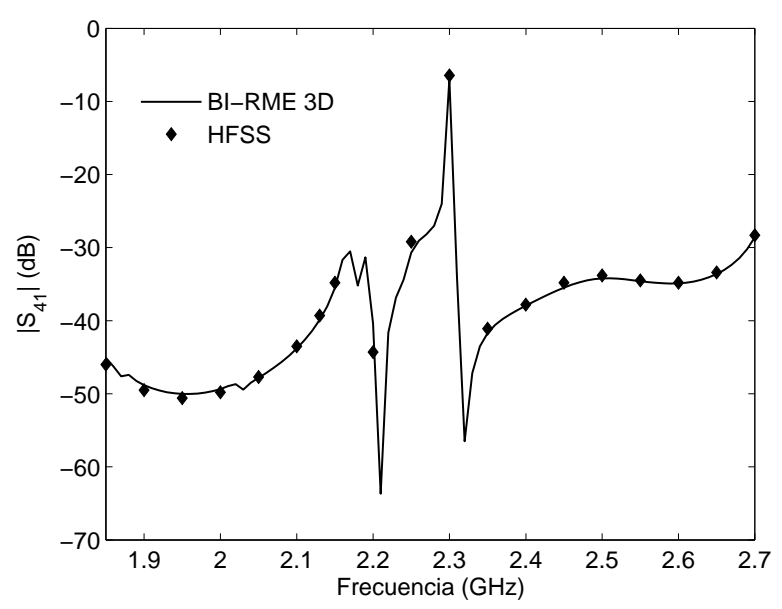

c)

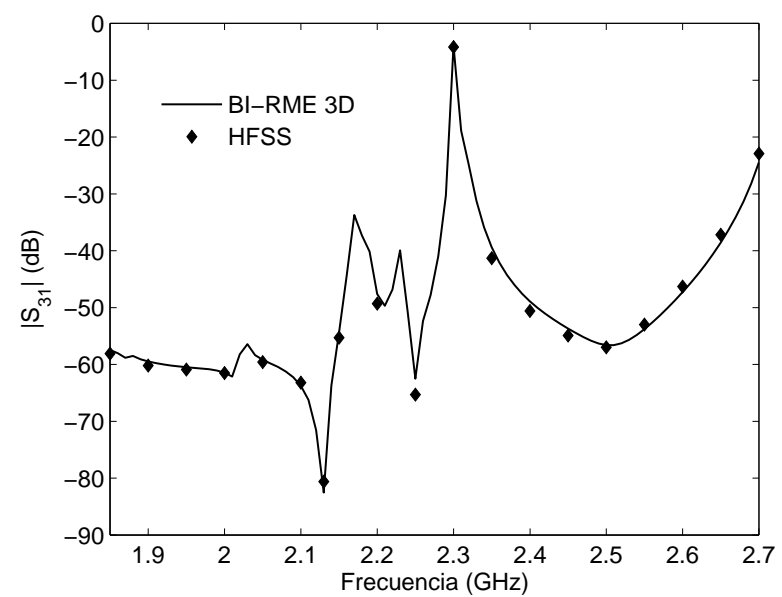

b)

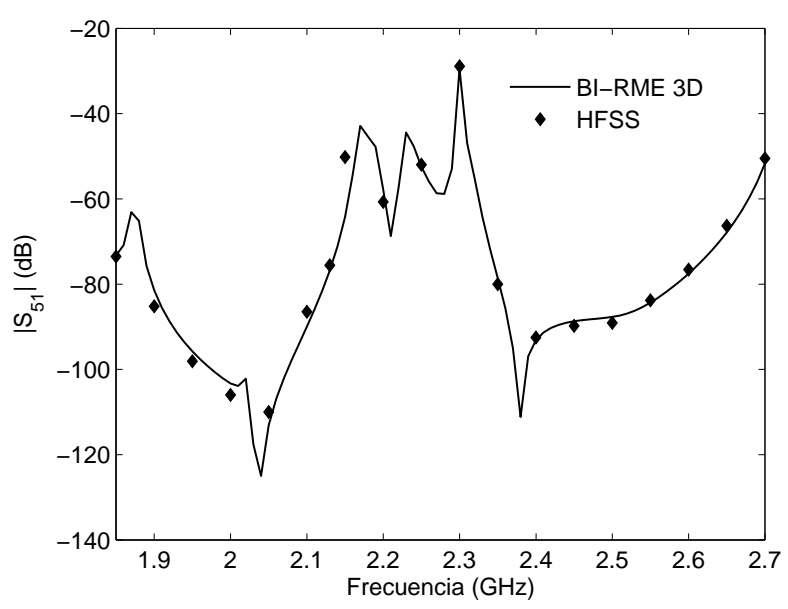

d)

Figura 5.20: Módulo de los parámetros de dispersión relativos al dispositivo comb-line de 11 cavidades y 5 puertos de acceso representado en la figura 5.19. (a) $\left|S_{21}\right|$ (dB); (b) $\left|S_{31}\right|$ (dB); (c) $\left|S_{41}\right|$ (dB); (d) $\left|S_{51}\right|(\mathrm{dB})$.

de vaso y postes en forma de champiñón. Además, también encontramos tornillos de sintonía en diferentes resonadores. Por otro lado, el puerto (1) es de tipo coaxial, y los puertos (2) y (3) son puertos rectangulares cuyas dimensiones coinciden con las de las caras laterales de los resonadores en que se sitúan. De nuevo, se asume que el puerto (1) se encuentra situado en el acceso local (1) del resonador $(1,1)$, con lo que los puertos (2) y (3) se encuentran localizados en los resonadores $(1,4)$ y $(3,2)$, respectivamente. En este caso, las dimensiones de todos los resonadores son $a=19,05 \mathrm{~mm}, b=9,525 \mathrm{~mm}$ y $c=a$. Por otra parte, el radio externo del puerto coaxial, el cual se encuentra en posición centrada, es $r_{1}=2,4 \mathrm{~mm}$; el 


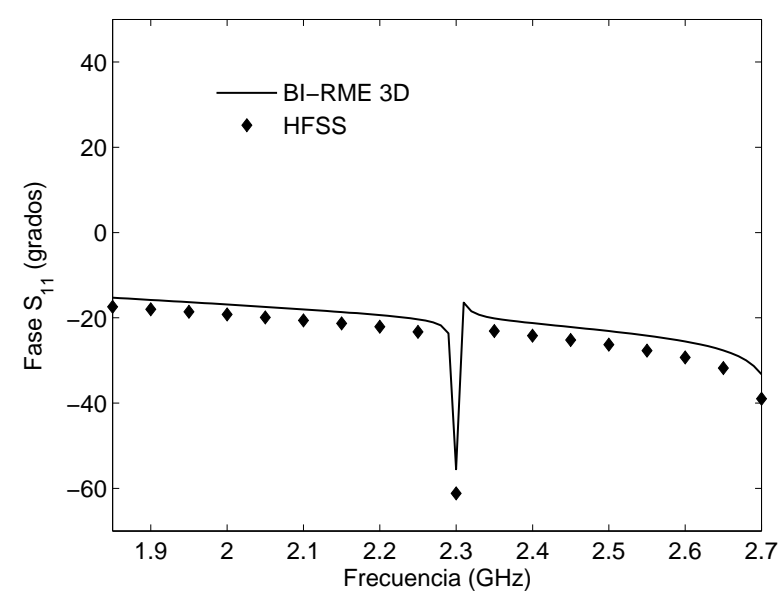

a)

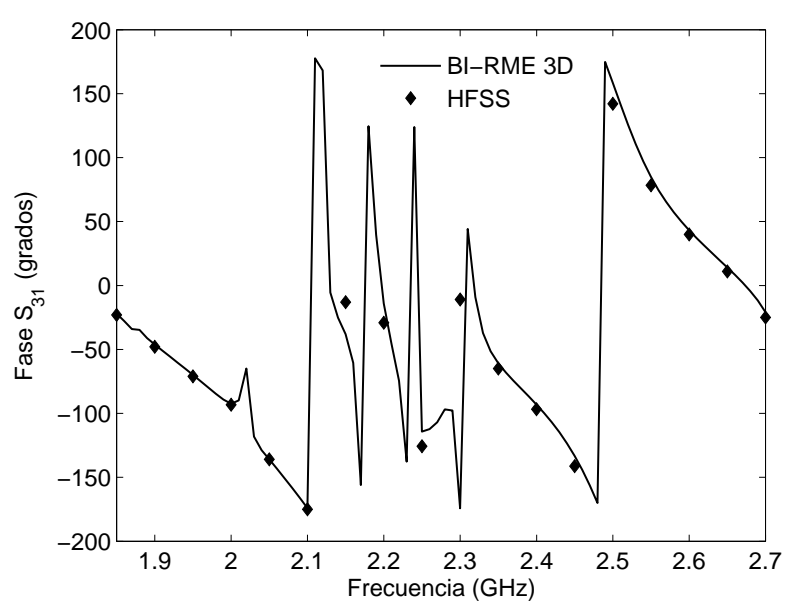

c)

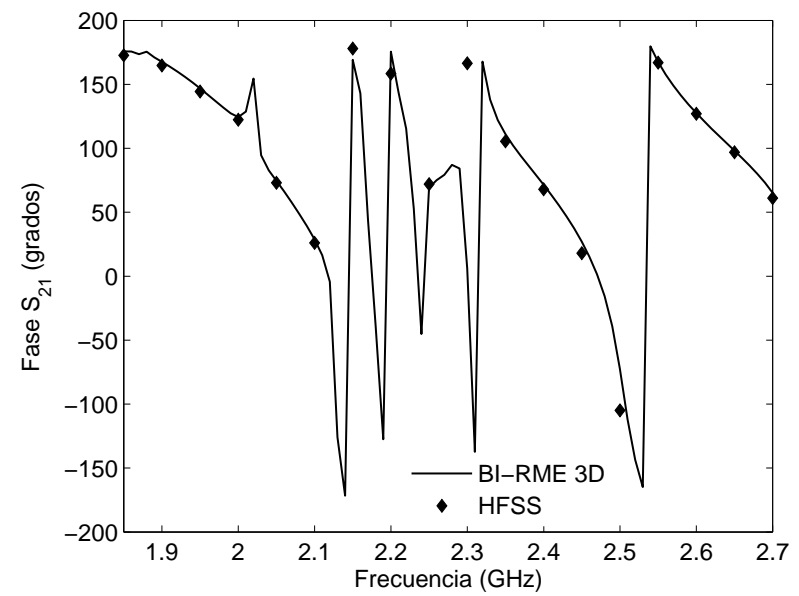

b)

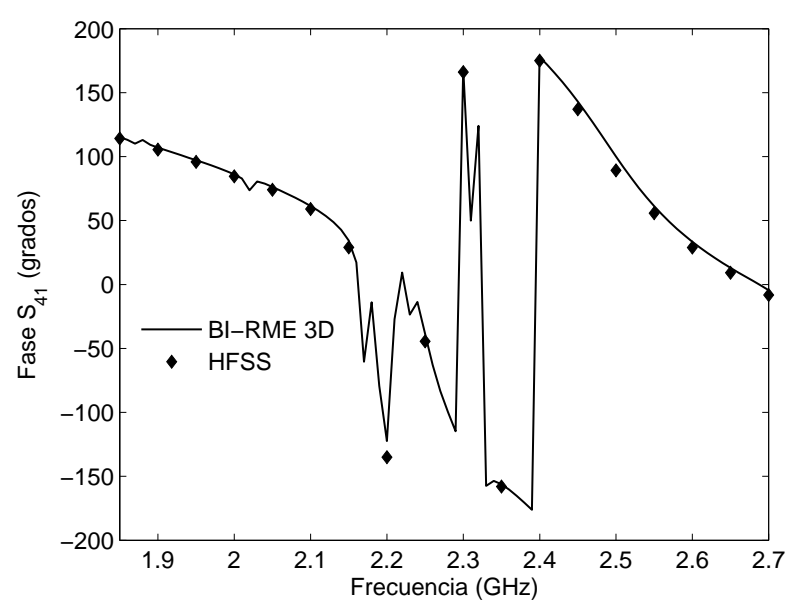

d)

Figura 5.21: Fase de los parámetros de dispersión relativos al dispositivo comb-line de 11 cavidades y 5 puertos de acceso representado en la figura 5.19. (a) $\arg \left(S_{11}\right)$ (grados); (b) $\arg \left(S_{21}\right)$ (grados); (c) $\arg \left(S_{31}\right)$ (grados); (d) $\arg \left(S_{41}\right)$ (grados).

radio interno es $r_{2}=1,2 \mathrm{~mm}$, la profundidad de penetración es $h_{p}=3,5 \mathrm{~mm}$ y se considera que $\varepsilon_{r}=2,6$. En cuanto a las dimensiones de los irises de acoplamiento (todos ellos en posición centrada), se tiene que, con respecto al que conecta el resonador $(1,1)$ con el resonador $(1,2)$, sus dimensiones transversales son $a_{i r}=17,0 \mathrm{~mm}$ y $b_{i r}=8,5 \mathrm{~mm}$. Las dimensiones del resto de los irises son todas iguales, siendo su valor de $a_{i r}=14,0 \mathrm{~mm}$ y $b_{i r}=7,0 \mathrm{~mm}$; por otra parte, la longitud de todos los irises del dispositivo es $l_{i r}=2,0 \mathrm{~mm}$. Las dimensiones de los postes conductores de geometría cilíndrica se recogen en la tabla 5.7, las de los postes en forma de vaso se encuentran en la tabla 5.8, y las de los postes en forma de champiñón se presentan en la tabla 5.9. Finalmente, en la tabla 5.10 se encuentran las dimensiones de los tornillos de sintonía presentes en la estructura. 


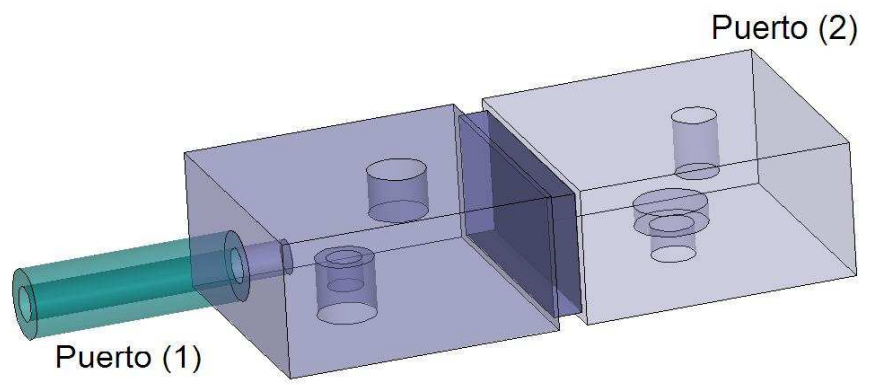

Figura 5.22: Filtro comb-line de 2 cavidades y 2 puertos de acceso, siendo uno de ellos de tipo coaxial y el otro de tipo rectangular. Esta estructura se utiliza para validar la herramienta de simulación relativa a la etapa 4 .

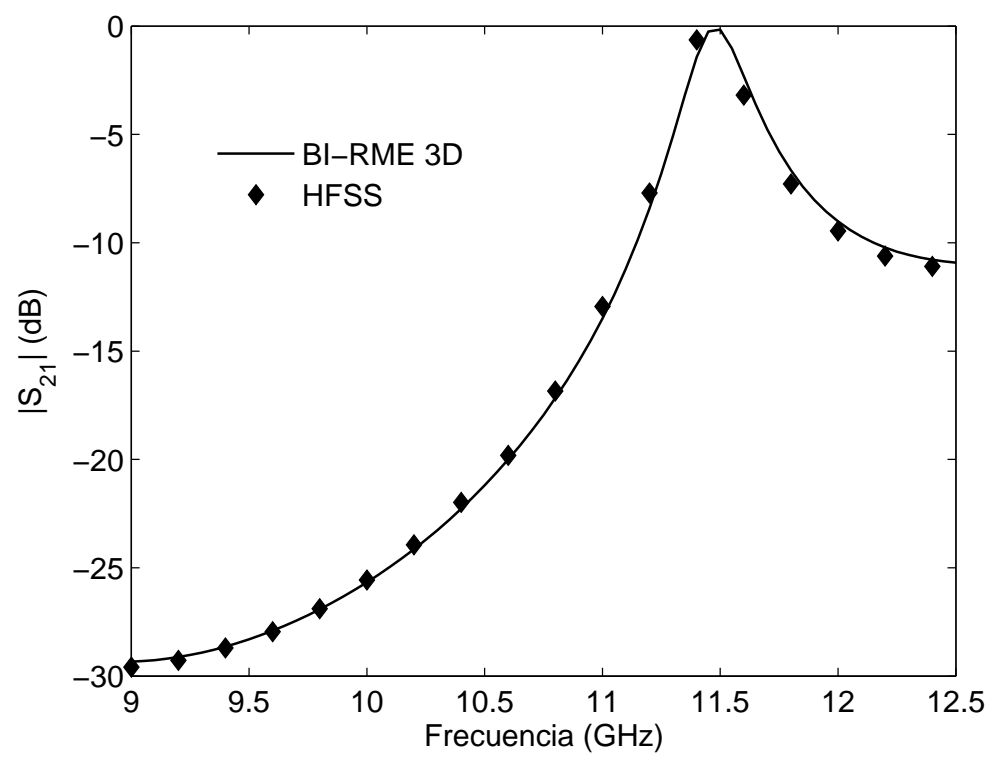

Figura 5.23: Módulo del parámetro $S_{21}$ para el filtro comb-line de la figura 5.22. Los resultados obtenidos con la herramienta de simulación se comparan con los datos proporcionados por HFSS.

El módulo de los parámetros de dispersión asociados a la estructura de la figura 5.24 se han representado en la figura 5.25, donde los resultados obtenidos se comparan satisfactoriamente con los datos proporcionados por HFSS. Por otro lado, las fases de los parámetros de dispersión se presentan en la figura 5.26. En este caso, la estructura se simuló en 8 minutos y 31 segundos para un total de 241 puntos en frecuencia, mientras que HFSS utilizó 82 minutos para 23 puntos en frecuencia. Los dos últimos ejemplos presentados validan por completo la herramienta de simulación correspondiente a la etapa 4 . 


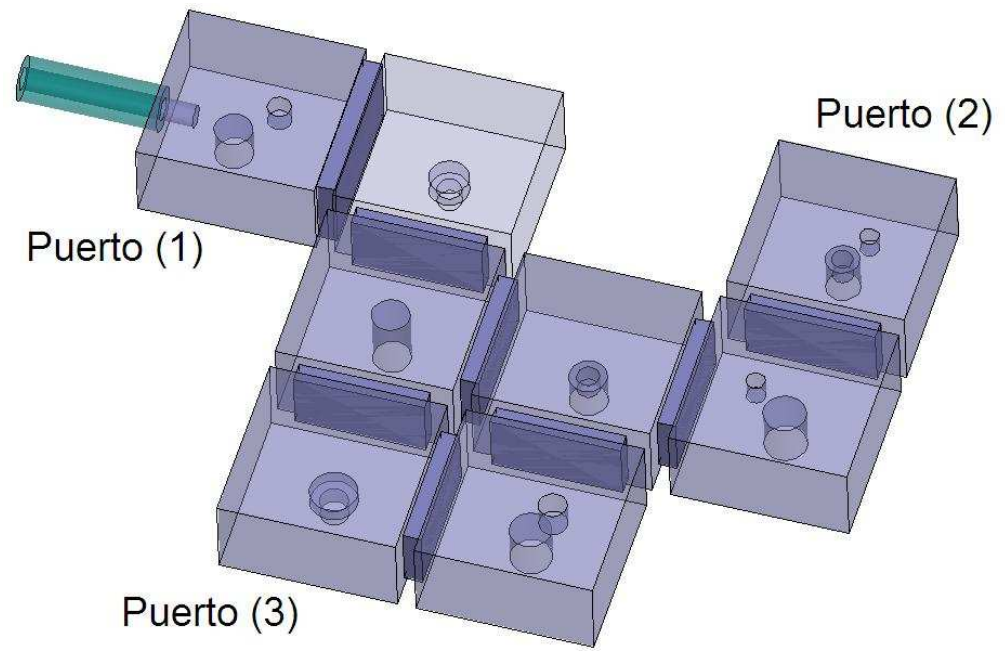

Figura 5.24: Dispositivo comb-line de 8 cavidades y 3 puertos de acceso, siendo uno de ellos de tipo coaxial y dos de tipo rectangular. Esta estructura se utiliza para validar la herramienta de simulación relativa a la etapa 4 .

\begin{tabular}{|c|c|c|c|}
\hline \hline Resonador & \multicolumn{3}{|c|}{ Dimensiones de los postes cilíndricos (mm) } \\
\cline { 2 - 4 }$(i, j)$ & Radio $r_{c}$ & Altura $h_{c}$ & Posición $\left(d_{x, c}, d_{z, c}\right)$ \\
\hline$(1,1)$ & 2,0 & 4,0 & $(0,-2)$ \\
\hline$(2,2)$ & 2,0 & 7,0 & $(0,0)$ \\
\hline$(2,4)$ & 2,25 & 5,0 & $(0,0)$ \\
\hline$(3,3)$ & 2,2 & 4,5 & $(0,0)$ \\
\hline \hline
\end{tabular}

Tabla 5.7: Dimensiones y posición relativa $(\mathrm{mm})$ de los postes conductores de geometría cilíndrica del dispositivo comb-line de 8 resonadores de la figura 5.24.

\begin{tabular}{|c|c|c|c|}
\hline \hline \multirow{2}{*}{$\begin{array}{c}\text { Resonador } \\
(i, j)\end{array}$} & \multicolumn{3}{|c|}{ Dimensiones de los postes en forma de vaso (mm) } \\
\cline { 2 - 4 } & Radios $r_{v, 1} \mathbf{y} r_{v, 2}$ & Alturas $h_{v, 1} \mathbf{y} h_{v, 2}$ & Posición $\left(d_{x, c}, d_{z, c}\right)$ \\
\hline$(1,4)$ & $1,1 \mathrm{y} 1,8$ & $2,1 \mathrm{y} 2,5$ & $(0,0)$ \\
\hline$(2,3)$ & $1,2 \mathrm{y} 2,0$ & $2,0 \mathrm{y} 2,0$ & $(0,0)$ \\
\hline \hline
\end{tabular}

Tabla 5.8: Dimensiones y posición relativa $(\mathrm{mm})$ de los postes conductores en forma de vaso del dispositivo comb-line de 8 resonadores de la figura 5.24.

\subsubsection{Validación de la herramienta de la etapa 5}

Seguidamente, se procede a la validación de la herramienta de la etapa 5. En este caso, se permite la existencia de irises de acoplamiento de sección transversal circular. Para validar esta herramienta, se ha escogido, en primer lugar, el filtro de 2 resonadores y 2 puertos de acceso de la figura 5.27. En este caso, el puerto (1) es de tipo coaxial (asumimos que se encuentra situado en el acceso local $(1)$ del resonador $(1,1)$ ), mientras que el puerto $(2)$ 


\begin{tabular}{|c|c|c|c|}
\hline \hline \multirow{2}{*}{$\begin{array}{c}\text { Resonador } \\
(i, j)\end{array}$} & \multicolumn{3}{|c|}{ Dimensiones de los postes en forma de champiñón (mm) } \\
\cline { 2 - 4 } & Radios $r_{c h, 1} \mathbf{y} r_{c h, 2}$ & Alturas $h_{c h, 1} \mathbf{y} h_{c h, 2}$ & Posición $\left(d_{x, c}, d_{z, c}\right)$ \\
\hline$(1,2)$ & 1,3 y 2,1 & 1,9 y 2,0 & $(0,0)$ \\
\hline$(3,2)$ & 1,5 y 2,5 & 2,2 y 1,5 & $(0,0)$ \\
\hline \hline
\end{tabular}

Tabla 5.9: Dimensiones y posición relativa $(\mathrm{mm})$ de los postes conductores en forma de champiñón del dispositivo comb-line de 8 resonadores de la figura 5.24.

\begin{tabular}{|c|c|c|c|}
\hline \hline \multirow{2}{*}{$\begin{array}{c}\text { Resonador } \\
(i, j)\end{array}$} & \multicolumn{3}{|c|}{ Dimensiones de los tornillos de sintonía (mm) } \\
\cline { 2 - 4 } & Radio $r_{t}$ & Altura $h_{t}$ & Posición $\left(d_{x, t}, d_{z, t}\right)$ \\
\hline$(1,1)$ & 1,25 & 2,2 & $(0,3,0)$ \\
\hline$(1,4)$ & 1,0 & 2,0 & $(0,3,0)$ \\
\hline$(2,4)$ & 1,0 & 2,5 & $(0,-3,0)$ \\
\hline$(3,3)$ & 1,5 & 3,0 & $(0,2,5)$ \\
\hline \hline
\end{tabular}

Tabla 5.10: Dimensiones y posición relativa $(\mathrm{mm})$ de los tornillos de sintonía del dispositivo comb-line de 8 resonadores de la figura 5.24.

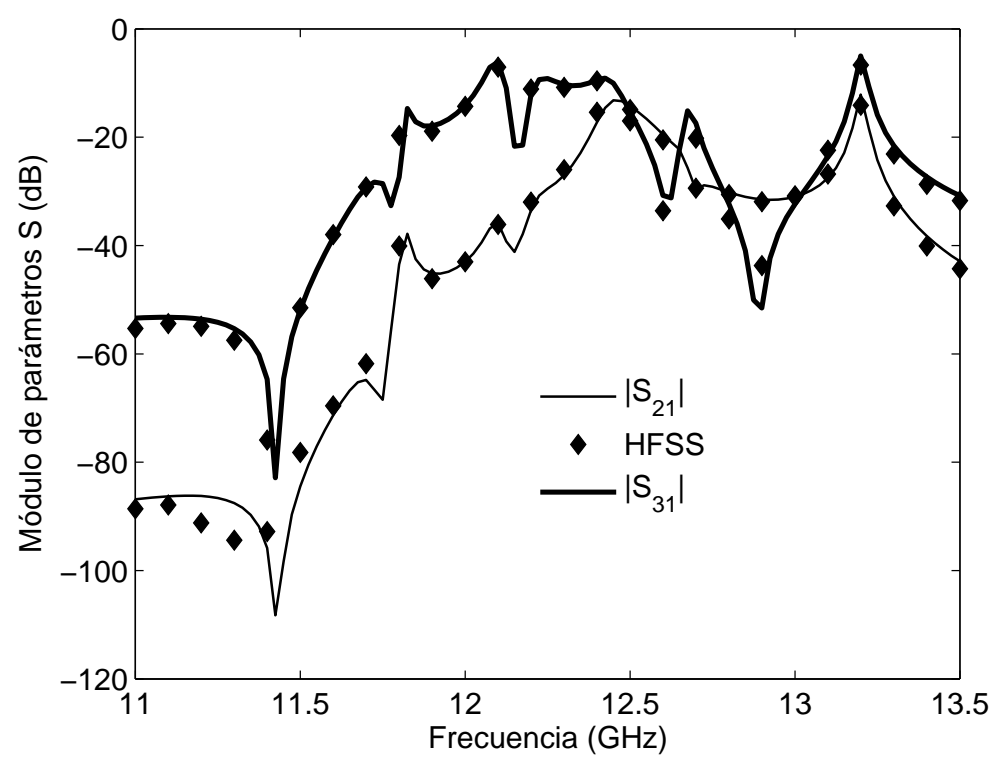

Figura 5.25: Módulo de los parámetros $S_{21}$ y $S_{31}$ para el dispositivo comb-line de la figura 5.24. Los resultados obtenidos con la herramienta de simulación se comparan con los datos proporcionados por HFSS.

es un puerto rectangular cuyas dimensiones coinciden con las de la cara lateral del resonador $(1,2)$ en que está situado. Las dimensiones de los dos resonadores son $a=19,05 \mathrm{~mm}$, $b=9,525 \mathrm{~mm}$ y $c=a$. Por otra parte, las dimensiones de la guía coaxial son las mismas que las utilizadas en los ejemplos anteriores: $r_{1}=2,4 \mathrm{~mm}, r_{2}=1,2 \mathrm{~mm}$ y $h_{p}=3,5 \mathrm{~mm}$; asi- 


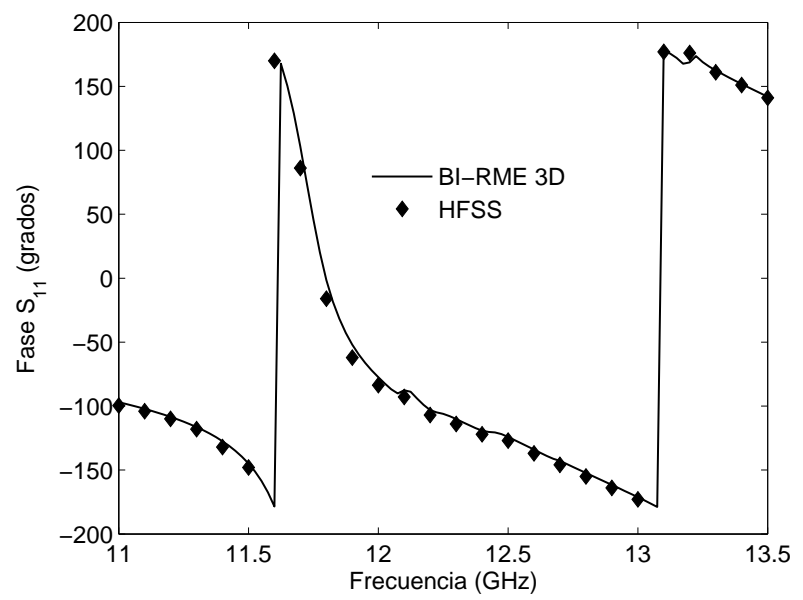

a)

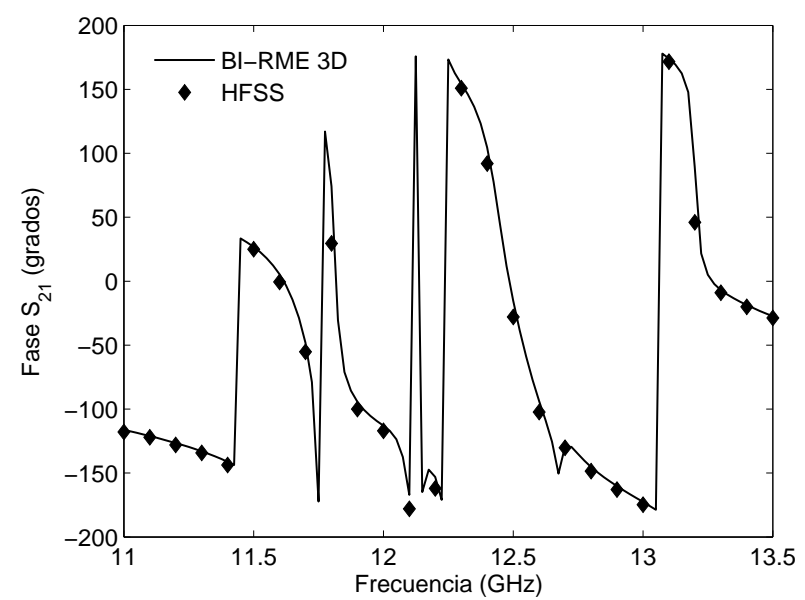

b)

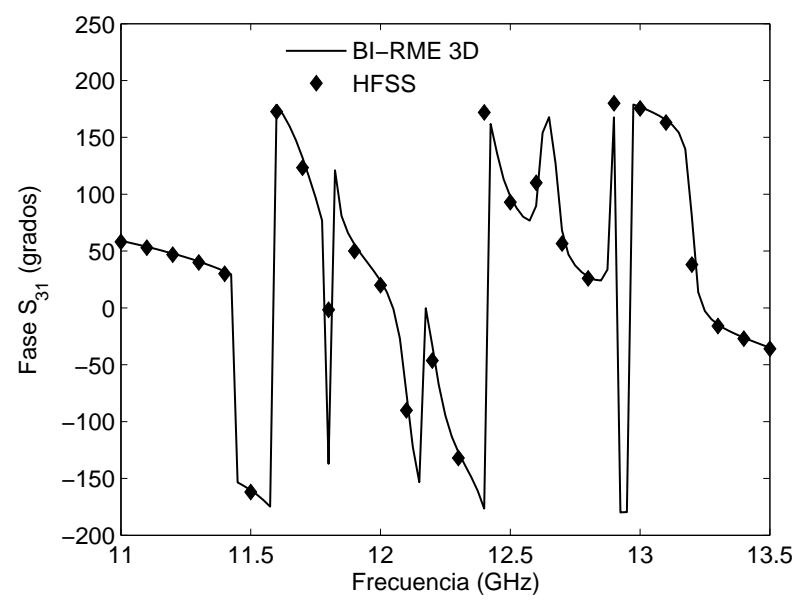

c)

Figura 5.26: Fase de los parámetros de dispersión relativos al dispositivo comb-line de 8 cavidades y 3 puertos de acceso representado en la figura 5.24. (a) $\arg \left(S_{11}\right)$ (grados); (b) $\arg \left(S_{21}\right)$ (grados); (c) $\arg \left(S_{31}\right)$ (grados).

mismo, se considera $\varepsilon_{r}=4,6$. Además, en el resonador $(1,1)$ se tiene un poste cilíndrico de radio $r_{c}=2,0 \mathrm{~mm}$ y altura $h_{c}=4,0 \mathrm{~mm}$ (posición relativa $(0,0,-2,0) \mathrm{mm}$ ); mientras que en el resonador $(1,2)$ encontramos un poste en forma de vaso en posición centrada, definido por los radios $r_{v, 1}=1,2 \mathrm{~mm}$ y $r_{v, 2}=2,0 \mathrm{~mm}$, y por las alturas $h_{v, 1}=2,0 \mathrm{~mm}$ y $h_{v, 2}=2,0 \mathrm{~mm}$. Adicionalmente, en el resonador $(1,2)$ se ha introducido un tornillo de sintonía de radio $r_{t}=1,5 \mathrm{~mm}$ y altura $h_{t}=2,0 \mathrm{~mm}$, situado en la posición relativa $(0, b, 2,0) \mathrm{mm}$. Finalmente, el iris de acoplamiento que interconecta los dos resonadores comb-line es de sección transversal circular, está situado en una posición centrada, su radio es $r_{i r}=3,0 \mathrm{~mm}$, su longitud es $l_{i r}=2,0 \mathrm{~mm}$ y se asume que la permitividad dieléctrica relativa del iris circular es $\varepsilon_{r}=8,0$. 


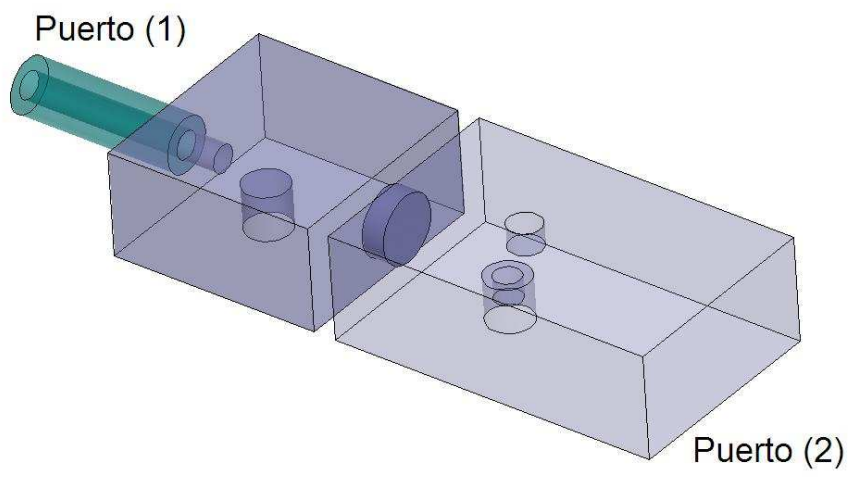

Figura 5.27: Filtro comb-line de 2 cavidades y 2 puertos de acceso, siendo uno de tipo coaxial y el otro de tipo rectangular. Esta estructura se utiliza para validar la herramienta de simulación relativa a la etapa 5 .

El módulo del parámetro de dispersión $S_{21}$ asociado al filtro de la figura 5.27 se representa en la figura 5.28, en donde se compara con éxito la respuesta obtenida con la proporcionada por HFSS. Por otra parte, es importante mencionar que el filtro se analizó en 171 segundos para un total de 241 puntos en frecuencia. Por su parte, HFSS utilizó 45 minutos para 31 puntos en frecuencia.

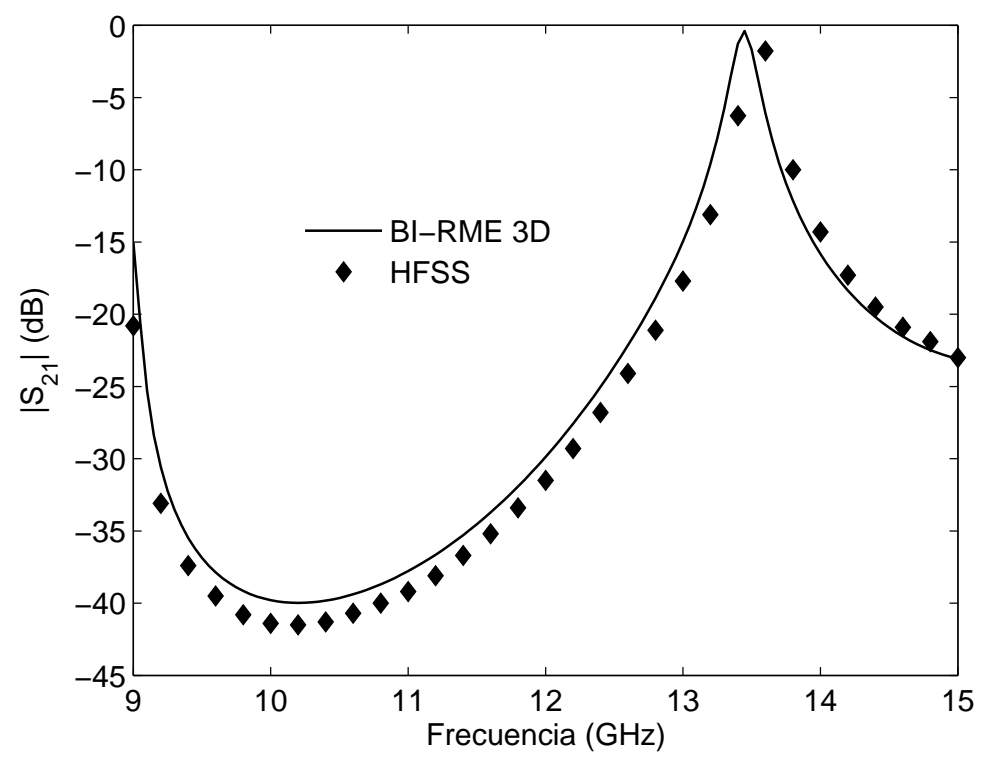

Figura 5.28: Módulo del parámetro $S_{21}$ para el filtro comb-line de la figura 5.27. Los resultados obtenidos con la herramienta de simulación se comparan con los datos proporcionados por HFSS.

El último ejemplo utilizado para validar la herramienta de la etapa 5 se presenta en la 
figura 5.29. Se puede observar que, en este caso, se ha partido de la estructura comb-line de la figura 5.24, en la que se han cambiado ciertos irises de sección transversal rectangular por irises de sección transversal circular. Por ese motivo, las dimensiones del dispositivo de la figura 5.29 son las mismas que las de la estructura de la figura 5.24. Por otro lado, en la tabla 5.11 se recogen las dimensiones de los irises de sección transversal circular (todos ellos se sitúan en una posición centrada).

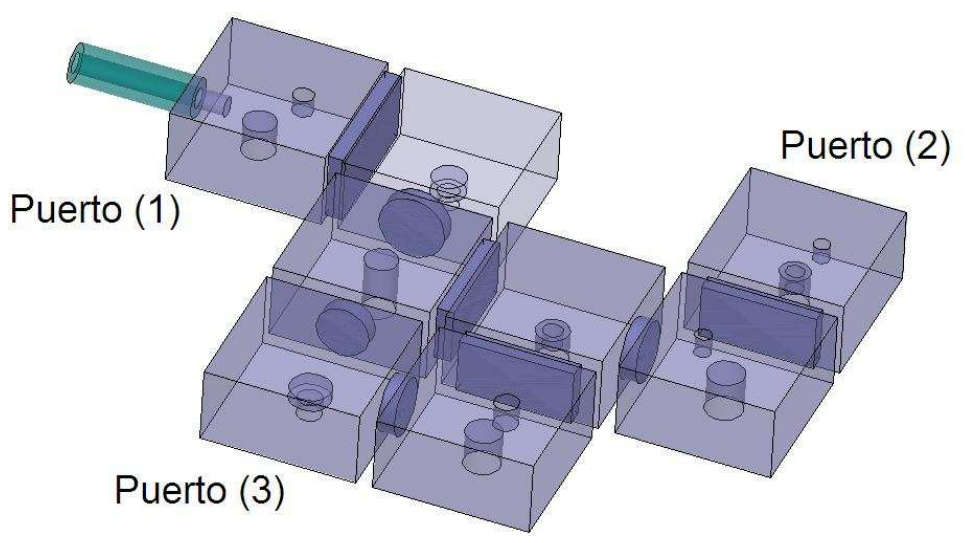

Figura 5.29: Dispositivo comb-line de 8 cavidades y 3 puertos de acceso, siendo uno de tipo coaxial y el resto de tipo rectangular. Esta estructura se utiliza para validar la herramienta de simulación relativa a la etapa 5 .

\begin{tabular}{|c|c|c|c|}
\hline \hline Resonadores que conecta & \multicolumn{3}{|c|}{ Dimensiones de los irises circulares (mm) } \\
\cline { 2 - 4 }$(i, j)-(k, l)$ & Radio $r_{i r}$ & Longitud $l_{i r}$ & Permitividad $\varepsilon_{r}$ \\
\hline$(1,2)-(2,2)$ & 4,0 & 2,0 & 8,0 \\
\hline$(2,3)-(2,4)$ & 3,7 & 2,0 & 12,0 \\
\hline$(2,2)-(3,2)$ & 3,2 & 2,0 & 10,0 \\
\hline$(3,2)-(3,3)$ & 3,5 & 2,0 & 16,0 \\
\hline \hline
\end{tabular}

Tabla 5.11: Dimensiones ( $\mathrm{mm}$ ) de los irises de sección transversal circular del dispositivo comb-line de 8 resonadores de la figura 5.29.

El módulo y las fases de los parámetros de dispersión del dispositivo se han representado en las figuras 5.30 y 5.31, respectivamente, donde también se presentan los resultados proporcionados por HFSS. Nuevamente, comprobamos que los resultados obtenidos presentan una excelente concordancia con HFSS. El análisis de esta estructura empleó 7 minutos y 32 segundos para 241 puntos en frecuencia, mientras que HFSS utilizó 105 minutos para 31 puntos en frecuencia.

\subsubsection{Otros ejemplos de validación}

Hasta ahora, se han presentado una serie de ejemplos de validación en los que no se ha perseguido el diseño de ningún tipo de respuesta eléctrica en particular. Así, el objetivo fun- 


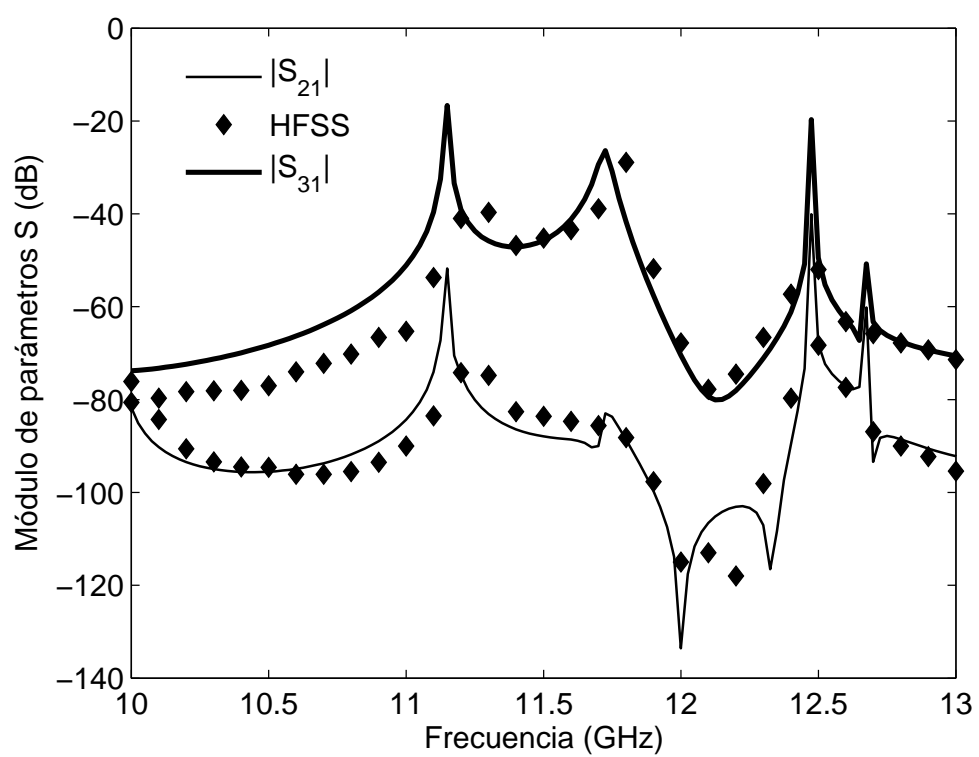

Figura 5.30: Módulo de los parámetros de dispersión del dispositivo comb-line de la figura 5.29. Los resultados obtenidos con la herramienta de simulación se comparan con los datos proporcionados por HFSS.

damental de dichos ejemplos ha consistido en la validación de la herramienta implementada. A continuación, se presentan dos diseños de filtros en configuración comb-line implementados en tecnología guiada. El primero de ellos, cuyas dimensiones han sido proporcionadas por la empresa Thales Alenia Space (Tres Cantos, Madrid), se representa en la figura 5.32. Consiste en un filtro de 4 resonadores y 2 puertos de acceso en guía coaxial (las dimensiones de las dos guías coaxiales, las cuales se encuentran en una posición centrada, son idénticas). Los resonadores están cargados con postes conductores en forma de vaso, y en todos ellos se han introducido tornillos de sintonía (los postes y los tornillos de sintonía del filtro son todos de idénticas dimensiones y están en una posición centrada). Por otro lado, los irises de acoplamiento son de sección transversal rectangular y están todos en una posición centrada (todos los irises son de las mismas dimensiones).

Las dimensiones de los resonadores del filtro son $a=b=c=12,0 \mathrm{~mm}$. En cuanto a las guías coaxiales, el radio externo es $r_{1}=1,46 \mathrm{~mm}$, el radio interno es $r_{2}=0,635 \mathrm{~mm}$, la profundidad de penetración de la sonda es de $h_{p}=2,8 \mathrm{~mm}$ y la permitividad dieléctrica relativa de las guías es $\varepsilon_{r}=1,0$. Por otro lado, los radios que definen los postes en forma de vaso son $r_{v, 1}=1,15 \mathrm{~mm}$ y $r_{v, 2}=1,65 \mathrm{~mm}$; mientras que las alturas son $h_{v, 1}=4,5 \mathrm{~mm}$ y $h_{v, 2}=6,0 \mathrm{~mm}$. Por su parte, el radio de los tornillos de sintonía es $r_{t}=1,0 \mathrm{~mm}$ y su altura $h_{t}=0,8 \mathrm{~mm}$. Finalmente, las dimensiones de la sección transversal de los irises son $a_{i r}=6,0 \mathrm{~mm}$ y $b_{i r}=4,0 \mathrm{~mm}$, siendo su longitud de $l_{i r}=1,0 \mathrm{~mm}$.

La respuesta eléctrica del filtro se recoge en la figura 5.33, donde se presenta el módulo del parámetro de transmisión $S_{21}$. Se aprecia que se trata de un filtro paso-banda en banda C centrado, aproximadamente, en 5,175 GHz. La respuesta obtenida se compara satisfactoria- 


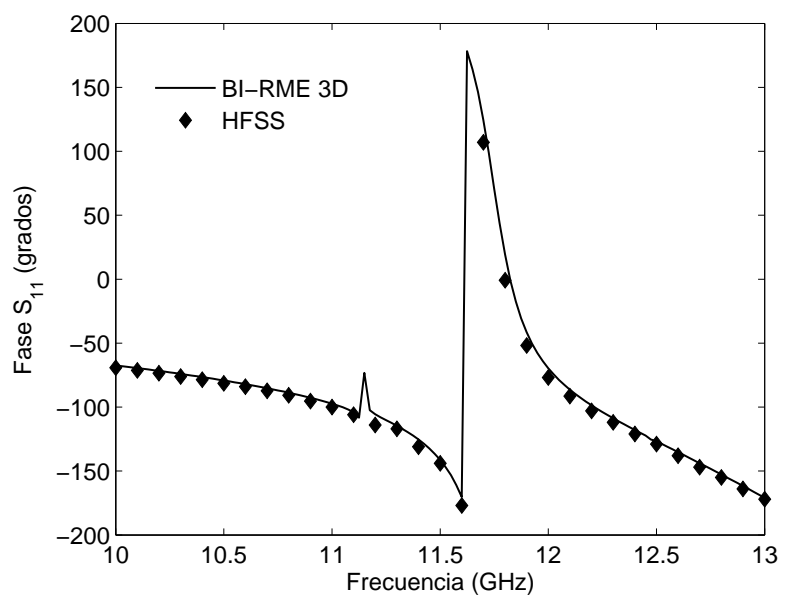

a)

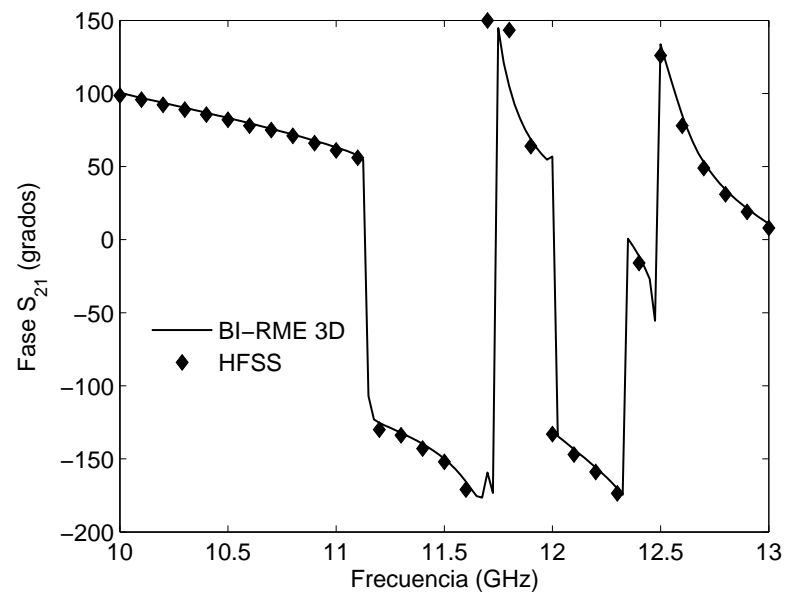

b)

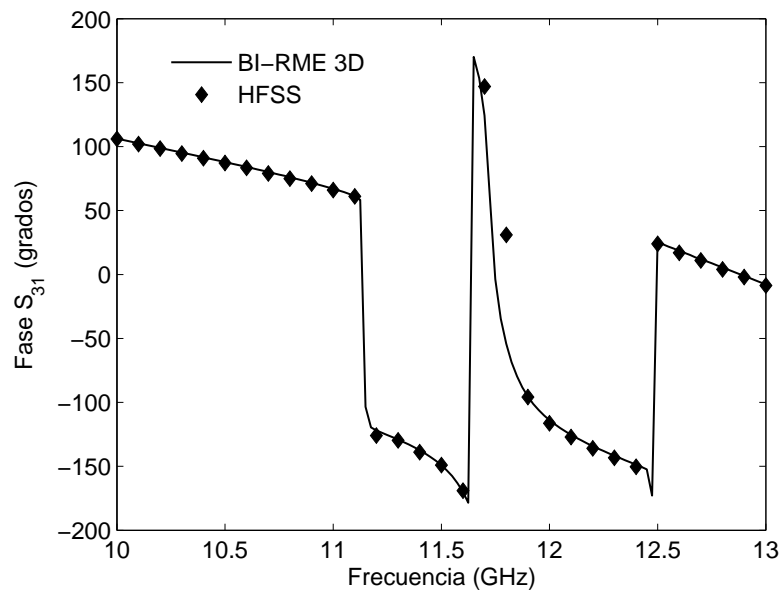

c)

Figura 5.31: Fase de los parámetros de dispersión relativos al dispositivo comb-line de 8 cavidades y 3 puertos de acceso representado en la figura 5.29. (a) $\arg \left(S_{11}\right)$ (grados); (b) $\arg \left(S_{21}\right)$ (grados); (c) $\arg \left(S_{31}\right)$ (grados).

mente con los datos proporcionados por HFSS. Por otro lado, el filtro se simuló en 12,5 minutos para un total de 601 puntos en frecuencia. Por su parte, la simulación con HFSS empleó 2 horas para alcanzar resultados precisos (39 puntos en frecuencia).

La segunda estructura que se presenta a continuación parte de un diseño que se recoge en [78]. En concreto, se trata de un filtro comb-line de 2 puertos de acceso de tipo coaxial compuesto por 6 resonadores en línea cargados con postes conductores de geometría cilíndrica $^{12}$. El filtro bajo análisis se ha representado en la figura 5.34. Las dimensiones de las guías coaxiales son las siguientes: radio externo $r_{1}=2,1 \mathrm{~mm}$, radio interno

\footnotetext{
${ }^{12}$ Se asume que el puerto coaxial (1) se encuentra en al acceso local (1) del resonador $(1,1)$.
} 


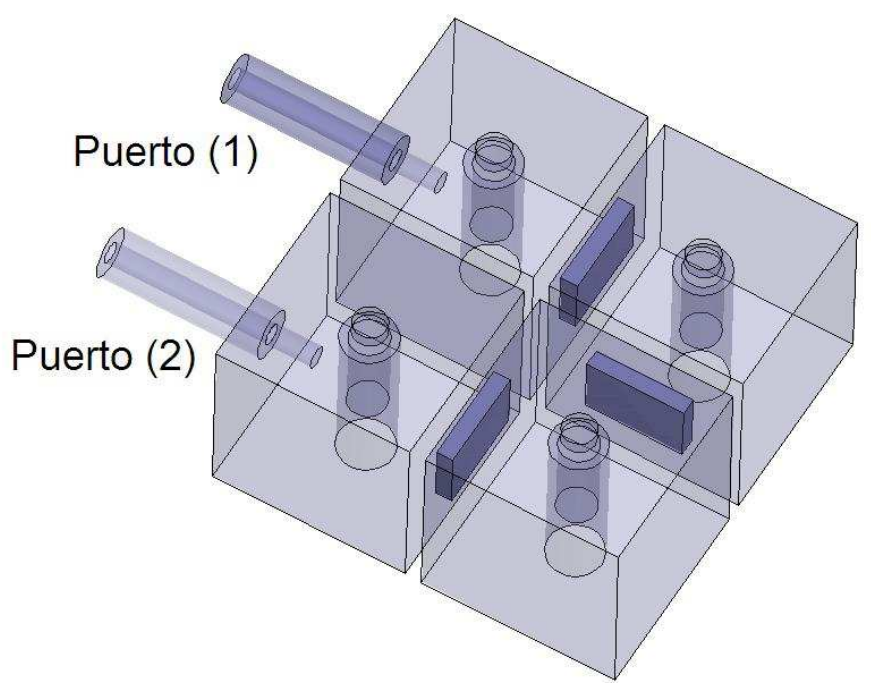

Figura 5.32: Filtro comb-line de 4 cavidades y 2 puertos de acceso de tipo coaxial. Los resonadores están cargados con postes conductores en forma de vaso y poseen todos ellos tornillos de sintonía.

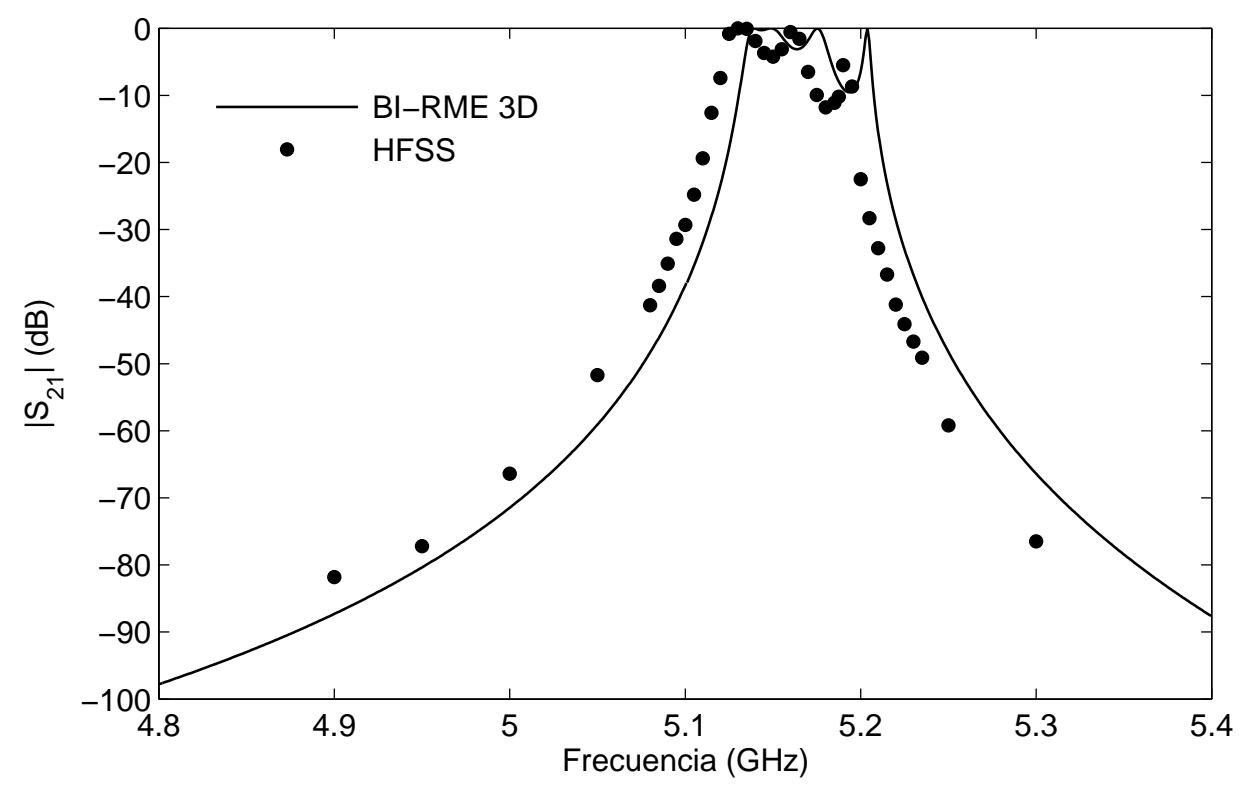

Figura 5.33: Módulo del parámetro de transmisión $S_{21}$ del filtro comb-line de la figura 5.32. Los resultados obtenidos con la herramienta de simulación se comparan con los datos proporcionados por HFSS.

$r_{2}=0,635 \mathrm{~mm}$, profundidad de penetración de la sonda coaxial $h_{p}=6,393 \mathrm{~mm}$ y permitividad dieléctrica relativa $\varepsilon_{r}=2,1$. Por otro lado, la posición relativa de los puertos coaxiales es $\left(d_{x}, d_{y}\right)=(0,-4,5405) \mathrm{mm}$. Por su parte, para los resonadores rectangulares se tiene que $a=c=25,0 \mathrm{~mm}$ (la altura $b$ de cada resonador se proporciona en la tabla 5.12). Por otra parte, todos los postes conductores se encuentran centrados en sus respectivos resonadores 
y el radio de todos ellos es $r_{c}=4,1 \mathrm{~mm}$ (la altura $h_{c}$ de cada poste se proporciona en la tabla 5.12). Por otro lado, los irises de acoplamiento están todos situados en la base del filtro, siendo su altura $b_{i r}=6,0 \mathrm{~mm}$ y su longitud $l_{i r}=1,5 \mathrm{~mm}$ (la anchura $a_{i r}$ de cada iris se proporciona en la tabla 5.12). La respuesta eléctrica del filtro se recoge en la figura 5.35,

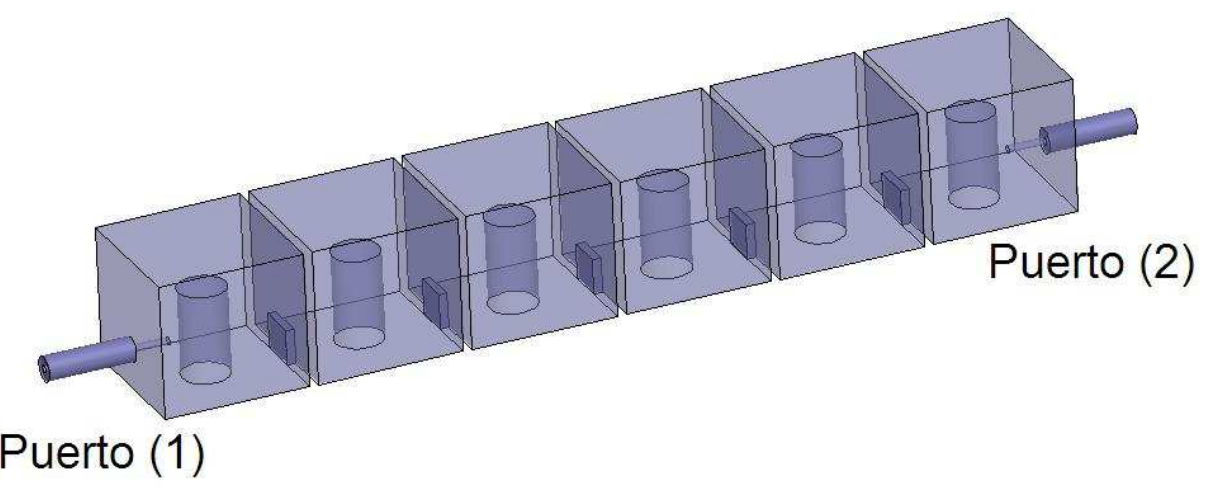

Figura 5.34: Filtro comb-line de 6 cavidades en línea y 2 puertos de acceso de tipo coaxial. Las dimensiones del filtro se han extraído de [78]. Los resonadores están cargados con postes conductores de geometría cilíndrica.

\begin{tabular}{|c|c|c|c|}
\hline \hline \multirow{2}{*}{$\begin{array}{c}\text { Resonador } \\
(i, j)\end{array}$} & \multicolumn{3}{|c|}{ Dimensiones del filtro (mm) } \\
\cline { 2 - 4 } & Altura de resonador $b$ & Altura de poste $h_{c}$ & Anchura de iris $a_{i r}$ \\
\hline$(1,1)$ & 23,081 & 15,081 & 7,256 \\
\hline$(1,2)$ & 23,248 & 15,248 & 6,496 \\
\hline$(1,3)$ & 23,269 & 15,269 & 6,396 \\
\hline$(1,4)$ & 23,269 & 15,269 & 6,496 \\
\hline$(1,5)$ & 23,248 & 15,248 & 7,256 \\
\hline$(1,6)$ & 23,081 & 15,081 & - \\
\hline \hline
\end{tabular}

Tabla 5.12: Dimensiones $(\mathrm{mm})$ del filtro comb-line de 6 resonadores en línea de la figura 5.34. Se asume que el iris de cada resonador es el que se conecta al puerto local (2) de dicho resonador.

donde se representa el módulo de los parámetros de dispersión del filtro y se comparan satisfactoriamente con los datos proporcionados por HFSS. En este caso, se trata de un filtro paso-banda en banda S centrado en 3,66 GHz. En relación con la eficiencia computacional de la herramienta, el filtro se simuló en 14 minutos para un total de 1001 puntos en frecuencia. Por su parte, HFSS empleó, aproximadamente, una hora para alcanzar resultados convergentes (17 puntos en frecuencia). 


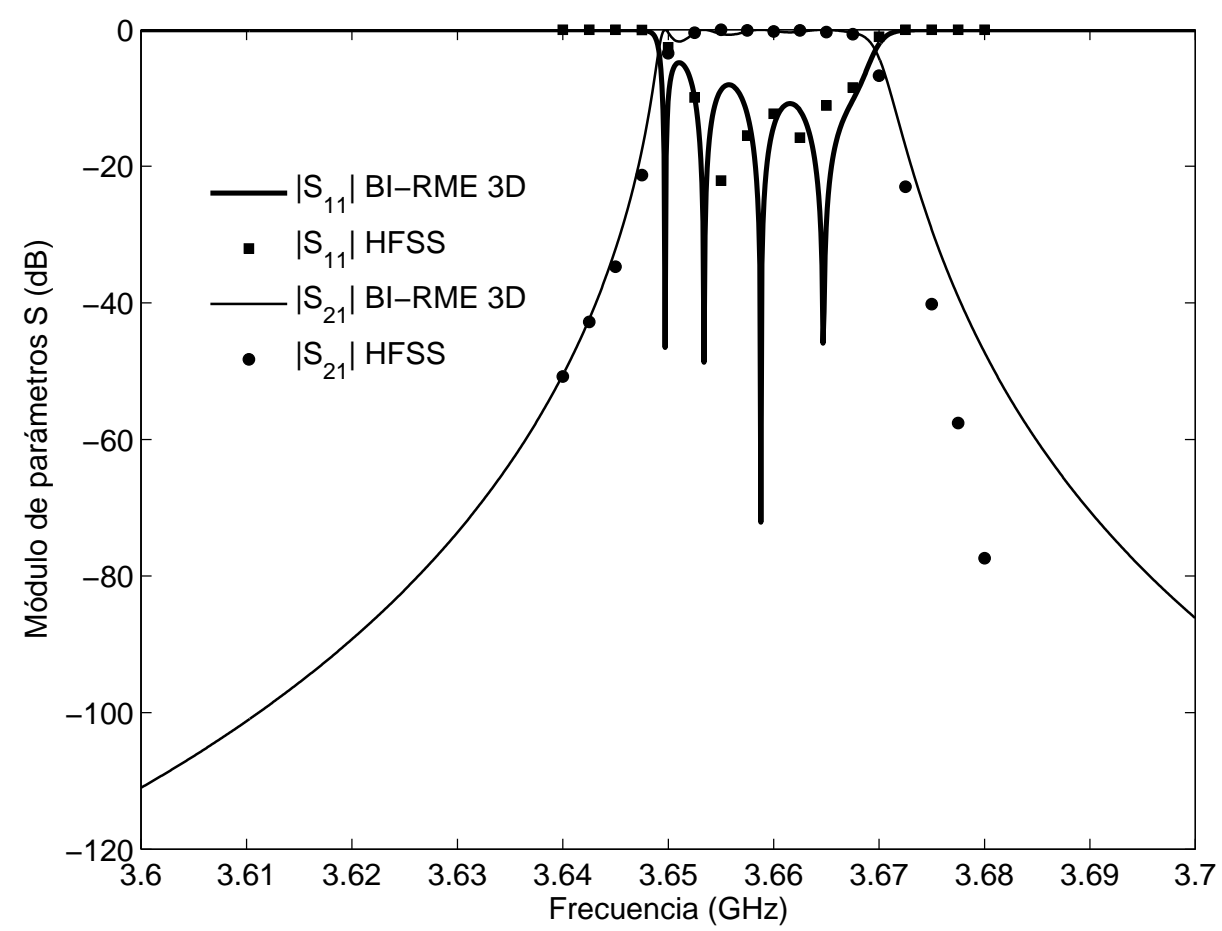

Figura 5.35: Módulo de los parámetros de dispersión del filtro comb-line de la figura 5.34. Los resultados obtenidos con la herramienta de simulación se comparan con los datos proporcionados por HFSS. 


\section{Capítulo 6}

\section{Conclusiones}

\subsection{Conclusiones y Principales Aportaciones}

En este capítulo se resumen las conclusiones más importantes del trabajo de investigación realizado, y se destacan las principales aportaciones originales que se derivan del estudio llevado a cabo en la presente tesis doctoral.

En primer lugar, se ha utilizado la técnica de la ecuación integral con el objetivo de caracterizar de forma rigurosa y eficiente diferentes tipos de uniones planares complejas, a saber: uniones entre guías rectangulares y guías coaxiales, uniones entre guías rectangulares y guías circulares, uniones entre dos guías coaxiales y uniones múltiples en guía rectangular. En particular, el análisis de la unión planar entre una guía rectangular y una guía coaxial ha resultado esencial para abordar con éxito la caracterización multimodal de la excitación coaxial de dispositivos en tecnología guiada utilizando el método BI-RME 3D. Asimismo, el análisis eficiente de la unión planar entre una guía rectangular y una guía coaxial ha precisado el desarrollo de una formulación alternativa para expresar la variación radial de las soluciones modales de la guía coaxial en términos de funciones sinusoidales. Por otro lado, el análisis de la unión planar entre una guía rectangular y una guía circular ha permitido la inserción de irises de acoplamiento de sección transversal circular como elemento de interconexión de los diferentes resonadores que integran los filtros en configuración comb-line. Además, al igual que en el caso de la guía coaxial, las funciones vectoriales modales de la guía circular se han expresado empleando una formulación alternativa a la solución clásica, de manera que la variación radial de las soluciones modales se ha expresado utilizando funciones sinusoidales. Los resultados obtenidos en esta primera parte de la tesis se han validado con éxito y han demostrado la eficiencia y la precisión de la técnica multimodal empleada.

En segundo lugar, en este trabajo de investigación se han analizado y diseñado diferentes tipos de uniones compensadas implementadas en guía rectangular utilizando el método BI-RME 3D. Las uniones en guía rectangular diseñadas (codos rectos, uniones en T, uniones $\mathrm{T}$ mágica y uniones turnstile) se emplean muy frecuentemente en numerosos dispositivos pasivos de microondas, por lo que su análisis eficiente y riguroso tiene un gran interés desde un punto de vista práctico. La aportación teórica fundamental de esta parte de la tesis con- 
siste en la ampliación de la teoría relativa al método BI-RME 3D para permitir el análisis de estructuras compensadas que posean puertos de acceso en guía rectangular ortogonales entre sí, ya que, hasta este momento, este método se había aplicado únicamente para caracterizar estructuras con puertos de acceso en guía rectangular paralelos entre sí. Todo ello ha permitido diseñar diferentes tipos de uniones implementadas en guía rectangular compensadas con postes conductores de geometría cilíndrica. En este sentido, gracias a la enorme flexibilidad del método BI-RME 3D, se ha permitido la compensación de estas estructuras utilizando postes conductores cilíndricos situados en una posición arbitraria, y se ha comprobado que la posición relativa del poste en la estructura es un parámetro de diseño muy importante que, hasta ahora, no se había tenido en cuenta en la literatura técnica dedicada a este tema (de hecho, en los trabajos de investigación más relevantes que se pueden encontrar en la literatura, el poste conductor se sitúa siempre en una posición centrada, de manera que los parámetros de diseño utilizados son el radio y la altura del poste exclusivamente). Gracias a estas aportaciones se han podido diseñar diferentes uniones con respuestas eléctricas óptimas, como por ejemplo codos rectos en plano $H$ y en plano $E$, uniones en $\mathrm{T}$ en plano $H$ y en plano $E$, uniones T mágica y uniones turnstile.

La siguiente parte de la tesis se ha dedicado al análisis riguroso de la excitación coaxial de dispositivos implementados en tecnología guiada mediante el método BI-RME 3D. Se han caracterizado dos tipos de excitación mediante sonda coaxial: la excitación coaxial clásica y la excitación en configuración colineal. Además, en ambos tipos de configuración se permite que la sonda coaxial pueda terminar en disco, posibilitándose así el diseño de transiciones de guía coaxial a guía rectangular con bajas pérdidas de retorno. La aportación fundamental de esta sección de la tesis consiste en la extensión de la teoría relativa al método BI-RME 3D para poder considerar puertos de acceso de sección transversal arbitraria, como es el caso de la guía coaxial. Asimismo, con el fin de evitar la dificultad matemática asociada a la integración analítica de las singularidades inherentes a las matrices que intervienen en el método BI-RME 3D, se ha presentado una estrategia que consiste en expresar las soluciones modales de la guía coaxial en términos de las funciones vectoriales modales de la guía rectangular a la que la sonda coaxial alimenta (en este caso, además, ha sido necesario recurrir a las integrales de acoplamiento modal derivadas en el capítulo dedicado al análisis de la unión planar entre una guía rectangular y una guía coaxial). Por otro lado, otra aportación importante consiste en el desarrollo de una herramienta de análisis de la excitación coaxial de dispositivos que, a diferencia de las que se presentan en la literatura técnica dedicada a este tema, posee una elevada eficiencia computacional. Además, gracias al carácter multimodal de la herramienta desarrollada, ésta podría ser integrada fácilmente en otras herramientas ya existentes destinadas al análisis de dispositivos de microondas más complejos, los cuales requieran un tratamiento multimodal de los diferentes elementos que integran el sistema. De hecho, en este capítulo se ha presentado el diseño de un filtro inductivo implementado en guía rectangular excitado mediante sonda coaxial, y ha quedado demostrada, tanto la eficiencia computacional de la herramienta desarrollada, como su enorme precisión.

Finalmente, en el último capítulo de la tesis doctoral se ha presentado una herramienta de simulación destinada al análisis y diseño de filtros y dispositivos en configuración comb-line 
implementados en guía rectangular. Este tipo de filtros, constituidos por la asociación de diferentes resonadores rectangulares cargados con postes conductores de geometría cilíndrica, son muy utilizados en diplexores o multiplexores para sistemas de comunicaciones espaciales. Además, la excitación de este tipo de filtros se suele realizar mediante sonda coaxial en configuración colineal. Por otro lado, es importante comentar que la empresa del sector aeroespacial Thales Alenia Space (Tres Cantos, Madrid) ha financiado el desarrollo de esta herramienta, ya que posee una elevada eficiencia computacional que permite minimizar enormemente el tiempo invertido en el proceso de análisis y diseño de este tipo de filtros. La herramienta implementada se basa en la técnica de la ecuación integral y en el método BI-RME 3D. En concreto, la técnica de la ecuación integral se usa para caracterizar las uniones planares (entre dos guías rectangulares o entre una guía rectangular y una guía circular) que surgen al caracterizar este tipo de filtros, mientras que el método BI-RME 3D se emplea en el análisis multimodal de los resonadores comb-line. La principal aportación de esta parte de la tesis consiste pues en el desarrollo de una herramienta de simulación muy versátil, de elevada precisión, y muy eficiente desde un punto de vista computacional. Así pues, las características más relevantes de la herramienta implementada son las siguientes:

- Permite el análisis de filtros y dispositivos con un número arbitrario de resonadores comb-line.

- Permite considerar un número arbitrario de puertos de acceso de diferentes tipos (entre ellos, puertos de acceso mediante sonda coaxial en configuración colineal terminada en disco).

- Los postes conductores de los resonadores comb-line pueden ser de geometría cilíndrica, así como tener forma de vaso o de champiñón.

- Los irises de acoplamiento que interconectan los diferentes resonadores comb-line pueden ser de sección transversal rectangular o circular.

- Se permite la introducción de tornillos metálicos de sintonía de geometría cilíndrica en los resonadores comb-line.

La herramienta implementada se ha verificado mediante diferentes ejemplos de validación, y se han presentado varios diseños que demuestran la precisión y la eficiencia de la herramienta de simulación desarrollada.

Por otro lado, es importante comentar que las publicaciones más relevantes relacionadas con la presente tesis doctoral se recogen en el Apéndice $\mathrm{H}$.

\subsection{Líneas Futuras de Investigación}

La realización de esta tesis doctoral deja abiertas una serie de líneas de investigación que se describen a continuación. En primer lugar, en relación con la sección de la tesis relacionada con el análisis de uniones compensadas en guía rectangular, sería interesante investigar si 
se puede mejorar la respuesta eléctrica de las uniones diseñadas añadiendo más postes conductores de geometría cilíndrica en dichas estructuras. Igualmente, sería conveniente estudiar si la consideración de postes conductores de geometría no cilíndrica (por ejemplo, postes en forma de vaso o en forma de champiñón) puede también optimizar los diseños presentados.

En segundo lugar, y en relación con la teoría presentada para caracterizar la excitación coaxial de dispositivos implementados en tecnología guiada, es importante recordar que, con el objetivo de evitar el cálculo de las singularidades que surgen al aplicar el método BI-RME 3D, se ha optado por una estrategia consistente en expresar las funciones vectoriales modales de la guía coaxial en términos de las soluciones modales de la guía rectangular de expansión a la que la sonda coaxial alimenta. Sin embargo, podría resultar muy interesante estudiar si se puede realizar la integración analítica (o numérica) de las singularidades mencionadas con el objetivo de mejorar aún más la eficiencia computacional de la herramienta implementada. Por otro lado, otra línea de investigación de gran interés consiste en integrar la herramienta de simulación relativa a la excitación coaxial de dispositivos en configuración colineal en otra herramienta, también basada en el método BI-RME 3D, que permita considerar en los resonadores postes de naturaleza dieléctrica. Finalmente, sería también de especial importancia integrar la herramienta desarrollada en otras herramientas ya existentes que permitan analizar y diseñar dispositivos de microondas más complejos (filtros, diplexores, multiplexores, etc.). De esta forma, desde el inicio del proceso de diseño, se podría tener en cuenta la introducción de la sonda coaxial de excitación, con todas las ventajas que ello supone.

Finalmente, con respecto a la sección de la tesis relacionada con el análisis de filtros en configuración comb-line, se abren también nuevas líneas de investigación. En primer lugar, tendría un enorme interés desarrollar una herramienta de diseño automatizado que se basara en la herramienta de análisis ya existente. En segundo lugar, es muy importante extender la teoría desarrollada con el fin de permitir que la sonda coaxial de excitación pueda contactar con el poste conductor del resonador comb-line. De esta manera, se podrían mejorar las pérdidas de retorno de este tipo de filtros y cambiar el tipo de acoplamiento en la excitación de dichos filtros. Finalmente, puede también resultar interesante generar una nueva herramienta que calcule el campo electromagnético en cada punto del filtro comb-line. De esta manera, dicha herramienta se podría emplear para predecir fenómenos de alta potencia (efecto multipactor y efecto corona) en este tipo de filtros. 


\section{Apéndice A}

\section{Matrices P y Q del Cálculo de los Modos de una Guía Coaxial usando el Método de los Momentos}

En el apartado 2.3.1 del capítulo 2 se han obtenido los modos $\mathrm{TM}^{z}$ y $\mathrm{TE}^{z}$ de una guía coaxial utilizando una técnica alternativa basada en el método de los momentos. En dicho cálculo, aparecen las denominadas matrices $\mathbf{P}$ y $\mathbf{Q}$ cuyos elementos se evalúan a continuación. Es importante resaltar que todos los resultados que se presentan en este apéndice se han calculado utilizando el paquete de cálculo simbólico que incorpora el software MATLAB.

\section{A.1. Modos TM ${ }^{2}$}

En primer lugar, se presentan los elementos de las matrices $\mathbf{P}^{\mathrm{TM}^{z}}$ y $\mathbf{Q}^{\mathrm{TM}^{z}}$ para el caso de los modos $\mathrm{TM}^{z}$ de una guía coaxial de radio externo $r_{1}$ y radio interno $r_{2}$. Las expresiones que definen estas matrices pueden encontrarse en las ecuaciones (2.83) y (2.84). Para el caso en que $r \neq t$ tendremos que ${ }^{1}$ :

$$
\begin{aligned}
P_{r, t}^{\mathrm{TM}^{z}}= & s^{2} \int_{r_{2}}^{r_{1}} \frac{1}{\rho} \sin \left(r \pi \frac{\rho-r_{2}}{r_{1}-r_{2}}\right) \sin \left(t \pi \frac{\rho-r_{2}}{r_{1}-r_{2}}\right) d \rho \\
& +\frac{r^{2}}{(r+t)^{2}(r-t)^{2}}\left[\frac{(-1)^{r-t}(r+t)^{2}-(-1)^{r+t}(r-t)^{2}}{2}-2 r t\right] \\
& +\frac{r}{(t+r)(t-r)}\left[\frac{(-1)^{t+r}(t-r)+(-1)^{t-r}(t+r)}{2}-t\right] \\
Q_{r, t}^{\mathrm{TM}^{z}}= & \frac{\left(r_{1}-r_{2}\right)^{2}}{(r+t)^{2}(r-t)^{2} \pi^{2}}\left[\frac{(-1)^{r-t}(r+t)^{2}-(-1)^{r+t}(r-t)^{2}}{2}-2 r t\right]
\end{aligned}
$$

\footnotetext{
${ }^{1}$ Hay que recordar que, en el caso de los modos $\mathrm{TM}^{z}, r$ y $t$ son diferentes de cero.
} 
Para el caso en que $r=t$ se obtiene:

$$
\begin{aligned}
P_{r, t}^{\mathrm{TM}^{z}} & =\frac{r_{1}+r_{2}}{r_{1}-r_{2}} \frac{(r \pi)^{2}}{4}+s^{2} \int_{r_{2}}^{r_{1}} \frac{1}{\rho} \sin \left(r \pi \frac{\rho-r_{2}}{r_{1}-r_{2}}\right) \sin \left(t \pi \frac{\rho-r_{2}}{r_{1}-r_{2}}\right) d \rho \\
Q_{r, t}^{\mathrm{TM}^{z}} & =\frac{1}{4}\left(r_{1}^{2}-r_{2}^{2}\right)
\end{aligned}
$$

La integral que aparece en las expresiones (A.1) y (A.3), la cual la denotaremos mediante $I_{P_{\mathrm{TM}}}(r, t)$, requiere un tratamiento numérico, tal y como se va a comprobar a continuación. En efecto, en caso de que $r \neq t$ se obtiene que:

$$
\begin{aligned}
I_{P_{\mathrm{TM}}}(r, t)= & \int_{r_{2}}^{r_{1}} \frac{1}{\rho} \sin \left(r \pi \frac{\rho-r_{2}}{r_{1}-r_{2}}\right) \sin \left(t \pi \frac{\rho-r_{2}}{r_{1}-r_{2}}\right) d \rho \\
= & \frac{1}{2} \cos \left(\pi r_{2} \frac{t-r}{r_{1}-r_{2}}\right)\left[\mathrm{Ci}\left(\pi r_{1} \frac{t-r}{r_{1}-r_{2}}\right)-\mathrm{Ci}\left(\pi r_{2} \frac{t-r}{r_{1}-r_{2}}\right)\right] \\
& +\frac{1}{2} \cos \left(\pi r_{2} \frac{t+r}{r_{1}-r_{2}}\right)\left[\mathrm{Ci}\left(\pi r_{2} \frac{t+r}{r_{1}-r_{2}}\right)-\mathrm{Ci}\left(\pi r_{1} \frac{t+r}{r_{1}-r_{2}}\right)\right] \\
& +\frac{1}{2} \sin \left(\pi r_{2} \frac{t-r}{r_{1}-r_{2}}\right)\left[\mathrm{Si}\left(\pi r_{1} \frac{t-r}{r_{1}-r_{2}}\right)-\mathrm{Si}\left(\pi r_{2} \frac{t-r}{r_{1}-r_{2}}\right)\right] \\
& +\frac{1}{2} \sin \left(\pi r_{2} \frac{t+r}{r_{1}-r_{2}}\right)\left[\mathrm{Si}\left(\pi r_{2} \frac{t+r}{r_{1}-r_{2}}\right)-\mathrm{Si}\left(\pi r_{1} \frac{t+r}{r_{1}-r_{2}}\right)\right]
\end{aligned}
$$

Si $r=t$, entonces:

$$
\begin{aligned}
I_{P^{\mathrm{TM}}}(r)= & \int_{r_{2}}^{r_{1}} \frac{1}{\rho} \sin ^{2}\left(r \pi \frac{\rho-r_{2}}{r_{1}-r_{2}}\right) d \rho \\
= & \frac{1}{2} \ln \left(\frac{r_{1}}{r_{2}}\right)+\frac{1}{2} \sin \left(\frac{2 \pi r r_{2}}{r_{1}-r_{2}}\right)\left[\operatorname{Si}\left(\frac{2 \pi r r_{2}}{r_{1}-r_{2}}\right)-\operatorname{Si}\left(\frac{2 \pi r r_{1}}{r_{1}-r_{2}}\right)\right] \\
& +\frac{1}{2} \cos \left(\frac{2 \pi r r_{2}}{r_{1}-r_{2}}\right)\left[\mathrm{Ci}\left(\frac{2 \pi r r_{2}}{r_{1}-r_{2}}\right)-\mathrm{Ci}\left(\frac{2 \pi r r_{1}}{r_{1}-r_{2}}\right)\right]
\end{aligned}
$$

En la expresión anterior, $\operatorname{Si}(x)$ y $\mathrm{Ci}(x)$ representan las denominadas integral seno e integral coseno, respectivamente, y sus definiciones son las siguientes:

$$
\begin{aligned}
\operatorname{Si}(x) & =\int_{0}^{x} \frac{\sin \tau}{\tau} d \tau \\
\operatorname{Ci}(x) & =\int_{\infty}^{x} \frac{\cos \tau}{\tau} d \tau
\end{aligned}
$$


Otras integrales de interés aparecen en el cálculo del factor de normalización asociado a los modos $\mathrm{TM}^{z}$, cuya expresión se recoge en (2.94). El cálculo de sus elementos se detalla a continuación. Sea:

$$
I^{\mathrm{TM}^{z}}(r, t)=\int_{r_{2}}^{r_{1}} \rho \cos \left(r \pi \frac{\rho-r_{2}}{r_{1}-r_{2}}\right) \cos \left(t \pi \frac{\rho-r_{2}}{r_{1}-r_{2}}\right) d \rho
$$

En caso de que $r \neq t$, se tiene que:

$$
I^{\mathrm{TM}^{z}}(r, t)=\frac{\left(r_{1}-r_{2}\right)^{2}}{\pi^{2}(r+t)^{2}(r-t)^{2}}\left[\frac{(-1)^{r-t}(r+t)^{2}+(-1)^{r+t}(r-t)^{2}}{2}-\left(r^{2}+t^{2}\right)\right]
$$

Por otro lado, si $r=t$ entonces:

$$
I^{\mathrm{TM}^{z}}(r)=\frac{1}{2 \epsilon_{r}}\left(r_{1}^{2}-r_{2}^{2}\right)
$$

En la expresión anterior, $\epsilon_{r}$ es la constante de Neumann definida en (C.6).

Finalmente, en el cálculo del factor de normalización deben resolverse también las siguientes integrales con respecto a la variable angular:

$$
\begin{aligned}
& \int_{0}^{2 \pi} \Phi_{(\xi), s}^{2}(\phi) d \phi= \begin{cases}2 \pi / \epsilon_{s} & \text { si }(\xi)=(c) \\
\pi\left(\epsilon_{s}-1\right) & \text { si }(\xi)=(s)\end{cases} \\
& \int_{0}^{2 \pi}\left(\Phi_{(\xi), s}^{\prime}(\phi)\right)^{2} d \phi= \begin{cases}\pi s^{2}\left(\epsilon_{s}-1\right) & \text { si }(\xi)=(c) \\
2 \pi s^{2} / \epsilon_{s} & \text { si }(\xi)=(s)\end{cases}
\end{aligned}
$$

\section{A.2. Modos TE}

A continuación, se calculan los elementos de las matrices $\mathbf{P}^{\mathrm{TE}}{ }^{z}$ y $\mathrm{Q}^{\mathrm{TE}}{ }^{z}$ para el caso de los modos $\mathrm{TE}^{z}$ de una guía coaxial de radio externo $r_{1}$ y radio interno $r_{2}$. Estas matrices se 
definieron en las expresiones (2.105) y (2.106). En caso de que $r \neq t$ se obtiene que ${ }^{2}$ :

$$
\begin{aligned}
P_{r, t}^{\mathrm{TE}}= & s^{2} \int_{r_{2}}^{r_{1}} \frac{1}{\rho} \cos \left(r \pi \frac{\rho-r_{2}}{r_{1}-r_{2}}\right) \cos \left(t \pi \frac{\rho-r_{2}}{r_{1}-r_{2}}\right) d \rho \\
& +\frac{r^{2}}{(r+t)^{2}(r-t)^{2}}\left[\frac{(-1)^{r-t}(r+t)^{2}+(-1)^{r+t}(r-t)^{2}}{2}-\left(r^{2}+t^{2}\right)\right] \\
& -\frac{r}{(r+t)(r-t)}\left[\frac{(-1)^{r+t}(r-t)+(-1)^{r-t}(r+t)}{2}-r\right] \\
Q_{r, t}^{\mathrm{TE}^{z}}= & \frac{\left(r_{1}-r_{2}\right)^{2}}{(r+t)^{2}(r-t)^{2} \pi^{2}}\left[\frac{(-1)^{r-t}(r+t)^{2}+(-1)^{r+t}(r-t)^{2}}{2}-\left(r^{2}+t^{2}\right)\right]
\end{aligned}
$$

expresión en la que se permite que $r$ o $t$ sean nulos. Para el caso en que $r=t$ se tiene que:

$$
\begin{aligned}
P_{r, t}^{\mathrm{TE}^{z}} & =\frac{(r \pi)^{2}}{2 \epsilon_{r}} \frac{r_{1}+r_{2}}{r_{1}-r_{2}}+s^{2} \int_{r_{2}}^{r_{1}} \frac{1}{\rho} \cos ^{2}\left(r \pi \frac{\rho-r_{2}}{r_{1}-r_{2}}\right) d \rho \\
Q_{r, t}^{\mathrm{TE} z} & =\frac{1}{2 \epsilon_{r}}\left(r_{1}^{2}-r_{2}^{2}\right)
\end{aligned}
$$

En estas expresiones, $\epsilon_{r}$ representa la constante de Neumann. Además, en caso de que $r=t=0$, la expresión (A.16) se reduce a la siguiente:

$$
\left.P_{r, t}^{\mathrm{TE}}\right|_{r=t=0}=s^{2} \ln \left(\frac{r_{1}}{r_{2}}\right)
$$

Las expresiones (A.14) y (A.16) se han dejado en función de una integral, que represen-

\footnotetext{
${ }^{2}$ En el caso de los modos $\mathrm{TE}^{z}$, los índices $r$ y $t$ pueden ser nulos.
} 
taremos mediante $I_{P \mathrm{TE}}(r, t)$, y que seguidamente calculamos. Para el caso en que $r \neq t$ :

$$
\begin{aligned}
I_{P^{\mathrm{TE}}}(r, t)= & \int_{r_{2}}^{r_{1}} \frac{1}{\rho} \cos \left(r \pi \frac{\rho-r_{2}}{r_{1}-r_{2}}\right) \cos \left(t \pi \frac{\rho-r_{2}}{r_{1}-r_{2}}\right) d \rho \\
= & \frac{1}{2} \cos \left(\pi r_{2} \frac{t-r}{r_{1}-r_{2}}\right)\left[\mathrm{Ci}\left(\pi r_{1} \frac{t-r}{r_{1}-r_{2}}\right)-\mathrm{Ci}\left(\pi r_{2} \frac{t-r}{r_{1}-r_{2}}\right)\right] \\
& +\frac{1}{2} \cos \left(\pi r_{2} \frac{t+r}{r_{1}-r_{2}}\right)\left[\mathrm{Ci}\left(\pi r_{1} \frac{t+r}{r_{1}-r_{2}}\right)-\mathrm{Ci}\left(\pi r_{2} \frac{t+r}{r_{1}-r_{2}}\right)\right] \\
& +\frac{1}{2} \sin \left(\pi r_{2} \frac{t-r}{r_{1}-r_{2}}\right)\left[\mathrm{Si}\left(\pi r_{1} \frac{t-r}{r_{1}-r_{2}}\right)-\mathrm{Si}\left(\pi r_{2} \frac{t-r}{r_{1}-r_{2}}\right)\right] \\
& +\frac{1}{2} \sin \left(\pi r_{2} \frac{t+r}{r_{1}-r_{2}}\right)\left[\operatorname{Si}\left(\pi r_{1} \frac{t+r}{r_{1}-r_{2}}\right)-\mathrm{Si}\left(\pi r_{2} \frac{t+r}{r_{1}-r_{2}}\right)\right]
\end{aligned}
$$

donde se permite que $r$ o $t$ sean nulos. En caso de que $r=t(\operatorname{con} r \neq 0)$, se obtiene que:

$$
\begin{aligned}
I_{P_{\mathrm{TE}}}(r)= & \int_{r_{2}}^{r_{1}} \frac{1}{\rho} \cos ^{2}\left(r \pi \frac{\rho-r_{2}}{r_{1}-r_{2}}\right) d \rho \\
= & \frac{1}{2} \ln \left(\frac{r_{1}}{r_{2}}\right)+\frac{1}{2} \sin \left(\frac{2 \pi r r_{2}}{r_{1}-r_{2}}\right)\left[\operatorname{Si}\left(\frac{2 \pi r r_{1}}{r_{1}-r_{2}}\right)-\operatorname{Si}\left(\frac{2 \pi r r_{2}}{r_{1}-r_{2}}\right)\right] \\
& +\frac{1}{2} \cos \left(\frac{2 \pi r r_{2}}{r_{1}-r_{2}}\right)\left[\mathrm{Ci}\left(\frac{2 \pi r r_{1}}{r_{1}-r_{2}}\right)-\mathrm{Ci}\left(\frac{2 \pi r r_{2}}{r_{1}-r_{2}}\right)\right]
\end{aligned}
$$

Si $r=t=0$, se tiene directamente que $I_{P^{\mathrm{TE}}}(r)=\ln \left(\frac{r_{1}}{r_{2}}\right)$.

Finalmente, en el cálculo del factor de normalización de los modos $\mathrm{TE}^{z}$, cuya expresión se define en (2.116), encontramos un nueva integral en la variable radial que se calcula a continuación. Sea:

$$
I^{\mathrm{TE}^{z}}(r, t)=\int_{r_{2}}^{r_{1}} \rho \sin \left(r \pi \frac{\rho-r_{2}}{r_{1}-r_{2}}\right) \sin \left(t \pi \frac{\rho-r_{2}}{r_{1}-r_{2}}\right) d \rho
$$

En el caso en que $r \neq t$ tendremos que:

$$
I^{\mathrm{TE}^{z}}(r, t)=\frac{\left(r_{1}-r_{2}\right)^{2}}{\pi^{2}(r+t)^{2}(r-t)^{2}}\left[\frac{(-1)^{r-t}(r+t)^{2}-(-1)^{r+t}(r-t)^{2}}{2}-2 r t\right]
$$

Si $r=t$ se obtiene:

$$
I^{\mathrm{TE}^{z}}(r)=\left(r_{1}^{2}-r_{2}^{2}\right) \frac{\epsilon_{r}-1}{4}
$$

siendo $\epsilon_{r}$ la constante de Neumann. Estas expresiones admiten que $r$ y $t$ sean nulos. 


\section{Apéndice B}

\section{Matrices P y Q del Cálculo de los Modos de una Guía Circular usando el Método de los Momentos}

En este apéndice se detallan los elementos de las matrices $\mathbf{P}$ y $\mathbf{Q}$ que se obtienen en la sección 2.4.1 tras utilizar el método de los momentos para la obtención de las funciones vectoriales modales de una guía de sección transversal circular. Es importante recordar que la expresión que adoptan estas matrices depende del valor que tome el índice modal angular $s$. Los cálculos presentados en este apéndice se han llevado a cabo utilizando el programa MATLAB.

\section{B.1. Modos $\mathbf{T M}^{z}$}

A continuación, se presentan los elementos de las matrices $\mathbf{P}^{\mathrm{TM}^{z}}$ y $\mathbf{Q}^{\mathrm{TM}^{z}}$ para el caso de los modos $\mathrm{TM}^{z}$ de una guía circular de radio $r_{1}$. La definición de estas matrices puede encontrarse en (2.156)-(2.157) para el caso en que $s \neq 0$, y en (2.166)-(2.167) para el caso en que $s=0$.

\section{Caso en que $s \neq 0$ :}

Si $r \neq t$, se tiene que ${ }^{1}$ :

$$
\begin{aligned}
P_{r, t}^{\mathrm{TM}^{z}}= & s^{2} \int_{0}^{r_{1}} \frac{1}{\rho} \sin \left(r \pi \frac{\rho}{r_{1}}\right) \sin \left(t \pi \frac{\rho}{r_{1}}\right) d \rho+\frac{r^{2}}{2}\left[\frac{(-1)^{r-t}-1}{(r-t)^{2}}-\frac{(-1)^{r+t}-1}{(r+t)^{2}}\right] \\
& +\frac{r}{2} \frac{(-1)^{r+t}(r-t)-(-1)^{r-t}(r+t)+2 t}{(r+t)(r-t)} \\
Q_{r, t}^{\mathrm{TM}^{z}}= & \frac{r_{1}^{2}}{2 \pi^{2}}\left[\frac{(-1)^{r-t}-1}{(r-t)^{2}}-\frac{(-1)^{r+t}-1}{(r+t)^{2}}\right]
\end{aligned}
$$

\footnotetext{
${ }^{1}$ En el caso de los modos $\mathrm{TM}^{z}$ con $s \neq 0$, los índices $r$ y $t$ no pueden ser nulos.
} 
En caso de que $r=t$ se obtiene:

$$
\begin{aligned}
P_{r, t}^{\mathrm{TM}^{z}} & =s^{2} \int_{0}^{r_{1}} \frac{1}{\rho} \sin ^{2}\left(r \pi \frac{\rho}{r_{1}}\right) d \rho+\left(\frac{r \pi}{2}\right)^{2} \\
Q_{r, t}^{\mathrm{TM}^{z}} & =\frac{r_{1}^{2}}{4}
\end{aligned}
$$

En las expresiones (B.1) y (B.3) aparecen unas integrales que se calculan seguidamente:

$$
\begin{gathered}
\int_{0}^{r_{1}} \frac{1}{\rho} \sin \left(r \pi \frac{\rho}{r_{1}}\right) \sin \left(t \pi \frac{\rho}{r_{1}}\right) d \rho=\frac{1}{2} \operatorname{Ci}(\pi(r-t))-\frac{1}{2} \operatorname{Ci}(\pi(r+t))+\frac{1}{2} \ln \left(\frac{r+t}{r-t}\right) \\
\int_{0}^{r_{1}} \frac{1}{\rho} \sin ^{2}\left(r \pi \frac{\rho}{r_{1}}\right) d \rho
\end{gathered}
$$

En estas expresiones, $\mathrm{Ci}(x)$ es la integral coseno definida en (A.8), y $\gamma$ representa la denominada constante de Euler cuyo valor aproximado es $\gamma \simeq 0,577215665$.

Por otro lado, en el cálculo del factor de normalización asociado a los modos $\mathrm{TM}^{z}$ con $s \neq 0$ encontramos la siguiente integral:

$$
I_{1}^{\mathrm{TM}^{z}}(r, t)=\int_{0}^{r_{1}} \rho \cos \left(r \pi \frac{\rho}{r_{1}}\right) \cos \left(t \pi \frac{\rho}{r_{1}}\right) d \rho
$$

En caso de que $r \neq t$ tendremos que:

$$
I_{1}^{\mathrm{TM}^{z}}(r, t)=\frac{r_{1}^{2}}{2 \pi^{2}}\left[\frac{(-1)^{r-t}-1}{(r-t)^{2}}+\frac{(-1)^{r+t}-1}{(r+t)^{2}}\right]
$$

Finalmente, si $r=t$ obtendremos:

$$
I_{1}^{\mathrm{TM}^{z}}(r, t)=\frac{r_{1}^{2}}{4}
$$

Caso en que $s=0$ :

En caso de que $r \neq t$, se concluye que ${ }^{2}$ :

$$
\begin{aligned}
P_{r, t_{(s=0)}}^{\mathrm{TM}^{z}}= & \frac{1}{2}\left(r+\frac{1}{2}\right)^{2}\left[\frac{(-1)^{r-t}-1}{(r-t)^{2}}+\frac{(-1)^{r+t+1}-1}{(r+t+1)^{2}}\right] \\
& +\left(r+\frac{1}{2}\right) \frac{(2 r+1)-(-1)^{r+t+1}(r-t)-(-1)^{r-t}(r+t+1)}{2(r+t+1)(r-t)} \\
Q_{r, t_{(s=0)}}^{\mathrm{TM}^{z}}= & \frac{r_{1}^{2}}{2 \pi^{2}}\left[\frac{(-1)^{r-t}-1}{(r-t)^{2}}+\frac{(-1)^{r+t+1}-1}{(r+t+1)^{2}}\right]
\end{aligned}
$$

\footnotetext{
${ }^{2}$ Para los modos $\mathrm{TM}^{z}$ con $s=0$, los índices $r$ y $t$ pueden ser nulos.
} 
Estas últimas expresiones son también válidas en el caso de que $r$ o $t$ sean nulos. Por otro lado, si $r=t$ (se permite que $r=t=0$ ) se tiene que:

$$
\begin{aligned}
P_{r, t_{(s=0)}}^{\mathrm{TM}^{z}} & =\frac{1}{4}\left[\pi^{2}\left(r+\frac{1}{2}\right)^{2}-1\right]+\frac{1}{2} \\
Q_{r, t_{(s=0)}}^{\mathrm{TM}^{z}} & =\frac{a^{2}}{\pi^{2}(2 r+1)^{2}}\left[\pi^{2}\left(r+\frac{1}{2}\right)^{2}-1\right]
\end{aligned}
$$

Finalmente, en la expresión del factor de normalización correspondiente a los modos $\mathrm{TM}^{z}$ con $s=0$ encontramos la siguiente integral:

$$
I_{2}^{\mathrm{TM}^{z}}(r, t)=\int_{0}^{r_{1}} \sin \left(\pi\left(r+\frac{1}{2}\right) \frac{\rho}{r_{1}}\right) \sin \left(\pi\left(t+\frac{1}{2}\right) \frac{\rho}{r_{1}}\right) \rho d \rho
$$

En caso de que $r \neq t$ obtenemos:

$I_{2}^{\mathrm{TM}^{z}}(r, t)=r_{1}^{2} \frac{(-1)^{t-r}\left[(r+t)^{2}+2(r+t)+1\right]-(-1)^{r+t+1}(t-r)^{2}-(4 r t+2(r+t)+1)}{2 \pi^{2}(r-t)^{2}(r+t+1)^{2}}$

Si $r=t$ se deduce que:

$$
I_{2}^{\mathrm{TM}^{z}}(r)=\frac{r_{1}^{2}}{4 \pi^{2}} \frac{\pi^{2}\left(r^{2}+r+\frac{1}{4}\right)+1}{r^{2}+r+\frac{1}{4}}
$$

En estas expresiones relativas a la integral $I_{2}^{\mathrm{TM}^{z}}(r, t)$ se permite que los índices $r$ y $t$ sean nulos.

\section{B.2. Modos TE}

Seguidamente, se proporcionan los elementos de las matrices $\mathbf{P}^{\mathrm{TE}}$ y $\mathbf{Q}^{\mathrm{TE}^{z}}$ definidas en las expresiones (2.180)-(2.181) para el caso en el que el índice angular $s$ es no nulo, y en las expresiones (2.188)-(2.189) para el caso en el que $s=0$. 
Caso en que $s \neq 0$ :

En este caso, los índices $r$ y $t$ pueden ser nulos. Así, si $r \neq t$ entonces:

$$
\begin{aligned}
P_{r, t}^{\mathrm{TE}^{z}}= & s^{2} \int_{0}^{r_{1}} \frac{1}{\rho} \sin \left(\pi\left(r+\frac{1}{2}\right) \frac{\rho}{r_{1}}\right) \sin \left(\pi\left(t+\frac{1}{2}\right) \frac{\rho}{r_{1}}\right) d \rho \\
& +\left(r+\frac{1}{2}\right)^{2} \frac{(-1)^{t-r}\left[(r+t)^{2}+2(r+t)+1\right]-(-1)^{r+t+1}(t-r)^{2}}{2(t-r)^{2}(t+r+1)^{2}} \\
& -\left(r+\frac{1}{2}\right)^{2} \frac{4 r t+2(r+t)+1}{2(t-r)^{2}(t+r+1)^{2}} \\
& +\left(r+\frac{1}{2}\right) \frac{(-1)^{r+t+1}(t-r)+(-1)^{t-r}(t+r+1)-(2 t+1)}{2(t-r)(t+r+1)} \\
Q_{r, t}^{\mathrm{TE}^{z}}= & r_{1}^{2} \frac{(-1)^{t-r}\left[(r+t)^{2}+2(r+t)+1\right]-(-1)^{r+t+1}(t-r)^{2}}{2 \pi^{2}(t-r)^{2}(t+r+1)^{2}} \\
& -r_{1}^{2} \frac{4 r t+2(r+t)+1}{2 \pi^{2}(t-r)^{2}(t+r+1)^{2}}
\end{aligned}
$$

En estas expresiones se incluyen los casos $r=0 \mathrm{y} t=0$. Por otra parte, si $r=t$ tendremos que:

$$
\begin{aligned}
P_{r, t}^{\mathrm{TE}^{z}} & =s^{2} \int_{0}^{r_{1}} \frac{1}{\rho} \sin ^{2}\left(\pi\left(r+\frac{1}{2}\right) \frac{\rho}{r_{1}}\right) d \rho+\left(r+\frac{1}{2}\right)^{2} \frac{\pi^{2}\left(r^{2}+r+\frac{1}{4}\right)+1}{(2 r+1)^{2}}-\frac{1}{2} \\
Q_{r, t}^{\mathrm{TE}^{z}} & =r_{1}^{2} \frac{\pi^{2}\left(r^{2}+r+\frac{1}{4}\right)+1}{\pi^{2}(2 r+1)^{2}}
\end{aligned}
$$

Las expresiones (B.19)-(B.20) incluyen el caso en que $r=0$.

Por otro lado, conviene observar que las expresiones (B.17) y (B.19) se han dejado en función de la siguiente integral ${ }^{3}$ :

$$
I_{1}^{\mathrm{TE}^{z}}(r, t)=\int_{0}^{r_{1}} \frac{1}{\rho} \sin \left(\pi\left(r+\frac{1}{2}\right) \frac{\rho}{r_{1}}\right) \sin \left(\pi\left(t+\frac{1}{2}\right) \frac{\rho}{r_{1}}\right) d \rho
$$

Así, en caso de que $r \neq t$ se tiene que:

$$
I_{1}^{\mathrm{TE}^{z}}(r, t)=\frac{1}{2} \mathrm{Ci}[\pi(r-t)]-\frac{1}{2} \mathrm{Ci}[\pi(r+t+1)]+\frac{1}{2} \ln \left(\frac{r+t+1}{r-t}\right)
$$

\footnotetext{
${ }^{3}$ La integral presente en (B.19) puede obtenerse a partir de la integral definida en (B.21) haciendo $r=t$.
} 
Por otro lado, si $r=t$ obtendremos:

$$
I_{1}^{\mathrm{TE}^{z}}(r)=\frac{1}{2} \gamma+\frac{1}{2} \ln 2+\frac{1}{2} \ln \left[\pi\left(r+\frac{1}{2}\right)\right]-\frac{1}{2} \mathrm{Ci}\left[2 \pi\left(r+\frac{1}{2}\right)\right]
$$

en donde $\gamma$ es la constante de Euler. Estas últimas expresiones admiten que los índices $r$ y $t$ sean nulos.

Finalmente, en el cálculo del factor de normalización asociado a los modos $\mathrm{TE}^{z}$ con $s \neq 0$ encontramos la siguiente integral:

$$
I_{2}^{\mathrm{TE}^{z}}(r, t)=\int_{0}^{r_{1}} \rho \cos \left(\pi\left(r+\frac{1}{2}\right) \frac{\rho}{r_{1}}\right) \cos \left(\pi\left(t+\frac{1}{2}\right) \frac{\rho}{r_{1}}\right) d \rho
$$

Así, cuando $r \neq t$ se obtiene que:

$$
I_{2}^{\mathrm{TE}^{z}}(r, t)=\frac{r_{1}^{2}}{2 \pi^{2}}\left[\frac{(-1)^{r-t}-1}{(r-t)^{2}}+\frac{(-1)^{r+t+1}-1}{(r+t+1)^{2}}\right]
$$

Por último, si $r=t$ se llega a que:

$$
I_{2}^{\mathrm{TE}^{z}}(r)=\frac{r_{1}^{2}}{\pi^{2}(2 r+1)^{2}}\left[\pi^{2}\left(r+\frac{1}{2}\right)^{2}-1\right]
$$

En las expresiones (B.25) y (B.26) se permite que $r$ y $t$ sean nulos.

Caso en que $s=0$ :

Para el caso en que $r \neq t$ se obtiene $e^{4}$

$$
\begin{aligned}
P_{r, t_{(s=0)}}^{\mathrm{TE}^{z}} & =\frac{r^{2}}{2}\left[\frac{(-1)^{r-t}-1}{(r-t)^{2}}+\frac{(-1)^{r+t}-1}{(r+t)^{2}}\right]-\frac{r}{2} \frac{(-1)^{t+r}(t-r)-(-1)^{t-r}(t+r)+2 r}{(t+r)(t-r)} \\
Q_{r, t_{(s=0)}}^{\mathrm{TE}^{z}} & =\frac{r_{1}^{2}}{2 \pi^{2}}\left[\frac{(-1)^{r-t}-1}{(r-t)^{2}}+\frac{(-1)^{r+t}-1}{(r+t)^{2}}\right]
\end{aligned}
$$

Por otro lado, si $r=t$ :

$$
\begin{aligned}
P_{r, t_{(s=0)}}^{\mathrm{TE}^{z}} & =\left(\frac{\pi r}{2}\right)^{2} \\
Q_{r, t_{(s=0)}}^{\mathrm{TE} z} & =\frac{r_{1}^{2}}{\alpha}
\end{aligned}
$$

${ }^{4}$ Los índices $r$ y $t$ pueden ser nulos en este caso. 
en donde $\alpha=4$ si $r \neq 0$, y $\alpha=2$ si $r=0$. Todas estas expresiones incluyen los casos en que $r=0$ o $t=0$.

Por otra parte, la expresión del factor de normalización de los modos $\mathrm{TE}^{z}$ para el caso $s=0$ se ha dejado en función de la siguiente integral:

$$
I_{3}^{\mathrm{TE}^{z}}(r, t)=\int_{0}^{r_{1}} \rho \sin \left(r \pi \frac{\rho}{r_{1}}\right) \sin \left(t \pi \frac{\rho}{r_{1}}\right) d \rho
$$

Para el caso en que $r \neq t$ se tiene que:

$$
I_{3}^{\mathrm{TE}^{z}}(r, t)=\frac{r_{1}^{2}}{2 \pi^{2}}\left[\frac{(-1)^{r-t}-1}{(r-t)^{2}}-\frac{(-1)^{r+t}-1}{(r+t)^{2}}\right]
$$

En caso de que $r=t$ entonces:

$$
I_{3}^{\mathrm{TE}^{z}}(r, t)=\frac{r_{1}^{2}}{4}
$$

Observe que si $r=0$ o $t=0$, entonces $I_{3}^{\mathrm{TE}^{z}}(r, t)=0$. 


\section{Apéndice C}

\section{Análisis Modal de Guías y Cavidades Rectangulares}

\section{C.1. Funciones Vectoriales Modales de Campo Magnético de una Guía Rectangular}

Sea una guía de ondas de sección transversal rectangular de dimensiones $l_{x_{\xi}} \times l_{y_{\xi}} \mathrm{y}$ sean $\left(x_{\xi}, y_{\xi}\right)$ las coordenadas transversales. Según [35], la función vectorial modal de campo magnético asociada a la $n$-ésima solución modal en dicha guía puede expresarse de manera general como sigue:

$$
\mathbf{h}_{n}^{(\xi)}(\mathbf{r})=\mathcal{N}_{x_{\xi}, n} F_{x_{\xi}, n}\left(x_{\xi}, y_{\xi}\right) \hat{\mathbf{x}}_{\xi}+\mathcal{N}_{y_{\xi}, n} F_{y_{\xi}, n}\left(x_{\xi}, y_{\xi}\right) \hat{\mathbf{y}}_{\xi}
$$

En la ecuación anterior, $\mathcal{N}_{x_{\xi}, n}$ y $\mathcal{N}_{y_{\xi}, n}$ representan unos factores de normalización cuyas expresiones deben particularizarse para los casos $\mathrm{TE}^{z_{\xi}} \mathrm{y} \mathrm{TM}^{z_{\xi} 1}[35]$ :

$$
\begin{aligned}
\mathcal{N}_{x_{\xi}, n}^{\mathrm{TE}} & =\frac{k_{x_{\xi}, n}}{k_{t, n}^{(\xi)}} \sqrt{\frac{\epsilon_{n_{x_{\xi}}} \epsilon_{n_{y_{\xi}}}}{l_{x_{\xi}} l_{y_{\xi}}}} \\
\mathcal{N}_{y_{\xi}, n}^{\mathrm{TE}} & =\frac{k_{y_{\xi}, n}}{k_{t, n}^{(\xi)}} \sqrt{\frac{\epsilon_{n_{x_{\xi}}} \epsilon_{n_{y_{\xi}}}}{l_{x_{\xi}} l_{y_{\xi}}}} \\
\mathcal{N}_{x_{\xi}, n}^{\mathrm{TM}} & =2 \frac{k_{y_{\xi}, n}}{k_{t, n}^{(\xi)}} \frac{1}{\sqrt{l_{x_{\xi}} l_{y_{\xi}}}} \\
\mathcal{N}_{y_{\xi}, n}^{\mathrm{TM}} & =-2 \frac{k_{x_{\xi}, n}}{k_{t, n}^{(\xi)}} \frac{1}{\sqrt{l_{x_{\xi}} l_{y_{\xi}}}}
\end{aligned}
$$

\footnotetext{
${ }^{1}$ De aquí en adelante, por simplicidad en la notación, se omitirá el superíndice $z_{\xi}$ al designar los modos $\mathrm{TE}^{z_{\xi}}$ y $\mathrm{TM}^{z_{\xi}}$.
} 
donde $\left(n_{x_{\xi}}, n_{y_{\xi}}\right)$ es la pareja de índices modales de la $n$-ésima solución modal relativa a las coordenadas $\left(x_{\xi}, y_{\xi}\right)$, respectivamente; $k_{x_{\xi}, n}=n_{x_{\xi}} \pi / l_{x_{\xi}}$ es el número de onda relativo a la coordenada $x_{\xi} ; k_{y_{\xi}, n}=n_{y_{\xi}} \pi / l_{y_{\xi}}$ es el número de onda relativo a la coordenada $y_{\xi}$; $\left(k_{t, n}^{(\xi)}\right)^{2}=k_{x_{\xi}, n}^{2}+k_{y_{\xi}, n}^{2}$ es el cuadrado del número de onda de corte del $n$-ésimo modo de la guía; y $\epsilon_{n}$ es el denominado factor de Neumann que se define como sigue:

$$
\epsilon_{n}=\left\{\begin{array}{l}
1, \text { si } n=0 \\
2, \text { si } n \neq 0
\end{array}\right.
$$

Por otro lado, las variaciones funcionales $F_{x_{\xi}, n}\left(x_{\xi}, y_{\xi}\right)$ y $F_{y_{\xi}, n}\left(x_{\xi}, y_{\xi}\right)$ que aparecen en (C.1) se definen del siguiente modo [35]:

$$
\begin{aligned}
& F_{x_{\xi}, n}\left(x_{\xi}, y_{\xi}\right)=\sin \left(k_{x_{\xi}, n} x_{\xi}\right) \cos \left(k_{y_{\xi}, n} y_{\xi}\right) \\
& F_{y_{\xi}, n}\left(x_{\xi}, y_{\xi}\right)=\cos \left(k_{x_{\xi}, n} x_{\xi}\right) \sin \left(k_{y_{\xi}, n} y_{\xi}\right)
\end{aligned}
$$

A continuación, estamos interesados en calcular la divergencia de las funciones vectoriales modales de campo magnético de tipo TE y de tipo TM. El campo magnético de tipo TE puede calcularse utilizando la siguiente relación:

$$
\mathbf{h}_{n}^{(\xi) \mathrm{TE}}(\mathbf{r})=\hat{\mathbf{z}_{\xi}} \times\left(\hat{\mathbf{z}_{\xi}} \times \frac{1}{k_{t, n}^{(\xi)}} \nabla_{t} \phi_{n}^{(\xi) \mathrm{TE}}(\mathbf{r})\right)
$$

siendo $z_{\xi}$ la coordenada axial que define la dirección de propagación en la guía; $\nabla_{t}$ el operador gradiente calculado sobre las coordenadas transversales $\left(x_{\xi}, y_{\xi}\right)$, y $\phi_{n}^{(\xi) \mathrm{TE}}(\mathbf{r})$ la siguiente función potencial ${ }^{2}$ :

$$
\phi_{n}^{(\xi) \mathrm{TE}}(\mathbf{r})=\sqrt{\frac{\epsilon_{n_{x_{\xi}}} \epsilon_{n_{y_{\xi}}}}{l_{x_{\xi}} l_{y_{\xi}}}} \cos \left(k_{x_{\xi}, n} x_{\xi}\right) \cos \left(k_{y_{\xi}, n} y_{\xi}\right)
$$

Entonces, es sencillo concluir que ${ }^{3}$ :

$$
\begin{aligned}
\nabla \cdot \mathbf{h}_{n}^{(\xi) \mathrm{TE}}(\mathbf{r})= & \nabla \cdot\left(\hat{\mathbf{z}_{\xi}} \times\left(\hat{\mathbf{z}_{\xi}} \times \frac{1}{k_{t, n}^{(\xi)}} \nabla_{t} \phi_{n}^{(\xi) \mathrm{TE}}(\mathbf{r})\right)\right)=\nabla \cdot\left(\hat{\mathbf{z}_{\xi}} \cdot \frac{1}{k_{t, n}^{(\xi)}} \nabla_{t} \phi_{n}^{(\xi) \mathrm{TE}}(\mathbf{r})\right) \hat{\mathbf{z}_{\xi}} \\
& -\frac{1}{k_{t, n}^{(\xi)}} \nabla \cdot \nabla_{t} \phi_{n}^{(\xi) \mathrm{TE}}(\mathbf{r})=-\frac{1}{k_{t, n}^{(\xi)}} \nabla_{t}^{2} \phi_{n}^{(\xi) \mathrm{TE}}(\mathbf{r})=k_{t, n}^{(\xi)} \phi_{n}^{(\xi) \mathrm{TE}}(\mathbf{r})
\end{aligned}
$$

Para el caso de los modos TM vamos a comprobar que la divergencia es nula. En efecto:

$$
\mathbf{h}_{n}^{(\xi) \mathrm{TM}}(\mathbf{r})=-\hat{\mathbf{z}_{\xi}} \times \nabla_{t} \phi_{n}^{(\xi) \mathrm{TM}}(\mathbf{r})
$$

\footnotetext{
${ }^{2}$ La función potencial (C.10) satisface la ecuación de ondas escalar.

${ }^{3}$ En este desarrollo se ha utilizado la identidad vectorial: $\mathbf{A} \times(\mathbf{B} \times \mathbf{C})=(\mathbf{A} \cdot \mathbf{C}) \mathbf{B}-(\mathbf{A} \cdot \mathbf{B}) \mathbf{C}$.
} 
siendo $\phi_{n}^{(\xi) \mathrm{TM}}(\mathbf{r})$ una adecuada función potencial. Entonces ${ }^{4}$ :

$$
\begin{aligned}
& \nabla \cdot \mathbf{h}_{n}^{(\xi) \mathrm{TM}}(\mathbf{r})=-\nabla \cdot\left(\hat{\mathbf{z}_{\xi}} \times \nabla_{t} \phi_{n}^{(\xi) \mathrm{TM}}(\mathbf{r})\right)=\nabla \cdot\left(\nabla_{t} \phi_{n}^{(\xi) \mathrm{TM}}(\mathbf{r}) \times \hat{\mathbf{z}_{\xi}}\right) \\
& =\nabla \cdot\left(\nabla_{t} \times \hat{\mathbf{z}_{\xi}} \phi_{n}^{(\xi) \mathrm{TM}}(\mathbf{r})-\phi_{n}^{(\xi) \mathrm{TM}}(\mathbf{r}) \nabla \times \hat{\mathbf{z}_{\xi}}\right)=0
\end{aligned}
$$

\section{C.2. Funciones de Green de tipo Magnético de una Cavi- dad Rectangular}

Considérese una cavidad rectangular de dimensiones $l_{x_{\xi}} \times l_{y_{\xi}} \times l_{z_{\xi}}$. Sean $\left(x_{\xi}, y_{\xi}\right)$ una pareja de coordenadas transversales y sea $z_{\xi}$ la correspondiente coordenada axial. La función de Green escalar de tipo magnético de dicha cavidad puede calcularse de la siguiente manera [21]:

$$
\begin{aligned}
& g^{\mathrm{m}}\left(\mathbf{r}, \mathbf{r}^{\prime}\right)=\sum_{n_{x_{\xi}}, n_{y_{\xi}}, n_{z_{\xi}}=0}^{\infty} \frac{\epsilon_{n_{z_{\xi}}}}{\kappa^{2} l_{z_{\xi}}} \phi_{n_{x_{\xi}, n_{y_{\xi}}}}\left(x_{\xi}, y_{\xi}\right) \phi_{n_{x_{\xi}, n_{y_{\xi}}}}\left(x_{\xi}^{\prime}, y_{\xi}^{\prime}\right) \cos \left(k_{z_{\xi}} z_{\xi}\right) \cos \left(k_{z_{\xi}} z_{\xi}^{\prime}\right) \\
& n_{x_{\xi}}=n_{y_{\xi}}=n_{z_{\xi}}=0 \text { excluido }
\end{aligned}
$$

donde $\epsilon_{n}$ es el factor de Neumann introducido en (C.6) y además se ha definido:

$$
\begin{aligned}
\kappa^{2} & =k_{x_{\xi}}^{2}+k_{y_{\xi}}^{2}+k_{z_{\xi}}^{2} \\
k_{\vartheta_{\xi}} & =\frac{n_{\vartheta_{\xi}} \pi}{l_{\vartheta_{\xi}}}, \operatorname{con} \vartheta=(x, y, z) \\
\phi_{n_{x_{\xi}}, n_{y_{\xi}}}\left(x_{\xi}, y_{\xi}\right) & =\sqrt{\frac{\epsilon_{n_{x_{\xi}}} \epsilon_{n_{y_{\xi}}}}{l_{x_{\xi}} l_{y_{\xi}}}} \cos \left(k_{x_{\xi}} x_{\xi}\right) \cos \left(k_{y_{\xi}} y_{\xi}\right)
\end{aligned}
$$

siendo $\kappa$ el número de onda de resonancia de la cavidad rectangular. Por otro lado, es importante observar que en la expresión de la función de Green escalar interviene la función potencial utilizada en (C.9) para obtener los modos TE de una guía de sección transversal rectangular de dimensiones $l_{x_{\xi}} \times l_{y_{\xi}}{ }^{5}$.

Asimismo, la parte estática de la función de Green diádica de tipo magnético puede expresarse como [21]:

$$
\begin{aligned}
\overline{\mathbf{G}}_{0}^{\mathrm{F}}\left(\mathbf{r}, \mathbf{r}^{\prime}\right)= & \sum_{n_{x_{\xi}}, n_{y_{\xi}}=0}^{\infty} \sum_{n_{z_{\xi}}=1}^{\infty} \frac{\mathbf{H}^{\mathrm{TE}}\left(x_{\xi}, y_{\xi}, z_{\xi}\right) \mathbf{H}^{\mathrm{TE}}\left(x_{\xi}^{\prime}, y_{\xi}^{\prime}, z_{\xi}^{\prime}\right)}{\kappa^{2}} \\
& +\sum_{n_{x_{\xi}}, n_{y_{\xi}}=1}^{\infty} \sum_{n_{z_{\xi}}=0}^{\infty} \frac{\mathbf{H}^{\mathrm{TM}}\left(x_{\xi}, y_{\xi}, z_{\xi}\right) \mathbf{H}^{\mathrm{TM}}\left(x_{\xi}^{\prime}, y_{\xi}^{\prime}, z_{\xi}^{\prime}\right)}{\kappa^{2}}
\end{aligned}
$$

\footnotetext{
${ }^{4}$ Aquí se ha empleado la siguiente propiedad: $\nabla \psi \times \mathbf{A}=\nabla \times(\psi \mathbf{A})-\psi \nabla \times \mathbf{A}$.

${ }^{5}$ De hecho, obsérvese que $\phi\left(x_{\xi}, y_{\xi}\right)=\phi_{n}^{(\xi) \mathrm{TE}}\left(x_{\xi}, y_{\xi}\right)$.
} 
donde $\mathbf{H}^{\mathrm{TE}}(\mathbf{r})$ y $\mathbf{H}^{\mathrm{TM}}(\mathbf{r})$ representan las funciones vectoriales modales normalizadas de campo magnético de los modos TE y TM de una cavidad rectangular ${ }^{6}$, respectivamente, y sus expresiones explícitas son las siguientes [35]:

$$
\begin{aligned}
\mathbf{H}^{\mathrm{TE}}(\mathbf{r})= & \frac{1}{\kappa} \sqrt{\frac{2}{l_{z_{\xi}}}}\left[k_{z_{\xi}} \cos \left(k_{z_{\xi}} z_{\xi}\right) \mathbf{h}_{n_{x_{\xi}}, n_{y_{\xi}}}^{\mathrm{TE}}(\mathbf{r})\right. \\
& \left.-\sqrt{k_{x_{\xi}}^{2}+k_{y_{\xi}}^{2}} \phi_{n_{x_{\xi}}, n_{y_{\xi}}}\left(x_{\xi}, y_{\xi}\right) \sin \left(k_{z_{\xi}} z_{\xi}\right) \hat{\mathbf{z}}_{\xi}\right] \\
\mathbf{H}^{\mathrm{TM}}(\mathbf{r})= & \sqrt{\frac{\epsilon_{n_{z_{\xi}}}}{l_{z_{\xi}}}} \cos \left(k_{z_{\xi}} z_{\xi}\right) \mathbf{h}_{n_{x_{\xi}}, n_{y_{\xi}}}^{\mathrm{TM}}(\mathbf{r})
\end{aligned}
$$

Finalmente, el rotacional de la parte estática de la función de Green diádica de tipo magnético puede escribirse como [21]:

$$
\begin{aligned}
\nabla \times \overline{\mathbf{G}}_{0}^{\mathrm{F}}\left(\mathbf{r}, \mathbf{r}^{\prime}\right)= & \sum_{n_{x_{\xi}}, n_{y_{\xi}}=0}^{\infty} \sum_{n_{z_{\xi}}=1}^{\infty} \frac{\mathbf{E}^{\mathrm{TE}}\left(x_{\xi}, y_{\xi}, z_{\xi}\right) \mathbf{H}^{\mathrm{TE}}\left(x_{\xi}^{\prime}, y_{\xi}^{\prime}, z_{\xi}^{\prime}\right)}{\kappa} \\
& +\sum_{n_{x_{\xi}}, n_{y_{\xi}}=1}^{\infty} \sum_{n_{z_{\xi}}=0}^{\infty} \frac{\mathbf{E}^{\mathrm{TM}}\left(x_{\xi}, y_{\xi}, z_{\xi}\right) \mathbf{H}^{\mathrm{TM}}\left(x_{\xi}^{\prime}, y_{\xi}^{\prime}, z_{\xi}^{\prime}\right)}{\kappa}
\end{aligned}
$$

donde las funciones vectoriales modales normalizadas de campo eléctrico de un resonador rectangular son [35]:

$$
\begin{aligned}
\mathbf{E}^{\mathrm{TE}}(\mathbf{r})= & \sqrt{\frac{2}{l_{z_{\xi}}}}\left(\mathbf{h}_{n_{x_{\xi}}, n_{y_{\xi}}}^{\mathrm{TE}}(\mathbf{r}) \times \hat{\mathbf{z}}_{\xi}\right) \sin \left(k_{z_{\xi}} z_{\xi}\right) \\
\mathbf{E}^{\mathrm{TM}}(\mathbf{r})= & \frac{1}{\kappa} \sqrt{\frac{\epsilon_{n_{z_{\xi}}}}{l_{z_{\xi}}}}\left[\left(\mathbf{h}_{n_{x_{\xi}}, n_{y_{\xi}}}^{\mathrm{TM}}(\mathbf{r}) \times \hat{\mathbf{z}}_{\xi}\right) k_{z_{\xi}} \sin \left(k_{z_{\xi}} z_{\xi}\right)\right. \\
& \left.+\frac{2}{l_{x_{\xi}} l_{y_{\xi}}} \sqrt{k_{x_{\xi}}^{2}+k_{y_{\xi}}^{2}} \sin \left(k_{x_{\xi}} x_{\xi}\right) \sin \left(k_{y_{\xi}} y_{\xi}\right) \cos \left(k_{z_{\xi}} z_{\xi}\right) \hat{\mathbf{z}}_{\xi}\right]
\end{aligned}
$$

Es importante destacar que los campos eléctrico y magnético de un resonador rectangular de dimensiones $l_{x_{\xi}} \times l_{y_{\xi}} \times l_{z_{\xi}}$ se han expresado en términos de las funciones vectoriales modales $\mathbf{h}_{n}(\mathbf{r})$ de una guía rectangular de dimensiones $l_{x_{\xi}} \times l_{y_{\xi}}$, las cuales ya se definieron en (C.9) y (C.12).

\footnotetext{
${ }^{6}$ En el primer sumatorio doble de la expresión (C.18) referido a los modos de tipo TE, los subíndices $n_{x_{\xi}} \mathrm{y}$ $n_{y_{\xi}}$ no pueden ser cero simultáneamente.
} 


\section{Apéndice D}

\section{Series y Funciones Auxiliares utilizadas en el Análisis de Uniones Compensadas}

\section{D.1. Series utilizadas en el Análisis de Uniones Compensa- das}

En el cálculo de la matrices $G_{m, n}^{(\gamma, \xi)}, T_{m, n}^{(\gamma, \xi)}$ y $L_{m, n}^{(\xi)}$ que se lleva a cabo en el capítulo 3 intervienen un conjunto de series infinitas cuya solución se detalla a continuación [66]:

$$
\begin{aligned}
\sum_{n_{z}=0}^{\infty} \frac{\epsilon_{n_{z}} \cos \left(k_{z} z\right)}{k_{t}^{2}+k_{z}^{2}} & =\frac{l_{z}}{k_{t}} \frac{\cosh \left[k_{t}\left(l_{z}-z\right)\right]}{\sinh \left(k_{t} l_{z}\right)} \\
\sum_{n_{z}=0}^{\infty}(-1)^{n_{z}} \frac{\epsilon_{n_{z}} \cos \left(k_{z} z\right)}{k_{t}^{2}+k_{z}^{2}} & =\frac{l_{z}}{k_{t}} \frac{\cosh \left(k_{t} z\right)}{\sinh \left(k_{t} l_{z}\right)} \\
\sum_{n_{z}=0}^{\infty} \frac{k_{z} \sin \left(k_{z} z\right)}{k_{t}^{2}+k_{z}^{2}} & =\frac{l_{z}}{2} \frac{\sinh \left[k_{t}\left(l_{z}-z\right)\right]}{\sinh \left(k_{t} l_{z}\right)} \\
\sum_{n_{z}=0}^{\infty}(-1)^{n_{z}} \frac{k_{z} \sin \left(k_{z} z\right)}{k_{t}^{2}+k_{z}^{2}} & =-\frac{l_{z}}{2} \frac{\sinh \left(k_{t} z\right)}{\sinh \left(k_{t} l_{z}\right)} \\
\sum_{n_{z}=0}^{\infty} \frac{k_{z}^{2}}{\left(k_{t}^{2}+k_{z}^{2}\right)^{2}} & =\frac{l_{z}}{4 k_{t}}\left(\operatorname{coth}\left(k_{t} l_{z}\right)-\frac{k_{t} l_{z}}{\sinh ^{2}\left(k_{t} l_{z}\right)}\right) \\
\sum_{n_{z}=0}^{\infty}(-1)^{n_{z}} \frac{k_{z}^{2}}{\left(k_{t}^{2}+k_{z}^{2}\right)^{2}} & =\frac{l_{z}}{4 k_{t} \sinh \left(k_{t} l_{z}\right)}\left(1-\frac{k_{t} l_{z}}{\tanh \left(k_{t} l_{z}\right)}\right)
\end{aligned}
$$

En todas estas expresiones se tiene que $k_{z}=\frac{n_{z} \pi}{l_{z}}$. 


\section{D.2. Funciones Auxiliares utilizadas en el Análisis de Unio- nes Compensadas}

Las expresiones de las matrices $G_{m, n}^{(\gamma, \xi)}$ y $T_{m, n}^{(\gamma, \xi)}$ obtenidas en las ecuaciones (3.30) y (3.52)-(3.55) del capítulo 3 se han descrito en términos de las siguientes funciones auxiliares:

$$
\begin{aligned}
& \rho_{m, n}^{(\gamma, \xi)}=\left\{\begin{array}{l}
\sqrt{\epsilon_{m_{x_{\gamma}}} \epsilon_{n_{x_{\xi}}}}, \text { si } \gamma=1,2 \mathrm{y} \xi=3,4 \\
\sqrt{\epsilon_{m_{y_{\gamma}}} \epsilon_{n_{x_{\xi}}}}, \text { si } \gamma=1,2 \mathrm{y} \xi=5 \\
\sqrt{\epsilon_{m_{y_{\gamma}}} \epsilon_{n_{y_{\xi}}}}, \text { si } \gamma=3,4 \mathrm{y} \xi=5
\end{array}\right. \\
& \psi_{m, n}^{(\gamma, \xi)}=\left\{\begin{array}{l}
\left(-\nu_{\gamma}\right)^{n_{x_{\xi}}} \nu_{\xi}^{m_{x_{\gamma}}} \delta_{m_{y_{\gamma}}, n_{y_{\xi}}}, \text { si } \gamma=1,2 \mathrm{y} \xi=3,4 \\
\left(-\nu_{\gamma}\right)^{n_{x_{\xi}}}\left(-\nu_{\xi}\right)^{m_{y_{\gamma}}} \delta_{m_{x_{\gamma}}, n_{y_{\xi}}}, \operatorname{si} \gamma=1,2 \mathrm{y} \xi=5 \\
\nu_{\gamma}^{n_{y_{\xi}}}\left(-\nu_{\xi}\right)^{m_{y_{\gamma}}} \delta_{m_{x_{\gamma}}, n_{x_{\xi}}}, \text { si } \gamma=3,4 \mathrm{y} \xi=5
\end{array}\right. \\
& \chi_{m, n}^{(\gamma, \xi)}=\left\{\begin{array}{l}
k_{n_{y_{\xi}}}^{2} \frac{\epsilon_{n_{\xi}}\left(\epsilon_{n_{y_{\xi}}}-1\right)}{\epsilon_{m_{x_{\gamma}}}}, \text { si } \gamma=1,2 \mathrm{y} \xi=3,4 \\
k_{n_{y_{\xi}}}^{2} \frac{\epsilon_{y_{\xi}}\left(\epsilon_{n_{y_{\xi}}}-1\right)}{\epsilon_{m_{y_{\gamma}}}}, \text { si } \gamma=1,2 \mathrm{y} \xi=5 \\
k_{n_{x_{\xi}}}^{2} \frac{\epsilon_{n_{x_{\xi}}}\left(\epsilon_{n_{x_{\xi}}}-1\right)}{\epsilon_{n_{y_{\gamma}}}}, \text { si } \gamma=3,4 \mathrm{y} \xi=5
\end{array}\right. \\
& \varphi_{m, n}^{(\gamma, \xi)}=\left\{\begin{array}{l}
\epsilon_{m_{x_{\gamma}}}-1, \text { si } \gamma=1,2 \text { y } \xi=3,4 \\
\epsilon_{m_{y_{\gamma}}}-1, \text { si } \gamma=1,2,3,4 \text { y } \xi=5
\end{array}\right. \\
& \zeta_{m, n}^{(\gamma, \xi)}=\left\{\begin{array}{l}
k_{n_{y_{\xi}}} \sqrt{\epsilon_{n_{x_{\xi}}}}, \text { si } \gamma=1,2 \mathrm{y} \xi=3,4,5 \\
k_{n_{x_{\xi}} \sqrt{\epsilon_{n_{y_{\xi}}}},} \text { si } \gamma=3,4 \mathrm{y} \xi=5
\end{array}\right. \\
& \theta_{m, n}^{(\gamma, \xi)}=\left\{\begin{array}{l}
\sqrt{\epsilon_{m_{x \gamma}}}, \text { si } \gamma=1,2 \mathrm{y} \xi=3,4 \\
\sqrt{\epsilon_{m_{y \gamma}}}, \text { si } \gamma=1,2 \mathrm{y} \xi=5 \\
-\sqrt{\epsilon_{m_{y \gamma}}}, \text { si } \gamma=3,4 \text { y } \xi=5
\end{array}\right.
\end{aligned}
$$




$$
\begin{gathered}
\tau^{(\gamma, \xi)}=\left\{\begin{array}{l}
1, \text { si } \gamma=1,2 \mathrm{y} \xi=3,4 \\
-1, \text { si } \gamma=1,2,3,4 \mathrm{y} \xi=5
\end{array}\right. \\
\lambda_{m, n}^{(\gamma, \xi)}=\left\{\begin{array}{l}
k_{n_{x_{\xi}}}, \text { si } \gamma=1,2 \mathrm{y} \xi=3,4,5 \\
-k_{n_{y_{\xi}}}, \text { si } \gamma=3,4 \mathrm{y} \xi=5
\end{array}\right.
\end{gathered}
$$

En estas expresiones, $\epsilon_{n}$ representa el factor de Neumann definido en (C.6); $\delta_{m, n}$ es la delta de Kronecker, y $\nu_{\xi}$ se definió en (3.24). 
246 Series y Funciones Auxiliares utilizadas en el Análisis de Uniones Compensadas 


\section{Apéndice E}

\section{Conexión de Matrices de Impedancias y Admitancias Generalizadas}

El objetivo de este apéndice es la caracterización de la conexión de una matriz de admitancias generalizada (MAG) y de una matriz de impedancias generalizada (MIG) a través de un sistema de ecuaciones equivalente, que relacione las corrientes y tensiones modales en cada uno de los puertos de acceso de la unión sin necesidad de invertir ninguna de las dos matrices multimodales. Así pues, considérese la figura E.1. En dicha figura, $Y_{\mathrm{A}}^{(\delta, \gamma)}$ es una MAG de dos puertos asociada a cierta unión $A$, y $Z_{\mathrm{B}}^{(\delta, \gamma)}$ es una MIG de dos puertos que caracteriza a cierta unión $B(\delta, \gamma=1,2)$. En concreto, la unión $A$ procede de la caracterización de una unión de dos puertos a través del método BI-RME 3D desarrollado en el capítulo 3, mientras que la unión $B$ proviene de la caracterización de una unión planar de dos puertos a partir de la técnica de la ecuación integral presentada en el capítulo 2. Por otro lado, $N$ es el número de modos considerados en todos los puertos de la unión; $V_{A, i}^{(\xi)}$ e $I_{A, i}^{(\xi)}$ (con $i=1, \ldots, N$ y $\xi=1,2$ ) representan, respectivamente, los voltajes y corrientes modales equivalentes en el puerto $(\xi)$ de la unión $A ; V_{B, i}^{(\xi)}$ e $I_{B, i}^{(\xi)}$ (con $i=1, \ldots, N$ y $\xi=1,2$ ) son los voltajes y corrientes modales equivalentes en el puerto $(\xi)$ de la unión $B$, respectivamente; e $\hat{Y}_{B, i}^{(\xi)}(\operatorname{con} i=1, \ldots, N$ y $\xi=1,2)$ son las admitancias modales asintóticas correspondientes al puerto $(\xi)$ de la unión $B$ cuyo significado se detalla en el capítulo 2 .

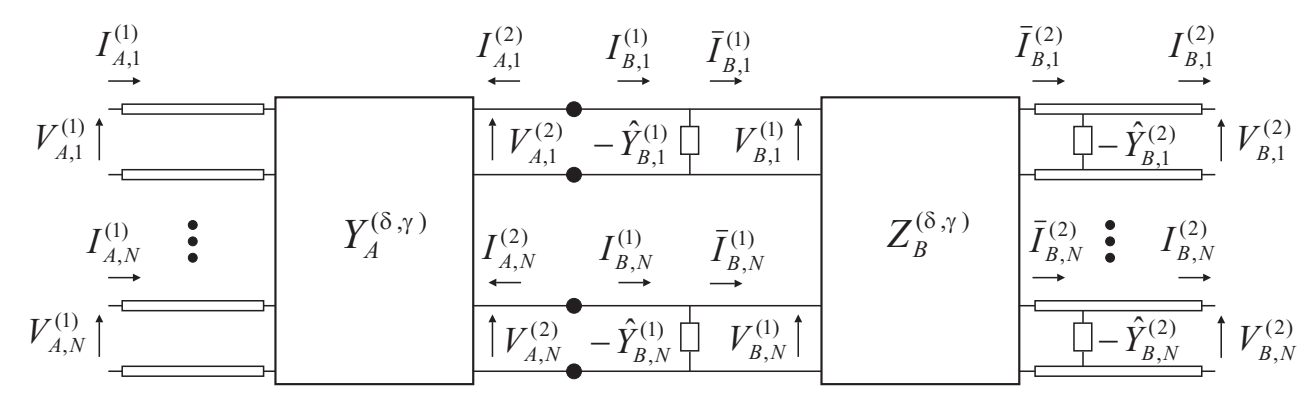

Figura E.1: Conexión de una matriz de admitancias generalizada y una matriz de impedancias generalizada. 
Las corrientes y voltajes modales de las uniones $A$ y $B$ se relacionan entre sí a partir de las correspondientes matrices multimodales ${ }^{1}$ :

$$
\begin{aligned}
& {\left[\begin{array}{c}
I_{A}^{(1)} \\
I_{A}^{(2)}
\end{array}\right]=\left[\begin{array}{cc}
Y_{A}^{(1,1)} & Y_{A}^{(1,2)} \\
Y_{A}^{(2,1)} & Y_{A}^{(2,2)}
\end{array}\right]\left[\begin{array}{c}
V_{A}^{(1)} \\
V_{A}^{(2)}
\end{array}\right]} \\
& {\left[\begin{array}{c}
V_{B}^{(1)} \\
V_{B}^{(2)}
\end{array}\right]=\left[\begin{array}{ll}
Z_{B}^{(1,1)} & Z_{B}^{(1,2)} \\
Z_{B}^{(2,1)} & Z_{B}^{(2,2)}
\end{array}\right]\left[\begin{array}{c}
\bar{I}_{B}^{(1)} \\
-\bar{I}_{B}^{(2)}
\end{array}\right]}
\end{aligned}
$$

Además, las diferentes corrientes definidas en la unión $B$ satisfacen las siguientes ecuaciones:

$$
\begin{aligned}
& \bar{I}_{B}^{(1)}=I_{B}^{(1)}+\hat{Y}_{B}^{(1)} V_{B}^{(1)} \\
& I_{B}^{(2)}=\bar{I}_{B}^{(2)}+\hat{Y}_{B}^{(2)} V_{B}^{(2)}
\end{aligned}
$$

donde $\hat{Y}_{B}^{(\xi)}(\xi=1,2)$ son matrices diagonales cuyos elementos son las admitancias modales asintóticas $\hat{Y}_{B, i}^{(\xi)}$, con $i=1,2, \ldots, N$.

En primer lugar, la resolución de la conexión matricial anterior requiere escoger un conjunto de incógnitas. Las incógnitas elegidas son: $V_{A}^{(1)}, \bar{I}_{B}^{(1)} \mathrm{y}-\bar{I}_{B}^{(2)}$. Para encontrar la primera ecuación del sistema final de ecuaciones partimos de la siguiente expresión extraída de (E.1):

$$
I_{A}^{(1)}=Y_{A}^{(1,1)} V_{A}^{(1)}+Y_{A}^{(1,2)} V_{A}^{(2)}
$$

Por otro lado, la continuidad de voltajes en la conexión de las uniones $A$ y $B$ impone que:

$$
V_{A}^{(2)}=V_{B}^{(1)}=Z_{B}^{(1,1)} \bar{I}_{B}^{(1)}+Z_{B}^{(1,2)}\left(-\bar{I}_{B}^{(2)}\right)
$$

donde se ha utilizado (E.2). Si ahora insertamos (E.6) en (E.5), deduciremos la primera ecuación del sistema de ecuaciones buscado:

$$
I_{A}^{(1)}=Y_{A}^{(1,1)} V_{A}^{(1)}+Y_{A}^{(1,2)} Z_{B}^{(1,1)} \bar{I}_{B}^{(1)}+Y_{A}^{(1,2)} Z_{B}^{(1,2)}\left(-\bar{I}_{B}^{(2)}\right)
$$

La obtención de la segunda ecuación del sistema parte de la siguiente expresión extraída de (E.1):

$$
I_{A}^{(2)}=Y_{A}^{(2,1)} V_{A}^{(1)}+Y_{A}^{(2,2)} V_{A}^{(2)}=Y_{A}^{(2,1)} V_{A}^{(1)}+Y_{A}^{(2,2)} Z_{B}^{(1,1)} \bar{I}_{B}^{(1)}+Y_{A}^{(2,2)} Z_{B}^{(1,2)}\left(-\bar{I}_{B}^{(2)}\right)
$$

donde hemos utilizado la relación (E.6). Por otro lado, introduciendo (E.6) en (E.3) deducimos que:

$$
I_{B}^{(1)}=\bar{I}_{B}^{(1)}-\hat{Y}_{B}^{(1)} V_{B}^{(1)}=\bar{I}_{B}^{(1)}-\hat{Y}_{B}^{(1)} Z_{B}^{(1,1)} \bar{I}_{B}^{(1)}-\hat{Y}_{B}^{(1)} Z_{B}^{(1,2)}\left(-\bar{I}_{B}^{(2)}\right)
$$

\footnotetext{
${ }^{1}$ Las matrices multimodales de las expresiones (E.1)-(E.2) son de dimensiones $2 N \times 2 N$.
} 
Además, la continuidad de corrientes en la conexión de las uniones $A$ y $B$ fuerza la siguiente condición:

$$
I_{A}^{(2)}=-I_{B}^{(1)} \Longrightarrow I_{A}^{(2)}+I_{B}^{(1)}=0
$$

con lo que recuperando ahora las expresiones (E.8) y (E.9) obtendremos la segunda ecuación buscada:

$$
Y_{A}^{(2,1)} V_{A}^{(1)}+\left[U+\left(Y_{A}^{(2,2)}-\hat{Y}_{B}^{(1)}\right) Z_{B}^{(1,1)}\right] \bar{I}_{B}^{(1)}+\left(Y_{A}^{(2,2)}-\hat{Y}_{B}^{(1)}\right) Z_{B}^{(1,2)}\left(-\bar{I}_{B}^{(2)}\right)=0
$$

siendo $U$ la matriz identidad de orden $N$.

Por último, la última ecuación del sistema se deduce directamente de (E.2):

$$
V_{B}^{(2)}=Z_{B}^{(2,1)} \bar{I}_{B}^{(1)}+Z_{B}^{(2,2)}\left(-\bar{I}_{B}^{(2)}\right)
$$

Así pues, el sistema matricial de ecuaciones que caracteriza la conexión se expresa de la siguiente manera:

$$
\left[\begin{array}{c}
I_{A}^{(1)} \\
0 \\
V_{B}^{(2)}
\end{array}\right]=\left[\begin{array}{ccc}
Y_{A}^{(1,1)} & Y_{A}^{(1,2)} Z_{B}^{(1,1)} & Y_{A}^{(1,2)} Z_{B}^{(1,2)} \\
Y_{A}^{(2,1)} & U+\left(Y_{A}^{(2,2)}-\hat{Y}_{B}^{(1)}\right) Z_{B}^{(1,1)} & \left(Y_{A}^{(2,2)}-\hat{Y}_{B}^{(1)}\right) Z_{B}^{(1,2)} \\
0 & Z_{B}^{(2,1)} & Z_{B}^{(2,2)}
\end{array}\right]\left[\begin{array}{c}
V_{A}^{(1)} \\
\bar{I}_{B}^{(1)} \\
-\bar{I}_{B}^{(2)}
\end{array}\right]
$$

La resolución del sistema anterior permitirá calcular fácilmente los parámetros de dispersión de la unión considerada [48].

Aunque la matriz de admitancias generalizada $Y_{\mathrm{A}}^{(\delta, \gamma)}$ estudiada en la conexión de la figura E.1 está asociada a una unión de dos puertos, en los ejemplos de aplicación que se presentan en el capítulo 3, la MAG que caracteriza a las diferentes estructuras bajo análisis puede llegar a ser de hasta cinco puertos. Así por ejemplo, el análisis eficiente de la unión turnstile compensada (ver apartado 3.3.4) requiere conectar una MAG de cinco puertos con una MIG de dos puertos. Por ello, es importante comentar que la teoría presentada en este apéndice puede ampliarse fácilmente para el caso en que la MAG tenga más de dos accesos. Así, a continuación se presenta como ejemplo el sistema matricial de ecuaciones que caracteriza la conexión eficiente de una MAG de cinco puertos y una MIG de dos puertos:

$$
\left[\begin{array}{c}
I_{A}^{(1)} \\
I_{A}^{(2)} \\
I_{A}^{(3)} \\
I_{A}^{(4)} \\
0 \\
V_{B}^{(2)}
\end{array}\right]=\mathbf{H} \cdot\left[\begin{array}{c}
V_{A}^{(1)} \\
V_{A}^{(2)} \\
V_{A}^{(3)} \\
V_{A}^{(4)} \\
\bar{I}_{B}^{(1)} \\
-\bar{I}_{B}^{(2)}
\end{array}\right]
$$


donde la matriz híbrida $\mathbf{H}$ se expresa de la siguiente manera:

$$
\mathbf{H}=\left[\begin{array}{cccccc}
Y_{A}^{(1,1)} & Y_{A}^{(1,2)} & Y_{A}^{(1,3)} & Y_{A}^{(1,4)} & Y_{A}^{(1,5)} Z_{B}^{(1,1)} & Y_{A}^{(1,5)} Z_{B}^{(1,2)} \\
Y_{A}^{(2,1)} & Y_{A}^{(2,2)} & Y_{A}^{(2,3)} & Y_{A}^{(2,4)} & Y_{A}^{(2,5)} Z_{B}^{(1,1)} & Y_{A}^{(2,5)} Z_{B}^{(1,2)} \\
Y_{A}^{(3,1)} & Y_{A}^{(3,2)} & Y_{A}^{(3,3)} & Y_{A}^{(3,4)} & Y_{A}^{(3,5)} Z_{B}^{(1,1)} & Y_{A}^{(3,5)} Z_{B}^{(1,2)} \\
Y_{A}^{(4,1)} & Y_{A}^{(4,2)} & Y_{A}^{(4,3)} & Y_{A}^{(4,4)} & Y_{A}^{(4,5)} Z_{B}^{(1,1)} & Y_{A}^{(4,5)} Z_{B}^{(1,2)} \\
Y_{A}^{(5,1)} & Y_{A}^{(5,2)} & Y_{A}^{(5,3)} & Y_{A}^{(5,4)} & U+\left(Y_{A}^{(5,5)}-\hat{Y}_{B}^{(1)}\right) Z_{B}^{(1,1)} & \left(Y_{A}^{(5,5)}-\hat{Y}_{B}^{(1)}\right) Z_{B}^{(1,2)} \\
0 & 0 & 0 & 0 & Z_{B}^{(2,1)} & Z_{B}^{(2,2)}
\end{array}\right]
$$




\section{Apéndice F}

\section{Series Auxiliares utilizadas en el Análisis de la Excitación Coaxial de Dispositivos}

El objetivo de este apéndice es el cálculo analítico de las series (4.32)-(4.33) obtenidas en la sección 4.2.2 del capítulo 4, dedicado al análisis riguroso de la excitación coaxial de dispositivos. Las series bajo estudio son las siguientes:

$$
\begin{aligned}
& S^{(1)}(z)=\sum_{r=1}^{\infty}(-1)^{r} \frac{k_{z}^{2}}{\left(k_{t}^{2}+k_{z}^{2}\right)^{2}} \cos \left(k_{z} z\right) \\
& S^{(2)}(z)=\sum_{r=1}^{\infty}(-1)^{r} \frac{k_{z}}{\left(k_{t}^{2}+k_{z}^{2}\right)^{2}} \sin \left(k_{z} z\right)
\end{aligned}
$$

donde $k_{z}=r \pi / c$. En primer lugar, se trabajará con la serie (F.1) y partiremos del siguiente resultado que se deduce en [66]:

$$
\gamma \frac{\cosh (\gamma z)}{\sinh (\gamma c)}=\frac{2}{c} \sum_{r=1}^{\infty}(-1)^{r} \frac{k_{z}^{2}}{k^{2}-k_{r}^{2}} \cos \left(k_{z} z\right)
$$

donde $k^{2}=\omega^{2} \mu \varepsilon, k_{z}=r \pi / c, k_{r}^{2}=k_{t}^{2}+k_{z}^{2} \mathrm{y} \gamma=\sqrt{k_{t}^{2}-k^{2}}$. Seguidamente, se extrae de cada miembro de la igualdad (F.3) el término correspondiente para $k=0$ (término estático o independiente de la frecuencia), con lo que se obtiene:

$$
\begin{aligned}
\gamma \frac{\cosh (\gamma z)}{\sinh (\gamma c)}-k_{t} \frac{\cosh \left(k_{t} z\right)}{\sinh \left(k_{t} z\right)} & =\frac{2}{c}\left[\sum_{r=1}^{\infty}(-1)^{r} \frac{k_{z}^{2}}{k^{2}-k_{r}^{2}} \cos \left(k_{z} z\right)-\sum_{r=1}^{\infty}(-1)^{r} \frac{k_{z}^{2}}{\left(-k_{r}^{2}\right)} \cos \left(k_{z} z\right)\right] \\
& =\frac{2}{c} k^{2} \sum_{r=1}^{\infty}(-1)^{r} \frac{k_{z}^{2}}{k_{r}^{2}\left(k^{2}-k_{r}^{2}\right)} \cos \left(k_{z} z\right)
\end{aligned}
$$

En consecuencia, de la expresión (F.4) se deduce:

$$
\sum_{r=1}^{\infty}(-1)^{r} \frac{k_{z}^{2}}{k_{r}^{2}\left(k^{2}-k_{r}^{2}\right)} \cos \left(k_{z} z\right)=\frac{c}{2 k^{2}}\left[\gamma \frac{\cosh (\gamma z)}{\sinh (\gamma c)}-k_{t} \frac{\cosh \left(k_{t} z\right)}{\sinh \left(k_{t} z\right)}\right]
$$


Así pues, podemos escribir ahora que:

$$
\lim _{k \rightarrow 0} \sum_{r=1}^{\infty}(-1)^{r} \frac{k_{z}^{2}}{k_{r}^{2}\left(k^{2}-k_{r}^{2}\right)} \cos \left(k_{z} z\right)=-\sum_{r=1}^{\infty}(-1)^{r} \frac{k_{z}^{2}}{k_{r}^{4}} \cos \left(k_{z} z\right)
$$

donde el miembro de la derecha de la igualdad anterior se corresponde con la expresión de la serie bajo estudio $S^{(1)}(z)$. Entonces, si ahora se introduce (F.5) en (F.6) se obtiene:

$$
\lim _{k \rightarrow 0} \sum_{r=1}^{\infty}(-1)^{r} \frac{k_{z}^{2}}{k_{r}^{2}\left(k^{2}-k_{r}^{2}\right)} \cos \left(k_{z} z\right)=\frac{c}{2} \lim _{k \rightarrow 0} \frac{f(k)}{k^{2}}
$$

donde hemos definido:

$$
f(k)=\sqrt{k_{t}^{2}-k^{2}} \frac{\cosh \left(z \sqrt{k_{t}^{2}-k^{2}}\right)}{\sinh \left(c \sqrt{k_{t}^{2}-k^{2}}\right)}-k_{t} \frac{\cosh \left(k_{t} z\right)}{\sinh \left(k_{t} c\right)}
$$

Dado que $f(k) \rightarrow 0$ cuando $k \rightarrow 0$, el cálculo del límite de la expresión (F.7) se lleva a cabo utilizando el teorema de L'Hôpital:

$$
\lim _{k \rightarrow 0} \frac{f(k)}{k^{2}}=\lim _{k \rightarrow 0} \frac{1}{2 k} \frac{d f(k)}{d k}=\frac{c}{2} \frac{\cosh \left(k_{t} z\right) \cosh \left(k_{t} c\right)}{\sinh ^{2}\left(k_{t} c\right)}-\frac{z}{2} \frac{\sinh \left(k_{t} z\right)}{\sinh \left(k_{t} c\right)}-\frac{1}{2 k_{t}} \frac{\cosh \left(k_{t} z\right)}{\sinh \left(k_{t} c\right)}
$$

Finalmente, de las expresiones (F.6) y (F.9) se concluye:

$$
\begin{aligned}
S^{(1)}(z) & =\sum_{r=1}^{\infty}(-1)^{r} \frac{k_{z}^{2}}{\left(k_{t}^{2}+k_{z}^{2}\right)^{2}} \cos \left(k_{z} z\right) \\
& =\frac{c}{4 \sinh \left(k_{t} c\right)}\left\{z \sinh \left(k_{t} z\right)+\left[\frac{1}{k_{t}}-c \operatorname{coth}\left(k_{t} c\right)\right] \cosh \left(k_{t} z\right)\right\}
\end{aligned}
$$

A continuación, se procede al cálculo analítico de la serie $S^{(2)}(z)$, cuya expresión se recoge en (F.2). Para ello, se parte del siguiente resultado extraído de [66]:

$$
\frac{\sinh (\gamma z)}{\sinh (\gamma c)}=\frac{2}{c} \sum_{r=1}^{\infty}(-1)^{r} \frac{k_{z}}{k^{2}-k_{r}^{2}} \sin \left(k_{z} z\right)
$$

donde la explicación de las diferentes variables es la misma que la que se ha dado para la expresión (F.3). Si se extrae de cada miembro de (F.11) el término correspondiente para $k=0$ (término estático o independiente de la frecuencia) se obtiene:

$$
\begin{aligned}
\frac{\sinh (\gamma z)}{\sinh (\gamma c)}-\frac{\sinh \left(k_{t} z\right)}{\sinh \left(k_{t} c\right)} & =\frac{2}{c}\left[\sum_{r=1}^{\infty}(-1)^{r} \frac{k_{z}}{k^{2}-k_{r}^{2}} \sin \left(k_{z} z\right)-\sum_{r=1}^{\infty}(-1)^{r} \frac{k_{z}}{\left(-k_{r}^{2}\right)} \sin \left(k_{z} z\right)\right] \\
& =\frac{2}{c} k^{2} \sum_{r=1}^{\infty}(-1)^{r} \frac{k_{z}}{k_{r}^{2}\left(k^{2}-k_{r}^{2}\right)} \sin \left(k_{z} z\right)
\end{aligned}
$$


de donde se deduce la siguiente expresión:

$$
\sum_{r=1}^{\infty}(-1)^{r} \frac{k_{z}}{k_{r}^{2}\left(k^{2}-k_{r}^{2}\right)} \sin \left(k_{z} z\right)=\frac{c}{2 k^{2}}\left[\frac{\sinh (\gamma z)}{\sinh (\gamma c)}-\frac{\sinh \left(k_{t} z\right)}{\sinh \left(k_{t} c\right)}\right]
$$

Si partimos de (F.13), podemos afirmar que:

$$
\lim _{k \rightarrow 0} \sum_{r=1}^{\infty}(-1)^{r} \frac{k_{z}}{k_{r}^{2}\left(k^{2}-k_{r}^{2}\right)} \sin \left(k_{z} z\right)=-\sum_{r=1}^{\infty}(-1)^{r} \frac{k_{z}}{k_{r}^{4}} \sin \left(k_{z} z\right)
$$

donde el miembro de la derecha de la igualdad anterior se corresponde con la serie $S^{(2)}(z)$ bajo estudio. Seguidamente, se introduce (F.13) en (F.14) para obtener:

$$
\lim _{k \rightarrow 0} \sum_{r=1}^{\infty}(-1)^{r} \frac{k_{z}}{k_{r}^{2}\left(k^{2}-k_{r}^{2}\right)} \sin \left(k_{z} z\right)=\frac{c}{2} \lim _{k \rightarrow 0} \frac{g(k)}{k^{2}}
$$

donde se ha definido:

$$
g(k)=\frac{\sinh \left(z \sqrt{k_{t}^{2}-k^{2}}\right)}{\sinh \left(c \sqrt{k_{t}^{2}-k^{2}}\right)}-\frac{\sinh \left(k_{t} z\right)}{\sinh \left(k_{t} c\right)}
$$

Puesto que $g(k) \rightarrow 0$ cuando $k \rightarrow 0$, podemos aplicar nuevamente el teorema de L'Hôpital para calcular el límite de la expresión (F.15). Entonces, se tiene que:

$$
\lim _{k \rightarrow 0} \frac{g(k)}{k^{2}}=\lim _{k \rightarrow 0} \frac{1}{2 k} \frac{d g(k)}{d k}=\frac{1}{2 k_{t}}\left[c \frac{\cosh \left(k_{t} c\right) \sinh \left(k_{t} z\right)}{\sinh ^{2}\left(k_{t} c\right)}-z \frac{\cosh \left(k_{t} z\right)}{\sinh \left(k_{t} c\right)}\right]
$$

Finalmente, de las expresiones (F.14) y (F.17) se obtiene el resultado buscado:

$$
\begin{aligned}
S^{(2)}(z) & =\sum_{r=1}^{\infty}(-1)^{r} \frac{k_{z}}{\left(k_{t}^{2}+k_{z}^{2}\right)^{2}} \sin \left(k_{z} z\right) \\
& =\frac{c}{4 k_{t} \sinh \left(k_{t} c\right)}\left[z \cosh \left(k_{t} z\right)-c \frac{\cosh \left(k_{t} c\right) \sinh \left(k_{t} z\right)}{\sinh \left(k_{t} c\right)}\right]
\end{aligned}
$$




\section{Apéndice G}

\section{Integrales Auxiliares utilizadas en el Análisis de la Excitación Coaxial de Dispositivos}

La sección 4.2.2 del capítulo 4 se dedica al cálculo de los elementos del bloque $T_{m n}^{(1,2)}$ para el caso de la excitación coaxial en configuración clásica. Del mismo modo, en la sección 4.3.2 del mismo capítulo, se calculan los elementos de los bloques $T_{m n}^{(1, \xi)}(\xi=1,2,3,4)$ de la matriz $\mathbf{T}$ para el caso de la excitación coaxial en configuración colineal. Las expresiones de todos estos elementos se han dejado en función de una serie de integrales de superficie en coordenadas cilíndricas que se han resuelto utilizando métodos numéricos (método de cuadratura de Gauss-Legendre). Por otra parte, es importante comentar que, en todas las integrales que se presentan seguidamente, los límites de integración inferior y superior para la variable radial $\rho$ son $r_{2}$ y $r_{1}$, respectivamente (radios interno y externo de la guía coaxial); y para la variable angular $\phi$ son 0 y $2 \pi$, respectivamente. Las diferentes integrales utilizadas se definen a continuación.

\section{G.1. Excitación Coaxial de Dispositivos en Configuración Clásica}

Caso $\mathrm{TEM}^{z}-\mathrm{TE}^{z}$ del bloque $T_{m n}^{(1,2)}$

$$
\begin{aligned}
I_{m_{\mathrm{TEM}}, n_{\mathrm{TE}}}^{(1)} & =\int_{\rho} \int_{\phi} \sin \left(k_{x, n}^{(2)}\left(d_{x}+\rho \sin \phi\right)\right) S_{n}^{(1)}(\rho, \phi) \cos \phi d \rho d \phi \\
I_{m_{\mathrm{TEM}}, n_{\mathrm{TE}}}^{(2)} & =\int_{\rho} \int_{\phi} \cos \left(k_{x, n}^{(2)}\left(d_{x}+\rho \sin \phi\right)\right) S_{n}^{(2)}(\rho, \phi) \sin \phi d \rho d \phi
\end{aligned}
$$


Caso $\mathrm{TE}^{z}-\mathrm{TE}^{z}$ del bloque $T_{m n}^{(1,2)}$

$$
\begin{aligned}
I_{m_{\mathrm{TE}}, n_{\mathrm{TE}}}^{(r),(1)} & =\int_{\rho} \int_{\phi} \rho \sin \left(k_{x, n}^{(2)}\left(d_{x}+\rho \sin \phi\right)\right) s_{r}(\rho) S_{n}^{(1)}(\rho, \phi) \Phi_{(\zeta), s}(\phi) \sin \phi d \rho d \phi \\
I_{m_{\mathrm{TE}}, n_{\mathrm{TE}}}^{(r),(2)} & =\int_{\rho} \int_{\phi} \rho \cos \left(k_{x, n}^{(2)}\left(d_{x}+\rho \sin \phi\right)\right) s_{r}(\rho) S_{n}^{(2)}(\rho, \phi) \Phi_{(\zeta), s}(\phi) \cos \phi d \rho d \phi \\
I_{m_{\mathrm{TE}}, n_{\mathrm{TE}}}^{(r),(3)} & =\int_{\rho} \int_{\phi} \sin \left(k_{x, n}^{(2)}\left(d_{x}+\rho \sin \phi\right)\right) c_{r}(\rho) S_{n}^{(1)}(\rho, \phi) \Phi_{(\zeta), s}^{\prime}(\phi) \cos \phi d \rho d \phi \\
I_{m_{\mathrm{TE}}, n_{\mathrm{TE}}}^{(r),(4)} & =\int_{\rho} \int_{\phi} \cos \left(k_{x, n}^{(2)}\left(d_{x}+\rho \sin \phi\right)\right) c_{r}(\rho) S_{n}^{(2)}(\rho, \phi) \Phi_{(\zeta), s}^{\prime}(\phi) \sin \phi d \rho d \phi
\end{aligned}
$$

$\underline{\text { Caso } \mathrm{TM}^{z}-\mathrm{TE}^{z} \text { del bloque } T_{m n}^{(1,2)}}$

$$
\begin{aligned}
I_{m_{\mathrm{TM}}, n_{\mathrm{TE}}}^{(r),(1)} & =\int_{\rho} \int_{\phi} \sin \left(k_{x, n}^{(2)}\left(d_{x}+\rho \sin \phi\right)\right) s_{r}(\rho) S_{n}^{(1)}(\rho, \phi) \Phi_{(\zeta), s}^{\prime}(\phi) \sin \phi d \rho d \phi \\
I_{m_{\mathrm{TM}}, n_{\mathrm{TE}}}^{(r),(2)} & =\int_{\rho} \int_{\phi} \cos \left(k_{x, n}^{(2)}\left(d_{x}+\rho \sin \phi\right)\right) s_{r}(\rho) S_{n}^{(2)}(\rho, \phi) \Phi_{(\zeta), s}^{\prime}(\phi) \cos \phi d \rho d \phi \\
I_{m_{\mathrm{TM}}, n_{\mathrm{TE}}}^{(r),(3)} & =\int_{\rho} \int_{\phi} \rho \sin \left(k_{x, n}^{(2)}\left(d_{x}+\rho \sin \phi\right)\right) c_{r}(\rho) S_{n}^{(1)}(\rho, \phi) \Phi_{(\zeta), s}(\phi) \cos \phi d \rho d \phi \\
I_{m_{\mathrm{TM}}, n_{\mathrm{TE}}}^{(r),(4)} & =\int_{\rho} \int_{\phi} \rho \cos \left(k_{x, n}^{(2)}\left(d_{x}+\rho \sin \phi\right)\right) c_{r}(\rho) S_{n}^{(2)}(\rho, \phi) \Phi_{(\zeta), s}(\phi) \sin \phi d \rho d \phi
\end{aligned}
$$

$\underline{\text { Caso } \mathrm{TEM}^{z}-\mathrm{TM}^{z} \text { del bloque } T_{m n}^{(1,2)}}$

$$
I_{m_{\mathrm{TEM}}, n_{\mathrm{TM}}}=\int_{\rho} \int_{\phi} \sin \left(k_{x, n}^{(2)}\left(d_{x}+\rho \sin \phi\right)\right) \cosh \left(k_{t, n}^{(2)}\left(d_{z}-\rho \cos \phi\right)\right) \cos \phi d \rho d \phi
$$


Caso $\mathrm{TE}^{z}-\mathrm{TM}^{z}$ del bloque $T_{m n}^{(1,2)}$

$$
\begin{aligned}
& I_{m_{\mathrm{TE}}, n_{\mathrm{TM}}}^{(r),(1)}=\int_{\rho} \int_{\phi} \rho \sin \left(k_{x, n}^{(2)}\left(d_{x}+\rho \sin \phi\right)\right) s_{r}(\rho) S_{n}(\rho, \phi) \Phi_{(\zeta), s}(\phi) \sin \phi d \rho d \phi \\
& I_{m_{\mathrm{TE}}, n_{\mathrm{TM}}}^{(r),(2)}=\int_{\rho} \int_{\phi} \sin \left(k_{x, n}^{(2)}\left(d_{x}+\rho \sin \phi\right)\right) c_{r}(\rho) S_{n}(\rho, \phi) \Phi_{(\zeta), s}^{\prime}(\phi) \cos \phi d \rho d \phi
\end{aligned}
$$

Caso $\mathrm{TM}^{z}-\mathrm{TM}^{z}$ del bloque $T_{m n}^{(1,2)}$

$$
\begin{aligned}
& I_{m_{\mathrm{TM}}, n_{\mathrm{TM}}}^{(r),(1)}=\int_{\rho} \int_{\phi} \sin \left(k_{x, n}^{(2)}\left(d_{x}+\rho \sin \phi\right)\right) s_{r}(\rho) S_{n}(\rho, \phi) \Phi_{(\zeta), s}^{\prime}(\phi) \sin \phi d \rho d \phi \\
& I_{m_{\mathrm{TM}}, n_{\mathrm{TM}}}^{(r),(2)}=\int_{\rho} \int_{\phi} \rho \sin \left(k_{x, n}^{(2)}\left(d_{x}+\rho \sin \phi\right)\right) c_{r}(\rho) S_{n}(\rho, \phi) \Phi_{(\zeta), s}(\phi) \cos \phi d \rho d \phi
\end{aligned}
$$

\section{G.2. Excitación Coaxial de Dispositivos en Configuración Colineal}

Caso $\mathrm{TEM}^{z}-\mathrm{TE}^{z}$ del bloque $T_{m n}^{(1,2)}$

$$
\begin{aligned}
I_{m_{\mathrm{TEM}}, n_{\mathrm{TE}}}^{(1,2)^{(a)}} & =\int_{\rho} \int_{\phi} \sin \left(k_{x, n}^{(2)}\left(d_{x}+\rho \cos \phi\right)\right) \cos \left(k_{y, n}^{(2)}\left(d_{y}+\rho \sin \phi\right)\right) \sin \phi d \rho d \phi \\
I_{m_{\mathrm{TEM},}, n_{\mathrm{TE}}}^{(1,2)^{(b)}} & =\int_{\rho} \int_{\phi} \cos \left(k_{x, n}^{(2)}\left(d_{x}+\rho \cos \phi\right)\right) \sin \left(k_{y, n}^{(2)}\left(d_{y}+\rho \sin \phi\right)\right) \cos \phi d \rho d \phi
\end{aligned}
$$

$\underline{\text { Caso } \mathrm{TE}^{z}-\mathrm{TE}^{z} \text { del bloque } T_{m n}^{(1,2)}}$

$I_{m_{\mathrm{TE}}, n_{\mathrm{TE}}}^{(r),(1,2)}=\int_{\rho} \int_{\phi} \rho \sin \left(k_{x, n}^{(2)}\left(d_{x}+\rho \cos \phi\right)\right) \cos \left(k_{y, n}^{(2)}\left(d_{y}+\rho \sin \phi\right)\right) s_{r}(\rho) \Phi_{(\zeta), s}(\phi) \cos \phi d \rho d \phi$

$I_{m_{\mathrm{TE}}, n_{\mathrm{TE}}}^{(r),(1,2)}=\int_{\rho} \int_{\phi} \sin \left(k_{x, n}^{(2)}\left(d_{x}+\rho \cos \phi\right)\right) \cos \left(k_{y, n}^{(2)}\left(d_{y}+\rho \sin \phi\right)\right) c_{r}(\rho) \Phi_{(\zeta), s}^{\prime}(\phi) \sin \phi d \rho d \phi$ 
$I_{m_{\mathrm{TE}}, n_{\mathrm{TE}}}^{(r),(1,2)^{(c)}}=\int_{\rho} \int_{\phi} \rho \cos \left(k_{x, n}^{(2)}\left(d_{x}+\rho \cos \phi\right)\right) \sin \left(k_{y, n}^{(2)}\left(d_{y}+\rho \sin \phi\right)\right) s_{r}(\rho) \Phi_{(\zeta), s}(\phi) \sin \phi d \rho d \phi$

$I_{m_{\mathrm{TE}}, n_{\mathrm{TE}}}^{(r),(1,)^{(d)}}=\int_{\rho} \int_{\phi} \cos \left(k_{x, n}^{(2)}\left(d_{x}+\rho \cos \phi\right)\right) \sin \left(k_{y, n}^{(2)}\left(d_{y}+\rho \sin \phi\right)\right) c_{r}(\rho) \Phi_{(\zeta), s}^{\prime}(\phi) \cos \phi d \rho d \phi$

Caso $\mathrm{TM}^{z}-\mathrm{TE}^{z}$ del bloque $T_{m n}^{(1,2)}$

$I_{m_{\mathrm{TM}}, n_{\mathrm{TE}}}^{(r),(1,2)^{(a)}}=\int_{\rho} \int_{\phi} \sin \left(k_{x, n}^{(2)}\left(d_{x}+\rho \cos \phi\right)\right) \cos \left(k_{y, n}^{(2)}\left(d_{y}+\rho \sin \phi\right)\right) s_{r}(\rho) \Phi_{(\zeta), s}^{\prime}(\phi) \cos \phi d \rho d \phi$

$I_{m_{\mathrm{TM}}, n_{\mathrm{TE}}}^{(r),(1,2)}=\int_{\rho} \int_{\phi} \rho \sin \left(k_{x, n}^{(2)}\left(d_{x}+\rho \cos \phi\right)\right) \cos \left(k_{y, n}^{(2)}\left(d_{y}+\rho \sin \phi\right)\right) c_{r}(\rho) \Phi_{(\zeta), s}(\phi) \sin \phi d \rho d \phi$

$I_{m_{\mathrm{TM}}, n_{\mathrm{TE}}}^{(r),(1,2)}=\int_{\rho} \int_{\phi} \cos \left(k_{x, n}^{(2)}\left(d_{x}+\rho \cos \phi\right)\right) \sin \left(k_{y, n}^{(2)}\left(d_{y}+\rho \sin \phi\right)\right) s_{r}(\rho) \Phi_{(\zeta), s}^{\prime}(\phi) \sin \phi d \rho d \phi$

$I_{m_{\mathrm{TM}}, n_{\mathrm{TE}}}^{(r),(1,2)^{(d)}}=\int_{\rho} \int_{\phi} \rho \cos \left(k_{x, n}^{(2)}\left(d_{x}+\rho \cos \phi\right)\right) \sin \left(k_{y, n}^{(2)}\left(d_{y}+\rho \sin \phi\right)\right) c_{r}(\rho) \Phi_{(\zeta), s}(\phi) \cos \phi d \rho d \phi$

Caso $\mathrm{TEM}^{z}-\mathrm{TE}^{z}$ del bloque $T_{m n}^{(1,3)}$

$$
\begin{aligned}
I_{m_{\mathrm{TEM}}, n_{\mathrm{TE}}}^{(1,3)^{(a)}} & =\int_{\rho} \int_{\phi} \sin \left(k_{y, n}^{(3)}\left(d_{y}+\rho \sin \phi\right)\right) S_{n}^{(3)}(\rho, \phi) \cos \phi d \rho d \phi \\
I_{m_{\mathrm{TEM},}, n_{\mathrm{TE}}}^{(1,3)^{(b)}} & =\int_{\rho} \int_{\phi} \cos \left(k_{y, n}^{(3)}\left(d_{y}+\rho \sin \phi\right)\right) S_{n}^{(4)}(\rho, \phi) \sin \phi d \rho d \phi
\end{aligned}
$$

Caso $\mathrm{TE}^{z}-\mathrm{TE}^{z}$ del bloque $T_{m n}^{(1,3)}$

$$
\begin{aligned}
& I_{m_{\mathrm{TE}}, n_{\mathrm{TE}}}^{(r),(1,)^{(a)}}=\int_{\rho} \int_{\phi} \rho \sin \left(k_{y, n}^{(3)}\left(d_{y}+\rho \sin \phi\right)\right) s_{r}(\rho) S_{n}^{(3)}(\rho, \phi) \Phi_{(\zeta), s}(\phi) \sin \phi d \rho d \phi \\
& I_{m_{\mathrm{TE}}, n_{\mathrm{TE}}}^{(r),(1,3)^{(b)}}=\int_{\rho} \int_{\phi} \sin \left(k_{y, n}^{(3)}\left(d_{y}+\rho \sin \phi\right)\right) c_{r}(\rho) S_{n}^{(3)}(\rho, \phi) \Phi_{(\zeta), s}^{\prime}(\phi) \cos \phi d \rho d \phi
\end{aligned}
$$




$$
\begin{aligned}
& I_{m_{\mathrm{TE}}, n_{\mathrm{TE}}}^{(r),(1,3)^{(c)}}=\int_{\rho} \int_{\phi} \rho \cos \left(k_{y, n}^{(3)}\left(d_{y}+\rho \sin \phi\right)\right) s_{r}(\rho) S_{n}^{(4)}(\rho, \phi) \Phi_{(\zeta), s}(\phi) \cos \phi d \rho d \phi \\
& I_{m_{\mathrm{TE}}, n_{\mathrm{TE}}}^{(r),(1,3)(d)}=\int_{\rho} \int_{\phi} \cos \left(k_{y, n}^{(3)}\left(d_{y}+\rho \sin \phi\right)\right) c_{r}(\rho) S_{n}^{(4)}(\rho, \phi) \Phi_{(\zeta), s}^{\prime}(\phi) \sin \phi d \rho d \phi
\end{aligned}
$$

$\underline{\text { Caso } \mathrm{TM}^{z}-\mathrm{TE}^{z} \text { del bloque } T_{m n}^{(1,3)}}$

$$
\begin{aligned}
& I_{m_{\mathrm{TM}}, n_{\mathrm{TE}}}^{(r),(1,3)(a)}=\int_{\rho} \int_{\phi} \sin \left(k_{y, n}^{(3)}\left(d_{y}+\rho \sin \phi\right)\right) s_{r}(\rho) S_{n}^{(3)}(\rho, \phi) \Phi_{(\zeta), s}^{\prime}(\phi) \sin \phi d \rho d \phi \\
& I_{m_{\mathrm{TM}}, n_{\mathrm{TE}}}^{(r),(1,3)^{(b)}}=\int_{\rho} \int_{\phi} \rho \sin \left(k_{y, n}^{(3)}\left(d_{y}+\rho \sin \phi\right)\right) c_{r}(\rho) S_{n}^{(3)}(\rho, \phi) \Phi_{(\zeta), s}(\phi) \cos \phi d \rho d \phi \\
& I_{m_{\mathrm{TM}}, n_{\mathrm{TE}}}^{(r),(1,3)(c)}=\int_{\rho} \int_{\phi} \cos \left(k_{y, n}^{(3)}\left(d_{y}+\rho \sin \phi\right)\right) s_{r}(\rho) S_{n}^{(4)}(\rho, \phi) \Phi_{(\zeta), s}^{\prime}(\phi) \cos \phi d \rho d \phi \\
& I_{m_{\mathrm{TM}}, n_{\mathrm{TE}}}^{(r),(1,3)^{(d)}}=\int_{\rho} \int_{\phi} \rho \cos \left(k_{y, n}^{(3)}\left(d_{y}+\rho \sin \phi\right)\right) c_{r}(\rho) S_{n}^{(4)}(\rho, \phi) \Phi_{(\zeta), s}(\phi) \sin \phi d \rho d \phi
\end{aligned}
$$

$\underline{\text { Caso } \mathrm{TEM}^{z}-\mathrm{TM}^{z} \text { del bloque } T_{m n}^{(1,3)}}$

$$
I_{m_{\mathrm{TEM}}, n_{\mathrm{TM}}}^{(1,3)}=\int_{\rho} \int_{\phi} \sin \left(k_{y, n}^{(3)}\left(d_{y}+\rho \sin \phi\right)\right) \cosh \left(k_{t, n}^{(3)}\left(a-d_{x}-\rho \cos \phi\right)\right) \cos \phi d \rho d \phi
$$

Caso $\mathrm{TE}^{z}-\mathrm{TM}^{z}$ del bloque $T_{m n}^{(1,3)}$

$$
\begin{aligned}
& I_{m_{\mathrm{TE}}, n_{\mathrm{TM}}}^{(r),(1,3)^{(a)}}=\int_{\rho} \int_{\phi} \rho \sin \left(k_{y, n}^{(3)}\left(d_{y}+\rho \sin \phi\right)\right) \cosh \left(k_{t, n}^{(3)}\left(a-d_{x}-\rho \cos \phi\right)\right) s_{r}(\rho) \Phi_{(\zeta), s}(\phi) \sin \phi d \rho d \phi \\
& I_{m_{\mathrm{TE}}, n_{\mathrm{TM}}}^{(r),(1,3)}=\int_{\rho} \int_{\phi} \sin \left(k_{y, n}^{(3)}\left(d_{y}+\rho \sin \phi\right)\right) \cosh \left(k_{t, n}^{(3)}\left(a-d_{x}-\rho \cos \phi\right)\right) c_{r}(\rho) \Phi_{(\zeta), s}^{\prime}(\phi) \cos \phi d \rho d \phi
\end{aligned}
$$


Caso $\mathrm{TM}^{z}-\mathrm{TM}^{z}$ del bloque $T_{m n}^{(1,3)}$

$I_{m_{\mathrm{TM}}, n_{\mathrm{TM}}}^{(r),(1,3)^{(a)}}=\int_{\rho} \int_{\phi} \sin \left(k_{y, n}^{(3)}\left(d_{y}+\rho \sin \phi\right)\right) \cosh \left(k_{t, n}^{(3)}\left(a-d_{x}-\rho \cos \phi\right)\right) s_{r}(\rho) \Phi_{(\zeta), s}^{\prime}(\phi) \sin \phi d \rho d \phi$

$I_{m_{\mathrm{TM}}, n_{\mathrm{TM}}}^{(r),(1,3)}=\int_{\rho} \int_{\phi} \rho \sin \left(k_{y, n}^{(3)}\left(d_{y}+\rho \sin \phi\right)\right) \cosh \left(k_{t, n}^{(3)}\left(a-d_{x}-\rho \cos \phi\right)\right) c_{r}(\rho) \Phi_{(\zeta), s}(\phi) \cos \phi d \rho d \phi$

Caso $\mathrm{TEM}^{z}-\mathrm{TE}^{z}$ del bloque $T_{m n}^{(1,4)}$

$$
\begin{aligned}
I_{m_{\mathrm{TEM},}, n_{\mathrm{TE}}}^{(1,4)(a)} & =\int_{\rho} \int_{\phi} \sin \left(k_{y, n}^{(4)}\left(d_{y}+\rho \sin \phi\right)\right) S_{n}^{(1)}(\rho, \phi) \cos \phi d \rho d \phi \\
I_{m_{\mathrm{TEM},}, n_{\mathrm{TE}}}^{(1,4)(b)} & =\int_{\rho} \int_{\phi} \cos \left(k_{y, n}^{(4)}\left(d_{y}+\rho \sin \phi\right)\right) S_{n}^{(2)}(\rho, \phi) \sin \phi d \rho d \phi
\end{aligned}
$$

Caso $\mathrm{TE}^{z}-\mathrm{TE}^{z}$ del bloque $T_{m n}^{(1,4)}$

$$
\begin{aligned}
I_{m_{\mathrm{TE}}, n_{\mathrm{TE}}}^{(r),(1)} & =\int_{\rho} \int_{\phi} \rho \sin \left(k_{y, n}^{(4)}\left(d_{y}+\rho \sin \phi\right)\right) s_{r}(\rho) S_{n}^{(1)}(\rho, \phi) \Phi_{(\zeta), s}(\phi) \sin \phi d \rho d \phi \\
I_{m_{\mathrm{TE}}, n_{\mathrm{TE}}}^{(r),(2)} & =\int_{\rho} \int_{\phi} \sin \left(k_{y, n}^{(4)}\left(d_{y}+\rho \sin \phi\right)\right) c_{r}(\rho) S_{n}^{(1)}(\rho, \phi) \Phi_{(\zeta), s}^{\prime}(\phi) \cos \phi d \rho d \phi \\
I_{m_{\mathrm{TE}}, n_{\mathrm{TE}}}^{(r),(3)} & =\int_{\rho} \int_{\phi} \rho \cos \left(k_{y, n}^{(4)}\left(d_{y}+\rho \sin \phi\right)\right) s_{r}(\rho) S_{n}^{(2)}(\rho, \phi) \Phi_{(\zeta), s}(\phi) \cos \phi d \rho d \phi \\
I_{m_{\mathrm{TE}}, n_{\mathrm{TE}}}^{(r),(4)} & =\int_{\rho} \int_{\phi} \cos \left(k_{y, n}^{(4)}\left(d_{y}+\rho \sin \phi\right)\right) c_{r}(\rho) S_{n}^{(2)}(\rho, \phi) \Phi_{(\zeta), s}^{\prime}(\phi) \sin \phi d \rho d \phi
\end{aligned}
$$

Caso $\mathrm{TM}^{z}-\mathrm{TE}^{z}$ del bloque $T_{m n}^{(1,4)}$

$$
\begin{aligned}
I_{m_{\mathrm{TM}}, n_{\mathrm{TE}}}^{(r),(1)} & =\int_{\rho} \int_{\phi} \sin \left(k_{y, n}^{(4)}\left(d_{y}+\rho \sin \phi\right)\right) s_{r}(\rho) S_{n}^{(1)}(\rho, \phi) \Phi_{(\zeta), s}^{\prime}(\phi) \sin \phi d \rho d \phi \\
I_{m_{\mathrm{TM}}, n_{\mathrm{TE}}}^{(r),(2)} & =\int_{\rho} \int_{\phi} \rho \sin \left(k_{y, n}^{(4)}\left(d_{y}+\rho \sin \phi\right)\right) c_{r}(\rho) S_{n}^{(1)}(\rho, \phi) \Phi_{(\zeta), s}(\phi) \cos \phi d \rho d \phi
\end{aligned}
$$




$$
\begin{aligned}
I_{m_{\mathrm{TM}}, n_{\mathrm{TE}}}^{(r),(3)} & =\int_{\rho} \int_{\phi} \cos \left(k_{y, n}^{(4)}\left(d_{y}+\rho \sin \phi\right)\right) s_{r}(\rho) S_{n}^{(2)}(\rho, \phi) \Phi_{(\zeta), s}^{\prime}(\phi) \cos \phi d \rho d \phi \\
I_{m_{\mathrm{TM}}, n_{\mathrm{TE}}}^{(r),(4)} & =\int_{\rho} \int_{\phi} \rho \cos \left(k_{y, n}^{(4)}\left(d_{y}+\rho \sin \phi\right)\right) c_{r}(\rho) S_{n}^{(2)}(\rho, \phi) \Phi_{(\zeta), s}(\phi) \sin \phi d \rho d \phi
\end{aligned}
$$

Caso $\mathrm{TEM}^{z}-\mathrm{TM}^{z}$ del bloque $T_{m n}^{(1,4)}$

$$
I_{m_{\mathrm{TEM},}, n_{\mathrm{TM}}}=\int_{\rho} \int_{\phi} \sin \left(k_{y, n}^{(4)}\left(d_{y}+\rho \sin \phi\right)\right) \cosh \left(k_{t, n}^{(4)}\left(d_{x}+\rho \cos \phi\right)\right) \cos \phi d \rho d \phi
$$

$\underline{\text { Caso } \mathrm{TE}^{z}-\mathrm{TM}^{z} \text { del bloque } T_{m n}^{(1,4)}}$

$$
\begin{array}{r}
I_{m_{\mathrm{TE}}, n_{\mathrm{TM}}}^{(r),(1)}=\int_{\rho} \int_{\phi} \rho \sin \left(k_{y, n}^{(4)}\left(d_{y}+\rho \sin \phi\right)\right) s_{r}(\rho) \cosh \left(k_{t, n}^{(4)}\left(d_{x}+\rho \cos \phi\right)\right) \Phi_{(\zeta), s}(\phi) \sin \phi d \rho d \phi \\
(\mathrm{G} .52) \\
I_{m_{\mathrm{TE}}, n_{\mathrm{TM}}}^{(r),(2)}=\int_{\rho} \int_{\phi} \sin \left(k_{y, n}^{(4)}\left(d_{y}+\rho \sin \phi\right)\right) c_{r}(\rho) \cosh \left(k_{t, n}^{(4)}\left(d_{x}+\rho \cos \phi\right)\right) \Phi_{(\zeta), s}^{\prime}(\phi) \cos \phi d \rho d \phi
\end{array}
$$

Caso $\mathrm{TM}^{z}-\mathrm{TM}^{z}$ del bloque $T_{m n}^{(1,4)}$

$I_{m_{\mathrm{TM}}, n_{\mathrm{TM}}}^{(r),(1)}=\int_{\rho} \int_{\phi} \sin \left(k_{y, n}^{(4)}\left(d_{y}+\rho \sin \phi\right)\right) s_{r}(\rho) \cosh \left(k_{t, n}^{(4)}\left(d_{x}+\rho \cos \phi\right)\right) \Phi_{(\zeta), s}^{\prime}(\phi) \sin \phi d \rho d \phi$

$I_{m_{\mathrm{TM}}, n_{\mathrm{TM}}}^{(r),(2)}=\int_{\rho} \int_{\phi} \rho \sin \left(k_{y, n}^{(4)}\left(d_{y}+\rho \sin \phi\right)\right) c_{r}(\rho) \cosh \left(k_{t, n}^{(4)}\left(d_{x}+\rho \cos \phi\right)\right) \Phi_{(\zeta), s}(\phi) \cos \phi d \rho d \phi$

En todas estas expresiones, $s_{r}(\rho)$ y $c_{r}(\rho)$ representan las funciones sinusoidales definidas en (4.102) y (4.103), respectivamente. Además, la función $S_{n}(\rho, \phi)$ se encuentra definida en (4.41); mientras que la expresión para las funciones $S_{n}^{(\alpha)}(\rho, \phi)(\alpha=1,2,3,4)$ puede encontrarse en (4.32), (4.33), (4.151) y (4.152), respectivamente. Finalmente, la función $\Phi_{(\zeta), s}(\phi)$ se ha definido en $(2.71)$, y $\Phi_{(\zeta), s}^{\prime}(\phi)$ representa su derivada con respecto a la variable angular $\phi$. 


\section{Apéndice H}

\section{Publicaciones realizadas relacionadas con la Tesis Doctoral}

A continuación, se enumeran las publicaciones realizadas relacionadas con la presente tesis doctoral.

\section{Publicaciones realizadas en revistas internacionales}

A. A. San Blas, F. Mira, V. E. Boria, B. Gimeno, M. Bressan and P. Arcioni, "On the fast and rigorous analysis of compensated waveguide junctions using off-centered partial-height metallic posts", IEEE Transactions on Microwave Theory and Techniques, vol. 55, no. 1, pp. 168-175, January 2007.

S. Cogollos, V. E. Boria, P. Soto, A. A. San Blas, B. Gimeno and M. Guglielmi, "Direct computation of the admittance parameters of a cubic junction with arbitrarily shaped access ports using the BI-RME method", Proc. Inst. Elect. Eng. - Microwave, Antennas, Propagation, vol. 150, no. 2, pp. 111-119, April 2003.

\section{Publicaciones realizadas en congresos internacionales}

F. Mira, A. A. San Blas, V. E. Boria and B. Gimeno, "Fast and accurate analysis and design of substrate integrated waveguide (SIW) filters", Proceedings of the 37th European Microwave Conference, Munich, Germany, September 2007, pp. 170-173.

A. A. San Blas, F. Mira, V. E. Boria, B. Gimeno, G. Conciauro, M. Bressan and P. Arcioni, "Efficient $\mathrm{CAD}$ of generalized coaxial probes in rectangular waveguide using the 3D BI-RME method", Proceedings of the 36th European Microwave Conference, Manchester, United Kingdom, September 2006, pp. 1163-1166. 
F. Mira, A. A. San Blas, V. E. Boria, B. Gimeno, M. Bressan and G. Conciauro, "Fast and accurate $\mathrm{CAD}$ tool of passive rectangular waveguide devices with cylindrical conducting posts", Proceedings of the 35th European Microwave Conference, Paris, France, September 2005, pp. 939-942.

A. A. San Blas, F. Mira, V. E. Boria, B. Gimeno, M. Bressan, G. Conciauro and P. Arcioni, "Efficient CAD of optimal multi-port junctions loaded with partial-height cylindrical posts using the 3D BI-RME method", 2005 IEEE MTT-S International Microwave Symposium Digest, Long Beach, United States, June 2005, pp. 67-70.

A. A. San Blas, A. Coves, M. Bressan, V. E. Boria, B. Gimeno and J. V. Morro, "Full-wave analysis of coaxial probes in rectangular waveguide: preliminary results for metal-loaded cavities and coaxial to rectangular waveguide transitions", 2004 IEEE AP-S International Symposium Digest, Monterrey, United States, June 2004, vol. 2, pp. 2143-2146.

A. A. San Blas, F. Mira, A. Coves, M. Taroncher, V. E. Boria, B. Gimeno and M. Bressan, "Fast calculation of the Green's functions of a boxed cavity resonator by means of Ewald's technique", Book of Abstracts of ISMOT 2003 (International Symposium on Microwave and Optical Technology), Ostrava, Czech Republic, August 2003, pp. 20.

\section{Publicaciones realizadas en congresos nacionales}

A. A. San Blas, F. Mira, V. E. Boria, B. Gimeno y M. Bressan, "Excitación coaxial generalizada de dispositivos pasivos de microondas usando el método BI-RME 3D", enviado al XXIII Simposium Nacional de la Unión Científica Internacional de Radio (URSI), Madrid, Septiembre 2008 (pendiente de aceptación).

F. Mira, A. A. San Blas, V. E. Boria, B. Gimeno, S. Cogollos, M. Bressan y L. Perregrini, "Desarrollo eficiente en serie de polos de la matriz generalizada de impedancias para uniones planares", Libro de Actas del XXI Simposium Nacional de la Unión Científica Internacional de Radio (URSI), Oviedo, Septiembre 2006, pp. 828-831.

F. Mira, A. A. San Blas, V. E. Boria, B. Gimeno, M. Bressan y G. Conciauro, "Herramienta de diseño eficiente y preciso de dispositivos pasivos de microondas con postes conductores", Libro de Actas del XX Simposium Nacional de la Unión Científica Internacional de Radio (URSI), Gandía, Septiembre 2005, pp. 437-440.

A. A. San Blas, F. Mira, V. E. Boria, B. Gimeno, A. Coves y M. Bressan, "Análisis eficiente de estructuras compensadas en guía rectangular: aplicación al diseño optimizado de divisores de potencia", Libro de Actas del XX Simposium Nacional de la Unión Científica Internacional de Radio (URSI), Gandía, Septiembre 2005, pp. 117-120. 
A. A. San Blas, A. Coves, J. V. Morro, V. E. Boria, S. Marini, B. Gimeno y J. Gil, "Análisis multimodal eficiente de uniones planares entre guías arbitrarias: aplicación a la excitación coaxial de dispositivos", Libro de Actas del XIX Simposium Nacional de la Unión Científica Internacional de Radio (URSI), Barcelona, Septiembre 2004, pp. 53.

A. A. San Blas, A. Coves, M. Bressan, G. Conciauro, V. E. Boria, J. V. Morro y B. Gimeno, "Cálculo acelerado de las funciones de Green de una cavidad rectangular para su aplicación a la excitación coaxial de dispositivos en guía”, Libro de Actas del XVIII Simposium Nacional de la Unión Científica Internacional de Radio (URSI), La Coruña, Septiembre 2003, pp. 105. 


\section{Bibliografía}

[1] T. Itoh, Numerical Techniques for Microwave and Millimeter-Wave Passive Structures, Wiley, New York, 1989.

[2] E. Sharp, "An exact calculation for a T-junction of rectangular waveguides having arbitrary cross sections", IEEE Transactions on Microwave Theory and Techniques, vol. 15, no. 2, pp. 109-116, February 1967.

[3] A. Alvarez, G. Connor, and M. Guglielmi, "New simple procedure for the computation of the multimode admittance or impedance matrix of planar waveguide junctions", IEEE Transactions on Microwave Theory and Techniques, vol. 44, no. 3, pp. 413-418, March 1996.

[4] P. Silvester, "A general high-order finite-element waveguide analysis program", IEEE Transactions on Microwave Theory and Techniques, vol. 17, no. 4, pp. 204-210, April 1969.

[5] M. Guglielmi, G. Gheri, M. Calamia, and G. Pelosi, "Rigorous multimode network numerical representation of inductive step", IEEE Transactions on Microwave Theory and Techniques, vol. 42, no. 2, pp. 317-325, February 1994.

[6] A. Alvarez and M. Guglielmi, "Multimode network representation of two dimensional steps in rectangular waveguides", in Proceedings of the 24th European Microwave Conference, Cannes, France, September 1994, pp. 1943-1948.

[7] G. Gerini, M. Guglielmi, and G. Lastoria, "Efficient integral equation formulations for the computation of the multimode admittance or impedance matrix of planar waveguide junctions", in IEEE MTT-S International Microwave Symposium Digest, Baltimore (MD), United States, June 1998, vol. 3, pp. 1747-1750.

[8] X. Liang, K. A. Zaki, and A. E. Atia, "A rigorous three plane mode-matching technique for characterizing waveguide T-junctions, and its application in multiplexer design”, IEEE Transactions on Microwave Theory and Techniques, vol. 39, no. 12, pp. 2138-2147, December 1991.

[9] T. Sieverding and F. Arndt, "Modal analysis of the magic tee", IEEE Microwave and Guided Wave Letters, vol. 3, no. 5, pp. 150-152, May 1993. 
[10] W. Wessel, T. Sieverding, and F. Arndt, "Mode-matching analysis of general waveguide multiport junctions", in IEEE MTT-S International Microwave Symposium Digest, Anaheim (CA), United States, June 1999, pp. 1273-1276.

[11] M. Bozzi, M. Bressan, and L. Perregrini, "Generalized Y-matrix of arbitrary 3D waveguide junctions by the BI-RME method", in IEEE MTT-S International Microwave Symposium Digest, Anaheim (CA), United States, June 1999, pp. 1269-1272.

[12] P. Arcioni, M. Bozzi, M. Bressan, G. Conciauro, and L. Perregrini, "Frequency/timedomain modeling of 3D waveguide structures by a BI-RME approach", Int. Journal of Numerical Modelling: Electronic Networks, Devices and Fields, vol. 15, pp. 3-21, 2002.

[13] A. G. Williamson and D. V. Otto, "Cilyndrical antenna in a rectangular waveguide driven from a coaxial line", Electronics Letters, vol. 8, no. 22, pp. 545-547, November 1972.

[14] Y. Leviatan, P. G. Li, A. T. Adams, and J. Perini, "Single-post inductive obstacle in rectangular waveguide", IEEE Transactions on Microwave Theory and Techniques, vol. 31, no. 10, pp. 806-811, October 1983.

[15] R. B. Keam and A. G. Williamson, "Broadband design of a coaxial line /rectangular waveguide probe transition", IEE Proceedings - Microwaves, Antennas and Propagation, vol. 141, no. 1, pp. 53-58, February 1994.

[16] M. E. Bialkowski, "Analysis of a coaxial-to-waveguide adaptor including a discended probe and a tuning post", IEEE Transactions on Microwave Theory and Techniques, vol. 43, no. 2, pp. 344-349, February 1995.

[17] J. F. Liang, H. C. Chang, and K. A. Zaki, "Coaxial probe modeling in waveguides and cavities", IEEE Transactions on Microwave Theory and Techniques, vol. 40, no. 12, pp. 2172-2180, December 1992.

[18] H. W. Yao and K. A. Zaki, "Modeling of generalized coaxial probes in rectangular waveguides", IEEE Transactions on Microwave Theory and Techniques, vol. 43, no. 12, pp. 2805-2811, December 1995.

[19] R. Bunger and F. Arndt, "Moment-method analysis of arbitrary 3D metallic $N$-port waveguide structures", IEEE Transactions on Microwave Theory and Techniques, vol. 48, no. 4, pp. 531-537, April 2000.

[20] J. Uher, J. Bornemann, and U. Rosenberg, Waveguide Components for Antenna Feed Systems. Theory and CAD, Artech House, Norwood, 1993.

[21] G. Conciauro, M. Guglielmi, and R. Sorrentino, Advanced Modal Analysis - CAD Techniques for Waveguides Components and Filters, Wiley, Chichester, 2000. 
[22] R. R. Mansour and R. H. Macphie, "An improved transmission matrix formulation of cascaded discontinuities and its application to E-plane circuits", IEEE Transactions on Microwave Theory and Techniques, vol. 34, no. 12, pp. 1490-1498, December 1986.

[23] F. Alessandri, M. Mongiardo, and R. Sorrentino, "Computer-aided design of beam forming networks for modern satellite antennas", IEEE Transactions on Microwave Theory and Techniques, vol. 40, no. 6, pp. 1117-1127, June 1992.

[24] M. Guglielmi, "Simple CAD procedure for microwave filters and multiplexers", IEEE Transactions on Microwave Theory and Techniques, vol. 42, no. 7, pp. 1347-1352, July 1994.

[25] V. E. Boria, M. Bozzi, D. Camilleri, A. Coves, H. Esteban, B. Gimeno, M. Guglielmi, and L. Polini, "Contributions to the analysis and design of all-inductive filters with dielectric resonators", in Proceedings of the 33rd European Microwave Conference, Munich, Germany, September 2003, pp. 1247-1250.

[26] F. Arndt and U. Papziner, "Modal S-matrix design of microwave filters composed of rectangular and circular waveguide elements", in IEEE MTT-S International Microwave Symposium Digest, Boston (MA), United States, June 1991, pp. 535-538.

[27] K. Wu, "An optimal circular-waveguide dual-mode filter without tuning screws", IEEE Transactions on Microwave Theory and Techniques, vol. 47, no. 3, pp. 271276, March 1999.

[28] N. Marcuvitz, Waveguide Handbook, Peter Peregrinus Ltd., London, 1986.

[29] A. Wexler, "Solution of waveguide discontinuities by modal analysis", IEEE Transactions on Microwave Theory and Techniques, vol. 15, pp. 508-517, September 1967.

[30] M. Leroy, "On the convergence of numerical results in modal analysis", IEEE Transactions on Antennas and Propagation, vol. 31, pp. 655-659, July 1983.

[31] R. Sorrentino, M. Mongiardo, F. Alessandri, and G. Schiavon, "An investigation of the numerical properties of the mode-matching technique", International Journal of Numerical Modelling, vol. 4, pp. 19-43, April 1991.

[32] B. Gimeno and M. Guglielmi, "Multimode equivalent network representation for $\mathrm{H}$ - and E-plane uniform bends in rectangular waveguide", IEEE Transactions on Microwave Theory and Techniques, vol. 44, no. 10, pp. 1679-1687, October 1996.

[33] K. S. Yee, "Numerical solution of initial boundary value problems involving Maxwell's equations in isotropic media", IEEE Transactions on Antennas and Propagation, vol. 14, no. 3, pp. 302-307, May 1966. 
[34] M. Guglielmi and G. Gheri, "Rigorous multimode network representation of capacitive steps", IEEE Transactions on Microwave Theory and Techniques, vol. 42, no. 4, pp. 622-628, April 1994.

[35] R. E. Collin, Field Theory of Guided Waves, IEEE Press, New York, 1991.

[36] B. Gimeno and M. Guglielmi, "Multimode equivalent network representation for junctions between coaxial and circular waveguides", International Journal of Microwave and Millimeter-Wave Computer-Aided Engineering, vol. 7, no. 2, pp. 180194, March 1997.

[37] R. E. Collin, Foundations for Microwave Engineering, McGraw-Hill, New York, 1992.

[38] R. F. Harrington, Time-Harmonic Electromagnetic Fields, McGraw Hill, New York, 1961.

[39] D. M. Pozar, Microwave Engineering, Wiley, New York, 1997.

[40] L. Jackson Laslett and W. Lewish, "Evaluation of the zeros of cross-product Bessel functions", Mathematics of Computation, vol. 16, no. 78, pp. 226-232, April 1962.

[41] G. G. Gentili, "Properties of TE-TM mode-matching techniques", IEEE Transactions on Microwave Theory and Techniques, vol. 39, no. 9, pp. 1669-1673, September 1991.

[42] G. Figlia and G. G. Gentili, "On the line-integral formulation of mode-matching technique", IEEE Transactions on Microwave Theory and Techniques, vol. 50, no. 2, pp. 578-580, February 2002.

[43] M. Bozzi, G. Conciauro, and L. Perregrini, "On the evaluation of modal coupling coefficients by contour integrals", IEEE Transactions on Microwave Theory and Techniques, vol. 50, no. 7, pp. 1853-1855, July 2002.

[44] M. Abramowitz and I. A. Stegun, Handbook of Mathematical Functions with Formulas, Graphs, and Mathematical Tables, Dover, New York, 1964.

[45] R. Vahldieck, J. Bornemann, F. Arndt, and D. Grauerholz, "Optmized waveguide Eplane metal insert filters for millimeter-wave applications", IEEE Transactions on Microwave Theory and Techniques, vol. 31, no. 1, pp. 65-69, January 1983.

[46] Y. C. Shih, "Design of waveguide E-plane filters with all-metal inserts", IEEE Transactions on Microwave Theory and Techniques, vol. 32, no. 7, pp. 695-704, July 1984.

[47] V. Postoyalko and D. S. Budimir, "Design of waveguide E-plane filters with all-metal inserts by equal ripple optimization", IEEE Transactions on Microwave Theory and Techniques, vol. 42, no. 2, pp. 217-222, February 1994. 
[48] Ángel A. San Blas Oltra, Caracterización de Codos Circulares en Guía Rectangular bajo Excitación Multimodal, Proyecto Fin de Carrera, Universidad Politécnica de Valencia, Noviembre 2000.

[49] V. E. Boria, G. Gerini, and M. Guglielmi, "An efficient inversion technique for banded linear systems", in IEEE MTT-S International Microwave Symposium Digest, Denver (CO), United States, June 1997, pp. 1567-1570.

[50] Y. Rong, H. Yao, K. A. Zaki, and T. G. Dolan, "Millimeter-wave $K a$-band $H$-plane diplexers and multiplexers", IEEE Transactions on Microwave Theory and Techniques, vol. 47, no. 12, pp. 2325-2330, December 1999.

[51] F. Alessandri, M. Mongiardo, and R. Sorrentino, "A technique for the fullwave automatic synthesis of waveguide components: application to fixed phase shifters", IEEE Transactions on Microwave Theory and Techniques, vol. 40, no. 7, pp. 1484-1495, July 1992.

[52] V. E. Boria and M. Guglielmi, "Efficient admittance matrix representation of a cubic junction of rectangular waveguides", in IEEE MTT-S International Microwave Symposium Digest, Baltimore (MD), United States, June 1998, pp. 1751-1754.

[53] T. Sieverding and F. Arndt, "Rigorous analysis of the rectangular waveguide six-port cross junction", IEEE Microwave and Guided Wave Letters, vol. 3, no. 7, pp. 224-226, July 1993.

[54] V. E. Boria, S. Cogollos, H. Esteban, M. Guglielmi, and B. Gimeno, "Efficient analysis of a cubic junction of rectangular waveguides using the admittance-matrix representation", Proc. Inst. Elect. Eng. - Microw., Antennas, Propag., vol. 147, no. 6, pp. 417-422, December 2000.

[55] S. Cogollos, V. E. Boria, P. Soto, A. A. San Blas, B. Gimeno, and M. Guglielmi, "Direct computation of the admittance parameters of a cubic junction with arbitrarily shaped access ports using the BI-RME method", Proc. Inst. Elect. Eng. - Microw., Antennas, Propag., vol. 150, no. 2, pp. 111-119, April 2003.

[56] J. Hirokawa, K. Sakurai, M. Ando, and N. Goto, "An analysis of a waveguide Tjunction with an inductive post", IEEE Transactions on Microwave Theory and Techniques, vol. 39, no. 3, pp. 563-566, March 1991.

[57] F. Alessandri, M. Dionigi, and R. Sorrentino, "Rigorous analysis of compensated Eplane junctions in rectangular waveguide", in IEEE MTT-S International Microwave Symposium Digest, Orlando (FL), United States, May 1995, pp. 987-990.

[58] C. Wang and K. A. Zaki, "Full wave modeling of generalized double ridge waveguide T-junctions", IEEE Transactions on Microwave Theory and Techniques, vol. 44, no. 12, pp. 2536-2542, December 1996. 
[59] $\mathrm{K}$. Wu and $\mathrm{H}$. Wang, "A rigorous modal analysis of $H$-plane waveguide T-junction loaded with a partial-height post for wide-band applications", IEEE Transactions on Microwave Theory and Techniques, vol. 49, no. 5, pp. 893-901, May 2001.

[60] J. Ritter and F. Arndt, "Efficient FDTD/matrix-pencil method for the full-wave scattering parameter analysis of waveguiding structures", IEEE Transactions on Microwave Theory and Techniques, vol. 44, no. 12, pp. 2450-2456, December 1996.

[61] A. A. San Blas, F. Mira, V. E. Boria, B. Gimeno, M. Bressan, G. Conciauro, and P. Arcioni, "Efficient CAD of optimal multi-port junctions loaded with partial-height cylindrical posts using the 3D BI-RME method", in IEEE MTT-S International Microwave Symposium Digest, Long Beach (CA), United States, June 2005, pp. 67-70.

[62] A. A. San Blas, F. Mira, V. E. Boria, B. Gimeno, M. Bressan, and P. Arcioni, "On the fast and rigorous analysis of compensated waveguide junctions using off-centered partial-height metallic posts", IEEE Transactions on Microwave Theory and Techniques, vol. 55, no. 1, pp. 168-175, January 2007.

[63] P. Arcioni, M. Bressan, and L. Perregrini, "A new boundary integral approach to the determination of the resonant modes of arbitrarily shaped cavities", IEEE Transactions on Microwave Theory and Techniques, vol. 43, no. 8, pp. 1848-1856, August 1995.

[64] P. P. Ewald, "Die berechnung optischer und electroststischer gitterpotentiale", Ann. der Physik, vol. 64, 1921.

[65] M. Bressan, L. Perregrini, and E. Regini, "BI-RME modeling of 3D waveguide components enhanced by the Ewald technique", in IEEE MTT-S International Microwave Symposium Digest, Boston (MA), United States, June 2000, pp. 1097-1100.

[66] Fermín E. Mira Pérez, Análisis Modal Eficiente de Guías de Onda Rectangulares con Postes Conductores, Tesis Doctoral, Universidad Politécnica de Valencia, Marzo 2005 .

[67] F. Mira, M. Bressan, G. Conciauro, B. Gimeno, and V. E. Boria, "Fast $S$-domain modeling of rectangular waveguides with radially symmetric metal insets", IEEE Transactions on Microwave Theory and Techniques, vol. 53, no. 4, pp. 1294-1303, April 2005.

[68] S. M. Rao, D. R. Wilton, and A. W. Glisson, "Electromagnetic scattering by surfaces of arbitrary shape", IEEE Transactions on Antennas and Propagation, vol. 30, pp. 409-418, May 1982.

[69] F. Arndt, I. Ahrens, U. Papziner, U. Wiechmann, and R. Wilkeit, "Optimized Eplane T-junction series power dividers", IEEE Transactions on Microwave Theory and Techniques, vol. 35, no. 11, pp. 1052-1059, November 1987. 
[70] M. A. Meyer and H. B. Goldberg, "Applications of the turnstile junction", IEEE Transactions on Microwave Theory and Techniques, pp. 40-45, December 1955.

[71] A. Navarrini and R. L. Plambeck, "A turnstile junction waveguide orthomode transducer", IEEE Transactions on Microwave Theory and Techniques, vol. 54, no. 1, pp. 272-277, January 2006.

[72] B. A. Auld, "The synthesis of symmetrical waveguide circulators", IEEE Transactions on Microwave Theory and Techniques, pp. 238-246, April 1959.

[73] M. El Sabbagh, K. A. Zaki, H. W. Yao, and M. Yu, "Full-wave analysis of coupling between combline resonators and its application to combline filters with canonical configurations", IEEE Transactions on Microwave Theory and Techniques, vol. 49, no. 12, pp. 2384-2393, December 2001.

[74] J. A. Ruiz-Cruz, K. A. Zaki, J. R. Montejo-Garai, and J. M. Rebollar, "Rectangular waveguide elliptic filters with capacitive and inductive irises and integrated coaxial excitation", in IEEE MTT-S International Microwave Symposium Digest, Long Beach (CA), United States, June 2005, pp. 269-272.

[75] A. Morini, G. Venanzoni, and T. Rozzi, "A new adaptive prototype for the design of side-coupled coaxial filters with close correspondence to the physical structure", IEEE Transactions on Microwave Theory and Techniques, vol. 54, no. 3, pp. 11461153, March 2006.

[76] V. E. Boria, G. Gerini, M. Guglielmi, and P. Arcioni, "Computer aided design of reentrant coaxial filters including coaxial excitation", in IEEE MTT-S International Microwave Symposium Digest, Anaheim (CA), United States, June 1999, vol. 3, pp. 1131-1134.

[77] G. Gerini, F. Diaz, and M. Guglielmi, "Triple mode filters with coaxial excitation", in IEEE MTT-S International Microwave Symposium Digest, Boston (MA), United States, June 2000, vol. 3, pp. 1763-1766.

[78] A. Morini, G. Venanzoni, M. Farina, and T. Rozzi, "Modified adaptive prototype inclusive of the external couplings for the design of coaxial filters", IEEE Transactions on Microwave Theory and Techniques, vol. 55, no. 9, pp. 1905-1911, September 2007.

[79] Y. Leviatan, D. H. Shau, and A. T. Adams, "Numerical study of the current distribution on a post in a rectangular waveguide", IEEE Transactions on Microwave Theory and Techniques, vol. 32, no. 10, pp. 1411-1415, October 1984.

[80] J. M. Jarem, "A multifilament method-of-moments solution for the input impedance of a probe-excited semi-infinite waveguide", IEEE Transactions on Microwave Theory and Techniques, vol. 35, no. 1, pp. 14-19, January 1987. 
[81] J. M. Jarem, "A method-of-moments analysis and a finite-difference time-domain analysis of a probe-sleeve fed rectangular waveguide cavity", IEEE Transactions on Microwave Theory and Techniques, vol. 39, no. 3, pp. 444-451, March 1991.

[82] M. E. Bialkowski, "Analysis of a coaxial-to-waveguide adaptor incorporating a dielectric coated probe", IEEE Microwave Guided Wave Letters, vol. 1, no. 8, pp. 211-214, August 1991.

[83] J. H. Lee, H. J. Eom, and K. H. Jun, "Reflection of a coaxial line radiating into a parallel plate", IEEE Microwave Guided Wave Letters, vol. 6, no. 3, pp. 135-137, March 1996.

[84] M. S. Leong, L. W. Li, P. S. Kooi, T. S. Yeo, and S. L. Ho, "Input impedance of a coaxial probe located inside a rectangular cavity: Theory and experiment", IEEE Transactions on Microwave Theory and Techniques, vol. 44, no. 7, pp. 1161-1164, July 1996.

[85] L. W. Li, P. S. Kooi, M. S. Leong, T. S. Yeo, and S. L. Ho, "Input impedance of a probe-excited semi-infinite rectangular waveguide with arbitrary multilayered loads: Part I - dyadic Green's functions", IEEE Transactions on Microwave Theory and Techniques, vol. 43, no. 7, pp. 1559-1566, July 1995.

[86] L. W. Li, P. S. Kooi, M. S. Leong, T. S. Yeo, and S. L. Ho, "Input impedance of a probe-excited semi-infinite rectangular waveguide with arbitrary multilayered loads: Part II - A full-wave analysis", IEEE Transactions on Microwave Theory and Techniques, vol. 45, no. 3, pp. 321-329, March 1997.

[87] K. W. Leung, Z. N. Chen, K. M. Luk, and E. K. N. Yung, "On the probe-fed dielectric resonator inside the parallel-plate waveguide", IEEE Transactions on Microwave Theory and Techniques, vol. 47, no. 7, pp. 1113-1117, July 1999.

[88] M. E. Bialkowski, "On the link between top-hat monopole antennas, disk-resonator diode mounts, and coaxial-to-waveguide transitions", IEEE Transactions on Antennas and Propagation, vol. 48, no. 6, pp. 1011-1013, June 2000.

[89] Z. N. Chen, K. Hirasawa, and K. Wu, "A broad-band sleeve monopole integrated into a parallel-plate waveguide", IEEE Transactions on Microwave Theory and Techniques, vol. 48, no. 7, pp. 1160-1163, July 2000.

[90] S. M. Saad, "A more accurate analysis and design of coaxial-to-rectangular waveguide end launcher", IEEE Transactions on Microwave Theory and Techniques, vol. 38, no. 2, pp. 129-134, February 1990.

[91] W. W. S. Lee and E. K. N. Yung, "The input impedance of a coaxial line fed probe in a cilyndrical waveguide", IEEE Transactions on Microwave Theory and Techniques, vol. 42, no. 8, pp. 1468-1473, August 1994. 
[92] E. K. N. Yung, Z. M. Xie, and R. S. Chen, "Input impedance of a coaxial-line fed probe in a thick coaxial-line waveguide", IEEE Transactions on Microwave Theory and Techniques, vol. 48, no. 10, pp. 1707-1711, October 2000.

[93] G. Gerini and M. Guglielmi, "Full-wave CAD of a rectangular waveguide filter with integrated coaxial excitation", IEEE Transactions on Microwave Theory and Techniques, vol. 49, no. 5, pp. 986-990, May 2001.

[94] J. Van Bladel, Singular Electromagnetic Fields and Sources, Clarendon Press, Oxford, 1991.

[95] P. Arcioni, M. Bressan, and L. Perregrini, "On the evaluation of the double surface integrals arising in the application of the boundary integral method to 3-D problems", IEEE Transactions on Microwave Theory and Techniques, vol. 45, no. 3, pp. 436-439, March 1997.

[96] Chen-To Tai, General Vector and Dyadic Analysis: Applied Mathematics in Field Theory, IEEE Press, New York, 1997.

[97] H. W. Yao, K. A. Zaki, A. E. Atia, and R. Hershtig, "Full wave modeling of conducting posts in rectangular waveguides and its applications to slot coupled combline filters", IEEE Transactions on Microwave Theory and Techniques, vol. 43, no. 12, pp. 2824 2830, December 1995.

[98] G. L. Matthaei, "Comb-line band-pass filters of narrow or moderate bandwidth", Microwave Journal, vol. 6, pp. 82-91, August 1963.

[99] R. Levy and J. D. Rhodes, "A comb-line elliptic filter", IEEE Transactions on Microwave Theory and Techniques, vol. 19, pp. 26-29, January 1971.

[100] C. Wang, K. A. Zaki, A. E. Atia, and T. G. Dolan, "Dielectric combline resonators and filters", IEEE Transactions on Microwave Theory and Techniques, vol. 46, no. 12, pp. 2501-2506, December 1998. 